\title{
Sandia Combustion Research
}

SAND $87-8502$

\section{When printing a copy of any digitized SAND Report, you are required to update the markings to current standards.}

Annual Report 1986 
Printed in the United States of America Available from

National Technical Information Service

5285 Port Royal Road

Springfield, VA 22161

NTIS price codes

Printed copy: A11

Microfiche copy: A01 


\section{Sandia National Laboratories}

Livermore, California 94550

date: September 21, 1987

to: Distribution

from: P. L. Mattern, 8300

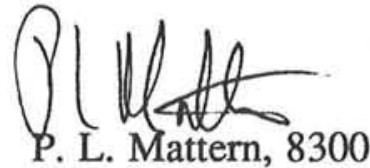

6314 J. R. Tillerson

subject: CRF Annual Report

This year's Annual Report should be a source of great pleasure and pride to all Sandians, and most particularly to you who shared in its preparation. Authors, editors, secretaries, tech artists, layouters, composers, proofreaders, interfacerswith-printers, all the rest of you who contributed, stand and take several bows. This is a truly professional, first-rate production demonstrating clearly the depth, breadth, and quality of the accomplishments of our scientific staff. Personally, I believe this Report sets a new Sandia standard of excellence in technical publication.

But we won't stand pat. The next issue will build on this year's graphic themes, establishing a thread of continuity into the future. (There are even non-accidental links to our sister publication CRF News!) Moreover, Pat Smith and her group are striving to take increasing advantage of the economies of production and improvements in design offered in the latest generation of "desktop publishing" hardware and software. Your ideas and comments are welcome!

Looking (maybe not so) far ahead, it seems to me that the right kind of communication with the outside world is essential to the future of our laboratory. You have established a benchmark against which future efforts can be judged. Congratulations! 


\author{
DISTRIBUTION: \\ Phyllis Bullock, Staff Builders \\ 8245 R. J. Kee \\ 8245 A. E. Lutz \\ 8265 All Division Personnel \\ 8301 L. Y. Armijo \\ 8313 L. R. Thorne \\ 8342 All Division Personnel \\ 8343 M. L. Koszykowski \\ 8343 A. M. F. Lau \\ 8350 All Department Personnel \\ 8360 All Department Personnel \\ COPY TO: \\ 1 I. Welber \\ 10 O. E. Jones \\ 30 J. A. Cantwell \\ 100 P.M. Stanford \\ 400 A. N. Blackwell (10) \\ 1000 V. Narayanamurti \\ 1100 F. L. Vook \\ 1120 J. B. Gerardo \\ 1124 A. Owyoung \\ 1124 P. Esherick \\ 1126 A. W. Johnson \\ 1130 G. A. Samara \\ 1150 J. E. Schirber \\ 1151 D. Emin \\ 1152 D. W. Schaefer \\ 1200 J. P. VanDevender \\ 1248 G. M. Loubriel \\ 1400 E. H. Barsis \\ 1412 K. T. Stalker \\ 1500 W. Herrmann \\ 1510 J. W. Nunziato \\ 1511 A. M. Kraynik \\ 1512 J. C. Cummings, Jr. \\ 1513 D. W. Larson \\ 1533 D. J. Chen \\ 1800 R. L. Schwoebel \\ 1811 R. L. Clough \\ 1840 R. J. Eagan \\ 2000 L. K. Anderson \\ 2311 H. D. Pruett \\ 2500 D. B. Hayes \\ 2515 Y. M. Loyola \\ 2531 M. M. Robertson
}




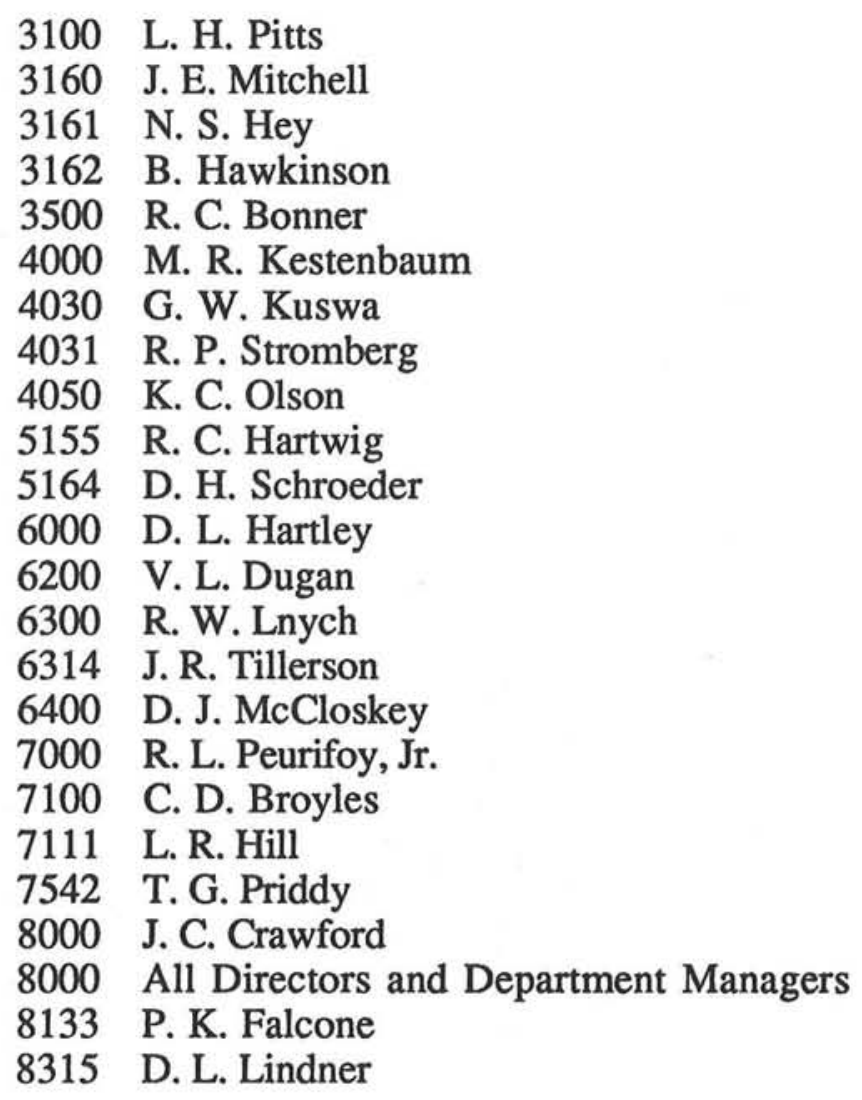


SAND87-8502

UC-96

\section{Sandia Combustion Research Program Annual Report \\ 1986}

Prepared by Sandia National Laboratories, Livermore, California 94550 for the U. S. Department of Energy under Contract DE-AC04-76DP00789 


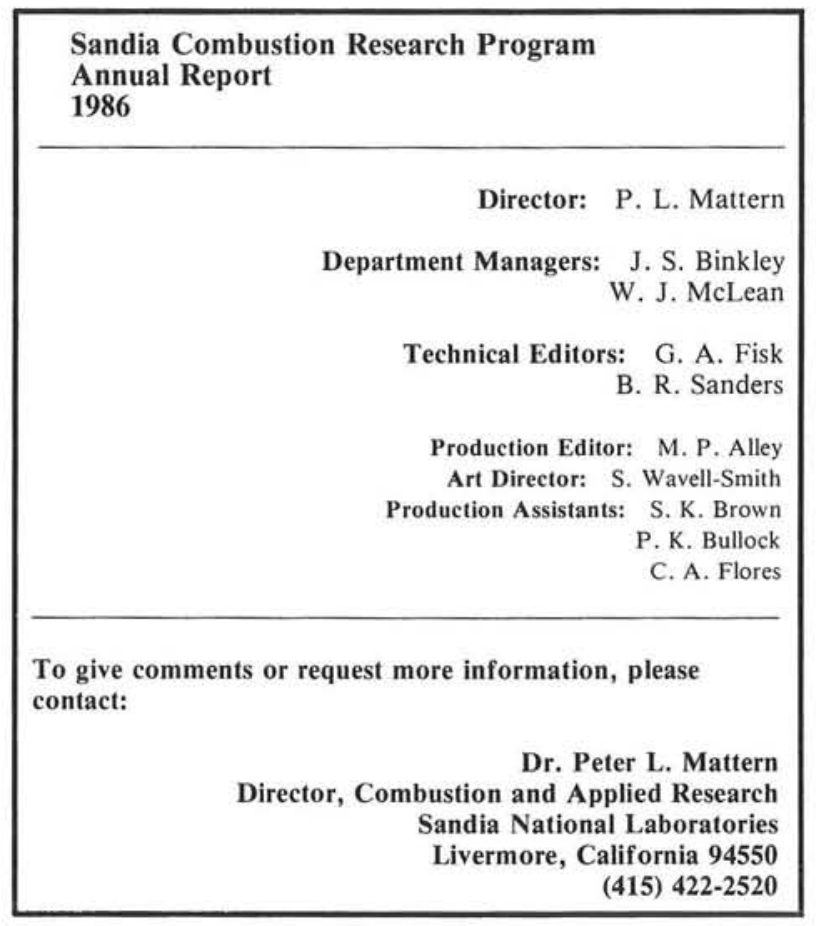




\section{Contents}

Combustion Research at Sandia

P. L. Mattern

\section{Section 1. Facilities and Special Equipment}

Facility Laser System,

R. L. Schmitt

Facility Computers,

D. E. Benthusen

\section{Section 2. Combustion Diagnostics}

High-Resolution CARS Measurements in a Flame,

R. L. Farrow

Measurements of the Nonresonant Third-Order Susceptibilities of Gases

Using CARS, R. L. Farrow, R. P. Lucht, L. A. Rahn

CARS Measurements of Temperature Profiles and Pressure in a

Tungsten Lamp, $\quad$ R. L. Farrow, F. P. Trebino

Three-Laser CARS Measurements of Two Species,

R. P. Lucht, R. E. Palmer, M. A. Maris

Studies of Nitrogen Self-Broadening at High Temperature With Inverse

Raman Spectroscopy,

L. A. Rahn, R. E. Palmer

Comparison of Rotationally Inelastic Collision Models for Q-Branch

Raman Spectra of $\mathrm{N}_{2}$,

L. A. Rahn, R. E. Palmer, M. L. Koszykowski, D. A. Greenhalgh

Collision-Induced Resonances in Four-Wave-Mixing and Induced-Grating

Processes, F. P. Trebino, L. A. Rahn

Photochemical Effects in 205-nm, Two-Photon-Excited Fluorescence

Detection of Atomic Hydrogen in Flames,

J. E. M. Goldsmith

Comparison of Laser-Induced Fluorescence Methods for Measurement of

Hydroxyl Concentration in Flames,

N. M. Laurendeau, J. E. M. Goldsmith

Laser-Induced Fluorescence and Resonance-Enhanced Multiphoton

Ionization of Jet-Cooled Chloronaphthalenes, E. A. Rohlfing, D. W. Chandler 
High Speed Thermometry Using Two-Line Atomic Fluorescence,

J. E. Dec, J. O. Keller

\section{Section 3. Combustion Chemistry}

Rapid Reduction of Nitrogen Oxides in Exhaust Gas Streams,

R. A. Perry, D. L. Siebers

Rotational Excitation of $\mathrm{NH}\left(\mathrm{a}^{1} \Delta\right)$ From Photodissociation of HNCO,

T. Spiglanin, D. W. Chandler

Nitric Oxide-Hydrocarbon Interactions in Low Pressure Flames,

L. R. Thorne, M. C. Branch, D. W. Chandler, R. J. Kee, J. A. Miller

A Theoretical Analysis of the Reaction Between Hydroxyl and Hydrogen

Cyanide at High Temperature, J. A. Miller, C. F. Melius

A Theoretical Analysis of the Overtone-Induced Isomerization of Methyl

Isocyanide, J. A. Miller, D. W. Chandler

Hydrogen-Atom Abstraction From Isobutane by $\mathrm{OH}$,

F. P. Tully, J. E. M. Goldsmith, A. T. Droege

Hydrogen-Atom Abstraction From n-Butane by $\mathrm{OH}$,

A. T. Droege, F. P. Tully

Determination of $\mathrm{H}, \mathrm{OH}$, and $\mathrm{O}$ Species Concentrations by Deuterium

Scavenging,

J. V. Volponi, W. J. McLean, R. Fristrom, Z. Munir

Double-Resonance Laser-Ionization Spectroscopy of Molecular Hydrogen

in the Region of the Second Dissociation Limit,

D. W. Chandler, D. H. Parker, J. O. Buck

\section{Section 4. Reacting Flows}

Velocity Measurements in a Turbulent Nonpremixed Bluff-Body

Stabilized Flame, $\quad$ R. W. Schefer, M. Namazian, J. Kelly

$\mathrm{CH}_{4}$ and $\mathrm{CH}$ Concentration Imaging in a Bluff-Body Stabilized Flame,

M. Namazian, R. W. Schefer, J. Kelly, M. B. Long

Two-Wavelength Single Laser $\mathrm{CH}$ and $\mathrm{CH}_{4}$ Imaging in a Turbulent

Diffusion Flame, M. Namazian, R. L. Schmitt, M. B. Long

Combustion-Torch Ignition: $\mathrm{NO}_{2}$ Fluorescence Imaging,

R. J. Cattolica 
Measurement of Joint Probability of Velocity and Concentration in a

Turbulent Nonpremixed Flame, $\quad$ R. W. Dibble, P. Magre,

R. W. Schefer, J. Y. Chen, V. Hartmann, W. Kollmann

Effects of Finite Chemical Kinetics in Subsonic Nonpremixed

Turbulent Flames in Hydrogen, $\quad$ P. Magre, R. W. Dibble

Soot Production in Axisymmetric Laminar Diffusion Flames at Pressures

From One to Ten Atmospheres, W. L. Flower, C. T. Bowman

Observations on the Soot Formation Mechanism in Laminar Ethylene-Air

Diffusion Flames at One and Two Atmospheres,

W. L. Flower, C. T. Bowman

In Situ Measurement of Flame-Formed Silica Particles Using Dynamic

Light Scattering, W. L. Flower, A. Hurd

Effects of Diffusional Stratification on Soot Formation in a Counterflow

Diffusion Flame, $\quad$ R. L. Axelbaum, W. L. Flower, C. K. Law

Turbulent Nonpremixed Flames of Methane Near Extinction: Probability

Density Functions, A. R. Masri, R.W. Dibble, R. W. Bilger

Combustion of Toxic Liquid Droplets,

R. R. Steeper, N. W. Sorbo, C. K. Law

\section{Section 5. Energetic Materials}

Plasma-Torch Ignition of Liquid Propellant,

R. J. Cattolica, N. Klein

Liquid Propellant Combustion Research,

S. R. Vosen

The Structure of Cyanogen-Nitrogen Dioxide Premixed Flames,

L. R. Thorne, O. I. Smith

HMX Thermal Decomposition by Simultaneous Thermogravimetry, Modulated-Molelcular-Beam Mass Spectrometry, $\quad R$. Behrens

RDX Thermal Decomposition by Simultaneous Thermogravimetry, Modulated-Molecular-Beam Mass Spectrometry, $\quad R$. Behrens

Influences of Two-Phase Flow in the Deflagration of Homogeneous Solids, S. B. Margolis, F. A. Williams, R. C. Armstrong

Percolation Model of Polydisperse Composite Solid Propellant Combustion, A. R. Kerstein 


\section{Section 6. Combustion Modeling}

Vortex Simulation of Unsteady Wrinkled Laminar Flames, W. T. Ashurst

Numerical Simulation of Turbulent Structure with Non-Unity Lewis Number,

W. T. Ashurst, N. Peters, M. D. Smooke

Numerical Study of Vortex Reconnection,

W. T. Ashurst, D. I. Meiron

Simulation of "Tulip" Flame Formation in a Closed Vessel,

P. K. Barr

A Detailed Study of Burning Fuel Droplets,

H. A. Dwyer, B. R. Sanders

Interaction of Pulsating and Spinning Waves in Nonadiabatic Flame

Propagation, $\quad$ M. R. Booty, S. B. Margolis, B. J. Matkowsky

On Nonadiabatic Condensed Phase Combustion,

H. F. Kaper, G. K. Leaf, S. B. Margolis, B. J. Matkowsky

Modeling of Large-Scale Flame Acceleration Experiments,

K. D. Marx

Ignition Modeling,

A. E. Lutz, R. J. Kee, H. A. Dwyer

New Computer Packages for Expanded Chemical Kinetics Modeling

Capabilities R. J.Kee, J. F. Grcar, M. E. Coltrin, J. A. Miller,

M. D. Smooke, G. Dixon-Lewis, J. Warnatz, P. Glarborg

\section{Section 7. High-Temperature Interfaces}

Techniques for Measuring Film Thickness: Interference Effects in Raman

Scattering From Thin, Growing Deposits,

K. F. McCarty, R. J. Anderson

Deposit-Forming Reactions of Pyrite and Sodium in Seeded Flames: An

In Situ Study, J. C. Hamilton, K. F. McCarty

Nonlinear Optical Surface Probe of Adsorption and High-Temperature

Segregation, $\quad$ R. J. Anderson, J. C. Hamilton, G. F. Foltz

Nitrogen CARS Study of a Flame Containing Silica Particles,

M. D. Allendorf, R. P. Lucht, E. Potkay 


\title{
Combustion Research at Sandia
}

\author{
P. L. Mattern
}

More than a decade ago, in response to a national energy crisis, Sandia proposed to the U. S. Department of Energy a new, ambitious program in combustion research. Our strategy was to apply the rapidly increasing capabilities in lasers and computers to combustion science and technology. Shortly thereafter, the Combustion Research Facility was established at Sandia's Livermore location. Designated a "User Facility," the charter of the Facility was to develop and maintain special-purpose resources to support a nationwide initiativeinvolving U. S. universities, industry, and national laboratories - to improve our understanding and control of combustion.

Today, the Combustion Research Facility occupies a unique niche among the Department of Energy "User Facilities." Our resources are diverse and include specialized lasers, computers, and more than two dozen laboratories optimized for the study of combustion-related processes. One of our strongest assets is our resident technical staff, with backgrounds and skills covering a broad spectrum of disciplines in the combustion sciences.

The Office of Basic Energy Sciences within the U. S. Department of Energy (DOE) provides support for the operation of the Facility, for individual and collaborative visits by users, and for fundamental research by Sandia staff in combustion-related chemistry, reacting flows, and advanced (usually laser-based) diagnostics methods. These basic studies are complemented by technology-oriented programs supported by DOE's Offices of Conservation and Fossil Energy. Occasionally, we carry out relevant work in support of the Department of Defense, NASA, and the NRC.

Fundamental to our charter is a commitment to the meaningful involvement of universities and industry at the Facility. One of our primary means of achieving these interactions is our Visiting Scientist Program. As is evident in Figure 2 of Section 10, more than fifty scientists were in residence at the Combustion Research Facility in 1986 for an extended period to accomplish specific research objectives. This number represents the continuation of a steady increase in national - and internationalparticipation at the Facility.

In addition to the Visiting Scientists Program, we are vigorously pursuing other means of establishing closer ties to the academic and industrial communities. One objective is to enlarge substantially the number of post-doctoral researchers and graduate students in residence at the Facility. Newly established seminar series, involving joint participation by Sandians and local universities, are another example of increased interactions with academia.

Our interactions with U.S. industry are highlighted by the health and vitality of several "Working Groups." These are composed of key members of industry, university, and national laboratory staff who meet regularly to discuss progress in research applied to specific combustion-related technologies. The first of these-focused on combustion in direct-injection, stratified charge engines - has just celebrated its 25 th biannual meeting. We believe these Working Groups represent an important model for the effective (and mutual) transfer of technology between national laboratories and U.S. industry.

The body of this Report presents research results of the past year, divided thematically into some ten categories. Publications and presentations arising from this work are included in the appendix. While the preponderance of our activities emphasizes fundamental knowledge, we actively seek to identify "practical" applications of this knowledge. In this regard our highlighted accomplishment of the year is the announcement of the discovery and demonstration of the RAPRENOx process by the inventor Robert A. Perry and his colleague Dennis 
L. Siebers. This new mechanism for the elimination of nitrogen oxides from essentially all kinds of combustion exhausts shows promise for commercialization, and may eventually make a significant contribution to our nation's ability to control smog and acid rain.

What's in the future for the Combustion Research Facility? It should be pointed out that the technology level of the Facility's original major equipment is now nearly two decades old. Shortly, we will be seeking feedback from the user community on our plans for next-generation facility resources. Our current ideas for lasers include a femtosecond source for chemistry studies, and a pulse-burst laser that is optimized for high-speed imaging. To enhance our support of users in computationally intensive research, we are addressing the possibility of adding a Class VI mainframe to our existing computer network.

Our basic research will continue to stress the use of cutting-edge laser technology to probe the chemistry and fluid dynamics of combustion. Research themes will include ultra-high resolution spectroscopies, complemented by dynamics studies extending into the femtosecond regime. Kinetics measurements will press toward more realistic pressures and temperatures, with added emphasis on rich hydrocarbon combustion. A small fraction of our effort will be invested in exploring fundamental scientific issues involved with new (to us) combustion-related activities that span the range from the design of hypersonic flight vehicles to toxic waste management.

Themes for our technology programs will include developing the knowledge base necessary for the effective application of RAPRENOx technology, for the understanding and control of engine knock, and for the computer-based design of advanced pulse combustors. We are actively participating in an attempt to establish a new program of collaborative research involving National Laboratories and universities, intended to help develop the technology base needed for the clean and effective use of coal beyond the year 2000. Finally, we anticipate that some of our research will be of increased benefit to the
Department of Defense, particularly in understanding the fundamentals of solid and liquid propellant combustion.

Hopefully, this Annual Report will spark your interest in work going on at the Combustion Research Facility. Please give me a call or write with questions or comments. I look forward to hearing from you. 


\section{Section 8. Combustion in Engines and Furnaces}

Direct Measurement of the Turbulent Burning Velocity in a Homogeneous-

Charge Engine, $\quad$ P. O. Witze, J. Mendes-Lopes

CARS Measurements of Temperature Profiles Near a Wall in an Internal

Combustion Engine, R. P. Lucht, M. A. Maris

CARS Measurements of Unburned Gas Temperatures in an Internal

Combustion Engine,

R. P. Lucht, R. M. Green, R. E. Palmer, C. Ferguson, R. E. Teets

Autoignition of Methanol and Ethanol Sprays Under Diesel Engine

Particle Seeding for Mie Scattering in Combusting Flows,

Response of a Pulse Combustor to Changes in Fuel Composition,

Systematic Study of the Oscillating Flow in the Tailpipe of a Pulse

Combustor, J. E. Dec, P. K. Barr, J. O. Keller

Mixing of Hot Products and Cold Reactants in a Pulse Combustor,

T. T. Bramlette

Development of Non-Linear Model of Pulse Combustion Processes, P. K. Barr

Two-Dimensional Flame Visualization in an Internal Combustion Engine,

R. M. Green, T. A. Baritaud

\section{Section 9. Coal Combustion}

Experimentally Determined Overall Burning Rates of Coal Chars,

R. E. Mitchell

Experimentally Determined Overall Burning Rates of Pulverized-Coal

Chars in Specified $\mathrm{O}_{2}$ and $\mathrm{CO}_{2}$ Environments,

R. E. Mitchell, O. Madsen

Agglomeration During Combustion of Coal/Water Slurries as a Function

of Coal Rank, D. Dunn-Rankin, J. Hoornstra, T. H. Fletcher, D. J. Holve

Numerical Simulation of Particle Size Distribution Evolution During Pulverization Char Combustion, D. Dunn-Rankin, A. R. Kerstein

Laser Spark Spectroscopy for In Situ Analysis of Particles in Combustion

Flows, D. K. Ottesen 
Determination of Coal Devolatization Rates at High Temperatures and

Evolution of Char Morphology and Mineral Matter Transformations

During the Controlled Combustion of a Bituminous Coal,

D. A. Nissen, F. Greulich

Percolation Model for Simulation of Char Oxidation and Fragmentation

Time-Histories, A. R. Kerstein, B. F. Edwards

\section{Section 10. Technology Transfer}

Cooperative Working Groups,

G. B. Drummond

Visiting Scientist Program,

G. B. Drummond

\section{Appendix}

Publications 1986 


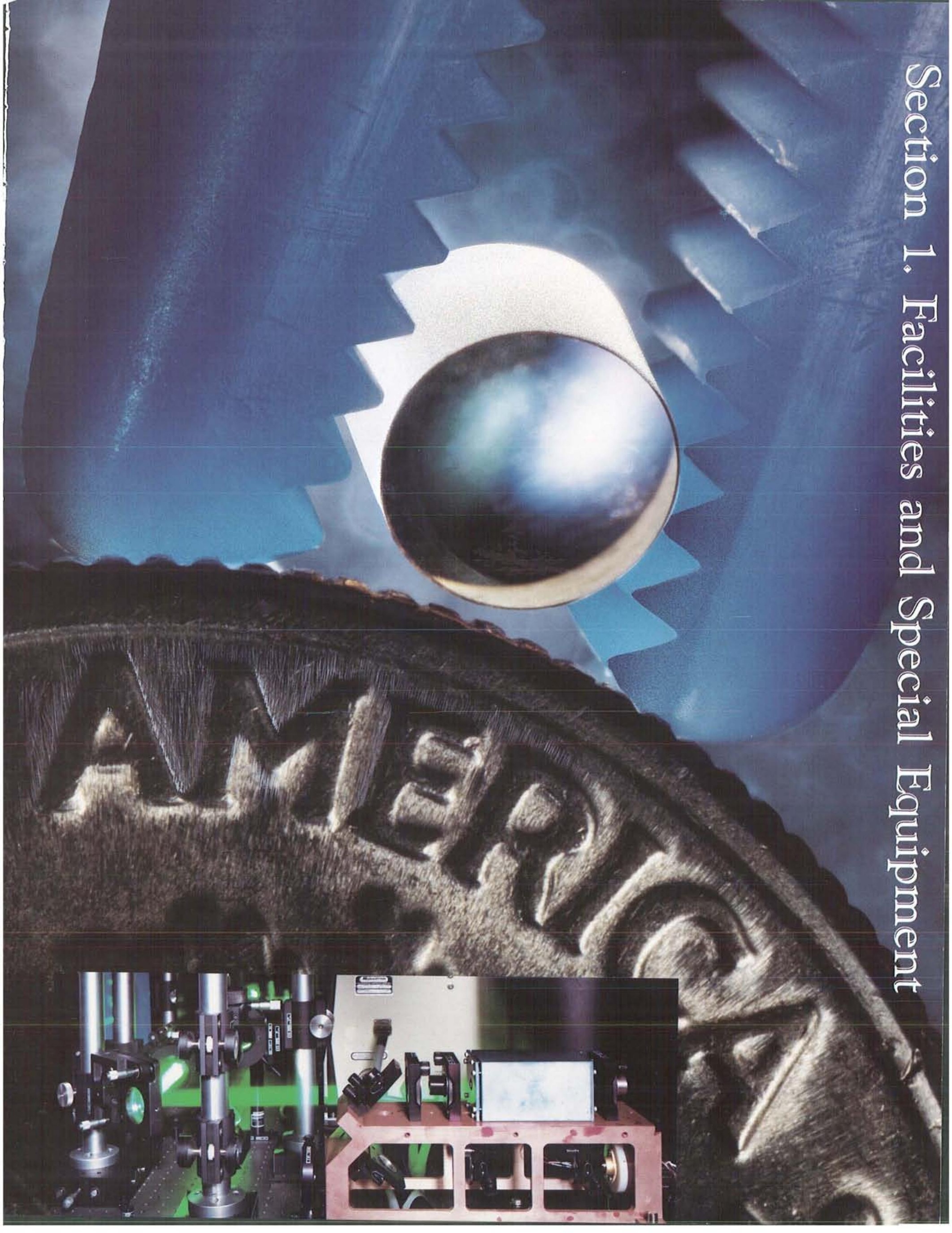


The small Nd:YAG crystal shown on the preceding page has mirrors polished and coated directly on its end faces to form a miniature laser cavity. Pumped by a small $\mathrm{GaAlAs}$ diode laser, this monolithic Nd:YAG laser produces stable, single-frequency light needed to injection-seed powerful Q-switched lasers. The injection seeding system, shown in the foreground of the inset photograph, integrates the diode-laserpumped monolithic Nd:YAG laser with a permanent magnet Faraday isolator to provide a convenient and reliable technique for insuring single frequency operation of (normally multi-mode) Q-switched Nd:YAG lasers. Single frequency Nd:YAG lasers, such as the one in the backgound of the inset photograph, are required for high resolution spectroscopies such as coherent anti-Stokes Raman spectroscopy (CARS) and inverse Raman spectroscopy. 


\section{Section 1 \\ Facilities and Special Equipment}

The Combustion Research Facility is a 51,500 square-foot, four building complex forming an eastward extension of Sandia National Laboratories Livermore. The Facility, which includes an office building, laboratory, auditorium, and mechanical equipment building, provides a highly sophisticated research environment for the resident staff and visiting researchers. It is located outside the Sandia security area to permit easy access for visitors.

A number of unique features have been incorporated in the facility: three central facility laser systems that provide state-of-the-art diagnostics capabilities, access to a broad range of computers ranging from minis and superminis to 2-million-word Cray systems, and a sophisticated, microprocessorcontrolled laboratory safety system.

\section{Facility Laser System}

\section{R. L. Schmitt}

The Facility Laser System consists of three centrally located lasers and a facility beam distribution (periscope) system. The three facility lasers are: Diana, a flashlamp-pumped dye laser; Sirius, a Nd:YAG laser and dye laser system; and, Dye Blaster, a general-purpose Nd:YAG laser. These lasers may be transmitted to any of the laboratories by using the Facility's periscope system.

\section{Diana}

Diana is a flashlamp-pumped dye laser system designed for single-shot temperature and species concentration measurements in turbulent environments. This laser delivers the high pulse energy required for single-shot spontaneous Raman scattering measurements in a relatively long pulse $(\sim 2 \mu \mathrm{s})$ that prevents dielectric breakdown in the focused beam. Diana consists of a prism-tuned oscillator followed by three amplifiers, and produces $200 \mathrm{~mJ}$ to $5 \mathrm{~J}$ per pulse depending on the dye used. Diana may be tuned from $400 \mathrm{~nm}$ to $600 \mathrm{~nm}$ using a variety of dyes.

Mehdi Namazian, a visiting scientist from Altex Technologies, has used Diana and the Raman imaging system to study the flow and mixing structures in bluff-body-stabilized flames. For most of the experiments, Diana was tuned to $444 \mathrm{~nm}$ (using Coumarin 440) and injected into a multipass cell for two-dimensional Raman scattering measurements from methane. Mehdi and Professor Marshall Long, from Yale University, also used Diana for simultaneous mapping of Raman scattering from $\mathrm{CH}_{4}$ and fluorescence from $\mathrm{CH}$. For these experiments, Diana was modified to lase on two wavelengths simultaneously ( 431.5 and $444 \mathrm{~nm}$ ). The shorter wavelength was used to excite fluorescence from $\mathrm{CH}$ and the other was used for spontaneous Raman scattering.

Bob Dibble (8351) and Asaad Masri, a visiting scientist from the University of Sydney, used Diana to perform simultaneous spaceand time-resolved concentration measurements of major species $\left(\mathrm{CH}_{4}, \mathrm{O}_{2}, \mathrm{~N}_{2}, \mathrm{H}_{2} \mathrm{O}\right.$, and $\left.\mathrm{H}_{2}\right)$ 
in the blue region of a turbulent methane nonpremixed flame. For these experiments, Diana was tuned to $532 \mathrm{~nm}$ (using Coumarin 521), and operated at $750 \mathrm{~mJ}$ per pulse. The 532$\mathrm{nm}$ wavelength was chosen to permit use of a Nd:YAG laser operating at $10 \mathrm{~Hz}$ (the Dye Blaster) for the time-consuming alignment of the multi-channel Raman spectrograph.

Bob Dibble (8351) and Phillipe Magre, a visiting scientist from ONERA, used Diana to perform instantaneous spatially resolved measurement of major species $\left(\mathrm{H}_{2}, \mathrm{O}_{2}, \mathrm{H}_{2} \mathrm{O}\right.$, and $\mathrm{N}_{2}$ ) to study the effects of finite chemical kinetics in turbulent, nonpremixed hydrogen flames. For these measurements, the laser was tuned to $514.5 \mathrm{~nm}$ and operated at $1.0 \mathrm{~J}$ per pulse.

Most recently, Professor Marshall Long and Brandon Yip (also from Yale University) used Diana for instantaneous three-dimensional imaging measurements in turbulent jets. In this experiment, the beam from Diana was formed into a thin sheet using cylindrical lenses, and this sheet was translated through the measurement volume by a high speed scanning mirror. A high speed framing camera (up to 20 frames $/ \mu \mathrm{s}$ ), synchronized to the laser pulse, captured a series of two-dimensional images during the laser pulse. Each frame was spatially separated from the previous frame by $\sim 0.2 \mathrm{~mm}$. The series of frames was then digitized and stored in computer memory. The frames will be used to construct three-dimensional images needed to study flame structure.

\section{Sirius}

Sirius is a multi-purpose laser system for nonlinear optical techniques such as CARS (Coherent anti-Stokes Raman Spectroscopy) and IRS (Inverse Raman Spectroscopy). The Sirius laser system consists of a Molectron MY34 Qswitched Nd:YAG laser system, a Quanta-Ray narrow-band dye laser, and a broadband dye laser. The Molectron Nd:YAG laser is capable of producing $250 \mathrm{~mJ}$ at $532 \mathrm{~nm}$ in $\sim 15$-ns-long pulses. A portion of the 532-nm light is used to pump either the narrow-band tunable dye laser or the broadband dye laser, depending on the requirements of the experiment. The 532-nm
Nd:YAG laser beam and the dye laser beam are transmitted side-by-side through the periscope system to the remote lab for the CARS experiments. Both the Nd:YAG laser energy and the dye laser wavelength are remotely controlled using a CAMAC serial highway. Operation of remote CARS experiments up to 60 meters from the Sirius laboratory have become routine.

We are currently installing a diode-laserpumped Nd:YAG laser injection seeding system ${ }^{1}$ on the Sirius laser to provide reliable singlemode laser pulses for high resolution CARS and other applications requiring single-frequency $\mathrm{Nd}$ :YAG lasers. At the same time, we will be modifying the laser cavity to improve the spatial beam profile. The modifications will improve the performance of the Sirius laser and allow it to be used to pump the dye amplifiers of pulseamplified ring dye lasers used in high-resolution CARS experiments.

This year, Mark Allendorf (8361), Bob Lucht (8362), and Gene Potkay, a visiting scientist from AT\&T, used Sirius for remote broadband nitrogen CARS to measure the temperature of silica-producing flames. These "glassmaking" flames are used in the commercial production of glass for optical communication fiber.

\section{Dye-Blaster}

The Dye Blaster is Q-switched, frequencydoubled Nd:YAG laser that provides additional capability to pump dye lasers located in remote user laboratories. This laser has been useful both for users who lack a Nd:YAG laser, and for users conducting experiments that require several lasers. The Dye Blaster is a Quanta-Ray DCR-2 that produces up to $350 \mathrm{~mJ}$ at $532 \mathrm{~nm}$ in $\sim 7$-ns-long pulses. It can be synchronized with other lasers or experimental apparatus as required.

The Dye Blaster has been heavily used this year for pumping remote dye lasers. In most cases, the Dye Blaster is not the only laser in the experiment, but it is used in combination with other lasers. For example, Eric Rohlfing and Dave Chandler (both 8353) used the Dye 
Blaster in experiments to study the state-tostate energy transfer rates in molecules. For these experiments, the $532-\mathrm{nm}$ beam from Dye Blaster was slit into two parts; one part of the beam was used to pump a tunable dye laser and the other part was used in the experiment directly. The tunable dye laser beam and the 532$\mathrm{nm}$ beam were used to produce vibrationally excited, rotationally selected molecules. Later, a second laser probed the energy of the molecules to measure the state-to-state energy transfer rates.

Tom Spiglanin and Dave Chandler (both 8353) used the Dye Blaster to pump a frequencydoubled dye laser, which was used for laserinduced-fluorescence measurements (LIF) on $\mathrm{NH}$. Another laser produced the $\mathrm{NH}$ by laser photolysis of HNCO. The LIF was used to probe the internal state distribution of the $\mathrm{NH}$ molecules.

Table I

Examples of Work Using Facility Lasers This Year

\section{DIANA: \\ Two-Wavelength Single Laser \\ Effects of Finite Chemical Kinetics \\ $\mathrm{CH}_{4}$ and $\mathrm{CH}$ Concentration Imaging \\ Spontaneous Raman}

M. Namazian, R. Schmitt, and $M$. Long

P. Magre and R. W. Dibble

M. Namazian, R. Schefer, J. Kelly, and M. Long

R. Dibble and A. Masri

SIRIUS:

Nitrogen CARS Studies of a Flame

M. Allendorf, R. Lucht, and E. Potkay

\section{DYE BLASTER:}

\begin{tabular}{|c|c|}
\hline $\begin{array}{l}\text { aser-Induced Fluorescence } \\
\text { and Resonance }\end{array}$ & E. Rohlfing and D. Chandler \\
\hline otational Excitation of $\mathrm{NH}$ & \\
\hline
\end{tabular}

\section{Reference}

1. R. L. Schmitt and L. A. Rahn, "Diode-LaserPumped Nd:YAG Laser Injection Seeding System," Appl. Opt. 25, 629 (1986).

\section{Facility Computers}

\section{E. Benthusen}

Computing capabilities at the Combustion Research Facility have been further enhanced this year. We have added new central processing units, disks, printers, and software. Since June 1980, a DEC VAX system has been in operation in the Combustion Research Facility. The system currently includes two VAX 11/780 machines in the Facility's computer center, a VAX 8600 in Sandia's central computing facility, and ten microVAX IIs used to support individual experiments with experiment control, data reduction, or theoretical modeling services. One of the VAX 11/780 machines is for scientific calculations; the other is for word processing.

The VAX 8600 is new this year. This machine has a central-processing-unit speed 4.2 times that of the VAX 11/780s. Although this machine is located in Sandia's central computing facility, there is terminal access to all Combustion Research Facility residents. The machine is on the Ethernet used by the Facility's machines so that files can be transferred easily. The VAX 8600 has 3 Gigabytes of rotating storage, a magnetic tape drive, and impact and laser printers. DEC is currently developing a concept known as local-area-clusters that will make all disks local to this 8600 appear to be part of the Facility's cluster disk farm. This change means that all disks connected to any of these machines would be equally available to both VAX 11/780s without the need for complicated file transfers.

Other hardware changes include the addition of several LN03 laser printers, making various graphic and high-quality print output available at several convenient locations. Also, new disks have been added to provide a total of nearly 7 Gigabytes of rotating storage.

The current operating system for the VAX network is VMS V4.4. A greatly enhanced Fortran 77 compiler (V4.5) is also available. Other 
system software includes Pascal, 'C', Lisp, Decalc, All-in-One, FMS, Datatrieve, and the Common-Data-Dictionary. The DEC data-base manager and three commonly used "Math Program Libraries" are also running on the scientific machine. The Muse word-processing software that had been available on the wordprocessing system has been replaced by the greatly enhanced Word-Marc Composer. The $\mathrm{TeX}$ typesetting software continues to be heavily used, and its output can now be produced on LN03 laser printers as well as the QMS laser printer/plotter.

A wealth of graphics software is available to VAX users. Issco's Disspla can be used to produce plots on a variety of output devices such as Tektronix 4014's, Talaris, and Printronix printer-plotters as well as the newer LN03s. Tell-a-Graf and Cuechart (also from Issco) are also running on both VAX $11 / 780$ s. These graphics systems accept plain English commands and can display input data in the form of a plot, bar-chart, or pie-chart. A Sandia-designed program called Text is also available and is very useful for making high quality viewgraphs. All of these systems are now capable of producing color graphics when used in conjunction with our AED and Tektronix color graphics terminals. The above-mentioned products can make use of a Sandia-designed software system called the "Virtual Graphics Device." This device allows powerful graphics editing as well as the ability to write files that can be processed directly on one of the Dicomed Computer Output Microfilm systems to produce journal-quality output in either black-and-white or color.

We plan to continue expanding our computer capabilities in the coming year. A new high-speed printer that is capable of interpreting PostScript, a powerful typesetting language widely used for desktop publishing, will soon be installed on the Ethernet. Several more microVAX II's will be installed in the laboratories, and they, too, will be added to the Ethernet. We are also planning to improve our capacity for high-speed computing by adding a supercomputer or array processor to our network. 
$\infty$

(B)

总.

N

$\Omega$

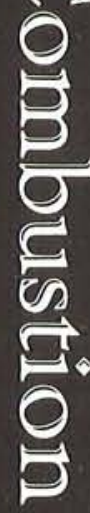

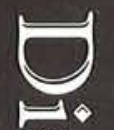

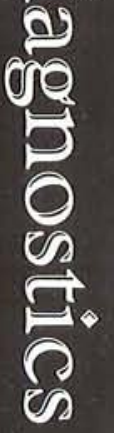

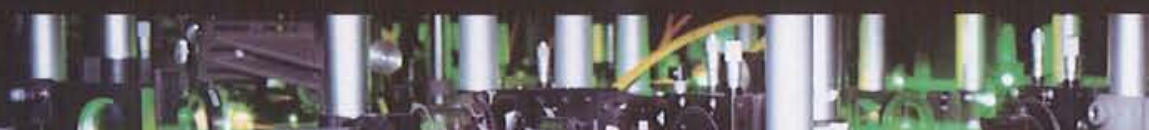

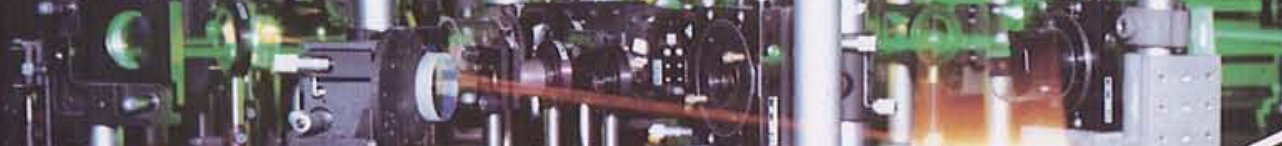

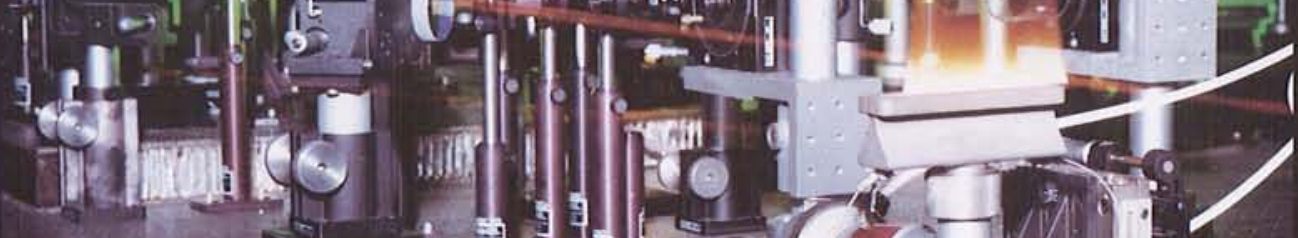

410 की

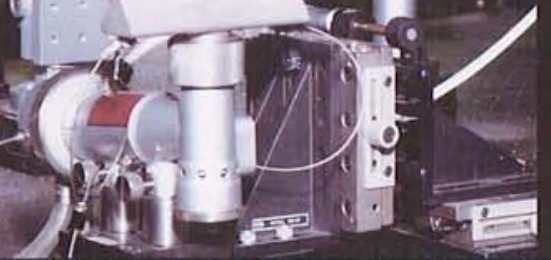


Four-wave mixing and induced-grating techniques (see related article, page 2-14) offer the prospect of using collisioninduced processes to study molecules during collisions, and thus during reactions, in combustion environments. The enlarged photograph on the preceding page shows three laser beams focused into a hydrogen-air flame seeded with sodium (hence the orange color); the nonlinear interaction between the beams and sodium atoms generates a fourth beam whose intensity is monitored as the wavelength of one of the input beams is scanned. The inset photograph shows a broader view of the burner (right) and a portion of the beamsteering optics to direct the beams to the flame. 


\section{Section 2 \\ Combustion Diagnostics}

One of the primary thrusts of the combustion research program at Sandia National Laboratories is the development of diagnostic techniques that take advantage of the latest advances in modern technology. This development effort has three objectives: innovative methods for measurements in combustion systems, a quantitative understanding of the usefulness and limitations of these methods, and the transfer of technology from research to applications environments.

Because of their nonintrusive nature and great versatility, laser-based optical techniques - both point-resolved and two-dimensional imaging-receive the strongest emphasis. Laser-induced fluorescence, spontaneous and coherent Raman spectroscopy, laser Doppler velocimetry, and multiphoton optogalvanic spectroscopy are but a few of the diagnostic methods that are being developed to measure temperature, species concentrations, velocity flow fields, and other parameters of key importance to the understanding of combustion phenomena. The capabilities of such techniques depend directly on the availability of laser sources with unique spatial, temporal, and spectral properties; thus, an important feature of the combustion diagnostic program is the development of high-performance lasers that meet the requirements of specialized situations. In addition to laser-based optical probes, intrusive probes are also being developed and evaluated.

Sandia's efforts to develop advanced combustion diagnostics, especially advanced laser-based optical probes, are supported primarily by the Chemical Sciences Division of the Department of Energy's Office of Basic Energy Sciences. Work in this program is complemented by work in the applied research programs to develop diagnostic techniques for specific combustion applications.

\section{High-Resolution CARS Measurements in a Flame ${ }^{\dagger}$}

\section{R. L. Farrow}

Among many optical techniques currently under development for concentration and temperature measurements in hostile combustion environments, coherent anti-Stokes Raman spectroscopy (CARS) has received perhaps the widest application. The continued diagnostic development of CARS calls for improved knowledge of

$\dagger$ Twenty-first (International) Symposium on Combustion (The Combustion Institute, 1986), in press. many spectroscopic parameters, including Raman cross-sections, linewidths, lineshapes, transition frequencies, and nonresonant susceptibilities. High-resolution CARS systems (based on single-frequency lasers) are capable of providing much of this information, but have not been widely applied to combustion problems.

We have constructed a high-resolution CARS system and have used it to measure fully resolved CARS spectra in a rich methane/air flat flame. Using a single-frequency Nd:YAG laser and a pulse-amplified, single-frequency $\mathrm{cw}$ dye laser, we measured $Q$-branch and $O$-branch transitions of $\mathrm{CO}$ and $\mathrm{N}_{2}$, respectively. Raman linewidths analyzed from these data are compared to infrared linewidth measurements. ${ }^{1}$ In 
addition, an accurate frequency is obtained for the $O(25)$ transition of $\mathrm{N}_{2}$. The results demonstrate the capabilities offered by high-resolution CARS as a spectroscopic tool for combustion diagnostic development.

The experimental layout has been described in detail elsewhere. We used a crossed-beam, three-dimensional phase-matching geometry to obtain spatial resolution of approximately $4 \mathrm{~mm}$ $\times 0.1 \mathrm{~mm}^{2}$. A VAX 11/730 computer acquired the digitized anti-Stokes signal and normalized the signal strength for each laser pulse by the measured Stokes energy and the square of the pump energy; $5-8$ pulses were averaged for each Stokes frequency step. The pump radiation was provided by the frequency-doubled output of a modified Molectron MY-34 Nd:YAG laser equipped with a MY-SAM single-axial-mode option. We estimate the pulse-averaged linewidth (HWHM) of the laser to be less than $25 \mathrm{MHz}$ at $532 \mathrm{~nm}$, based on measurements of other singlemode Molectron Nd:YAG lasers. For the Stokes radiation, $60 \mathrm{~mJ}$ of the total $90 \mathrm{~mJ} 532-\mathrm{nm}$ output was used to pulse-amplify the $\mathrm{cw}$ beam from a coherent 699-29 actively stabilized ringdye laser. The linewidth (HWHM) of this laser after three dye amplifier stages was measured to be $\leq 35 \mathrm{MHz}$.

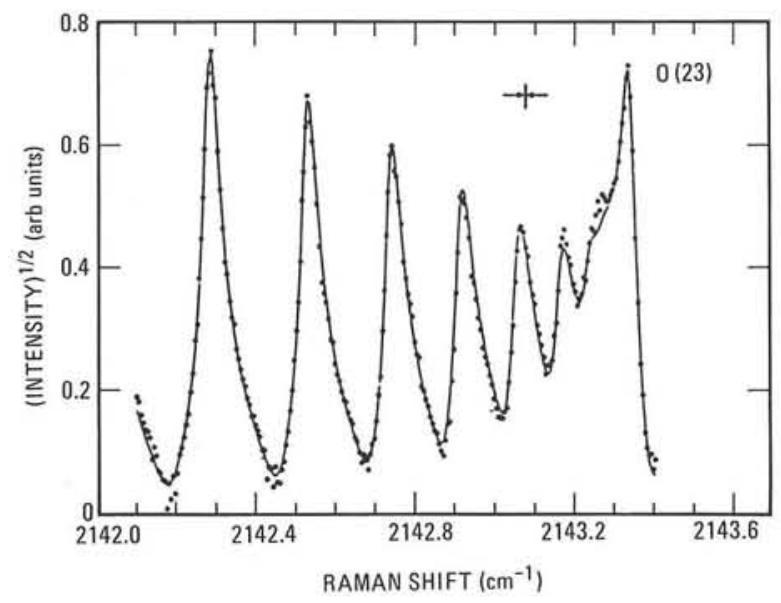

Figure 1. Experimental spectrum of the CO bandhead region in the flame. The labeled peak is an $O$-branch transition of $\mathrm{N}_{2}$, and the solid curve is a theoretical spectrum with CO Raman linewidths and relative concentrations varied for best fit.
An experimental measurement of the $\mathrm{CO}$ bandhead region is shown by the data points in Figure 1; the prominent peak at $2143.35 \mathrm{~cm}^{-1}$ is the $O(23)$ transition of $\mathrm{N}_{2}$. The solid line is a theoretical spectrum obtained by varying the linewidth scaling factor for the $\mathrm{CO}$ linewidths and the concentration relative to $\mathrm{N}_{2}$ for best fit. The agreement between theory and experiment is much improved over earlier low-resolution studies, which were hampered by linewidth and frequency inaccuracies in modeling the $\mathrm{N}_{2}$ transitions.

CO $Q$-branch linewidths were measured for $\mathrm{J}=2$ to 17 by fitting individual transitions to Lorentzian line profiles as discussed above. The results, normalized to 760 torr, are given by the data points in Figure 2.

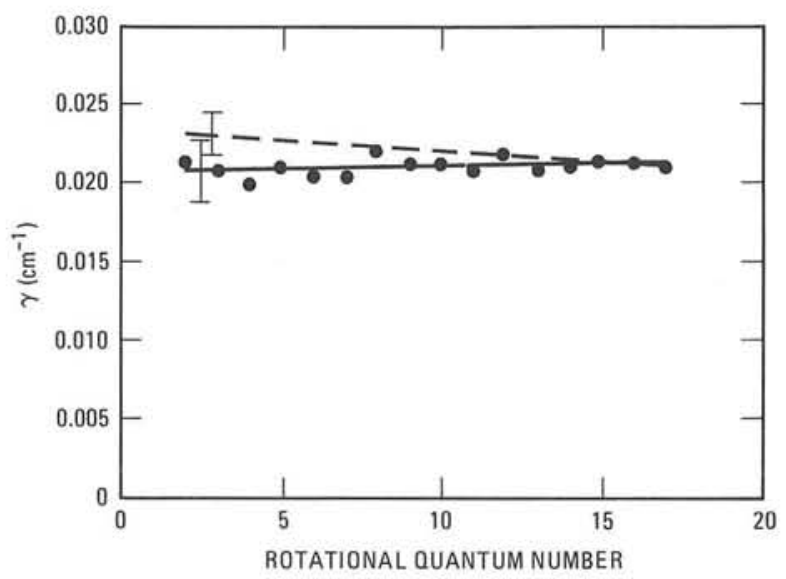

Figure 2. Raman linewidths of $Q(\mathrm{~J})$ transitions of $\mathrm{CO}$ in the flame, indicated by data points and referenced to 760 torr. The solid curve is a straight-line fit to the data: $\gamma(\mathrm{J})=0.02074+$ $3.72 \times 10^{-5} \mathrm{~J} \mathrm{~cm}^{-1}$. (From regression analysis, the data are also consistent with a horizontal line.) The broken curve is a fit to the infrared linewidths reported by Varghese and Hanson, ${ }^{1}$ corrected for our flame temperature (1690 K) and including an estimated Doppler width of $0.001 \mathrm{~cm}^{-1}$.

The solid curve is a straight-line fit to the data. The uncertainty indicated for the data points is principally due to random errors such as statistical noise, baseline errors, and fit errors (the uncertainty is twice as large for $\mathrm{J}=$ 2). The CO Raman linewidths were comparable to infrared P- and R-branch collisional widths 
of CO measured in methane/air flat flames by Varghese and Hanson. ${ }^{1}$ A straight-line fit ${ }^{1}$ to the infrared collisional widths was corrected for our flame temperature of $1690 \mathrm{~K}$ using Jdependent temperature exponents reported in the same reference. (Since full linewidths were not reported, an additional $0.001 \mathrm{~cm}^{-1}$ was added to each width to account for Doppler contributions). The results are indicated by a broken line in Figure 2. The infrared linewidths appear to be somewhat larger, at least for low J, than the corresponding Raman linewidths. This result is not unreasonable since the infrared transitions are subject to additional broadening by pure rotational dephasing. However, given the uncertainties in both sets of measurements, the differences are not considered significant.

These results demonstrate that high-resolution CARS is a viable technique for spectroscopic investigations of many species important in combustion. It appears that high-resolution CARS can provide greater accuracy in concentration measurements, compared to conventional CARS experiments, due to a reduced uncertainty in the Raman linewidths. We are currently working to extend the continuous scanning range of this experiment and are planning further investigations of $\mathrm{CO}$ and other combustion-related species.

\section{References}

1. P. L. Varghese and R. K. Hanson, "Collision Width Measurements of CO in Combustion Gases Using a Tunable Diode Laser," $J$. Quant. Spectrosc. Radiat. Transfer 26, 339 (1981).

2. R. L. Farrow, R. P. Lucht, G. L. Clark, and R. E. Palmer, "Species Concentration Measurements Using CARS with Nonresonant Susceptibility Normalization," Appl. Opt. 24, 2241 (1985).

\section{Measurements of the Nonresonant Third-Order Susceptibilities of Gases Using CARS}

\author{
R. L. Farrow, R. P. Lucht, L. A. Rahn
}

Coherent anti-Stokes Raman Spectroscopy (CARS) is widely used to characterize the temperature and composition of gas mixtures via measurements of the bulk third order susceptibility, $\chi^{(3)}$. In general, $\chi^{(3)}=\chi_{r}+\chi_{n r}$, where $\chi_{r}$ indicates resonant ro-vibrational contributions due to Raman transitions, and $\chi_{n r}$ indicates nonresonant electronic contributions. In combustion applications of CARS, $\chi_{n r}$ can be a non-negligible, if not dominant, component of a spectrum. Thus, for accurate data analysis, and for concentrations measurements referenced to the nonresonant background, $\chi_{n r}$ must be known independently.

Currently, reliable data for the nonresonant susceptibilities of combustion gases are incomplete; measurements for many significant combustion-related gases have not been reported. In this paper, we present measurements of nonresonant susceptibilities of propane $\left(\mathrm{C}_{3} \mathrm{H}_{8}\right)$, n-butane $\left(\mathrm{n}-\mathrm{C}_{4} \mathrm{H}_{10}\right)$, and $\mathrm{H}_{2} \mathrm{O}$ vapor. These results were obtained from scanned CARS spectra of binary mixtures of $\mathrm{N}_{2}$ and the subject gases. The resonant susceptibility of the $\mathrm{N}_{2} Q-$ branch, which served as a calibration standard, was compared to $\chi_{n r}$ by measuring the spectral profile produced by the interference of resonant and nonresonant amplitudes. We used a CARS fitting code to extract the least-squares value of $\chi_{n r}$, with $\chi_{r}$ computed by extending a previously described exponential-gap susceptibility model. ${ }^{1}$ Raman transition linewidths were obtained from separate measurements by inverse Raman spectroscopy (IRS).

The pump laser was a frequency-doubled, single-mode Nd:YAG laser with a FWHM linewidth estimated to be $50 \mathrm{MHz}$. The Stokes radiation was obtained from a Lamda Physik 2002-EC dye laser pumped by the Nd:YAG 
laser. The dye laser had a linewidth of $1.1 \mathrm{GHz}$ with an intracavity etalon inserted.

Spectra of $\mathrm{N}_{2}$ from binary gas mixtures of $4.9 \pm 0.2 \% \mathrm{~N}_{2}$ in $\mathrm{n}-\mathrm{C}_{4} \mathrm{H}_{10}$ and $5.2 \pm 0.2 \% \mathrm{~N}_{2}$ in $\mathrm{C}_{3} \mathrm{H}_{8}$ were measured in a gas cell at $296 \mathrm{~K}$. To measure off-resonant contributions from Raman transitions of the buffer gas, we first subtracted all $\chi_{n r}$ contributions using polarization techniques (see Figure 1).

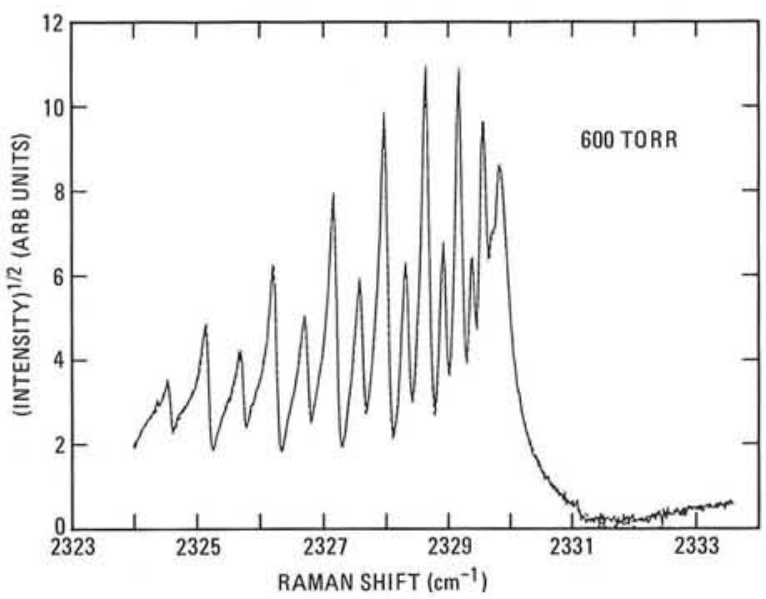

Figure 1. Spectrum of the $\mathrm{N}_{2} Q$-branch (solid line) and fit (dashed line) from a gas mixture of $5.1 \pm 0.1$ mole $\% \mathrm{~N}_{2}$ in $\mathrm{C}_{3} \mathrm{H}_{8}$ at $296 \mathrm{~K}$ and a pressure of 600 torr. The resolution was 0.03 $\mathrm{cm}^{-1}$, sufficient to fully resolve the Raman lineshapes. A polarizer angle of 60 degrees was used to suppress nonresonant electronic intensities, so that the remaining background was due to offresonant $\mathrm{C}_{3} \mathrm{H}_{8}$ Raman transitions.

The resonant susceptibility of $\mathrm{N}_{2}$ in the buffer (including J-dependent linewidths and pressure-narrowing effects) was analyzed from this data by using a modified version of the exponential gap model of Koszykowski et al. ${ }^{1}$ The polarization analyzer was then rotated to $50 \pm 0.5$ degrees to additionally observe $\chi_{n r}$ contributions (see Figure 2).

Varying the total background for best fit gave a value for $\chi_{n r}\left(\mathrm{C}_{3} \mathrm{H}_{8}\right)$ of $8.0 \pm 1.0 \times 10^{-17}$ $\mathrm{cm}^{3} / \mathrm{erg}$ (STP), not including the off-resonant
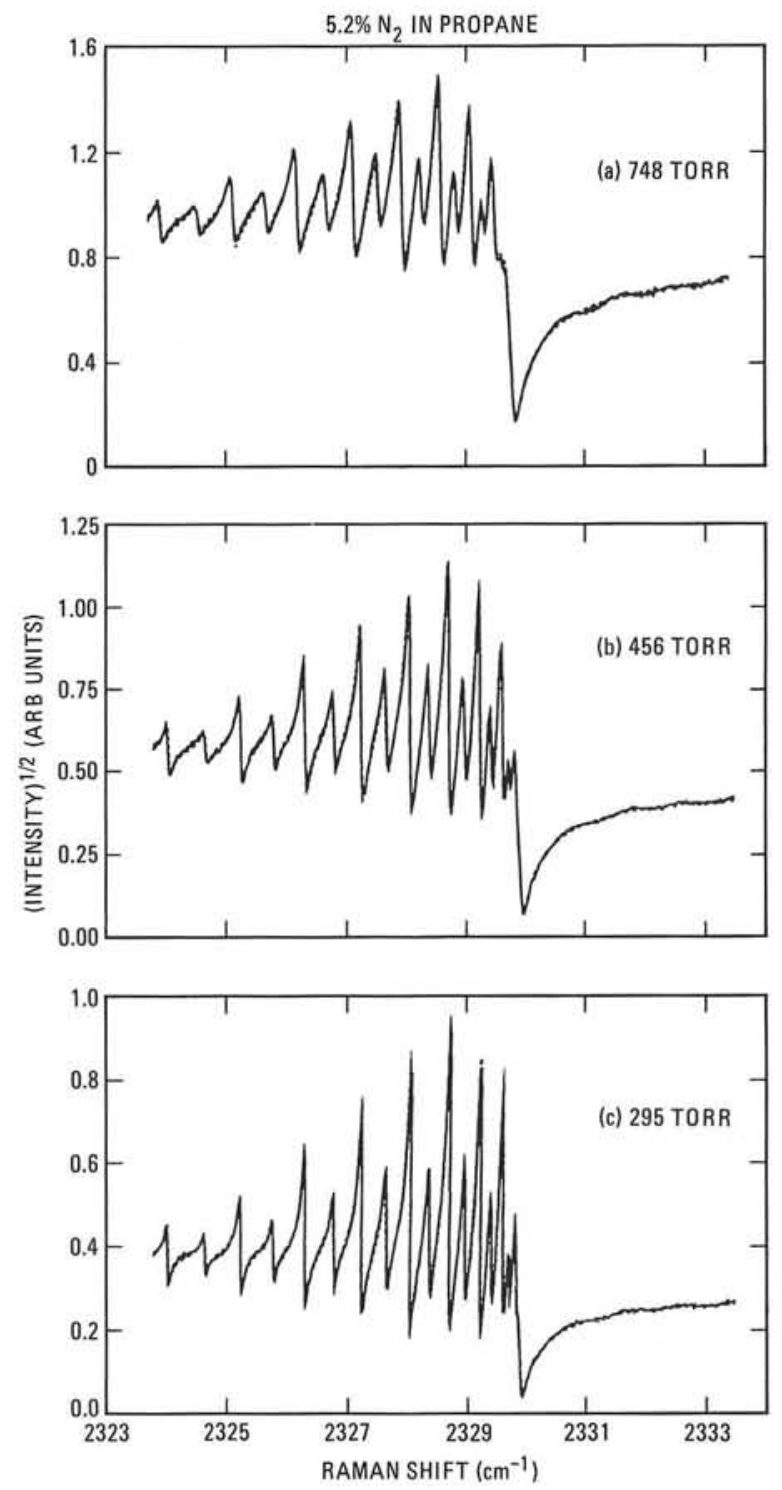

Figure 2. Spectrum of the $\mathrm{N}_{2} Q$-branch (solid line) and fit (dashed line) at various pressures, from the same gas as Figure 1. Here, the polarizer angle was 50 degrees.

Raman contribution from $\mathrm{C}_{3} \mathrm{H}_{8}, 1.8 \pm 0.5 \times$ $10^{-17} \mathrm{~cm}^{3} /$ erg $\left(\chi_{1111}\right.$ component quoted). Similarly, we found $\chi_{n r}\left(\mathrm{n}-\mathrm{C}_{4} \mathrm{H}_{10}\right)=1.0 \pm 0.12 \times$ $10^{-16} \mathrm{~cm}^{3} / \mathrm{erg}$, after subtracting an off-resonant Raman contribution, $2.2 \pm 0.6 \times 10^{-17} \mathrm{~cm}^{3} / \mathrm{erg}$. For parallel-polarized CARS measurements, the $\chi_{1111}$ component of the Raman susceptibility can be added to $\chi_{n r}$ to obtain an effective nonresonant background. 
We have also measured $\chi_{n r}\left(\mathrm{H}_{2} \mathrm{O}\right)$ from CARS spectra of $\mathrm{N}_{2}$ in flame gases containing $67 \% \mathrm{H}_{2} \mathrm{O}$ and $33 \% \mathrm{~N}_{2}$. The gas temperature was determined to be $1570 \mathrm{~K}$ from analysis of background-subtracted $\mathrm{N}_{2} Q$-branch spectra (we did not detect any off-resonant Raman intensity from $\mathrm{H}_{2} \mathrm{O}$ ). Since the dye laser linewidth was not narrow enough to resolve the $\mathrm{N}_{2}$ lineshapes in the flame, we used collisional widths measured with IRS for the same flame. Analysis of flame $\mathrm{N}_{2}$ spectra yielded an STP value for $\chi_{n r}\left(\mathrm{H}_{2} \mathrm{O}\right)$ of $1.85 \pm 0.18 \times 10^{-17} \mathrm{~cm}^{3} /$ erg.

\section{Reference}

1. M. L. Koszykowski, L. A. Rahn, R. E. Palmer, and M. E. Coltrin, "Theoretical and Experimental Studies of High-Resolution Inverse Raman Spectra of $\mathrm{N}_{2}$ at 1-10 atm," J. Phys. Chem. 91, 41 (1987).

\section{CARS Measurements of Temperature Profiles and Pressure in a Tungsten Lamp}

\section{R. L. Farrow, F. P. Trebino}

The measurement of temperature and pressure inside incandescent lamps is important for understanding heat and mass transport processes and, consequently, lamp efficiency and longevity. Traditional methods, however, are often inadequate. Radiation and optical pyrometers measure the temperature of emitting materials only; thermocouples cannot survive the temperatures of interest, and interferometric techniques measure only integrated quantities. Pressure-measurement devices, such as transducers and barometers, have similar problems. Coherent anti-Stokes Raman Spectroscopy (CARS), on the other hand, allows temperature and pressure measurements with none of these drawbacks and is a potential alternative technique.
Previous work has established CARS as an accurate temperature probe in a variety of hostile environments, ranging from laboratory flames to jet engine exhausts. In addition, Devonshire et $a l^{1}$ have performed CARS measurements in specially constructed incandescent lamps. Very little work has been done, however, to establish CARS as an accurate pressure probe. To our knowledge, only Gustafson and Byer ${ }^{2}$ have attempted such measurements, doing so in a 60 -atm deuterium fusion pellet. In this paper, we describe the use of CARS to measure the temperature profile and pressure in nitrogen-filled, high-intensity lamps. We employ an off-the-shelf General Electric lamp, with no special windows or polish, and measure the CARS $Q$-branch spectrum of the nitrogen fill. We find temperatures that range from below $1000 \mathrm{~K}$ beneath the lamp filament to over $2000 \mathrm{~K}$ near the top of the filament, with an experimental accuracy of approximately $5 \%$. In addition, we obtain values of the pressure to within $5 \%$, verified in a specially constructed lamp that permitted independent measurement of the pressure.

The General Electric 1000-W FEL lamp's envelope had a roughly cylindrical geometry: 50 $\mathrm{mm}$ long and $18 \mathrm{~mm}$ in diameter. Its filament took the shape of a coil $18 \mathrm{~mm}$ long and 7.0 $\mathrm{mm}$ in diameter. The lamp's internal pressure at room temperature was known in advance to be about $2.5 \mathrm{~atm}$. In this study, the lamp voltage and current were 44 volts and 4.7 amps, respectively. Using a previously described CARS apparatus $^{3}$ (but with a single-axial-mode pump laser), we probed $0.1 \mathrm{~mm} \times 0.1 \mathrm{~mm} \times 1 \mathrm{~mm}$ regions in a horizontally mounted lamp along a radial direction from just below to just above the filament. The probed regions were about midway between the lamp's base and its apex (see Figure 1).

Figure 2 shows a typical nitrogen $Q$-branch $(\Delta J=0)$ spectrum for a region centered within the lamp filament. Evident in the spectrum are the $\mathrm{v}=0 \rightarrow 1$ band and, below Raman shifts of about $2300 \mathrm{~cm}^{-1}$, the $\mathrm{v}=1 \rightarrow 2$ hot band. In the lower regions of the filament and below, spectra exhibit weaker line strengths in the hot band 


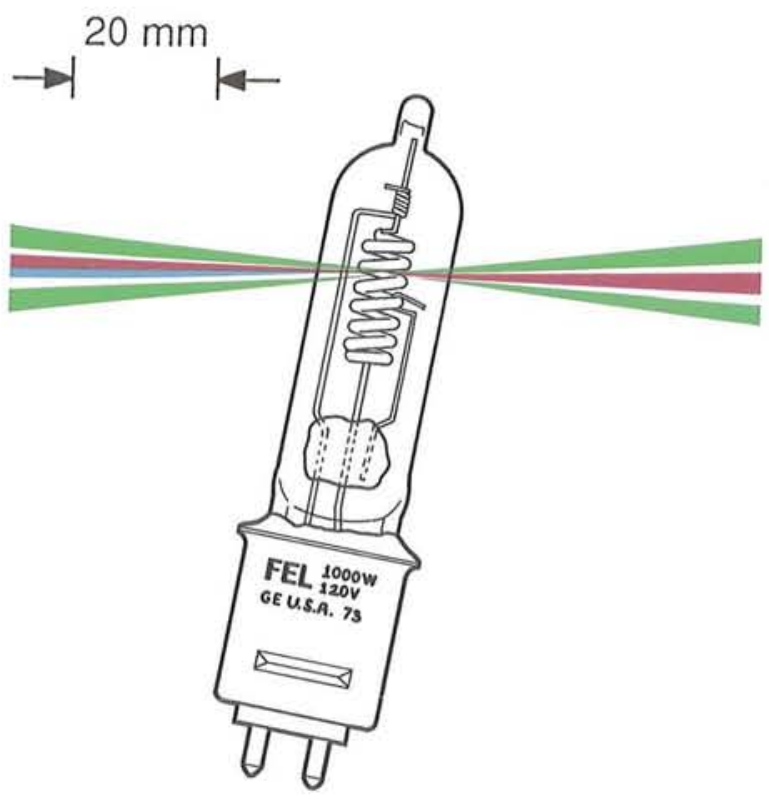

Figure 1. Schematic diagram of GE FEL lamp used in these studies. The laser beams were focused in the midplane of the lamp, which was translated vertically to probe different locations in the region of the filament. The red, blue, and green colors indicate the Stokes, anti-Stokes, and pump beams, respectively.

and a steeper decrease of line strength with increasing J-value, indicative of lower temperatures. All spectra show similar signal-to-noise ratios.

Figure 3 shows the measured nitrogen CARS temperature profile in the lamp, obtained from the CARS spectra using a least-squares curve-fitting procedure based on an exponentialgap model for the J-dependent molecular linewidths. ${ }^{4}$

Temperatures range from $970 \mathrm{~K}$ just below the filament to $2210 \mathrm{~K}$ near the top of the filament, consistent with a radiation pyrometer reading of the filament temperature of $2260 \mathrm{~K}$. Marked asymmetry with respect to the lamp center exists in the profile, indicating that the effects of convection are significant, as expected for the existing temperature and pressure conditions. To perform pressure measurements, we used a specially constructed lamp with an open fill tube, allowing independent measurement of

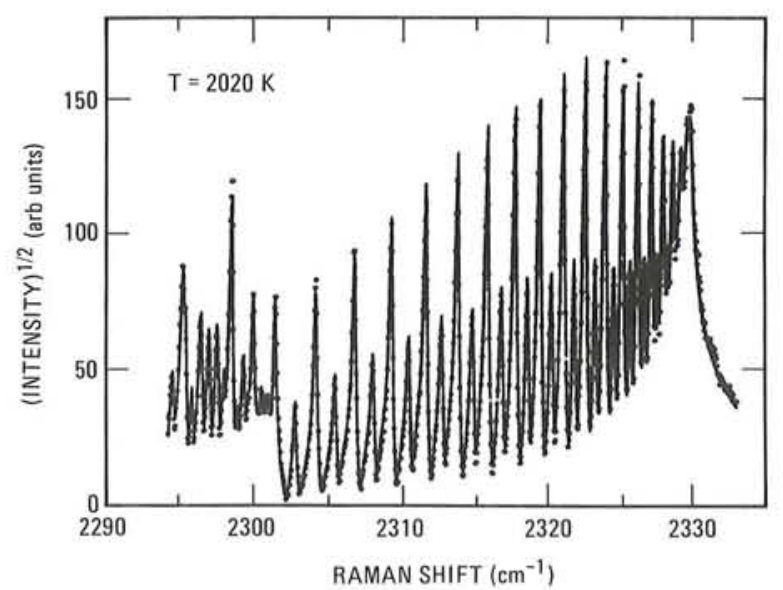

Figure 2. Experimental (data points) and theoretical (solid line) spectra of the nitrogen $Q$ branch at a point centered within the filament. Least-squares curve-fitting yields a temperature of $2020 \mathrm{~K}$ for this region.

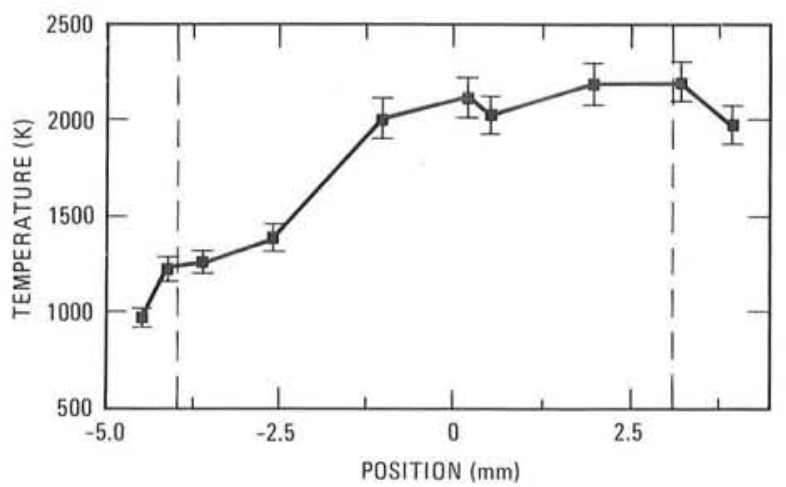

Figure 3. Temperature profile across lamp, measured using CARS. The coiled filament extended from $-3.9 \mathrm{~mm}$ (low side) to $+3.1 \mathrm{~mm}$ (high side). The lamp envelope walls occurred at $-9.1 \mathrm{~mm}$ and $+8.9 \mathrm{~mm}$.

the lamp pressure with a Baratron gauge with an estimated accuracy of $\pm 3 \%$. Because the exponential-gap model we initially tried underpredicts the CARS lineshapes at high temperatures $(>1500 \mathrm{~K}),{ }^{4}$ we employ a slightly modified model that better predicts high-temperature lineshapes and, hence, better predicts the pressure. For three CARS scans with known lamp pressures of $5.46,5.41$, and $5.43 \mathrm{~atm}$, we find fitted CARS values of 5.60, 5.57, and $5.43 \mathrm{~atm}$, respectively, i.e., an average difference of less 
than $3 \%$ and an overall accuracy of about $5 \%$. A high-resolution spectrum in the standard lamp yields a pressure of 8.75 atm, a reasonable value, but one that we are unable to corroborate due to the unavailability of alternative techniques. We anticipate that CARS temperature and pressure measurements may be useful for studies of other hostile environments, including lamps with more extreme internal conditions, such as high-intensity arc lamps. Conversely, commercial lamps could provide convenient sample chambers for gas-phase nonlinearoptical spectroscopy at high temperature and pressure.

\section{References}

1. R. Devonshire, I. S. Dring, G. Hoey, F. M. Porter, D. R. Williams, and D. A. Greenhalgh, "Accurate CARS Measurement and FluidFlow Modeling of the Temperature Distribution Around a Linear Incandescent Filament," Chem. Phys. Lett. 129, 191 (1986).

2. E. K. Gustafson and R. L. Byer, "Coherent Anti-Stokes Raman Scattering from Small Volumes," Opt. Lett. 9, 220 (1984).

3. R. L. Farrow, R. P. Lucht, G. L. Clark, and R. E. Palmer, "Species Concentration Measurements Using CARS with Nonresonant Susceptibility Normalization," Appl. Opt. 24, 2241 (1985).

4. L. A. Rahn and R. E. Palmer, "Studies of Nitrogen Self-Broadening at High Temperature with Inverse Raman Spectroscopy," J. Opt. Soc. Am. B 3, 1164 (1986).

\section{Three-Laser CARS Measurements of Two Species}

\author{
R. P. Lucht, R. E. Palmer, M. A. Maris *
}

A three-laser, coherent anti-Stokes Raman scattering (CARS) technique for the simultaneous acquisition of spectra from two species has been developed. A narrowband, tunable dye laser beam is used as one of the CARS pump beams. The frequency spacing between the spectra of the two species can be adjusted by changing the frequency of the dye laser pump beam, enabling the spectra to be displayed at high resolution $\left(0.5 \mathrm{~cm}^{-1}\right)$ on a single intensified diode array detector.

Coherent anti-Stokes Raman scattering (CARS) has been used extensively to measure temperature and species concentrations in combustion media. Until recently, one disadvantage of CARS was that only one species at a time could be measured, except when Raman lines would accidentally overlap. This was in contrast to spontaneous Raman scattering, where several groups have demonstrated simultaneous measurements of multiple species on the same laser shot.

Recently, however, Eckbreth and Anderson ${ }^{1}$ developed a technique in which two broadband Stokes lasers are used to acquire CARS signals from several species simultaneously. Teets ${ }^{2}$ developed a three-laser CARS technique with two pump beams at fixed frequencies, $\omega_{1}$ and $\omega_{2}$. The use of pump beams at two different frequencies allows generation of CARS signals from species which have Raman transitions at either $\omega_{1}-\omega_{s}$ or $\omega_{2}-\omega_{s}$.

We have developed a three-laser CARS technique in which one of the pump beams is a tunable, narrowband dye laser. The technique shares the advantage with the three-laser CARS technique of Teets $;^{2}$ that the CARS signals from the two species, which are probed, are generated at nearly the same frequency. Because one of

\footnotetext{
* Computer Genetics Corporation, Wakefield, MA.
} 
the pump beams is tunable, however, the frequency separation between the CARS spectra of the probed species is adjustable. In addition, the use of the continuously tunable dye laser as one of the CARS pump beams allows virtually any pair of species to be probed.

The three-laser CARS method used for measuring nitrogen and oxygen simultaneously is illustrated schematically in Figure 1.

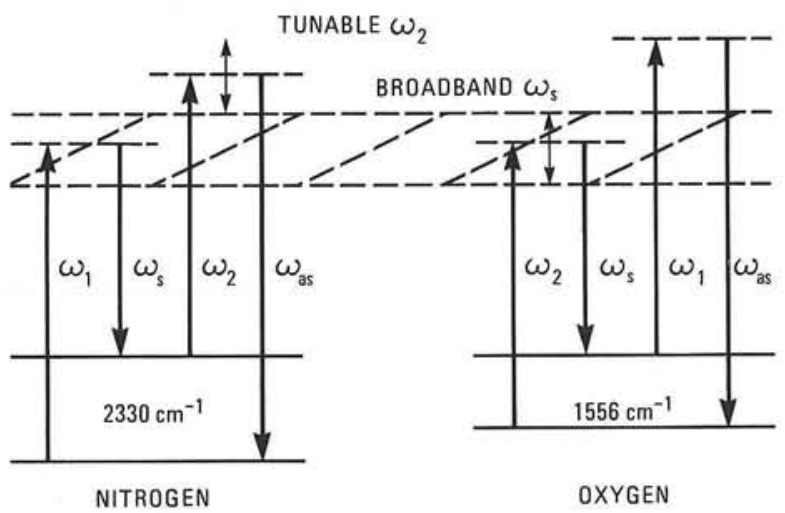

Figure 1. Energy level schematic for three-laser CARS measurement of oxygen and nitrogen.

To acquire spectra from the nitrogen and oxygen $Q$-branches at 2330 and $1556 \mathrm{~cm}^{-1}$, respectively, $532 \mathrm{~nm}$ radiation from a frequencydoubled Nd:YAG laser (Quanta-Ray DCR-2) was used for pump beam $1\left(\omega_{1}=18800 \mathrm{~cm}^{-1}\right)$. The frequency-doubled Nd:YAG laser radiation was used to pump a narrowband (Quanta-Ray PDL-2) dye laser which lased at $555 \mathrm{~nm}\left(\omega_{2}=\right.$ $\left.18000 \mathrm{~cm}^{-1}\right)$, with a bandwidth of $0.2 \mathrm{~cm}^{-1}$. The Nd:YAG laser radiation was also used to pump a broadband dye laser, centered at 607 $\mathrm{nm}\left(\omega_{s}=16470 \mathrm{~cm}^{-1}\right)$ with a spectral bandwidth (FWHM) of $100 \mathrm{~cm}^{-1}$.

The broadband dye laser was used as the Stokes laser, and the narrowband dye laser was used for pump beam 2. The Raman transition in nitrogen was excited by pump beam 1 and the Stokes beam, and pump beam 2 was scattered from the excited Raman polarization to generate an anti-Stokes signal at $491 \mathrm{~nm}$ (Figure 1, left). For oxygen, the Raman transition was excited by pump beam 2 and the Stokes beam, and pump beam 1 was scattered from the excited Raman polarization to generate an antiStokes signal at $491 \mathrm{~nm}$ (Figure 1, right). The CARS signal was focused onto the entrance slit of a $0.85-\mathrm{m}$ double spectrometer, and detected at the exit plane using an intensified diode array. A three-laser CARS spectrum of oxygen and nitrogen in a heated cell is shown in Figure 2. The dashed line in Figure 2 is the theoretical fit: The Sandia CARS code has been modified for calculation of three-laser CARS spectra.

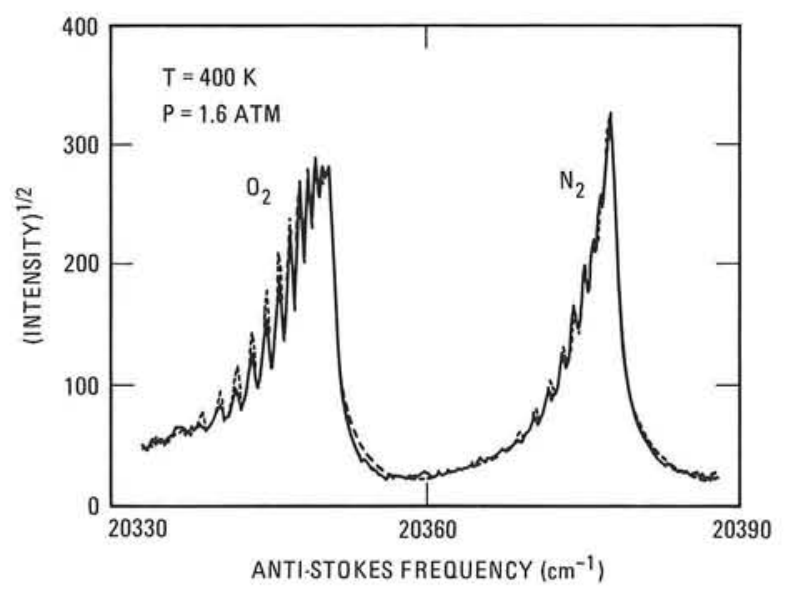

Figure 2. Three-laser CARS spectrum of nitrogen and oxygen obtained from cell at $400 \mathrm{~K}$ and $1.6 \mathrm{~atm}$. The spectrum was averaged on the intensified diode array for $\mathbf{5 0}$ laser shots. The narrowband dye laser wavelength was $554.06 \mathrm{~nm}$. The solid line is the experimental curve and the dashed line is the theoretical fit.

Using a narrowband dye laser at $550 \mathrm{~nm}$ and a broadband dye laser at $630 \mathrm{~nm}$, threelaser CARS spectra of propane and nitrogen in a fuel-injected engine were also acquired. We also acquired simultaneously pure rotational and vibrational CARS spectra from nitrogen. ${ }^{4} \mathrm{~A}$ narrowband dye laser at $602.5 \mathrm{~nm}$ and a broadband dye laser at $607 \mathrm{~nm}$ were used to generate the spectrum shown in Figure 3.

In conclusion, a new technique for performing multiple-species CARS measurements has been demonstrated. CARS spectra from two species can be acquired at high resolution on a single multichannel detector, allowing simultaneous determination of temperature and relative mole fractions. 


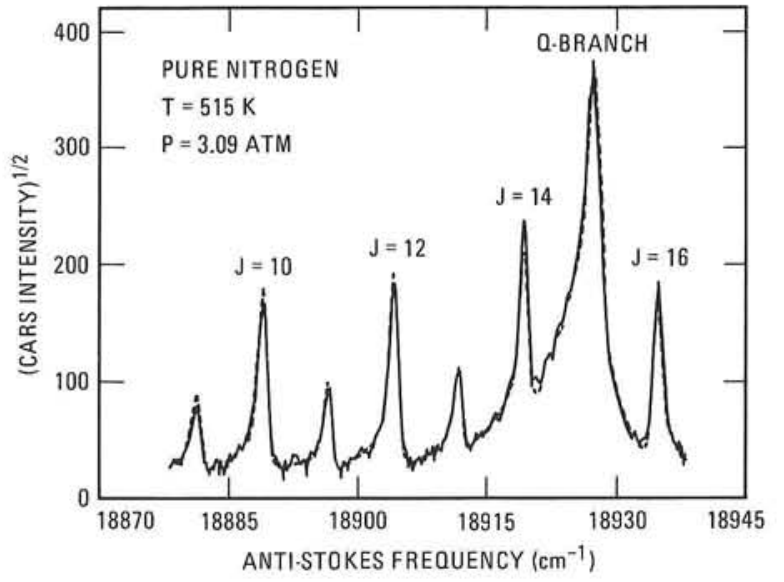

Figure 3. Three-laser CARS spectrum of pure rotational and vibrational $Q$-branch transitions in nitrogen. The spectrum was averaged on the intensified diode array detector for 120 laser shots. The narrowband dye laser wavelength was $602.50 \mathrm{~nm}$. The solid line is the experimental curve and the dashed line is the theoretical fit.

\section{References}

1. A. C. Eckbreth and T. J. Anderson, "Dual Broadband CARS for Simultaneous, Multiple Species Measurements," Appl. Opt. 24, 2731 (1985).

2. R. E. Teets, Unpublished presentation at the International Laser Science Conference, (1985).

3. R. P. Lucht, "Three-Laser Coherent AntiStokes Raman Scattering Measurements of Two Species," Opt. Lett. 12, 78 (1987).

4. R. P. Lucht, R. E. Palmer, and M. A. Maris, "Simultaneous Acquisition of Pure Rotational and Vibrational Nitrogen Spectra Using ThreeLaser CARS," to appear in Opt. Lett. (1987).

\section{Studies of Nitrogen Self-Broadening at High Temperature With Inverse Raman Spectroscopy}

\author{
L. A. Rahn, R. E. Palmer
}

Precise, high-resolution Raman spectra of combustion-related molecules have been measured using inverse Raman spectroscopy (IRS). The analysis of these measurements provides broadening coefficients, validation of collisional narrowing models, and, in conjunction with coherent anti-Stokes Raman spectroscopy (CARS), nonresonant susceptibilities. This information allows significant improvement in our ability to extract accurate temperatures and concentrations from CARS measurements in combustion systems.

The "quasi-cw" inverse Raman spectroscopy (IRS) technique used in this study is similar to that first developed by Owyoung. ${ }^{1}$ In the IRS technique, a pulsed pump laser induces a small absorption in a cw probe laser beam via the stimulated Raman effect. The primary advantages of IRS are that the signal intensity has a linear dependence on the imaginary part of the third-order nonlinear susceptibility, and the spectral resolution is limited only by the linewidths of the lasers used. High resolution is attained in the present experiment by using single-frequency, argon-ion probe and tunable $\mathrm{cw}$ dye pump lasers. The pump laser is amplified to a peak power of $\sim 1 \mathrm{MW}$ by the frequency-doubled output of an injection-seeded Nd:YAG laser operating at $20 \mathrm{~Hz}$. The resulting $\sim 45 \mathrm{MHz}\left(0.0015 \mathrm{~cm}^{-1}\right)$ resolution is complemented by a Raman shift accuracy of \pm 0.001 $\mathrm{cm}^{-1}$, determined with a traveling corner cube wavemeter.

Much of our work is focused on molecular nitrogen, since it is often studied in CARS thermometry and in relative concentration measurements. As part of the development of a comprehensive predictive capability for the nitrogen Raman spectrum in combustion environments, a complete study of $\mathrm{N}_{2} Q$-branch self-broadening 
coefficients for temperatures from $500 \mathrm{~K}$ to 1500 $\mathrm{K}$ and for $\mathrm{J}=2$ to $\mathrm{J}=30$ has been performed. Measurements were made in a furnace at pressures from 20 Torr to 760 Torr and were analyzed using the Galatry line profile to account for the Doppler effect. The resulting collisional self-broadening coefficients (FWHM) are compared to the $295 \mathrm{~K}$ values reported by Rosasco et $a l^{2}{ }^{2}$ in Table I.

We have compared these linewidths to predictions from a modified exponential gap scaling law that predicts transition rates $\gamma_{i j}$ between individual rotational states of nitrogen. The rate from state $\mathrm{J}_{i}$ to state $\mathrm{J}_{j}(i<j)$ is given by

$$
\begin{aligned}
\gamma_{\mathrm{ji}}= & \alpha \rho \frac{1-e^{-m}}{1-e^{-m T / T_{0}}}\left(\frac{T_{0}}{T}\right)^{0.5} \\
& \times\left(\frac{1+1.5 E_{i} / k T \delta}{1+1.5 E_{i} / k T}\right)^{2} \exp \left(-\beta\left|\Delta E_{i j}\right| / k T\right)
\end{aligned}
$$

Here, $\mathrm{p}$ is the pressure, $\mathrm{T}_{0}$ is a reference temperature, $\mathrm{E}_{i}$ is the rotational term energy (in $\mathrm{cm}^{-1}$ ) for state $\mathrm{J}_{i}$, and $\Delta \mathrm{E}_{i j}$ is the difference in rotational term energy between states $J_{i}$ and $\mathrm{J}_{j}$. The fitting parameters are $\alpha=0.0231 \mathrm{~cm}^{-1}$ $\mathrm{atm}^{-1}, \beta=1.67, \delta=1.21$, and $\mathrm{m}=0.1487$ at $\mathrm{T}_{0}=295 \mathrm{~K}$. The rate $\gamma_{i j}$ from $\mathrm{J}_{j}$ to $\mathrm{J}_{i}$ is determined by microscopic reversibility. Using these values, Equation 1 can be used to predict collisional broadening coefficients. The values from the IRS experiment (symbols) are compared to the scaling law predictions (solid lines) in Figure 1. As seen in the figure, agreement between the experimental values and scaling law predictions is excellent. Recent experimental results at 1000 $\mathrm{K}$ and $20 \mathrm{~atm}$ are also well described by this model.

High-resolution inverse Raman spectroscopy is proving to be a valuable tool for combustionrelated molecular spectroscopy. This technique provides important spectroscopic parameters such as J-dependent line-widths. In the present work, we have measured collisional self-broadening coefficients for the nitrogen $Q$-branch as a function of temperature and rotational quantum number. These measurements and the success of the modified exponential-gap scaling law in predicting linewidths are yielding significant

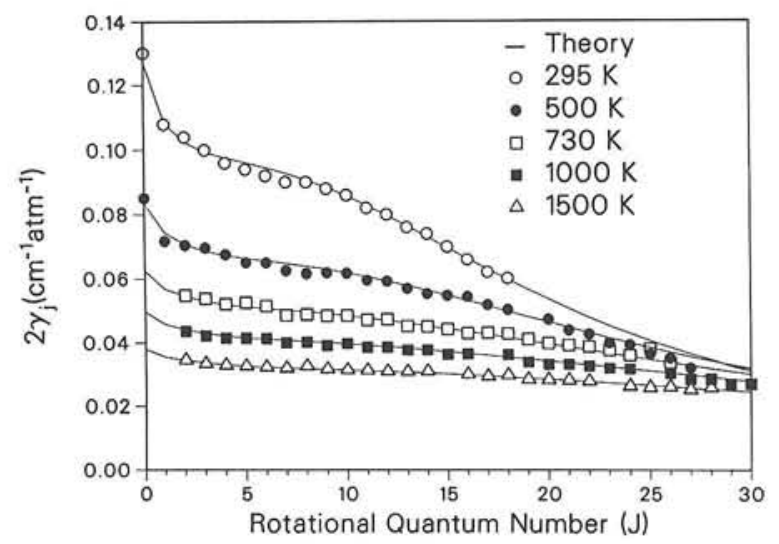

Figure 1. $\mathrm{N}_{2}$ collisional self-broadening coefficients (FWHM) as a function of temperature and rotational state. The solid curves are predictions from the modified exponential gap scaling law discussed in the text. The $295 \mathrm{~K}$ data is from Reference 2.

new information that is expected to aid both combustion measurements as well as our understanding of collisional relaxation processes. Presently, the scaling law discussed above predicts only collisional self-broadening coefficients for nitrogen. In actual flames, however, other collision partners are present. Two notable examples are $\mathrm{H}_{2} \mathrm{O}$ and $\mathrm{CO}_{2}$. Preliminary inverse Raman measurements have been made of $\mathrm{N}_{2}$ $Q$-branch transitions in the post-flame gases of an $\mathrm{H}_{2}-\mathrm{O}_{2}-\mathrm{N}_{2}$ flame, where the major (66\%) collision partner is $\mathrm{H}_{2} \mathrm{O}$ at $1570 \mathrm{~K}$. Other work in progress includes measurements of $\mathrm{CO}$ selfbroadening and broadening of $\mathrm{N}_{2}$ by hydrocarbons.

\section{References}

1. A. Owyoung, "High Resolution Coherent Raman Spectroscopy of Gases," in Laser Spectroscopy IV, ed. by $\mathrm{H}$. Walther and K. W. Rothe (Springer-Verlag, New York, NY, 1979), p. 175 .

2. G. J. Rosasco, W. Lempert, and W. S. Hurst, "Line Interference Effects in the Vibrational $Q$-Branch Spectra of $\mathrm{N}_{2}$ and CO," Chem. Phys. Lett. 97, 435 (1983). 
Table I

Collisional Self-Broadening Coefficients (FWHM) for the $\mathrm{N}_{2} Q$-Branch as a Function of Temperature and Rotational Quantum Number

\begin{tabular}{|c|c|c|c|c|c|}
\hline \multicolumn{6}{|c|}{$2 \gamma_{j}\left(\mathrm{~cm}^{-1} \mathrm{~atm}^{-1}\right)$} \\
\hline $\mathrm{J}$ & $295 \mathrm{~K}^{a}$ & $500 \mathrm{~K}$ & $730 \mathrm{~K}$ & $1000 \mathrm{~K}$ & $1500 \mathrm{~K}$ \\
\hline 0 & $0.130(30)$ & $0.0851(46)$ & - & - & - \\
\hline 1 & $0.108(12)$ & $0.0717(33)$ & - & - & - \\
\hline 2 & $0.104(4)$ & $0.0705(27)$ & $0.0547(22)$ & $0.0436(14)$ & $0.0345(14)$ \\
\hline 3 & $0.100(6)$ & $0.0697(44)$ & $0.0537(19)$ & $0.0422(14)$ & $0.0333(16)$ \\
\hline 4 & $0.096(4)$ & $0.0676(25)$ & $0.0520(20)$ & $0.0415(13)$ & $0.0328(12)$ \\
\hline 5 & $0.094(4)$ & $0.0651(21)$ & $0.0525(19)$ & $0.0414(14)$ & $0.0326(16)$ \\
\hline 6 & $0.092(4)$ & $0.0650(32)$ & $0.0514(17)$ & $0.0413(13)$ & $0.0321(18)$ \\
\hline 7 & $0.090(8)$ & $0.0625(26)$ & $0.0485(23)$ & $0.0399(13)$ & $0.0318(16)$ \\
\hline 8 & $0.090(4)$ & $0.0615(22)$ & $0.0488(20)$ & $0.0401(13)$ & $0.0324(11)$ \\
\hline 9 & $0.088(8)$ & $0.0618(21)$ & $0.0483(16)$ & $0.0390(13)$ & $0.0314(14)$ \\
\hline 10 & $0.086(6)$ & $0.0613(24)$ & $0.484(17)$ & $0.0397(15)$ & $0.0311(11)$ \\
\hline 11 & $0.082(10)$ & $0.0595(20)$ & $0.0471(18)$ & $0.0385(14)$ & $0.0310(14)$ \\
\hline 12 & $0.080(4)$ & $0.0591(21)$ & $0.0472(19)$ & $0.0385(12)$ & $0.0307(11)$ \\
\hline 13 & $0.076(6)$ & $0.0569(18)$ & $0.0451(23)$ & $0.0377(13)$ & $0.0305(11)$ \\
\hline 14 & $0.074(6)$ & $0.0551(21)$ & $0.0452(22)$ & $0.077(12)$ & $0.0306(10)$ \\
\hline 15 & $0.070(10)$ & $0.0546(18)$ & $0.0442(16)$ & $0.0362(12)$ & - \\
\hline 16 & $0.066(8)$ & $0.0542(17)$ & $0.0429(18)$ & $0.0363(11)$ & $0.0297(10)$ \\
\hline 17 & $0.062(16)$ & $0.0517(17)$ & $0.0430(16)$ & - & 0.0290 (11) \\
\hline 18 & $0.060(12)$ & $0.0502(18)$ & $0.0428(17)$ & $0.0361(12)$ & $0.2940(11)$ \\
\hline 19 & - & - & $0.0410(24)$ & $0.0336(12)$ & 0.0279 (13) \\
\hline 20 & - & $0.0472(15)$ & $0.0397(20)$ & $0.0329(11)$ & $0.0278(13)$ \\
\hline 21 & - & $0.0438(15)$ & $0.0391(25)$ & $0.0329(12)$ & $0.0276(13)$ \\
\hline 22 & - & $0.0426(13)$ & $0.0386(13)$ & $0.0323(10$ & $0.0273(10)$ \\
\hline 23 & - & $0.0398(14)$ & $0.0375(14)$ & $0.0316(12)$ & - \\
\hline 24 & - & $0.0392(12)$ & $0.0359(21)$ & $0.0313(10)$ & $0.0258(11)$ \\
\hline 25 & - & $0.0362(15)$ & $0.0380(17)$ & - & $0.0253(10)$ \\
\hline 26 & - & 0.0349 (11) & 0.0333 (12) & $0.0299(11)$ & $0.0255(9)$ \\
\hline 27 & - & $0.0316(16)$ & - & $0.0280(15)$ & $0.0247(12)$ \\
\hline 28 & - & - & - & $0.0280(10)$ & $0.0253(10)$ \\
\hline 29 & - & - & - & $0.0264(15)$ & - \\
\hline 30 & - & - & - & $0.0264(10)$ & - \\
\hline
\end{tabular}




\section{Comparison of Rotationally Inelastic Collision Models for $Q$-Branch Raman Spectra of $\mathrm{N}_{2}$}

\author{
L. A. Rahn, R. E. Palmer, M. L. Koszykowski, D. \\ A. Greenhalgh *
}

Recently, several fitting laws have been shown ${ }^{1-4}$ to successfully describe the selfbroadened linewidths of the $Q$-branch Raman spectrum of $\mathrm{N}_{2}$ over a wide temperature range for pressures at or below one atmosphere. These descriptions are similar in that they model stateto-state rotationally inelastic rates, which are then summed to yield the total rate out of a particular rotational state. This total outward rate determines the width of the corresponding $Q$-branch line. Thus, the Raman linewidths are assumed to be due only to rotationally inelastic collisions; i.e., vibrational relaxation and other sources of vibrational dephasing are assumed unimportant. Although the total rate out of each rotational state is essentially the same for each of these fitting laws, the laws are distinguished by two different assumptions about the scaling of the state-to-state rates with rotational energy defect. The modified exponential energy-gap (MEG) and the differential energygap (DEG) ${ }^{4}$ models are based on an exponential energy-gap scaling law, whereas the polynomial energy-gap (PEG) model $^{2}$ is based on a power law. At high pressures, collisional narrowing of the $Q$-branch becomes important and the spectrum is sensitive to the actual state-to-state rotationally inelastic rates. In this communication, we compare the ability of the MEG and PEG fitting laws to predict $\mathrm{N}_{2}$ inverse Raman spectra (IRS) acquired at $295 \mathrm{~K}$ and $10 \mathrm{~atm}$, as well as at $1000 \mathrm{~K}$ and $20 \mathrm{~atm}$.

Both the MEG and PEG fitting laws predict state-to-state rates that can be used to calculate collisionally narrowed $\mathrm{N}_{2} Q$-branch spectra with the S-matrix (also called the Gmatrix) method. The MEG law has been previously compared to high-pressure $\mathrm{N}_{2}$ Raman

* Engineering Sciences Division, AERE, Harwell, Oxfordshire, England. spectra acquired at $295 \mathrm{~K}$, while the PEG law remains untested in this respect. The DEG law has also been used to fit low-resolution CARS spectra acquired in an internal combustion engine at $824 \mathrm{~K}$ and $17.5 \mathrm{~atm} .{ }^{4}$ Only the MEG and PEG laws will be compared here, since the DEG law is essentially equivalent to the former. The IRS spectra of the $\mathrm{N}_{2} Q$-branch to which we compare these fitting laws are the $295-\mathrm{K}, 10$ atm spectrum, as well as a spectrum recently measured at $1000 \mathrm{~K}$ and $20 \mathrm{~atm}$. The IRS experiment used to make these measurements is described in Reference 1 and will not be discussed further here.

Inverse Raman spectra taken at $295 \mathrm{~K}$ and $10 \mathrm{~atm}$ are compared with the results of the calculations of the MEG and PEG models in Figure 1. Measurements at $1000 \mathrm{~K}$ and 20 atm are compared with the two theories in Figure 2. It is clear from Figure 1 that the PEG model does not treat collisional narrowing at $10 \mathrm{~atm}$ as well as the MEG model. In particular, it appears that the former underpredicts the effects of collisional narrowing. At $1000 \mathrm{~K}$ (Figure 2), the MEG model is still clearly superior, although it, too, is beginning to underpredict the degree of narrowing at this temperature.

The fact that both models predict the linewidths well, but differ greatly in their ability to predict collisional narrowing, is related to their different descriptions of the energy-gap scaling of the state-to-state rates. For both temperatures, the PEG rates fall off more slowly with energy defect than those of the MEG law. Because the sum of the rates must equal the linewidth for both laws, the slower fall-off for the PEG law results in lower rates at low energy defect, compared with the MEG law rates. The rates corresponding to the smallest energy gaps and S-matrix elements closest to the diagonal, are, at these pressures, the most important for collisional narrowing. Greater collisional narrowing is thus predicted by the MEG law, in agreement with the measured spectra.

In conclusion, a significant difference in the ability of two fitting laws to predict highpressure Raman spectra has been found. Even 


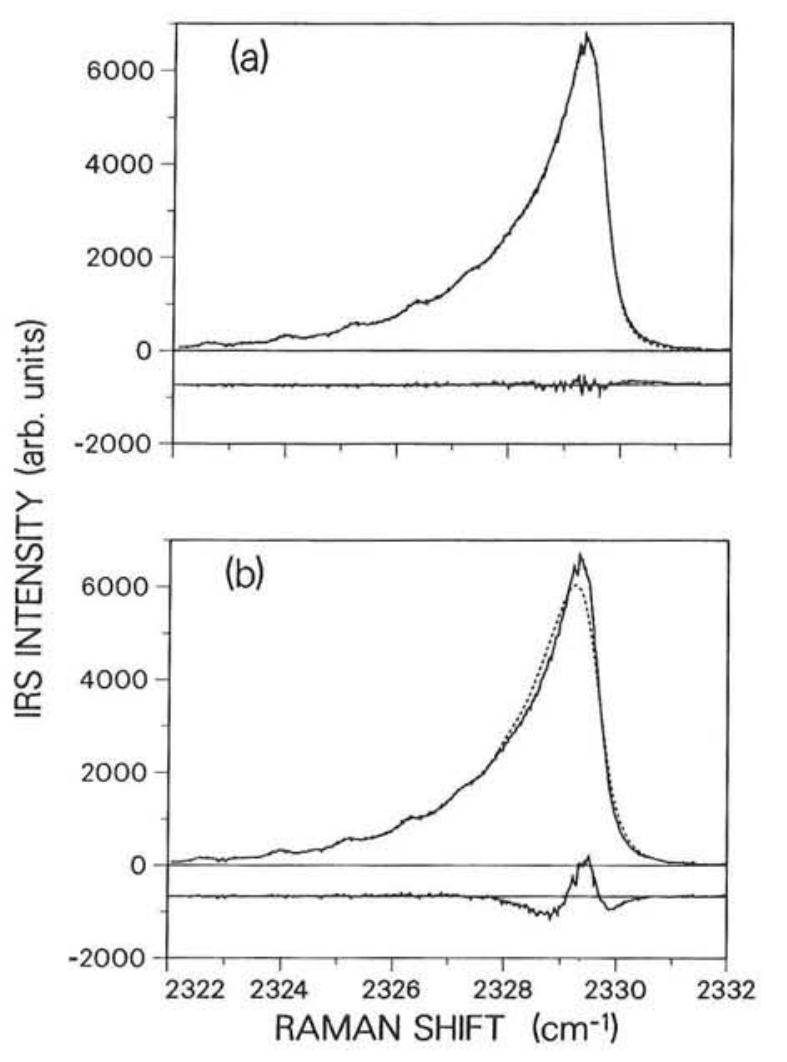

Figure 1. Inverse Raman spectra of $\mathrm{N}_{2}$ at 295 $K$ and 10 atm. In (a), the experimental spectrum (solid line) is compared with a calculation (dashed line) based on the MEG law (see text). The lower plot in (a) is the difference between experiment and theory, and has been shifted down for clarity. The plots in (b) are the same as in (a), but compared with theory based on the PEG law.

though a scaling law can not be uniquely defined by these measurements since they test only the S-matrix elements closest to the diagonal, the success of the exponential energy-gap laws is very encouraging. It is hoped that, as measurements at higher pressures with better signal-to-noise ratios are made, information on more and more elements of the S-matrix can be used to gradually build up a picture of the full S-matrix. The temperature dependence of these rates, in particular, requires more investigation. Both experimental and theoretical work in these areas is continuing and will be reported in a later publication.

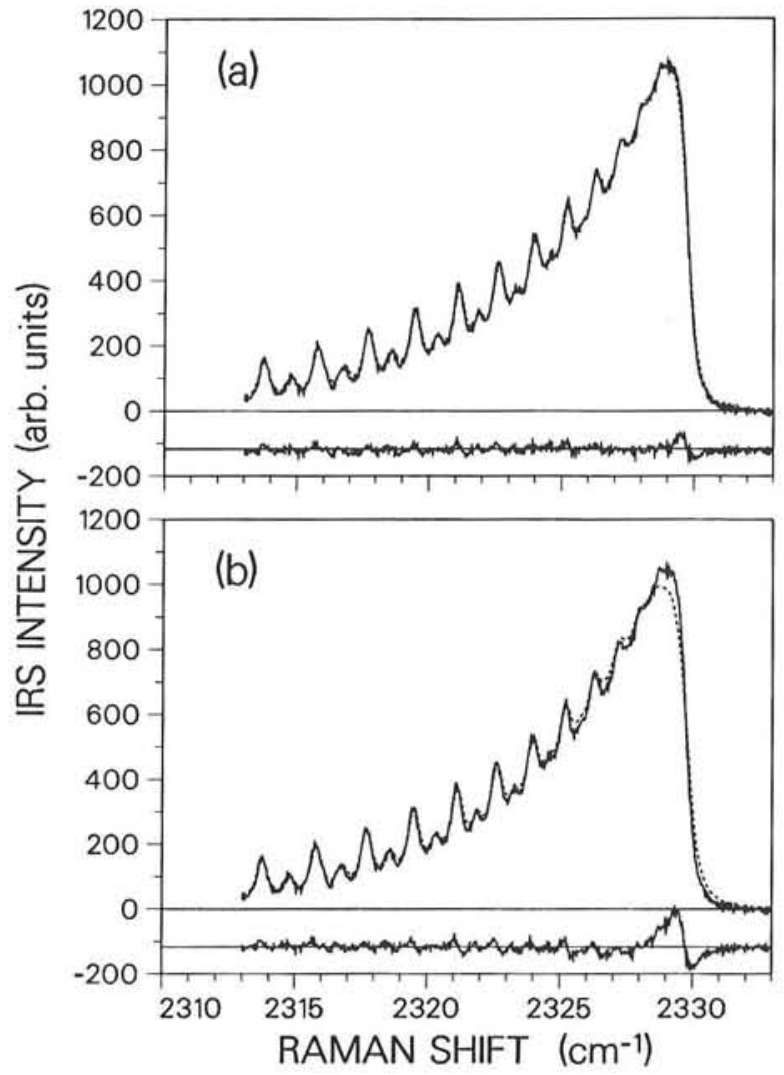

Figure 2. Inverse Raman spectra of $\mathrm{N}_{2}$ at 1000 $\mathrm{K}$ and 20 atm. In (a), the experimental spectrum (solid line) is compared with a calculation (dashed line) based on the MEG law (see text). The lower plot in (a) is the difference between experiment and theory, and has been shifted down for clarity. The plots in (b) are the same as in (a), but compared with theory based on the PEG law.

\section{References}

1. L. A. Rahn and R. E. Palmer, "Studies of Nitrogen Self-Broadening at High Temperature with Inverse Raman Spectroscopy," J. Opt. Soc. Am. B 3, 1164 (1986).

2. D. A. Greenhalgh, F. M. Porter and S. A. Barton, "A Polynomial Energy-Gap Model for Molecular Linewidths," J. Quant. Spectrosc. Radiat. Transfer 34, 95 (1985).

3. B. Lavoral, G. Millot, R. Saint-Loup, C. Wenger, H. Berger, J. P. Sala, J. Bonamy, and D. Robert, "Rotational Collisional Line Broadening at High Temperatures in the $\mathrm{N}_{2}$ Fundamental $Q$-Branch Studied with Stimulated Raman Spectroscopy," J. Physique 47, 417 (1986). 
4. D. A. Greenhalgh and F. M. Porter, "CARS Applications in Chemical Reactors, Combustion and Heat Transfer," Advances in Laser Science-1, edited by W. C. Stwalley and M. Lapp (AIP, New York, NY, 1985), p. 273.

\section{Collision-Induced Resonances in Four-Wave-Mixing and Induced-Grating Processes ${ }^{\dagger}$}

\author{
F. P. Trebino, L. A. Rahn
}

In four-wave-mixing and induced-grating processes, collisions can dephase quantum-mechanical amplitudes that would normally destructively interfere. ${ }^{1}$ As a result, otherwise forbidden "extra resonances" occur in these nonlinearoptical processes, having strengths that increase with pressure. In particular, Raman-type processes have, in addition to the well-known term proportional to the population difference, $\rho_{11}{ }^{(0)}$ $-\rho_{22}{ }^{(0)}$, an additional collision-induced term ${ }^{2}$ proportional to $\rho_{11}{ }^{(0)}+\rho_{22}{ }^{(0)}$. Thus, collisions can induce a Raman-type resonance between equally populated levels. In addition, collisions can cause population gratings to occur under nonresonant pumping conditions. ${ }^{3}$ In this process, it is the distortion introduced in the energy-level structure of the atom or molecule by collisions that allows the necessary population transfer to occur.

Collision-induced processes, such as these, are of great interest for several reasons. First, their existence confirms the validity of the perturbation-expansion approach used to calculate wave-mixing intensities. Second, they yield information on the microscopic nature of the collision process and allow the study of collisions in the absence of other obscuring processes. Specifically, the use of large detunings from resonance (of greater than the inverse collision

$\dagger$ Proceedings of the Second International Laser Science Conference, (1986), in press. duration) offers information regarding the nonimpact regime, i.e., about intracollisional events. Third, these processes could potentially form the basis of diagnostic techniques for studying various properties of the medium. For example, the resonance lineshapes indicate the temporal behavior of the longitudinal and transverse relaxation processes occurring in the medium. In addition, the population gratings mentioned above can take the form of ground-state-spin gratings - extremely long-lived effects (in the particularly useful geometry of a grating) that could be used in labelling applications.

Previous experimental work ${ }^{1-3}$ has demonstrated these and other collision-induced effects in sodium in the presence of various buffer gases. We have extended these measurements to a flame environment, where collision-sensitive processes are of great interest. Specifically, we have studied a sodium-seeded hydrogen-air flame using a range of detunings $\Delta$ from 0.5 to 100 $\mathrm{cm}^{-1}$. In this flame, we have observed populationgrating resonances and (Raman-type) Zeeman and hyperfine coherences.

In one set of experiments, parallel-polarized excitation beams illuminate the flame from slightly different angles. These beams are tuned to frequencies 2 to $100 \mathrm{~cm}^{-1}$ below the $\mathrm{Na} 3^{2} \mathrm{~S}_{1 / 2}-3^{2} \mathrm{P}_{1 / 2}$ transition, so they excite only sodium atoms undergoing collisions. Relaxation of excited atoms to the ground electronic state then occurs by collisional quenching. The efficiency of this process varies with hyperfine quantum numbers, $\left|F, m_{F}\right\rangle$, so the individual hyperfine population densities (equal in the dark) change as the intensity is turned up. Because the parallel-polarized excitation beams give rise to a spatially sinusoidal intensity, the effect of the optical excitation is to induce population gratings.

Diffraction from such gratings yields fourwave-mixing spectra in the flame typified by that shown in Figure 1, having both a broad and a very narrow component. 


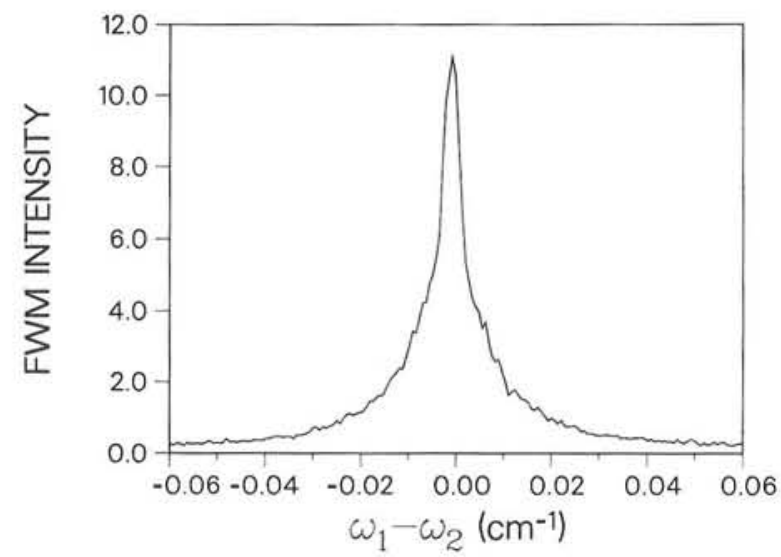

Figure 1. Four-wave-mixing spectrum of collisioninduced population gratings in sodium in a flame. Two components - a broad pedestal and a narrow spike - are present, corresponding to a rapidly decaying collision-quenched excited-electronicstate grating and a slowly decaying ground-statespin grating, respectively.

The very narrow component represents a ground-state-spin grating. ${ }^{3}$ Its width is mostly due to the laser linewidths and residual Doppler broadening, indicating an extremely long lifetime, which is reasonable in view of the spin-flip required for relaxation between the $\mathrm{F}=1$ and $\mathrm{F}=2$ hyperfine levels. The broad component is thought to be due to an excited-electronic-state $\left(3{ }^{2} \mathrm{P}_{1 / 2}\right)$ grating, the width of which is the inverse of the collisional quenching time of this state. The observed linewidth of this component indicates a collision-quenching timescale of 200 psec.

In another set of experiments, orthogonally polarized excitation beams, at frequencies a few wavenumbers above or below the $\mathrm{Na} 3^{2} \mathrm{~S}_{1 / 2^{-}}$ $3^{2} \mathrm{P}_{1 / 2}$ transition, illuminate the flame. Because orthogonally polarized beams do not interfere, no gratings can form, but now the two Ramantype effects, Zeeman and hyperfine coherences, do occur. In addition, collision-induced optical pumping of these levels is known to contribute to the four-wave-mixing lineshape. ${ }^{2}$ For zero magnetic field, the resonance condition for the Zeeman coherence is $\omega_{1}-\omega_{2}=0$, and for the ground-state hyperfine coherence in sodium, resonance occurs at $\omega_{1}-\omega_{2}= \pm 1772 \mathrm{MHz}$.

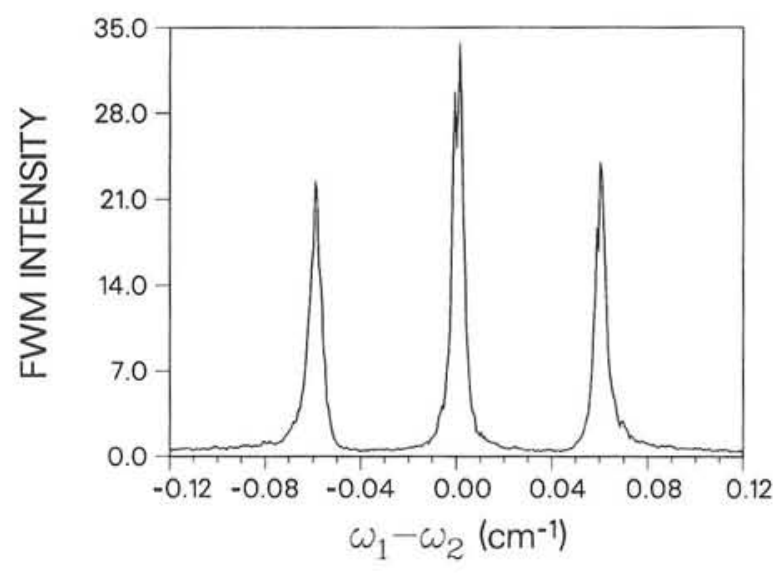

Figure 2. Four-wave-mixing spectrum of collisioninduced Zeeman $\left(\omega_{1}-\omega_{2}=0\right)$ and hyperfine $\left(\omega_{1}-\omega_{2}= \pm \omega_{\text {hyperfine }}= \pm 0.059 \mathrm{~cm}^{-1}\right)$ coherences. All linewidths are limited by residual Doppler broadening and the laser linewidths.

The effect of a magnetic field on the spectrum of collision-induced coherences is shown in Figure 3. For this experiment, a field of $350 \mathrm{G}$ was applied parallel to the polarization of the light at frequency $\omega_{1}$. Each hyperfine coherence splits into four components, and the Zeeman coherence splits into two groups of three barely resolved components.

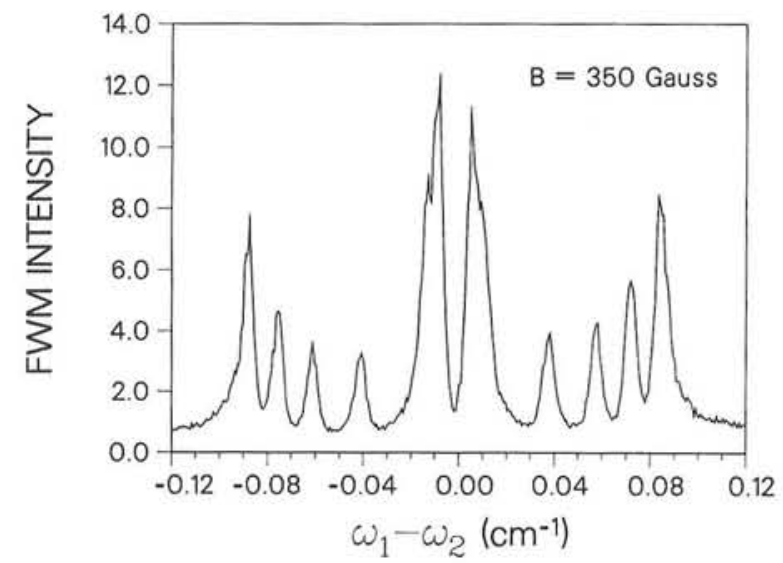

Figure 3. Four-wave-mixing spectrum of collisioninduced Zeeman $\left(\omega_{1}-\omega_{2}=0\right)$ and hyperfine $\left(\omega_{1}-\omega_{2}= \pm \omega_{\text {hyperfine }}= \pm 0.059 \mathbf{c m}^{-1}\right)$ coherences in a magnetic field of 350 Gauss. The hyperfine coherences split into four spectral lines each, while the Zeeman coherence splits into two barely resolvable triplets. 
Our studies show that collision-induced extra resonances in four-wave mixing (long-lived ground-state-spin gratings, Zeeman coherences and hyperfine coherences) are easily observed in flames. Such work should help to clear the way for diagnostic applications of these effects.

\section{References}

1. Y. Prior, A. R. Bogdan, M. Dagenais, and N. Bloembergen, "Pressure-Induced Extra Resonances in Four-Wave Mixing," Phys. Rev. Lett. 46, 111 (1981).

2. L. J. Rothberg and N. Bloembergen, "HighResolution Four-Wave Light-Mixing Studies of Collision-Induced Coherence in Na Vapor," Phys. Rev. A 30, 820 (1984).

3. L. J. Rothberg and N. Bloembergen, "HighResolution Studies of Collision-Induced Population Grating Resonances in Optical FourWave Mixing in Sodium Vapor," Phys. Rev. A 30, 2327 (1984).

\section{Photochemical Effects in 205-nm, Two-Photon-Excited Fluorescence Detection of Atomic Hydrogen in Flames ${ }^{\dagger}$}

\section{J. E. M. Goldsmith}

Multiphoton excitation using high-power pulsed UV laser systems is proving to be very useful in combustion studies, because conventional single-photon excitation of several species that are very important in such studies (e.g., atomic hydrogen) would otherwise require vacuum-ultraviolet radiation. There has been little discussion, however, of potential photochemical effects caused by the intense UV pulses. This report describes photochemical effects observed in two-photon-excited fluorescence detection of atomic hydrogen in flames, where the effect is

† Opt. Lett. 11, 416 (1986). especially serious because one of the species being produced photochemically is (supposedly) being measured in the experiment.

The atomic hydrogen measurements described in this report use two-photon excitation of the $\mathrm{n}=1 \rightarrow \mathrm{n}=3$ transition with $205-\mathrm{nm}$ radiation, and subsequent detection of Balmer- $\alpha$ radiation $(656 \mathrm{~nm})$ from $\mathrm{n}=3 \rightarrow \mathrm{n}=2$ decay. We used this scheme to measure relative concentration profiles in a lean (equivalence ratio 0.8 ), atmospheric-pressure, premixed hydrogenoxygen flame for comparison with profiles measured in the same flame using other techniques. Two such profiles are shown in Figure 1. (Note that these are relative concentration profiles only.)

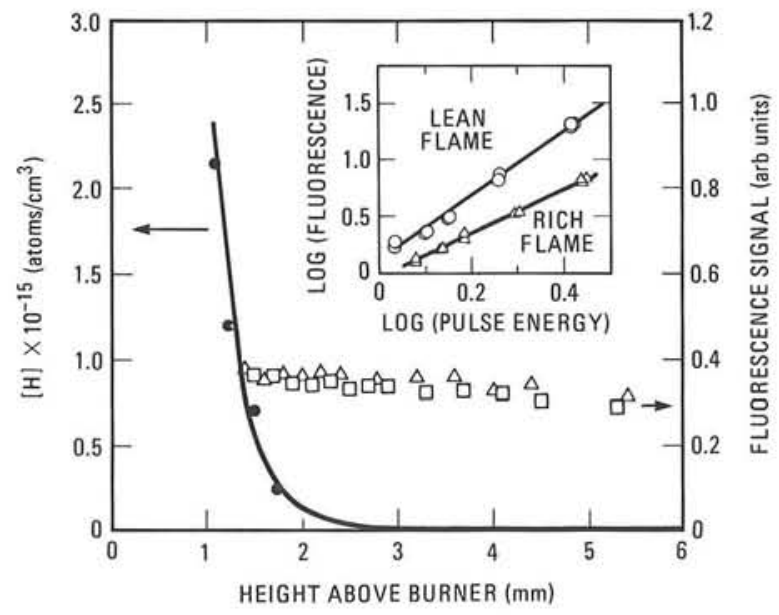

Figure 1. Atomic hydrogen concentration profiles in a lean (equivalence ratio 0.8), atmosphericpressure hydrogen-oxygen flame. The solid curve (absolute concentration) was calculated from the measured $\mathrm{OH}$ concentration assuming partial equilibrium, and the solid circles were measured using resonant multiphoton optogalvanic spectroscopy. Two-photon-excited fluorescence spectroscopy was used to measure the relative profiles represented by the squares (interference-filter fluorescence isolation) and triangles (spectrometer fluorescence isolation). Inset: intensity dependences of the fluorescence signals in the lean and in a rich hydrogen-oxygen flame.

For comparison, the solid curve in Figure 1 represents an absolute profile that was calculated from the $\mathrm{OH}$ concentration measured using laser absorption spectroscopy and assuming partial equilibrium, and the circles represent a relative concentration profile measured using resonant multiphoton optogalvanic spectroscopy. 
The profiles measured using fluorescence detection are not at all consistent with the other profiles, and are far too constant to represent a realistic atomic hydrogen concentration profile in this flame.

To further investigate this anomalous profile, we next measured the intensity dependence of the hydrogen fluorescence signal by varying the intensity of the 205-nm beam with neutraldensity filters. Log-log plots of two such measurements are shown in the inset in Figure 1. The rich-flame measurement was recorded $\sim 1.5$ $\mathrm{mm}$ above the burner surface in a rich (equivalence ratio 1.2) hydrogen-oxygen flame, a region with a relatively high natural atomic hydrogen concentration. The least-squares-fit slope of 1.9 \pm 0.1 ( $3 \sigma$ error estimate) for the log-log plot is consistent with the expected quadratic intensity dependence for the two-photon excitation process. The lean-flame measurement was recorded $\sim 3 \mathrm{~mm}$ above the burner surface in the lean (equivalence ratio 0.8) hydrogen-oxygen flame, a region with a natural atomic hydrogen concentration of $\sim 10^{14}$ atoms $/ \mathrm{cm}^{3}$ (see Figure 1). This point has a very similar water concentration $(\approx$ $90 \%)$ to the point used in the rich flame, but a roughly fifty-times lower atomic hydrogen concentration, as estimated from relative measurements using a different excitation scheme. The least-squares-fit slope of $2.8 \pm 0.2$ in the lean flame is consistent with a cubic intensity dependence, suggesting that an additional singlephoton process is involved in generating the signal.

We next set up an experiment to determine if photolysis of flame species could be causing the flat $\mathrm{H}$-atom concentration profile and cubic intensity dependence in the lean flame. A second laser system was used to produce $310-\mathrm{nm}$ radiation coincident with the $\mathrm{OH}(0,0)$ $\mathrm{P}_{1}(5)$ transition. This beam was combined spatially with the 205 -nm beam by a dichroic mirror, with the $310-\mathrm{nm}$ beam delayed by $5 \mathrm{~ns}$ and focused inside the larger waist of the $205-\mathrm{nm}$ beam. The $\mathrm{OH}$ fluorescence induced by the 310 $\mathrm{nm}$ beam was monitored at $310 \mathrm{~nm}$ as a function of the wavelengths of the two beams, with care taken that no observable fluorescence was created by the $205-\mathrm{nm}$ beam.

Two $\mathrm{OH}$ excitation spectra recorded with this configuration are shown in Figure 2.

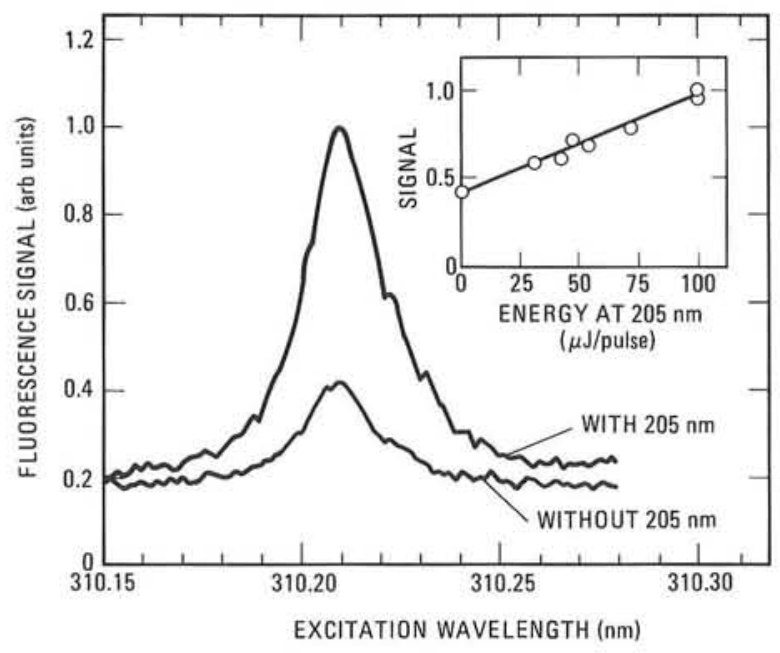

Figure 2. Fluorescence excitation spectra of the $\mathrm{OH}(0,0) \mathrm{P}_{1}(5)$ transition with and without an additional 205-nm beam present. Inset: Intensity dependence of the enhancement of the $\mathrm{OH}$ fluorescence signal produced by the $205-\mathrm{nm}$ beam.

The lower spectrum was recorded with the 205$\mathrm{nm}$ beam blocked; the background level in the wings of the spectrum is from scattered 310 $\mathrm{nm}$ laser radiation. The upper spectrum was recorded under identical conditions, but with the 205-nm beam present, which caused the $\mathrm{OH}$ signal to increase nearly fourfold. We were not able to observe any significant variation in $\mathrm{OH}$ enhancement with wavelength over the region $204.6-205.9 \mathrm{~nm}$. The inset shows the dependence of the increase of the peak $\mathrm{OH}$ signal (with scattered light subtracted) on the pulse energy of the 205-nm beam, indicating a linear dependence of the enhanced $\mathrm{OH}$ signal (and therefore presumably concentration) on the 205$\mathrm{nm}$ pulse energy.

The magnitude of the enhanced $\mathrm{OH}$ signal did not vary significantly as a function of height above the burner over the range $2-10$ $\mathrm{mm}$. Combined with the flat atomic hydrogen concentration profile observed in the lean flame (Figure 1), this result suggests that a stable flame product is being photolyzed, since the concentration of flame radicals decreases sharply 
over this range of heights. From this and other evidence, we conclude that the $205-\mathrm{nm}$ beam is producing $\mathrm{OH}$ and $\mathrm{H}$ via single-photon photolysis of water produced in the flame. We made a rough estimate of the photolysis cross-section by using the scheme described above to measure the change in observed $\mathrm{OH}$ fluorescence for a known pulse energy and spot size in a flame with known concentrations of $\mathrm{OH}$ and water (the same lean hydrogen/oxygen flame studied previously). The result was the creation of 1.4 $\times 10^{15}$ mole- cules $/ \mathrm{cm}^{3}$ of $\mathrm{OH}$, and a photolysis cross-section of $10^{-20} \mathrm{~cm}^{2}$. This seems like a rather large value, since extrapolation to longer wavelengths of the absorption coefficient of water at $0^{\circ} \mathrm{C}$ in the vacuum ultraviolet would suggest a value of less than $10^{-21} \mathrm{~cm}^{2}$. It has been estimated that the first vibrationally excited state of water has an absorption cross-section $\approx 500$ times larger than the ground state at 193 $\mathrm{nm}$. Thus, it is not surprising that the photolysis cross-section is substantially higher than $10^{-21} \mathrm{~cm}^{2}$ in a flame, which has a much higher population of excited vibrational states than water at $0^{\circ} \mathrm{C}$.

Photolysis of water to create $1.4 \times 10^{15}$ molecules $/ \mathrm{cm}^{3}$ of $\mathrm{OH}$ implies the creation of the same number of hydrogen atoms. As shown in Figure 1, this value is greater than the natural atomic hydrogen concentration in the same flame in the range of heights accessible in the current study ( $>1.5 \mathrm{~mm}$ above the burner). Since the temperature and water concentration change very little in the post-flame gases, it is not surprising that the atomic hydrogen flame profiles measured using fluorescence detection are essentially flat.

This study demonstrates one of several types of photochemical effects that can cause erroneous results if considerable care is not exercised in the use of high-power UV laser pulses for making spectroscopic measurements. In particular, the pulses can create artificially high concentrations, via photolysis processes, of the species being measured, even in a hydrogenoxygen flame. This problem may be even more serious in hydrocarbon flames, which contain a larger number of chemical species. Determination of detection limits for any given method thus cannot be based solely on signal-to-noise considerations, but must also take into account the degree to which the species being measured is also being produced by the laser pulse. Alternatively, photolytic production of radicals in excess (but known) concentrations in flames may provide the possibility of calibrating detection methods for making absolute concentration measurements in flame environments.

\section{Comparison of Laser-Induced Fluorescence Methods for Measurement of Hydroxyl Concentration in Flames ${ }^{\dagger}$}

\section{N. M. Laurendeau, ${ }^{*}$ J. E. M. Goldsmith}

In the last decade, a variety of laser-induced fluorescence methods have become available for measurement of intermediate species in flames. However, few comparisons have been made to assess the relative advantages and disadvantages of competing diagnostic methods, and to determine the best method for a given application to combustion. In this paper, we provide such a comparison for five distinct fluorescence methods. The comparison is based on measured $\mathrm{OH}$ concentration profiles obtained with four nonsaturating methods in a lean (equivalence ratio 0.6) $\mathrm{H}_{2} / \mathrm{O}_{2} / \mathrm{Ar}$ flame at 72 Torr that has previously been used for $\mathrm{OH}$ measurements via laser-saturated fluorescence (LSF). ${ }^{1}$

The five fluorescence techniques all operate by exciting an individual rovibronic transition within the $\mathrm{A}^{2} \Sigma^{+}-\mathrm{X}^{2} \Pi$ sys-tem of $\mathrm{OH}$. In addition to the LSF method, the four nonsaturating methods are: (1) single-photon excitation within the $(0,0)$ band followed by broadband detection of the entire $(0,0)$ manifold; (2) single-photon excitation within the $(0,0)$ system

\footnotetext{
$\dagger$ Submitted to Applied Optics (1986).

* Purdue University, West Lafayette, IN.
} 
followed by detection of the $(0,1)$ manifold; $(3)$ single-photon excitation within the $(1,0)$ band followed by broadband detection of the $(1,1)$ manifold; and (4) two-photon excitation within the $(0,0)$ system followed by detection of the $(0,0)$ manifold. For simplicity in the remainder of this paper, the four non-saturating methods are labeled $\operatorname{SPF}(0,0), \operatorname{SPF}(0,1), \operatorname{SPF}(1,1)$, and TPF, respectively, where the values of $\left(\mathrm{v}^{\prime}, \mathrm{v}^{\prime \prime}\right)$ refer to the $\mathrm{A} \rightarrow \mathrm{X}$ vibronic bands that produce the detected fluorescence.

In general, the measurements agree within our limits of reproducibility $(\sim 5 \%)$, especially considering the major changes in the apparatus required to switch between the TPF and SPF methods. The $\operatorname{SPF}(0,0)$ and the $\operatorname{SPF}(0,1)$ profiles are most amenable to comparison, since the only change made between the two was the insertion of an additional filter in front of the photomultiplier. The $\operatorname{SPF}(0,1)$ profile is approximately $10 \%$ higher near the peak owing to negligible self-absorption of radiation in the detected $(0,1)$ band. The $\operatorname{SPF}(1,1)$ measurement was made at a later date, after the gas-flow system had been modified, so a detailed comparison between the $\operatorname{SPF}(1,1)$ profile and the other profiles is hampered by uncertainties with respect to exact reproduction of the flame conditions. Under identical conditions, we would expect the peak of the $\operatorname{SPF}(1,1)$ profile to be slightly higher than that of the $\operatorname{SPF}(0,1)$ profile due to negligible absorption of the laser beam before it reaches the probe volume.

The experimental flame was modeled with the Sandia flat-flame code assuming no entrainment and negligible radiative heat losses above the burner. We found that a burner surface temperature of $400 \mathrm{~K}$ gave a theoretical temperature profile to within $50 \mathrm{~K}$ of an experimental profile ${ }^{1}$ for the same flame. Using laser absorption spectroscopy, we measured a peak $\mathrm{OH}$ concentration of $1.8 \times 10^{15} \mathrm{~cm}^{-3}$ in this flame, assuming a $40 \%$ decrease in effective optical path length relative to physical path length as determined in a previous study. This value is in excellent agreement with the calculated peak $\mathrm{OH}$ concentration of $2.0 \times 10^{15} \mathrm{~cm}^{-3}$.
The $\mathrm{OH}$ concentration and temperature profiles resulting from the model are plotted as the solid curves in Figure 1.

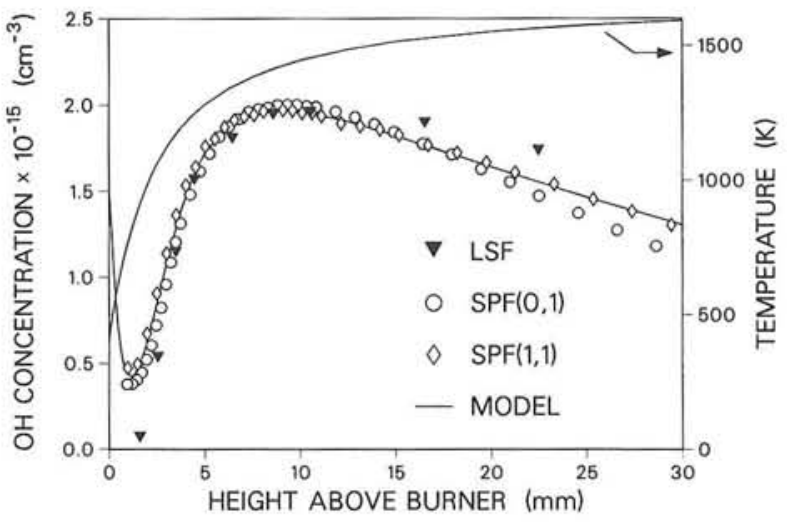

Figure 1. $\mathrm{OH}$ concentration and temperature profiles in the lean $\mathrm{H}_{2} / \mathrm{O}_{2} / \mathrm{Ar}$ flame at a total pressure of 72 Torr. The relative $\operatorname{SPF}(0,1)$ and $\operatorname{SPF}(1,1)$ profiles and the relative LSF profile have been scaled vertically for best agreement with the calculated absolute profile (solid curve).

The most relevant characteristics of these five methods with respect to selection for a given application are summarized in Table I. All methods but $\operatorname{SPF}(0,0)$ are capable of rejecting scattered laser radiation, which is of special importance near surfaces and in particleladen flows. Self-absorption of emitted $\mathrm{OH}$ fluorescence and laser-beam attenuation by $\mathrm{OH}$ absorption can cause difficulties in flames containing relatively high concentrations of $\mathrm{OH}$; SPF $(1,1)$ most successfully circumvents these potential problems. The most important characteristic of LSF is its insensitivity to electronic quenching, a feature not shared by any of the other methods. Other LSF characteristics include the capability for single-pulse measurements and for absolute concentration measurements without in situ calibration via absorption. These characteristics are especially useful for determination of probability density functions in turbulent flames, where calibration in the flame itself is nearly impossible and variations in quenching rate arise due to turbulent fluctuations.

The final column of Table I lists detection limits, which are estimated for the SPF 


\section{Table I}

Detection Characteristics and Estimated Detection Limits for Measurements of $\mathrm{OH}$ Fluorescence

\begin{tabular}{cccccc}
\hline Method & $\begin{array}{c}\text { Sensitive to } \\
\text { Scattered } \\
\text { Light }\end{array}$ & $\begin{array}{c}\text { Sensitive to } \\
\text { Self- } \\
\text { Absorption }\end{array}$ & $\begin{array}{c}\text { Sensitive to } \\
\text { Laser } \\
\text { Absorption }\end{array}$ & $\begin{array}{c}\text { Sensitive to } \\
\text { Quenching }\end{array}$ & $\begin{array}{c}\text { Detection } \\
\text { Limit }\left(\mathrm{cm}^{-3}\right)^{a}\end{array}$ \\
\hline $\operatorname{SPF}(0,0)$ & Yes & Yes & Yes & Yes & $10^{12}$ \\
$\operatorname{SPF}(0,1)$ & No & No & Yes & Yes & $10^{12}$ \\
$\operatorname{SPF}(1,1)$ & No & No & No & Yes & $10^{11}$ \\
TPF & No & Yes & No & Yes & $10^{13}$ \\
LSF & No & Yes & No & No & $10^{12}$ \\
\hline
\end{tabular}

${ }^{a}$ At 72 Torr total pressure.

and TPF methods by measuring the signal-tonoise ratio of excitation scans across relevant $\mathrm{OH}$ transitions. The detection limit for LSF is obtained from the estimated signal-to-noise ratio for an $\mathrm{OH}$ fluorescence spectrum in a rich $\mathrm{CH}_{4} / \mathrm{O}_{2} / \mathrm{N}_{2}$ flame, for which the background is mostly due to rotational Raman scattering from $\mathrm{N}_{2}$. These estimated detection limits are for $\mathrm{H}_{2} / \mathrm{O}_{2} /$ Ar flames similar to that used in this investigation, and thus the quoted values are relevant to a total pressure of 72 Torr with water as the primary collision partner. Because the rate coefficient for electronic quenching varies approximately linearly with pressure, the $\mathrm{OH}$ number density at the detection limit is also proportional to the total pressure, with the mole-fraction detectivity being roughly independent of pressure. LSF is not sensitive to quenching, so the value given is essentially independent of both pressure and collision partner. TPF has the lowest sensitivity because of the weakness of the two-photon excitation process. The $\operatorname{SPF}(0,1)$ and $\operatorname{SPF}(1,1)$ methods have similar detection limits, with a higher limit for $\operatorname{SPF}(0,0)$ because of its sensitivity to scattered laser radiation. LSF provides less sensitivity at 72 Torr than the optimum value obtained with the $\operatorname{SPF}(0,1)$ or $\operatorname{SPF}(1,1)$ methods because the LSF fluorescence signal originates from a single rovibronic transition, whereas that for the SPF methods encompasses the entire vibrational band. However, at higher pressures, electronic quenching reduces the SPF signal; hence, above approximately $1 \mathrm{~atm}$, LSF offers both the least sensitivity to quenching and the best detection limit.

\section{Reference}

1. R. P. Lucht, D. W. Sweeney, and N. M. Laurendeau, "Laser-Saturated Fluorescence Measurements of $\mathrm{OH}$ Concentration in Flames," Comb. Flame 50, 189 (1983). 


\section{Laser-Induced Fluorescence and Resonance-Enhanced Multiphoton Ionization of Jet-Cooled Chloronaphthalenes}

\section{E. A. Rohlfing, D. W. Chandler}

The ultrasensitive detection of chlorinated aromatic hydrocarbons, such as polychlorinated biphenyls (PCB's) and polychlorinated dibenzop-dioxins (PCDD's), is currently a problem of intense concern due to the toxic and/or carcinogenic nature of these species and their widespread presence in the environment. It is now known that combustion is a major source of PCDD's and that a wide variety of combustors produce PCDD's, including municipal waste incinerators, coal and fossil fuel plants, chemical waste burners, fireplaces, and wood stoves. We are investigating methods for the detection of chlorinated aromatics in the gas phase. There are three requirements for a detection technique. First, it must be ultrasensitive (ppb level or less). Second, it must be isomerically selective, which means that it must differentiate between molecules that contain the same number of chlorine atoms but that differ in the position of the chlorine substituents on the aromatic rings.

Such isomers often have dramatically different toxicities. Third, the detection technique should be real-time or nearly real-time so that it can provide feedback to the combustor in order to optimize the destruction of the chlorinated aromatics.

In this paper, we report on the use of laserinduced fluorescence (LIF) and resonance-enhanced multiphoton ionization (REMPI) for the detection of mono- and dichloro-naphthalenes. ${ }^{1}$ These molecules are similar in nature to PCB's and are good surrogates for more complicated species such as PCDD's. In our experiments, chloronaphthalene vapor is diluted to approximately $10 \mathrm{ppm}$ in 3-4 atm helium and allowed to expand freely through the $0.5 \mathrm{~mm}$ orifice of a pulsed valve into vacuum. This free jet expansion cools the molecules to very low rotational temperatures (typically $<10 \mathrm{~K}$ ) and dramatically simplifies the LIF or REMPI excitation spectrum by narrowing rotational contours and eliminating vibrational hot bands. The expansion is crossed at approximately 50 orifice diameters downstream by a frequency doubled dye laser beam. For LIF studies, the fluorescence is collected, filtered, and imaged onto a photomultiplier tube; the photomultiplier signal is amplified and processed with a boxcar averager. For REMPI studies, the jet expands into the ionization region of a small time-of-flight (TOF) mass spectrometer. Photoions generated by the UV laser are accelerated, allowed to drift, and detected with a dual microchannel plate; this signal is also amplified and processed with a boxcar averager. In all cases, the initial one photon excitation step is to the first excited singlet state, an $\mathrm{S}_{1}-\mathrm{S}_{0}$ transition. Since the $\mathrm{S}_{1}$ state lies less than halfway to the ionization potential, additional absorption of two photons is necessary to produce parent ions in a $1+2$ REMPI process.

In Figure 1, the LIF spectrum of jet-cooled 2-chloro-naphthalene $(2 \mathrm{CN})$ is displayed. Also indicated in Figure 1 are the origin and first vibronic band of naphthalene, which was present at a small concentration in the pulsed valve. This spectrum clearly demonstrates that $2 \mathrm{CN}$ can be differentiated from naphthalene based on the red shift of its origin band. In naphthalene, the purely electronic $\mathrm{S}_{1}-\mathrm{S}_{0}$ transition is weak and the spectrum derives much of its intensity from vibronic interaction with the second excited singlet state, $\mathrm{S}_{2}$. Thus the origin is weak and the most intense bands are the vibronic bands that correspond to the antisymmetric vibrational modes that couple $\mathrm{S}_{1}$ and $\mathrm{S}_{2}$. In $2 \mathrm{CN}$, however, the origin is by far the strongest band and the vibronic bands are now dominated by progressions in totally symmetric vibrational modes. Clearly, the addition of a $\mathrm{Cl}$ substituent greatly increases the $\mathrm{S}_{1}-\mathrm{S}_{0}$ oscillator strength. The addition of $\mathrm{Cl}$ also enhances the rate of intersystem crossing to the triplet manifold, thereby reducing the lifetime of the $\mathrm{S}_{1}$ state (from $\sim 300 \mathrm{~ns}$ in naphthalene to $\sim 30 \mathrm{~ns}$ in 2CN) and fluorescence quantum yield (from $\sim 0.24$ in naphthalene to $\sim 0.007$ in $2 \mathrm{CN}$ ). The 
reduced quantum yield makes LIF far less sensitive toward $2 \mathrm{CN}$ than it is toward naphthalene and explains why the naphthalene bands are seen so strongly in Figure 1. Nonetheless, we estimate a detection limit of $\sim 30 \mathrm{ppb}$ for $2 \mathrm{CN}$ under our experimental conditions.

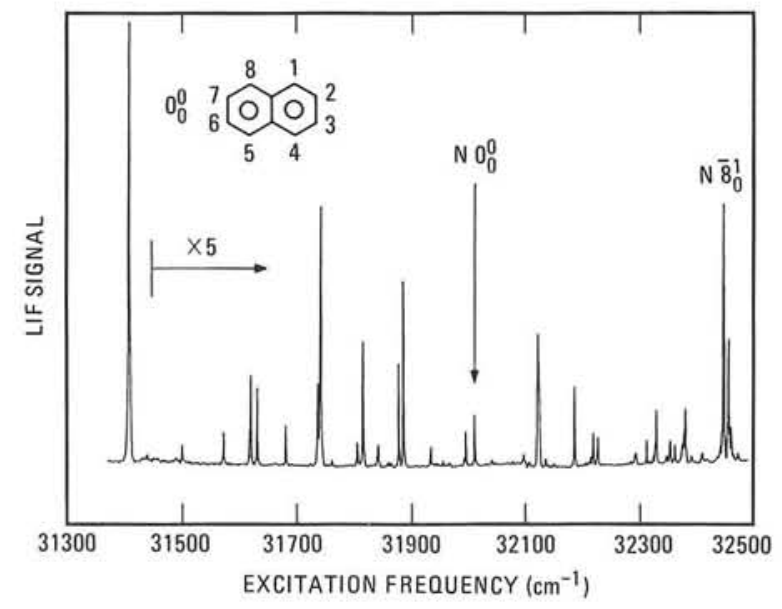

Figure 1. The jet-cooled LIF spectrum of 2chloro-naphthalene (2CN) seeded at approximately $10 \mathrm{ppm}$ in $3 \mathrm{~atm}$ helium. The $\mathrm{S}_{1}-\mathrm{S}_{0}$ origin band is indicated as are the origin and first vibronic band of naphthalene (N), which was present in low concentration in the pulsed valve. Also shown is the labelling scheme for the chloronaphthalene derivatives.

The addition of a second chlorine substituent to naphthalene further reduces the $\mathrm{S}_{1}$ lifetime and fluorescence quantum yield, so much so, in fact, that we were unable to observe fluorescence from any of the dichloronaphthalenes (DCN's). However, REMPI was found to be much more sensitive under similar conditions than LIF, and we have obtained jet-cooled REMPI spectra of a series of DCN's. In Figure 2, we display the parent ion $\left(\mathrm{C}_{10} \mathrm{H}_{6} \mathrm{Cl}_{2}{ }^{+}\right)$ REMPI spectra of 1,8 and 1,4 DCN.

Unlike the $\mathrm{S}_{1}-\mathrm{S}_{0}$ spectra of the monochloronaphthalenes, the origin bands are not dominant in the DCN spectra, indicating that the purely electronic transition is weaker in the DCNs and that vibronically-induced transition strength may again be important. This could explain the dense vibronic structure, especially in the 1,8 spectrum, where there may be a combination of progressions in totally symmetric

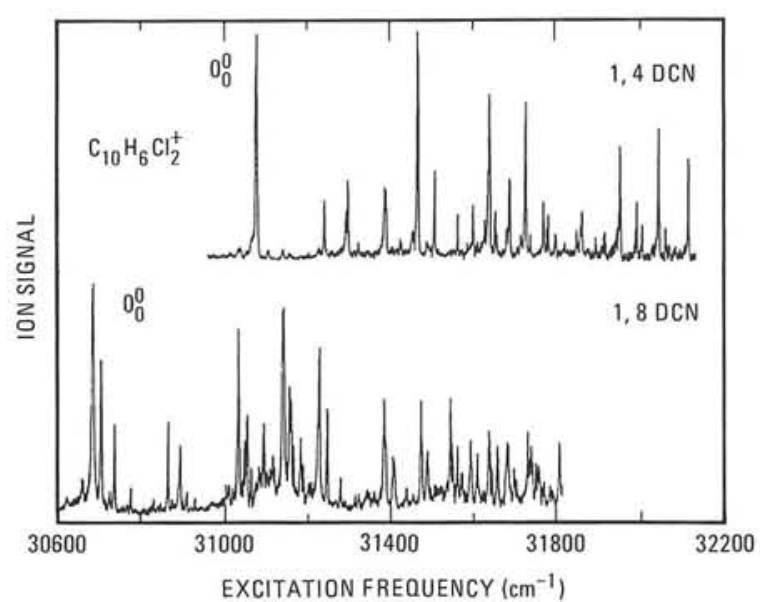

Figure 2. The jet-cooled resonance-enhanced multiphoton ionization (REMPI) spectra of 1,4 and 1,8 dichloronaphthalene (DCN). Expansion conditions are similar to those in Figure 1 and the REMPI process is a one-photon excitation of the $\mathrm{S}_{1}-\mathrm{S}_{0}$ transition followed by two-photon ionization. The ion signal monitored is the parent ion, $\mathrm{C}_{10} \mathrm{H}_{6} \mathrm{Cl}_{2}{ }^{+}$, and the origin bands are labelled.

modes and false origins due to vibronically active modes. At the laser intensities necessary to effect $1+2$ REMPI, there is substantial fragmentation of the DCN parent ion into small fragments. For all the DCN's the relative intensities of the $\mathrm{C}_{1}{ }^{+}-\mathrm{C}_{10}{ }^{+}$fragments are similar. However, the DCN's in which the two chlorines are on adjacent carbons (1,2 and 2,3 DCN) show a larger ratio of fragment to parent ion intensities. We speculate that in these DCN's, $\mathrm{C}_{10} \mathrm{H}_{6}{ }^{+}$is formed via the concerted loss of $\mathrm{Cl}_{2}$ from the parent ion, a process that is energetically possible when the parent ion absorbs one UV photon (an overall four photon process). Conversely, in DCN's that have the chlorines separated by more than one carbon, $\mathrm{C}_{10} \mathrm{H}_{6}{ }^{+}$ is formed by sequential loss of two $\mathrm{Cl}$ atoms from the parent ion, a process which requires the absorption of two photons (an overall five photon process). Subsequent fragmentation to smaller fragments then occurs independently of how $\mathrm{C}_{10} \mathrm{H}_{6}{ }^{+}$was initially formed.

The jet-cooled REMPI spectra in Figure 2 clearly show that the 1,4 and $1,8 \mathrm{DCN}$ isomers can be distinguished based on the positions of their $\mathrm{S}_{1}-\mathrm{S}_{0}$ origins. The jet-cooled LIF and REMPI origin red shifts for the mono- and dichloro-naphthalenes are displayed in Table 
I, where they are compared to the shifts observed for these species in a cryogenic naphthalene host matrix. ${ }^{2}$ Agreement between gas phase and matrix data is fair, except for the 1,5 DCN isomer. The data in Table I demonstrate that isomeric selectivity for the CN's and DCN's is possible via jet-cooled $\mathrm{S}_{1}-\mathrm{S}_{0}$ spectroscopy. Jetcooling is absolutely essential for this selectivity; the small differences in origin position for the DCN's could never be resolved at room temperature.

Table I

$\mathrm{S}_{1}-\mathrm{S}_{0}$ Origin Red Shifts for Chloronaphthalenes

Red shift from naphthalene $\left(\mathrm{cm}^{-1}\right)$

\begin{tabular}{ccc}
\hline Compound & Gas Phase & Matrix $^{a}$ \\
\hline 1 CN & 436 & $436^{b}$ \\
2 CN & 601 & 618,666 \\
1,2 DCN & 903 & 1069 \\
1,4 DCN & 933 & 995 \\
1,5 DCN & 906 & 1426 \\
1,8 DCN & 1327 & 1152,1453 \\
2,3 DCN & 1013 & 957 \\
2,7 DCN & 907 & 995 \\
\multicolumn{2}{c}{} \\
${ }^{a}$ LIF and TPE in naphthalene host crystal, from Refer- \\
ence 2. \\
${ }^{b}$ Adjusted to match gas phase 1 CN shift. \\
\hline
\end{tabular}

\section{References}

1. E. A. Rohlfing and D. W. Chandler, "Laser Spectroscopy of Jet-Cooled Chlorinated Aromatic Hydrocarbons," Proceedings of the 1986 International Laser Science Conference, (1986), in press.

2. C. F. Pace and J. R. Maple, "Laser-Induced Fluorescence Spectrometry of Aromatic Hydrocarbon Derivatives in Vapor-Deposited Parent Molecule Matrices," Anal. Chem. 57, 940 (1985).

\section{High Speed Thermometry Using Two-Line Atomic Fluorescence $^{\dagger}$}

\author{
J. E. Dec, ${ }^{*}$ J. O. Keller
}

The study of turbulent combustion, and turbulent heat transfer from combustion products, requires accurate, spatially and temporally resolved temperature measurements. Although several nonintrusive optical temperature techniques have been developed, two-line atomic fluorescence (TLAF), with continuous-wave (cw) lasers, has several advantages. Among these are high accuracy and precision, applicability to low temperature flows $(<1000 \mathrm{~K})$, measurements in the presence of strong elastic scattering, high data rates, and the ability to calculate temperatures at near real time.

The principles of the TLAF technique are straightforward. The flow is seeded with an atomic species (indium in this study) which has suitable electronic energy levels. The first excited state (level 1) must be close to the ground state (level 0) so both are populated at the temperature to be measured ( 500 to $2500 \mathrm{~K}$ in this study). The second excited state (level 2) must be sufficiently high so that its natural population is virtually zero. However, level 2 must have allowed transitions with 0 and 1 . The probe volume is alternately illuminated with lasers whose wavelengths are tuned to pump the $0-2(410.18 \mathrm{~nm})$ and the $1-2(451.13 \mathrm{~nm})$ transitions. While the 0-2 transition is pumped, the spontaneous fluorescence of the 2-1 decay is measured. Similarly, while the 1-2 transition is pumped, the 2-0 fluorescence is measured. The ratio of the fluorescence powers is proportional to the original atomic population distribution between energy levels 0 and 1 . The population distribution is then related to the temperature through Boltzmann statistics.

\footnotetext{
† Sandia Report SAND86-8622; also, Twenty-first (International) Symposium on Combustion (The Combustion Institute, 1986), in press.

* University of Michigan at Ann Arbor.
} 
The required theoretical development is discussed in the literature by Alkemade ${ }^{1}$ and others. This development shows that the temperature may be obtained from the ratio of the fluorescence powers by a simple one-equation calculation. Thus, with a laboratory computer, the temperature and temperature statistics are calculated from the fluorescence signals in a matter of seconds (near real time). Furthermore, this relationship is independent of the collisional quenching coefficient and atomic seed number density, terms often required in other fluorescence temperature techniques that are difficult to determine accurately.

An error analysis showed that indium is the element best suited for use as the atomic seed at typical combustion and post combustion temperatures $(500-2500 \mathrm{~K})$. With this seed, the error analysis showed that, at $2500 \mathrm{~K}$ temperatures a precision of $\pm 2 \%$ can be obtained at data rates as high as $10 \mathrm{kHz}$, and at $500 \mathrm{~K}$, a precision of $\pm 5 \%$ is possible at rates up to 5 $\mathrm{kHz}$. These speeds are fast enough to follow turbulent temperature fluctuations inmost flows, and the accuracies are as good as or better than those available from other nonintrusive techniques. As will be seen below, the actual experimental errors were slightly higher than predicted. A minimum standard deviation of $3.75 \%$ was obtained at about $2000 \mathrm{~K}$ whereas the error analysis predicted $\pm 3.0 \%$. It is believed that this discrepancy arises from mode shifting of the laser in frequency space.

Figure 1 shows the TLAF experimental setup. Fluorescence of the indium is induced by two cw standing wave dye lasers, each pumped with an argon ion laser. The laser beams are modulated by a mechanical chopper at frequencies up to $5 \mathrm{kHz}$. The beams are positioned with respect to the chopper blade so that while one beam passes through, the output of the opposite beam is completely blocked. A single lens focuses both beams into the probe volume creating a common focused beam diameter of about $400 \mu \mathrm{m}$, which defines the cross-sectional area of the probe volume. Laser powers are monitored by a single photodiode, while a second photodiode is used to generate a synchronizing trigger pulse for the computer. The fluorescence is collected at $90^{\circ}$ to the incident beam, and imaged onto an $800 \mu \mathrm{m}$ slit that defines the probe volume length. A dichroic mirror then splits the fluorescence into the $451.13 \mathrm{~nm}$ and $410.18 \mathrm{~nm}$ components. Band-pass filters $(10 \mathrm{~nm})$, centered at the fluorescence wavelengths, remove room background noise and most flame emission before the fluorescence enters the photomultiplier tubes (PMTs).

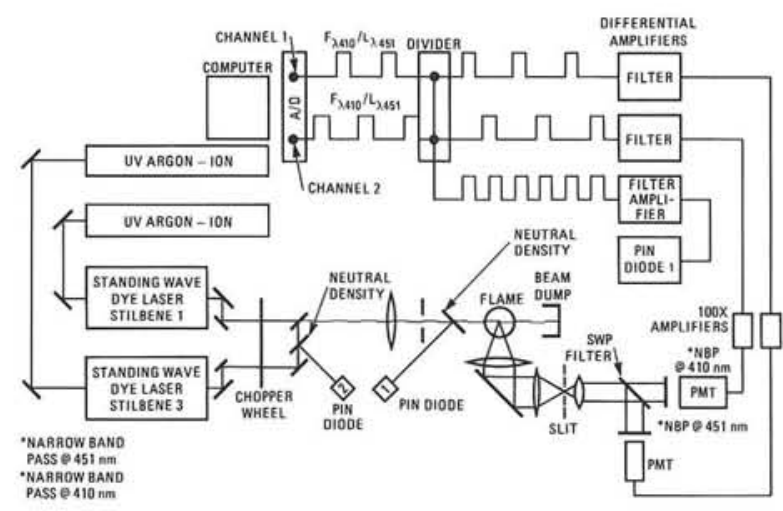

Figure 1. Experimental setup and block diagram of signal processing arrangement.

Results from two burners are presented: a circular premixed laminar flat flame burner (30-mm diameter); and a dual premixed burner (consisting of two $22 \mathrm{~mm}$ square burners separated by a common wall). By varying the mass flow rates to each side of the dual burner, and adding nitrogen to one side, adjacent flows of different velocities and temperatures are created. The shearing between these different velocity flows develops into turbulence, and the temperature difference causes turbulent temperature fluctuations. Methane is the fuel used in all burners. The output of a submicron constantoutput pneumatic nebulizer seeds the main air supply with a dilute solution of indium chloride in distilled water. As the indium chloride passes through the flame, it is disassociated into the required atomic indium. Care was taken to keep the indium seed concentration sufficiently low so that attenuation of the incident laser power and reabsorption (trapping) of the fluorescence were negligible. 
Since the validity of TLAF temperatures has been previously established by Omenetto et $a l,{ }^{2}$ the system was not calibrated on an absolute scale. A calibration coefficient was determined by forcing the mean temperature of a TLAF sample (encompassing an average of 2000 individual measurements) to match a radiationcorrected thermocouple reading, at one point in the flow. This method limits the TLAF accuracy to that of the thermocouple, but does not affect its precision or time resolution. With this method, only a simple one-point calibration is required to compensate for changes in experimental conditions.

Figure 2a shows radial temperature profiles in the premixed laminar burner. Each TLAF data point is the mean of 2000 individual temperature realizations, while the thermocouple data points result from a single long-timeaverage ( $>1 \mathrm{~s}$ ) manual reading. With the low band-pass filters at either $10 \mathrm{kHz}$ or $30 \mathrm{kHz}$ filters on the PMT signal $(1 \mathrm{kHz}$ or $5 \mathrm{kHz}$ data rates, respectively), the average TLAF temperatures fall very close to the thermocouple readings across the burner. The difference in the temperatures at the flame edge is believed to be due to the lateral shifting (flicker) of the flame between measurements. The root mean square of the differences between the temperatures and the temperature mean $\left(\mathrm{T}_{\mathrm{RMS}}\right)$ at the two TLAF data rates are presented in Figure $2 \mathrm{~b}$. Also plotted in this figure is a $\mathrm{T}_{\mathrm{RMS}}$ obtained with Rayleigh scattering. An argon ion laser, lasing at 1 watt of power at $488 \mathrm{~nm}$, was used to produce the Rayleigh scattering, and the Rayleigh PMT signal was filtered with a $10 \mathrm{kHz}$ low band-pass filter. As expected, the faster data with the higher frequency filter has a higher $\mathrm{T}_{\mathrm{RMS}}$; however, it is still much lower than the $\mathrm{T}_{\mathrm{RMS}}$ of the Rayleigh data. This is due to the smaller effect of shot noise in the stronger TLAF signal. It should be noted that these measurements were made in a lean flame where the atomic seed concentration was approximately 10 times less than the concentration at which trapping becomes significant. At an equivalence ratio near unity, higher atomic seed concentrations resulted, and a $\mathrm{T}_{\mathrm{RMS}}$ of about 75 $\mathrm{K}$ was obtained with the $10 \mathrm{kHz}$ filter. All the
$\mathrm{T}_{\mathrm{RMS}}$ 's increase greatly at the edge of the flame due to flicker.
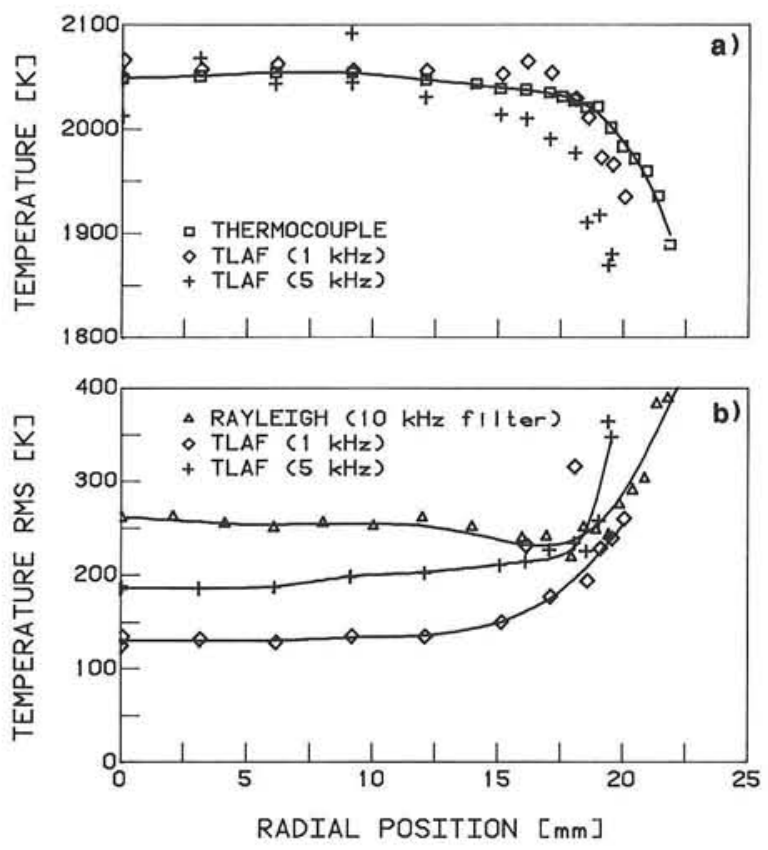

Figure 2. a) TLAF and thermocouple radial temperature profiles, $30 \mathrm{~mm}$ above the surface of the premixed laminar burner. The line is a spline fit to the thermocouple data. b) Standard deviation of temperature $\left(\mathrm{T}_{R M S}\right)$ for TLAF and Rayleigh measurements at the same conditions as Figure 2a.

Although a substantial portion of the $\mathrm{T}_{\mathrm{RMS}}$ is due to shot noise, and other sources as mentioned above, Figure 3 shows that, within the uncertainties from these other sources, TLAF can follow turbulent temperature fluctuations. This figure shows a scan across the two flows in the dual burner that was taken at a $2 \mathrm{kHz}$ data (chopper) rate with 10 $\mathrm{kHz}$ filters, and an equivalence ratio of unity. The TLAF temperatures (means of 2000 individual realizations) are constant at both ends of the profile and are connected by a gradient region. The $\mathrm{T}_{R M S}$ is at a background level of about $75 \mathrm{~K}$ across the regions of no temperature gradient. However, in the region of temperature gradient the $\mathrm{T}_{R M S}$ increases, reaching a maximum of about $125 \mathrm{~K}$. This increase in the $\mathrm{T}_{R M S}$ indicates that the TLAF technique has responded to the turbulent temperature fluctuations in the shear region between the two flows. 


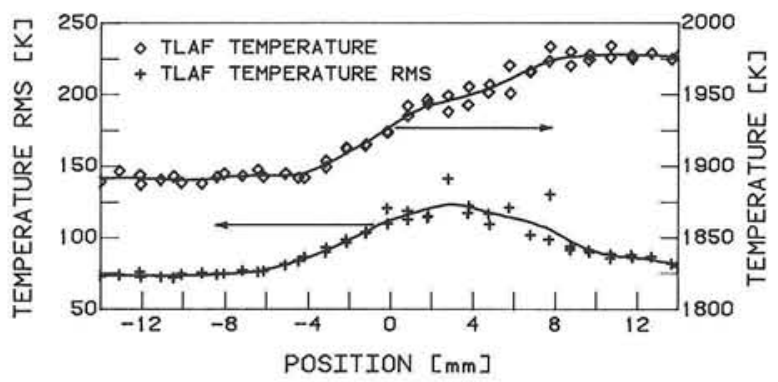

Figure 3. TLAF temperature and standard deviation of temperature ( $T_{R M S}$ ) profiles across a dual flow premixed burner. The velocity ratio of the two flows is about $3: 1$ and the vertical position is $42 \mathrm{~mm}$ above the burner exit.

\section{References}

1. C. T. J. Alkemade, "A Theoretical Discussion on Some Aspects of Atomic Fluorescence Spectroscopy in Flames," Pure and Appl. Chem. 23, 73 (1970).

2. N. Omenetto, R. Browner, and J. Winefordner, "'Color Temperature' Atomic Fluorescence Method for Flame Temperature Measurement," Anal. Chem. 44, 1683 (1972). 
$\omega$

$\Omega$

0

B

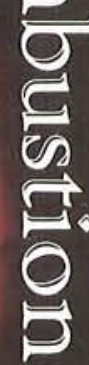

15.

$\Omega$

官.

量

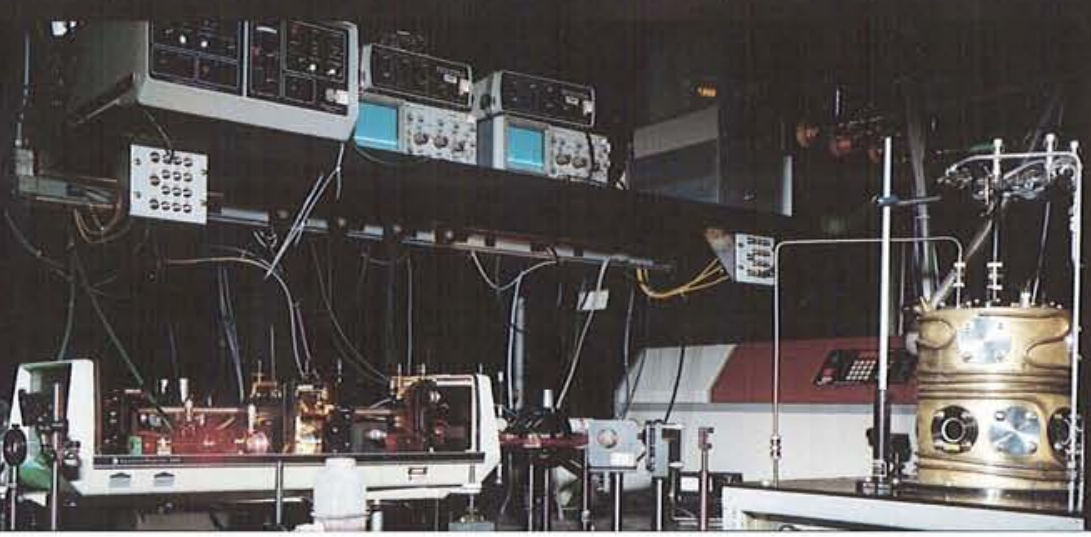


An important aspect of research in combustion chemistry is the determination of the rates of individual chemical reactions that play significant roles in the conversion of fuel to products. The inset photograph on the preceding page shows an apparatus in which an excimer laser (background) photolytically creates a population of $\mathrm{OH}$ radicals in the cell at the right. The rate of reaction between $\mathrm{OH}$ and a fuel is determined by monitoring the concentration of $\mathrm{OH}$ as a function of time. This is done by exciting $\mathrm{OH}$ with the laser shown in the foreground and collecting the resulting fluorescence. The enlarged picture shows the heart of the excitation system: a device in which the frequency of the laser's light is doubled to allow it to excite $\mathrm{OH}$ (see related articles, pages 3-12 and 3-15). 


\section{Section 3 \\ Combustion Chemistry}

Under the sponsorship of the Department of Energy's Office of Basic Energy Sciences, Sandia National Laboratories undertakes fundamental experimental and computational research in combustion chemistry. This work provides information needed to understand the complex chemical processes involved when fuels are burned. The program emphasizes measurements of reaction rates using state-ofthe-art diagnostics, and stresses the close coupling of experiment, theory, and modeling.

Staff and visitors investigate elementary reactions important to the burning of fuels or the production of pollutants. Typical experiments utilize slow-flow reactors together with such techniques as laser photolysis and laser-induced fluorescence to generate and detect, respectively, reactive species. Using mass spectrometry and Fourier-transform infrared spectroscopy as diagnostic tools for experiments conducted in low-pressure flames, researchers study the kinetic mechanisms involved in prototypical combustion systems. Other experiments and related theoretical efforts address fundamental questions of molecular dynamics - questions that must be understood to develop adequate models of kinetic processes.

Theoretical calculations of potential energy surfaces enable predictions of the product distributions that result when reactive species interact. These calculations are useful in extrapolating measured reaction rates. Computer modeling of the complete kinetics for combusting systems defines the dominant reaction pathways.

The experimental, theoretical, and modeling programs are focused on topics of importance to energyproducing technology. Themes of current emphasis include the formation and destruction of nitrogencontaining pollutants and the oxidation of hydrocarbons.

\section{Rapid Reduction of Nitrogen Oxides in Exhaust Gas Streams ${ }^{\dagger}$}

\section{R. A. Perry, D. L. Siebers}

Nitrogen oxides $\left(\mathrm{NO}_{\mathrm{x}}\right)$ play a major role in the formation of photochemical smog and in acid rain production. Some progress has been made in reducing $\mathrm{NO}_{\mathrm{x}}$ emissions through the use of combustion and exhaust control schemes, including 3-way catalyst, staged combustion, and ammonia injection. Nevertheless, estimates indicate that the production of nitrogen oxides will continue to increase through the end of the century and beyond, if current trends continue. We present here a new chemical process capable of completely removing $\mathrm{NO}_{\mathrm{x}}$ from the products of combustion. The effectiveness of this process is established in flow tube experiments. Further, we demonstrate the practical feasibility of this method by eliminating $\mathrm{NO}_{\mathrm{x}}$ from a portion of the exhaust from a single-cylinder diesel engine. Based on these encouraging results we conclude

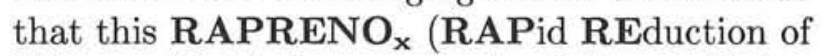
Nitrogen $\mathbf{O}_{\mathbf{x}}$ ides) process potentially could play a major role in controlling $\mathrm{NO}_{\mathrm{x}}$ emissions from most combustion devices.

\footnotetext{
† Nature 324, 657 (1986).
} 
Nitrogen oxide formation in combustion has received a great deal of attention. The recognition $^{1}$ that ammonia can be used to reduce nitric oxide in exhaust gases led to major efforts to characterize reactions important in the Thermal DeNO process. The mechanisms and models that resulted have greatly improved our understanding of nitrogen chemistry in combustion. For example, this research has led to the prediction of nitric oxide concentrations in ammonia injection schemes, as well as in general nitrogen chemistry in combustion. Experimental efforts have continued to increase our understanding of the radical species important in the conversion of fuel-bound nitrogen to $\mathrm{N}_{2}$ and $\mathrm{NO}$ during combustion. Perry ${ }^{2}$ studied NCO radical reactions with combustion species. NCO radicals formed from the photolysis of isocyanic acid (HNCO) react very rapidly with nitric oxide. Perry proposed that this reaction might be important in nitrous oxide formation in combustion. These observations, combined with previous insights into nitrogen chemistry, led to a new scheme for $\mathrm{NO}_{\mathrm{x}}$ reduction.

The proposed nitrogen oxide reduction scheme is based on the addition of isocyanic acid to an exhaust stream containing $\mathrm{NO}_{\mathrm{x}}$. Isocyanic acid is formed from the decomposition of cyanuric acid (a nontoxic, commercially available compound) when cyanuric acid is heated above approximately $330^{\circ} \mathrm{C}$,

$$
(\mathrm{HOCN})_{3} \stackrel{\Delta}{\rightarrow} \mathrm{HNCO}
$$

When HNCO is mixed with an exhaust gas stream at temperatures greater than approximately $400^{\circ} \mathrm{C}$, a series of reactions occur that result in the loss of HNCO and NO. Using a long-pass optical cell with Fourier transform infrared (FTIR) detection, carbon monoxide, carbon dioxide, water, and nitrogen (by inference) are observed as products. Table I shows results obtained in initial flow-reactor experiments to study the overall chemistry of RAPRENO . This process has been used to remove more than $99 \%$ of the nitric oxide emissions in simulated and real exhaust gas streams containing from 250-3,000 ppm by volume of NO.
The flow reactor was a $3.8 \mathrm{~cm}$ diameter, 1 meter long, stainless steel tube enclosed in a furnace. The simulated exhaust gas was sampled with a 6.4-mm-quartz probe; the position of the probe tip was varied over a range of $70 \mathrm{~cm}$ by the use of a stepping motor. The flow rates of the gases used ( $\mathrm{Ar} / \mathrm{NO} / \mathrm{HNCO})$ were controlled and measured with calibrated mass-flow controllers. Residence times in the flow reactor were determined from the equation $t=A d / V$ where $\mathrm{t}$ is time, $\mathrm{V}$ is flow rate, $\mathrm{A}$ is the cross sectional area of the tube, and $\mathrm{d}$ is the distance from the point of mixing. The temperature of the tube was measured using chromel/alumel thermocouples, and the pressure in the tube was measured using a capacitance manometer.

Although uncertainty exists concerning the initiation chemistry, certain observations regarding the overall chemical process can be made from the flow tube experiments. The most prominent feature of the results, as seen in Table I, is the decrease in NO and HNCO concentration and the formation of $\mathrm{CO}$ and $\mathrm{CO}_{2}$. It is evident from the data that the process occurs on a 1:1 molar basis.

The following possible reaction scheme which, when combined with previously developed models, may explain the experimental results.

$$
\begin{aligned}
\mathrm{HNCO} & \stackrel{\Delta}{\rightarrow} \mathrm{NH}+\mathrm{CO} \\
\mathrm{NH}+\mathrm{NO} & \rightarrow \mathrm{H}+\mathrm{N}_{2} \mathrm{O} \\
\mathrm{H}+\mathrm{HNCO} & \rightarrow \mathrm{NH}_{2}+\mathrm{CO} \\
\mathrm{OH}+\mathrm{CO} & \rightarrow \mathrm{H}+\mathrm{CO}_{2} \\
\mathrm{NH}_{2}+\mathrm{NO} & \rightarrow \mathrm{N}_{2} \mathrm{H}+\mathrm{OH} \\
& \rightarrow \mathrm{N}_{2}+\mathrm{H}_{2} \mathrm{O} \\
\mathrm{N}_{2} \mathrm{H} & \rightarrow \mathrm{N}_{2}+\mathrm{H} .
\end{aligned}
$$

This reaction scheme implies that following initiation the sequence through $\mathrm{N}_{2} \mathrm{H}$ produces a growth of 1 hydrogen atom per cycle to sustain the chain. One important aspect of the chemistry of RAPRENO ${ }_{x}$ is the absence of the need for controlled amounts of oxygen, suggesting that it could be readily implemented as a control process if the presence of oxygen does not greatly change the mechanism. 
Table I

Flow Data Obtained From Stainless Steel Flow Reactor Using Simulated Exhaust Gases (Ar/NO/HNCO Mixture)

\begin{tabular}{|c|c|c|c|c|c|c|c|c|}
\hline $\begin{array}{l}\text { Temperature } \\
\left({ }^{\circ} \mathrm{C}\right)\end{array}$ & $\begin{array}{l}\text { Pressure } \\
\text { (torr) }\end{array}$ & $\begin{array}{c}(\mathrm{NO})_{i} \\
\text { (p.p.m.) }\end{array}$ & $\begin{array}{c}(\mathrm{HNCO})_{i}{ }^{*} \\
\text { (p.p.m.) }\end{array}$ & $\begin{array}{l}(\mathrm{NO})_{f}^{\dagger} \\
\text { (p.p.m.) }\end{array}$ & $\begin{array}{c}(\mathrm{CO})^{\ddagger} \\
\text { (p.p.m.) }\end{array}$ & $\begin{array}{l}\left(\mathrm{CO}_{2}\right)^{\ddagger} \\
\text { (p.p.m.) }\end{array}$ & $\begin{array}{c}(\mathrm{HNCO})_{f} \\
\text { (p.p.m.) }\end{array}$ & $\begin{array}{c}\text { Time } \\
\text { (s) }\end{array}$ \\
\hline 585 & 508 & 0 & 660 & 0 & 420 & 190 & 50 & 0.30 \\
\hline 582 & 512 & 740 & 610 & 410 & 30 & 500 & 80 & 0.30 \\
\hline 639 & 540 & 725 & 695 & 120 & 100 & 520 & 75 & 0.30 \\
\hline 638 & 539 & 500 & 635 & 70 & 130 & 425 & 80 & 0.59 \\
\hline 647 & 637 & 370 & 350 & 100 & 40 & 250 & 60 & 0.15 \\
\hline 647 & 537 & 365 & 485 & 95 & 80 & 345 & 60 & 0.72 \\
\hline 640 & 537 & 725 & 720 & 80 & 130 & 525 & 65 & 1.15 \\
\hline 643 & 532 & 725 & 805 & 45 & 160 & 600 & 60 & 1.40 \\
\hline 663 & 537 & 670 & 925 & 40 & 80 & 800 & 20 & 2.00 \\
\hline 651 & 603 & 530 & 555 & 115 & 65 & 450 & 40 & 0.35 \\
\hline 644 & 537 & 725 & 855 & 25 & 210 & 600 & 45 & 1.70 \\
\hline 643 & 537 & 725 & 820 & 45 & 160 & 600 & 60 & 1.40 \\
\hline
\end{tabular}

* $(\mathrm{HNCO})_{i}$ is the initial HNCO concentrations calculated assuming carbon mass balance from the equation $(\mathrm{HNCO})_{i}=(\mathrm{HNCO})_{f}+\left(\mathrm{CO}_{2}\right)+(\mathrm{CO})$.

${ }^{\dagger}(\mathrm{NO})$ determined by $\mathrm{NO}_{x}$ chemiluminescent analyser.

$\ddagger(\mathrm{CO}),\left(\mathrm{CO}_{2}\right)$ and $(\mathrm{HNCO})$ measured using absorption at selected lines precalibrated with known concentrations of $\mathrm{CO}, \mathrm{CO}_{2}, \mathrm{HNCO}$

To test the effect of excess oxygen on the process, and to develop a technology for exhaust conditions that are presently untreatable by existing technologies, such as 3-way catalyst and ammonia injection, we used the proposed method to reduce the nitrogen oxides in a portion of a diesel engine exhaust gas stream. The experiments used a single cylinder, $7.2 \mathrm{hp}$ Onan diesel engine. The engine was operated at a medium load condition (an overall equivalence ratio of 0.5 ) and an engine speed of 1800 rpm. A continuous sample of unfiltered exhaust gas was drawn at a rate of one liter per minute and passed over cyanuric acid in a 14.6-cm-long by $4.72-\mathrm{cm}$-diameter stainless steel tube with a volume of $256 \mathrm{~cm}^{-3}$ (the cyanuric acid bed). The sample then flowed through a 17.8-cmlong by 2.2 -cm-diameter tube packed with two sizes of carbon steel spheres, 0.55 and 0.64 cm-diameter (the packed bed), which had an open volume of 31 cubic centimeters and the total surface area including steel spheres of 1630 square centimeters. The packed bed was used to test the effect of surface-to-volume ratio and residence times on the chemistry. Finally, the exhaust gas flowed through a $\mathrm{NO}_{x}$ chemiluminescence analyzer. Samples also were taken of the treated and untreated exhaust gas for FTIR analysis.

Figure 1 shows the results of the diesel engine experiments. The NO concentration measured in the treated exhaust gas is plotted as a function of the cyanuric acid bed temperature. Figure 1 shows that for cyanuric acid bed temperatures above $360^{\circ} \mathrm{C}$ the $\mathrm{NO}_{x}$ was essentially eliminated. These results were insensitive to packed bed temperatures between $450^{\circ} \mathrm{C}$ and $900^{\circ} \mathrm{C}$, provided that the cyanuric acid bed temperature was greater than $360^{\circ} \mathrm{C}$. At lower acid bed temperatures, nitric oxide levels in excess of that produced by the diesel engine (410 ppm) were observed (Figure 1). Surface interactions of HNCO with oxygen are believed to be important in this effect, and suggest the need to minimize the surface area in designing a practical device. Experiments, using gaseous HNCO injection in oxidizing environments, show that NO reduction takes place efficiently in the presence of excess oxygen. 


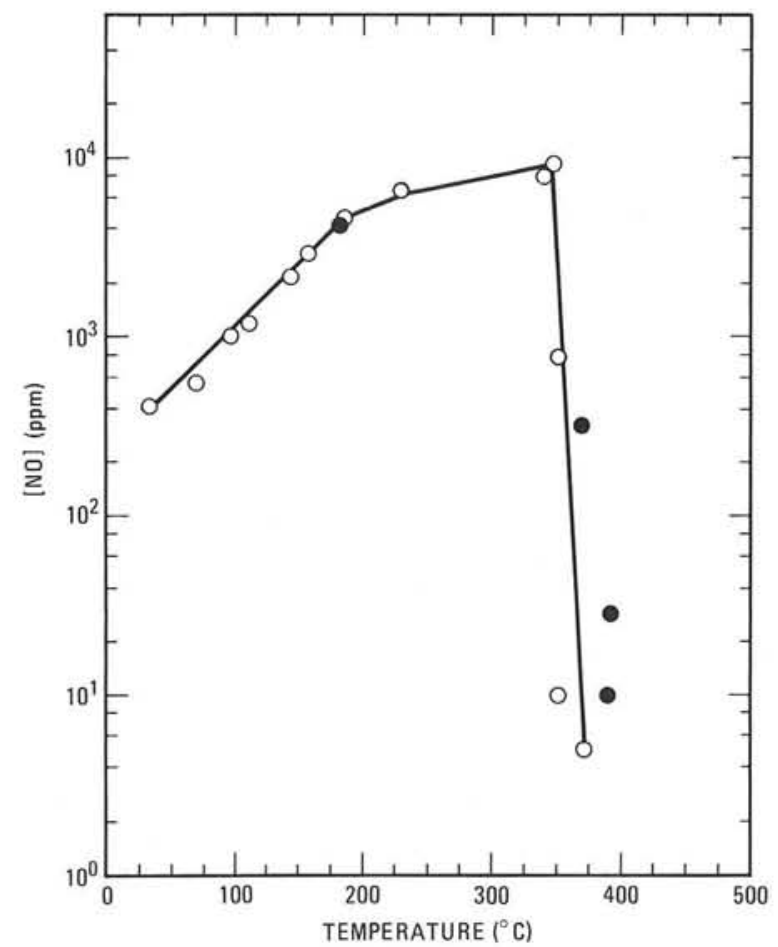

Figure 1. Plot of NO concentration (ppm) in treated portion (1.0 liter/minute) of diesel engine exhaust gas versus cyanuric acid bed temperature $\left({ }^{\circ} \mathrm{C}\right)$. The diesel engine was operated at medium load (overall equivalence ratio of 0.5 ) and 1800 RPM. Instrumentation sensitivity is limited to approximately $5 \mathrm{ppm}$. Data were obtained on successive days, with solid circles taken initially and open circles obtained in separate experiments.

In summary, we have discovered a chemical means of removal of $\mathrm{NO}_{x}$ from the products of combustion or industrial emissions, and we have demonstrated its feasibility on a portion of the exhaust from a diesel engine. This process has important implications for a wide spectrum of energy conversion technologies and for industrial applications that presently produce $\mathrm{NO}_{x}$ as exhaust byproducts.

\section{References}

1. R. K. Lyon, U.S. Patent No. 3,900,554 (August 1975)

2. R. A. Perry, "Kinetics of the Reactions of NCO Radicals with $\mathrm{H}_{2}$ and NO Using Laser Photolysis-Laser Induced Fluorescence," $J$. Chem. Phys. 82, 5485 (1985).
3. J. A. Miller, M. D. Smooke, R. M. Green, and R. J. Kee, "Kinetic Modeling of the Oxidation of Ammonia in Flames," Comb. Sci. Tech. 34, 149 (1983).

\section{Rotational Excitation of $\mathrm{NH}\left(\mathrm{a}^{1} \Delta\right)$ From Photodissociation of $\mathrm{HNCO}^{\dagger}$}

\author{
T. A. Spiglanin, D. W. Chandler
}

Unimolecular dissociations occur in hightemperature environments and may contribute to the initiation of combustion processes. Understanding the dynamics of these fragmentations helps us to better understand some details of the molecular potential energy surfaces that are relevant to both the dissociative and the reverse associative processes. Our approach to learning about fragmentation dynamics is to initiate a dissociation by absorption of light and determine the fate of the energy input via the photon as rotational, vibrational, translational, and electronic excitation of the products before they collide with other molecules. Knowing the distribution of this energy allows us to formulate mechanisms for the dissociation.

Isocyanic acid (HNCO) is of interest to us because it produces radicals that are important in the combustion of nitrogen-containing hydrocarbons. It is also interesting as a molecule for which the total energy distribution in each of the fragments of dissociation can be determined. In a recent study, we determined the dissociation energy of the process

$$
H N C O \rightarrow N H\left(a^{1} \Delta\right)+C O\left(X^{1} \Sigma^{+}\right)
$$

to be $41530 \mathrm{~cm}^{-1},{ }^{1}$ corresponding to $241 \mathrm{~nm}$ light. In another study, we measured the distribution of energy in rotation of the CO fragment of this dissociation and found that $\sim 20 \%$ of the

\footnotetext{
$\dagger$ Submitted to J. Chem. Phys. (1986).
} 
available energy (the fraction of the energy carried by the photon that exceeds the bond dissociation energy) resides in $\mathrm{CO}$ rotation. In the present study, we determine the distribution of the available energy in rotation of the $\mathrm{NH}\left(\mathrm{a}^{1} \Delta\right)$ fragment.

We perform these experiments using two tunable ultraviolet laser beams. A pulsed laser beam of wavelength shorter than $241 \mathrm{~nm}$ first induces HNCO dissociation, and then a second pulsed laser beam of wavelength near $324 \mathrm{~nm}$ induces fluorescence from the $\mathrm{NH}\left(\mathrm{a}^{1} \Delta\right)$. Measuring the intensity of the laser-induced fluorescence versus photon wavelength near $324 \mathrm{~nm}$, we obtain fluorescence excitation spectra (absorption monitored by fluorescence) of the NH fragment produced at each wavelength chosen for photolysis. Several of these spectra are shown in Figure 1 along with the available energy.

We determine rotational state distributions from these and other similar data. Figure 2 displays three of these distributions corresponding to available energies of $4270 \mathrm{~cm}^{-1}, 6650 \mathrm{~cm}^{-1}$, and $10764 \mathrm{~cm}^{-1}$.

Surprisingly, only a small fraction of the available energy resides in the rotation of $\mathrm{NH}\left(\mathrm{a}^{1} \Delta\right)$. If the available energy were to distribute statistically among all of the product degrees of freedom, the rotational state distributions would appear as the dashed curves in Figure 2. Instead, an impulsive explanation is more appropriate: the rotational excitation of the fragments being imparted by a repulsive force along the breaking $\mathrm{N}-\mathrm{C}$ bond. Because this force is applied at the $\mathrm{N}$ atom of $\mathrm{NH}$, very little rotational excitation results since the $\mathrm{N}$ atom is near the center of mass of that fragment. Fits of a model assuming impulsive dissociation to the data appear in Figure 2 along with the data. Clearly, better agreement results from the impulsive mechanism than from the statistical model. The impulsive nature of the dissociation is consistent with the rotational excitation that we observed for $\mathrm{CO}$ produced by HNCO photodissociation, although a change in the NCO configuration is required on excitation (from nearly linear to near $120^{\circ}$ ) to explain CO rotational excitation.

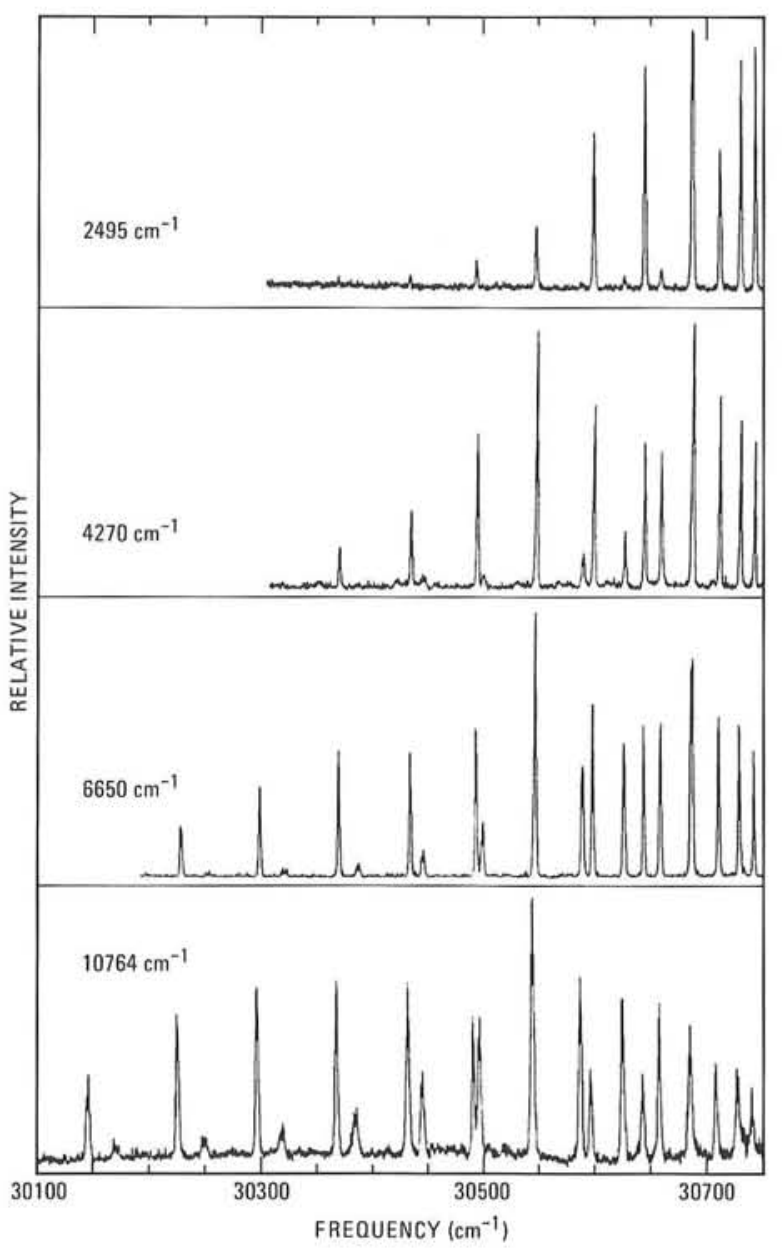

Figure 1. Fluorescence excitation spectra of $\mathrm{NH}\left(\mathrm{a}^{1} \Delta\right)$ produced by photodissociation of HNCO at several wavelengths. The amount by which the energy of the photolysis photon exceeds the bond dissociation energy is indicated in each frame of the figure.

The average rotational excitation of the $\mathrm{NH}$ and of the $\mathrm{CO}$ relative to the energy in excess of the bond dissociation energy are roughly constant over a wide range of photolysis energies $(20 \%$ for CO rotation and $12 \%$ for NH rotation), implying that the mechanism of dissociation is the same over the frequency range $41530 \mathrm{~cm}^{-1}-52300 \mathrm{~cm}^{-1}$. Vibrational excitation of $\mathrm{CO}$ following $\mathrm{HNCO}$ photodissociation with $10200 \mathrm{~cm}^{-1}$ available energy accounts for about $13 \%$ of the available energy, ${ }^{2}$ and $\mathrm{NH}$ is not vibrationally excited for even this highenergy photolysis. ${ }^{3}$ The remaining $55 \%$ of the 

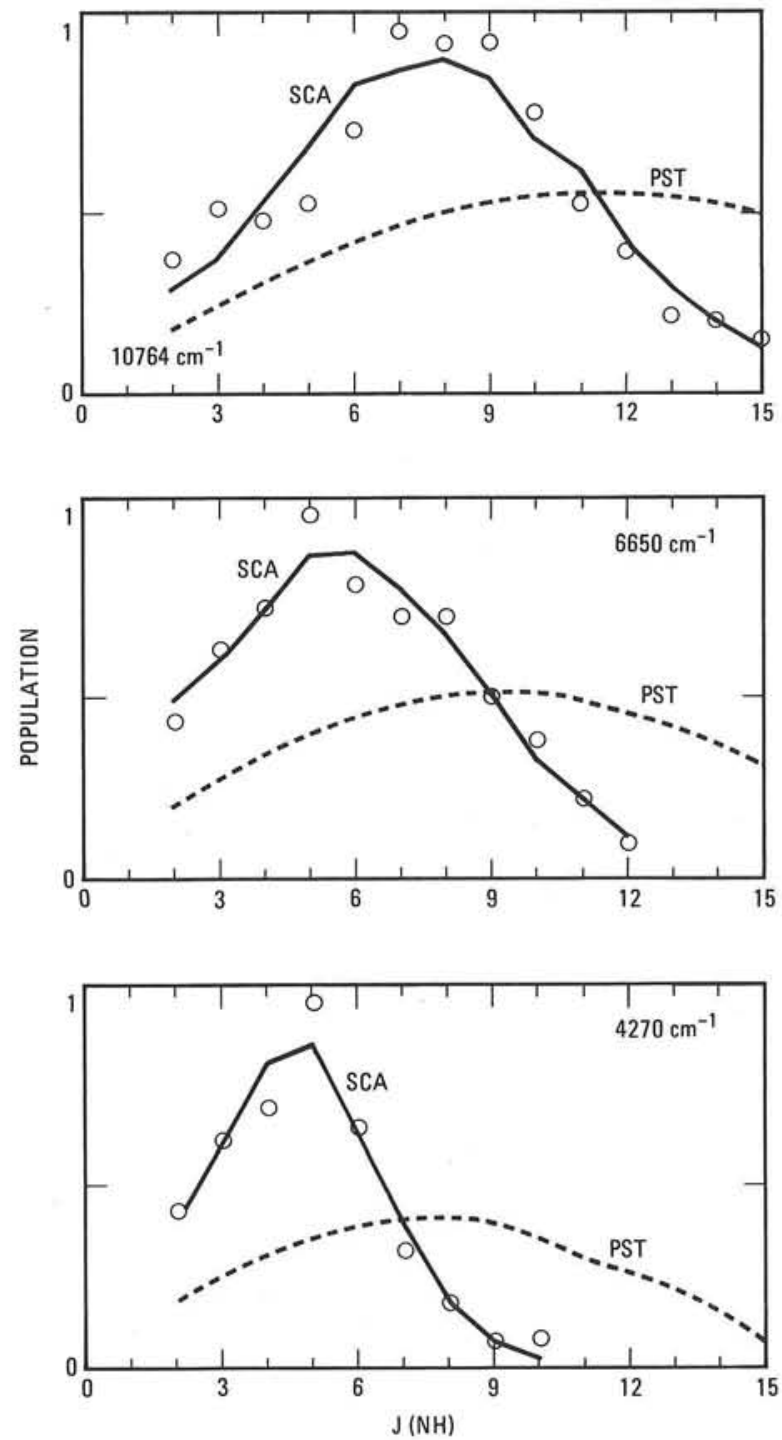

Figure 2. Rotational state distributions of $\mathrm{NH}\left(\mathrm{a}^{1} \Delta\right)$ produced by photodissociation of HNCO derived from fluorescence excitation spectra (Figure 1). The available energy for each distribution is indicated in each frame. The curve labeled PST is the predicted distribution based on a statistical model and the curve SCA (that passes through most of the points) is a fit to the data using a model that assumes impulsive dissociation.

energy must reside in translation of the fragments away from the center of mass. This translational energy $\left(1000 \mathrm{~cm}^{-1}\right.$ for $1930 \mathrm{~cm}^{-1}$ photolysis) corresponds to very high translational temperatures.

\section{References}

1. T. A. Spiglanin, R. A. Perry, and D. W. Chandler, "Photodissociation Studies of HNCO:

Heat of Formation and Product Branching Ratios," J. Phys. Chem. 90, 6184 (1986).

2. G. T. Fujimoto, M. E. Umstead, and M. C. Lin, "Dynamics of CO Formation in the Photodissociation of $\mathrm{HNCO}$ and $\mathrm{CH}_{2} \mathrm{CO}$ at 193 nm," Chem. Phys. 65, 197 (1982).

3. W. S. Drozdoski, A. P. Baronavski, and J. R. McDonald, "Photodissociation of $\mathrm{HNCO}$ at $193 \mathrm{~nm}$. NH( $\left(\mathrm{a}^{-1} \Delta\right)$ Internal Energy Distribution and Reaction Rate with $\mathrm{O}_{2}$," Chem. Phys. Lett. 64, 421 (1979).

\section{Nitric Oxide-Hydrocarbon Interactions in Low-Pressure Flames}

\author{
L. R. Thorne, M. C. Branch, ${ }^{*} D$. W. Chandler, R. \\ J. Kee, J. A. Miller
}

Recent studies in the low-pressure flame laboratory have concentrated on the fate of fuelbound nitrogen in combustion processes. Work at other laboratories showed that a significant fraction of fuel-bound nitrogen is converted directly to HCN during the early stages of combustion. This has motivated studies relating to the gas phase combustion chemistry of HCN. Among these studies is an earlier one in this laboratory. ${ }^{1}$ This study showed that HCN doped into a low-pressure $\mathrm{H}_{2} / \mathrm{O}_{2}$ flame produces either $\mathrm{N}_{2}$ or NO depending on whether the flame is rich or lean. The major sequence in the nitrogen pathway was identified as $\mathrm{HCN} \rightarrow \mathrm{NCO} \rightarrow \mathrm{NH}$ $\rightarrow \mathrm{N} \rightarrow \mathrm{NO}$ or $\mathrm{N}_{2}$.

As an extension of this earlier work, we have recently completed a study ${ }^{2}$ in which our objective was to determine the importance of a "nitrogen-recycle mechanism" that may exist in hydrocarbon flames in which NO is present.

\footnotetext{
* University of Colorado at Boulder.
} 
This mechanism could play an important role in determining the level of NO emission under rich conditions. As in the flames studied previously, not only is HCN consumed, but in addition there is the possibility that HCN may be regenerated from the reaction of $\mathrm{NO}$ or $\mathrm{NH}_{\mathrm{i}}(\mathrm{i}=$ $0,1,2)$ with hydrocarbon free radicals. To test the importance of these reactions in recycling nitrogen, we have doped $\mathrm{NO}$ and $\mathrm{C}_{2} \mathrm{H}_{2}$ into a $\mathrm{H}_{2} / \mathrm{O}_{2} / \mathrm{Ar}$ flame. This flame was chosen because it has a high hydrocarbon-free radical concentration resulting from the high yield of the primary $\mathrm{C}_{2} \mathrm{H}_{2}$ oxidation mechanism: $\mathrm{O}+\mathrm{C}_{2} \mathrm{H}_{2} \rightarrow \mathrm{CH}_{2}$ $+\mathrm{CO}$.

Our current approach is similar to the earlier work in which concentration and temperature profiles of the flame are measured in the laboratory, and then a chemical kinetic model is developed and refined so as to reproduce the measured profiles. In this way, details of the of the combustion process that cannot be easily measured directly in the laboratory, such as the important chemical reaction sequences, can be deduced from the kinetic model.

The laboratory measurements were made using the low-pressure flame apparatus consisting of a 9.7-cm-diameter, porous-plug burner enclosed in a vacuum chamber. The burner is fed by a manifold in which the gases are premixed. The chamber is operated at a pressure of 25 Torr and is pumped by a 300 -cfm Stokes pump. A quadrupole mass spectrometer is mounted on the vacuum chamber above the burner and has a quartz microprobe which is oriented along the vertical axis of the burner and samples the combustion gases. Concentration profiles of stable species in the flame are made by translating the burner vertically relative to the microprobe. Relative concentration profiles for $\mathrm{CH}$ and $\mathrm{CN}$ are made by laser-induced fluorescence (LIF) using a tunable, UV dye laser that is pumped by a cw Ar-ion laser. The dye laser is tunable over a range of $350 \mathrm{~nm}$ to $400 \mathrm{~nm}$, which permits excitation of the B-X transitions of $\mathrm{CH}$ and $\mathrm{CN}$. Temperature profiles are determined spectroscopically from the rotational line intensities of the B-X R-branch transitions in the CN LIF spectra. Because of the pervasiveness of $\mathrm{CN}$ in the flame, this permits temperature determinations to be made from $0.2 \mathrm{~cm}$ above the burner surface to well into the post-flame gases.

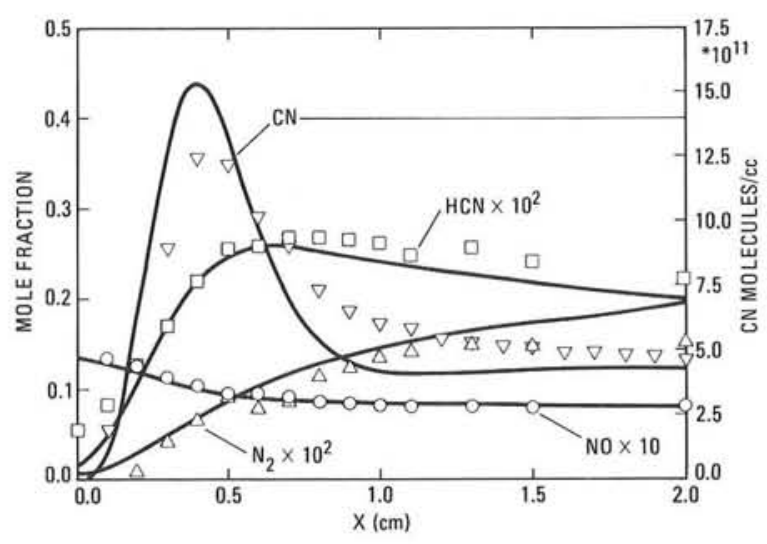

Figure 1. Concentration profiles for nitrogencontaining species in the $\mathrm{NO} / \mathrm{C}_{2} \mathrm{H}_{2}$-doped $\mathrm{H}_{2} / \mathrm{O}_{2} / \mathrm{Ar}$ flame. The symbols give the observed profiles; the lines give the computed profiles.

Figure 1 shows the observed (symbols) and calculated (lines) concentration profiles for nitrogen-containing species for a 25 Torr flame with a mass flow rate of $4.67 \times 10^{-3} \mathrm{gm}$ $\mathrm{cm}^{-2} \mathrm{sec}^{-1}$, having a composition of the following mole fractions (X): $\mathrm{X}_{\mathrm{Ar}}=0.5978, \mathrm{X}_{\mathrm{H}_{2}}=$ $0.277, \mathrm{X}_{\mathrm{O}_{2}}=0.092, \mathrm{X}_{\mathrm{C}_{2} \mathrm{H}_{2}}=0.0197$, and $\mathrm{X}_{\mathrm{NO}}$ $=0.0135$. The removal of NO, intermediate production of $\mathrm{HCN}$ and $\mathrm{CN}$, and ultimate production of $\mathrm{N}_{2}$ are observed and modeled well. Perhaps the most striking feature of these results is the peak in the CN profile at $0.4 \mathrm{~cm}$. This early peak can be modeled adequately only if the reaction $\mathrm{C}+\mathrm{NO} \rightarrow \mathrm{CN}+\mathrm{O}$ is included in the mechanism. Without this reaction, the CN profile is predicted to rise gradually and plateau as in the HCN-doped flames studied earlier. This is strong evidence for the importance of C-atom reactions in the production of $\mathrm{CN}$ and, subsequently, $\mathrm{HCN}$ in NO-doped rich hydrocarbon flames.

The model indicates that the primary reactions of the nitrogen-recycle mechanism that produce $\mathrm{CN}$ and $\mathrm{HCN}$ are $\mathrm{CH}_{n}+\mathrm{NO}$, with $\mathrm{n}=$ $0,1,2$. The reaction involving $\mathrm{CH}_{2}$ is less important than those involving $\mathrm{CH}$ and $\mathrm{C}$. Rapid equilibrium is established between $\mathrm{HCN}$ and 
CN primarily through the reaction $\mathrm{CN}+\mathrm{H}_{2} \rightarrow$ $\mathrm{HCN}+\mathrm{H}$. A schematic diagram of important reaction sequences is shown in Figure 2.

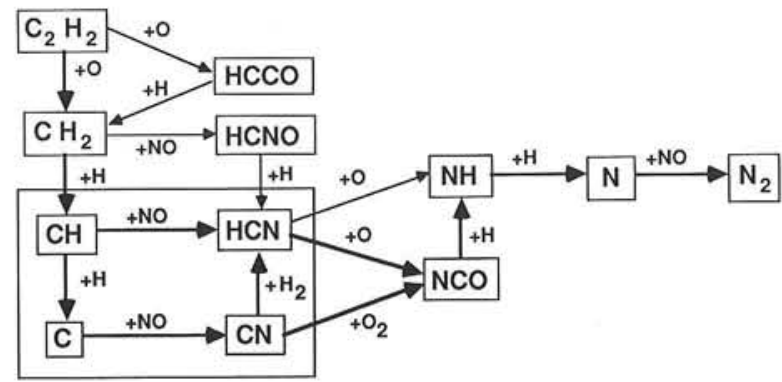

Figure 2. Schematic diagram showing the important reaction sequences in the $\mathrm{NO} / \mathrm{C}_{2} \mathrm{H}_{2}$-doped, $\mathrm{H}_{2} / \mathrm{O}_{2} / \mathrm{Ar}$ flame. Heavy arrows show the most important reaction routes. The reactions of NO with $\mathrm{CH}_{\mathrm{n}}(\mathrm{n}=0,1,2)$ are the major route in the production of cyanides in this flame and are important in the "nitrogen-recycle" mechanism. These are highlighted in the shaded box.

As in the case of HCN-doped flames, NCO and $\mathrm{NH}$ play a major role in the NO-doped flames and the principle nitrogen-containing end product is $\mathrm{N}_{2}$. Furthermore, the major route for production of cyanides in the NO-doped flame is the reaction of NO with small hydrocarbon free radicals. Reactions of $\mathrm{NH}_{\mathrm{i}}(\mathrm{i}=0,1,2)$ with hydrocarbons are much less important in cyanide production.

\section{References}

1. J. A. Miller, M. C. Branch, W. J. McLean, D. W. Chandler, M. D. Smooke, and R. J. Kee, "The Conversion of HCN to $\mathrm{NO}$ and $\mathrm{N}_{2}$ in $\mathrm{H}_{2}-\mathrm{O}_{2}-\mathrm{HCN}-\mathrm{Ar}$ Flames at Low Pressure," Twentieth Symposium (International) on Combustion (The Combustion Institute, 1984), p. 673.

2. L. R. Thorne, M. C. Branch, D. W. Chandler, R. J. Kee, and J. A. Miller, "Nitric OxideHydrocarbon Interactions in Low-Pressure Flames," Twenty-First Symposium (International) on Combustion (The Combustion Institute, 1986), in press.

\section{A Theoretical Analysis of the Reaction Between Hydroxyl and Hydrogen Cyanide at High Temperature ${ }^{\dagger}$}

\author{
J. A. Miller, C. F. Melius
}

In a number of experimental investigations of hydrogen cyanide decay in the postflame gases of atmospheric flames, it has been deduced that a reaction that is first order in $\mathrm{OH}$ under partially-equilibrated conditions plays an important role in the HCN conversion. The only plausible candidates for such a reaction are

$$
\begin{aligned}
\mathrm{OH}+\mathrm{HCN} & \stackrel{k_{a}}{\longrightarrow} \mathrm{HOCN}+\mathrm{H} \\
& \stackrel{k_{b}}{\longrightarrow} \mathrm{HNCO}+\mathrm{H} \\
\stackrel{k_{c}}{\longrightarrow} & \mathrm{NH}_{2}+\mathrm{CO} .
\end{aligned}
$$

However, in a recent paper on HCN conversion in low-pressure $\mathrm{H}_{2} / \mathrm{O}_{2} / \mathrm{Ar}$-HCN flames, Miller et $a l^{1}$ have shown that HCN removal occurs primarily through reaction with oxygen atoms and that reaction with $\mathrm{OH}$ is negligible. These investigators also pointed out an apparent inconsistency between the low-temperature result for the high-pressure limit rate coefficient of the addition reaction, $\mathrm{OH}+\mathrm{HCN} \rightarrow \mathrm{HOHCN}$, and the flame results for $k_{T}$ where $k_{T}=k_{a}+k_{b}+k_{c}$. The present investigation was initiated to shed some light on this point.

In the present paper, we use BAC-MP4 (bond-additivity-corrected Moller-Plesset fourthorder perturbation theory) potential energy surface information and statistical-theoretical methods to predict the rate coefficients $k_{a}, k_{b}$, and $k_{c}$. We employ three levels of approximation in the statistical-theoretical treatment:

(i) canonical theory, CT (no energy or angular momentum conservation and no tunneling)

$\dagger$ Twenty-first Symposium (International) on Combustion (The Combustion Institute, 1986), in press. 
(ii) microcanonical/J-conservative theory, $\mu \mathrm{JT}$ (conserves both energy and angular momentum)

(iii) microcanonical/J-conservative theory with one-dimensional tunneling, $\mu \mathrm{JT}-\mathrm{T}$.

Figure 1 is a schematic diagram of the reaction pathways of interest. Reactions $a, b$, and $c$ all involve the formation and destruction of intermediate complexes, denoted by *, **, and *** on the diagram. The saddle-point regions, separating complexes from each other and from reactant and product regions of the potential, are numbered as shown in the figure. Note that we have omitted the dissociation of the ${ }^{* * *}$ complex into $\mathrm{HNCO}+\mathrm{H}$. In fact, this dissociation is a possibility. However, eliminating it from the analysis does not restrict our conclusions. The more energetically favored channel, $\mathrm{NH}_{2}+$ $\mathrm{CO}$, can only be overestimated somewhat. In fact, the $\mathrm{NH}_{2}+\mathrm{CO}$ channel, $k_{c}$, turns out to be completely negligible compared to $k_{a}$ and $k_{b}$ anyway. Also shown in Figure 1 is the adduct formed by $\mathrm{OH}$ addition to $\mathrm{HCN}$ on the nitrogen end. This adduct is highly unstable with respect to $\mathrm{HCN}+\mathrm{OH}$ (endothermic by $19 \mathrm{kcal} / \mathrm{mole}$ ). Consequently, products formed from this adduct have been excluded in the present analysis.

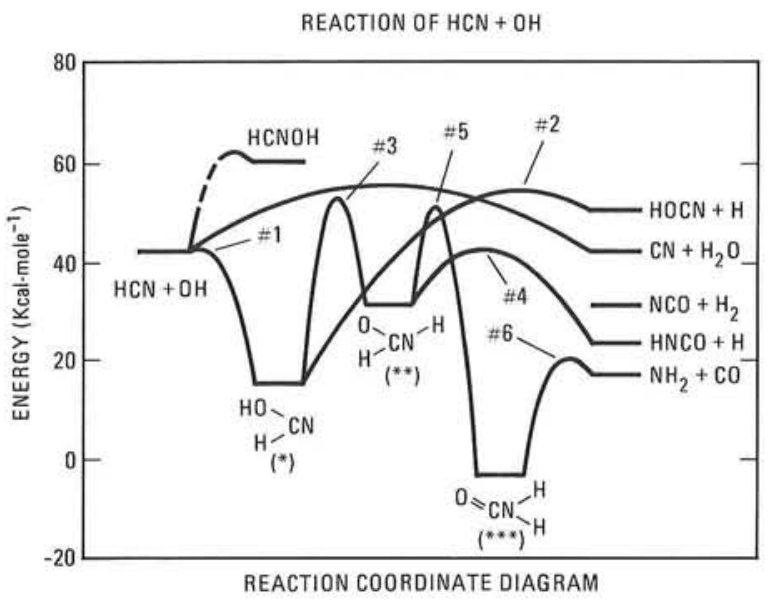

Figure 1. Schematic diagram of the possible reaction paths of the reaction $\mathrm{OH}+\mathrm{HCN} \rightarrow$ products. The asterisks designate complexes; the numbers designate transition-state dividing surfaces.
The results of all three theoretical treatments for the three different product channels are shown in Figure 2.

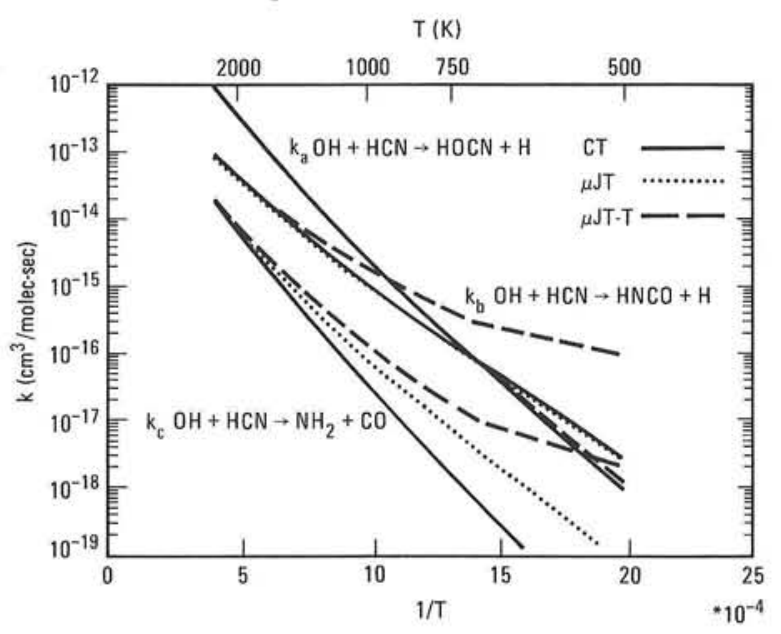

Figure 2. Arrhenius plot of $k_{a}, k_{b}$, and $k_{c}$ predicted by the three theoretical treatments: canonical theory (CT), microcanonical/J-conservative theory $(\mu \mathrm{JT})$, microcanonical/J-conservative theory with one-dimen-sional tunneling ( $\mu \mathrm{JT}-\mathrm{T})$.

The most important results are readily apparent. At temperatures below $900 \mathrm{~K}$, channel $b(\mathrm{HNCO}+\mathrm{H})$ is dominant, whereas at higher temperatures, channel $a(\mathrm{HOCN}+\mathrm{H})$ takes over. Channel $c\left(\mathrm{NH}_{2}+\mathrm{CO}\right)$ never plays a major role. Due to the dominance of a single transitionstate dividing surface (TSDS) in each case, $k_{a}$ and $k_{b}$ are insensitive to energy, and angularmomentum conservation ( $k_{a}$ is determined principally by TSDS- $2, k_{b}$ by TSDS-3). Tunneling in the 1,3 hydrogen shift (TSDS-3), however, significantly increases $k_{b}$ and $k_{c}$ at low temperature. It may well be a general rule that tunneling for 1,2 and 1,3 hydrogen transfers is very large; the imaginary frequency for such processes is normally $\sim 2000 i$, leading to the possibility of large tunneling fluxes. It should also be noted that, because of reaction-path curvature effects for the mass combination involved (heavy-light-heavy), our one-dimensional calculation probably underestimates the effect of tunneling in these processes.

In Figure 3, we have plotted the $\mu \mathrm{JT}-\mathrm{T}$ results for $k_{a}, k_{b}$ and $k_{T}$ along with the experimental results available from flame experiments. Also shown on the plot are the results of a simple transition-state theory calculation for the 
abstraction rate coefficient (using BAC-MP4 potential parameters),

$$
\mathrm{OH}+\mathrm{HCN} \stackrel{k_{d}}{\longrightarrow} \mathrm{CN}+\mathrm{H}_{2} \mathrm{O},
$$

and the experimental results available for $k_{d}$. The theoretical result for $k_{T}$ is in excellent agreement with Haynes' experimental determination, but it lies somewhat below Morley's results and even more below Fenimore's. Nevertheless, it seems reasonable to conclude that the present theoretical treatment supports Haynes' original conclusion about the importance of reaction (a) in atmospheric-pressure flames. However, it is important to realize that reaction $a$ (or $b$ or c) can be an important process only when the abstraction reaction is nearly equilibrated. Although the agreement between theory and the limited experimental data available for the abstraction is not as good as one might hope, it is sufficient to conclude that $k_{d} \gg k_{T}$ under virtually all flame conditions.

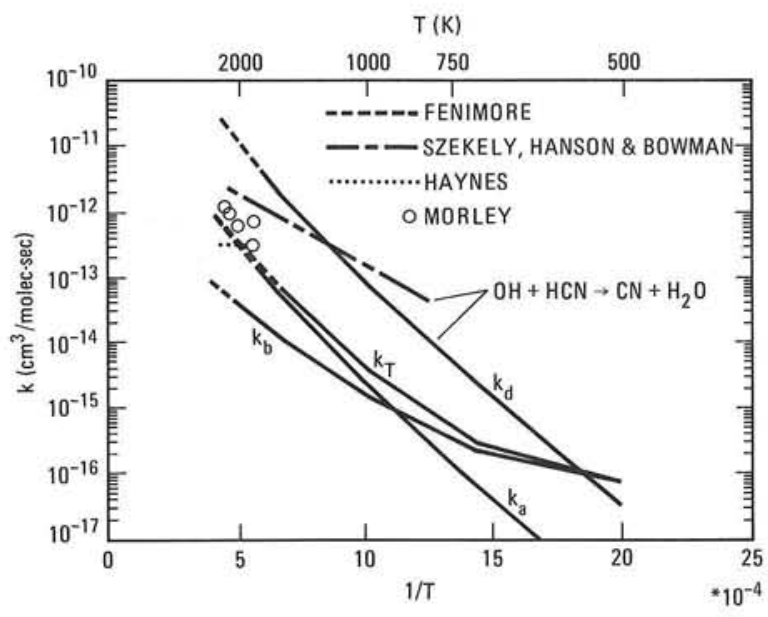

Figure 3. Comparison of experimental and theoretical results. The experimental results are from Fenimore, Haynes, Morley, and Szekely, Hanson, and Bowman. The result attributed to Fenimore on the plot is the result quoted in his abstract. His data actually are slightly larger. For detailed citations, see unabridged article.
The high-pressure-limit rate coefficient for the addition reaction,

$$
\mathrm{OH}+\mathrm{HCN} \stackrel{k_{A}}{\longrightarrow} \mathrm{HOHCN},
$$

is a rigorous upper bound on $k_{T}$. Consequently, it is of interest to compare a conventional transition-state-theory calculation for $k_{A}$ to the experimental result of Zellner and coworkers. ${ }^{2}$ This comparison is shown in Figure 4.

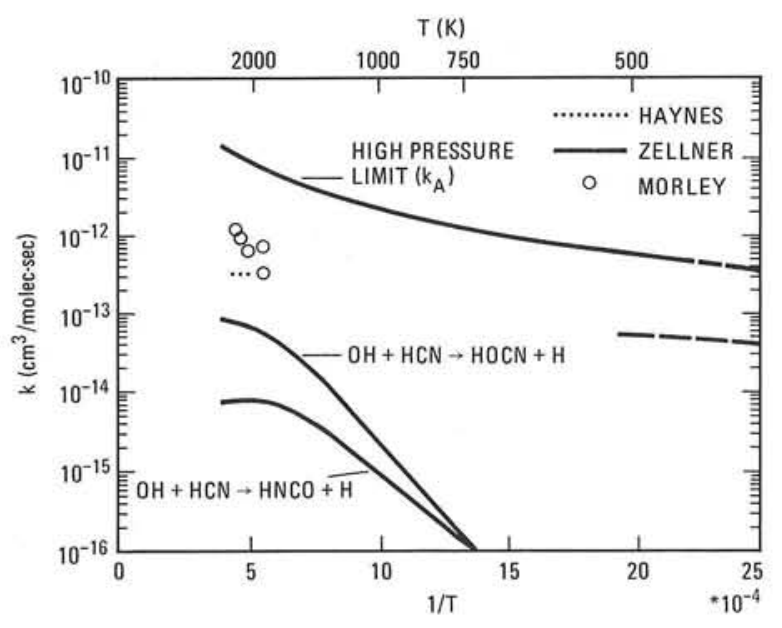

Figure 4. Arrhenius plot showing the effect of a low value of $k_{A}, \mathrm{OH}+\mathrm{HCN} \rightarrow \operatorname{HOHCN}(\mathrm{A})$, on the results for $k_{a}$ and $k_{b}$.

The theoretical result is higher in absolute value and increases more rapidly with temperature than the experiment. If the experiment is correct, the low rate coefficient (more specifically, the low A-factor) must be due to variational and/or non-RRKM effects. Our highly preliminary results with variational transitionstate theory indicate that variational effects do not significantly change the theoretical prediction, at least at low temperature.

If the experimental result of Reference 2 is correct and extrapolatable, it places a strict upper limit on the rate coefficients $k_{a}, k_{b}$ and $k_{c}$ at high temperature, as pointed out by Miller et $a .^{1}$ In fact, $k_{A}$ enters explicitly as a multiplicative factor in the canonical theory expressions for $k_{a}, k_{b}$ and $k_{c}$,

$$
k_{A}=g(T) \frac{k_{B} T}{h} \frac{Q_{1}^{\ddagger}(T)}{Q_{R}(T)} .
$$


Since it was shown above that canonical theory is an accurate representation of $k_{a}, k_{b}$ and $k_{c}$ at high temperature, we can use equation (III.1) to determine (formally) the reactive flux, i.e., $Q_{1}^{\ddagger}(T)$, implied by the experimental result for $k_{A}$ and then calculate $k_{a}$ and $k_{b}$ with this empirical $Q_{1}^{\ddagger}(T)$. The results of such a calculation are also shown in Figure 4. It is readily seen that the agreement of $k_{T}$ with Haynes' experiment is destroyed. Whereas the purely theoretical predictions are not limited by the rate of formation of the * complex, the semiempirical correction makes this process the rate-limiting one at high temperatures. We believe that Zellner et $a l^{2}$ may have incurred some error in extrapolating their data to the high-pressure limit.

\section{References}

1. J. A. Miller, M. C. Branch, W. J. McLean, D. W. Chandler, M. D. Smooke, and R. J. Kee, "The Conversion of $\mathrm{HCN}$ to $\mathrm{NO}$ and $\mathrm{N}_{2}$ in $\mathrm{H}_{2}-\mathrm{O}_{2}-\mathrm{HCN}-\mathrm{Ar}$ Flames at Low Pressure," Twentieth Symposium (International) on Combustion (The Combustion Institute, 1985), p. 673.

2. B. Fritz, K. Lorenz, W. Steinert, and R. Zellner, "Rate of Oxidation of Hydrogen Cyanide by Hydroxyl Radicals at Lower Temperatures," Oxidation Commun. 6, 363 (1984).

\section{A Theoretical Analysis of the Overtone-Induced Isomerization of Methyl Isocyanide ${ }^{\dagger}$}

\section{J. A. Miller, D. W. Chandler}

In a previous paper, we employed the masterequation formalism to analyze the problem of overtone photochemistry, and showed that the yield in such experiments is a strong function of the energy-transfer parameters, even for strong

† J. Chem. Phys. 85, 4502 (1986). colliders such as t-butyl hydroperoxide. The pressure dependence of the yield was found to be sensitive both to the shape of the energytransfer function and to the parameters chosen for the model (e.g., average energy transferred in a deactivating collision). From these results, we were able for the first time to extract the average energy transferred per collision from overtone-photochemistry data: $-1700 \mathrm{~cm}^{-1}$ for hot t-butyl hydroperoxide colliding with cold t-butyl hydroperoxide. In addition, we showed that an analysis of the shape of the "reactive distribution" explained the pressure dependence of the unimolecular reaction rate constant and, therefore, the curvature of the Stern-Volmer plots. The "reactive distribution" is nothing more than the normalized energy distribution of the product yield. It is a measure of the relative propensity of different energies to produce products. We also observed that, in the case of t-butyl hydroperoxide, not all states initially excited by the laser-produced products, and some states that are not initially prepared can lead to products if collisions subsequently populate them. In this paper, we use the concept of the reactive distribution to interpret quantitatively the pressure dependence in the $\mathrm{CH}_{3} \mathrm{NC}$ isomerization data of Reddy and Berry., ${ }^{1,2}$

We can summarize the principal results of our investigation as follows:

1. Stern-Volmer curvature in overtone photochemistry experiments can be understood in terms of the "reactive distribution." Negative curvature corresponds to an increase in the mean energy of the reactive distribution with increasing pressure. This is essentially a "strong-collider" effect. Positive curvature corresponds to a decrease of the mean energy of the reactive distribution with increasing pressure. This is necessarily a "weak-collider" effect. Usually, for a given excitation, one is able to detect only one type of curvature. However, in some cases (e.g., the fifth overtone of t-butyl hydroperoxide), there is positive curvature at low pressure and negative curvature at higher pressure, corresponding 
first to a shift down and then up of the reactive distribution.

2. Stern-Volmer plots are always linear at sufficiently high pressure due to the existence of a limiting high-pressure reactive distribution. This result is predicted analytically for the strong-collider case. It exists even for the weak collider case, although the limiting distribution attained is not the one predicted by the strong-collider model.

3. Excitation to $5 \nu_{\mathrm{CH}}$ in $\mathrm{CH}_{3} \mathrm{NC}$ produces a Stern-Volmer plot with negative curvature. Excitation to the $6 \nu_{\mathrm{CH}}$ produces a plot with positive curvature. Both cases are quantitatively predicted by the masterequation/RRKM model with a consistent set of parameters. However, the overtone results are consistent with RRKM theory only if the threshold energy of the 300 model (deduced by Rabinovitch and coworkers from thermal experiments) is increased by $200 \mathrm{~cm}^{-1}$. The average energy transferred in a collision of overtoneexcited $\mathrm{CH}_{3} \mathrm{NC}$ with room temperature $\mathrm{CH}_{3} \mathrm{NC}$ is determined to be $-1000 \mathrm{~cm}^{-1}$ if a single-exponential energy-transfer function is assumed (see Figure 1).

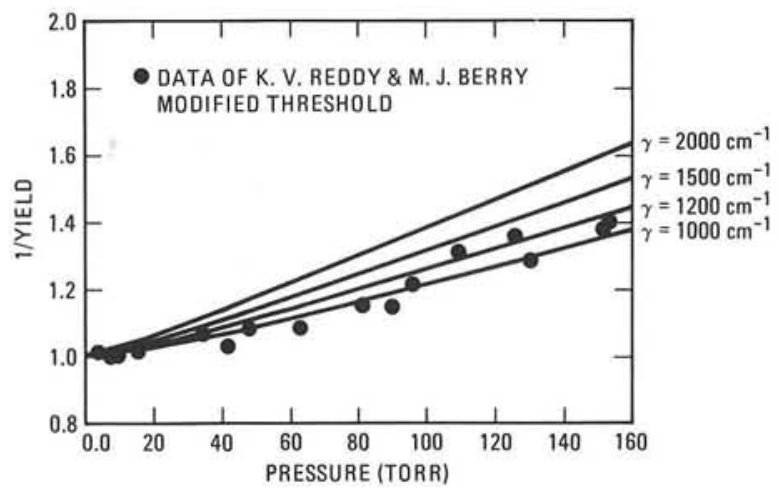

Figure 1. Stern-Volmer plot for the fifth overtone of $\mathrm{CH}_{3} \mathrm{NC}$. The master-equation calculations are for the modified $\mathbf{3 0 0}$ model. The yield is the fraction of the molecules that dissociate after having absorbed a photon.
4. Product yield as a function of pressure in overtone photochemistry experiments is a strong function of the average energy transferred per collision. These experiments are therefore a good method of obtaining information about this important parameter.

5. For fixed $\overline{\langle(\Delta E)\rangle_{o}}$, the average energy transferred per collision averaged over the initial distribution, the yield is sensitive to the shape of the energy transfer function both for excitation just above threshold (fourth overtone) and for excitation high above threshold (fifth overtone). Increased yield correlates directly with larger values of the second moment of $p\left(E, E^{\prime}\right)$, i.e., $\overline{\left.<(\Delta E)^{2}\right\rangle_{o}}$.

\section{References}

1. K. V. Reddy and M. J. Berry, "Intracavity CW Dye Laser Photoactivation of Unimolecular Reactants: Isomerization of State-Selected Methyl Isocyanide," Chem. Phys. Lett. 52, 111 (1977).

2. K. V. Reddy and M. J. Berry, "Reaction Dynamics of State-Selected Unimolecular Reactants. Energy Dependence of the Rate Coefficient for Methyl Isocyanide Isomerization," Faraday Discuss. of Chem. Soc. 67, 188 (1979).

\section{Hydrogen-Atom Abstraction From Isobutane by $\mathrm{OH}^{\dagger}$}

\author{
F. P. Tully, J. E. M. Goldsmith, A. T. Droege
}

Hydrogen-atom abstraction from hydrocarbons by hydroxyl radicals plays a fundamental role in the chemistry of atmospheric and combustion processes. Numerical modeling of these processes requires a large data base of accurate rate coefficients spanning a wide range of temperatures and pressures. Both the absolute rate

† J. Phys. Chem. 90, 5932 (1986). 
coefficients and the branching ratios for formation of isomeric product radicals are required input to chemical models. In previous reports, we described measurements of rate coefficients for $\mathrm{H}$ - and D-atom abstraction by $\mathrm{OH}$ from primary and/or secondary C-H and C-D sites in neopentane, 2,2,3,3-tetramethyl butane, ethane, and propane. The reactivities of individual abstraction sites were characterized and kinetic isotope effects were discussed. In this study, using the laser photo-lysis/laser-induced fluorescence experimental technique, we made kinetic measurements of the reactions of $\mathrm{OH}$ with four selectively deuterated isotopes of isobutane. We determined absolute rate coefficients, $\mathrm{k}_{1}-\mathrm{k}_{4}$, for the reactions

$$
\begin{gathered}
\mathrm{OH}+\left(\mathrm{H}_{3} \mathrm{C}\right)_{3} \mathrm{CH} \stackrel{\mathrm{k}_{1}}{\longrightarrow}\left\{\begin{array}{l}
\mathrm{H}_{2} \mathrm{O}+\left(\mathrm{H}_{3} \mathrm{C}\right)_{2} \mathrm{CHCH}_{2} \\
\mathrm{H}_{2} \mathrm{O}+\left(\mathrm{H}_{3} \mathrm{C}\right)_{3} \mathrm{C}
\end{array}\right. \\
\mathrm{OH}+\left(\mathrm{H}_{3} \mathrm{C}\right)_{3} \mathrm{CD} \stackrel{\mathrm{k}_{2}}{\longrightarrow}\left\{\begin{array}{l}
\mathrm{H}_{2} \mathrm{O}+\left(\mathrm{H}_{3} \mathrm{C}\right)_{2} \mathrm{CDCH}_{2} \\
\mathrm{HDO}+\left(\mathrm{H}_{3} \mathrm{C}\right)_{3} \mathrm{C}
\end{array}\right. \\
\mathrm{OH}+\left(\mathrm{D}_{3} \mathrm{C}\right)_{3} \mathrm{CH} \stackrel{\mathrm{k}_{3}}{\longrightarrow}\left\{\begin{array}{l}
\mathrm{HDO}+\left(\mathrm{D}_{3} \mathrm{C}\right)_{2} \mathrm{CHCD}_{2} \\
\mathrm{H}_{2} \mathrm{O}+\left(\mathrm{D}_{3} \mathrm{C}\right)_{3} \mathrm{C}
\end{array}\right. \\
\mathrm{OH}+\left(\mathrm{D}_{3} \mathrm{C}\right)_{3} \mathrm{CD} \stackrel{\mathrm{k}_{4}}{\longrightarrow}\left\{\begin{array}{l}
\mathrm{HDO}+\left(\mathrm{D}_{3} \mathrm{C}\right)_{2} \mathrm{CDCD}_{2} \\
\mathrm{HDO}+\left(\mathrm{D}_{3} \mathrm{C}\right)_{3} \mathrm{C}
\end{array}\right.
\end{gathered}
$$

at 400 Torr of helium pressure over the temperature range 293-864 K. We utilized the results of these experiments to calculate the branching ratios for formation of t-butyl and isobutyl radicals in Reaction (1) and to characterize the kinetic isotope effect at the tertiary abstraction site in isobutane.

The absolute rate coefficients determined in these studies are plotted in Figure 1. The solid curves in Figure 1 were generated by nonlinear-least-squares fits of our $\mathrm{k}_{i}(\mathrm{~T})$ data (weighted as $\left.\omega_{k}=1 /{\sigma_{k}}^{2}\right)$ to the modified Arrhenius expression $\mathrm{k}_{i}(\mathrm{~T})=\mathrm{A}_{i} \mathrm{~T}^{n_{i}} \exp \left(-\mathrm{E}_{i} / \mathrm{RT}\right)$. The optimized rate-coefficient expressions, in the units $\mathrm{cm}^{3}$ molecule ${ }^{-1} \mathrm{~s}^{-1}$, are

$$
\begin{aligned}
& \mathrm{k}_{1}(\mathrm{~T})=\left(4.31 \times 10^{-17}\right) \mathrm{T}^{1.80} \mathrm{e}^{\left(+348 \mathrm{cal} \mathrm{mole}^{-1} / \mathrm{RT}\right)} \\
& \mathrm{k}_{2}(\mathrm{~T})=\left(1.20 \times 10^{-16}\right) \mathrm{T}^{1.69} \mathrm{e}^{\left(-169 \mathrm{cal} \mathrm{mole}^{-1} / \mathrm{RT}\right)} \\
& \mathrm{k}_{3}(\mathrm{~T})=\left(1.08 \times 10^{-19}\right) \mathrm{T}^{2.57} \mathrm{e}^{\left(+1130 \mathrm{cal} \mathrm{mole}^{-1} / \mathrm{RT}\right)} \\
& \mathrm{k}_{4}(\mathrm{~T})=\left(9.12 \times 10^{-20}\right) \mathrm{T}^{2.63} \mathrm{e}^{\left(+699 \mathrm{cal} \mathrm{mole}^{-1} / \mathrm{RT}\right)}
\end{aligned}
$$

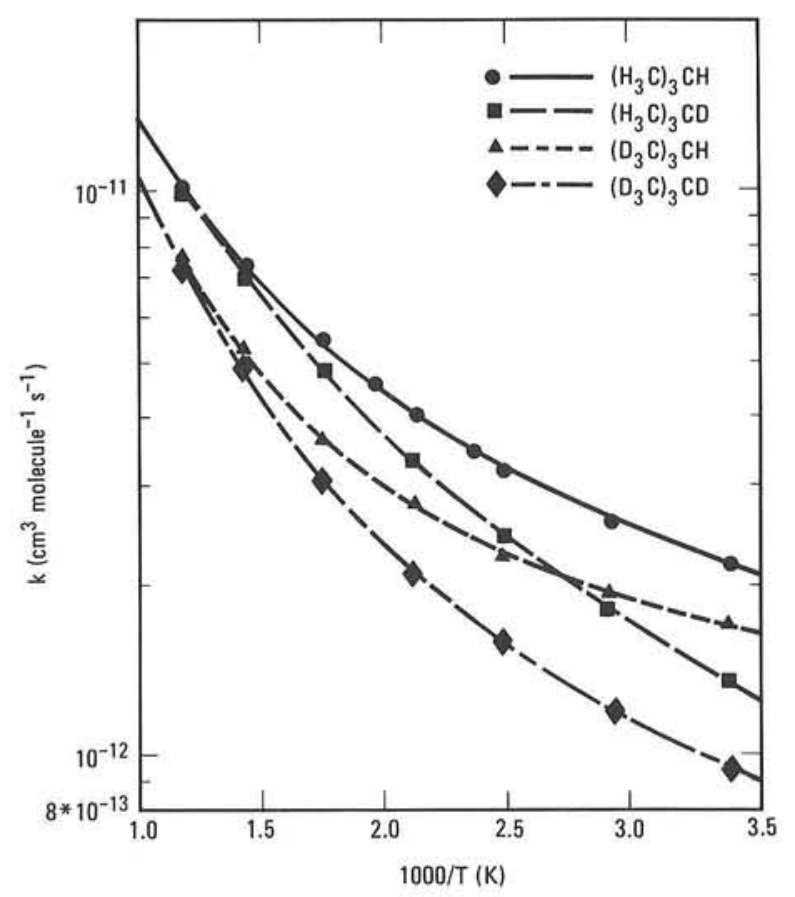

Figure 1. Arrhenius plot of our kinetic measurements on Reactions (1)-(4). The curves drawn through the data points plot the best-fit $k_{i}(T)$ expressions given in the text.

In our recent studies of the reactions of $\mathrm{OH}$ with ethane and with propane, we showed conclusively that the reactivity of a given methyl or methylene group could be suppressed by deuterium substitution within that group without altering the reactivity of the remaining $\mathrm{C}-\mathrm{H}$ or $\mathrm{C}-\mathrm{D}$ sites in the molecule. We anticipate analogous results for the reactions $\mathrm{OH}+$ isobutanes $\rightarrow$ products, and we make the assumption that the primary and tertiary $\mathrm{C}-\mathrm{H}$ and $\mathrm{C}-\mathrm{D}$ reactivities are separable. This assumption permits $\mathrm{k}_{1}$ to $\mathrm{k}_{4}$ to be written as follows:

$$
\begin{aligned}
& \mathrm{k}_{1}=3 \mathrm{k}_{\mathrm{H}}{ }^{\mathrm{P}}+\mathrm{k}_{\mathrm{H}}{ }^{\mathrm{T}} \\
& \mathrm{k}_{2}=3 \mathrm{k}_{\mathrm{H}}{ }^{\mathrm{P}}+\mathrm{k}_{\mathrm{D}}{ }^{\mathrm{T}} \\
& \mathrm{k}_{3}=3 \mathrm{k}_{\mathrm{D}}{ }^{\mathrm{P}}+\mathrm{k}_{\mathrm{H}}{ }^{\mathrm{T}} \\
& \mathrm{k}_{4}=3 \mathrm{k}_{\mathrm{D}}{ }^{\mathrm{P}}+\mathrm{k}_{\mathrm{D}}{ }^{\mathrm{T}}
\end{aligned}
$$

where $\mathrm{k}_{\mathrm{H}}{ }^{\mathrm{P}}, \mathrm{k}_{\mathrm{D}}{ }^{\mathrm{P}}, \mathrm{k}_{\mathrm{H}}{ }^{\mathrm{T}}$, and $\mathrm{k}_{\mathrm{D}}{ }^{\mathrm{T}}$ are site-specific rate coefficients for $\mathrm{H}$ - and $\mathrm{D}$-atom abstraction per primary $\mathrm{CH}_{3}$ - or $\mathrm{CD}_{3}$-group and per tertiary $\mathrm{CH}$ - or CD-group, respectively. For the above equations to be justified, the equality 


$$
\begin{aligned}
& \mathrm{k}_{1}(\mathrm{~T})+\mathrm{k}_{4}(\mathrm{~T})=\mathrm{k}_{2}(\mathrm{~T})+\mathrm{k}_{3}(\mathrm{~T}) \\
& \quad=3 \mathrm{k}_{\mathrm{H}}^{\mathrm{P}}(\mathrm{T})+3 \mathrm{k}_{\mathrm{D}}^{\mathrm{P}}(\mathrm{T})+\mathrm{k}_{\mathrm{H}}{ }^{\mathrm{T}}(\mathrm{T})+\mathrm{k}_{\mathrm{D}}{ }^{\mathrm{T}}(\mathrm{T})
\end{aligned}
$$

must hold for all temperatures $\mathrm{T}$. These sums are calculated from the experimental data, and Equation (II) is proven correct to within an average deviation of $1.2 \%$. This result clearly demonstrates that the total reaction-rate coefficient may be expressed as the sum of individual methyl- and methylidyne-group reactivities, each of which is independent of the extent of deuteration of the remainder of the isobutane molecule.

Because Equality (II) is valid, Equation (I) reduces to a set of three independent equations with four unknowns. Therefore, in order to obtain values for $k_{H}{ }^{P}, k_{D}{ }^{P}, k_{H}{ }^{T}$, and $k_{D}{ }^{T}$, one further assumption is necessary. We have three choices for this additional constraint. First, results from earlier studies in this series indicate that the temperature dependence of the primary kinetic isotope effect, $\mathrm{k}_{\mathrm{H}}{ }^{\mathrm{P}} / \mathrm{k}_{\mathrm{D}}{ }^{\mathrm{P}}(\mathrm{T})$, is nearly identical for the reactions $\mathrm{OH}+$ ethane and $\mathrm{OH}$ + neopentane. Since isobutane and neopentane are similar compounds, we assume that the expression derived from the $\mathrm{OH}+$ neopentane results, $\mathrm{k}_{\mathrm{H}}{ }^{\mathrm{P}} / \mathrm{k}_{\mathrm{D}}{ }^{\mathrm{P}}(\mathrm{T})=(0.94 \pm 0.09) \exp (938 \pm$ $92 \mathrm{cal} \mathrm{mole}-1 / \mathrm{RT})$, is also appropriate for primary $\mathrm{H}$ - and $\mathrm{D}$-atom abstraction from isobutane by $\mathrm{OH}$. Note that this relation constrains the relative abstraction-rate coefficients from $\mathrm{CH}_{3}$ and $\mathrm{CD}_{3}$-groups, but not their absolute magnitudes. Also, in recent calculations Walker ${ }^{1}$ has shown that reaction-rate coefficients for $\mathrm{OH}+$ neopentane may be used to approximate $\mathrm{CH}_{3}$ group reactivities in inter- mediate-sized hydrocarbons. Thus, as second and third possibilities for choosing a fourth independent equation, we assume that $\mathrm{k}_{\mathrm{H}}{ }^{\mathrm{P}}$ and $\mathrm{k}_{\mathrm{D}}{ }^{\mathrm{P}}$ in isobutane are given by the relations $3 \mathrm{k}_{\mathrm{H}}{ }^{\mathrm{P}}=0.75 \mathrm{k}\left(\mathrm{OH}+\right.$ neo- $\left.C_{5} H_{12}\right)$ and $3 \mathrm{k}_{\mathrm{D}}{ }^{\mathrm{P}}=0.75 \mathrm{k}\left(\mathrm{OH}+\right.$ neo- $\left.C_{5} D_{12}\right)$, where the $\mathrm{OH}+$ neopentane data are taken from our previous work. The $\mathrm{k}_{\mathrm{H}}{ }^{\mathrm{T}}$ and $\mathrm{k}_{\mathrm{D}}{ }^{\mathrm{T}}$ values that result from the above three solutions to Equation (I) are, within their propagated $\pm 2 \sigma$ error limits, mutually consistent.

Utilizing the assumptions justified above, we may generate best-fit representations for the site-specific rate coefficients. The resulting expressions, in the units $\mathrm{cm}^{3}$ molecule ${ }^{-1} \mathrm{~s}^{-1}$, are

$$
\begin{aligned}
& \mathrm{k}_{\mathrm{H}}^{\mathrm{T}}(\mathrm{T})=\left(9.52 \times 10^{-14}\right) \mathrm{T}^{0.51} \mathrm{e}^{\left(-63 \mathrm{cal} \mathrm{mole}^{-1} / \mathrm{RT}\right)} \\
& \mathrm{k}_{\mathrm{D}}{ }^{\mathrm{T}}(\mathrm{T})=\left(1.05 \times 10^{-15}\right) \mathrm{T}^{1.16} \mathrm{e}^{\left(+29 \mathrm{cal} \mathrm{mole}^{-1} / \mathrm{RT}\right)} \\
& 3 \mathrm{k}_{\mathrm{H}}{ }^{\mathrm{P}}(\mathrm{T})=\left(3.81 \times 10^{-16}\right) \mathrm{T}^{1.53} \mathrm{e}^{\left(-776 \mathrm{cal} \mathrm{mole}^{-1} / \mathrm{RT}\right)} \\
& \left.3 \mathrm{k}_{\mathrm{D}}{ }^{\mathrm{P}}(\mathrm{T})=\left(4.13 \times 10^{-20}\right) \mathrm{T}^{2.79} \mathrm{e}^{(-433 \mathrm{cal} \mathrm{mole}} \mathrm{e}^{-1} / \mathrm{RT}\right) \text {. }
\end{aligned}
$$

These best-fit expressions for $\mathrm{k}_{\mathrm{H}}{ }^{\mathrm{T}}(\mathrm{T}), \mathrm{k}_{\mathrm{D}}{ }^{\mathrm{T}}(\mathrm{T})$, $3 \mathrm{k}_{\mathrm{H}}{ }^{\mathrm{P}}(\mathrm{T})$, and $3 \mathrm{k}_{\mathrm{D}}{ }^{\mathrm{P}}(\mathrm{T})$ are represented graphically in Figure 2. Tertiary $\mathrm{H}$-atom abstraction dominates the reactivity at low temperatures, but primary $\mathrm{H}$-atom transfer becomes most important above $500 \mathrm{~K}$.

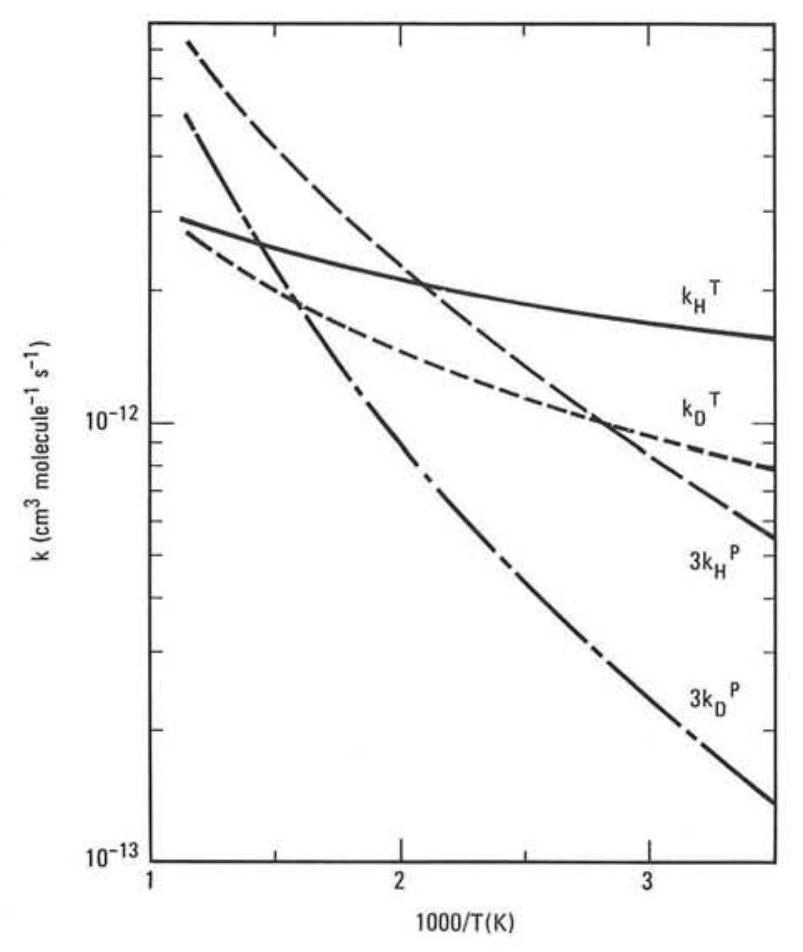

Figure 2. Arrhenius plot of the total rate coefficients for $\mathrm{H}$ - and D-atom transfer to $\mathrm{OH}$ from primary and tertiary abstraction sites in isobutane.

One other interesting feature of this work concerns the magnitudes of the kinetic-isotope effects that we find for $\mathrm{H}$ - and D-atom abstraction by $\mathrm{OH}$ from primary-, secondary- and tertiaryreactive sites in alkanes. For the reactions of $\mathrm{OH}$ with neopentane and with ethane, we determined primary-kinetic-isotope effects which changed from 5.0 to 1.5 as $\mathrm{T}$ varied over the 
temperature range 290-903 K. For reaction of $\mathrm{OH}$ at the secondary site in propane, $\mathrm{k}_{\mathrm{H}} \mathrm{s} / \mathrm{k}_{\mathrm{D}} \mathrm{s}$ varies from 2.6 to 1.5 over a comparable temperature range. For reaction of $\mathrm{OH}$ at the tertiary site in isobutane, $\mathrm{k}_{\mathrm{H}}^{\mathrm{T}} / \mathrm{k}_{\mathrm{D}}{ }^{\mathrm{T}}$ varied from 1.9 to near (or below) the classical limit of 1.4 as the temperature increased from $300 \mathrm{~K}$ to $750 \mathrm{~K}$. Theoretical attempts to reproduce this trend in $\mathrm{k}_{\mathrm{H}} / \mathrm{k}_{\mathrm{D}}$ magnitudes are in progress.

\section{Reference}

1. R. W. Walker, "Temperature Coefficients for Reactions of $\mathrm{OH}$ Radicals with Alkanes Between 300 and $1000 \mathrm{~K}$," Int. J. Chem. Kinet. 17, 573 (1985).

\section{Hydrogen-Atom Abstraction From n-Butane by $\mathrm{OH}^{\dagger}$}

\section{A. T. Droege, F. P. Tully}

Hydrogen-atom abstraction from alkanes by hydroxyl radicals is the principal mechanism of fuel consumption in lean and stoichiometric alkane/air flames. Numerical modeling of combustion processes requires both the absolute rate coefficients and the branching ratios for the formation of product radicals in these reactions in order to accurately model the subsequent isomeric radical chemistry. In the previous four papers in this series, we described measurements of the rate coefficients for $\mathrm{H}$ - and D-atom abstraction by $\mathrm{OH}$ from primary, secondary, and tertiary C-H and C-D sites in neopentane, 2,2,3,3tetramethylbutane, ethane, propane, and isobutane. The reactivities of individual abstraction sites were found to be independent of the hydrogen isotopic content of remote alkyl groups within the alkane molecule. Kinetic isotope effects were characterized, and, for the reactions $\mathrm{OH}+\mathrm{C}_{3} \mathrm{H}_{8} \rightarrow$ products and $\mathrm{OH}+$ iso- $\mathrm{C}_{4} \mathrm{H}_{10}$

† J. Phys. Chem. 90, 5937 (1986). $\rightarrow$ products, branching ratios for the formation of isomeric product radicals were determined.

In this study we report kinetic measurements on the reactions of $\mathrm{OH}$ with n-butane and nbutane- $\mathrm{d}_{10}$ using the laser photolysis/laserinduced fluorescence experimental technique. Using several assumptions based on the results of our previous $\mathrm{OH}+$ alkane studies, these data permit us to determine site-specific rate coefficients for $\mathrm{H}$ - and $\mathrm{D}$-atom abstraction by $\mathrm{OH}$ from the methyl and methylene groups in nbutane. We characterize the kinetic isotope effect for abstraction of $\mathrm{H}$ - and D-atoms from the methylene sites in n-butane and compare it to our previous measurement for $\mathrm{H}$ - and $\mathrm{D}$ atom transfer to $\mathrm{OH}$ from the secondary site in propane.

We measured absolute rate coefficients for the reaction of $\mathrm{OH}$ with $\mathrm{n}-\mathrm{C}_{4} \mathrm{H}_{10}(1)$ and $\mathrm{n}-\mathrm{C}_{4} \mathrm{D}_{10}$ (2) at 400 Torr of helium pressure over the temperature ranges 294-509 $\mathrm{K}\left(\mathrm{k}_{1}\right)$ and 294-599 K $\left(\mathrm{k}_{2}\right)$. The rate coefficients determined in these studies are plotted in Figure 1.

The solid curves in Figure 1 were generated by a nonlinear-least-squares fit of our $\mathrm{k}_{1}(\mathrm{~T})$ and $\mathrm{k}_{2}\left(\mathrm{~T}\right.$ ) data (weighted as $\omega_{k}=1 / \sigma_{k}{ }^{2}$ ) to the modified-Arrhenius expression $\mathrm{k}(\mathrm{T})=\mathrm{AT}^{n}$ $\exp (-\mathrm{E} / \mathrm{RT})$, with the result $\mathrm{k}_{1}(\mathrm{~T})=(2.34 \mathrm{x}$ $\left.10^{-17}\right) \mathrm{T}^{1.95} \exp \left(+267 \mathrm{cal} \mathrm{mole}^{-1} / \mathrm{RT}\right) \mathrm{cm}^{3}$ molecule ${ }^{-1} \mathrm{~s}^{-1}$ and $\mathrm{k}_{2}(\mathrm{~T})=\left(2.92 \times 10^{-18}\right) \mathrm{T}^{2.20}$ $\exp \left(+65 \mathrm{cal} \mathrm{mole}^{-1} / \mathrm{RT}\right) \mathrm{cm}^{3}$ molecule $\mathrm{c}^{-1} \mathrm{~s}^{-1}$.

In the preceding studies in this series, we showed conclusively that the reactivity of an individual methyl, methylene, or methylidyne group could be suppressed by deuterium substitution within that group without altering the reactivity of the remaining $\mathrm{C}-\mathrm{H}$ or C-D sites in the molecule. In our investigations of the $\mathrm{OH}+$ propane and $\mathrm{OH}+$ isobutane reactions, we characterized the kinetics of selectively deuterated hydrocarbon isotopes. These data permitted us to derive the temperature-dependent branching ratios for formation of the isomeric radical products of these reactions. In the present study, 


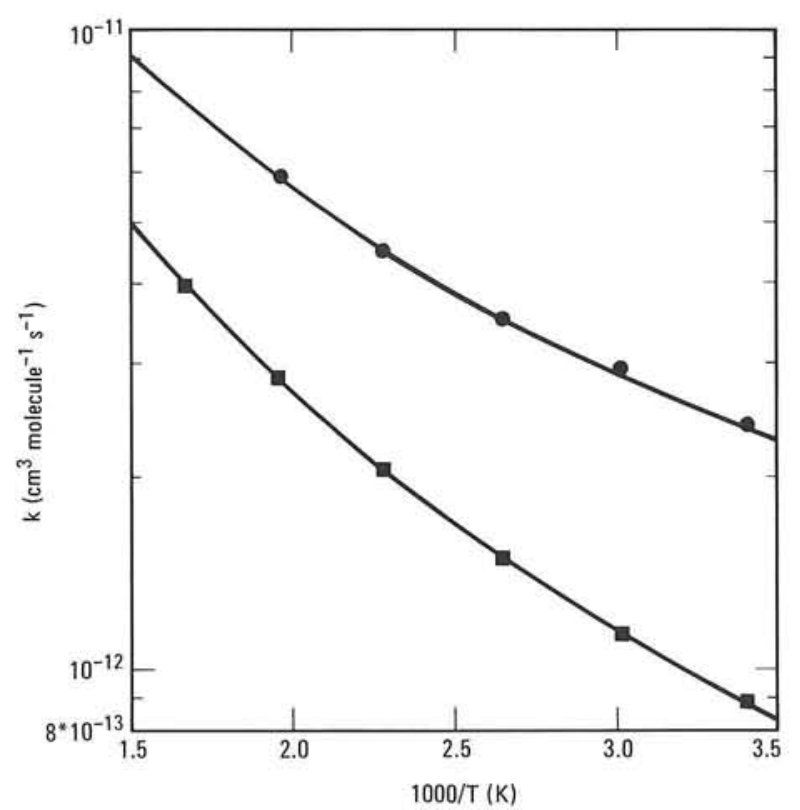

Figure 1. Arrhenius plot of our measurements for reaction (1), $\bigcirc$ and (2), $\square$. The curve drawn through the data points represents the best-fit $\mathbf{k}_{i}(\mathrm{~T})$ expression given in the text.

only the reactions of $\mathrm{OH}$ with $\mathrm{n}-\mathrm{C}_{4} \mathrm{H}_{10}$ and n$\mathrm{C}_{4} \mathrm{D}_{10}$ were investigated. Following the procedures of our earlier studies, we may write the equations

$$
\begin{aligned}
& \mathrm{k}_{1}=2 \mathrm{k}_{\mathrm{H}}{ }^{\mathrm{P}}+2 \mathrm{k}_{\mathrm{H}}{ }^{\mathrm{s}} \\
& \mathrm{k}_{2}=2 \mathrm{k}_{\mathrm{D}}{ }^{\mathrm{P}}+2 \mathrm{k}_{\mathrm{D}} \mathrm{s}
\end{aligned}
$$

where $\mathrm{k}_{\mathrm{H}}{ }^{\mathrm{P}}, \mathrm{k}_{\mathrm{D}}{ }^{\mathrm{P}}, \mathrm{k}_{\mathrm{H}} \mathrm{s}$, and $\mathrm{k}_{\mathrm{D}}{ }^{\mathrm{s}}$ are site-specific rate coefficients for $\mathrm{H}$ - and D-atom abstraction per primary $\mathrm{CH}_{3}$ - or $\mathrm{CD}_{3}$-group and per secondary $\mathrm{CH}_{2}$ - or $\mathrm{CD}_{2}$-group, respectively. For the reactions of $\mathrm{OH}$ with ethane, propane, and isobutane, analogous separations of reactivity were proven valid to within average deviations of $1.2 \%$; the equations are undoubtedly appropriate for the reaction of $\mathrm{OH}$ with n-butane. As written, the above two equations contain four unknowns, $\mathrm{k}_{\mathrm{H}}{ }^{\mathrm{P}}, \mathrm{k}_{\mathrm{D}}{ }^{\mathrm{P}}, \mathrm{k}_{\mathrm{H}}{ }^{\mathrm{S}}$, and $\mathrm{k}_{\mathrm{D}}{ }^{\mathrm{S}}$, and two additional relations among these quantities are needed to produce a solution.

Results from earlier studies in this series indicate that the temperature dependence of the primary kinetic isotope effect, $\mathrm{k}_{\mathrm{H}}{ }^{\mathrm{P}} / \mathrm{k}_{\mathrm{D}}{ }^{\mathrm{P}}(\mathrm{T})$, is nearly identical for the reactions $\mathrm{OH}+$ ethane and $\mathrm{OH}+$ neopentane. As a third independent equation, then, we make the same assumption that we used in analyzing our $\mathrm{OH}+$ propane data: that the primary kinetic isotope effect appropriate for the $\mathrm{OH}+\mathrm{n}$-butane reaction is identical to that found for the $\mathrm{OH}+$ ethane reaction, and $\mathrm{k}_{\mathrm{H}}{ }^{\mathrm{P}} / \mathrm{k}_{\mathrm{D}}{ }^{\mathrm{P}}(\mathrm{T})=(1.01 \pm 0.06) \exp (907 \pm$ $52 \mathrm{cal} \mathrm{mole}-1 / \mathrm{RT})$. In our $\mathrm{OH}+$ propane and $\mathrm{OH}+$ isobutane studies, we determined branching ratios for the formation of $\mathrm{n}_{-} \mathrm{C}_{3} \mathrm{H}_{7}$ and i$\mathrm{C}_{3} \mathrm{H}_{7}$ radicals and $\mathrm{t}-\mathrm{C}_{4} \mathrm{H}_{9}$ and $\mathrm{i}-\mathrm{C}_{4} \mathrm{H}_{9}$ radicals, respectively. Recently, Atkinson ${ }^{1}$ derived generalized formulae for calculating both total and site-specific rate coefficients for reactions involving $\mathrm{H}$-atom transfer to $\mathrm{OH}$. Atkinson's approach accounts for the influence of neighboring alkyl groups on reactivity, and, over the temperature range $300-1000 \mathrm{~K}$, the branching ratios calculated using his formulae agree with our $\mathrm{OH}+$ propane and $\mathrm{OH}+$ isobutane determinations to within an average deviation of $\approx 4 \%$. Because of this excellent agreement with our previous work, we assume that the branching ratios for n-butyl and sec-butyl radical production from $\mathrm{OH}+\mathrm{n}$ butane calculated using Atkinson's formulae are correct. This assumption yields the fourth equation, $\mathrm{k}_{\mathrm{H}}{ }^{\mathrm{P}} / \mathrm{k}_{\mathrm{H}}{ }^{\mathrm{S}}(\mathrm{T})=1.035 \exp (-536 \mathrm{~K} / \mathrm{T})$, needed to produce a solution for $\mathrm{k}_{\mathrm{H}}{ }^{\mathrm{P}}, \mathrm{k}_{\mathrm{D}}{ }^{\mathrm{P}}, \mathrm{k}_{\mathrm{H}} \mathrm{S}$, and $\mathrm{k}_{\mathrm{D}} \mathrm{s}$. Site-specific rate coefficients for H- and Datom abstraction from two methyl or methylene groups in n-butane are plotted in Figure 2.

Also displayed are optimized fits of these data (weighted as $\omega_{\mathrm{k}}=1 /{\sigma_{\mathrm{k}}}^{2}$ ) to the modified Arrhenius equation $\mathrm{k}(\mathrm{T})=\mathrm{AT} \mathrm{T}^{n} \exp (-\mathrm{E} / \mathrm{RT})$. The resulting expressions, in the units $\mathrm{cm}^{3}$ molecule $\mathrm{e}^{-1}$ $\mathrm{s}^{-1}$, are

$$
\begin{aligned}
2 \mathrm{k}_{\mathrm{H}}{ }^{\mathrm{S}}(\mathrm{T}) & =\left(1.20 \times 10^{-16}\right) \mathrm{T}^{1.64} \mathrm{e}^{\left(+247 \mathrm{cal} \mathrm{mole}^{-1} / \mathrm{RT}\right)} \\
2 \mathrm{k}_{\mathrm{D}}{ }^{\mathrm{S}}(\mathrm{T}) & =\left(1.49 \times 10^{-18}\right) \mathrm{T}^{2.24} \mathrm{e}^{\left(+300 \mathrm{cal} \mathrm{mole}^{-1} / \mathrm{RT}\right)} \\
2 \mathrm{k}_{\mathrm{H}}{ }^{\mathrm{P}}(\mathrm{T}) & =\left(6.86 \times 10^{-17}\right) \mathrm{T}^{1.73} \mathrm{e}^{\left(-753 \mathrm{cal} \mathrm{mole}^{-1} / \mathrm{RT}\right)} \\
2 \mathrm{k}_{\mathrm{D}}{ }^{\mathrm{P}}(\mathrm{T}) & =\left(6.78 \times 10^{-17}\right) \mathrm{T}^{1.73} \mathrm{e}^{\left(-1659 \mathrm{cal} \mathrm{mole}^{-1} / \mathrm{RT}\right)} .
\end{aligned}
$$

Clearly, H- and D-atom abstractions from the secondary sites in $\mathrm{n}-\mathrm{C}_{4} \mathrm{H}_{10}$ and $\mathrm{n}-\mathrm{C}_{4} \mathrm{D}_{10}$ dominate the reactivity in reactions (1) and (2) throughout the temperature range of our measurements. 


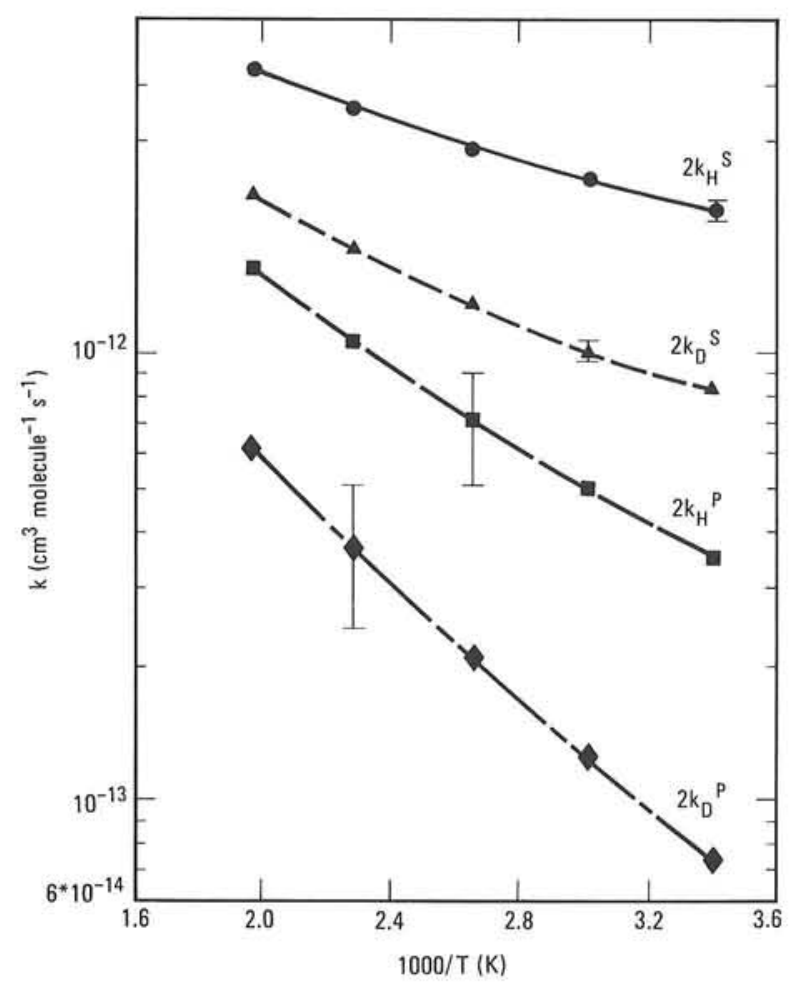

Figure 2. Arrhenius plot of the total rate coefficients for $\mathrm{H}$ - and $\mathrm{D}$-atom abstraction by $\mathrm{OH}$ from primary and secondary abstraction sites in n-butane. Error limits represent $\pm 2 \sigma$ estimates of the total experimental uncertainty and are shown for only one point in each data set for pictorial clarity.

From the data, we may compute $\mathrm{k}_{\mathrm{H}} \mathrm{s} / \mathrm{k}_{\mathrm{D}} \mathrm{s}$ for the five temperatures at which $\mathrm{k}_{1}$ and $\mathrm{k}_{2}$ were both measured. These quantities are compiled in Table I along with comparable-temperature $\mathrm{k}_{\mathrm{H}} \mathrm{s} / \mathrm{k}_{\mathrm{D}} \mathrm{s}$ values determined in our previous study of the $\mathrm{OH}+$ propane $\rightarrow$ products reaction.

Within cumulative error estimates, we obtain identical values of $\mathrm{k}_{\mathrm{H}} \mathrm{s} / \mathrm{k}_{\mathrm{D}} \mathrm{s}$ for the two reactions. This finding is interesting in light of the fact that the values of $\mathrm{k}_{\mathrm{H}} \mathrm{s}$ for $\mathrm{OH}+\mathrm{n}$-butane exceed those for $\mathrm{OH}+$ propane by factors of $1.2-1.3$. We found an entirely analogous result in our previous studies of the reactions $\mathrm{OH}+$ ethane $\rightarrow$ products and $\mathrm{OH}+$ neopentane $\rightarrow$ products. Although the values $\mathrm{k}_{\mathrm{H}}^{\mathrm{P}}(\mathrm{T})$ are very different for these two processes, the quantities $\mathrm{k}_{\mathrm{H}}{ }^{\mathrm{P}} / \mathrm{k}_{\mathrm{D}}{ }^{\mathrm{P}}(\mathrm{T})$ are nearly identical.
Table I

Comparison of Kinetic Isotope Effects at Methylene Reaction Sites for the Reactions $\mathrm{OH}+\mathrm{n}$-Butane $\rightarrow$ Products and $\mathrm{OH}+$ Propane $\rightarrow$ Products

\begin{tabular}{ccc}
\hline & $\begin{array}{c}\text { n-butane } \\
\mathrm{k}_{\mathrm{H}} \mathrm{s} / \mathrm{k}_{\mathrm{D}}{ }^{\mathrm{s}}\end{array}$ & $\begin{array}{c}\text { propane } \\
\mathrm{k}_{\mathrm{H}} \mathrm{s} / \mathrm{k}_{\mathrm{D}}{ }^{\mathrm{s}}\end{array}$ \\
\hline 294 & $2.52 \pm 0.17^{a}$ & - \\
295 & - & $2.62 \pm 0.49$ \\
328.5 & - & $2.56 \pm 0.77$ \\
332 & $2.43 \pm 0.16$ & - \\
376.5 & - & $2.22 \pm 0.19$ \\
377 & $2.21 \pm 0.16$ & - \\
437.2 & - & $2.08 \pm 0.29$ \\
439 & $2.06 \pm 0.16$ & - \\
503.5 & - & $2.08 \pm 0.15$ \\
509 & $1.91 \pm 0.15$ & - \\
\hline
\end{tabular}

${ }^{a}$ Uncertainties represent $\pm 2 \sigma$ estimates of the total experimental error.

As we found in our earlier investigations, $\mathrm{k}_{\mathrm{H}} \mathrm{s} /$ $\mathrm{k}_{\mathrm{D}}{ }^{\mathrm{S}}$ may be well represented by the functional form $\mathrm{k}_{\mathrm{H}} \mathrm{s} / \mathrm{k}_{\mathrm{D}} \mathrm{s}(\mathrm{T})=\mathrm{I} \exp (\mathrm{C} / \mathrm{RT})$. Best-fit values of $\mathrm{I}$ and $\mathrm{C}$ and their associated $\pm 2 \sigma$ uncertainties are $1.31 \pm 0.12$ and $390 \pm 64 \mathrm{cal} \mathrm{mole}^{-1}$, respectively. As we have noted previously, the magnitudes of $\mathrm{k}_{\mathrm{H}} / \mathrm{k}_{\mathrm{D}}$ appear to fall into groups according to the relation $\mathrm{k}_{\mathrm{H}} / \mathrm{k}_{\mathrm{D}}$ (primary) > $\mathrm{k}_{\mathrm{H}} / \mathrm{k}_{\mathrm{D}}$ (secondary) $>\mathrm{k}_{\mathrm{H}} / \mathrm{k}_{\mathrm{D}}$ (tertiary). Theoretical analysis of this trend continues.

\section{Reference}

1. R. Atkinson, "Estimation of $\mathrm{OH}$ Radical Rate Constants for $\mathrm{H}$-atom Abstraction from $\mathrm{C}-\mathrm{H}$ and O-H Bonds Over the Temperature Range 250-1000 K.," Int. J. Chem. Kinet. 18, 555 (1986). 


\section{Determination of $\mathrm{H}, \mathrm{OH}$, and $O$ Species Concentrations by Deuterium Scavenging ${ }^{\dagger}$}

\author{
J. V. Volponi, W. J. McLean, R. M. Fristrom, * \\ Z. A. Munir ${ }^{* *}$
}

Measurements of the concentration profiles of stable and radical species in low pressure acetylene-oxygen-argon flames have been made by the scavenger probe technique using deuterium as the scavenging agent. The use of the deuterium species offers the advantage over previously examined scavengers ${ }^{1}$ of rapid reaction with the most common flame radicals $-\mathrm{H}, \mathrm{OH}$, and $\mathrm{O}$ - to yield a unique product for each radical by the reactions :

$$
\begin{gathered}
\mathrm{D}_{2}+\mathrm{H} \longrightarrow \mathrm{DH}+\mathrm{D} \\
\mathrm{D}_{2}+\mathrm{OH} \longrightarrow \mathrm{DOH}+\mathrm{D} \\
2 \mathrm{D}_{2}+\mathrm{O} \longrightarrow \mathrm{D}_{2} \mathrm{O}+2 \mathrm{D}
\end{gathered}
$$

Determining the $\mathrm{DH}, \mathrm{DOH}$, and $\mathrm{D}_{2} \mathrm{O}$ concentrations in the scavenged flame with a mass spectrometer gives a direct indication of the $\mathrm{H}, \mathrm{OH}$, and $\mathrm{O}$ concentrations, respectively, ${ }^{1}$ provided that the scavenging reactions sufficiently take place rapidly near the probe entrance that the above reactions go to completion before $\mathrm{H}, \mathrm{OH}$ and $\mathrm{O}$ are removed via other homogeneous or heterogeneous reactions.

The experimental system used in this study consists of a low-pressure flame chamber which houses a 10-cm-diameter flat flame burner. Flames of equivalence ratio $0.99,1.58$, and 0.75 were stabilized at 25,18 , and 76 torr, respectively. A quartz probe, placed to sample from the center of the vertically translatable burner, transferred gas samples to a quadrupole mass spectrometer that was operated in a differentially pumped, chopped, molecular-beam mode. The deuterium was added through an injector probe that was placed through the center of the

$\dagger$ Comb. Flame 65, 243 (1986).

* Johns Hopkins University, Laurel, MD.

** University of California at Davis. sampling probe, as close to the entrance to the sampling probe as possible, to insure reaction with the largest quantity of radicals, and fabricated to prevent deuterium flow out of the sampling probe tip. The molar flow rate needed to cause effective reaction was found to be approximately 3 times the sample flow rate.

The experimentally determined stable and radical species concentration profiles for the $\phi=0.99$ flame are shown in Figure 1.

Plotted along with the experimental data are profiles computed from a detailed chemical kinetic model. ${ }^{2}$ In general, the measured and predicted stable-species profiles are in good agreement with differences attributable to the combined effects of experimental error and uncertainties in the model predictions due to errors in temperature measurement. The computed and experimental $\mathrm{H}$ atom profiles are in good agreement, but the $\mathrm{O}$ and $\mathrm{OH}$ radical profiles are not. The radical profiles for the $\phi=1.58$ flame exhibit similar behavior when compared to the calculated profiles.

The differences between the predicted $\mathrm{O}$ and $\mathrm{OH}$ concentrations and the experimentally determined $\mathrm{O}$ and $\mathrm{OH}$ concentrations may be explained by the occurrence of additional reactions in the scavenger probe and transfer line. The concentration of $\mathrm{DH}, \mathrm{DOH}$, and $\mathrm{D}_{2} \mathrm{O}$ formed in the scavenging process from the reaction of $\mathrm{D}_{2}$ with the $\mathrm{H}, \mathrm{OH}$, and $\mathrm{O}$ flame radicals may change in the probe or transfer line if additional reactions take place. Possible interfering reactions are the isotopic exchange reactions occurring between the deuterated species and the non-deuterated species. The isotopic exchange reaction, $\mathrm{D}_{2} \mathrm{O}+\mathrm{H}_{2} \mathrm{O} \longrightarrow \mathrm{DOH}+\mathrm{DOH}$, was found to be predominantly occurring in the transfer line, and consequently influencing the $\mathrm{OH}$ and $\mathrm{O}$ measurements. The results of this occurrence will be an addition to the measured $\mathrm{DOH}$ concentration and a decrease in the measured $\mathrm{D}_{2} \mathrm{O}$ concentration, causing the observed differences with the model predictions for $\mathrm{O}$ and $\mathrm{OH}$. Consequently, the exact determination of the $\mathrm{O}$ and $\mathrm{OH}$ concentrations will be in error, although a comparison can be made between the experimental and modeled $\mathrm{O}$ and $\mathrm{OH}$ profiles 

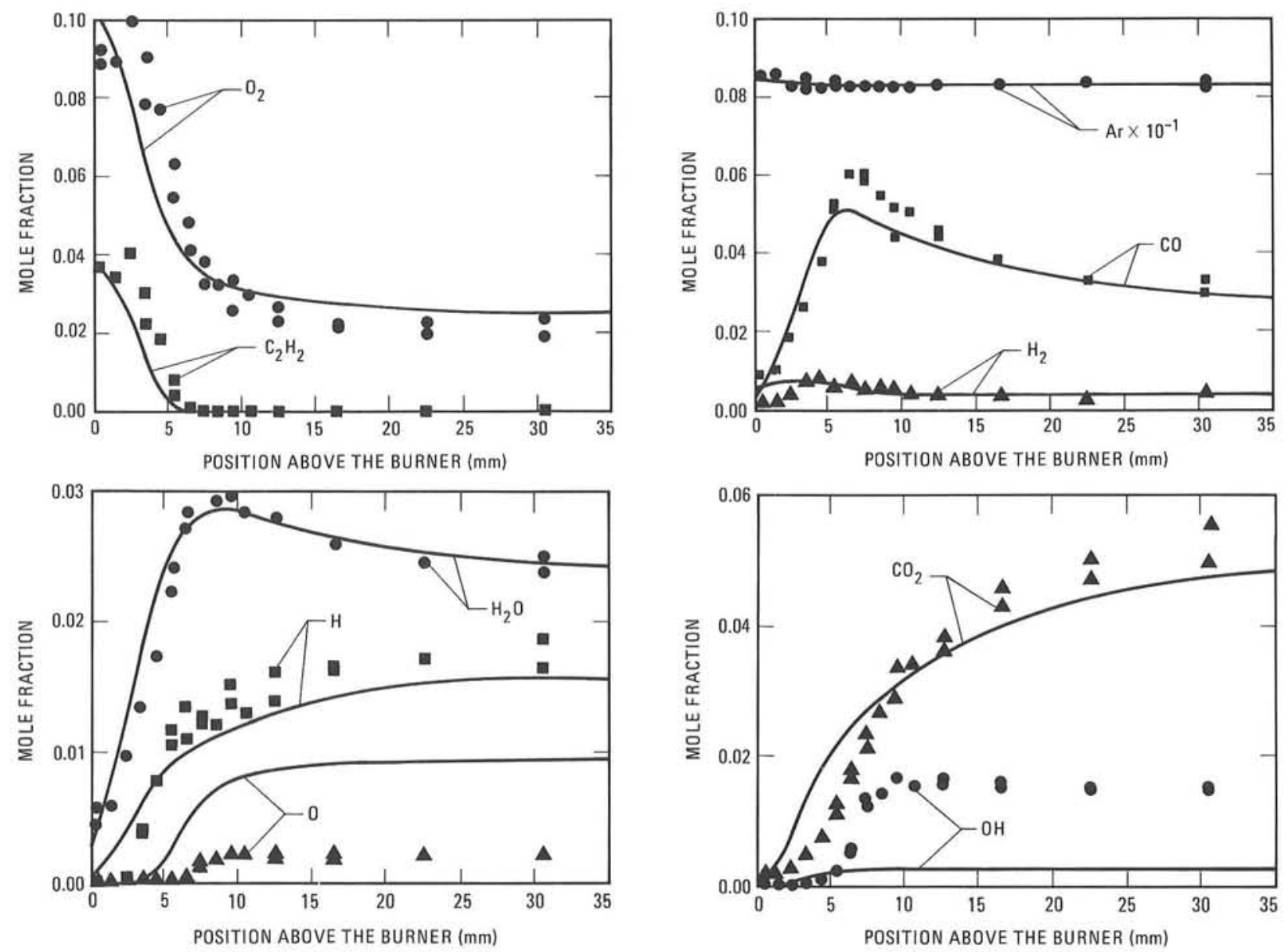

Figure 1. Comparison of the experimentally determined and computer-modeled profiles for the stable and radical species found in the near stoichiometric $(\phi=0.99) \mathrm{C}_{2} \mathrm{H}_{2} / \mathrm{O}_{2} / \mathrm{Ar}$ 30-torr flame. The symbols represent the experimental data, while the solid lines represent the theoretical calculations.

that will account for this exchange reaction occurring. By converting the $\mathrm{D}_{2} \mathrm{O}$ concentrations to $\mathrm{DOH}$, assuming that all $\mathrm{D}_{2} \mathrm{O}$ is converted to $\mathrm{DOH}$ from the exchange reaction $\mathrm{D}_{2} \mathrm{O}+\mathrm{H}_{2} \mathrm{O}$ $\longrightarrow \mathrm{DOH}+\mathrm{DOH}$, and adding this to the $\mathrm{DOH}$ concentrations, $\mathrm{OH}+2(\mathrm{O})$, an indication of the total $\mathrm{O}$ and $\mathrm{OH}$ concentration in the flame is found. A comparison between the experimentally determined total $\mathrm{O}$ and $\mathrm{OH}$ concentrations and the total calculated from the model is shown in Figure 2. The computed and measured total $\mathrm{O}$ and $\mathrm{OH}$ radical concentrations are in good agreement. No interfering reactions were found for the DH measurements.

No evidence was found for the occurrence of any reactions between the added deuterium and the stable species formed in the $\phi=0.99$ and $\phi=1.58$ flames. However, in the $\phi=0.75$ flame the added deuterium visibly burned with the excess oxygen present which caused the scavenging results to be in error. The deuterium, also, was found to indirectly interfere with the subsequent reactions,

$$
\begin{gathered}
\mathrm{CO}+\mathrm{OH} \longrightarrow \mathrm{CO}_{2}+\mathrm{H} \\
\mathrm{H}+\mathrm{OH}+\mathrm{M} \longrightarrow \mathrm{H}_{2} \mathrm{O}+\mathrm{M} \\
\mathrm{H}+\mathrm{H}+\mathrm{M} \longrightarrow \mathrm{H}_{2}+\mathrm{M}
\end{gathered}
$$

in the $\phi=0.99$ and $\phi=1.58$ flames. These reactions would otherwise proceed in or on the probe but are eliminated by the scavenging of the $\mathrm{H}, \mathrm{OH}$, and $\mathrm{O}$ radicals. As the radical species are consumed, the absence of these reactions leads to increased $\mathrm{CO}$ levels and decreased $\mathrm{CO}_{2}, \mathrm{H}_{2} \mathrm{O}$, and $\mathrm{H}_{2}$ levels compared with levels observed when these reactions take place in the probe. Thus, the scavenging probe can also be 
argued to lead to more accurate measurements of the major stable species.

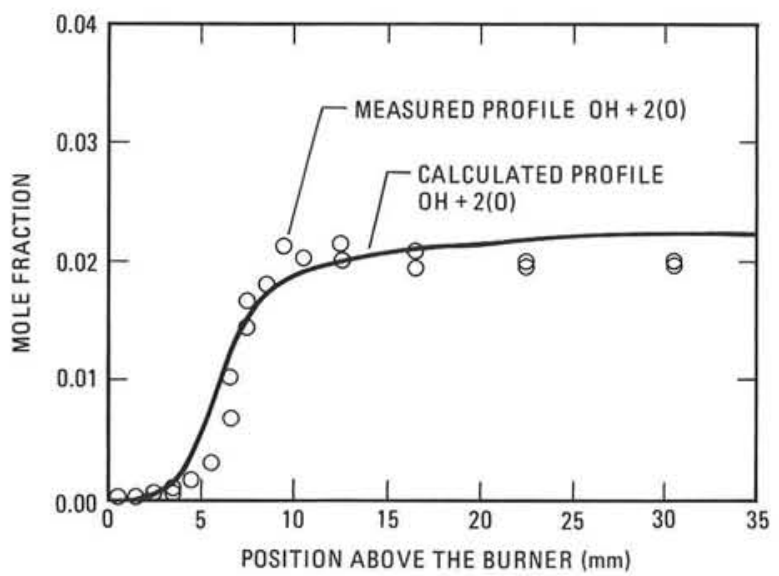

Figure 2. Comparison of the experimentally determined and computer modeled $\mathrm{OH}+2(\mathrm{O})$ total concentration profiles found in the near stoichiometric $(\phi=0.99) \mathrm{C}_{2} \mathrm{H}_{2} / \mathrm{O}_{2} / \mathrm{Ar} 30$-torr flame. (Converting all O's to OH's needs a factor of 2 from $\mathrm{D}_{2} \mathrm{O}+\mathrm{H}_{2} \mathrm{O} \rightarrow 2 \mathrm{DOH}$ ). The symbols represent the experimental total, while the solid line represents the theoretically calculated total.

The use of deuterium as a scavenging agent can provide some help in distinguishing among the radicals in the total radical pool of flames that do not contain excess oxygen, which is an advantage compared to previously tested scavenger species. $\mathrm{H}$ radicals, in particular, can be scavenged reliably with deuterium; no evidence was found for reactions which interfered with the HD concentration. However, the $\mathrm{OH}$ and O separation is evidently limited in our apparatus by wall exchange reactions. As would be expected, the accuracy of the measurement of stable-species concentrations as determined via sampling probes can be improved by addition of a scavenging agent, because interfering radicalmolecule reactions within the probe can be eliminated by the addition of a large excess of $\mathrm{D}_{2}$. Thus, the scavenging probe technique may prove useful in situations where rapid atom and radical reactions in the probe would otherwise cause serious errors in stable-species determinations, even if the technique is not used to determine labile species concentrations.

\section{References}

1. R. M. Fristrom, "Comments on Quenching Mechanisms in the Microprobe Sampling of Flames," Comb. Flame 50, 239 (1983).

2. J. A. Miller, R. E. Mitchell, M. D. Smooke, and R. J. Kee, "Toward a Comprehensive Chemical Kinetic Mechanism for the Oxidation of Acetylene: Comparison of Model Predictions with Results from Flame and Shock Tube Experiments," Nineteenth Symposium (International) on Combustion (The Combustion Institute, 1982), p. 181.

\section{Double-Resonance Laser- Ionization Spectroscopy of Molecular Hydrogen in the Region of the Second Dissociation Limit ${ }^{\dagger}$}

\section{W. Chandler, D. H. Parker, ${ }^{*}$ J. Buck*}

We report ionization spectra of $\mathrm{H}_{2}$ prepared in the E,F $\left({ }^{1} \Sigma_{g}^{+}\right)$state. Ionization is enhanced by bound states near the second dissociation limit, as shown schematically in Figure 1.

The E,F state is prepared with a pulsed tunable UV laser, and mass-selected ionization spectra are generated by scanning the wavelength of a pulsed, visible probe laser. A set time delay of 40 nsec separates the two pulses. Vibrational levels of the $\mathrm{B}, \mathrm{B}^{\prime}$ and $\mathrm{C}$ states, resonant with the visible laser, enhance the probability of further absorption to produce $\mathrm{H}_{2}^{+}$, or to dissociative states leading, for example, to excited $(n=3) H$ atoms. We obtained spectra originating from various rovibrational levels of the E,F states of $\mathrm{H}_{2}, \mathrm{HD}$, and $\mathrm{D}_{2}$, usually over the 117,200 to $118,500 \mathrm{~cm}^{-1}$ total energy range. Here we highlight transitions from $\mathrm{J}=1$ of the $\mathrm{E}, \mathrm{F}(\mathrm{v}=5$ and 6$)$ states of $\mathrm{H}_{2}$, that lie at similar

\footnotetext{
$\dagger$ Submitted to J. Phys. Chem. (1986).

- University of California at Santa Cruz.
} 
energies but whose average internuclear separations center in the outer and inner wells of the $\mathrm{E}, \mathrm{F}$ state, respectively.

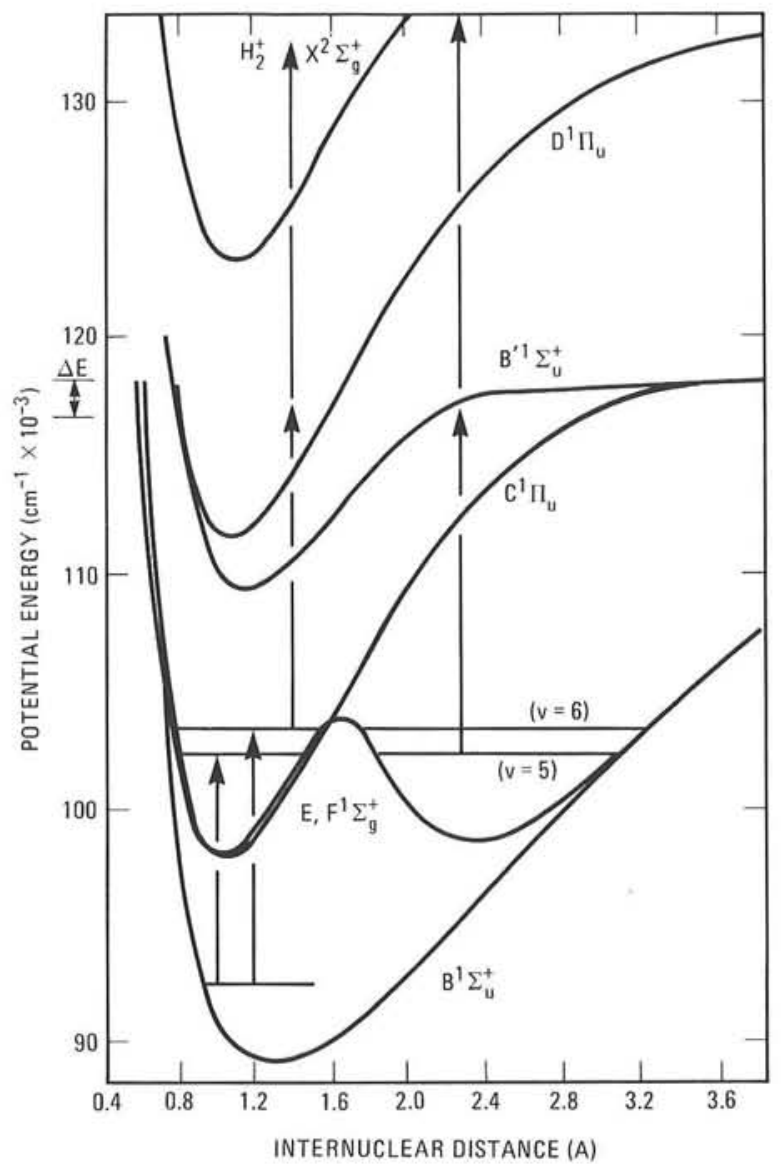

Figure 1. Energy level diagram of $\mathrm{H}_{2}$ showing observed states and resonances. $\Delta \mathrm{E}$ on energy axis represents the energies of the states accessed at the three photon step of the multiply resonant ionization process.

Details of the experimental apparatus have been published previously. Briefly, the E,F state of $\mathrm{H}_{2}$ is prepared by two-photon absorption and ionized 40 nsec later by an unfocused, pulsed, tunable dye laser beam $\left(\sim 10^{6} \mathrm{~W} / \mathrm{cm}^{2}, 0.2 \mathrm{~cm}^{-1}\right.$ bandwidth). Ions are extracted into a time-offlight mass spectrometer and the separate $\mathrm{H}^{+}$ and $\mathrm{H}_{2}^{+}$signals, generated by the time-delayed probe laser are recorded as the probe laser, wavelength is scanned. Gases from Cambridge Isotopes and Matheson were used at 3 microbar chamber pressure.
Figure 2 shows the $\mathrm{H}^{+}$and $\mathrm{H}_{2}^{+}$ionization spectra near the second dissociation limit $\left(118,377.6 \mathrm{~cm}^{-1}\right)$.

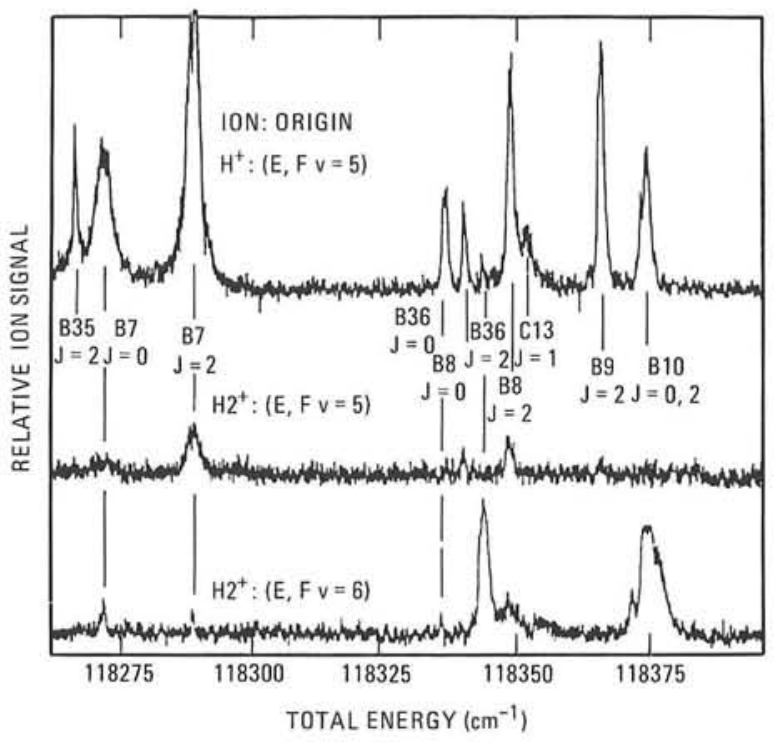

Figure 2. Doubly resonant spectra observed for the $\mathrm{H}^{+}$and the $\mathrm{H}_{2}^{+}$ions. Labeled features indicate resonance in the third-photon step of the multiphoton process. The origin level indicates the appropriate E,F level populated by initial two-photon process.

Ionization signal intensity is plotted versus the sum of the probe laser photon energy plus the term value of the $\mathrm{E}, \mathrm{F}$ intermediate state $\left(102,790\right.$ and $103,606 \mathrm{~cm}^{-1}$ for $\mathrm{v}=5$ and 6$)$. The assigned peaks correspond to resonances in a stepwise photoionization process involving two or more photons. The ionization potential of $\mathrm{H}_{2}\left(124,417 \mathrm{~cm}^{-1}\right)$ is less than the energy sum of two visible probe laser photons from the E,F level.

Glass-Maujean ${ }^{1}$ has calculated transition moments from $\mathrm{J}=0$ of these E,F levels using the transition dipole function of Wolniewicz and Dressler. ${ }^{2}$ Transitions to B levels were predicted to be $\sim 10^{7}$ weaker than to $\mathrm{B}^{\prime}$ and $\mathrm{C}$ levels for the outer well $\mathrm{E}, \mathrm{F}(\mathrm{v}=6)$ state, and $\sim 10^{4}$ weaker for the inner well $v=5$ state. Similar calculations for the $\mathrm{J}=1$ states are in general agreement with Glass-Maujean. Transition strengths to vibrational levels near the dissociation limit were not predicted for levels higher 
than $\mathrm{B}(\mathrm{v}=35), \mathrm{B}^{\prime}(\mathrm{v}=8)$, or $\mathrm{C}(\mathrm{v}=13)$, although higher bound levels are known for the B state and the $\mathrm{B}^{\prime}$ state.

We observe these relative trends in spectra to lower rovibrational states of the $\mathrm{B}, \mathrm{B}^{\prime}$, and $\mathrm{C}$ electronic states as predicted in the calculation of Glass-Maujean, but the relative strength of the $\mathrm{E}, \mathrm{F} \rightarrow \mathrm{B}$ and weakness of the $\mathrm{E}, \mathrm{F} \rightarrow \mathrm{C}$ transitions in the region near the second dissociation limit reported here is surprising. To achieve better agreement between theory and experiment, the nature of the excitation source used to obtain the spectra must be considered and further refinements of the calculations, including the use of a more completely determined dipole moment function and extension to and beyond the dissociation limits may be warranted.

We observe the production of $\mathrm{H}^{+}$from $\mathrm{H}_{2}$ prepared in the $\mathrm{E}, \mathrm{F}(\mathrm{v}=5)$ state. Ionization of $\mathrm{n}=3 \mathrm{H}$ atoms created by photodissociation from the observed resonances is responsible for the $\mathrm{H}^{+}$. Production of $\mathrm{H}$ atoms in the $\mathrm{n}=3$ state via resonant two-photon absorption of probe laser photons is possible (at total energies above $\left.118205 \mathrm{~cm}^{-1}\right)$ from the $\mathrm{E}, \mathrm{F}(\mathrm{v}=5)$ state; this mechanism is not energetically accessible from the $\mathrm{E}, \mathrm{F}(\mathrm{v}=6)$ state because lower energy photons are used to reach the intermediate $\mathrm{B}, \mathrm{B}^{\prime}$ or $\mathrm{C}$ state. Further, we observe a propensity for molecules in which a $\mathrm{B}^{\prime}$ resonance is involved to dissociate less, i.e., to form more $\mathrm{H}_{2}^{+}$, than do molecules whose ionization is enhanced through the B manifold. Photodissociation processes which generate $\mathrm{H}^{+}$signal from the E,F states will be discussed further, separately.

From the observed spectra, a reassignment of $\mathrm{B}(\mathrm{v}=37, \mathrm{~J}=2)$ to $\mathrm{B}^{\prime}(\mathrm{v}=9, \mathrm{~J}=2)$ as suggested by Namioka ${ }^{3}$ is supported because the resonance at $118,366 \mathrm{~cm}^{-1}$ produces more $\mathrm{H}_{2}^{+}$than do the transitions involving other $\mathrm{B}$ levels, and the resonance is completely absent from the spectrum when the spectrum originates from the $\mathrm{E}, \mathrm{F}(\mathrm{v}=6)$ states where the dominant resonances are B state levels. Strong $\mathrm{B}^{\prime}$ transitions are seen, and predicted, when the $\mathrm{E}, \mathrm{F}(\mathrm{v}=5, \mathrm{~J}=1)$ state is the starting state, in accord with this reassignment.
Resonances appear near the dissociation limit from both E,F starting states. A broad peak at $118,374 \mathrm{~cm}^{-1}$ is seen in the spectrum originating from $\mathrm{E}, \mathrm{F}(\mathrm{v}=5)$ which we assign as the last bound resonance of the $\mathrm{B}^{\prime}$ state $(\mathrm{v}=10)$. From the $\mathrm{E}, \mathrm{F}(\mathrm{v}=6)$ level, a broad peak is seen that extends to the dissociation limit, and corresponds well with the $\mathrm{B}(\mathrm{v}=36), \mathrm{B}(\mathrm{v}=37)$ and $\mathrm{B}(\mathrm{v}=38)$ levels. These final bound states are obscured in the ground state absorption spectrum by underlying continua from dissociation of higher J levels. Analysis of the transition lineshapes of double-resonance spectra provides a sensitive test of the accuracy of the weakly bound excited state wavefunction. Our spectra exhibit ac Stark splitting which, at higher intensities, can also be used to extract molecular and photophysical parameters of the REMPI process. We have demonstrated the ability to explore unstudied Franck-Condon regions, providing new spectroscopic and dynamic information on this most fundamental molecule.

\section{References}

1. M. Glass-Maujean, "Transition Probabilities for the $\mathrm{D}$ and $\mathrm{B}^{\prime}$ Vibrational Levels to the $\mathrm{X}$ Vibrational Levels and Continuum of Molecular Hydrogen," At. Data Nucl. Tables 30, 275 (1984).

2. L. Wolniewicz and K. Dressler, "Electronic Transition Moments Between Excited Singlet States of the Hydrogen Molecule," J. Mol. Spectrosc. 96, 195 (1982).

3. T. Namioka, "Absorption Spectra of $\mathrm{H}_{2}$ in the Vacuum Ultraviolet Region (I) Lyman and the Werner Bands," J. Chem. Phys. 40, 3154 (1964); "(II) B'-X, B"-X, D-X, and D'X Bands," J. Chem. Phys. 41, 2141 (1964); "(III) Potential Energy Curves for the B ${ }^{1} \Sigma_{u}^{+}$, $\mathrm{C}^{\prime} \Pi_{u}, \mathrm{~B}^{\prime 1} \Sigma_{u}^{+}$, and $\mathrm{D}^{\prime} \Pi_{u}$ states," J.Chem. Phys. 43, 1636 (1965). 


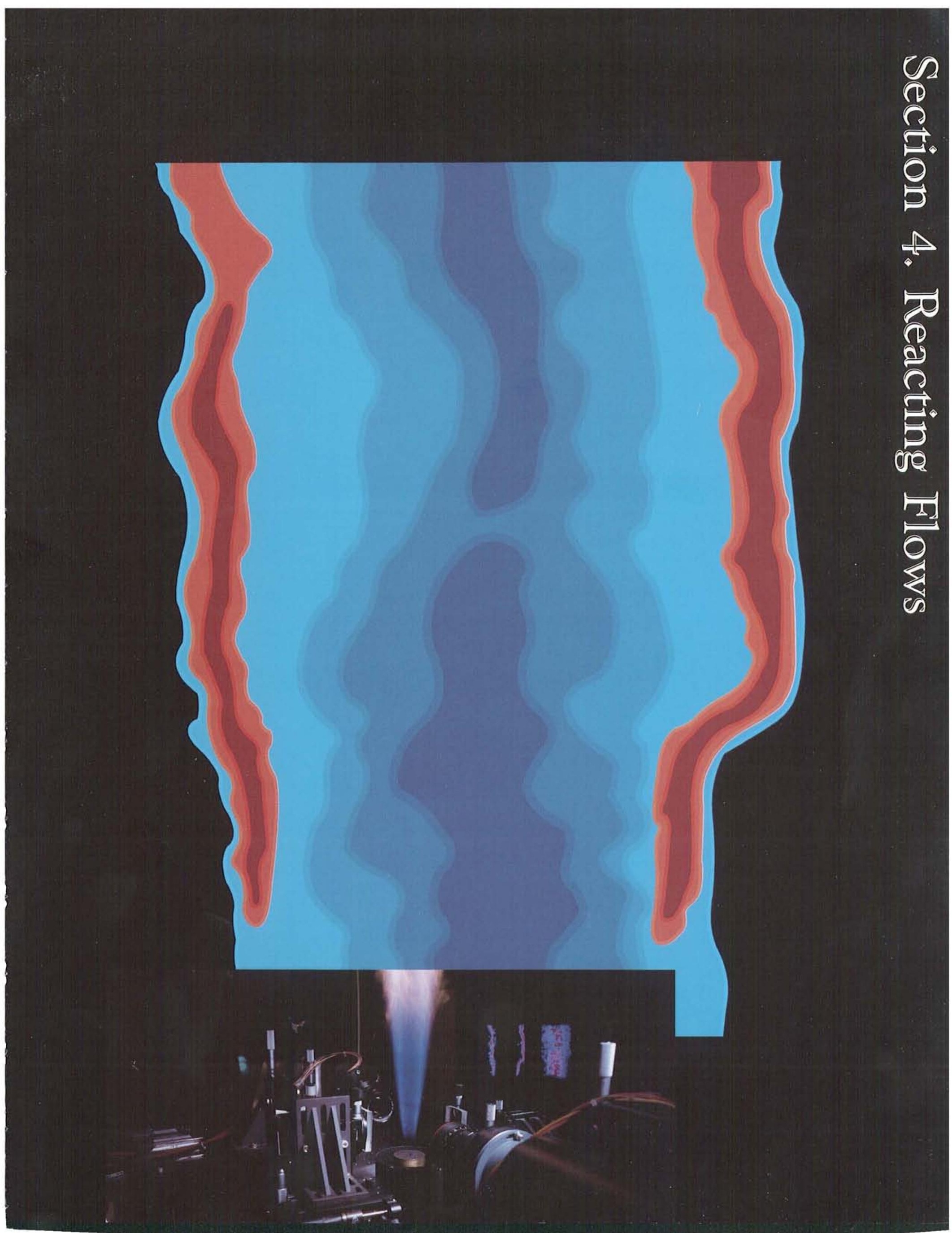


Simultaneous two-dimensional imaging of major and minor species provides insight into the effect of turbulence on the instantaneous flame front. The photograph on the preceding page shows the distribution of $\mathrm{CH}_{4}$ (fuel) and $\mathrm{CH}$ (a minor species marking the flame front) in a lifted turbulent diffusion flame obtained using a two-wavelength laser (see related article, page 4-6). The inset photograph shows the flame, the two-camera imaging setup, and the separated $\mathrm{CH}_{4}$ and $\mathrm{CH}$ concentration images on the background monitor. 


\section{Section 4 Reacting Flows}

A multidisciplinary program in reacting flows establishes an important link between fundamental studies of combustion chemistry and the "real" world of practical combustion. The objective of the program, which is sponsored by the Department of Energy's Office of Basic Energy Science, is to use experimentation and computer modeling to increase our understanding of the fundamental interactions between chemistry and fluid dynamics in chemically reacting flows. Research results will be used in improving predictive capabilities for turbulent combustion of hydrocarbon fuels. Experimental studies include flows involving complex fluid mechanics but simplified chemistry; these flows are used to probe primary turbulent transport mechanisms. Studies of flows with complex chemistry but simplified fluid mechanics help identify combustion mechanisms responsible for the formation and growth of soot. Concomitant modeling studies employ both stochastic approaches to predict turbulent flow quantities and deterministic approaches to predict unsteady flame structure and effects of flow fields on soot growth and transport.

An important element of the experimental program is the development of new laser diagnostic techniques capable of imaging a planar slice through the flow field in a temporally-resolved manner. With these diagnostics, it is possible to obtain two-dimensional measurements of major and minor chemical species and velocity during time scales that are short compared with the characteristic chemical or fluid dynamical time scales involved in combustion. Obtaining such measurements at high frequency makes it possible to follow the temporal development of unsteady reacting flows.

Regular workshops are held with scientists from universities and industry who are involved in the fundamental aspects of reacting flow research. These meetings provide a forum for informal discussion of new developments and research opportunities in the field.

\section{Velocity Measurements in a Turbulent, Nonpremixed, Bluff-Body-Stabilized Flame}

\author{
R. W. Schefer, M. Namazian, ${ }^{*}$ J. Kelly *
}

Bluff-body-stabilized flames are characterized by high turbulence intensities, enhanced turbulent mixing rates, and upstream recirculation of high temperature combustion products for improved flame stabilization and combustion characteristics. Because of their relevance to many practical combustion devices, considerable interest has been focused on the development of numerical models for these flows.

Mixing and combustion in bluff-bodystabilized flames has been studied previously using direct flame photography, planar flow visualization, and concentration-imaging techniques. More recently, we have made velocity measurements in this flow using laser Doppler velocimetry. Data have been obtained under both noncombusting and combusting conditions to better characterize the interaction between turbulence and combustion heat release. The objective of the measurements has been to supplement the

* Altex Technologies Corporation, San Jose, CA. 
existing $\mathrm{CH}_{4}$ and $\mathrm{CH}$ data and to provide quantitative information on important turbulence quantities related to turbulent mixing.

A schematic of the axisymmetric bluffbody burner is shown in Figure 1. Methane fuel was supplied through the center of the bluff body at a nominal velocity of $21 \mathrm{~m} / \mathrm{sec}$, giving a Reynolds number of 14,000 . Air was supplied through a coaxial jet surrounding the bluff body at a velocity of $25 \mathrm{~m} / \mathrm{s}$.

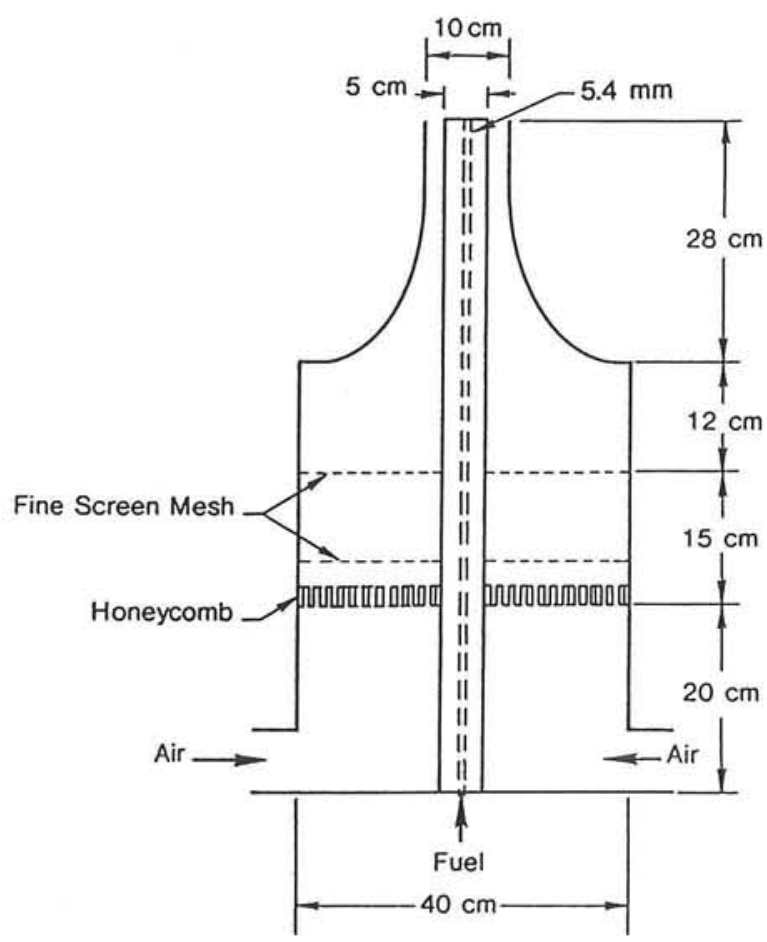

Figure 1. Schematic of apparatus used to study turbulent mixing and combustion chemistry in bluff-body-stabilized flames.

The laser Doppler velocimetry (LDV) measurements were made with a conventional twocolor dual-beam system with frequency shifting to eliminate directional ambiguity. With this system the axial velocity $u$ could be measured simultaneously with either the radial velocity $v$ or the azimuthal velocity $w$. Both the fuel and air streams were seeded with nominal $0.85-\mu \mathrm{m}$ diameter alumina particles. Light scattered from the particles was collected at an angle of 15 degrees to the forward direction and focused onto two pinhole-photomultiplier tube combinations. The output signal from each photomultiplier tube was filtered, processed, and validated using a counter-type processor.

Figure 2 shows the time-averaged velocity vector plots of the flow field determined from the axial and radial velocity measurements. Results are shown for both the noncombusting (Figure 2a) and combusting (Figure 2b) cases. In both cases the annular air converges toward the centerline downstream of the centerbody and forms a wake region which extends approximately one centerbody downstream of the bluff-body face. A toroidal recirculating flow region is formed within the wake region by the interaction of the outer annular air with the central jet flow. The existence of two stagnation points located along the centerline is clearly indicated in the noncombusting case. The upstream fuel stagnation point corresponds to the point where the fuel jet stagnates and is turned upstream by the opposing reverse flow of the annular air. The downstream air stagnation point corresponds to the end of the vortex region established by the annular air flow.

The combusting case (Figure $2 \mathrm{~b}$ ) shows a downstream shift in the extent of the recirculation region due to expansion of high temperature combustion gases. In addition, the well-defined fuel and air stagnation points located along the centerline in the noncombusting flow have moved downstream and formed a single stagnation region (in a time-averaged sense) where the centerline velocity has a near-zero value at approximately $70 \mathrm{~mm}$ downstream of the bluff body.

These results can be related to the two extremes of operating conditions for the bluff-body combustor, which are determined by whether the annular air or the fuel jet dominates the flow. When the annular air dominates, the momentum of the air flow is sufficient to establish a fuel jet stagnation zone along the centerline and a reverse jet which flows radially outward and upstream toward the centerbody. When the central jet dominates, the outer air flow reaching the centerline is insufficient to stagnate the 
(a) NONCOMBUSTING

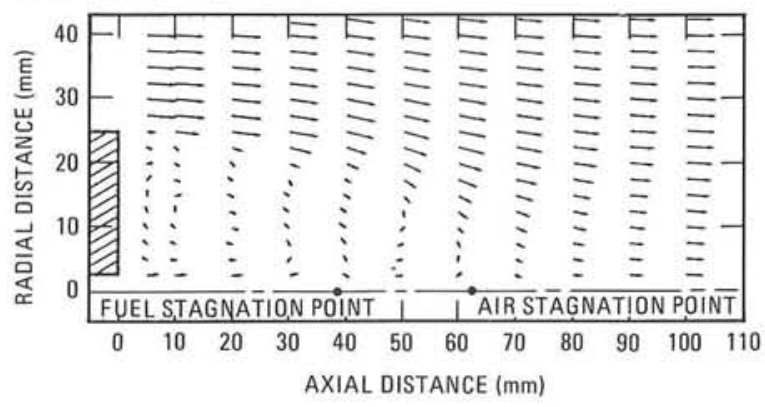

(b) COMBUSTING

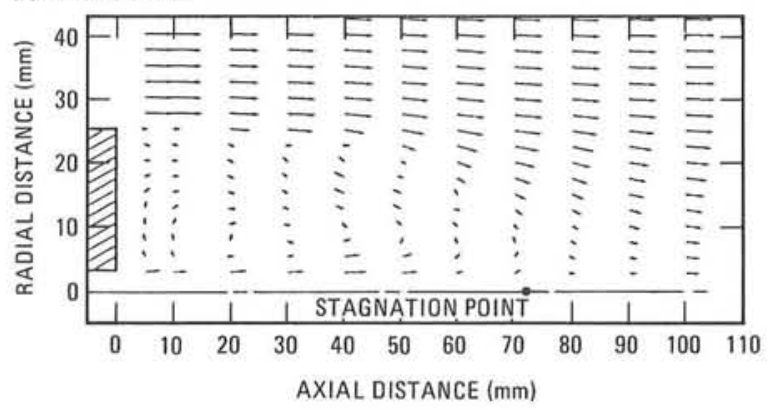

Figure 2. Laser Doppler velocimetry measurements of the time-averaged velocity fleld in a bluffbody-stabilized methane jet: (a) noncombusting flow; (b) combusting flow. Fuel jet velocity $=21$ $\mathrm{m} / \mathrm{s}$; annular air velocity $=25 \mathrm{~m} / \mathrm{s}$.

central jet flow and, at sufficiently high jet momentum, the fuel jet penetrates the downstream end of the recirculation zone. From Figure 2, the noncombusting case more closely approaches the air-dominated flow field as can be seen from the two well-defined stagnation points located along the centerline. The combusting case corresponds to an intermediate flow condition in which neither the central jet nor the annular air flow is dominant.

Measurements of the turbulent kinetic energy are important to development and validation of modeling approaches to turbulent flows. The turbulent kinetic energy $\mathrm{k}=\left({u^{\prime}}^{2}+{v^{\prime}}^{2}+\right.$ \left.${w^{\prime}}^{2}\right) / 2$ was determined from measured values of the three velocity components.

Shown in Figure 3 are contour lines of constant $\mathrm{k}$ for the combusting case. The regions of maximum kinetic energy occur 1) in the shear layer between the outer air flow and the recirculation zone, and 2) along the centerline downstream of the fuel jet exit. Production of $\mathrm{k}$ between the outer air flow and the recirculation zone is directly related to the high shear generated by the velocity differences across this region. The centerline maximum coincides closely with the respective location of the fuel-jet stagnation point in each flow and is related to largescale oscillations in the axial location of the centerline stagnation zone. These axial oscillations result in a situation in which the fixed measurement volume alternately contains reverse-flowing air and downstream-flowing jet fluid. The high resulting values of the velocity fluctuations are consistent with the high values of $\mathrm{k}$ measured along the centerline in Figure 3.

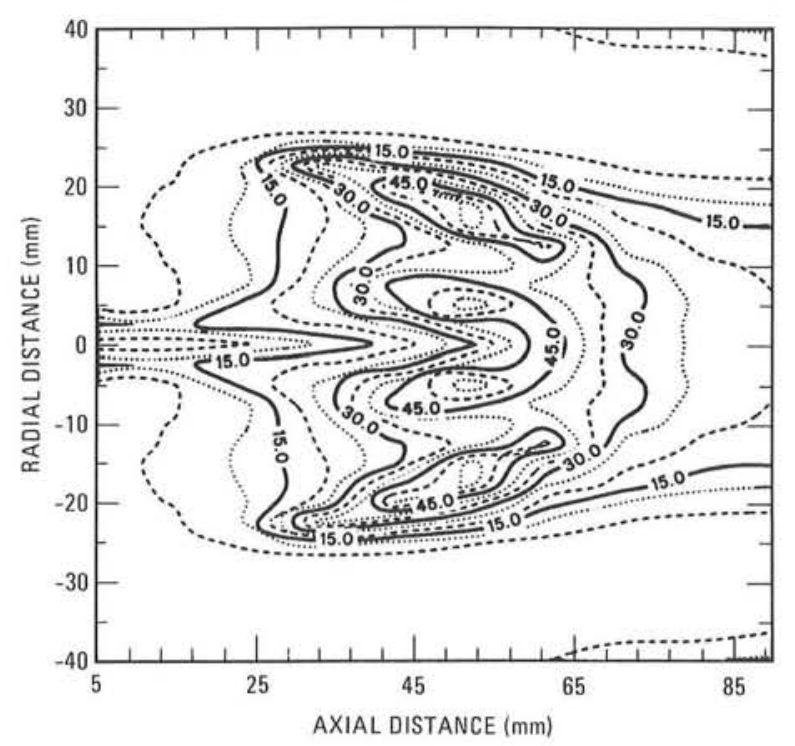

Figure 3. Contours of turbulent kinetic energy measured using laser Doppler velocimetry in a bluff-body-stabilized methane flame. Fuel jet velocity $=21 \mathrm{~m} / \mathrm{s}$; annular air velocity $=25 \mathrm{~m} / \mathrm{s}$. 


\section{$\mathrm{CH}_{4}$ and $\mathrm{CH}$ Concentration Imaging in a Bluff-Body- Stabilized Flame}

\author{
M. Namazian, ${ }^{*}$ R. W. Schefer, J. Kelly* \\ M. B. Long**
}

Two-dimensional imaging techniques have proven to be a valuable tool with which to study turbulent reacting flows. Previous imaging measurements in a bluff-body-stabilized flame using Raman scattering from $\mathrm{CH}_{4}$ and Mie-scattering from small-diameter seed particles have shown that mixing in these flows is characterized by large-scale, irregular, and time-varying structures. ${ }^{1}$ To more fully understand the processes by which the large structures initiate and sustain combustion in bluff-body burner flames, a two-dimensional imaging technique for the simultaneous measurement of $\mathrm{CH}_{4}$ and $\mathrm{CH}$ was developed and applied to this flow. Measurements of the $\mathrm{CH}_{4}$ concentration provide data on fuel mixing and its subsequent consumption during the combustion process. $\mathrm{CH}$ is an important intermediate species that characteristically exists at high concentrations in the flame zone where rapid combustion reactions occur. Using $\mathrm{CH}$ as an indicator of the location of the flame zone, the two-species technique simultaneously provides both a map of the fuel mixing characteristics and defines the relationship between turbulent flow structure and the instantaneous flame zone.

Instantaneous planar images of $\mathrm{CH}_{4}$ and $\mathrm{CH}$ were obtained by simultaneously recording $\mathrm{CH}$ fluorescence and $\mathrm{CH}_{4}$ Raman scattering with an intensified, low-light-level camera. Figure 1 shows the details of the optical imaging set up.

A 0.2 -mm-thick sheet of laser light was created by focusing the laser beam into a multipass cylindrical light cell. The laser beam is produced by a flashlamp-pumped dye laser which is located in the CRF. For the combined $\mathrm{CH}_{4}-\mathrm{CH}$ experiments, the laser was operated

* Both with Altex Technologies Corp., San Jose, CA.

** Yale University, New Haven, CT. at a 431.5-nm wavelength (5 Joules/pulse) and the laser sheet was used to vibrationally excite $\mathrm{CH}_{4}$ molecules and simultaneously induce fluorescence in $\mathrm{CH}$ molecules. The scattered light from $\mathrm{CH}$ and $\mathrm{CH}_{4}$ was collected at a right angle to the laser sheet and focused onto a computercontrolled, intensified vidicon detector that recorded the intensity of the scattered light. The light-collection system consisted of a 24mm-focal length, f/1.4 collection lens closest to the laser sheet that focused the Raman-scattered light onto the $12-\mathrm{mm} \times 12-\mathrm{mm}$ camera. With this optical system, the $100 \times 100$ pixel format of the detector provided a spatial resolution of $500 \mu \mathrm{m} \times 500 \mu \mathrm{m}$. The temporal resolution was $1.8 \mu \mathrm{sec}$, which was the laser pulse duration. The effects of ambient light and flame background luminescence were minimized by gating the camera and intensification stage of the camera to $4 \mu \mathrm{sec}$. In addition, a 10-nm-widebandpass interference filter centered at $490 \mathrm{~nm}$ was used in the collection optics to selectively pass both the $\mathrm{CH}_{4}$ Raman-scattered light and $\mathrm{CH}$ laser-induced fluorescence while rejecting light at other wavelengths.

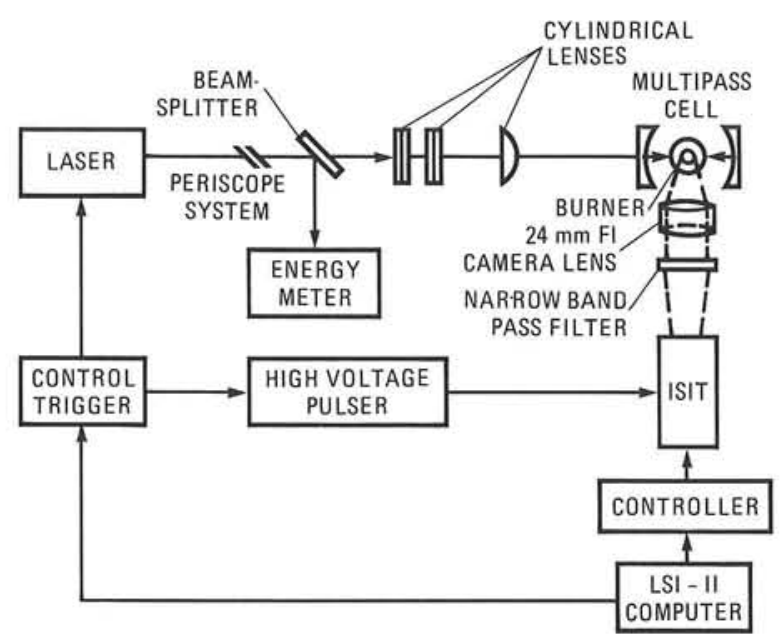

Figure 1. Imaging system for measuring combined $\mathrm{CH}-\mathrm{CH}_{4}$ in bluff-body-stabilized flames.

In regions where high $\mathrm{CH}$ concentrations were present, the signal typically exceeded the expected signal level from 100-percent $\mathrm{CH}_{4}$ at room temperature. This relatively strong signal provided a simple method for identifying zones 
in which abundant $\mathrm{CH}$ was present. Large $\mathrm{CH}$ signal levels were used to qualitatively define the location of the reaction zones.

The burner consisted of an axisymmetric bluff-body (50-mm diameter) with methane fuel supplied through a centrally located 5.4-mmdiameter tube. Air was supplied through a 20mm-thick coaxial jet surrounding the bluff body. Fuel and air velocities for all measurements were $21 \mathrm{~m} / \mathrm{sec}$ and $25 \mathrm{~m} / \mathrm{sec}$, respectively, which gives a fuel-jet Reynolds number of 14,000 .

The contour plot of an instantaneous image of combined $\mathrm{CH}_{4}$ and $\mathrm{CH}$ data is shown in Figure 2. The data are normalized by the Raman signal from pure methane at room temperature.

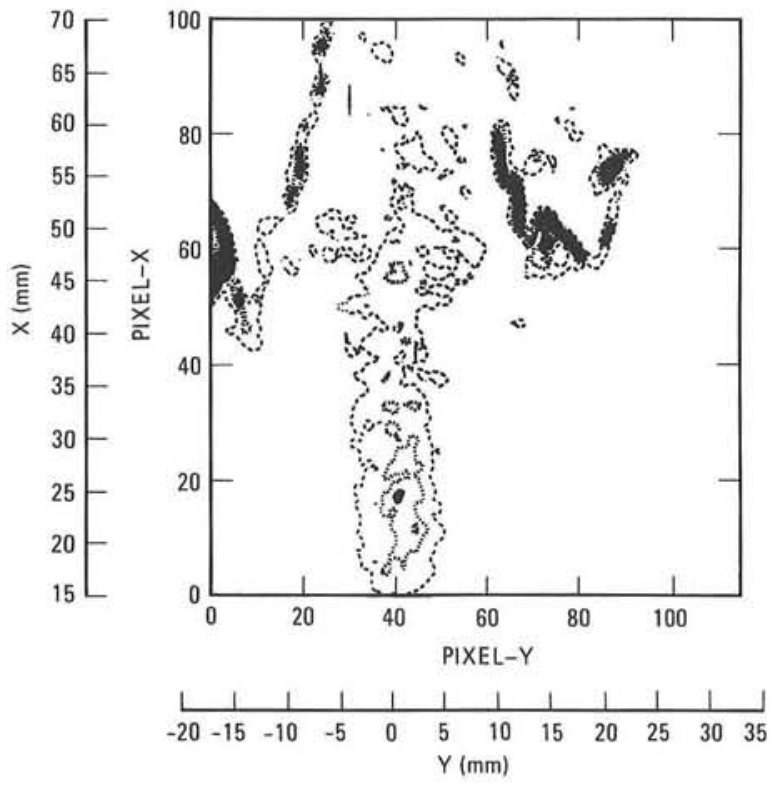

Figure 2. Instantaneous contours of signal level from combined $\mathrm{CH}-\mathrm{CH}_{4}$ in a bluff-body-stabilized flame. Signal is normalized by signal of pure methane at STP. Contour levels are $50 \%$ (dashed line), $80 \%$ (dotted line) in concentration. Higher levels $(>110 \%)$ indicated by solid lines are concentrated in the darker regions near the stagnation zone. Fuel-jet velocity $=21 \mathrm{~m} / \mathrm{s}$; annular-air velocity $=25 \mathrm{~m} / \mathrm{s}$.

Contours associated with levels greater than $100 \%$ are concentrated in narrow dark regions and define the locations where significant $\mathrm{CH}$ is present. The centrally located $50 \%$ and $80 \%$ contours define the main body of the fuel jet and clearly show the downstream dispersion of $\mathrm{CH}_{4}$ due to the combined effects of turbulent mixing with annular air and the consumption of $\mathrm{CH}_{4}$ during combustion. The $\mathrm{CH}$ is present in high concentrations only near the downstream end of central fuel jet. This location corresponds to the point where the fuel jet stagnates and is turned upstream by the opposing reverse flow of the outer annular air. The $\mathrm{CH}$ creates a somewhat disconnected envelope around the top portion of the fuel jet. Comparisons of several images show that the location, shape, and connectivity of this envelope is time varying and irregular. The disconnected appearance of the reaction zone appears to be the result of an intermittent high local strain rate that occasionally breaks the connectivity of the reaction zone. From this data it may be concluded that bluff-body flames develop in the stagnation zone where conditions are favorable for flame stability.

Further interpretation of the above results for the bluff-body-stabilized flame can be made through comparisons with the corresponding images obtained in a zero-air-flow turbulent diffusion flame. These results, shown in Figure 3, indicate a nearly continuous reaction zone that forms along the outer radial boundary of the central fuel jet.

The images shown are consistent with the general view of turbulent nonpremixed flames as ensembles of nonpremixed laminar flames that form along the mixing region separating the fuel jet and surrounding air. The comparable thickness of simple turbulent diffusion flames and the bluff-body flame zones shown in Figure 2 suggests that similar mechanisms control combustion in both configurations. That is, the flame zone in the bluff-body burner forms at the interface between larger-scale turbulent structures separating unmixed fuel and air. These "flamelets" are stabilized in the downstream fuel stagnation zone because of the lower velocities in this region. 


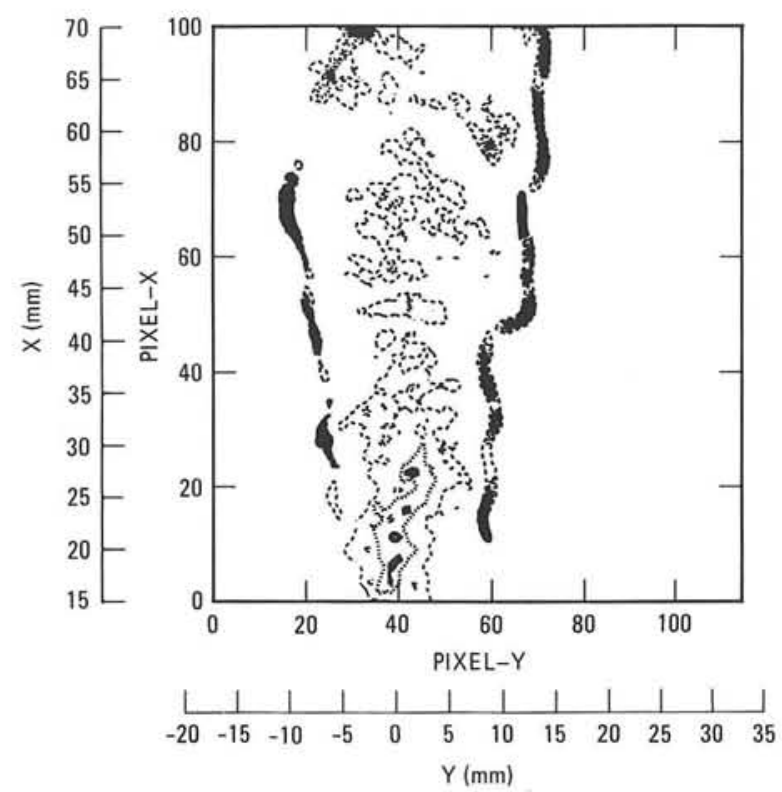

Figure 3. Instantaneous contours of signal level from combined $\mathrm{CH}-\mathrm{CH}_{4}$ in a turbulent diffusion flame. Signal is normalized by signal of pure methane at STP. Contour levels are $50 \%$ (dashed line), $80 \%$ (dotted line) in concentration. Higher levels $(>110 \%)$ indicated by solid lines are concentrated in the darker regions near the stagnation zone. Fuel-jet velocity $=21 \mathrm{~m} / \mathrm{s}$.

\section{Reference}

1. M. Namazian, J. Kelly, R. W. Schefer, S. C. Johnston, and M. B. Long, "Flow and Mixing Structures in Bluff-Body Stabilized Gas Flames," to appear in Proceedings of the International Gas Research Conference (1987).

\section{Two-Wavelength, Single Laser $\mathrm{CH}$ and $\mathrm{CH}_{4}$ Imaging in a Turbulent Diffusion Flame $^{\dagger}$}

\author{
M. Namazian, ${ }^{*}$ R. L. Schmitt, M. B. Long ${ }^{* *}$
}

The view that turbulent combusting flows consist of large-scale structures calls for multipoint measurement techniques that allow simultaneous imaging of many structures. Through the imaging of complete structures, a greater understanding of how turbulence influences combustion can be obtained. Two-dimensional measurements of single scalars, such as species concentration or temperature, using Mie, Rayleigh, fluorescence, and Raman scattering have contributed to the understanding of turbulent combustion. Recent simultaneous two-dimensional monitoring of temperature and fuel concentration, by combined Rayleigh and Raman scattering, ${ }^{1}$ and by Rayleigh and $\mathrm{C}_{2}$ fluorescence, ${ }^{2}$ proved to be valuable in characterizing turbulent combustion. To further understand the interaction between fuel mixing and combustion, a technique is required to map the fuel concentration and simultaneously define the combustion zone. This article describes a two-dimensional mapping technique that combines $\mathrm{CH}_{4}$ Raman scattering and $\mathrm{CH}$ fluorescence to measure fuel concentration and the location of the combustion zone, respectively.

In this experiment the $(0,0)$ band of the $A^{2} \Delta-X^{2} \Pi$ system of $\mathrm{CH}$ was pumped at $431.5 \mathrm{~nm}$ and fluorescence from the $(0,1)$ transition was observed at $\sim 489 \mathrm{~nm}$. A 10 -nm-bandwidth interference filter centered at $490 \mathrm{~nm}$ stopped elastically-scattered light from contaminating the fluorescence signal. Unfortunately, the Raman-scattered light from $\mathrm{CH}_{4}$ with 431.5-nm excitation coincides with the $\mathrm{CH}$ fluorescence wavelength. Thus, the signal passed by the interference filter was the sum of the $\mathrm{CH}_{4}$ Raman and $\mathrm{CH}$ fluorescence intensities.

$\dagger$ Submitted to Optics Letters (1986).

- Altex Technologies Corporation, San Jose, CA

** Yale University, New Haven, CT. 
Since the Raman-scattered light is shifted in frequency, an independent and simultaneous measurement of $\mathrm{CH}_{4}$ Raman scattering was made by using a second incident wavelength $(444 \mathrm{~nm})$ that did not excite fluorescence from $\mathrm{CH}$. The $\mathrm{CH}$ fluorescence measurement was obtained by subtracting the independent $\mathrm{CH}_{4}$ measurement from the combined $\mathrm{CH} / \mathrm{CH}_{4}$ measurement. This provided a simultaneous two-dimensional measurement of the $\mathrm{CH}_{4}$ and $\mathrm{CH}$ concentration.

To produce the two-wavelength laser beam required for this experiment, the cavity of the DIANA facility laser was modified to operate with two back mirrors, as shown schematically in Figure 1.

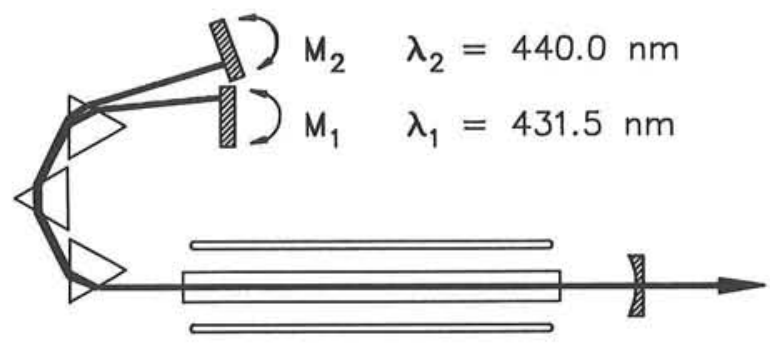

Figure 1. To produce the two-wavelength laser beam required for this experiment, the cavity of the Diana laser was operated with two back mirrors. The back mirrors were aligned to produce 431.5-nm and 444-nm wavelengths with $25 \mathrm{~mJ}$ and $30 \mathrm{~mJ}$ of energy, respectively. The laser pulse duration was $1.8 \mu \mathrm{sec}$.

As shown in Figure 2, the two-wavelength beam was formed into a sheet by a multipass cell consisting of a pair of cylindrical reflectors. The incoming beam was focused into a sheet at the center of the cell by a cylindrical lens and then refocused at each pass by the cylindrical reflectors, which were spaced to form a cylindrical cavity. The intensity of this sheet is 30 times that produced by a single cylindrical lens.

The light scattered from the sheet was collected by two identical 24-mm-focal-length f/1.4 camera lenses located on opposite sides of the sheet. The light collected on each side of the light sheet was passed through an appropriate interference filter and focused onto an image intensifier. A 10-nm-bandwidth interference filter centered at $510 \mathrm{~nm}$ was used in front of one

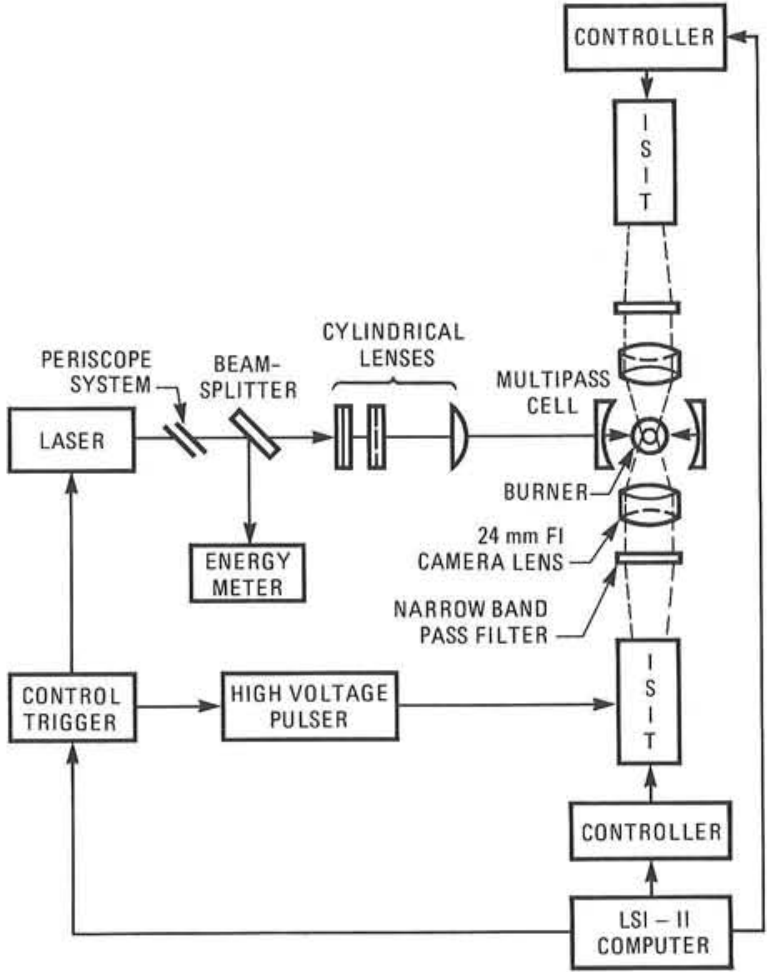

Figure 2. The two-dimensional imaging system consists of a multipass cell that forms the laser beam into a thin sheet and two low-light-level cameras that record the scattered light from the sheet. Narrow-band interference filters in front of the cameras selectively pass the wavelengths of interest.

camera to pass the $\mathrm{CH}_{4}$ Raman-scattered light generated by the 444-nm beam, and a $10-\mathrm{nm}$ bandwidth interference filter centered at $490 \mathrm{~nm}$ was used in front of the other detector to pass the combined $\mathrm{CH}$ laser-induced fluorescence and the $\mathrm{CH}_{4}$ Raman-scattered light generated by the 431.5-nm beam. The output face of each intensifier was coupled to a vidicon detector (PARC Model 1254) via a fiber optic coupler. To minimize the collected background emission, both intensifiers were gated to stay on for $4 \mu \mathrm{sec}$, which encompassed the $1.8 \mu \mathrm{sec}$ laser pulse duration. The two detectors were operated and controlled synchronously by a single LSI $11 / 23$ microcomputer, which triggered the laser and image intensifiers, and collected the data from both cameras. 
The area imaged by the two detectors was not identical because of differences in the magnification of the collection optics and the electronic adjustment of the detector heads. This was corrected by interpolating and spatially scaling the data so that each point of the images of the two cameras corresponded to the same spatial location. Each image was also corrected for the background signal, shot-to-shot variation of the laser energy, nonuniformity of the laser sheet, and camera response. The corrected $\mathrm{CH}_{4}$ image was then subtracted from the $\mathrm{CH} / \mathrm{CH}_{4}$ image to obtain the $\mathrm{CH}$ image.

As a system-test example, the technique was applied to a lifted methane diffusion flame. Figure 3 shows the decomposed images of $\mathrm{CH}(\mathrm{a})$ and $\mathrm{CH}_{4}(\mathrm{~b})$, each covering a 27.4-mm x 34.0$\mathrm{mm}$ field of view. Methane fuel was supplied through a 5.4-mm-inside-diameter tube at 21 $\mathrm{m} / \mathrm{sec}$, resulting in a jet Reynolds number of 11,000. The lower and upper edge of the images are 3 and 9.4 jet diameters above the burner face, respectively. The data are normalized by the Raman signal produced by pure methane at the standard condition and contour levels of $0.2,0.3$, and 0.4 are plotted. The CH signal, representing the instantaneous reaction zone, is concentrated in a narrow region located at the methane-air boundary. The flame is lifted from the nozzle, which results in a $\mathrm{CH}$-free region in the lower part of Figure 3(b).

In summary, this article describes a technique for simultaneous two-dimensional monitoring of methane and $\mathrm{CH}$ concentration in a combusting environment. This approach can also be used with other fuels. The application of this technique to combustion environments will provide new information on the interaction between turbulence and chemistry.

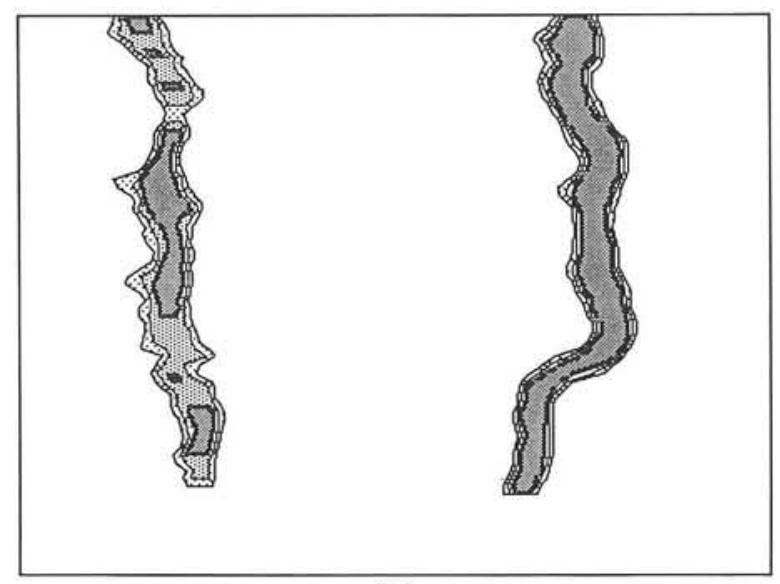

(a)

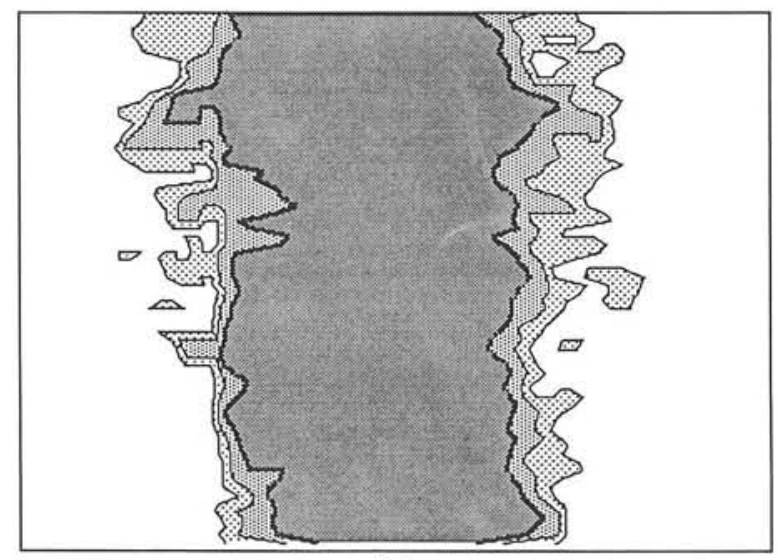

(b)

Figure 3. Decomposed images of $\mathrm{CH}(\mathrm{a})$ and $\mathrm{CH}_{4}$ (b) from a lifted methane diffusion flame. The data are normalized by the Raman signal of pure methane and contour levels of $0.2,0.3$, and 0.4 are plotted.

\section{References}

1. M. B. Long, P. S. Levin, and D. C. Fourguette, "Simultaneous Two-Dimensional Mapping of Species Concentration and Temperature in Turbulent Flames," Opt. Lett. 10, 267 (1985).

2. R. W. Dibble, M. B. Long, and A. Masri, "Two-Dimensional Imaging of $\mathrm{C}_{2}$ in Turbulent Nonpremixed Jet Flames," Progress in Astronautics and Aeronautics, V. 105, edited by J. R. Bowen et al (AIAA, New York, NY, 1986), p. 99 . 


\section{Combustion-Torch Ignition: $\mathrm{NO}_{2}$ Fluorescence Imaging}

\section{R. J. Cattolica}

A combustion torch uses chemical energy released by combustion reactions to mix hightemperature combustion products with unburned reactants as a means of initiating further combustion. Using combustion-torch ignition, improved performance near the lean flammability limit and increased rates of flame propagation have been observed.

In a previous study, ${ }^{1}$ the fluid mechanic structure of a combustion torch in a dividedchamber combustion vessel was systematically varied from laminar to turbulent conditions. In that work, both laser-schlieren videography and laser-fluorescence imaging of $\mathrm{OH}$ concentration were used to follow the combustion process. Intermediate radical species, nonuniform temperature distribution, and turbulent fluid motion of the combustion products can all contribute to the increased rates of flame propagation observed in the study.

To clarify the role of these elements in this complex combustion system, a planar-imaging technique based on laser-induced fluorescence (LIF) from nitrogen dioxide $\left(\mathrm{NO}_{2}\right)$ has been used to visualize the flame propagation associated with combustion-torch ignition. By doping the reactant gas with a trace concentration of $\mathrm{NO}_{2}$ and observing its disappearance with LIF imaging, the consumption of reactants by the flame can be observed directly. ${ }^{2}$

Combustion-torch ignition experiments were conducted in the divided-chamber combustion vessel ${ }^{1}$ (2 liter volume) depicted in Figure 1. The combustion torch was formed by a flamegenerated starting jet exhausting from a 44-cc cylindrical prechamber $(3.9-\mathrm{cm}$ diameter and $3.7-\mathrm{cm}$ length). For the lean methane-air equivalence ratio used in the experiment $(\phi=0.65)$, the overall nitrogen dioxide concentration $w$ as $700 \mathrm{ppm}$.

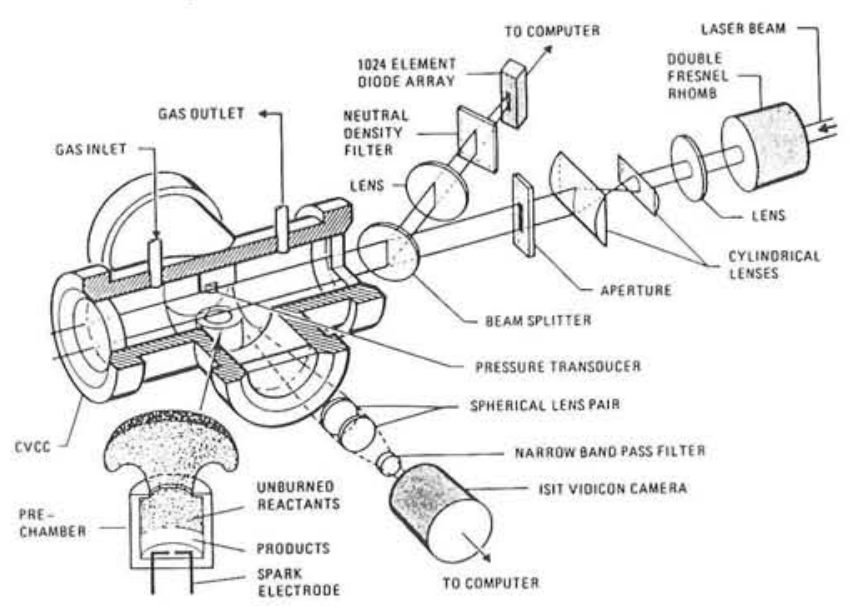

Figure 1. Laser-induced-fluorescence imaging experiment of a combustion-torch ignition in a constant volume combustion chamber.

The laser-excitation and fluorescence-detection system shown in Figure 1 was similar to that used in previous LIF imaging studies of $\mathrm{OH}$, but was modified for nitrogen dioxide. Since the $\mathrm{NO}_{2}$ molecule has a broad absorption spectrum with a high density of states, a tunable dye laser is not required to induce it to fluoresce. In this experiment, the second-harmonic output of a Nd:YAG laser $(532 \mathrm{~nm})$ was the excitation source. The laser beam was formed into a sheet of light ( $45 \mathrm{~mm} \times 1 \mathrm{~mm}$ ) by a negative (-63-mm f.l.) and positive (208-mm f.l.) cylindrical lens pair. An 8 nsec laser pulse with 100 $\mathrm{mJ}$ of energy was used to excite $\mathrm{NO}_{2}$. With this optical configuration, a linear relationship was obtained between the fluorescence signal and the laser pulse energy up to $100 \mathrm{~mJ}$. In this experiment, four prechamber orifice diameters were used to alter the structure of the flow field of the combustion torch from laminar to turbulent conditions. The gas velocity and Reynolds number of each combustion torch are listed in Table I. Also listed in Table I is the flame exit time (the time between spark ignition and the first appearance of the flame at prechamber exit) for each torch. The measured flame exit time was unchanged by the $\mathrm{NO}_{2}$ doping.

The overall effectiveness of combustiontorch ignition can be inferred from the temporal development of the pressure in the combustion vessel. The rate of pressure rise is tied directly 
to the rate of combustion. In Figure 2, the pressure histories are presented for combustion-torch ignition using the four orifice diameters.

Table I

Combustion Torch Gas Velocity, Exit Time, and Prechamber-Orifice Reynolds Number for a Lean Methane-Air Mixture $(\phi=0.65)$

\begin{tabular}{cccc}
\hline Orifice & Exit & $\begin{array}{c}\text { Exit } \\
\text { Velocity }\end{array}$ & $\begin{array}{c}\text { Reynolds } \\
\text { Number }\end{array}$ \\
\hline $25.4 \mathrm{~mm}$ & $20 \mathrm{msec}$ & $250 \mathrm{~cm} / \mathrm{sec}$ & 4290 \\
$17.9 \mathrm{~mm}$ & $18 \mathrm{msec}$ & $504 \mathrm{~cm} / \mathrm{sec}$ & 6095 \\
$12.7 \mathrm{~mm}$ & $16 \mathrm{msec}$ & $1001 \mathrm{~cm} / \mathrm{sec}$ & 8589 \\
$6.35 \mathrm{~mm}$ & $15 \mathrm{msec}$ & $4005 \mathrm{~cm} / \mathrm{sec}$ & 17183 \\
\hline
\end{tabular}

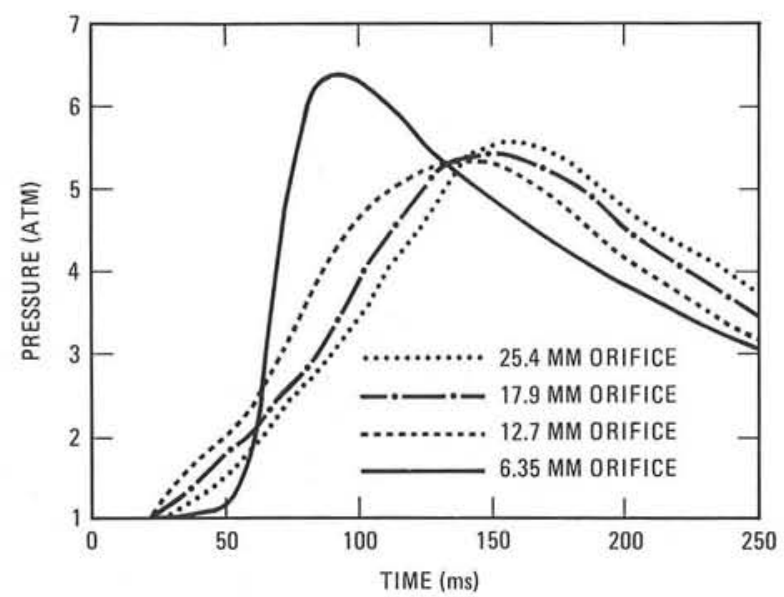

Figure 2. Pressure history for combustion-torch ignition of a lean methane-air mixture $(\phi=0.65)$ in a combustion vessel as a function of the diameter of the prechamber orifice.

The pressure histories obtained with the three largest diameter orifices were similar. The rate of pressure rise and peak pressure were nearly identical, with only a slight shift in the time scale due to increased velocities of the starting jets. For the smallest diameter orifice $(6.35 \mathrm{~mm})$ the pressure history was significantly different. The peak pressure was 20 percent higher and the rate of pressure rise was a factor of ten faster than for torch ignition at lower Reynolds number. The initial pressure rise was also shifted to longer time by an increased induction time.

The general explanation of the enhanced rate of combustion observed with the most turbulent combustion torch has been provided from the two-dimensional $\mathrm{OH}$ concentration measurements. ${ }^{1}$ In the case of the most turbulent combustion torch (6.35-mm diameter), the $\mathrm{OH}$ imaging measurements in the early development of the torch $(<26 \mathrm{~ms})$ showed that the interface between reactants and combustion products could not sustain a flame. The initially high strain rates in the combustion torch nearly extinguish the flame and allow combustion products from the prechamber to mix with the unburned reactants. As the strain rate in the combustion torch decreases, reignition occurs and a flame is reestablished. The process of mixing combustion products with the unburned reactants prior to reignition is responsible for increasing the rate of combustion.

In the mixing process, chemically reactive species, high-temperature gas, and turbulent momentum from the combustion torch are injected into the unburned reactants. Each of these elements can contribute to an increased rate of combustion. Since nitrogen dioxide molecules doped in the unburned reactants are removed rapidly by $\mathrm{H}$ - and $\mathrm{O}$-atom attack and temperatures above $700 \mathrm{~K}$, the effect of the presence of radicals and high-temperature gas mixed with unburned reactants can be observed with LIF imaging of $\mathrm{NO}_{2}$.

Two-dimensional LIF imaging measurements of $\mathrm{NO}_{2}$ during combustion-torch ignition using the four different orifice diameters were made. Figure 3 displays the results for the 6.36$\mathrm{mm}$ orifice.

The three-color fluorescence intensity scale to the right of each frame follows the three-tier, semi-quantitative concentration scale developed for flame visualization. ${ }^{2}$ The blue color in the scale corresponds to fluorescence signal levels between 90 to 100 percent of the signal from $700 \mathrm{ppm} \mathrm{NO} \mathrm{N}_{2}$ in the ambient fuel-air mixture. The yellow color denotes intermediate signal levels between 10 and 90 percent of the reference condition and indicates where $\mathrm{NO}_{2}$ is removed in the experiment. Fluorescence signals below ten percent of the ambient reference begin to approach the background noise level in the measurement ( 5 percent) and are marked by the color red. 


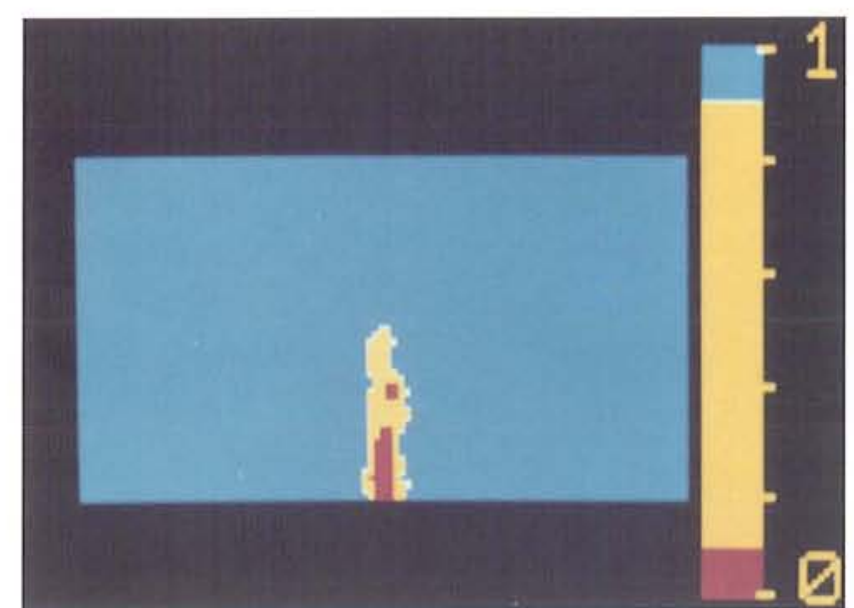

1.0 MS

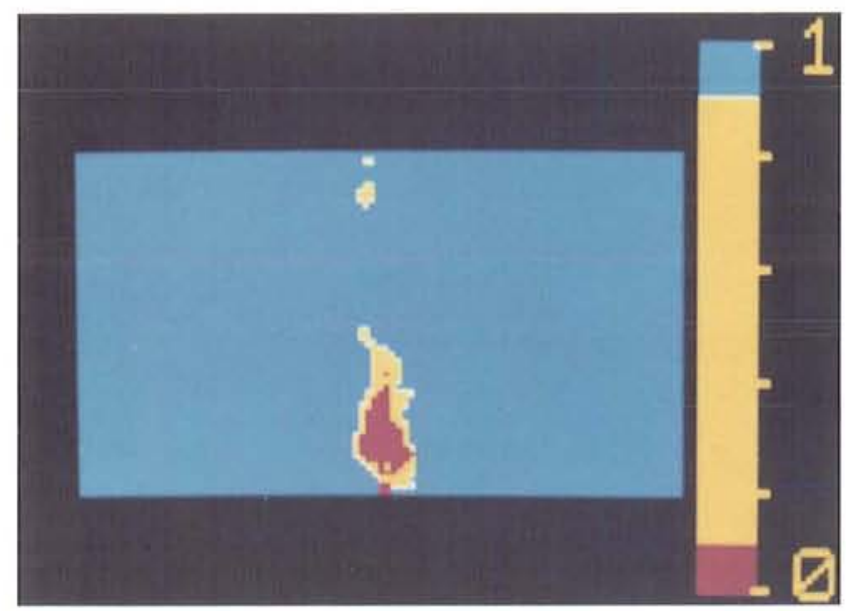

24.0 MS

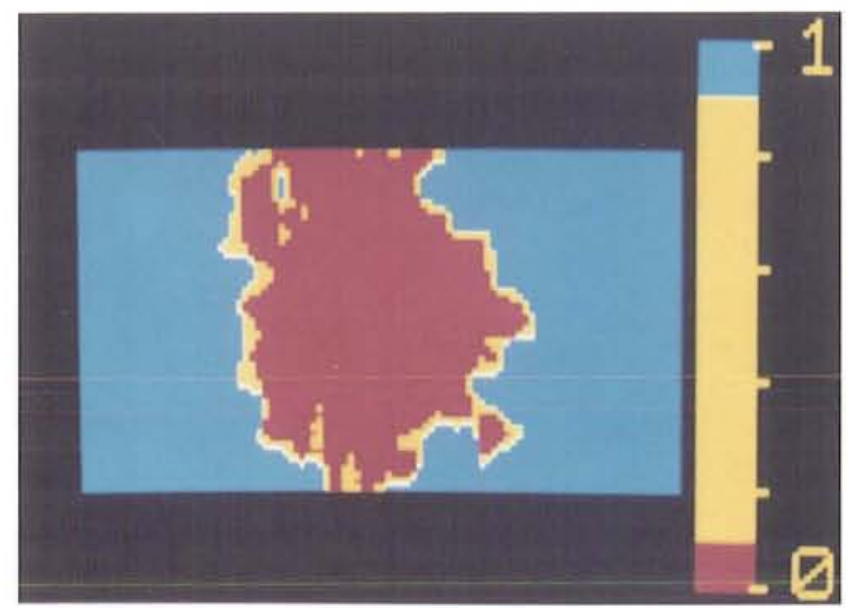

29.0 MS

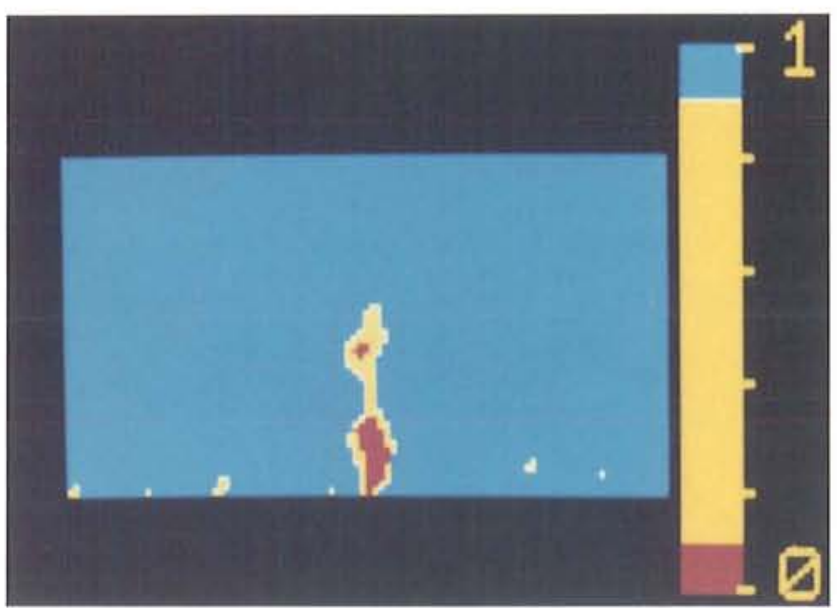

10.0 MS

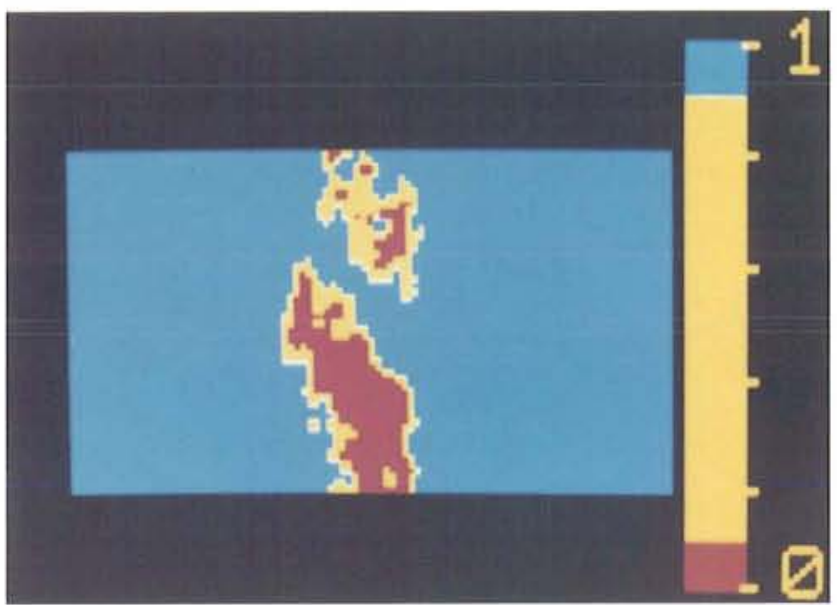

27.0 MS

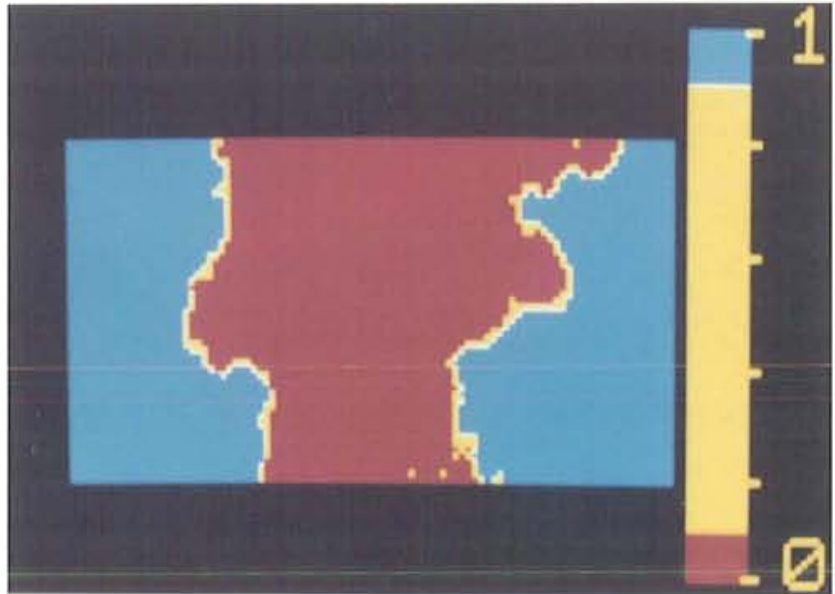

30.0 MS

Figure 3. Two-dimensional laser-induced fluorescence measurements of the $\mathrm{NO}_{2}$ molecules in a combustion torch from a $6.35-\mathrm{mm}$ orifice as a function of time after exiting the orifice. 
The $\mathrm{NO}_{2}$ fluorescence-imaging measurements shown in Figure 3 follow a pattern similar to that observed in the schlieren and $\mathrm{OH}$ imaging results. ${ }^{1}$ From 1 to $24 \mathrm{~ms}$ into the development of the combustion torch, low levels of $\mathrm{NO}_{2}$ were observed only in a region close to the prechamber exit. Combustion products ejected from the prechamber during this initial portion of the development of the combustion torch mix rapidly with the unburned reactants. During this mixing process, no reduction in the ambient $\mathrm{NO}_{2}$ concentration was observed outside the confined jet of combustion products exiting the prechamber. Flame propagation and growth begin as strain rates in the combustion torch subside, with diminished jetting from the prechamber, at $27 \mathrm{~ms}$ into ignition. The rapid propagation of the flame between the frames at $29 \mathrm{~ms}$ and $30 \mathrm{~ms}$ corresponds to the time in the pressure history of the torch ignition at which the rate of combustion increases substantially and the pressure rises rapidly.

The well-defined flame front and narrow region of $\mathrm{NO}_{2}$ consumption on the boundary of the combustion torch at $30 \mathrm{~ms}$ are of particular note. No reduction in the nitrogen dioxide concentration in the fuel-air mixture ahead of the flame front was observed in this image or in measurements at later time $(40 \mathrm{~ms})$. If chemically active species or high-temperature gas from the combustion torch had initiated any significant chemical reaction ahead of the flame, a reduction of the $\mathrm{NO}_{2}$ concentration would have been observed as a broadening of the reaction zone where $\mathrm{NO}_{2}$ was consumed in the flame front, or the appearance of pockets of intermediate concentration levels of $\mathrm{NO}_{2}$ ahead of the flame. Neither condition was observed in any of the $\mathrm{NO}_{2}$ imaging measurements.

The only remaining enhancement characteristic from the torch is the turbulent momentum transferred to the initially quiescent reactants. Turbulent fluid motion can affect the combustion rate by increasing the flame surface area or flame speed. Since no significant large scale flame wrinkling was observed, any effect of turbulence on the flame front using this combustion torch must be on a length scale comparable with the thickness of the flame front.

\section{References}

1. R. J. Cattolica and S. R. Vosen, "CombustionTorch Ignition: Fluorescence Imaging of $\mathrm{OH}$ Concentration," Comb. Flame (1987), in press.

2. R. J. Cattolica, "Visualization of Flame Propagation by Laser-Fluorescence Imaging of $\mathrm{Ni}$ trogen Dioxide," Comb. Sci. Tech. (1987), in press.

\section{Measurement of Joint Probability of Velocity and Concentration in a Turbulent Nonpremixed Flame}

\author{
R. W. Dibble, P. Magre, ${ }^{*}$ R. W. Schefer, J. Y. \\ Chen, V. Hartmann, ${ }^{* *}$ W. Kollmann ${ }^{* * *}$
}

Models of turbulent reactive flow contain submodels for the various underlying physical processes. Typically, one can identify a submodel for fluid turbulence, a submodel for chemical kinetics, a submodel for convective heat transfer, a submodel for radiative heat transfer, and often a submodel for droplet (or particle) vaporization and combustion. In addition, most practical flows are spatially complex, requiring a full three-dimensional solution of the conservation equations that include terms resulting from the postulates made in the above submodels. Straightforward numerical solution of the discretized conservation equations is rarely tractable without some degree of approximation to the exact solution. While much progress has been made in development of the

\footnotetext{
* ONERA, Chatillon, France.

** BMW AG, Munich, West Germany.

*** University of California at Davis.
} 
various submodels, and in improvement of numerical algorithms and computational speed, the principal problems confronting models of turbulent reacting flows are the need for an improved submodel of turbulence with large variations of density and a submodel that deals with temperature-sensitive chemical reactions in flow fields where the temperature has large fluctuations.

The Sandia Combustion Tunnel is designed so that the submodel for turbulent mixing in variable density flows is investigated with minimal interference from other physical processes such as radiation and heat transfer. The facility that achieves this submodel isolation consists of a vertical turbulent jet of hydrogen that is attached to a round tube surrounded by coflowing air in the test section of a wind tunnel. Because the fuel is gaseous, the need for a submodel for droplet or particle evaporation and combustion is eliminated.

Because hydrogen flames do not produce soot, a submodel for radiative heat transfer is unneccessary if molecular radiation may be ignored. Molecular radiation from the combustion products, mainly water, is negligible. ${ }^{1}$ While the nonsooting properties of hydrogen flames are advantageous from the point of view of eliminating a radiation submodel, the primary reason for using hydrogen fuel is the fast chemical kinetics that are associated with hydrogen-air flames. When these kinetic rates are large compared to rates of mixing (large Damköhler number), the chemical system responds rapidly to changes in the local properties caused by mixing; the chemical state remains at, or very near to, chemical equilibrium throughout the mixing process. Consequently, the submodel for chemical reaction rates, which can be very complicated, is replaced by a submodel that simply dictates the equilibrium compositions.

With the assumptions of adiabaticity, unity Lewis number, and large Damköhler number, all of the concentrations and temperatures can be related to a single conserved scalar. One such conserved scalar is the mixture fraction $f$, which is the local instantaneous mass fraction of fluid that originated from the nozzle. In the nozzle, $f$ has the value of unity, and in the free stream air, zero. Changes in $f$ are then due only to turbulent and molecular transport. Turbulent mixing of the conserved scalar is the unsolved problem in this limiting-case model. Conserved scalar mixing is quantified by the correlation between the velocity and the conserved scalar. This correlation is generated from the joint probability of velocity, $u$ and $v$, and the conserved scalar $P(u, v, f)$.

Figure 1 illustrates an apparatus that simultaneously measures velocity, using laser Doppler velocimetry, and mixture fraction, using laser Raman scattering. When the dye laser for the Raman scattering system is triggered by the random occurance of an LDV event, the instantaneous axial and radial velocities, $u$ and $v$, are obtained in addition to the mixture fraction $f$. From an ensemble of these events, a joint probability distribution $P(u, v, f)$ is generated.

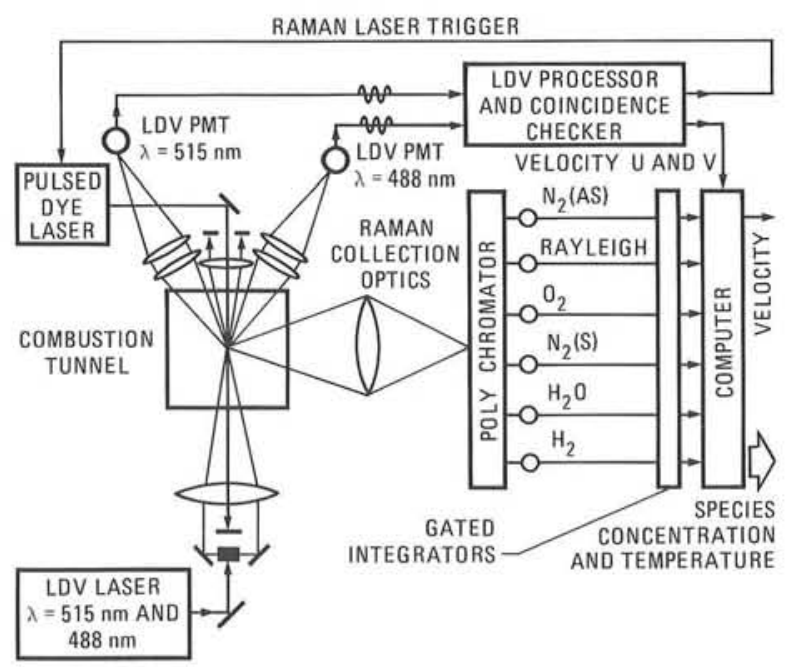

Figure 1. Schematic of the LDV-laser Raman apparatus

The events that are used to generate the distributions $P(u, v, f)$ are conditioned by the arrival of an LDV particle. As a consequence, the distributions $P(u, v, f)$ in mixing flows, such as the nonpremixed flame studied here, are sensitive to the relative number density of LDV seed particles added to the air and to the fuel. In order to establish extreme effects of this sensitivity, LDV-Raman experiments are performed once with LDV seed particles added to 
the fuel only and then again with the LDV particles added to the air only; the distributions resulting from these experiments are labeled $P_{f u e l}(u, v, f)$ and $P_{a i r}(u, v, f)$, respectively. Accordingly, there are two cases for each projection of a probability distribution. The top contour of Figure 2 shows the projections of the joint distribution generated from an ensemble of 3000 LDV-Raman events recorded at axial location $\mathrm{x} / \mathrm{D}=50$ and radial locations $\mathrm{y} / \mathrm{D}=6.0$, where most of the fluid is coflowing air. For the most part, fluid at this location is outside of the flame and is not turbulent, but it is unsteady as the distribution in axial (and radial) velocities indicates.

Figure 2b illustrates $P_{\text {fuel }}(u, f)$. Relative to the $P_{\text {air }}(u, f)$, Figure 2a, the distribution $P_{\text {fuel }}(u, f)$ shows both greater spread in the data and an increase in most probable value of mixture fraction and of axial velocity. These qualitative features, i.e., the differences between $P_{\text {air }}(u, f)$ and $P_{\text {fuel }}(u, f)$, are similar for all radial locations; however, quantitative differences are largest at larger radii. These features are consistent with the notion that the adding seed particles to the fuel will bias the data toward higher axial velocities and higher mixture fractions.

It is disconcerting that these measurements of velocity-scalar statistics are dependent on the origin of the particle that triggers the event. Fortunately, the unconditional distribution $P(u, v, f)$ can be generated from the two distributions $P_{\text {air }}(u, f)$ and $P_{\text {fuel }}(u, f)$, which represent the extremes in bias due to origin of seed particle: ${ }^{2}$

$$
P(u, v, f)=\bar{f} P_{f u e l}(u, v, f)+(1-\bar{f}) P_{a i r}(u, v, f)
$$

This equation shows that the unconditional distribution $P(u, v, f)$ approaches the conditional distribution $P_{a i r}(u, v, f)$, as $\bar{f}$ approaches zero. In most flames, the stoichiometric contour occurs at small values of $f$; e.g. $f=0.16$ for this flame and $f=0.05$ for most hydrocarbon flames. The equation indicates a substantial bias of the data if the air stream is not seeded.
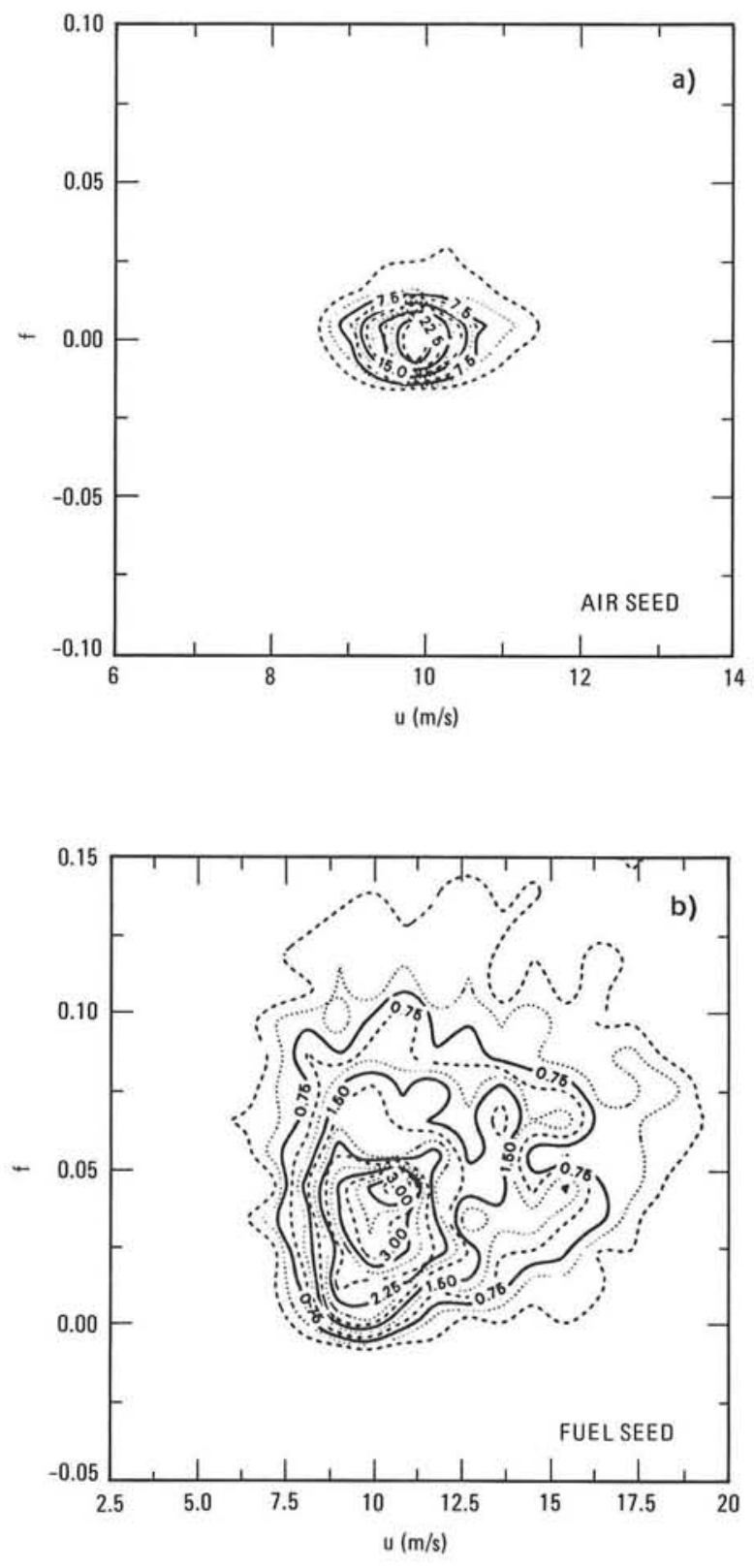

Figure 2. Contours of the joint probability distributions of mixture fraction and axial velocity $P(u, f)$ for a $\mathbf{H}_{2}$-Ar nonpremixed turbulent jet flame at $x / D=50$ and $y / D=6.0$. These contours show the effect of adding LDV seed to the coflow air stream only (top) and to the fuel jet stream only (bottom). Conditions are: $\mathrm{Re}+$ 9000 ; fuel jet velocity $=75 \mathrm{~m} / \mathrm{s}$; coflow air velocity $=9.2 \mathrm{~m} / \mathrm{s}$.

As noted above, the important correlations between velocity and mixture fraction are generated from joint probabilities such as illustrated in Figure 2. Figure 3 presents comparisons of model predictions and of correlations generated 
from the present data. For correlation $\widetilde{f^{\prime \prime} v^{\prime \prime}}$, the agreement between the model predictions and the experimental data is considered reasonable. However, the same is not true for correlation $\widetilde{f^{\prime \prime} u^{\prime \prime}}$; the model predicts considerably lower values than those indicated by the data.
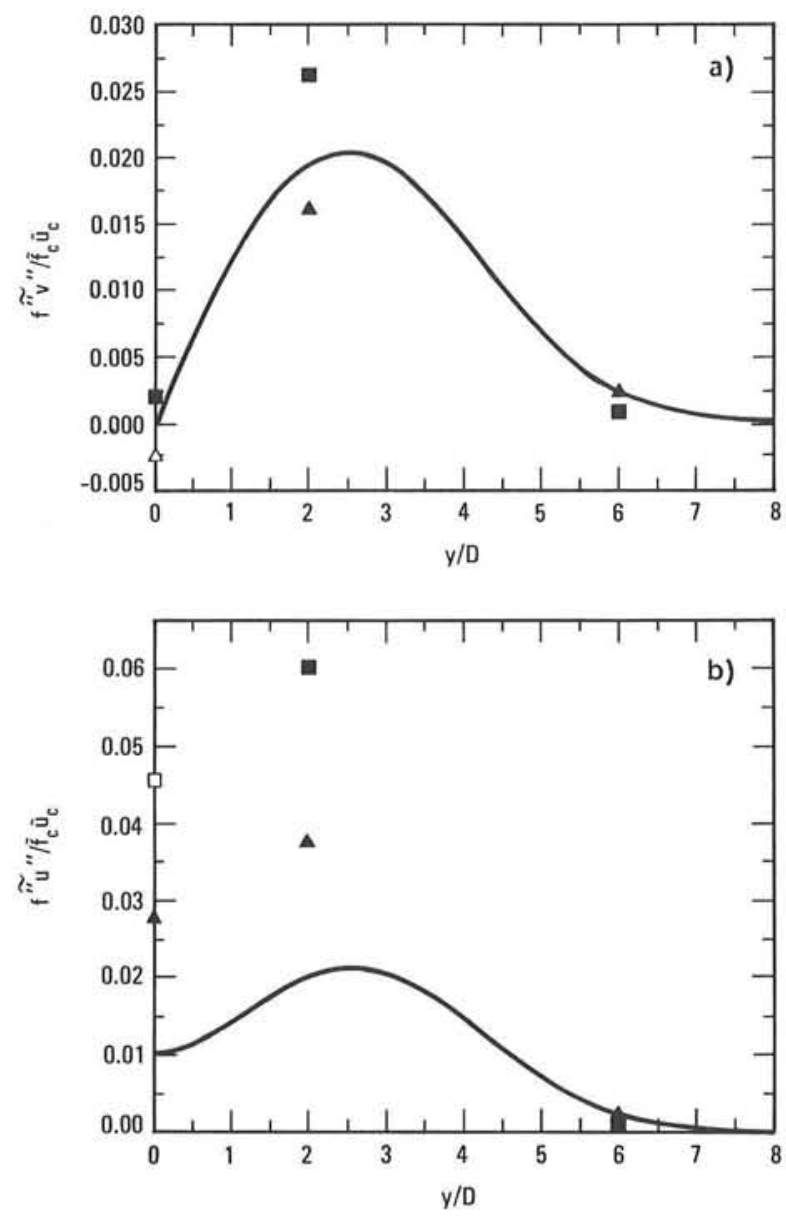

Figure 3. Radial profiles of the turbulent transport of mixture fraction in the radial direction, $\widetilde{f^{\prime \prime} v^{\prime \prime}}(\mathrm{a})$; and in the axial direction $\widetilde{f^{\prime \prime} u^{\prime \prime}}$ (b). Conditions are as in Figure 2.

In the past, development of predictive methods for these correlations has been at a slow pace primarily due to the lack of appropriate experimental data for model validation. One of the objectives of this paper is to demonstrate the use of statistical information deduced from the present joint distribution measurements in assessing the performance of a recently proposed turbulence model. ${ }^{3}$

\section{References}

1. M. C. Drake, R. W. Bilger, and S. H. Starner, "Raman Measurements and Conserved Scalar Modeling in Turbulent Diffusion Flames," Nineteenth Symposium (International) on Combustion (The Combustion Institute, 1983), p. 459.

2. R. W. Dibble, V. Hartmann, R. W. Schefer, and W. Kollman, "Conditional Sampling of Velocity and Scalars in Turbulent Flames Using Simultaneous LDV-Raman Scattering," $J$. of Experiments in Fluids (1986), in press; also, Sandia Report SAND84-8860.

\section{Effects of Finite Chemical Kinetics in Subsonic, Nonpremixed, Turbulent Flames of Hydrogen ${ }^{\dagger}$}

\author{
P. Magre, ${ }^{*}$ R. W. Dibble
}

Departures from chemical equilibrium appear in nonpremixed turbulent flames when the rate of mixing competes with the rate of chemical reaction. The ratio of these two rates is the Damköhler number, which is the characteristic time of the mixing over characteristic time of the chemical reaction. When the Damköhler number is large, the mixing rate is slow relative to the chemical rate and consequently the system is nearly at chemical equilibrium. As the Damköhler number is reduced, the chemical system departs from equilibrium.

In this experiment, we study the effects of Damköhler number in a turbulent nonpremixed jet flame of hydrogen or methane into air; this flame is illustrated in Figure 1. These flames are often erroneously considered to be in the large Damköhler number limit.

† Presented at the AIAA Twenty-fifth Aerospace Sciences Meeting (1987); to be submitted to Combustion and Flame (1987).

* ONERA, 92322 Chatillon, France. 


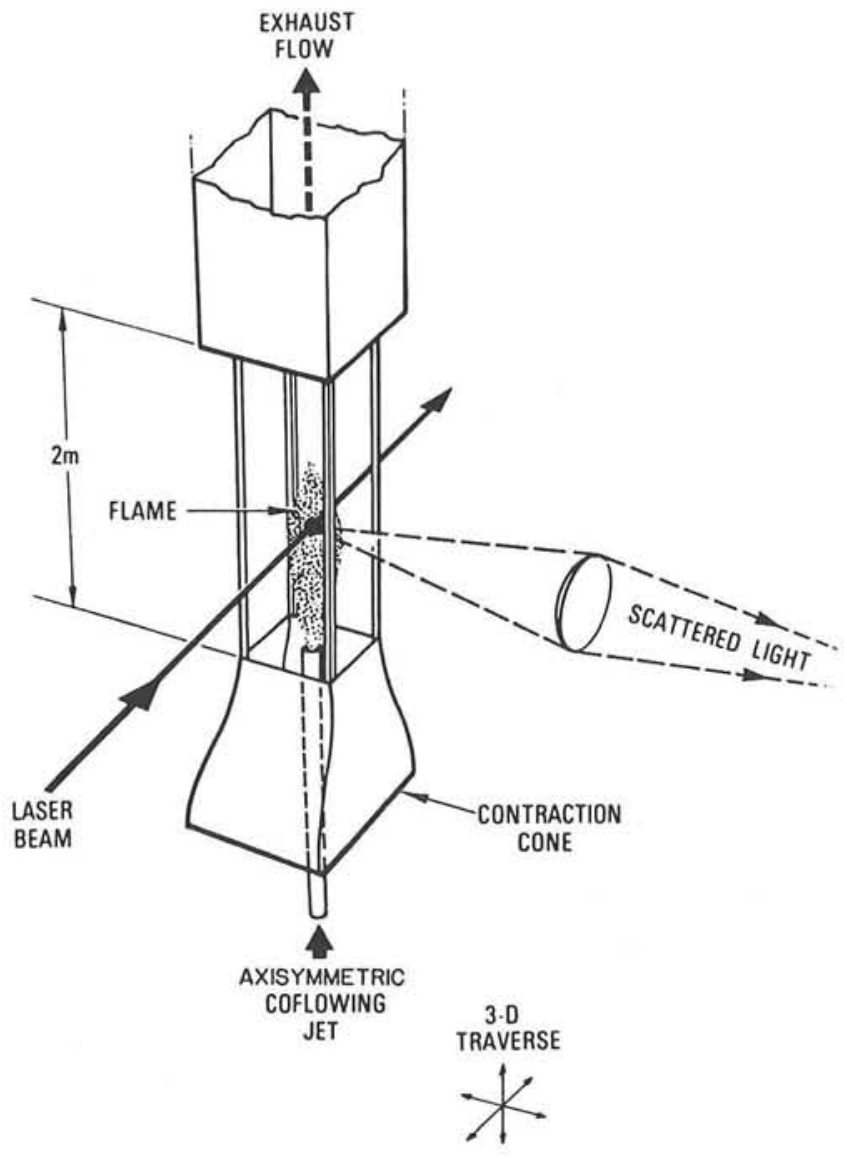

Figure 1. Illustration of the Sandia Combustion Tunnel Facility; note that the tunnel translates and the optics remain fixed.

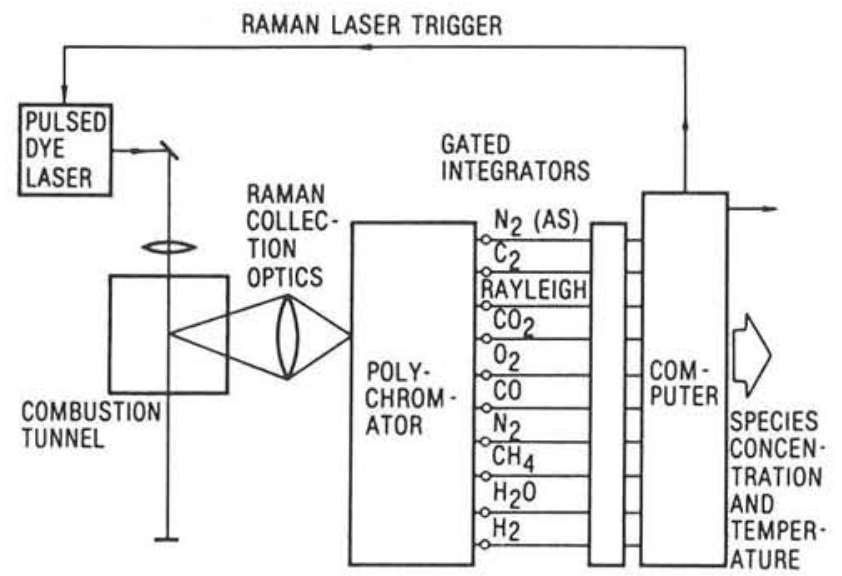

Figure 2. Multichannel laser Raman Scattering System.

We measure departures from chemical equilibrium with nonintrusive laser diagnostics. Instantaneous and spatially resolved measurements of major combustion species $\left(\mathrm{H}_{2}, \mathrm{O}_{2}\right.$,
$\mathrm{H}_{2} \mathrm{O}$, and $\mathrm{N}_{2}$ ), density, and temperature are determined from the Raman and Rayleigh scattering from a single 2-microsecond laser pulse; the Raman scattering apparatus is shown in Figure 2.
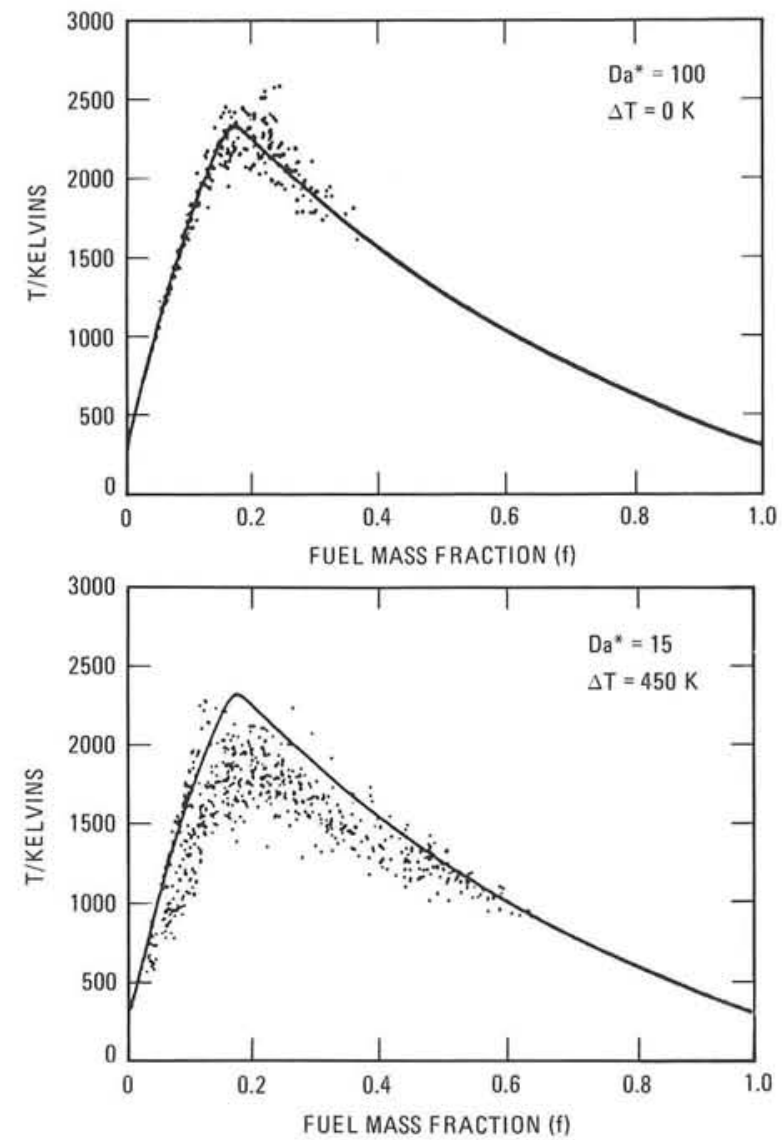

Figure 3. Each point in these two ensembles of simultaneous fuel mass fraction $f$ and temperature $\mathbf{T}$ measurements is from a single laser pulse. For the top case $U_{\text {jet }}=75 \mathrm{~m} / \mathrm{s}$ and $\mathrm{x} / \mathrm{D}=50$, the $f$-T points cluster around the equilibrium line; the difference between the measured temperature and the equilibrium temperature is essentially zero. For purpose of comparison, this $\Delta \mathbf{T}=0$ $\mathrm{K}$ case is assigned a relative Damköhler number $\mathrm{Da}^{*}$ of 100 . The bottom case, $U_{\text {jet }}=175 \mathrm{~m} / \mathrm{s}$ and $\mathrm{x} / \mathrm{D}=30$, has a relative Damköhler number of 15. Here, the temperature difference is as large as $450 \mathrm{~K}$, indicating large departures from chemical equilibrium.

From these Raman and Rayleigh measurements, we infer the local fuel mixture fraction $f$. Departure from chemical equilibrium is inferred from the comparison between the measured temperature and the equilibrium temperature deduced from the value of $f$. 
We vary the Damköhler number in two ways. First, we hold the fuel composition constant and change the mixing rate by steadily increasing the fuel velocity $\mathrm{U}_{\text {jet }}$; the Reynolds numbers explored are $\operatorname{Re}=8,500, \operatorname{Re}=17,000$, and $R e=20,000$. The experimental results show a dramatic effect of the Reynolds number on the departure of chemical equilibrium. An example of this effect is shown in Figure 3, which shows data obtained at $\mathrm{x} / \mathrm{D}=50$, where $\mathrm{x}$ is the downstream distance and D is the nozzle diameter.

Large differences between the measured temperature and the inferred equilibrium temperature are obtained as the flame approaches blow-off conditions; our measurements are summarized in Figure 4.

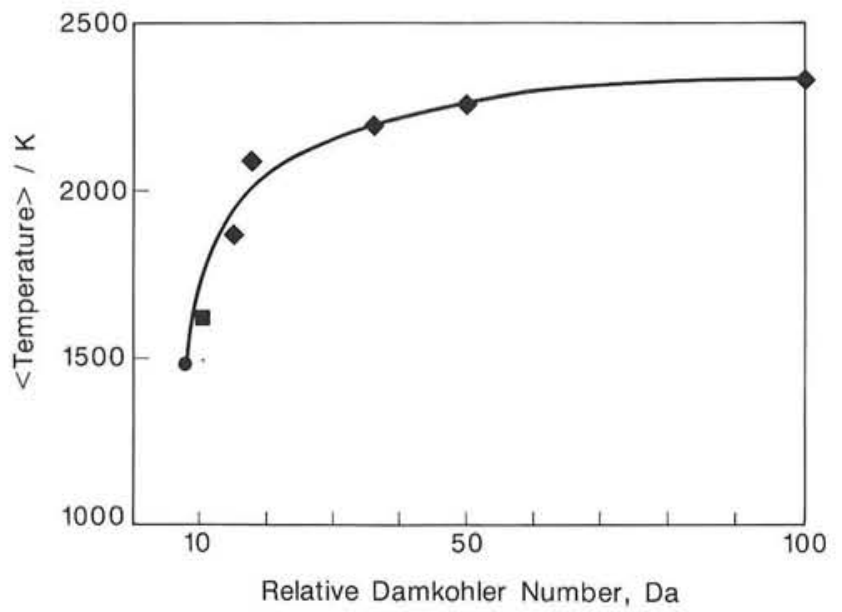

Figure 4. This plot of the mean flame temperature $\mathbf{T}_{\text {flame }}$ versus Damköhler number $\mathrm{Da}^{*}$ shows a decrease in $T_{\text {flame }}$ as $\mathrm{Da}^{*}$ is reduced. The diamonds represent measurements from experiments in which fuel is $22 \%$ argon-in-hydrogen while the square and the circle are from experiments in which the fuel is $60 \%$ and $70 \%$ nitrogen-inhydrogen, respectively.

Figure 4 includes results from experiments where we varied the Damköhler number by changing the fuel composition while maintaining a nearly constant mixing rate. In this second case, we alter the chemical reaction rate by diluting the fuel with increasing amounts of nitrogen. These last experiments also show a difference between measured temperature and the inferred equilibrium temperature.

\section{Soot Production in Axisymmetric Laminar Diffusion Flames at Pressures From One to Ten Atmospheres ${ }^{\dagger}$}

\author{
W. L. Flower, C. T. Bowman *
}

Laminar axisymmetric ethylene-air diffusion flames have been studied at pressures from 0.1 to $1.0 \mathrm{MPa}$ (1-10 standard atmospheres) in order to gain insight into the processes controlling the evolution of soot from such flames, including both soot formation and soot oxidation. Measurements of the attenuation of laser light across the flame diameter characterize the volume of soot present in the flame. Data were acquired as a function of axial position above the burner port, allowing independent identification and examination of regions of soot formation and soot oxidation.

The apparatus used here consists of a vertical 1.27-cm-diameter circular burner tube that is surrounded by a co-flowing air stream and is enclosed in a pressure vessel of rectangular cross section. Experiments were performed at a sequence of pressures in the range from 0.1 to 1.0 $\mathrm{MPa}$, holding fuel mass flow rate fixed. Ethylene was used as the fuel at rates ranging from 1.7 to 4.9 standard cubic centimeters per second (scc/s).

Optical access to the flame was provided by four circular ports fitted with fused-silica windows. The beam from an argon-ion laser was mechanically chopped before passing horizontally through the flame. A silicon photodiode measured the intensity of light transmitted

$\dagger$ Twenty-First Symposium (International) on Combustion (The Combustion Institute, 1986), in press.

* Stanford University, Stanford, CA. 
through the soot-laden flame. A lock-in amplifier referenced to the laser-chopping frequency measured the detector output.

The fraction of the initial laser intensity $I_{o}$ that propagates through the flame, $I / I_{o}$, is given by

$$
\ln \left\{I / I_{o}\right\}=-\int_{-\infty}^{+\infty} C_{\mathrm{ext}} N d r,
$$

where $r$ is the radial coordinate, $N$ is the particle number density and $C_{\text {ext }}$ is the particle extinction coefficient. For the flames studied here, the soot particles are sufficiently small so that it is reasonable to assume that they behave approximately as Rayleigh scatterers, in which case it follows from Eq. 1:

$$
\int_{-\infty}^{+\infty} f_{\mathrm{v}} d r=C(\lambda, m) \ln \left\{I / I_{o}\right\},
$$

where $f_{v}$ is the soot volume fraction. The proportionality factor $C(\lambda, m)$ depends on wavelength $\lambda$ and the particle complex refractive in$\operatorname{dex} m$.

Flames at atmospheric pressure were similar in appearance to those studied previously. For low fuel flow rates, the flame came to a relatively sharp tip at the vertical position where all the soot had been oxidized. For atmosphericpressure flames with fuel flow rates above 3.6 $\mathrm{scc} / \mathrm{s}$, there was no well-defined flame tip, and the flame luminosity became less intense with height due to cooling of the soot by radiative losses.

As pressure was increased at fixed fuel mass flow, flames that did not emit smoke at atmospheric pressure began to smoke at higher pressures. Figure 1 shows a sequence of photographs of flames at pressures from $0.13 \mathrm{MPa}$ to $0.5 \mathrm{MPa}$ for a fuel flow rate of $2.4 \mathrm{scc} / \mathrm{s}$.

The well defined luminous tip of the atmospheric-pressure flame disappears as pressure increases, and a darkening orange column of soot rises from the flame. At a fixed axial position, the diameter of the visible flame decreases with increasing pressure. Also, the opacity of the soot column increases with pressure, reflecting an increase in the integrated soot volume fraction across the flame diameter.
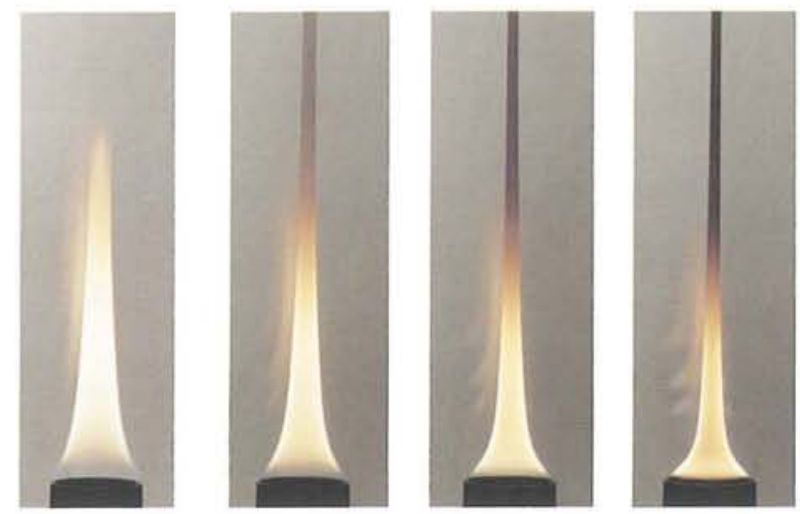

Figure 1. Photographs of axisymmetric laminar ethylene-air diffusion flames at a sequence of pressures. The ethylene mass flowrate is $\mathbf{2 . 4}$ standard cubic centimeters per second for each flame. Bright luminosity from soot is visible for each flame. At $0.13 \mathrm{MPa}$, the flame is close to the point of incipient soot breakthrough. For each of the flames at higher pressures, a significant amount of soot survives the oxidation region of the flame. In these cases, the luminosity from soot becomes noticeably less intense with increasing height above the burner tip. As pressure increases, the decrease in soot luminosity moves to lower positions in the flame.

Figure 2 shows measurements of $C(\lambda, m)$ $\times \ln \left\{I / I_{o}\right\}$ as a function of the nondimensional axial coordinate $\eta=\ln \left\{1+\frac{1}{S}\right\} D z / Q_{\text {fuel }}$, where $S$ is a stoichiometric constant, and $D$ is the gas diffusion coefficient. When the attenuation is attributable to soot absorption, the ordinate in Figure 2 is equal to the integral of soot volume fraction over the flame diameter, $\int_{-\infty}^{+\infty} f_{v} d r$ (Eq. $2)$. Shown are measurements for flames at pressures from 0.1 to $0.5 \mathrm{MPa}$ at a fixed ethylene flow rate of $1.7 \mathrm{scc} / \mathrm{s}$. At $0.1 \mathrm{MPa}, \int_{-\infty}^{+\infty} f_{v} d r$ increases during the soot-formation stage to a peak at $\eta=0.011$. The soot is then completely removed by oxidation at later times. The same processes are apparent at $0.2 \mathrm{MPa}$. Soot formation occurs more rapidly, leading to a greater peak integral of $f_{v}$. Only about half of the peak soot volume is oxidized before $\int_{-\infty}^{+\infty} f_{v} d r$ levels off. At $0.3 \mathrm{MPa}, \int_{-\infty}^{+\infty} f_{v} d r$ increases still more 
rapidly, and a smaller fraction of the peak soot volume is oxidized.

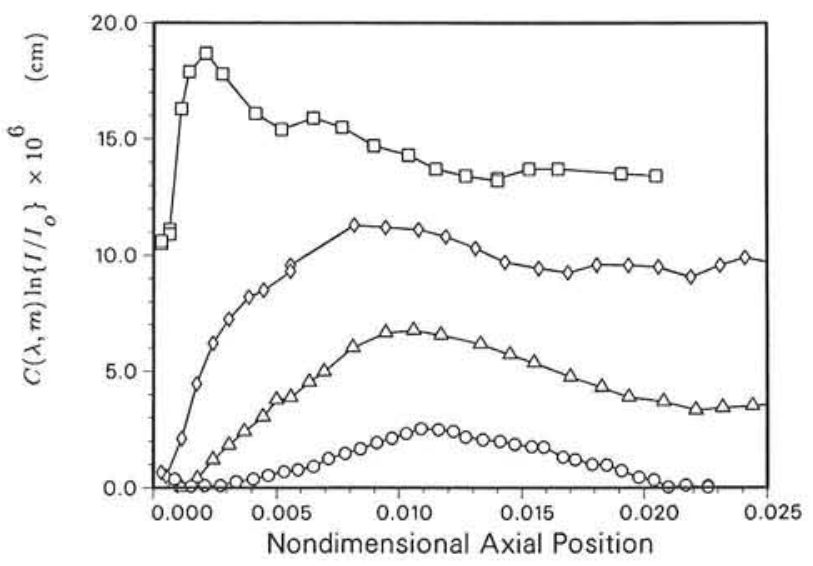

Figure 2. Values of $C(\lambda, m) \ln \left\{I / I_{o}\right\}$ inferred from measurements of the attenuation of laser light across the flame diameter as a function of the nondimensional axial position $\eta$. Shown are measurements obtained for flames with a fuel flow rate of $1.7 \mathrm{scc} / \mathrm{s}$ at pressures up to $0.5 \mathrm{MPa}$ : $\bigcirc-0.1 \mathrm{MPa} ; \triangle-0.2 \mathrm{MPa} ; \diamond-0.3 \mathrm{MPa} ; \square-$ $0.5 \mathrm{MPa}$. When the attenuation is attributable to absorption of light by soot particles, $C(\lambda, m)$ $\ln \left\{I / I_{o}\right\}$ is equal to the integral of the soot volume fraction across the flame diameter, $\int_{-\infty}^{+\infty} f_{v} d r$.

The behavior at $0.5 \mathrm{MPa}$ differs from the lower-pressure results. The peak in $C(\lambda, m) \times$ $\ln \left\{I / I_{o}\right\}$ is attained more quickly than at the lower pressures, followed by a rapid decay, a slight increase to a smaller local peak, and then a gradual decay. At the position of the first peak, the scattering of light is several orders of magnitude less than at higher positions in the same flame, where the measured extinction of light is smaller. We attribute the first peak to molecular absorption of light by hydrocarbon species. This interpretation of the early peak is consistent with our own observations at higher flow rates and pressures, discussed below, and with previous observations of molecular absorption and/or fluorescence in the region near the burner tip. The second local absorption maximum at $\eta \approx 0.0065$ corresponds to the actual peak in soot volume.

In Figure 3, the peak values of $\int_{-\infty}^{+\infty} f_{v} d r$ are plotted as a function of pressure for flames

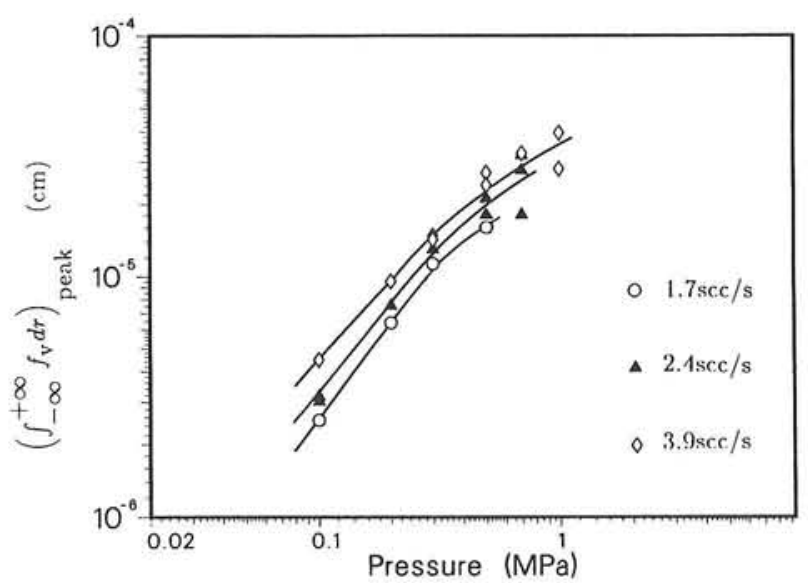

Figure 3. Peak values of the integral of the soot volume fraction across the flame diameter, $\int_{-\infty}^{+\infty} f_{v} d r$, as a function of pressure. Ethylene flow rates: $\bigcirc-1.7 \mathrm{scc} / \mathrm{s} ; \Delta-2.4 \mathrm{scc} / \mathrm{s} ; \diamond-3.9 \mathrm{scc} / \mathrm{s}$.

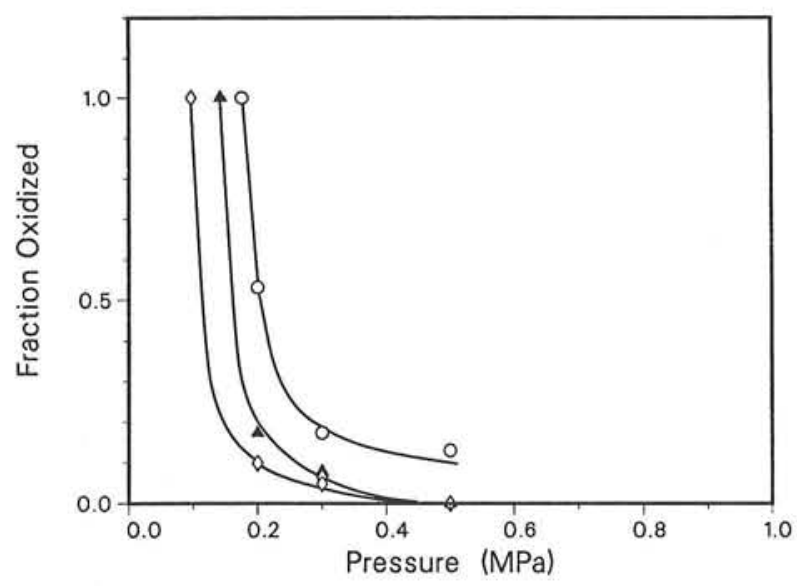

Figure 4. Fractional decrease of the integral of the soot volume fraction across the diameter between the peak value and the final late-time value,

$$
\left[\left(\int_{-\infty}^{+\infty} f_{v} d r\right)_{\text {peak }}-\left(\int_{-\infty}^{+\infty} f_{v} d r\right)_{\text {final }}\right] /\left(\int_{-\infty}^{+\infty} f_{v} d r\right)_{\text {peak }}
$$

displayed as a function of pressure. This quantity characterizes the fraction of the peak soot volume fraction which is removed by oxidation. Shown are results for flames with ethylene flow rates of $1.7 \mathrm{scc} / \mathrm{s}(\bigcirc), 2.4 \mathrm{scc} / \mathrm{s}(\triangle)$, and 3.9 $\mathrm{scc} / \mathrm{s}(\diamond)$.

at three different fuel flow rates. The data fit well with expressions of the form

$$
\int_{-\infty}^{+\infty} f_{v} d r \propto p^{n}
$$


with $n=1.2 \pm 0.1$. The data at each flow rate show a reduced dependence of $\int_{-\infty}^{+\infty} f_{v} d r$ on pressure at high pressures.

The extent of soot oxidation is plotted as a function of pressure in Figure 4. The removal of soot by oxidation ceases very quickly as pressure is increased, perhaps as a result of greater radiative cooling by the high soot concentrations present at higher pressures.

The experiments performed here provide insight into the pressure dependence of processes controlling the evolution of soot from diffusion flames. The quantity of soot emitted from a particular flame is ultimately determined by a competition between formation and oxidation processes. These experiments show that not only does the formation of soot increase strongly with pressure, but also that, as pressure increases, soot oxidation removes a decreasing fraction of the soot that is formed.

\section{Observations on the Soot Formation Mechanism in Laminar Ethylene-Air Diffusion Flames at One and Two Atmospheres ${ }^{\dagger}$}

\section{W. L. Flower, C. T. Bowman*}

The effect of pressure on the structure of sooting two-dimensional, laminar, ethylene-air diffusion flames has been investigated. Our first studies looked at the influence of pressure on total soot production, as well as local soot volume fraction, particle size, and number density. ${ }^{1}$ These soot measurements were later combined with measurements of the velocity field in the flame to determine local soot production rates. ${ }^{2}$ In the present paper, we use these same data

\footnotetext{
† To appear in Combustion Science Technology (1987).
}

* Stanford University, Stanford, CA. to show how pressure affects the mechanism of soot formation in diffusion flames. The approach we have taken is to examine the soot formation process along individual particle paths.

The experimental configuration considered here is a Wolfhard-Parker burner enclosed in a pressure chamber. The experiments and data have been described in detail previously. ${ }^{1,2}$

The size, number density, and volume fraction of the soot particles were determined from measurements of the scattering and extinction of light by soot particles. ${ }^{1}$ Velocity measurements were made using a laser-Doppler velocimeter. $^{2}$

The analysis of the data begins with the determination of flow streamlines. We used an iterative numerical procedure to determine streamlines that are tangent to the measured velocity vectors.

The next step in the data analysis is determination of the variation of the measured velocities and soot quantities along selected individual streamlines. The reciprocal streamwise velocities were curvefit as a function of distance $s$ along the streamlines, and residence times $t$ along the streamlines were calculated by integration:

$$
t=\int_{0}^{s} \frac{d s}{v_{\mathrm{s}}} .
$$

The velocity history before the first axial measurement location was determined from the relation

$$
v=\left\{v_{\mathrm{o}}^{2}+2 a z\right\}^{\frac{1}{2}},
$$

which provides a good prediction of the axial velocity in the region of high soot loading. ${ }^{2}$

Polynomial curvefits to soot volume fraction $f_{\mathrm{v}}$, mean particle diameter $d$, and particle number density $N$ were obtained along individual streamlines. These curvefits were then manipulated analytically to determine the evolution along the individual streamlines of various quantities characterizing the soot formation process.

For the present discussion, we choose the streamline that intersects the locus of maximum soot volume fraction at a height $2 \mathrm{~cm}$ above 
the burner, which is representative of streamlines in the region where most of the soot is located. Figure 1 shows the evolution of soot volume fraction at pressures of one and two atmospheres. At each pressure, soot volume fraction increases rapidly early in the flame, leveling off at later times. Soot volume fraction increases with pressure, as observed previously.

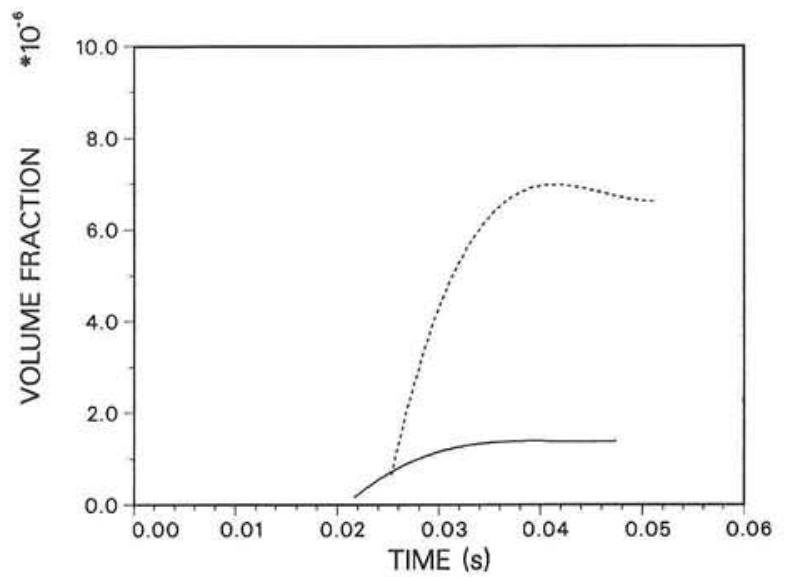

Figure 1. Soot volume fraction $f_{\mathrm{v}}$ as a function of time on representative streamlines in flames at 1 (solid line) and 2 (dashed line) atmospheres. The representative streamline at each pressure is the streamline that crosses the contour of maximum soot volume fraction at a height $2 \mathrm{~cm}$ above the burner.

The volumetric rate of soot formation $\dot{f}_{\mathrm{v}}=d f_{\mathrm{v}} / d t$ is obtained by differentiating the polynomial fit to $f_{\mathrm{v}}$. Along streamlines in the region of the flame containing significant quantities of soot, the volumetric soot formation rate is greater at two atmospheres than at the same residence time in the one-atmosphere flame (Figure 2).

The soot surface area per unit volume, $\sigma=6 f_{\mathrm{v}} / d=\pi N d^{2}$, is determined from the fits to the soot particle size and volume fraction. Along the representative streamlines, $\sigma$ increases at early times, leveling off at later times (Figure $3)$.

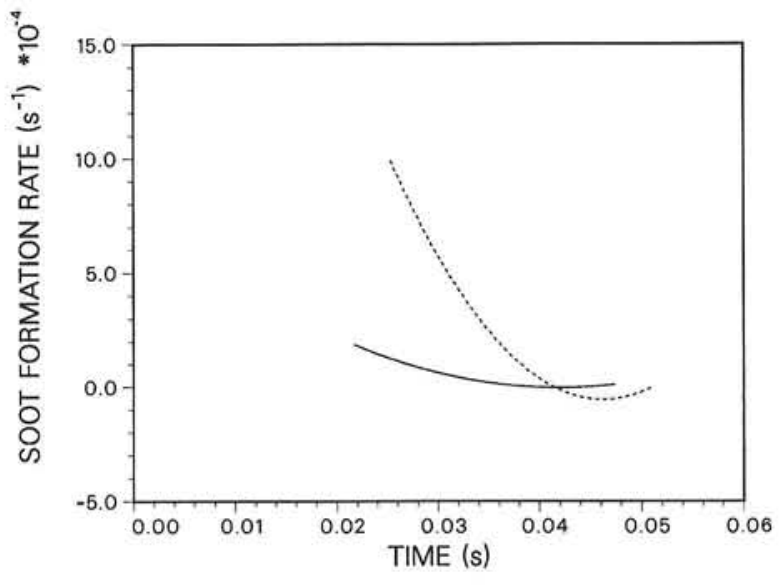

Figure 2. Volumetric soot formation rate, $\dot{f}_{\mathrm{v}}$ as a function of time on representative streamlines in flames at 1 (solid line) and 2 (dashed line) atmospheres. (See Figure 1 for the definition of the representative streamlines).

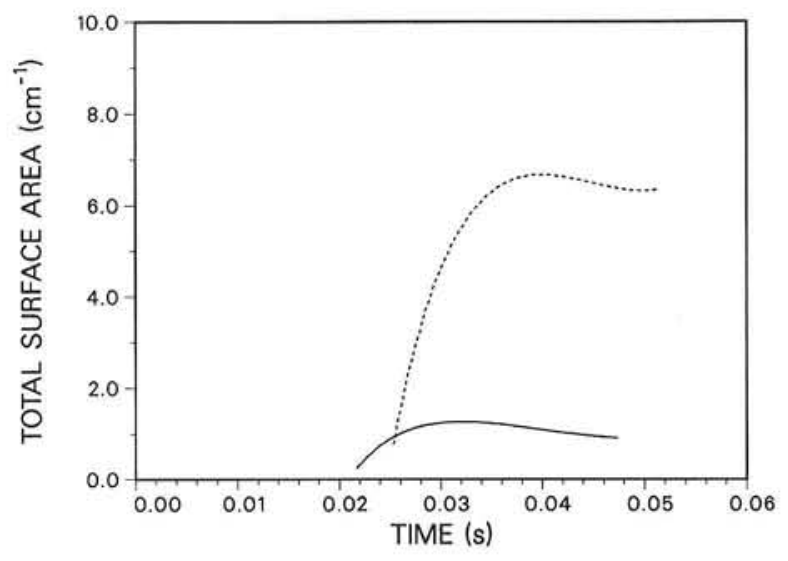

Figure 3. Total surface area of soot particles per unit volume in space, $\sigma$, as a function of time on representative streamlines in flames at 1 (solid line) and 2 (dashed line) atmospheres. (See Figure 1 for the definition of the representative streamlines).

The present data show a significant increase in the soot surface area per unit volume as the pressure is increased from one to two atmospheres, that cannot be explained by a change in particle size. Our measurements show that the increase in soot surface area per unit volume is largely due to an increase in the particle number density $N$ with pressure.

Normalizing the soot formation rate by $\sigma$, we obtain the specific soot formation rate, $\dot{f}_{\mathrm{v}} / \sigma$, which represents the volumetric rate of soot 
formation per unit surface area. The pressurenormalized specific soot formation rate, $\dot{f}_{\mathrm{v}} / \sigma P$, is shown in Figure 4.

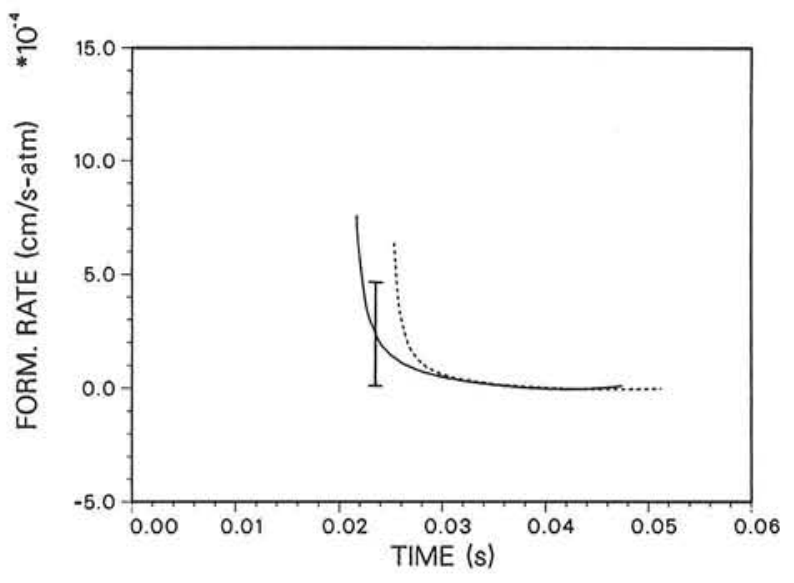

Figure 4. Pressure-normalized specific surface growth rate, $\dot{f}_{\mathrm{v}} / \sigma P$, as a function of time on representative streamlines in flames at 1 (solid line) and 2 (dashed line) atmospheres. (See Figure 1 for the definition of the representative streamlines.) The error bar represents the estimated uncertainty in $\dot{f}_{\mathrm{v}} / \sigma P$, as determined from the scatter of the measurements about the curvefits to $f_{\mathrm{v}}, d$, and $N$ that were used to determine $\dot{f}_{\mathrm{v}}$ and $\sigma$.

While the local volumetric rate of soot production $\left(\dot{f}_{\mathrm{v}}\right)$ is significantly larger at two atmospheres than at one, the specific soot production rate, normalized by pressure, is the same within experimental error for both pressures.

Harris and Weiner ${ }^{3}$ have proposed that the specific soot formation rate in premixed flames may be expressed in terms of a first-order rate equation,

$$
\dot{f}_{\mathrm{v}}=k \sigma P_{g}=k \sigma \chi_{g} P,
$$

where $k$ is the surface growth rate constant, $P_{g}$ is the partial pressure of the primary growth species, and $\chi_{g}$ represents the mole fraction of the growth species. The fact that the pressurenormalized specific surface growth rate, $\dot{f}_{\mathrm{v}} / \sigma P$, does not vary greatly with pressure for our experiments suggests that the surface growth kinetics can be reasonably represented by the first-order rate equation for the regions of the diffusion flame examined here.
For most streamlines investigated at both one and two atmospheres, the specific soot production rate decreases with residence time, as observed for the streamline shown in Figure 4. These results suggest a decrease in the reactivity of the soot particles or a decrease in the concentration of growth species with time.

The total soot surface area per unit volume $\sigma$ is much larger in two-atmosphere, ethylene-air diffusion flames than at atmospheric pressure. This increase is due largely to an increase in the number of soot particles present in the higher-pressure flame.

The soot formation rate $\dot{f}_{\mathrm{v}}$ is somewhat larger at two atmospheres than at one. However, the pressure-normalized specific soot formation rates $\dot{f}_{\mathrm{v}} / \sigma P$ are essentially the same at the two pressures.

We conclude that the observed increase in soot production results primarily from the increase in the total surface area available for particle growth. Since the increase in $\sigma$ is believed to result primarily from an increase in the number of particles with pressure, we conclude that variation in the particle inception rate with pressure appears to play a major role in governing the variation of soot formation rates with pressure.

\section{References}

1. W. L. Flower and C. T. Bowman, "Measurements of the Structure of Sooting Laminar Diffusion Flames at Elevated Pressure," Twentieth Symposium (International) on Combustion (The Combustion Institute, 1985), p. 1035 .

2. W. L. Flower, "The Effect of Elevated Pressure on the Rate of Soot Production in Laminar Diffusion Flames," Comb. Sci. Tech. 48, 31 (1986).

3. S. J. Harris and A. M. Weiner, "Determination of the Rate Constant for Soot Surface Growth," Comb. Sci. Tech. 31, 155 (1983). 


\section{In Situ Measurement of Flame-Formed Silica Particles Using Dynamic Light Scattering ${ }^{\dagger}$}

\author{
W. L. Flower, A. J. Hurd*
}

Silica $\left(\mathrm{SiO}_{2}\right)$ particles that are produced in flames have a number of commercial applications and also have become popular as a model system for studying the formation and structure of aggregated aerosols. Silica particles are formed in flames by the oxidation of silicon compounds that are introduced with the fuel. The composition of flame-synthesized silica can be very carefully controlled, allowing the production of material of exceptionally high purity or, through the introduction of dopants such as germanium, the manufacture of material with a specified refractive index. These attributes make flame-synthesized silica a desirable material for the manufacture of optical fibers.

Since the processes by which silica particles are formed and evolve in flames are topics of current research interest, it is desirable to have means of measuring the particles present during these processes, either as a research tool or as an instrument for process control in commercial applications. Examination of material collected by physical sampling can provide valuable information regarding the state of the particles. However, such analyses are not only time-consuming, but are also ambiguous, since it is unclear whether the collected samples are the same as the undisturbed particles in the flame.

An alternative means of examining flamesynthesized particles is to use optical diagnostic techniques. Particle diagnostic techniques that rely on the measurement of scattered light have been applied in studies of carbon-soot formation, coal combustion, and the combustion of liquid-fuel sprays. Among these other applications, flame-formed silica particles are most

† Submitted to Applied Optics (1986).

- Sandia National Laboratories, Albuquerque, NM. similar to carbon soot. However, unlike carbon soot, which has a complex refractive index and absorbs light, silica has a real refractive index, and the particles are non-light-absorbing.

A technique that is widely used for measuring carbon soot particles is to measure both the scattering and absorption of light by the soot particles, from which the size and number of particles can be inferred. However, this technique is not practical for measuring silica or other non-absorbing materials.

Dynamic-light-scattering techniques do not rely on the absorption of light and are insensitive to the optical properties of the particles. They are, therefore, an attractive alternative for the measurement of silica and other nonabsorbing particles. This article describes the demonstration of dynamic light scattering for the in situ measurement of flame-generated silica particles.

Dynamic light scattering relies on the fact that monochromatic light scattered from an ensemble of identical Brownian particles exhibits a Lorentzian lineshape with width directly proportional to the particle diffusion coefficient $D$. For parameter values typical of those encountered for silica particles in flames, the broadened linewidth may be only a few kilohertz wide and is therefore difficult to measure at optical frequencies. However, when a portion of the scattered light is focused on a photomultiplier tube, photoelectric mixing of the scattered light with itself results in photocurrent fluctuations, the power spectrum of which is also Lorentzian but is centered at zero frequency with halfwidth $\Delta f$ double that of the optical spectrum,

$$
\Delta f=\frac{16 \pi D}{\lambda_{0}^{2}} \sin ^{2}\left(\frac{\theta}{2}\right) .
$$

Here, $\theta$ is the scattering angle measured from the forward direction, and $\lambda_{0}$ is the wavelength of incident light. This power spectrum is readily measured using a spectrum analyzer.

For fine silica particles in flames, the relation between the particle diffusion coefficient 
$D$ and the particle diameter $d$ is determined by kinetic theory,

$$
D=\frac{3 k T l}{2 \mu \pi \alpha d^{2}}
$$

Here $l$ is the mean free path, $T$ is the gas temperature, $\mu$ is the gas viscosity, and the constant $\alpha$ characterizes the nature of the particle/molecule collisions.

We have studied the growth of silica particles in flames using an apparatus similar to that used in the study of the growth of carbon soot particles in a rich premixed flame. A schematic of the experimental setup is shown in Figure 1.

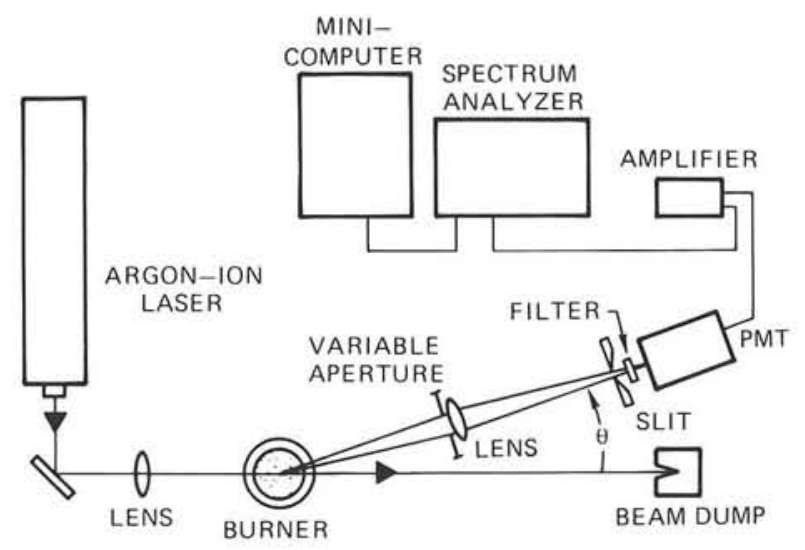

Figure 1. Schematic diagram of the optical system used for measuring soot size using dynamic light scattering.

A porous-plug flat-flame burner was used for the generation of silica particles in fuel-lean premixed methane-oxygen flames. Prior to mixing with oxygen, a portion of the methane flow was bubbled through a reservoir of liquid hexamethyldisiloxane, introducing a small amount of silicon-containing vapor into the fuel stream. Pure silica particles were produced in the flame according to the overall reaction

$$
\left(\mathrm{CH}_{3}\right)_{6} \mathrm{Si}_{2} \mathrm{O}+12 \mathrm{O}_{2} \rightarrow 2 \mathrm{SiO}_{2}+9 \mathrm{H}_{2} \mathrm{O}+6 \mathrm{CO}_{2} \text {. }
$$

This procedure is used commonly for the flamedeposition of non-catalytic silica coatings on thermocouples and serves as a useful model system for examining flame-synthesized silica.
The flame examined here has only faint blue luminosity, in contrast to the intense yellow luminosity emitted by carbon soot in fuelrich premixed flames. The only direct visual evidence of silica production is a white plume of smoke that is not visible until far above the flame. An argon-ion laser beam (514.5 nm) is not attenuated significantly when passing through the flame. However, strong scattering of laser light by the silica particles is distinctly visible to the eye.

An argon-ion laser was used as the probe source in these experiments. The laser power ranged from 0.5 to $3.0 \mathrm{~W}$ for the various experimental conditions. The detection optical system consisted of a 10-cm-focal-length collection lens, an adjustable vertical slit located at the image plane of the probe volume, and a photomultiplier tube. A variable aperture was used to vary the collection solid angle, and a narrow-bandpass interference filter provided spectral discrimination against light at wavelengths other than $514.5 \mathrm{~nm}$. The lens, aperture, slit, filter, and photomultiplier tube were fixed to an optical rail that pivoted about a point directly below the probe volume. The photomultiplier-tube output was amplified, and the photocurrent power spectrum was measured using a fast-Fourier-transform spectrum analyzer.

Most measurements were made at a scattering angle $7.5^{\circ}$ from the forward direction, with a few other measurements made at scattering angles up to $25^{\circ}$. The photocurrent power spectrum was averaged over a period of from 5 to 30 seconds. A sample power spectrum obtained at a position $10 \mathrm{~mm}$ above the burner surface (6-7 $\mathrm{mm}$ beyond the flame front) is displayed in Figure 2. Also shown is the Lorentzian. profile, plus a constant baseline term, that best fits the data obtained using a least-squares fitting procedure.

The average particle diffusion coefficient $D$ was determined from the width of the best-fit Lorentzian profile (Equation 1), and the effective particle diameter $d$ then was determined using Equation 2. The gas temperature was measured using a thermocouple. The mean free 
path and gas viscosity were determined from kinetic theory expressions evaluated at the measured temperature. The factor $\alpha$ was assumed to have a value of 1.44 , which is appropriate for diffuse collisions between gas molecules and particles.

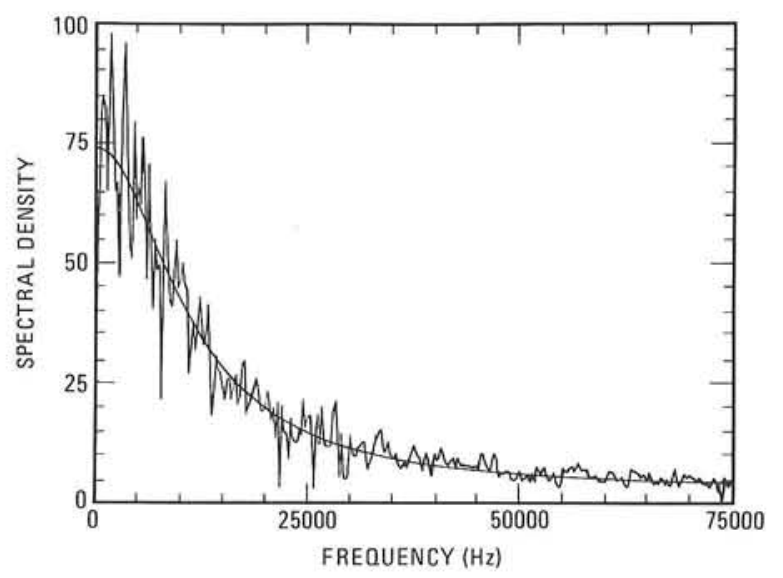

Figure 2. Measured photocurrent power spectrum (irregular solid curve) and the Lorentzian profile plus constant baseline that best fits the measured spectrum (smooth curve). The measured signal is due to scattering from silica particles that are generated in a lean methane-oxygen flame seeded with hexamethyldisiloxane. Measurements shown here were made $10 \mathrm{~mm}$ above the burner surface.

Measured silica particle sizes are plotted as a function of height above the burner surface in Figure 3. The flame front was located 3 to 4 $\mathrm{mm}$ above the burner surface, and 40 -nm particles were first measured at $6.0 \mathrm{~mm}$ above the burner. This is somewhat larger than the earliest carbon soot particles measured in the rich premixed flames. The early growth of the silica particles measured here is also more rapid than was observed for carbon soot. The largest silica particles were about $10 \%$ larger than the carbon particles in the richest premixed flame studied.

We also collected silica samples for later examination using a transmission electron microscope. Even at $6 \mathrm{~mm}$ above the burner, the collected silica appears to be an aggregate of individual, 30-nm-diameter spherical particles. The size measured, using dynamic light scattering, is $40 \mathrm{~nm}$, which is closer to the size of the individual spherical units than to the size of the

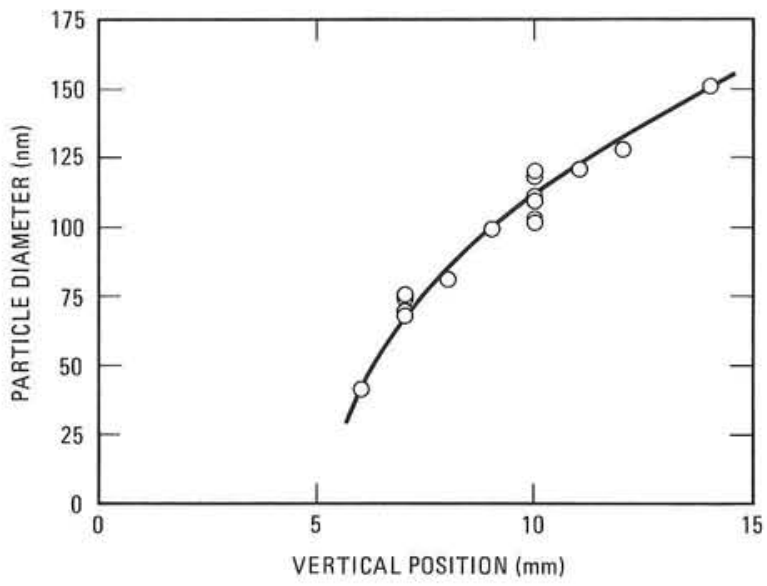

Figure 3. Measured silica particle size as a function of height above the burner surface.

aggregated cluster. This suggests that aggregation took place during sampling. At $14 \mathrm{~mm}$ above the burner, a typical aggregated cluster contains a greater number of fundamental particles. The particle size measured, using dynamic light scattering $(160 \mathrm{~nm})$, is, in this case, closer to the size of the aggregated cluster than to the 30 -nm-diameter fundamental spherical particles.

In summary, we have demonstrated the in situ measurement of flame-synthesized silica particles using dynamic light scattering. This technique is especially advantageous for the measurement of silica and other particles with real refractive indices, since, unlike many of the common static-light-scattering techniques, it does not rely on the absorption of light by the particles. In addition, the dynamic-lightscattering technique has the advantage of being insensitive to particle optical properties. 


\section{Effects of Diffusional Stratification on Soot Formation in a Counterflow Diffusion Flame}

\author{
R. L. Axelbaum, ${ }^{*}$ W. L. Flower, C. K. Law *
}

In diffusion, as well as premixed, flames, the reactant concentration and, thereby, the reaction intensity can be potentially modified through the physical processes of diffusional stratification due to flow nonuniformity and/or flame curvature. This point can be demonstrated by considering the following experimental anomaly. In his study of the influence of various diluents on the soot-formation process, McLintock ${ }^{1}$ varied the inert diluent on the oxidizer side of an ethylene coflow flame and found that the smoke points decreased in the order of helium, nitrogen, and argon. That is, the propensity to soot was least when the oxidizer diluent was helium and greatest when the diluent was argon. Because the heat capacities of argon and helium are the same, McLintock reasoned that the difference was due to the high mass diffusivity of helium, which caused the concentration of helium to be greater within the flame.

A completely opposite result, however, was reported by Schug et al, ${ }^{2}$ who found that the addition of helium to the fuel side of an ethylene coflow diffusion flame was not as effective as argon in suppressing soot. In fact, helium was the least effective additive for soot suppression. This behavior was attributed to the high thermal diffusivity of helium and its subsequent effects on the temperature distribution and thereby the fuel pyrolysis rate. From these two studies, we find that the relative effectiveness of helium as a soot-suppressing additive depends on whether helium is added to the fuel or to the oxidizer.

McLintock ${ }^{1}$ and Schug et al have, respectively, used concentration and temperature

\footnotetext{
* Both with University of California at Davis.
}

modification to interpret their results. We note, however, that both results can be explained, in a consistent manner, as a consequence of either concentration modification or temperature modification. In this study, we have systematically isolated influences from these two factors, and have demonstrated that concentration modification alone can adequately account for the anomalous results observed for helium addition. As such, this factor can not, in general, be overlooked in studies of soot formation in diffusion flames.

The counterflow diffusion flame, established in the forward stagnation region of a porous cylinder, has been used in this study. The burner consists of a porous cylinder mounted horizontally in a vertically oriented wind tunnel (Figure 1). The cylinder is mounted to a vertical translator which allows it to be moved in the streamwise direction. Measurements are taken along the forward stagnation streamline where the flame is essentially one-dimensional. The oxidizer gases are supplied by the wind tunnel while the fuel gases are ejected from the porous cylinder. Temperature measurements are obtained with a silica-coated Pt/Pt-13\%Rh thermocouple. Radiation corrections are not made because only relative temperatures are of interest. Soot volume fraction is obtained from the Lambert-Beer Law for extinction with the assumption that the soot particles behave as spherical Rayleigh scatterers. Figure 1 shows the optical system employed to measure extinction. The soot volume fraction is assumed uniform over the length of the burner, with no correction for end effects.

For the experimental conditions in this study, the flame location is a few millimeters on the oxidizer side of the stagnation point. The soot formation and growth region lies between the flame and the region of the stagnation point. Consequently, the flow field on the oxidizer side of the stagnation point is of primary importance for residence time considerations. Therefore, the upstream velocity of the oxidizer is maintained constant at $38 \mathrm{~cm} / \mathrm{s}$ during all studies. For a constant oxidizer velocity, flow field similarity is achieved by maintaining 


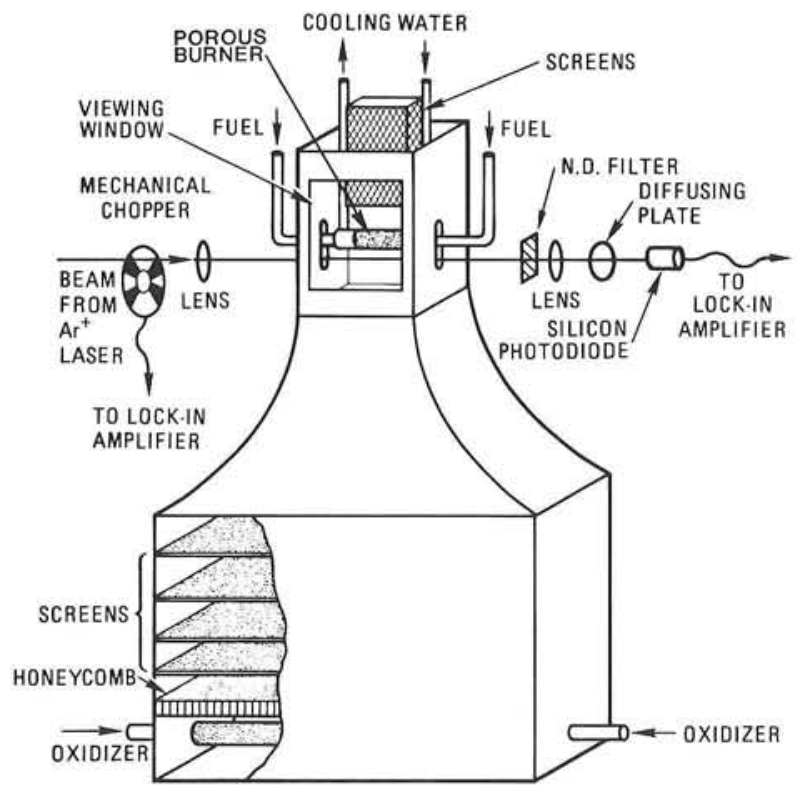

Figure 1. Experimental apparatus and optical system.

a fixed stagnation point. This is accomplished by holding the fuel ejection parameter constant. The fuel ejection parameter defined for variable gas densities is

$$
-\mathrm{f}_{\mathrm{w}}=\left(\rho_{\mathrm{w}} \mathrm{v}_{\mathrm{w}} / \rho_{\infty}\right)\left(\mathrm{a} \nu_{\infty}\right)^{-1 / 2}
$$

where $\rho_{w}$ and $\mathrm{v}_{w}$ are, respectively, the gas density and velocity at the burner surface, and $\rho_{\infty}$ and $\nu_{\infty}$ are, respectively, the free stream values of the density and viscosity for the oxidizer. The characteristic velocity gradient $a$ is defined as $a=2 \mathrm{U}_{\infty} / \mathrm{R}$, where $\mathrm{U}_{\infty}$ is the free stream velocity of the oxidizer and $R$ is the burner radius. The fuel ejection parameter defined in this way allows flow similarity to be maintained even when variable density inerts are added.

As previously mentioned, both concentration modification and temperature broadening will yield similar trends for inert addition to the coflow flame. Therefore, it is difficult to determine which, if either, mechanism is the dominant physical mechanism responsible for the observed trends. This is not the case for the counterflow diffusion flame. In the counterflow diffusion flame, the streamlines on both the fuel and oxidizer sides of the stagnation point are such that the heavier species will be preferentially swept away from the stagnation region when compared to the lighter species. Consequently, relative to argon addition, concentration modification should lead to a reduction in fuel concentration and thereby soot loading when helium is added to the fuel.

Soot burn-up does not occur in the forward stagnation region of the counterflow diffusion flame. As a result, if helium were to broaden the temperature distribution, the effect as reasoned in Reference 2 would be to increase the soot loading. Therefore, in the counterflow diffusion flame, the effect of concentration modification is opposite to that of temperature distribution broadening and we can compare the relative importance of the two mechanisms for this flame.

In addition to helium and argon, we have also used neon and krypton for diluents in this study. All diluents are therefore completely inert and, being monatomic, have the same influence on the adiabatic flame temperature.

Figure 2 shows the uncorrected temperature distributions for propane/inert mixtures. The maximum flame temperatures measured show slight increases with increases in the molecular weight (decreases in the mobility) of the inert; krypton yields the highest temperature $\left(1638^{\circ} \mathrm{C}\right)$ while helium the lowest $\left(1606^{\circ} \mathrm{C}\right)$. The temperature profiles are surprisingly similar with no significant broadening by the lighter inerts.

In Figure 3, the soot volume fraction distributions for the propane/inert mixtures are shown. The curves show a strong relationship between the mobility of the inert and the maximum soot loading for the flame. The ordering is anticipated from the concentration modification argument; krypton, the least mobile inert, yields the greatest soot loading $\left(5.4 \times 10^{-6}\right)$ while helium, the most mobile, yields the lowest soot loading $\left(2.4 \times 10^{-6}\right)$. 


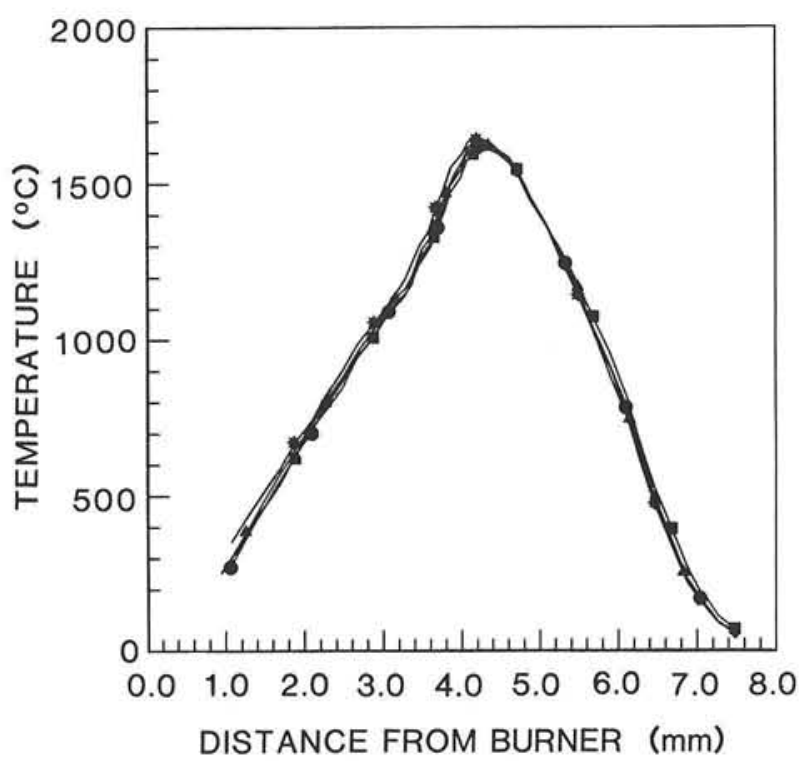

Figure 2. Temperature distributions for a propane/air counterflow diffusion flame with inert addition on the fuel side; symbols are located at every fifth data point, $\mathrm{f}_{w}=-3.0: \square=80 \%$ $\mathrm{C}_{3} \mathrm{H}_{8} / 20 \% \mathrm{He} ; \mathrm{O}=\mathbf{8 0 \%} \mathrm{C}_{3} \mathrm{H}_{8} / 20 \%$ Ne; $\Delta=$ $80 \% \mathrm{C}_{3} \mathrm{H}_{8} / 20 \% \mathrm{Ar} ; *=80 \% \mathrm{C}_{3} \mathrm{H}_{8} / 20 \% \mathrm{Kr}$.

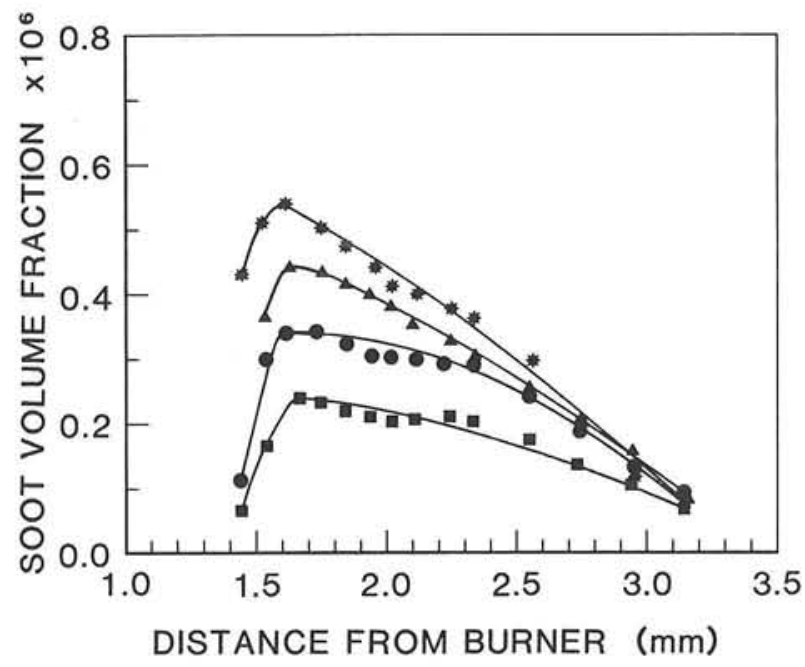

Figure 3. Soot volume fraction as a function of distance from burner surface for a propane/enriched air $\left(25 \% \mathrm{O}_{2}\right)$ counterflow diffusion flame with inert addition on the fuel side; $\mathbf{f}_{w}=-\mathbf{3 . 0}$ : $\square=80 \% \mathrm{C}_{3} \mathrm{H}_{8} / 20 \% \mathrm{He} ;=\mathbf{8 0 \%} \mathrm{C}_{3} \mathrm{H}_{8} / 20 \%$ Ne; $\triangle=80 \% \mathrm{C}_{3} \mathrm{H}_{8} / 20 \% \mathrm{Ar} ; *=80 \% \mathrm{C}_{3} \mathrm{H}_{8} / 20 \%$ Kr.

The $32^{\circ} \mathrm{C}$ difference in the maximum measured flame temperatures seen in Figure 2 does not account for the more than two-fold difference in soot loading. Temperature effects were investigated further in related work, ${ }^{3}$ and the results show that even with a $43^{\circ} \mathrm{C}$ increase in maximum measured temperature, the soot loading increases only $13 \%$.

The present results, then, demonstrate quite strongly that concentration modification due to diffusional stratification can play a significant role in the soot formation process, particularly for additives on the fuel side of the counterflow diffusion flame.

\section{References}

1. I. S. McLintock, "The Effect of Various Diluents on Soot Production in Laminar Ethylene Diffusion Flames," Comb. Flame 12, 217 (1968).

2. K. P. Schug, Y. Manheimer-Timnat, P. Yaccarino, and I. Glassman, "Sooting Behavior of Gaseous Hydrocarbon Flames and the Influence of Additives," Comb. Sci. Tech. 22, 235 (1980).

3. R. L. Axelbaum, C. K. Law, and W. L. Flower, "Effects of Diffusional Stratification and Oxygen Addition on Soot Formation in a Counterflow Diffusion Flame," presented at the Spring Technical Meeting of the Western States and Canadian Sections, The Combustion Institute (1986), Paper No. 86-67.

\section{Turbulent Nonpremixed Flames of Methane Near Extinction: Probability Density Functions ${ }^{\dagger}$}

\section{A. R. Masri, ${ }^{*}$ R. W. Bilger, ${ }^{*}$ R. W. Dibble}

In a recent paper, ${ }^{1}$ we reported the mean and rms fluctuations of temperature, density, mixture fraction $f$, and the mass fractions of $\mathrm{CH}_{4}, \mathrm{O}_{2}, \mathrm{H}_{2} \mathrm{O}, \mathrm{H}_{2}, \mathrm{CO}, \mathrm{CO}_{2}$, and $\mathrm{N}_{2}$ obtained by pulsed spontaneous Raman and Rayleigh scattering in the blue (visibly soot free) regions

$\dagger$ Submitted to Combustion and Flame (1986).

* Both with University of Sydney, N.S.W., Australia. 
of a pilot-stabilized turbulent nonpremixed flame of methane into a coflowing stream of air. In this paper, we report information on the probability density functions ( $p d f$ 's) of the scalars measured.

The burner which produces the flame consists of a central jet of fuel surrounded by a large annulus of coflowing premixed combustion products, and this is centered in a large coflowing stream of air. This arrangement has been designed to allow the study the strong turbulence effects on the chemistry under conditions approaching extinction in a flow which is fluiddynamically simple; a streaming flow describable by parabolic equations. Flame extinction is approached by increasing the fuel-jet velocity, and when extinction occurs, it leaves the pilot annulus alight with apparently some mixing and combustion of the fuel and outside air occurring over the first 15 diameters but not at a sufficient rate of heat release to propagate the flame further. At jet velocities below the extinction condition, the flame propagates into a full turbulent jet diffusion flame with a region near $x / D_{j}$ of 20 where mixing is intense $\left(D_{j}\right.$ is the fuel jet diameter), temperatures are lowest, and combustion conditions are most critical.

It is not possible to determine from the probe measurements alone the relative effects of unmixedness of the mixture fraction (high values of the rms of mixture fraction fluctuations $\left.\overline{f_{r m s}^{\prime}}\right)$ and chemical kinetics on the changes in the mean structure of the flame as it approaches extinction. Space and time resolved Raman and Rayleigh measurements can easily address these questions. The means and rms fluctuations of the Raman and Rayleigh data presented in Reference 1 established that at a fixed axial location in the flame, the radial profile of $\overline{f_{r m s}^{\prime}}$ and, hence, unmixedness remain almost unchanged and the change in the flame structure is due almost entirely to chemical kinetic effects. The probability density functions ( $p d f$ 's) presented in this paper will help clarify other issues concerning the turbulence-chemistry interactions and the local flame structure near extinction.
Figure 1 shows the joint pdf's ( $j p d f$ 's) for temperature T versus $f$. On each plot, a total of 19 colors are shown representing 19 levels for the probability density. The split between levels is near logarithmic, and the value of the probability density at each color level is shown next to the color bar. The area under each $j p d f$ equals one. All $j p d f$ 's are found to lie in the domain expected from fast and frozen chemistry for the $p d f$ 's of $f$ except for few overshoots in temperature and species mass fractions detected in the regions where the Raman signals are weak. For mixture fractions less than $\simeq 0.1$, the $j p d f$ taken at $r / R=3.32$ in flames $L$ and $M$, least intensely mixed and most intensely mixed, ${ }^{1}$ respectively, show a distinctly bimodal behavior with temperature following either a fully burnt line or a line representing only mixing with hot combustion products. This bimodality disappears in these figures for richer mixtures, the reactedness at any $f$ becoming centrally distributed.

The $j p d f$ 's of all reactive scalars, including the ones not presented in this paper are consistent with one another and show that in regions of low mixing rates most of the data collected lie close to the fast chemistry limit indicating complete combustion. When the mixing rates become intense, as $x / D_{j}=20$ in flame $M$, the majority of the samples are either partially reacted or mixed with hot combustion products and lie on an asymptote slightly shifted from the cold mixing one. A number of hot fully burnt pockets are still encountered, however, and the probability densities of such pockets are about an order of magnitude lower than those of flame $L$ at the same location. The existence of such hot streaks in regions of the flames, which are very close to extinction, indicates that they may be responsible for keeping the flame alight and that full flame blow-off will therefore take place when these hot streaks cease to occur, or when their probability density decreases below a certain limit.

In turbulent reacting flows, the understanding of the local, instantaneous structure 

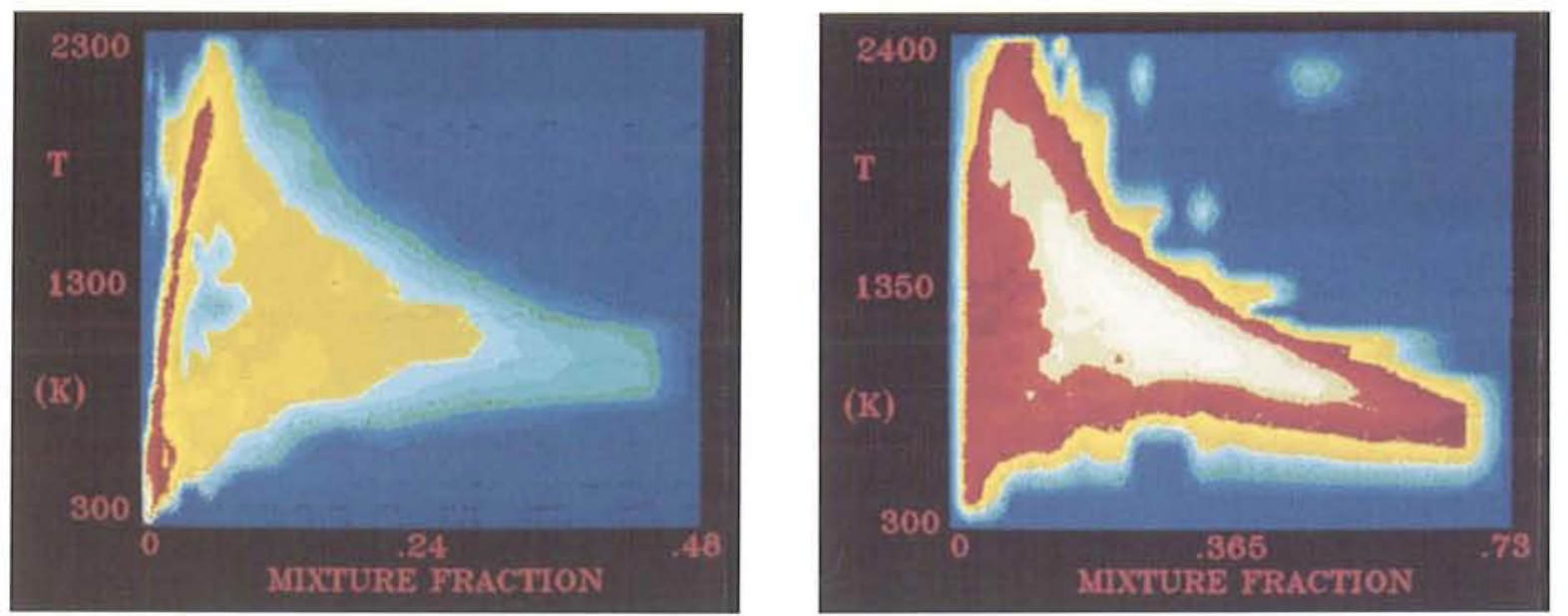

$\mathbf{L}$
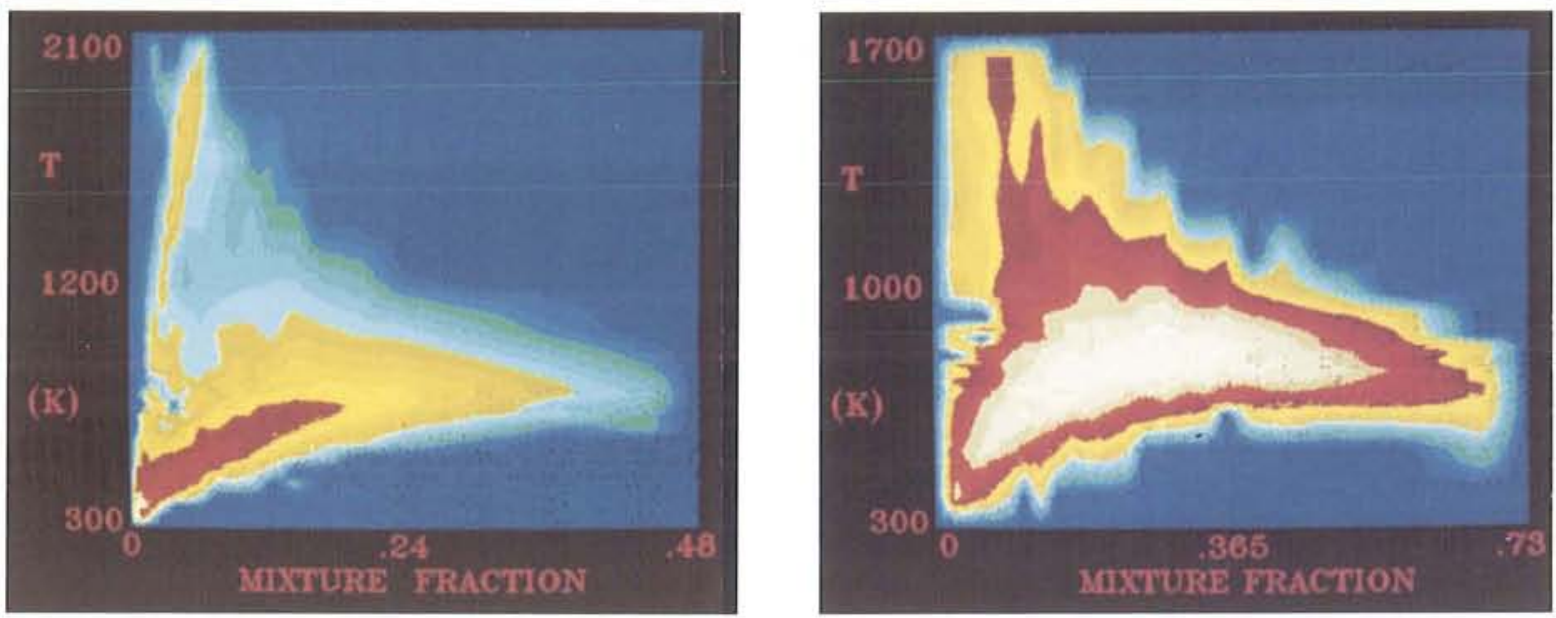

M

Figure 1. Joint $p d f$ 's of temperature T versus mixture fraction $f$ at $x / D_{j}=20$ in flames $L$ (top) and $M$ (bottom); the fuel jet velocity is highest in flame $M$. Plots on the left hand side are for $r / R=3.32$, fuellean, and on the right hand side are for $r / R=2.47$, fuel-rich.

of the flame as it approaches extinction is essential to the development of theoretical models that account for finite-rate chemistry effects and predict overall flame extinction. In the laminar flamelet model, the turbulent flame is conceived as an ensemble of moving laminar diffusion flamelets which can be extinguished as the local mixing rates become intense. Overall flame extinction occurs when the percentage of the extinguished flamelets exceeds a certain limit. At any instant of time the local mixtures can be either burnt or unburnt. This is consistent with the bimodal behavior seen in Figure 1 for $f$ less than $\sim 0.1$. The bimodality alone does not provide conclusive evidence that laminar flamelets prevail because, within the flammability limits $\left(0.028 \leq f \leq 0.087\right.$ for $\mathrm{CH}_{4}$-air mixtures $)$, well stirred reactor data would give a similar behavior. The data shows, however, that the bimodality extends beyond the flammability limits. This supports the laminar flamelet model, but it may also question the relevance of the flammability limit concept in this flow configuration. If the laminar flamelets exist, they are likely to be in the superlayer region which extends to mixture fractions greater than 0.2 . The existence of folded flamelets also cannot be excluded. 
For richer mixtures $(f>0.1)$, the data show a centered distribution with a high probability of intermediate states between burnt and unburnt flamelets. Such mixtures can represent any of three possibilities: (i) Non-stationary transitions between the fully burnt and extinguished flamelets (i.e., mixtures lying on the unsteady transition, middle branch of the $S$ shaped flame stability curve). (ii) Locally, partially premixed flamelets. Flamelets such as these were proposed by Peters ${ }^{2}$ as an extension to the laminar flamelet model and later adopted by Rogg et al, ${ }^{3}$ with detailed chemistry and utilization of various degrees of partial premixing to predict methane-air diffusion flames. (iii) Partially reacted mixtures of fuel, oxidant, and product, which lie beyond the rich flammability limit, have low rates of reaction and yield, therefore, a broad, distributed reaction zone. The existence of such distributed reaction zones in turbulent reacting flows requires that their thickness be larger than the smallest representative turbulence length scale. These questions are to be addressed more quantitatively in a forthcoming paper by considering conditionally sampled $p d f$ 's.

\section{References}

1. A. R. Masri, R. W. Dibble, and R. W. Bilger, "Turbulent Nonpremixed Flames of Methane Near Extinction: Mean Structure from Raman Measurements," submitted to Comb. Flame (1986).

2. N. Peters, "Partially Premixed Diffusion Flamelets in Non-Premixed Turbulent Combustion," Twentieth Symposium (International) on Combustion (The Combustion Institute, 1983), p. 353.

3. B. Rogg, F. Behrendt, and J. Warnatz, "Turbulent Nonpremixed Combustion in Partially Premixed Diffusion Flamelets with Detailed Chemistry," Twenty-first Symposium (International) on Combustion (The Combustion Institute, 1986), in press.

\section{Combustion of Toxic Liquid Droplets}

\author{
R. R. Steeper, N. W. Sorbo, ${ }^{*}$ C. K. Law
}

Large industrial stockpiles of toxic materials in the form of liquid wastes present an urgent demand for advanced research in disposal technology. The promise of incineration methods encourages fundamental studies of the mechanisms of toxic material combustion. The Combustion Control of Toxic Materials program at Sandia has been established to probe the physico-chemical processes which underlie the formation and destruction of toxic materials in combustion environments.

Combustion of these toxic materials involves multiple reaction steps occurring in widely varying environments found in the atomization, preheat, flame, and post-flame zones of an incinerator. Complications can occur when the principal organic hazardous constituents (POHC's) pass through the incinerator as incompletelyreacted rogue droplets or are partially reacted to form secondary hazardous species called products of incomplete combustion (PIC's). Our work focuses on the decomposition of POHC's in the high temperature, high radical concentration environment of the flame zone, followed by destruction of remaining POHC's and flameformed PIC's in the high-temperature, low radical post-flame environment.

In 1986, the Combustion Control of Toxic Materials program was broadened with the establishment of a collaborative agreement with Professors C. K. Law (Droplet Combustion) and D. P. Chang (Toxic Materials) of the University of California at Davis. Joint experiments have been outlined for the particle levitator and flatflame reactor at Sandia and the single droplet reactor at U. C. Davis.

To begin the experiments, we chose chlorinated hydrocarbons as test compounds because of the important role of chlorine as a reactioninhibiting radical scavenger as well as because of

* Both with University of California at Davis. 
the prevalence of organo-chlorides in industrial wastes. Because large scale incineration normally involves mixtures of POHC's with carrier fuels, we are including mixtures of chlorinated and unchlorinated hydrocarbons in our tests. Single droplets of the chosen mixture are generated with a piezoelectric device and injected into the post-flame zone of a downflow reactor. We use photo-micrography to measure the particle size history and sampling for the liquid phase composition history. To date, we have run about 50 mixtures that explore the effect of mixture fraction, volatility differential, chlorine loading, and carbon loading on the incinerability of the toxic compounds.

An encouraging development has emerged from the volatility differential studies. Our most recent results show that combustion times decrease significantly for proper mixtures of the chlorinated hydrocarbons with less volatile carriers. We compared the burning rate of tetrachloroethane (boiling point $146^{\circ} \mathrm{C}$ ) mixed with equally volatile nonane (bp $151^{\circ} \mathrm{C}$ ) with the rate for a mixture of tetrachloroethane plus less volatile hexadecane (bp $\left.287^{\circ} \mathrm{C}\right)$. In spite of the lower burning rate of pure hexadecane compared with pure nonane, the hexadecane in mixture with the chlorinated species promoted a distinctly accelerated combustion rate (see Figure 1).

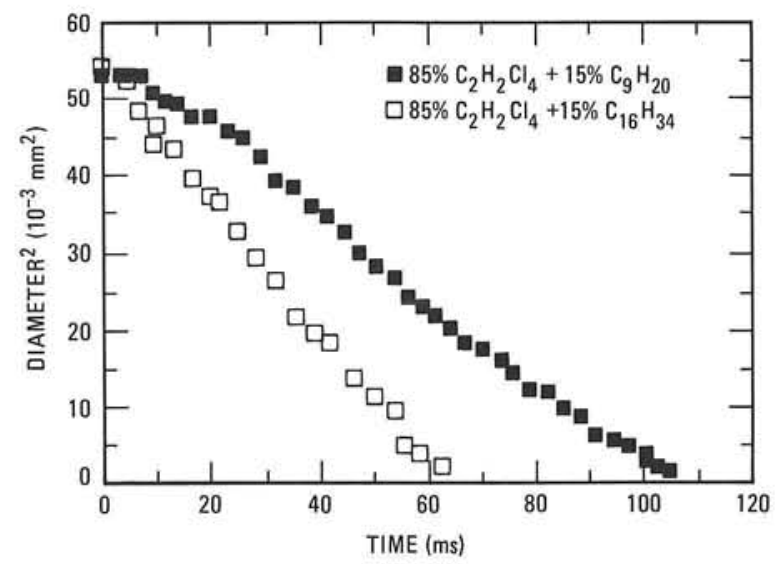

Figure 1. Burning droplet size histories for mixtures of two different alkanes with tetrachloroethane. 

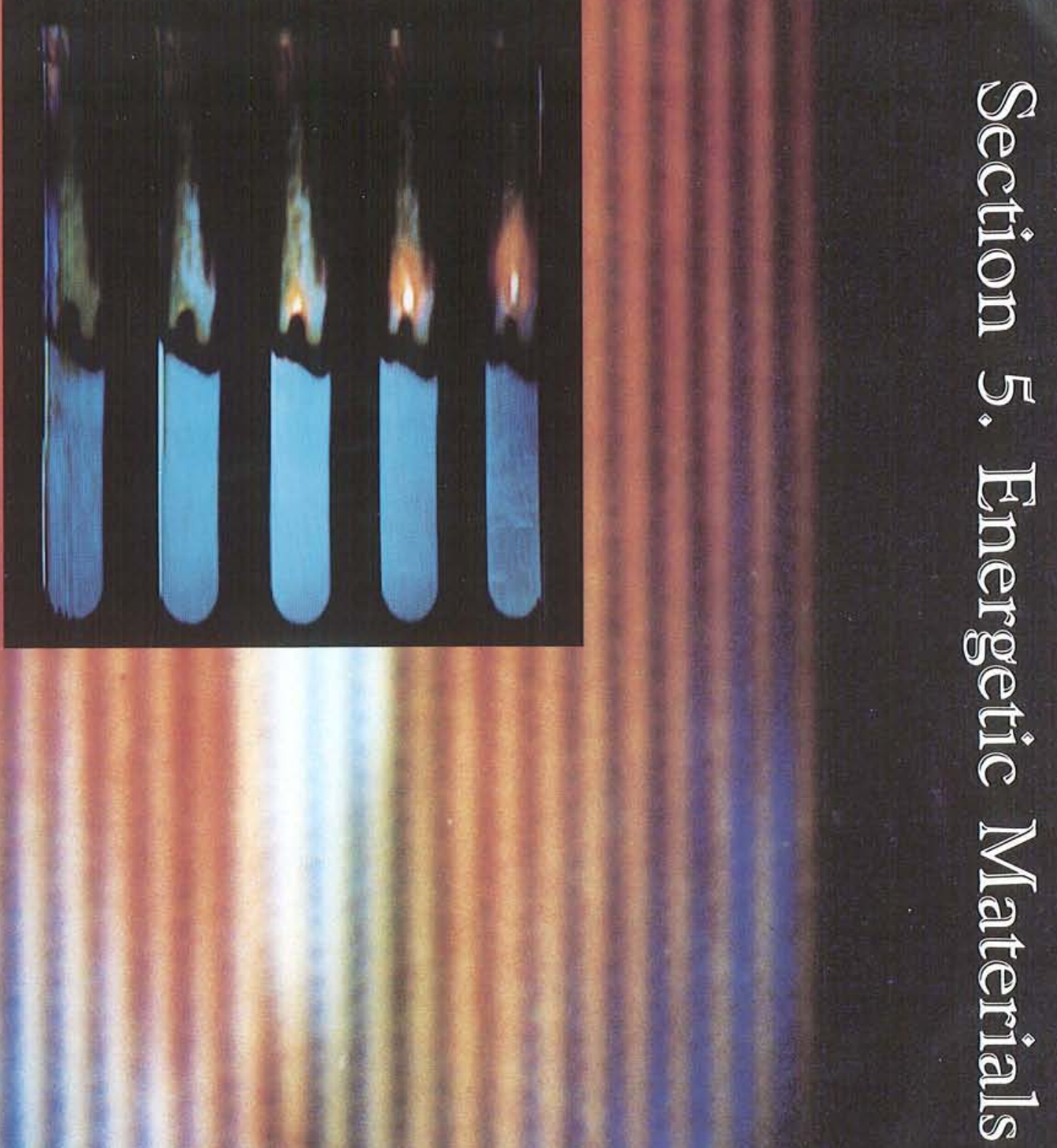
The fundamentals of combustion of solid and liquid propellants are being examined in an effort to improve the safety and performance of energetic materials. The preceding page shows the ignition of a liquid propellant and subsequent development and evolution of a luminous flame at high pressures (34 $\mathrm{MPa}$ or $5000 \mathrm{psi}$ ). The inset photograph was taken from a video tape with the time between each of the five flames being $1 / 300$ th of a second. The enlarged photograph is a portion of a flame as it begins to develop following ignition. These experiments are the first to visually record liquid propellant as it burns and, coupled with other efforts, will lead to an understanding of burning rates, flame stability, ignition, and other issues critical to the design and use of these propellants (see related article, page 5-5). 


\section{Section 5 Energetic Materials}

A research program involving the combustion of energetic materials emphasizes studies of a fundamental nature that are directed towards understanding the combustion chemistry and physics of energetic solids and liquids. The objective of the research is to apply advanced laser diagnostics and computer modeling to propellant combustion with the ultimate goal of improving propellant performance and establishing an understanding that could lead to the development of new propellants for guns and rockets. Program sponsorship is derived primarily through a Memorandum of Understanding between the Department of Energy and the Department of the Army.

The experimental effort consists of solid-propellant thermal decomposition and deflagration studies as well as liquid mono-propellant ignition and combustion studies. Related gas-phase flame experiments at reduced pressure are designed to investigate the two-stage flame character exhibited by many solid propellants, including chain-branching chemistry and thermal diffusion effects. Thermal decomposition studies of solid propellants using a modulated-molecular-beam mass spectrometer are carried out to determine quantitatively the molecular fragments and reaction products that result from the decomposition. Combustion experiments emphasizing deflagration of solid and liquid propellants under conditions of varying pressure and propellant composition are being performed using both spatially resolved and planar-imaging laser diagnostics.

A strong modeling program accompanies the experimental effort. Quantum chemistry calculations provide information about thermochemical heats of formation and possible reaction pathways for the decomposition of energetic molecules. Solid and liquid propellant deflagrations are being modeled analytically and through the use of multi-dimensional thermochemical codes. These studies include liquid mono-propellant droplet combustion calculations using a new code capability derived from hydrocarbon combustion work.

\section{Plasma-Torch Ignition of Liquid Propellant ${ }^{\dagger}$}

\section{R. J. Cattolica, N. Klein*}

Liquid monopropellants are fluids containing both fuel and oxidizer and, as such, are capable of self-contained combustion, thereby releasing substantial quantities of heat and gaseous

† Sandia Report SAND86-8647; submitted to Combustion Science and Technology (1986).

* U. S. Army Ballistic Research Laboratory, Aberdeen, MD. products. The design of systems in which liquid propellants are to be used requires detailed knowledge of their combustion characteristics. The ignition and combustion of a liquid propellant can be studied by using a plasma torch/plug device to ignite a small quantity of the material.

Experiments were conducted to study the ignition characteristics of an aqueous mixture of hydroxylammonium nitrate (HAN) and triethanol-ammonium nitrate (TEAN) in a 7:1 molar ratio using the plasma-torch igniter shown in Figure 1. 


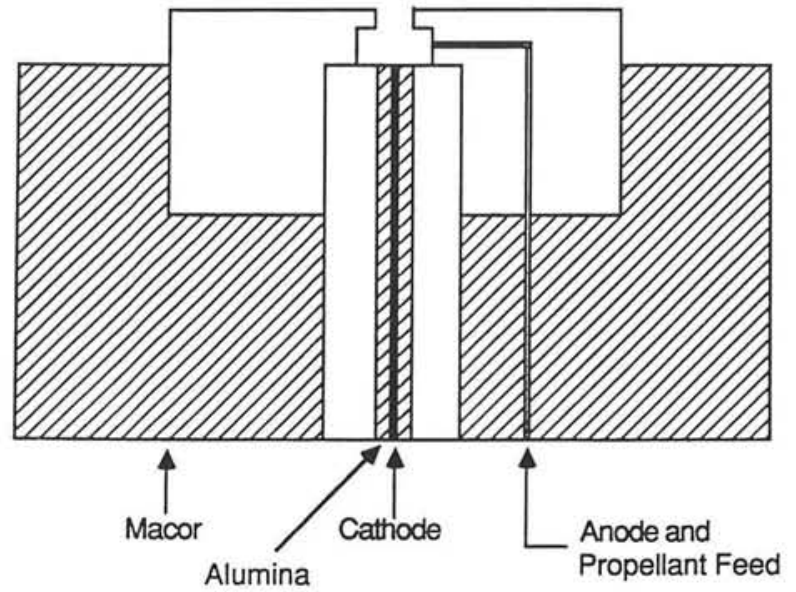

Figure 1. Sketch of plasma-torch igniter for liquidpropellant experiment.

The outer body of the plasma-torch chamber, which also functions as the anode in the high voltage circuit, is made of 316 stainless steel. The cathode at the bottom of the cylindrical chamber ( $30 \mu$ liter volume) is also made of 316 stainless steel and is insulated from the outer body. Liquid propellant is introduced into the plasma torch through a feed tube connected to an adjustable positive displacement pump. The outer body of the plasma torch is insulated from a 2 liter stainless steel combustion chamber.

The liquid propellant was ignited with the plasma torch, operating at 600 volts and $40 \mu \mathrm{F}$ capacitance ( 7.2 joules stored energy). The current trace in Figure 2 shows $200 \mu$ secs of low current discharge before the onset of the main high-current arc. This low-current regime corresponds to ohmic heating and subsequent decomposition of the liquid propellant with the formation of a gas sheath at the anode surface. The gas formation continues until the local resistance becomes high enough for an arc discharge to occur. With a 5-atm back pressure of argon in a containment chamber above the plasma torch, the gas sheath was formed repeatedly, so that an arc always occurred $200 \mu$ sec after the application of the high voltage. With this stable operating configuration, optical diagnostic techniques were used to probe the liquid-propellant exhaust plume from the plasma torch.

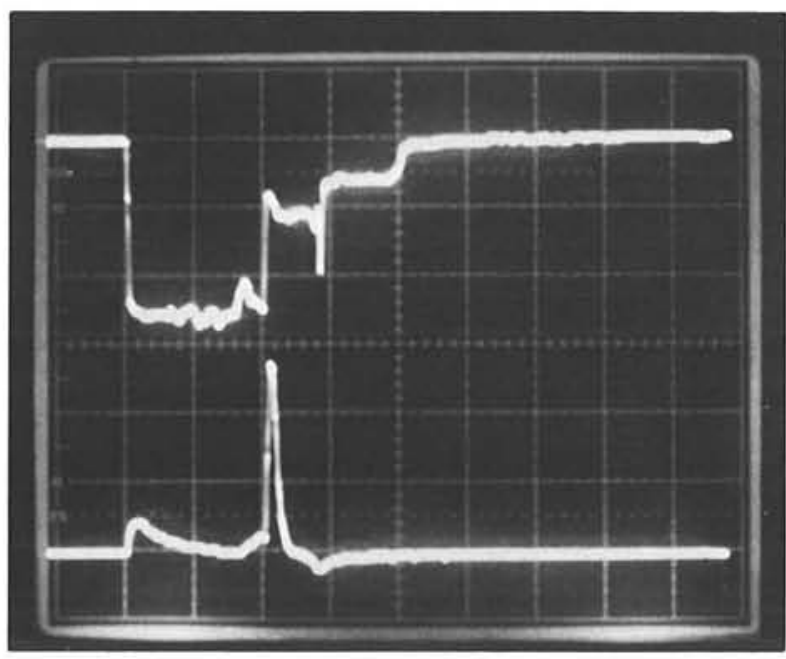

Figure 2. Oscilloscope trace of current-voltage characteristic of plasma-torch ignition of HAN (14.4 molar) with 75 psia back pressure of argon. Upper trace: vertical scale, 200 volts/division; horizontal scale, $\mathbf{1 0 0} \mu \mathrm{sec} /$ division. Lower trace: vertical scale, 50 amps/division; horizontal scale, $100 \mu \mathrm{sec} /$ division.

Laser shadowgraphy was used to observe the performance of the plasma torch and to visually define the operating conditions under which the spectroscopic measurements could be made. An argon-ion laser (2 W), operating at a wavelength of $514.9 \mathrm{~nm}$, was used to back-light the plasma plug exhaust plume. The laser light was formed into a series of $0.5 \mu$ sec pulses by an acousto-optic modulator synchronized with the framing rate of a high speed video camera (Kodak-Spin Physics Model SP2000). Sequential frames from a high speed video recording are shown in Figure 3.

After arc-discharge ignition (indicated at $0.0 \mathrm{~ms}$ ) an exhaust jet is seen exiting the $1-\mathrm{mm}$ diameter orifice of the plasma plug. The development of the exhaust plume was repeatable with the voltage-current characteristics shown in Figure 2.

LIF imaging of the $\mathrm{OH}$ molecule in the exhaust plume from the plasma-torch was attempted. Very large signal levels were observed in the measurements. These high signal levels could be obtained with the laser wavelength tuned off the $\mathrm{OH}$ absorption line used for excitation. The large signal observed in the exhaust plume was the result of Mie scattering. 


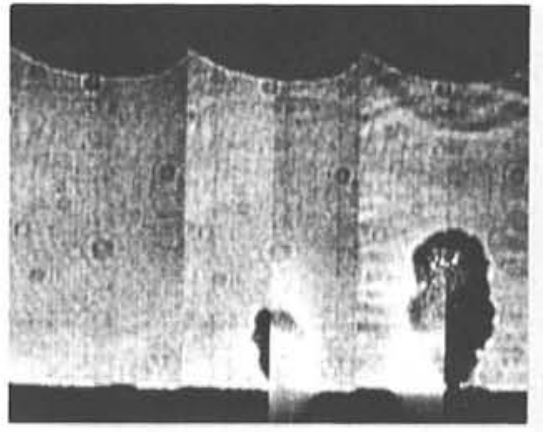

$0 \mathrm{~ms}$

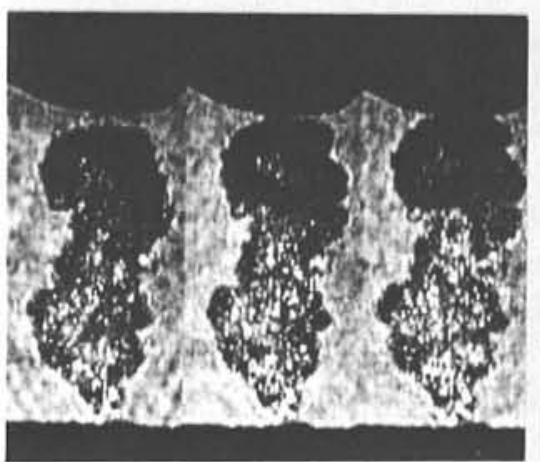

$1.5 \mathrm{~ms}$

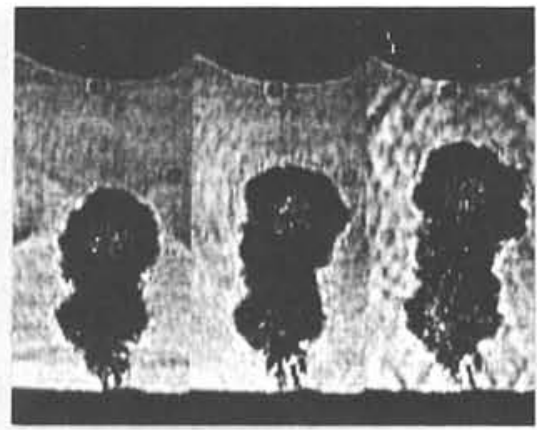

$0.5 \mathrm{~ms}$

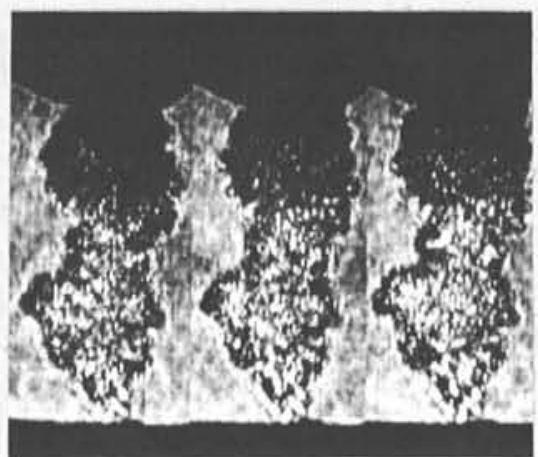

$2.0 \mathrm{~ms}$

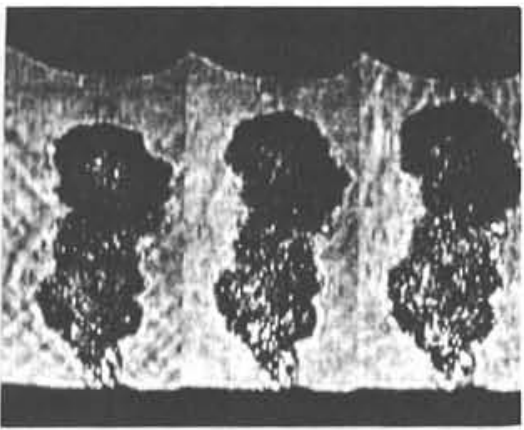

$1.0 \mathrm{~ms}$

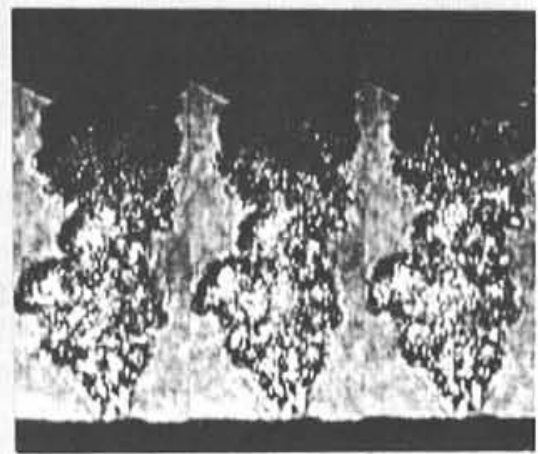

$2.5 \mathrm{~ms}$

Figure 3. Laser shadowgraph of the exhaust plume from the plasma-torch ignition of liquid propellant. The framing rate was $6 \mathrm{kHz}$ and exposure time $500 \mathrm{~ns}(0 \mathrm{msec}$ corresponds to initiation time of the highvoltage arc discharge).

Although liquid droplets have been observed in the plasma torch exhaust for the specific operating conditions that were selected, no evidence of droplets was observed. The main conclusion from these LIF imaging experiments is that a very large number of small (perhaps as small as 50 micron) droplets are found in the exhaust plume.

A time-resolved emission study was conducted to determine if the intense emission observed in the shadowgraph observations was the result of the plasma discharge or the subsequent chemiluminescence from combustion. Using a 0.25 meter monochromator, a photomultiplier, and a transient digitizer, a time-resolved emission spectrum from the plasma-torch ignition of liquid propellant was observed with a $200 \mathrm{nsec}$ temporal resolution, a $4 \mathrm{~nm}$ spectral resolution. The monochromator slit $(0.5 \mathrm{~mm} \times 12 \mathrm{~mm})$ was aligned along the axis of the plasma torch. In Figure 4, the simultaneous recording of the current in the plasma discharge and the emission from electronically excited $\mathrm{OH}$ molecules is shown.

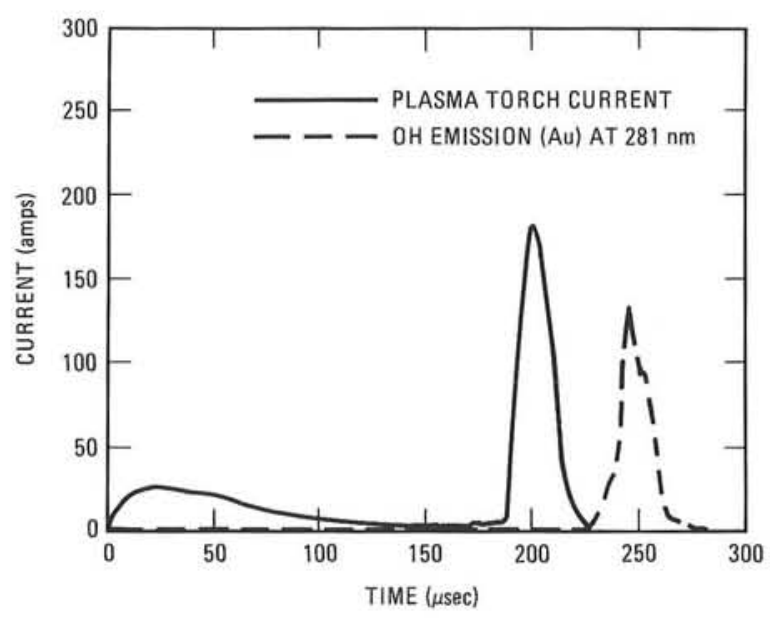

Figure 4. Simultaneous plasma-torch current history and emission from the $(1,0)$ band of $\mathrm{OH}$. 
The plasma-torch discharge current peaked at 185 amperes at $200 \mu \mathrm{sec}$ after application of high voltage to the liquid propellant. After the completion of the electrical discharge, chemiluminescence from the $\mathrm{OH}$ molecule in the $(1,0)$ band appears $15 \mu \mathrm{sec}$ later. Although this simple experiment indicated the presence of chemiluminescence, it could not give spatial information and was an inefficient way to survey the entire spectral range.

To provide more complete information on the spatial and spectral distribution of the emission from the plasma-torch liquid-propellant exhaust plume, an experiment was conducted with a gated ISIT camera at the focal plane of a $0.5 \mathrm{~m}$ monochromator. Emission from the experiment was recorded over a $35 \mathrm{~nm}$ range of wavelength with $0.7 \mathrm{~nm}$ spectral resolution. With the entrance slit of the monochromator aligned along the centerline of the plasma torch, a $30 \mathrm{~mm} \times 0.4 \mathrm{~mm}$ section of the exhaust plume was observed. The ISIT camera provided a spatial resolution of $0.3 \mathrm{~mm}$ along this section. By repeating the experiment with the center wavelength of the monochromator shifted by $25 \mathrm{~nm}$ each time, the spatially-resolved emission spectrum from the exhaust plume was measured over a spectral range from 225 to $600 \mathrm{~nm}$. Selected plots of these emission spectra appear in Figure 5.
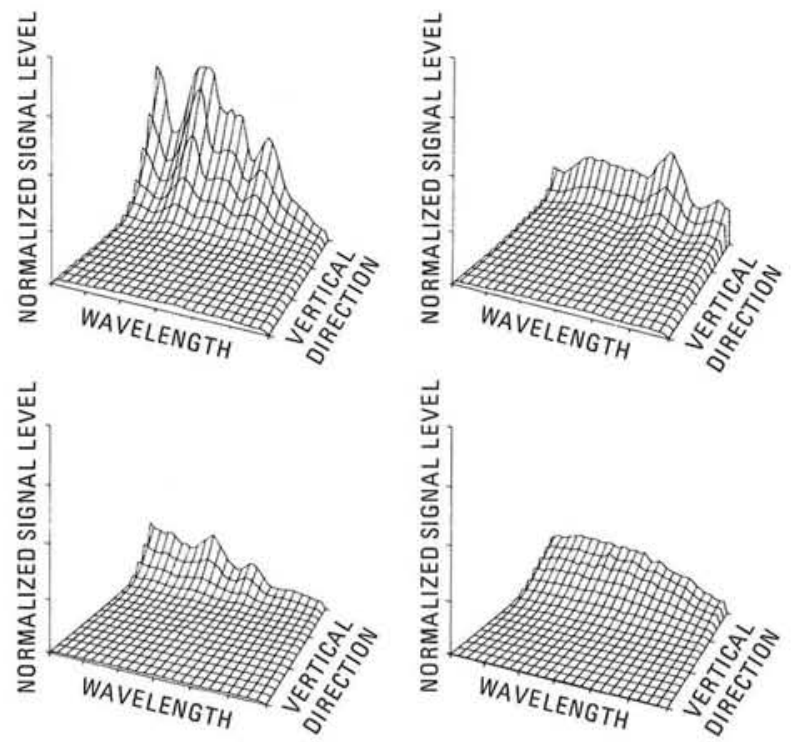

Figure 5. Spatially resolved emission of plasmatorch exhaust at different center wavelengths: a) $275 \mathrm{~nm}, \mathrm{~b}) 300 \mathrm{~nm}$, c) $325 \mathrm{~nm}$, and d) $450 \mathrm{~nm}$.
The measurements, starting at a wavelength of $225 \mathrm{~nm}$, show no significant emission until the high lying vibration bands of the A$\mathrm{X}$ electronic transition of the $\mathrm{OH}$ molecule begin to appear above $260 \mathrm{~nm}$. From $260 \mathrm{~nm}$ to $295 \mathrm{~nm}$ a number of strong $\mathrm{OH}$ emission features contribute to the spectrum: $267 \mathrm{~nm}(3,1)$, $275 \mathrm{~nm}(4,2), 281 \mathrm{~nm}(1,0), 287 \mathrm{~nm}(2,1)$, and $294 \mathrm{~nm}(3,2)$. The $(0,0)$ vibrational band at $306 \mathrm{~nm}$ is relatively weak in comparison to the higher lying vibrational levels populated during the ignition of the liquid propellant. Emission from the $(1,0)$ and $(0,0)$ in the A-X electronic transition from $\mathrm{NH}$ is observed at $335 \mathrm{~nm}$. Although some structure indicating the presence of chemiluminescence from $\mathrm{CH}$ can be identified, a low signal level at $431 \mathrm{~nm}$ is a very weak signal compared to the $\mathrm{OH}$ and $\mathrm{NH}$ emissions. Beginning at $450 \mathrm{~nm}$ and extending to longer wavelengths a strong continuum appears. Although $\mathrm{NO}_{2}$ has a strong broad-band emission signature throughout this region, it is not possible to identify the observed signal as being from $\mathrm{NO}_{2}$. Blackbody radiation at high combustion temperatures could also produce a broad spectral signature.

The plasma torch/plug is a viable device for safely studying the ignition and combustion of highly energetic liquid propellants. The small sample size employed limits the amount of energy available and even catastrophic reaction is incapable of serious damage to personnel or equipment. It thus becomes feasible to conduct experiments in close proximity to delicate diagnostic instrumentation. Recent work at substantially higher chamber pressure indicates that both combustion quenching and droplet concentrations are thus reduced, thereby producing the conditions necessary for successful exploitation of advanced diagnostic methods. 


\section{Liquid Propellant Combustion Research}

\section{S. R. Vosen}

The objectives of the liquid propellant combustion program are to obtain a fundamental understanding of the physical processes which are important in liquid propellant combustion and to identify chemical species which are present in the flame. The initial goals of this research were to obtain photographs of the liquid propellant burning in a strand burner at a constant, elevated pressure to determine how the liquid-gas interface moves and where gas phase reactions occur.

The propellants of interest are composed of a mixture of $\mathrm{N}_{2} \mathrm{H}_{4} \mathrm{O}_{4}$ (hydroxylammonium nitrate, or HAN) and an aliphatic amine nitrate. Each of these chemicals by itself is capable of combustion, but when they are combined in the proper stoichiometric proportion, the reaction proceeds to nitrogen, water, and carbon dioxide.

The aliphatic amine nitrate chosen for study is $\left(\mathrm{C}_{2} \mathrm{H}_{5} \mathrm{O}\right)_{3} \mathrm{NHNO}_{3}$ (triethanolammonium nitrate, or TEAN), which in stoichiometric proportions with HAN gives:

$$
2 \mathrm{C}_{6} \mathrm{H}_{16} \mathrm{~N}_{2} \mathrm{O}_{6}+7 \mathrm{~N}_{2} \mathrm{H}_{4} \mathrm{O}_{4} \rightarrow 6 \mathrm{CO}_{2}+8 \mathrm{~N}_{2}+22 \mathrm{H}_{2} \mathrm{O} \text {. }
$$

The liquid propellants are prepared by dissolving the HAN and TEAN salts with a small quantity of water (approximately $20 \%$ by weight). This liquid mixture may then be ignited thermally (as with a $\mathrm{CO}_{2}$ laser) or by an electric discharge.

Little is known of the physical processes which govern the combustion of HAN-TEAN mixtures. There is evidence that decomposition reactions occur in the liquid at high pressures and temperatures. As the reactants decompose or react in the liquid phase to form molecules with lower vapor pressures, the intermediaries will evolve into the gas phase, where reaction to completion will occur. It is also possible that there may be reactions at the liquid-gas interface. Since the propellant contains at least two reactive species which will decompose at different temperatures, the possibility of two-phase flow exists.

Experiments were performed in a strand burner-type arrangement, in which a vertical column of propellant is ignited at one end, and combustion proceeds through the propellant. The propellant was contained in a strand burner, shown in Figure 1.
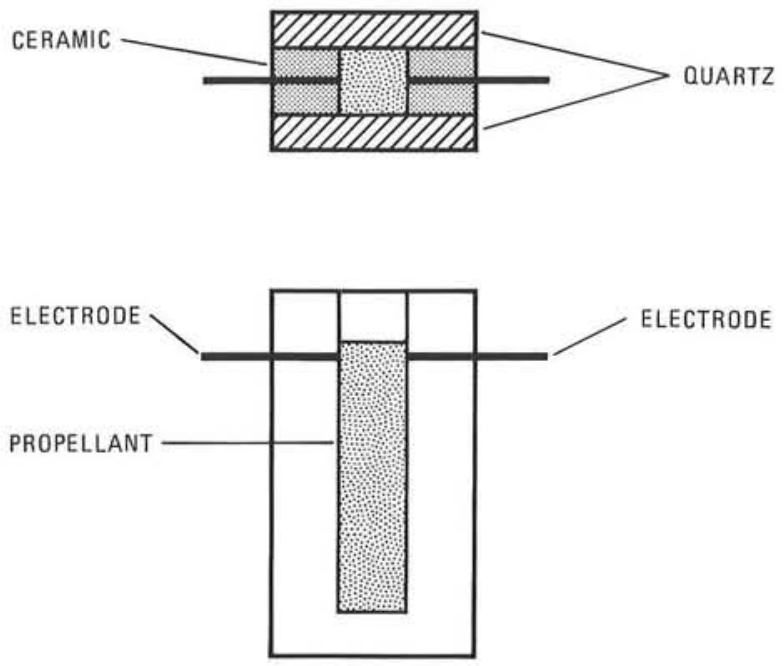

Figure 1. Schematic of strand burner used for liquid propellant combustion studies. The propellant sample is $5 \mathrm{~mm}$ wide and $20 \mathrm{~mm}$ high.

The burner has a 5-mm-square cross section, is $20 \mathrm{~mm}$ deep, and has quartz sides to provide optical access. Combustion is initiated by an electric spark, resulting in a deflagration of the propellant which propagated down the column. The strand burner is contained in a 13 liter pressure vessel which has acrylic windows for observing combustion of the propellant.

A series of stills from a movie are shown in Figure 2. The container is in a $33.3 \mathrm{MPa}$ atmosphere of argon, and is backlit with diffuse yellow light. The movie was taken with a standard video system (30 frames/ second) with the light source continuously on, giving an exposure time of approximately $1 / 30$ of a second. The first frame shows the LP prior to ignition. The meniscus is clearly visible near the top of the container, and the locations of the electrodes are indicated. In the succeeding frames, the liquid 


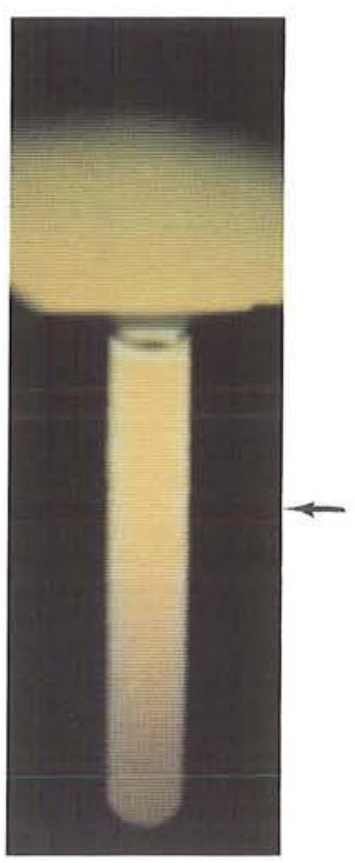

$t=0.0$

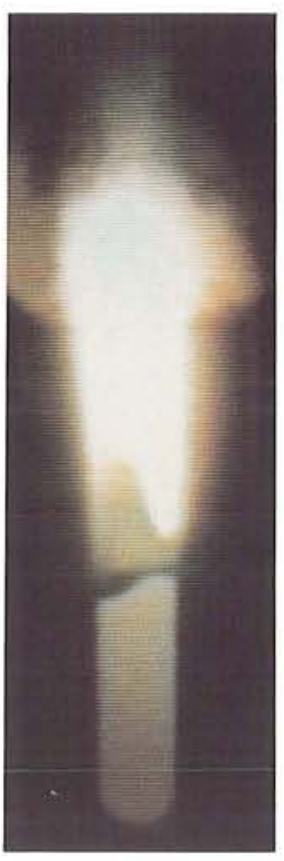

66.7

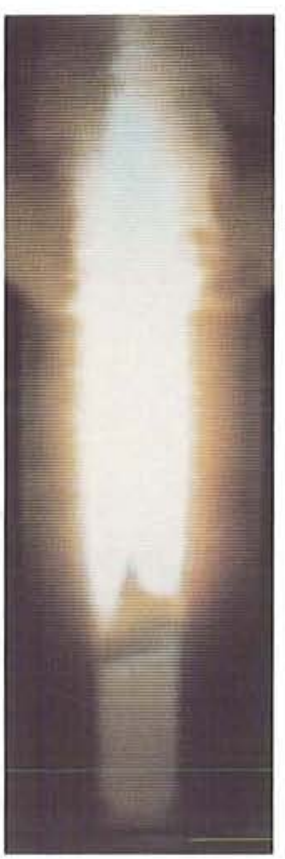

133.3

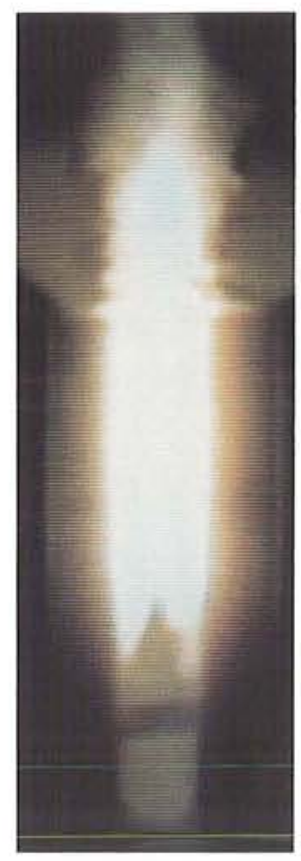

200.0

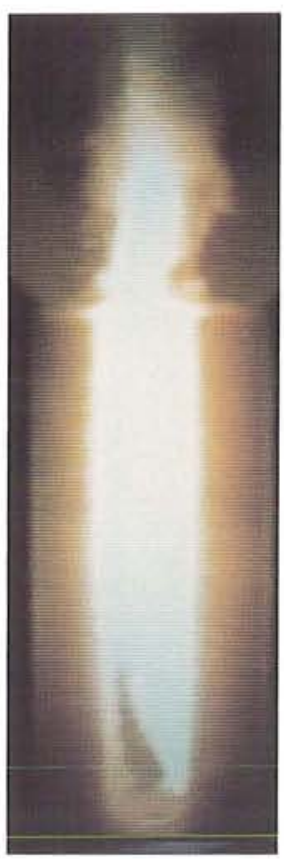

$266.7 \mathrm{msec}$

Figure 2. Combustion of a HAN/TEAN/water mixture in $33.3 \mathrm{MPa}$ argon atmosphere. The backlit photographs have an exposure of time of $33.3 \mathrm{msec}$. Time is given from electrode discharge. Electrode placement is indicated by the arrow. Container is $5 \mathrm{~mm}$ wide and $5 \mathrm{~mm}$ deep.

surface and a gas phase flame can be seen regressing through the sample. The meniscus appears to undulate as it regresses, possibly on a time scale shorter than the $1 / 30$ second exposure time. The visible flame also appears to undulate side to side as well as pulsate up and down.

Movies taken at higher framing rates and much shorter exposures reveal a much more complicated process than that shown in Figure 2. Figure 3 shows a series of 10 stills from a backlit movie taken at 300 frames/second with a $50 \mu$ second exposure. The shape of the liquid surface can be seen to vary considerably over the 1/30 exposure time of the stills in Figure 2. Combustion can be seen to be occurring both near the liquid surface and in the gas phase.

It is too soon to draw any specific conclusions from the data taken so far, but the following observations have been made:
1. Below $17 \mathrm{MPa}$ no visible flame is seen.

2. Even when no visible flame is present, the $\mathrm{LP}$ is converted to gases.

3. The regression rate decreases from 200 $\mathrm{mm} / \mathrm{sec}$ at $6.8 \mathrm{MPa}$ to $40 \mathrm{~mm} / \mathrm{sec}$ at 33.3 $\mathrm{MPa}$.

4. It is difficult to determine the burning rate from the regression rate because of the uncertainty of the shape of the liquid-gas interface.

5. The combustion process is very complicated, involving gas-phase and liquid-gasinterface reactions.

Work in this area is to continue, with the emphasis on understanding the structure of liquid propellant flames, to identify important chemical species, and to determine temperature profiles through the flame front. 


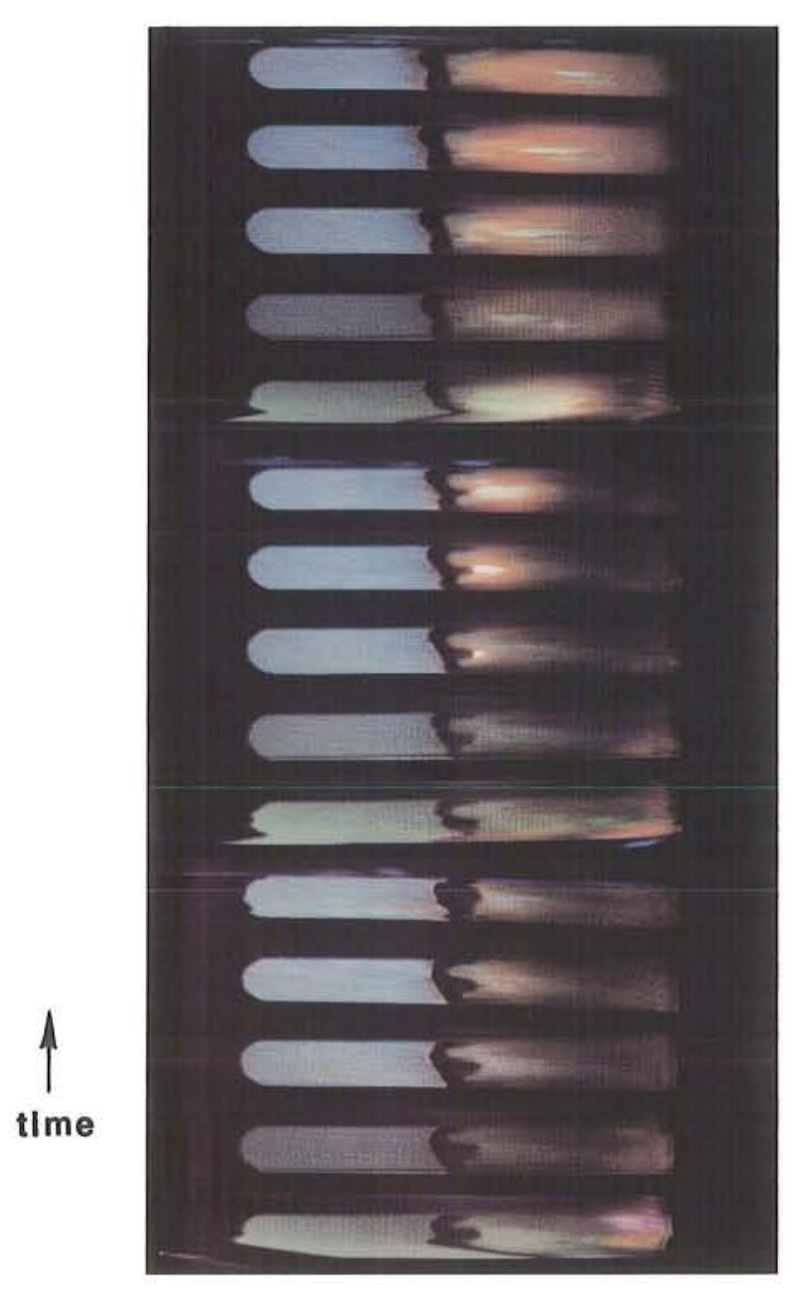

Figure 3. High speed backlit photographs of a HAN/TEAN/water mixture in $33.3 \mathrm{MPa}$ argon atmosphere. The pictures, taken at $\mathbf{3 0 0}$ frames/ second with an exposure time of $50 \mu \mathrm{sec}$, show the complex nature of LP combustion.

\section{The Structure of Cyanogen-Nitrogen Dioxide Premixed Flames ${ }^{\dagger}$}

\section{R. Thorne, O. I. Smith*}

We are continuing our studies of low-pressure flames of which gas-phase flame chemistry is similar to that the nitramines, RDX (hexahydro1,3,5-trinitro-s-triazine) and HMX (octa-hydro1,3,5,7-tetranitro-1,3,5,7-tetraazocine). Nitramines are an important class of solid energetic materials used as propellants and explosives. Previous studies have shown that during the initial stages of combustion, solid nitramines pyrolyze and fragment into smaller molecules that react either as fuels or oxidizers in the gas phase. Thus, the actual combustion of nitramines occurs primarily in the gas phase. This finding underscores the importance of understanding the gas phase combustion chemistry of nitramines and motivates our current investigations.

Because the combustion of solid nitramines involves a large number of species and chemical reactions, we have chosen to simplify the problem by studying a series of low-pressure flames made from one oxidizer and one fuel species previously identified in the gas phiase combustion of these materials. Specifically, HCN and $\mathrm{H}_{2} \mathrm{CO}$ have been identified as important fuels, and $\mathrm{NO}, \mathrm{NO}_{2}$, and $\mathrm{N}_{2} \mathrm{O}$ have been identified as important oxidizers. Recent theoretical results of Carl Melius suggest that $\mathrm{HCN}$ and $\mathrm{NO}_{2}$ are produced during rapid heating of nitramines such as RDX, and that $\mathrm{H}_{2} \mathrm{CO}$ and $\mathrm{N}_{2} \mathrm{O}$ are produced during slower heating. ${ }^{1}$ We have selected the $\mathrm{HCN} / \mathrm{NO}_{2}$ system for our initial lowpressure flame studies, but, because of the potential explosion hazards associated with the large quantities of liquid HCN that would be required for the experiment, we have chosen to study three flames whose flame chemistries are subsets of the $\mathrm{HCN} / \mathrm{NO}_{2}$ system: $\mathrm{C}_{2} \mathrm{~N}_{2} / \mathrm{NO}_{2}$, $\mathrm{H}_{2} / \mathrm{C}_{2} \mathrm{~N}_{2} / \mathrm{NO}_{2}$, and $\mathrm{H}_{2} / \mathrm{NO}_{2}$.

\footnotetext{
$\dagger$ Presented at the Fall Meeting of the Western States Section (The Combustion Institute, 1986).

- University of California at Los Angeles.
} 
Thus, an objective of the present study is to develop combustion mechanisms for the gas phase combustion of nitramines by first developing mechanisms for simple low-pressure flame systems whose gas phase chemistries are related to that of nitramines. Our approach follows that of earlier combustion mechanism development work in this laboratory involving $\mathrm{HCN}$ and NO. ${ }^{2}$ Species concentrations and temperatures are measured at various heights above the burner surface, and then these results are compared to the profiles calculated from a detailed model of the combustion process. The model is modified by adding or deleting reactions to or from the reaction set and changing the reaction rates within the bounds of their estimated uncertainties. When the calculated profiles agree with the observed profiles, the reaction-rate sensitivity coefficients and species-production rate information computed from the model can be used to deduce a combustion mechanism. This mechanism indicates how the elementary chemical reactions of the model lead from reactants through intermediates to products.

The model calculations make use of the computer programs developed by Miller et al. ${ }^{2}$ They include diffusion effects and incorporate detailed chemistry as a set of elementary bimolecular chemical reactions whose temperature dependence is modeled by a three-parameter Arrhenius form. Most of the rate constants used in this study are available from the literature. Those that are unavailable are estimated by theoretical methods.

The laboratory flame data are obtained using a 9.7-cm-diameter burner operated in a vacuum housing maintained at 25 -Torr pressure. The burner surface is a stainless-steel porous plug. The reactant gases are premixed in a manifold before entering the burner body where glass beads are used for additional mixing. The concentration profiles for the major stable species are measured using a quartz microprobe that continuously withdraws a small sample of the combustion gases and directs it into a quadrupole mass spectrometer for quantitative analysis. Data are taken at various heights above the burner by translating the burner vertically using a stepping motor. Laser adsorption is used to measure the relative concentration profiles of the $\mathrm{CN}$ radical using the $\mathrm{B}-\mathrm{X}, \mathrm{R}_{1}(14)$, and $\mathrm{R}_{2}(14)$ transitions. The temperature profile is obtained from the $\mathrm{CN}$ rotational temperature which is determined from a Boltzman plot of the B-X, R-branch adsorption line intensities. The temperature of the burner surface is measured with a thermocouple.

We have concentrated our initial efforts on modeling two rich $\mathrm{C}_{2} \mathrm{~N}_{2} / \mathrm{NO}_{2}$ flames of equivalence ratio 2.50 and 1.37. Typical measured and computed profiles for the richer flame are shown in Figure 1.

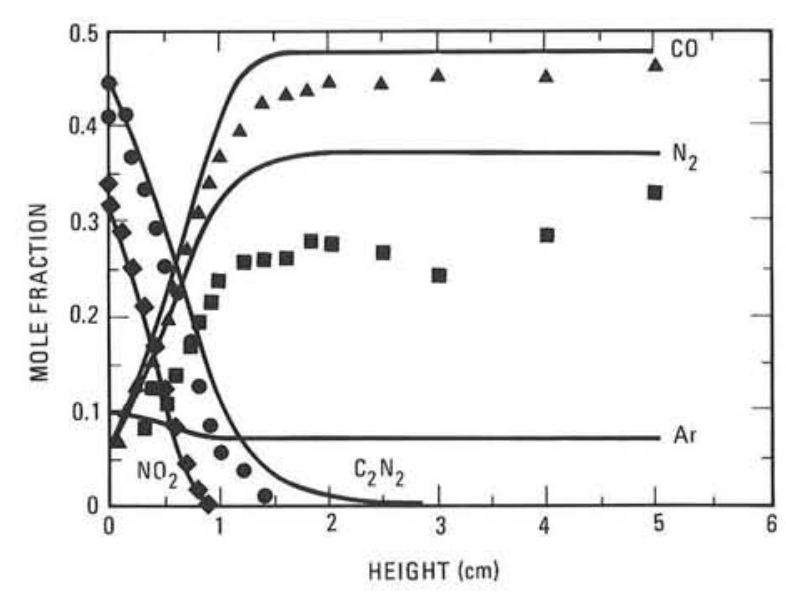

Figure 1. Observed (symbols) and computed profiles for the $\mathrm{C}_{2} \mathrm{~N}_{2} / \mathrm{NO}_{2}$ flame with equivalence ratio of 2.50 .

The computed profiles are based on a set of 51 reactions involving 15 reactants: $\mathrm{C}_{2} \mathrm{~N}_{2}$, $\mathrm{CN}, \mathrm{NO}, \mathrm{NO}_{2}, \mathrm{~N}_{2} \mathrm{O}, \mathrm{N}_{2}, \mathrm{CO}, \mathrm{CO}_{2}, \mathrm{NCO}, \mathrm{N}, \mathrm{O}$, $\mathrm{O}_{2}, \mathrm{C}_{2} \mathrm{~N}, \mathrm{CN}_{2}$, and $\mathrm{C}$. The combustion mechanism deduced for nitrogen-containing species is shown in Figure 2; that for the carbon-containing species is shown in Figure 3.

We have observed that the richer flame is more stable than the leaner flame. The combustion mechanism provides a basis for explaining this observation. O-atoms in the leaner flame react with $\mathrm{NO}_{2}$ to give $\mathrm{NO}$ and $\mathrm{O}_{2}$. This produces two relatively unreactive free radicals, NO and $\mathrm{O}_{2}$, and consumes one very reactive radical, $\mathrm{O}$, thereby depleting the "reactive" radical pool. In the richer flame, $\mathrm{NO}_{2}$ is depleted by way of 


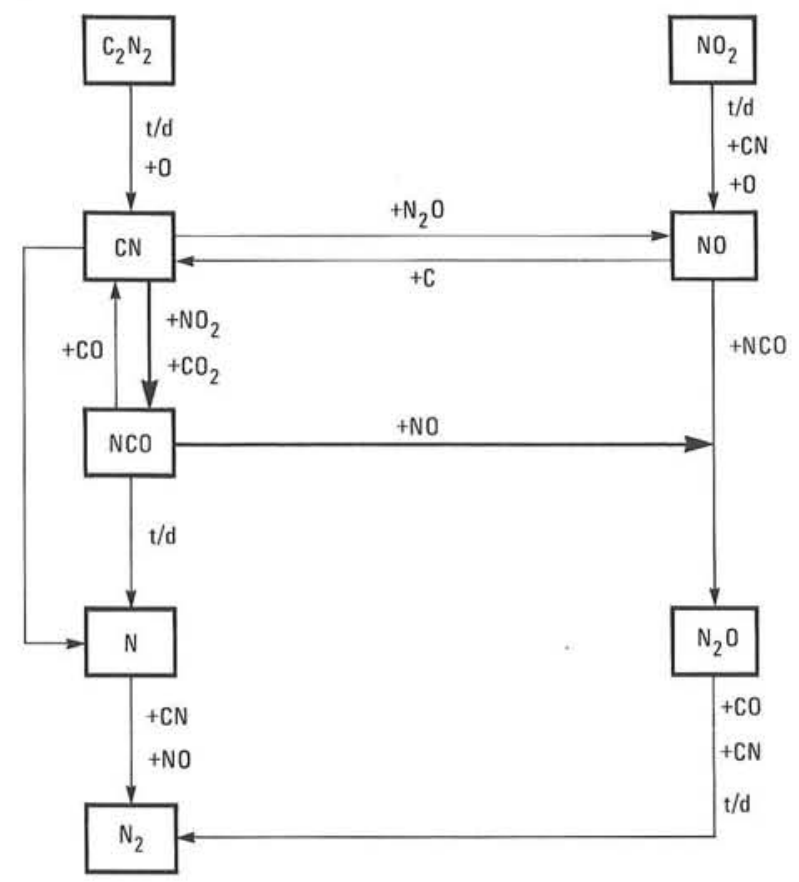

Figure 2. Combustion mechanism for nitrogencontaining species in the $\mathrm{NO}_{2} / \mathrm{C}_{2} \mathrm{~N}_{2}$ flame having an equivalence ratio of 2.50. The heavy arrows indicate the major reaction routes, $t / d$ indicates thermal decomposition.

thermal decomposition, producing $\mathrm{NO}$ and $\mathrm{O}$. This adds $\mathrm{O}$-atoms to the "reactive" radical pool and stabilizes the flame.

The major CN loss mechanism in these flames leads to the formation of NCO which, in turn, is depleted primarily by reacting with NO or by disproportionation. Both of these reactions serve to stabilize the flame. The first of these reaction releases a substantial amount of heat and the second is a major channel for $\mathrm{N}$ atom production.

The largest differences between the observed and calculated profiles occur for NO far down stream and for $\mathrm{CN}$ in the richer flame. These discrepancies are probably due to the lack of appropriate sink reactions for $\mathrm{C}$ and $\mathrm{CN}$ in

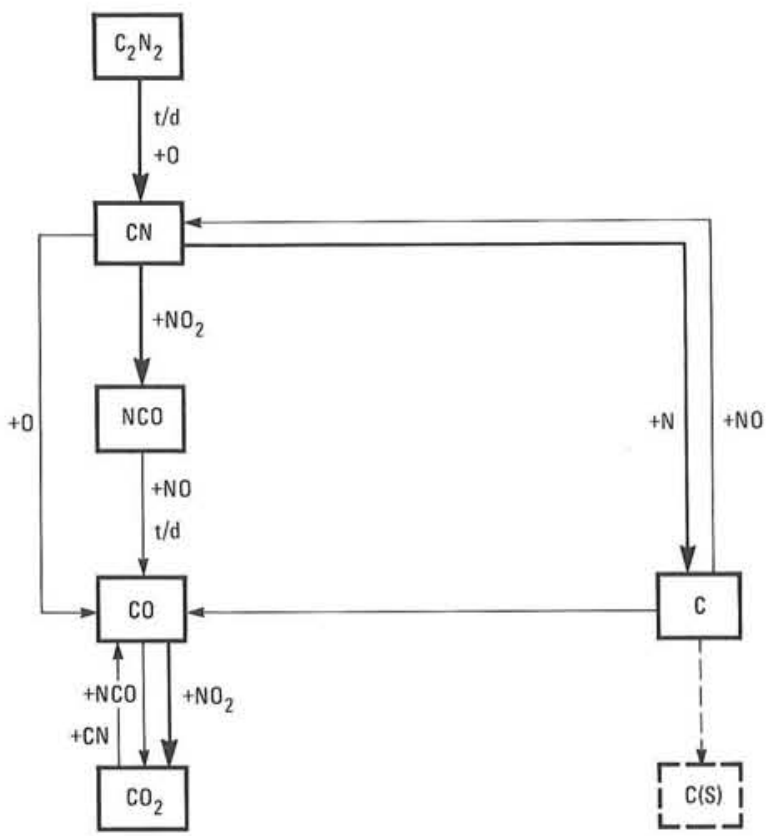

Figure 3. Combustion mechanism for carboncontaining species in the $\mathrm{NO}_{2} / \mathrm{C}_{2} \mathrm{~N}_{2}$ flame with equivalence ratio of 2.50 . The heavy arrows indicate major reaction routes, $t / d$ indicates thermal decomposition, $\mathrm{C}(\mathrm{s})$ indicates solid carbon.

the model. Work is in progress to make additional measurements and to further refine the flame model and combustion mechanism for the $\mathrm{HCN} / \mathrm{NO}_{2}$ flame system.

\section{References}

1. C. F. Melius and J. S. Binkley, "Thermochemistry of the Decomposition of Nitramines in the Gas Phase," Twenty-first Symposium (International) on Combustion (The Combustion Institute, 1986), in press.

2. L. R. Thorne, M. C. Branch, D. W. Chandler, R. J. Kee and J. A. Miller, "Nitric OxideHydrocarbon Interactions in Low Pressure Flames," Twenty-first Symposium (International) on Combustion (The Combustion Institute, 1986), in press. 


\section{HMX Thermal Decomposition by Simultaneous}

Thermogravimetry, Modulated-Molecular-Beam Mass Spectrometry ${ }^{\dagger}$

\section{R. Behrens}

After many thermal decomposition studies of octahydro-1,3,5,7-tetranitro- 1,3,5,7-tetrazocine (HMX),<smiles>O=[N+]([O-])N1CN([N+](=O)[O-])CN([N+](=O)[O-])CN([N+](=O)[O-])C1</smiles>

there are still unresolved questions, such as whether the decomposition occurs in the gas or in the condensed phase, whether the initial step involves the breaking of a C-N or an N$\mathrm{NO}_{2}$ bond, and whether autocatalysis plays an important role in the decomposition process. To answer these questions, it is important to identify and measure the concentrations of the various decomposition products during different stages of the decomposition of an HMX sample. Mass spectrometry has been used by a number of investigators to measure the products evolved during an HMX decomposition. Although it is sensitive to all gas species that evolve during the decomposition, mass spectrometry is limited by difficulties in associating a measured ion signal with a particular decomposition product and in providing quantitative measurements. We use simultaneous thermogravimetry modulated beam mass spectrometry (STMBMS) and timeof-flight (TOF) velocity spectra measurements to overcome these limitations. The STMBMS measurements provide a means of quantifying the mass spectrometric data and, in conjunction with TOF velocity spectra measurements, allow the measured ion signals to be associated

$\dagger$ To appear in Chemical Propulsion Information Agency publication of 23rd JANNAF Combustion Meeting (1986). with either the evaporating reactant or the thermal decomposition products evolving during the HMX sample decomposition.

A schematic of the apparatus and data analysis procedures are described in detail elsewhere. ${ }^{1}$ Briefly, however, a $10-\mathrm{mg}$ HMX sample is placed in a reaction cell that may be either heated at controlled rates or held at a constant temperature. A small orifice in the cell allows a portion of the gas phase molecules to escape into a high vacuum environment. The relative number of molecular collisions in the reaction cell is controlled by varying the size of the orifice. The gas-phase species concentrations in the reaction cell are determined as a function of time by the STMBMS and TOF velocity spectra measurements on the gas exiting the cell.

The approximate molecular weight of the thermal decomposition products is obtained by convoluting a velocity distribution that characterizes the flow from the reaction cell with the TOF gating function for different trial molecular weights to obtain the best fit to the TOF velocity spectra.

Appearance potential measurements ${ }^{2}$ have been used to eliminate the interference of HMX ion-dissociation fragments on HMX thermal decomposition product measurements. The results indicated that the thermal decomposition of HMX produces ring-fragmentation products that have the same molecular formulas as iondissociation fragments formed in the mass spectrum of HMX. These results are very interesting since they indicate that the thermal decomposition reaction mechanisms may be similar to the ion-dissociation fragmentation pathways. However, since some of the bonds in HMX are relatively weak and may be susceptible to ion fragmentation even at low electron energies, we made STMBMS and TOF velocity spectra measurements to determine whether or not HMX undergoes ion dissociation at the low electron energies used in appearance potential measurements.

The data was collected using a $0.1 \mathrm{~cm}$ diameter orifice and a reaction cell temperature of $225^{\circ} \mathrm{C}$. The electron energies ranged from 12.4 
ev to $70 \mathrm{ev.} \mathrm{All} \mathrm{of} \mathrm{the} \mathrm{ion} \mathrm{signals} \mathrm{measured} \mathrm{in}$ these experiments were highly temporally correlated and remained constant while the sample evaporated from the cell. This indicates that HMX undergoes ion dissociation at 12.4 ev, about $3 \mathrm{ev}$ above the appearance potential. To substantiate that HMX fragments at 12.4 ev, the TOF velocity spectra of the different ion masses were measured and a representative sample of the results are shown in Figure 1.

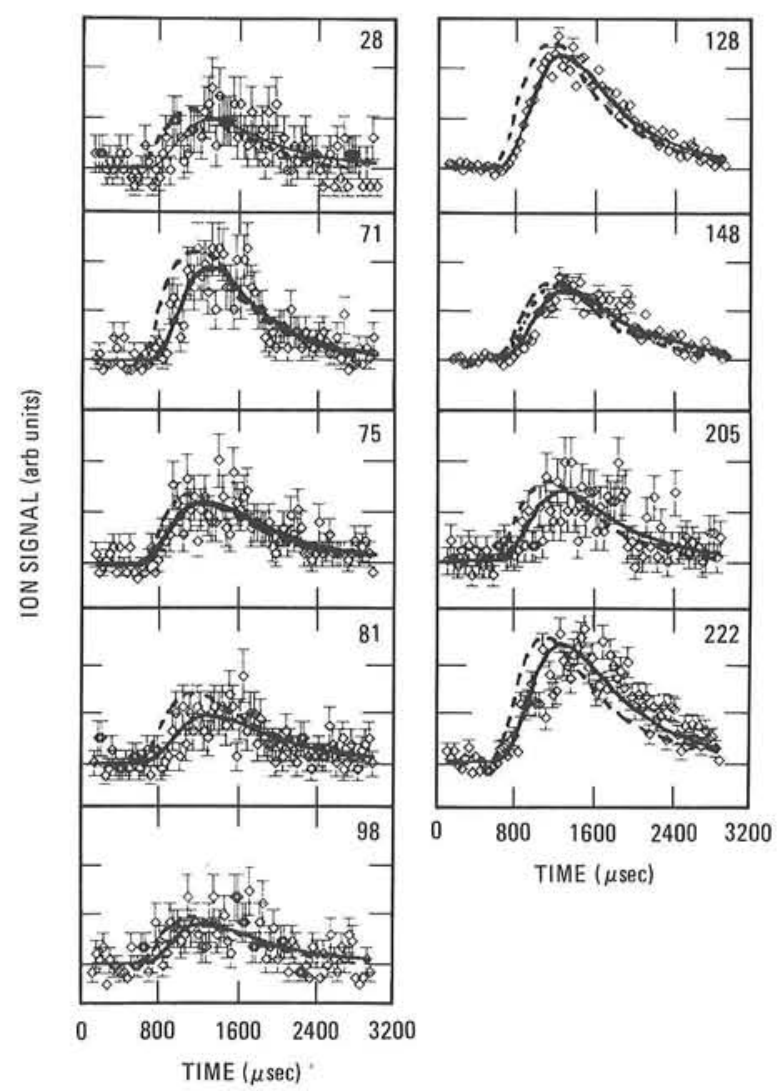

Figure 1. The TOF velocity spectra of HMX ion dissociation products are shown. The numbers on each graph are the ion masses. The solid and dashed curves are fits to the data using molecular weights of 296 and 222, respectively, in the convoluted velocity distributions.

The solid curves, which are convoluted fits of a velocity distribution characterized by the molecular weight of HMX (296), provide the best fit to the data. The dashed curves, which are convoluted velocity distributions characterized by a molecular weight of 222 , are shown for reference. From these results, we conclude that under the selected reaction cell conditions no HMX thermal decomposition occurs and that HMX undergoes extensive ion dissociation at $3 \mathrm{ev}$ above its appearance potential, making it useless for eliminating HMX ion fragment interference in thermal decomposition measurements.

Thermal decomposition is promoted by decreasing the reaction cell orifice to $0.01 \mathrm{~cm}$, thus containing the HMX sample in the reaction cell longer.

An autocatalytic reaction is evident from the weight-loss data as shown in Figure 2. The major ion signals in the higher molecular weight region $(75,120,128,148$, and 222$)$ are highly correlated and remain relatively constant throughout the sample decomposition. This indicates that they are formed from ion dissociation of HMX and correlate with the HMX vapor pressure in the cell.

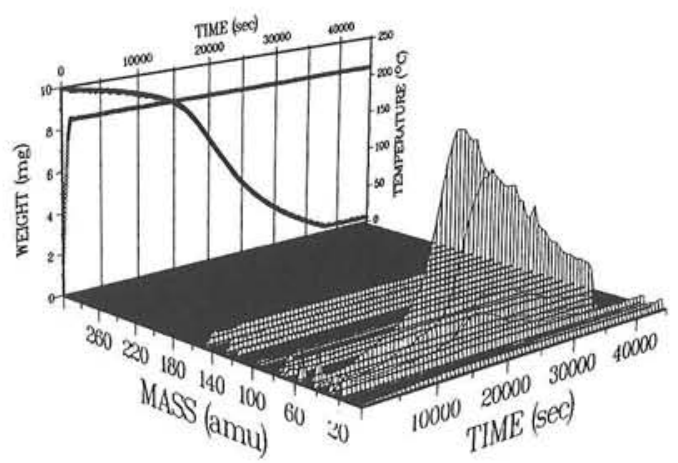

Figure 2. The ion signals and sample weight from an isothermal HMX thermal decomposition experiment are shown. The constant ion signals at higher molecular weights are ion-dissociation products from the HMX reactant and the varying ion signals at the lower molecular weights are either parent ions or ion-dissociation products from HMX thermal decomposition products.

The major ion signals that are associated with the autocatalytic behavior are at masses $18,27,29,30,44,46,47,74$, and 132 . To determine the molecular weight of the molecules leading to the formation of these ions, TOF velocity spectra were measured. The results show the following association between ion masses and thermal decompostion products: $\mathrm{H}_{2} \mathrm{O}(18)$, 
HCN (27), $\mathrm{H}_{2} \mathrm{CO}(29,30), \mathrm{N}_{2} \mathrm{O}(44)$, and HMX $(75,120,128,148,222)$. We also find that the ion signal at mass 74 originates from a molecule with a molecular weight of 74 which is probably $\mathrm{N}$-nitroformimine $\left(\mathrm{H}_{2} \mathrm{C}=\mathrm{N}-\mathrm{NO}_{2}\right)$, and the ion signal at mass 132 originates from a product with a molecular weight of 280 that may be a product from $\mathrm{HMX}$ where one of the $\mathrm{NO}_{2}$ groups has been reduced to a NO group.

By combining the STMBMS and TOF velocity spectra data, the partial pressure of each species in the reaction cell is calculated and the results are shown in Figure 3. In addition, when autocatalysis is observed, four percent of the original sample remains as a carbonaceous residue.

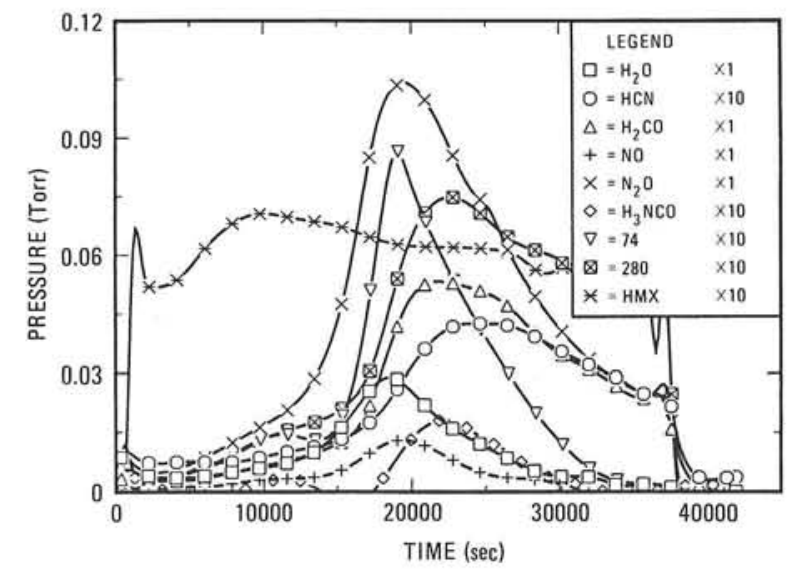

Figure 3. The partial pressures of the reactant and each thermal decomposition product during the course of an HMX sample decomposition are shown.

The data suggest that there are two condensed-phase thermal-decomposition mechanisms. One involves the formation of $\mathrm{N}_{2} \mathrm{O}, \mathrm{H}_{2} \mathrm{CO}, \mathrm{N}$ nitroformimine, and a carbonaceous residue. A reaction scheme that explains the data is the formation of a residue that reacts with HMX.

The other condensed phase reaction involves the formation of a decomposition product that is only slightly lighter than the HMX and undergoes ion dissociation in the mass spectrometer to mass 132. Assuming a stoichiometry of $\mathrm{C}_{2} \mathrm{H}_{4} \mathrm{~N}_{4} \mathrm{O}_{3}$ and a cracking pattern similar to HMX, this suggests that one of the nitro groups may be reduced to a nitroso group and the intermediate would have a molecular weight of 280 . The temporal correlation between the $\mathrm{HCN}$ and $132^{+}$signal indicates that HCN may be formed in the condensed phase through this intermediate.

Two different reaction mechanisms have been identified for HMX thermal decomposition. The products from these reactions have been carefully identified using STMBMS and TOF velocity spectra measurements to eliminate the possibility of misinterpreting an ion-dissociation fragment from either the reactant or a thermaldecomposition product as a parent ion from a thermal-decomposition product.

\section{References}

1. R. Behrens, Jr., "A New Simultaneous Thermogravimetry and Modulated Molecular Beam Mass Spectrometry Apparatus for Quantitative Thermal Decomposition Studies," to appear in Rev. Sci. Instr. (1987).

2. M. Farber and R.D. Srivastava, "Mass Spectrometric Studies of the Thermal Decomposition of 1,3,5,7-Tetranitro-1,3,5,7Tetraazacyclooctane (HMX)," Chem. Phys. Lett. 80, 345 (1981). 


\section{RDX Thermal Decomposition by Simultaneous \\ Thermogravimetry, Modulated-Molecular-Beam Mass Spectrometry}

\section{R. Behrens}

After many thermal decomposition studies of hexahydro-1,3,5-trinitro- s-triazine (RDX),<smiles>O=[N+]([O-])N1CN([N+](=O)[O-])CN([N+](=O)[O-])C1</smiles>

there are still unresolved questions, such as whether the decomposition occurs in the gas or condensed phase, whether the initial step involves the breaking of a C-N or an $\mathrm{N}-\mathrm{NO}_{2}$ bond, and whether autocatalysis plays an important role in the decomposition process. In contrast to our HMX decomposition studies (see p. 5-10 this issue) in which we observed two condensedphase reactions leading to the measured-reaction products, the RDX studies show reactions in both the gas phase and condensed phase.

The RDX decomposition products are characterized by simultaneous thermogravimetric, modulated-beam mass spectrometry (STMBMS), and time-of-flight (TOF) velocity spectra measurements. The details of the experimental technique have been presented elsewhere. ${ }^{1}$

The results from a STMBMS experiment with a 26-mg RDX sample show that a number of ion masses are temporally correlated and gradually increase in intensity as the sample is heated from $155^{\circ} \mathrm{C}$ to $180^{\circ} \mathrm{C}$. (See Figure 1 ). These are ion-dissociation products from RDX. The major fragments include ion masses 205, $148,128,120,102,101,98,75,71,46,42,30$, and 28. TOF velocity spectra at ion masses 205 , $148,128,120,75$, and 46 confirm these as iondissociation fragments. Two other features are apparent: First, above $180^{\circ} \mathrm{C}$, a number of new ion signals appear, the most obvious being at

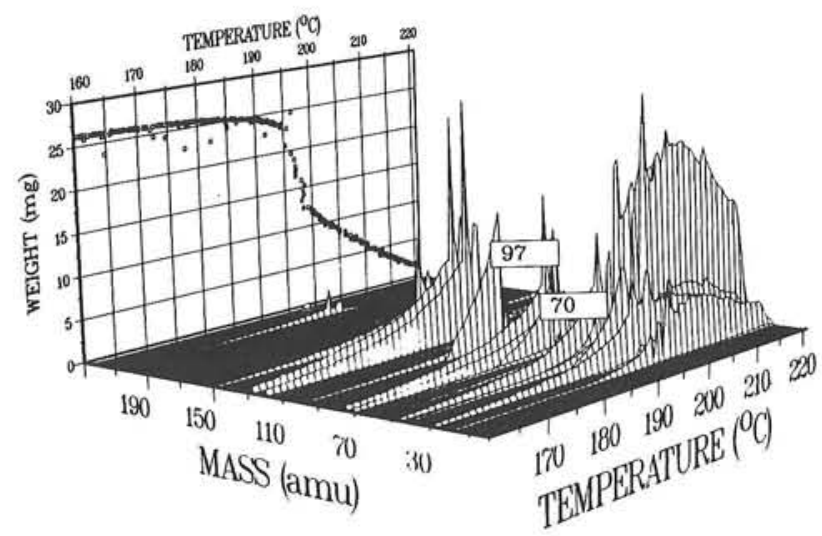

Figure 1. The weight and ion signals from the thermal decomposition of a 26-mg sample of RDX are shown. The rapid weight loss at $195^{\circ} \mathrm{C}$ is due to molten sample being expelled from the crucible and forming a pool on top of the reaction cell orifice. The data points below the weight curve are noise in the data.

masses 70 and 97 . These ions are formed from RDX thermal-decomposition products. Second, the sample loses weight very rapidly starting at $195^{\circ} \mathrm{C}$. From similar experiments with $25 \mathrm{mg}$ samples and visual examination of the reaction cell after the rapid weight loss, we found that the rapid weight loss arises when molten sample flows through the reaction cell orifice and forms a pool on top of the orifice. The evaporation rate from the pool is much greater than that through the reaction cell orifice. This provides useful information regarding the phases in which the different decomposition products are formed. For example, if different products are formed in the solid and gas phases prior to the molten sample's flowing through the orifice, then ion signals from products formed in the gas phase should decrease because the orifice is partially blocked, and the ion signals from products formed in the condensed phase should increase because the molten material evaporates more rapidly outside of the reaction cell.

The temperature dependence of the ion signals from the major RDX thermal decomposition products, after they are corrected for the RDX ion-dissociation fragment contribution, are shown in Figure 2. 


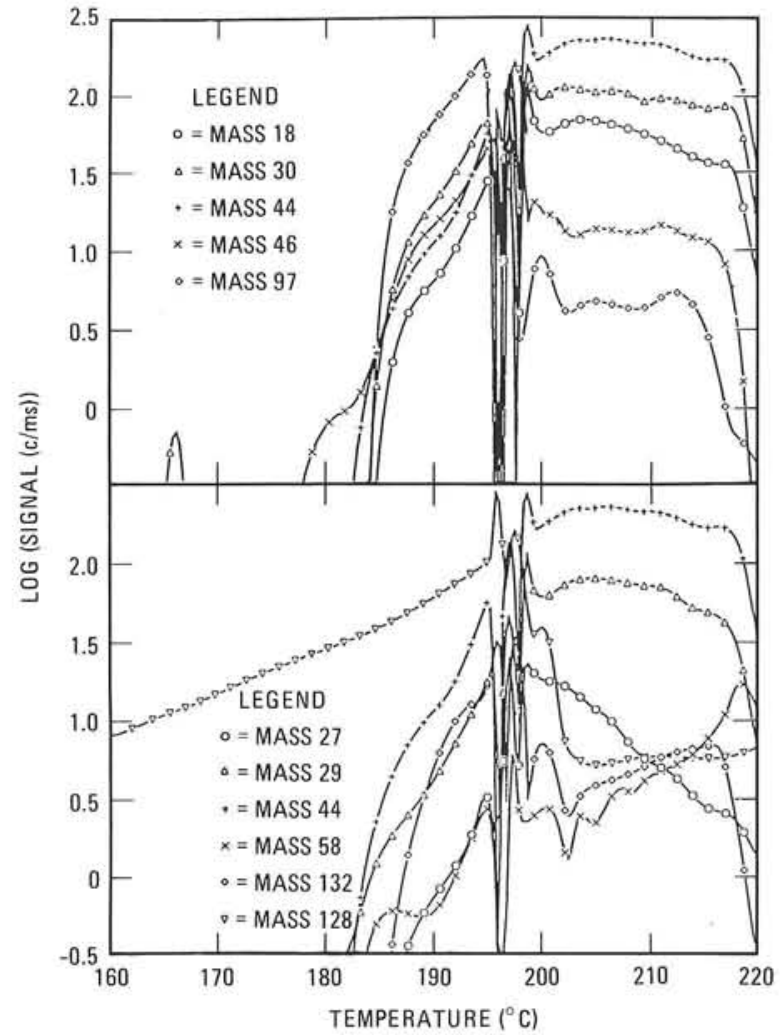

Figure 2. The ion signals from the 26-mg RDX thermal-decomposition experiment are shown. The ion masses are listed in the legend. These signals are associated with $\mathrm{H}_{2} \mathrm{O}(18), \mathrm{HCN}(27)$, $\mathrm{NO}(30), \mathrm{H}_{2} \mathrm{CO}(29), \mathrm{N}_{2} \mathrm{O}(44), \mathrm{NO}_{2}(46)$, hy-droxys-triazine( 97 ), hexahydro-1-nitroso-3,5-dinitro-stri-azine(132), and RDX(128).

To determine the approximate molecular weight of the parent molecule leading to the formation of these ions at different temperatures, the TOF velocity spectra were measured below and above the temperature where ion signals at masses 70 and 97 first appeared. The results are shown in Figure 3.

From the STMBMS and TOF velocityspectra data, the thermal decomposition products are assigned to the ion mass signals shown in Figure 2 as follows: $\mathrm{H}_{2} \mathrm{O}$ (18), $\mathrm{HCN}(27)$, $\mathrm{H}_{2} \mathrm{CO}(29)$, NO (30), $\mathrm{N}_{2} \mathrm{O}(44), \mathrm{NO}_{2}$ (46), hydroxys-triazine (HST) (97),<smiles>Oc1ncncn1</smiles>

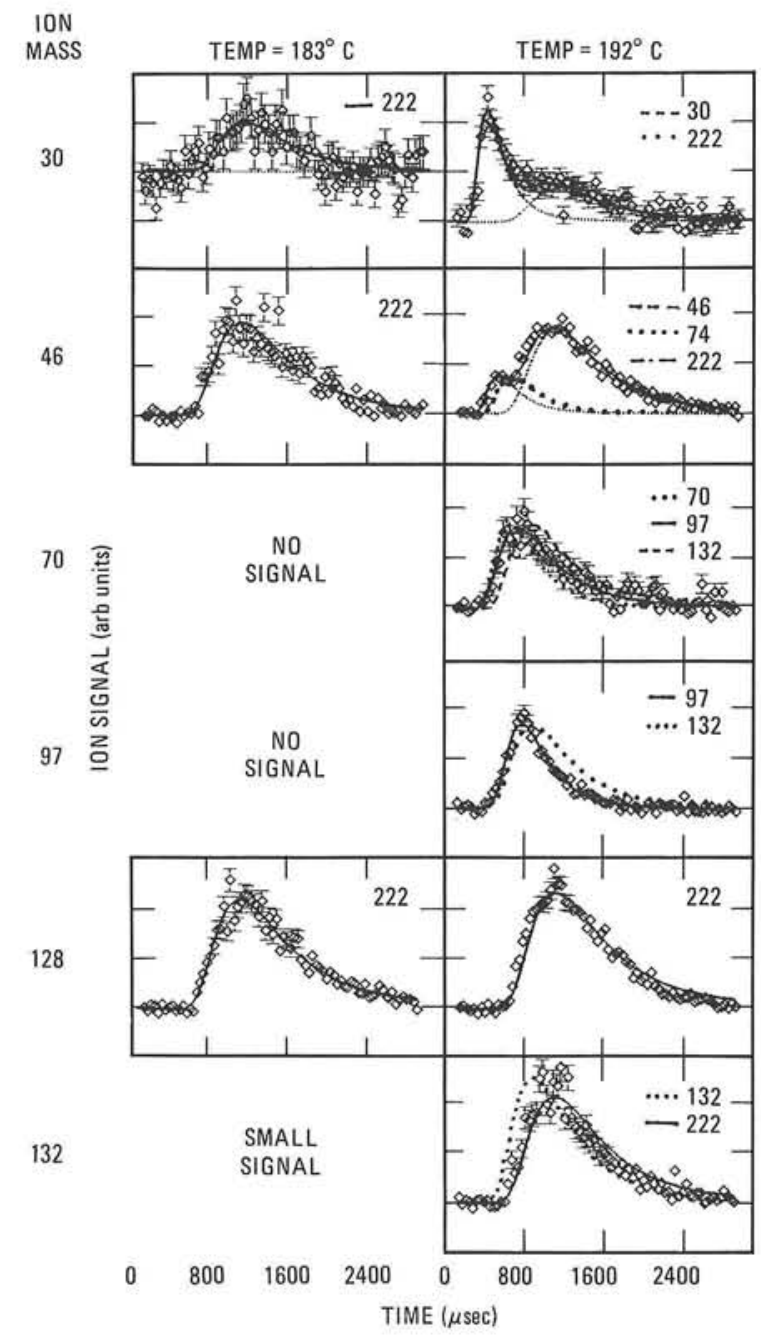

Figure 3. The TOF velocity spectra of RDX recorded before $\left(183^{\circ} \mathrm{C}\right)$ and after $\left(192^{\circ} \mathrm{C}\right)$ the appearance of ion signals at 70 and 97 . The ion masses are listed in the left column and the molecular weight used to characterize the velocity distributions which are used to fit the data are shown on each graph.

and probably hexahydro-1-nitroso-3,5-dinitro-striazine (132).<smiles>O=[N+]([O-])N1CN([N+](=O)[O-])CN([N+](=O)[O-])C1</smiles>

The results of the thermal decomposition experiments up to $195^{\circ} \mathrm{C}$ may be summarized 
as follows: below $184^{\circ} \mathrm{C}$, no thermal decomposition is observed and the ion signals arise from ion dissociation of RDX; at $187^{\circ} \mathrm{C}$, reactions involving gas phase species start and the products observed include $\mathrm{H}_{2} \mathrm{O}, \mathrm{NO}, \mathrm{N}_{2} \mathrm{O}, \mathrm{NO}_{2}$, and hydroxy-s-triazine; condensed-phase reactions generate formaldehyde and $\mathrm{N}_{2} \mathrm{O}$ and also a product that may be hexahydro-1-nitroso3,5-dinitro-s-triazine. One of the major products of subsequent HST decomposition will be HCN. Hence, these results show that the relative amounts of $\mathrm{N}_{2} \mathrm{O}$ /formaldehyde and $\mathrm{HCN} / \mathrm{NO}_{2}$ will depend on whether the decomposition occurs in the condensed or in the gas phase.

The temperature dependence of the HST concentration in the reaction cell indicates that its rate of formation is not controlled by a unimolecular decomposition. Although the reaction mechanism leading to the formation of HST is not established by the present measurements, quantum chemical calculations ${ }^{2}$ suggest that hydrogen atom extraction from the $\mathrm{CH}_{2}$ group will lead to N-N bond breaking because hydrogen atom loss from the $\mathrm{CH}_{2}$ group decreases the adjacent $\mathrm{N}-\mathrm{N}$ bond strength from 48 to 2 $\mathrm{kcal} / \mathrm{mole}$. Hydrogen abstraction by a radical, such as $\mathrm{OH}$, would lead to the formation of<smiles>O=[N+]([O-])N1C=NCN([N+](=O)[O-])C1</smiles>

The quantum chemical calculations also indicate that the $\mathrm{C}-\mathrm{H}$ bond strength in RDX that has lost a HONO group decreases by 10 $\mathrm{kcal} / \mathrm{mole}$. This makes the abstraction of subsequent hydrogen atoms by $\mathrm{NO}_{2}$ to form $\mathrm{HONO}$ more favorable. The HONO may decompose by $2 \mathrm{HONO} \longrightarrow \mathrm{H}_{2} \mathrm{O}+\mathrm{NO}+\mathrm{NO}_{2}$ to form the other measured gas phase reaction products. Quantum mechanical calculations ${ }^{3}$ on benzene and striazine analogs indicate that hydroxy-s-triazine should be more stable than s-triazine.

The results show that different decomposition reactions occur in the gas and condensed phases. The gas phase reactions lead to $\mathrm{HCN}, \mathrm{NO}_{2}$, and $\mathrm{NO}$ products, and the condensed phase reactions lead to formaldehyde and $\mathrm{N}_{2} \mathrm{O}$ products. The gas phase reactions form a newly observed intermediate product, hydroxy-s-triazine.

\section{References}

1. R. Behrens, Jr., "A New Simultaneous Thermogravimetry and Modulated Molecular Beam Mass Spectrometry Apparatus for Quantitative Thermal Decomposition Studies," to appear in Rev. Sci. Inst. (1987).

2. C. F. Melius and J. S. Binkley, "Thermochemistry of the Decomposition of Nitramines in the Gas Phase," Twenty-first (International) Symposium (The Combustion Institute, 1986), in press.

\section{Influences of Two-Phase Flow in the Deflagration of Homogeneous Solids ${ }^{\dagger}$}

\section{S. B. Margolis, F. A. Williams, ${ }^{*}$ R. C. Armstrong}

During combustion, most homogeneous solid propellants develop thin layers at their surfaces in which finite-rate exothermic reactions occur in a region of two-phase flow. The two-phase region typically begins through nucleation in a softened "melt" to form a foam and results later in dispersion of condensed material as a fizz. Because of difficulties in describing phenomena of two-phase flow, progress in the theoretical analysis of such deflagrations that include these effects has been slow. A review of most of this research has been given by Merzhanov. ${ }^{1}$ The present work ${ }^{2}$ extends these studies.

The character of the two-phase flow varies widely for different propellants. Nitrocellulose exhibits little softening so that the two-phase

$\dagger$ To appear in Combustion and Flame (1987).

* Princeton University, Princeton, NJ. 
region is mainly a fizz zone containing dispersed solid-like propellant particles, while nitramines, for example, show little fizz but extensive bubbling in the foam layer. The present study addresses questions specifically related to the latter type of two-phase region by considering processes that would occur in a liquid layer containing bubbles.

To be able to focus clearly on the effects of two-phase flow (Figure 1), the description of the chemistry will be simplified significantly here. In particular, a one-step exothermic process

$$
R(c) \rightarrow P(g)
$$

will be considered, where $R(c)$ is the condensedphase reactant, and $P(g)$ is the gaseous product. Thus, each phase is a pure species, and gasphase reactions are either neglected or assumed to be remote. Although these assumptions are not satisfied for all propellants that exhibit a dispersed phase, such as the nitramines, this simple chemical model will serve to clarify certain two-phase effects. The introduction of more elaborate chemistry is then a relatively straightforward application.

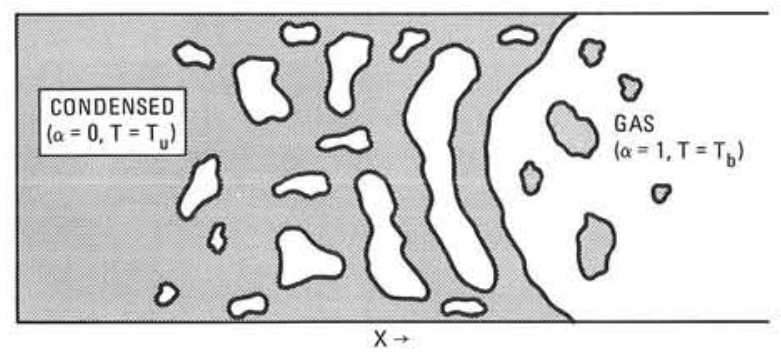

Figure 1. Sketch of the two-phase region during propellant deflagration. The shaded areas denote condensed unburned propellant, and the clear areas denote gaseous burned products.

Previous deflagration studies, which have included distributed two-phase effects, have required velocities to be the same in each phase. An excellent example is the early paper of Maksimov and Merzhanov. ${ }^{3}$ In the present work, the possibility of different velocities in different phases is addressed, with mechanisms identified that support the velocity differences. The specific mechanisms included here are pressure gradients, sur-face-tension gradients, and viscous forces. The estimated importance of these mechanisms in the deflagration of nitramine propellants motivated their consideration. Since the chemical mechanism considered, however, corresponds to that of Maksimov and Merzhanov ${ }^{3}$ with no condensed-phase products, the present work may be viewed as extending theirs to address influences of different velocities in different phases.

The major results that we derive are formulas for the mass burning rate as a function of the various parameters in the problem. For simplicity, we indicate here the results we have obtained for large values of the interphase heattransfer parameter. The dimensional velocity of each phase is given by

$$
u_{c}=\frac{m}{\rho_{c}}(1-s \alpha), \quad u_{g}=\frac{m}{\rho_{g}}(1+s-s \alpha),
$$

where $u_{c}$ and $u_{g}$ denote the velocities in the condensed and gas phases, $\rho_{c}$ and $\rho_{g}$ are the corresponding densities in each phase, $\alpha$ is the gas-phase volume fraction, $m$ is the (constant) total mass flux, and $s$ is a velocity-perturbation parameter which incorporates viscous and surfacetension gradient effects (the latter typically dominates over the former). An analysis in the limit of large activation energy then determines an expression for the burning rate eigenvalue $\Lambda$, which is inversely proportional to the square of the mass burning rate $\left(\mathrm{m}^{2}\right)$, as an expansion in $k^{-1}$ for large values of the nondimensional heattransfer parameter $k$. The result for the case of equal thermal conductivities for the two phases is

$$
\Lambda \sim\left(1-\frac{1}{2} s+\frac{1}{6} s^{2}\right)-\frac{1}{k}\left(\frac{1}{30}+\frac{b}{6 a}\right)+\cdots,
$$

in which the last term is further restricted to $s=0$. In that term, $b$ is the ratio of gas-phase heat capacity to that of the condensed phase, and $a=1-T_{u} / T_{b}$, where $T_{u}$ and $T_{b}$ denote the unburned and burned temperatures, respectively.

From the above expression, we conclude that $m^{2}$ increases as $s$ increases for small values of $s$. That is, the effects of the gradient of surface tension increase the burning velocity. Since viscous drag in the gas opposes the effects of surface-tension gradients, increasing the gas viscosity tends to decrease $m^{2}$. The solution for $s=0$ gives a value of $m^{2}$ approximately 
$\rho_{c} / \rho_{g}$ times that obtained with the alternative assumption that $u_{c}=u_{g}$. Therefore, pressuregradient effects, which then account for this velocity difference, also increase the burning velocity. The surface-tension gradient and pressuregradient influences both apparently can be quite significant in practice.

The influence of the interphase heat-transfer rate can be inferred from the last term in the expression for $\Lambda$, which was derived for the case $s=0$. Since this term is negative, $\Lambda$ is decreased (i.e., $m^{2}$ is increased) as $k$ is decreased (i.e., as the resistance to heat transfer increases). Physically, this occurs because the resistance to heat transfer causes the temperature in the condensed phase to exceed that of the gas phase in the reaction zone. This is expected because the resistance keeps more of the heat released in the condensed phase, where the reaction is occurring, thereby increasing the temperature and the reaction rate there. However, it may be inferred that this tendency for $m^{2}$ to decrease as the heat-transfer coefficient increases may not apply for all values of $b, \ell$ (the ratio of the gas-phase thermal conductivity to that of the condensed phase), and $s$. For example, if $\ell$ is sufficiently large, or if $b$ is sufficiently small, then the resistance to heat transfer causes the gas to become hotter than the condensed material (because of its higher thermal conductivity or lower heat capacity) through heat conduction from the burned products. The consequent reduction in temperature in the reaction material would then be expected to decrease $m^{2}$.

In summary, therefore, the results of our two-phase theory for the deflagration of solid propellants show that within the dispersedphase region, temperature and velocity differences between the two phases arise. In particular, the influences of pressure gradients and surface-tension gradients produce gas velocities in excess of liquid velocities. For steady, planar burning of the propellant, formulas for the burning rate, which were derived by the method of activation-energy asymptotics, show that there are a variety of influences of two-phase flow on solid-propellant deflagration which have been neglected by previous single-phase theories.

\section{References}

1. A. G. Merzhanov, "The Theory of Stable Homogeneous Combustion of Condensed Substances," Comb. Flame 13, 143 (1969).

2. S. B. Margolis, F. A. Williams, and R. C. Armstrong, "Influences of Two-Phase Flow in the Deflagration of Homogeneous Solids," to appear in Comb. Flame (1987).

3. E. I. Maksimov and A. G. Merzhanov, "Theory of Combustion of Condensed Substances," Combust. Explos. Shock Waves 2, 25 (1966).

\section{Percolation Model of Polydisperse Composite Solid Propellant Combustion $^{\dagger}$}

\section{A. R. Kerstein}

Local variations in the rate of advance of the burn front during the combustion of heterogeneous propellants may influence the overall burn rate. A new modeling approach has been formulated to represent this influence, thereby establishing a mechanistic basis for interpreting the measured composition dependence of the burn rate of composites. The composition dependence of the burn rate is predicted by analyzing the least-time path of the burn front through the propellant. The predicted burn rate is the average burn rate along the least-time path divided by the tortuosity, or arc length per streamwise interval, of the least-time path.

The composites considered here are polydispersions consisting of particles of $n$ different diameters $d_{1}<d_{2}<\cdots<d_{n}$ with volume fractions $f_{1}, f_{2}, \ldots, f_{n}$, respectively, whose sum is unity. The particles are assumed to have identical chemical composition, and the velocities $v_{1}>v_{2}>\cdots>v_{n}$ of the burn front through

\footnotetext{
$\dagger$ Submitted to Combustion and Flame (1986).
} 
the respective components are assumed to depend only on particle diameter. The latter inequalities reflect the experimentally observed ${ }^{1}$ decrease of burn rate with increasing particle size in ammonium perchlorate (AP) composites.

The model is introduced by first neglecting tortuosity considerations. In this preliminary formulation, the least-time burning path is taken to be a straight line in the streamwise direction, i.e., parallel to the mean burn rate vector. We consider a segment of length $L$ along such a path, where $L \gg d_{n}$. On average, a total length $f_{1} L$ of the segment intersects particles of size $d_{1}$, and similarly for the other sizes. The time for the burn front to advance a distance $L$ is the sum of the time intervals required to burn through each of the respective components. For instance, a time $t_{1}=f_{1} L / v_{1}$ is spent traversing component 1 , and so forth. Therefore, the overall burn rate is

$$
V=L / \sum_{i=1}^{n} t_{i}=\left(\sum_{i=1}^{n} \frac{f_{i}}{v_{i}}\right)^{-1} .
$$

This formula will be applied to propellants for which the component volume fractions are known, but only the qualitative trends governing the component burn rates are known. A convenient functional form consistent with experimentally determined trends is

$$
v=v_{\infty}\left(1+k e^{-d / d_{o}}\right)
$$

which contains three adjustable parameters. (The burn rate $v_{j}$ of the $j$ th component is obtained from equation (2) by substituting $d=d_{j}$ on the right hand side.) Complications associated with binder chemistry, etc., are subsumed within this phenomenological expression.

The parameters in this preliminary model, and in the percolation model discussed shortly, are assigned by fitting the models to burn rate measurements reported by Miller. ${ }^{2}$ Miller measured the burn rates of a suite of 29 AP propellants, each of which was a polydispersion of two or more particle sizes selected from a total of 8 available sizes. Here, his measurements at a pressure of 1000 psi are analyzed. The best fit of the preliminary model to Miller's data, corresponding to parameter values $v_{\infty}=0.97 \mathrm{~cm} / \mathrm{sec}$, $k=6$ and $d_{o}=30 \mu$, is shown in Figure 1 .

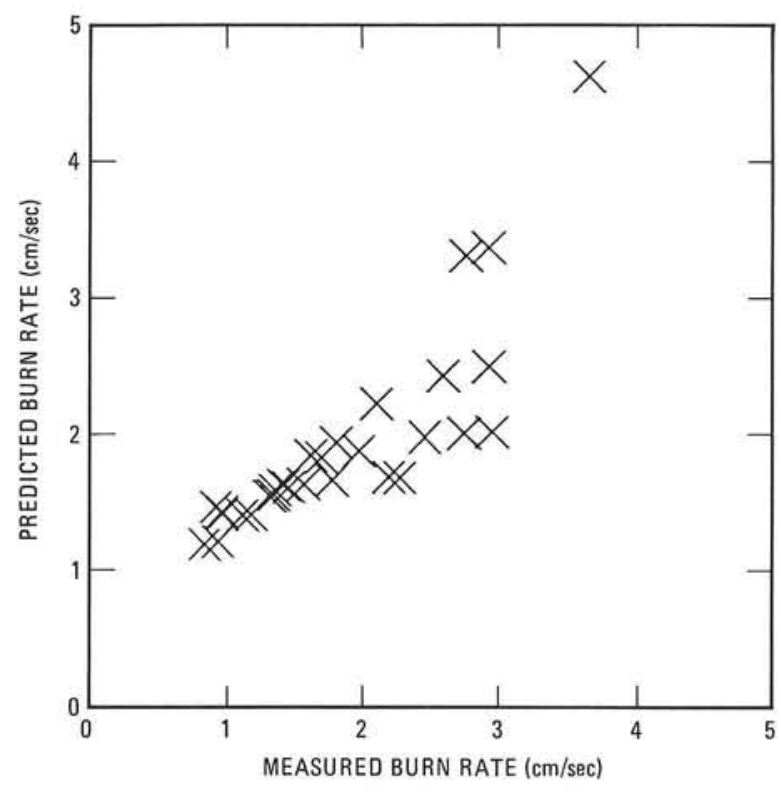

Figure 1. Burn rate predicted using the preliminary model versus experimental burn rate at a pressure of 1000 psi, measured by Miller. ${ }^{2}$

The model is augmented to include tortuosity by invoking percolation theory, ${ }^{3}$ which addresses the geometry of connected paths through irregular structures. In particular, percolation theory predicts that a two-component propellant with component burn rates $v_{1}>0$ and $v_{2}=0$ will burn without extinction only if $f_{1}>p_{c}$, where the numerical value of the "percolation threshold" $p_{c}$ is geometry-dependent. For $f_{1}>p_{c}$, application of the theory to the burn-rate problem gives the approximate expression

$$
\frac{V}{v_{1}}=\left(\frac{f_{1}-p_{c}}{1-p_{c}}\right)^{0.31}
$$

for the overall burn rate $V$, where the right hand side is a tortuosity factor.

An approximate method has been formulated to generalize equation (3) for application to $n$-component polydispersions. To apply the method to Miller's data, equation (2) is again adopted to relate $v_{j}$ to $d_{j}$. Since $p_{c}$ is not known a priori, it is an additional adjustable parameter. The best fit to Miller's data, shown in Figure 2, is obtained for parameter values $v_{\infty}=0.78 \mathrm{~cm} / \mathrm{sec}, k=6, d_{o}=30 \mu$, and $p_{c}=0.5$. 


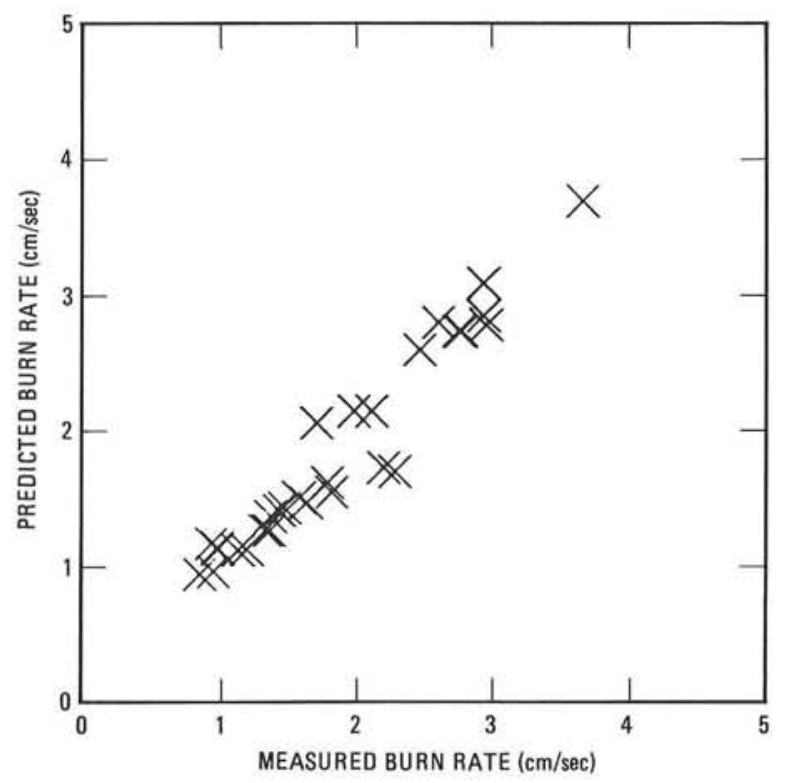

Figure 2. Burn rate predicted using the percolation model versus experimental burn rate at a pressure of 1000 psi, measured by Miller. ${ }^{2}$

In addition to achieving a markedly improved fit, the percolation model yields a value of $v_{\infty}$ which agrees with the value $0.8 \mathrm{~cm} / \mathrm{sec}$ of the bulk-AP burn rate measured ${ }^{1}$ at 1000 psi.

\section{References}

1. J. D. Hightower and E. W. Price, "Combustion of Ammonium Perchlorate," Eleventh Symposium (International) on Combustion (The Combustion Institute, 1967), p. 463.

2. R. R. Miller, "Effects of Particle Size on Reduced Smoke Propellant Ballistics," AIAA Paper No. 82-1096 (1982).

3. D. Stauffer, Introduction to Percolation Theory (Taylor and Francis, London, 1985). 

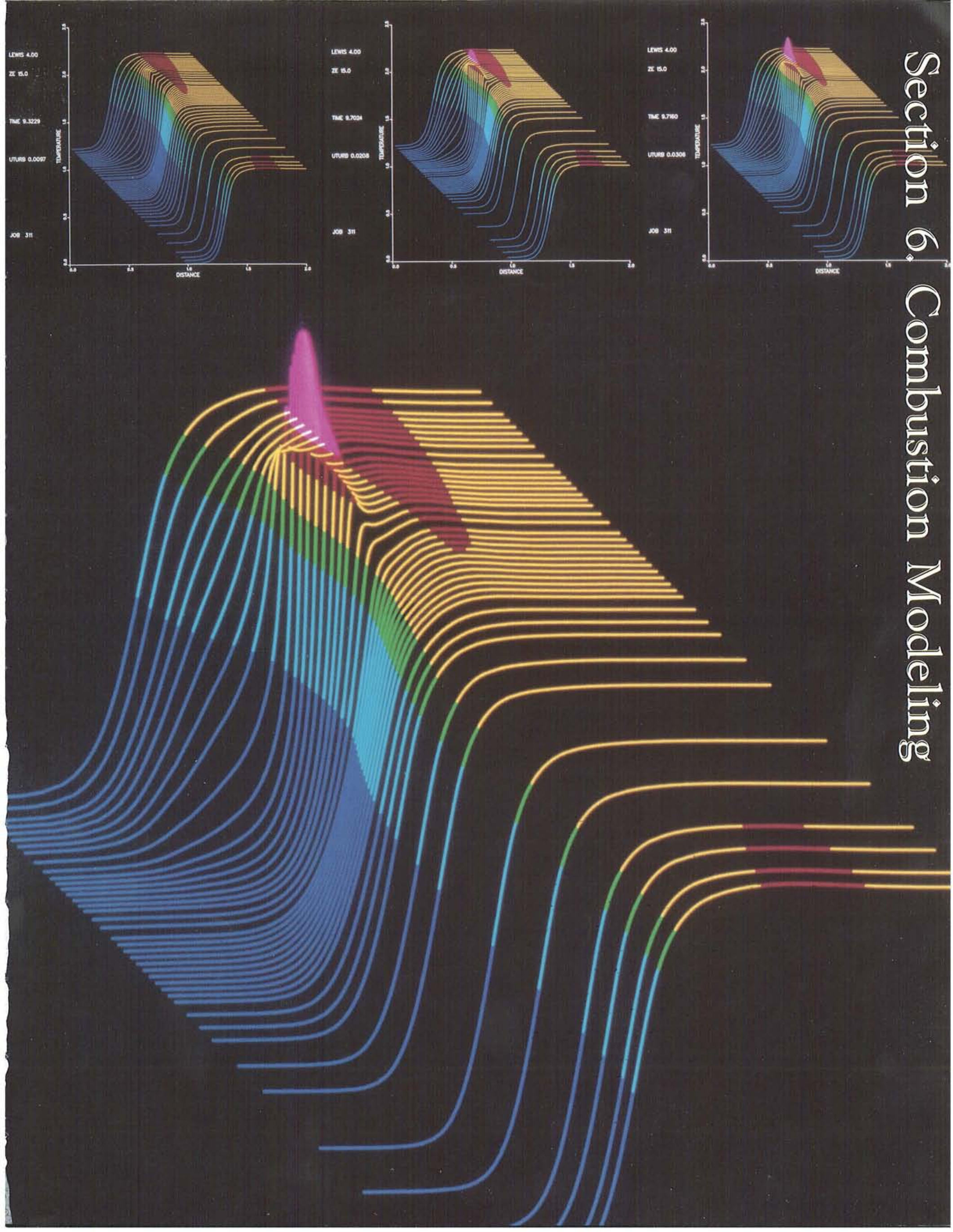
The computer simulation of combustion flows provides a unique opportunity for making controlled variations of modeled parameters. Shown on the preceding page is the perspective view of temperature in a calculated turbulent, two-dimensional premixed flame. The top pictures from left to right show the time evolution of a prominent hot spot, where the thermal diffusion has been made four times larger than the mass diffusion. Such a case leads to transient hot spots and a pulsating flame velocity. We compare the calculated turbulent reaction rate to that of a planar flame and find that differential diffusion can decrease or increase the reaction rate depending upon whether the ratio of diffusion rates is greater or less than unity (see related article, page 6-4). 


\section{Section 6 Combustion Modeling}

The combustion modeling programs at Sandia National Laboratories emphasize the application of innovative mathematical and numerical approaches to predict the mutual influences of reactions and fluid transport mechanisms. Most combustion applications include, in addition to chemical kinetics, complex fluid dynamics associated with a combination of multidimensional geometries, unsteady conditions, moving boundaries, and two-phase transport. Our current modeling research programs attempt to provide a fundamental understanding of these subprocesses so that future simulations will contain more realistic chemical and physical descriptions of combustion phenomena. The areas selected for study are strongly influenced by ongoing experimental research activities, in which direct comparison with data from well-characterized experiments verify and extend our combustion models.

A strong emphasis of the modeling programs supported by the DOE Office of Basic Energy Sciences is the numerical simulation of combustion processes. Since current computational limitations prevent a detailed analysis of both fluid mechanics and chemistry, the research projects tend to focus on one of these aspects. Reacting turbulent and two-phase flows emphasize the complex nature of fluids transport with simple-heat release, while the CHEMKIN family of codes provides users with comprehensive chemical kinetics and molecular transport capabilities.

\section{Vortex Simulation of Unsteady Wrinkled Laminar Flames ${ }^{\dagger}$}

\section{W. T. Ashurst}

Premixed flames stabilized by a flame holder in a turbulent flow are easy to create in the laboratory and have a long history in the literature. Early experimental investigations indicated that the flame motion has almost no dependence on the turbulence. These conclusions, however, must be restricted to those conditions under which the experiments were conducted: turbulent velocity fluctuations comparable to laminar flame speed and much less than the mean flow velocity and with turbulence length scales much greater than laminar flame thickness - in other words, a wrinkled laminar flame.

$\dagger$ To appear in Comb. Sci. Tech. (1987).
Two recent experimental investigations have shown the effect of low intensity turbulence on a premixed flame. In the first experiment, Rajan, Smith, and Rambach ${ }^{1}$ measured density along a line through the flame brush and showed that flame thickness remains at its laminar value. The apparent thickening of the flame with increasing distance from the flame holder is due to the flapping motion of the thin flame. In the second experiment, Cheng ${ }^{2}$ seeded the flow with oil droplets and solid particles in order to measure conditioned velocity statistics; i.e., he split the velocity data into burnt and unburnt values and showed that the apparent (unconditioned) turbulent shear stress is actually an artifact of flame motion.

Stimulated by this experimental information, we have performed a discrete vortex simulation of the V-shaped flame, in which the turbulence intensity is represented in a stochas- 
tic fashion by adding discrete vortices to the freestream flow. The flame is assumed to be of zero thickness and the flame speed is a specified function of flame curvature. The flame location is described by $x, y$ coordinates parameterized by arc length. Discrete volume sources were located along the flame in order to generate the proper density change. While the modeling assumptions are many, the computed motion of the flame produces velocity statistics that are in good agreement with Cheng's experimental values.

Examination of the mean velocities in the flame region shows that the hot products move faster than the cold reactants, and the angle of the flow is towards the midplane for products and away from the midplane for reactants. The conditioned turbulence quantities do not show the large rise in the flame region as is found in the unconditioned signals. It is the intermittent motion of the flame which generates the apparent turbulence. Therefore, time-averaged Eulerian information obtained without knowledge of the flame location is very misleading and the flame appears turbulent. Lagrangian information with respect to the flame reveals the dual nature of the flow. We now see that previous unconditioned velocity measurements have contributed to misleading time-average turbulence models.

We now describe in more detail the effects of flame motion on the velocities at a fixed space location. Figure 1 presents three views of the left half of the symmetric V-shaped flame (shown by a solid line).

The velocity vectors of the discrete vorticity are shown by scaled lines. We note in particular the increased velocity and the turning of the vortex trajectory for those vortices near the flame. The velocities are correlated with the flame location and its passage: flow is away from the midplane when unburnt and towards the midplane when burnt.

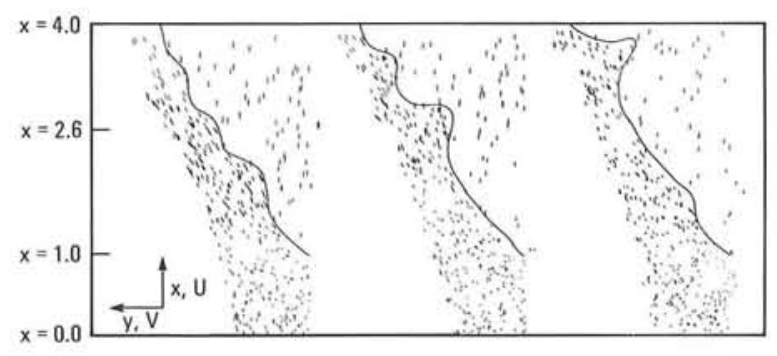

Figure 1. Discrete vorticity and flame sheet at three times, separated by a unit time (which equals unit distance divided by freestream velocity) with time increasing from left to right. Only the left half of the symmetric V-shaped flame is shown, the flame holder is at $x=1.0$ and the shear stress profile of Figure 3 was calculated at $x=2.6$. The flame sheet is composed of straight line segments with about 150 used in each picture. The formation of the cusp, prominent in the last picture, can be traced back in the earlier pictures and we find that this wrinkle in the flame sheet moves at the freestream velocity. The dash lines are scaled vorticity velocity vectors, and the acceleration across the flame with a turning towards the midplane can be seen. Because the flame is oblique to the freestream and the flow is not confined to a one-dimensional stream tube, the velocity jump at the flame is very much smaller than the density ratio effect produced in a one-dimensional flow - a factor of seven in this case.

Figure 2 is a scatter plot of velocity at one location within the flame brush region. For each discrete time-step, a dash is plotted in $U, V$ velocity space. The dash is horizontal if the gas is burnt and vertical if unburnt. In the scatter plot we see that the burnt and unburnt signals divide into two regions. The tic-marks along the axes represent conditioned and unconditioned velocity averages. With respect to the unconditioned mean, the burnt gas has a larger $U$ velocity and a smaller $V$ velocity with the opposite true for the unburnt gas. From the scatter plot, we see that the data falls mostly into the second and fourth quadrants formed by the unconditioned mean velocities. A cross correlation of all the data yields the unconditioned turbulent shear stress $\left\langle u^{\prime} v^{\prime}\right\rangle$, and, from the data location, this shear stress will be negative. This result is in agreement with Cheng's experimental results and, as he points out, implies counter-gradient 


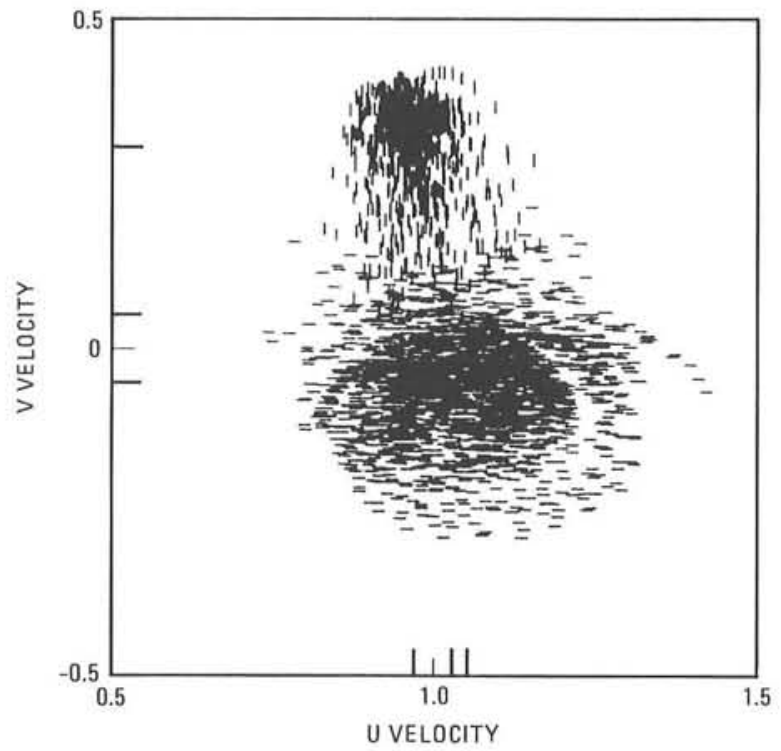

Figure 2. Scatter plot of burnt (horizontal dash) and unburnt (vertical dash) velocity signals. Ticmarks along the axes represent conditioned and unconditioned velocity averages. With respect to the unconditioned average velocities, the burnt and unburnt data lie in the second and fourth quadrants which produces a negative $\left\langle u^{\prime} v^{\prime}\right\rangle$ correlation for the turbulent shear stress.

transport since the signs of both mean velocity gradients produce a positive shear stress.

As in Cheng's results, the conditioned shear stresses are an order of magnitude smaller than the unconditioned. The profile of shear stress across the flame brush region is shown in Figure 3. In these figures the unconditioned values are the solid squares and are connected by a line; the conditioned unburnt values are the solid circles and the conditioned burnt values are the open triangles.

Comparison of fluctuation velocities with experiment is quite good and indicates that the flame dynamics have been duplicated: unconditioned shear stress peak is 0.013 for both experiment and calculated, $u^{\prime}$ is $0.128_{\text {exper }}$ and $0.118_{\text {calc }}, v^{\prime}$ is $0.164_{\text {exper }}$ and $0.166_{\text {calc }}$ which gives a kinetic energy of 0.021 in both cases. We find that the mean unconditioned velocities represent a weighted average between the conditioned values, which have an almost constant

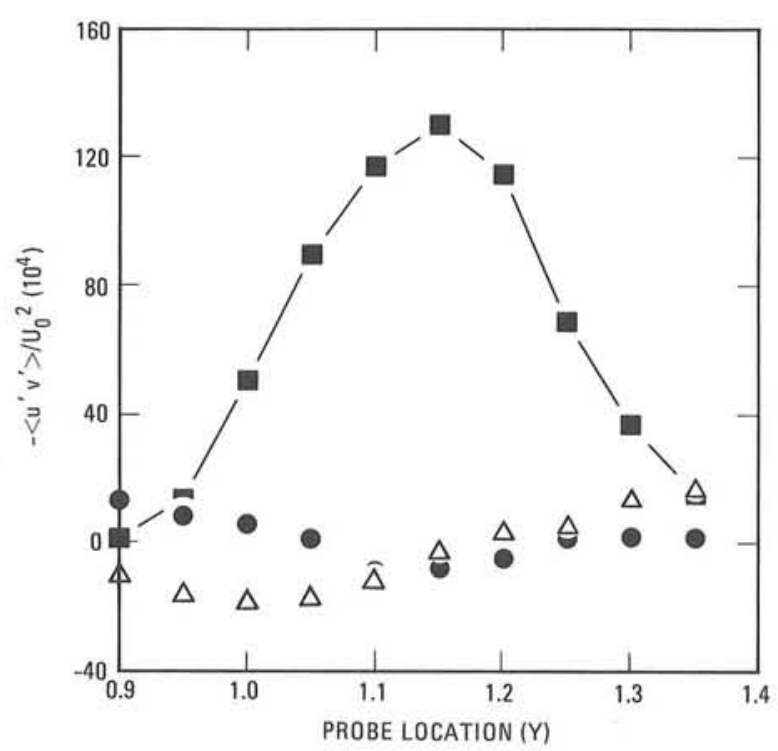

Figure 3. Time-average turbulent shear stress across the flame brush region: unconditioned (solid squares), unburnt (solid circles), and burnt (triangles). The conditioned shear stresses are essentially zero and the apparent shear stress in the unconditioned data is produced by the intermittent flame motion.

difference across the flame region. This constant difference explains the unconditioned shear stress, since the shear stress can be expressed by three terms:

$$
\begin{array}{r}
\left\langle u^{\prime} v^{\prime}\right\rangle=(1-\Omega)\left\langle u^{\prime} v^{\prime}\right\rangle_{r}+\Omega\left\langle u^{\prime} v^{\prime}\right\rangle_{b}+ \\
\Omega(1-\Omega)\left(U_{b}-U_{r}\right)\left(V_{b}-V_{r}\right)
\end{array}
$$

where the subscript $r$ is the conditioned unburnt reactant values and $b$ is the conditioned burnt values and $\Omega$ is the time-average burnt fraction which varies from zero to unity across the flame region. In Figure 3, the shape of the unconditioned shear stress is consistent with the calculated $\Omega$ dependence, and the peak magnitude of the unconditioned shear stress is in good agreement with the difference in the mean velocities. 


\section{References}

1. S. Rajan, J. R. Smith, and G. D. Rambach, "Internal Structure of a Premixed Turbulent Flame Using Rayleigh Scattering," Comb. Flame 57, 95 (1984).

2. R. K. Cheng, "Conditional Sampling of Turbulence Intensities and Reynolds Stress in Premixed Turbulent Flame," Comb. Sci. Tech. 41, 109 (1984).

\section{Numerical Simulation of Turbulent Flame Structure With Non-Unity Lewis Number ${ }^{\dagger}$}

\section{W. T. Ashurst, N. Peters, ${ }^{*}$ M. D. Smooke**}

The description of premixed turbulent combustion differs fundamentally from that of nonreacting turbulent flow. If the Kolmogorov length is greater than the flame thickness, then combustion takes place in thin layers embedded within the turbulent flow field. These thin layers are generally referred to as flamelets. In diffusion flames, for instance, the structure of laminar diffusion flamelets can be described by two parameters: the conserved scalar and the scalar dissipation, conditioned at stoichiometric mixture. The scalar dissipation, which is proportional to the square of the scalar gradient, characterizes the instantaneous local residence and diffusion time scales and can also be related to local stretching of the flame surface.

In premixed flames, the situation is more complicated: the position of the flamelet is not

$\dagger$ SAND86-8898 (1986); also, to appear in Combustion Science and Technology (1987).

* Institut fur Allgemeine Mechanik, Aachen, West Germany.

** Yale University, New Haven, CT. restricted to the vicinity of the surface of stoichiometric mixture, but the flame front propagates relative to the flow field. The flame velocity relative to the flow depends on local conditions and the interaction with the flow. There have been attempts to construct flamelet models for premixed flames, but they have ignored the possible changes in the internal flame structure due to turbulent flow induced strains. From analysis, we know that the local premixed flame speed depends strongly on flame stretch (flow divergence and/or flame surface curvature) when combined with differential diffusion between temperature and the deficient species; this is expressed by the Lewis number (ratio of thermal to mass diffusivity) being different from unity.

In this study, we numerically investigate the response of a premixed flame to various randomly defined flow fields, and gather statistics under conditions for which a theoretical understanding exists. We restrict ourselves to a onestep kinetic model with a large, but finite activation energy. To simplify the computations and to avoid the influence of the additional effect of thermal expansion, we assume the density to be constant and thus focus entirely on the Lewis number effect. Differential diffusion of energy and mass in a nonuniform flow introduces a change in the enthalpy which, in turn, leads, due to the large activation energy, to a change in the flame velocity. The enthalpy is therefore the second relevant scalar variable which appears in addition to the progress variable, on which the Bray-Moss-Libby formulation of premixed turbulent flames is based. A formulation similar to ours but for heat loss and unity Lewis number was recently derived by Bray et al. ${ }^{1}$

A Lagrangian-calculated velocity field is combined with an Eulerian description of scalar transport and reaction, on an adaptive mesh with implicit time-integration, to describe premixed flame structure in a two-dimensional mixing flow. Flames with Lewis numbers $(L e)$ of $1 / 2$ and 2 have been calculated. For comparison purposes, we first calculated the one-dimensional flame structure, and Figure 1 presents the enthalpy $h$ in terms of the progress variable $c$. 


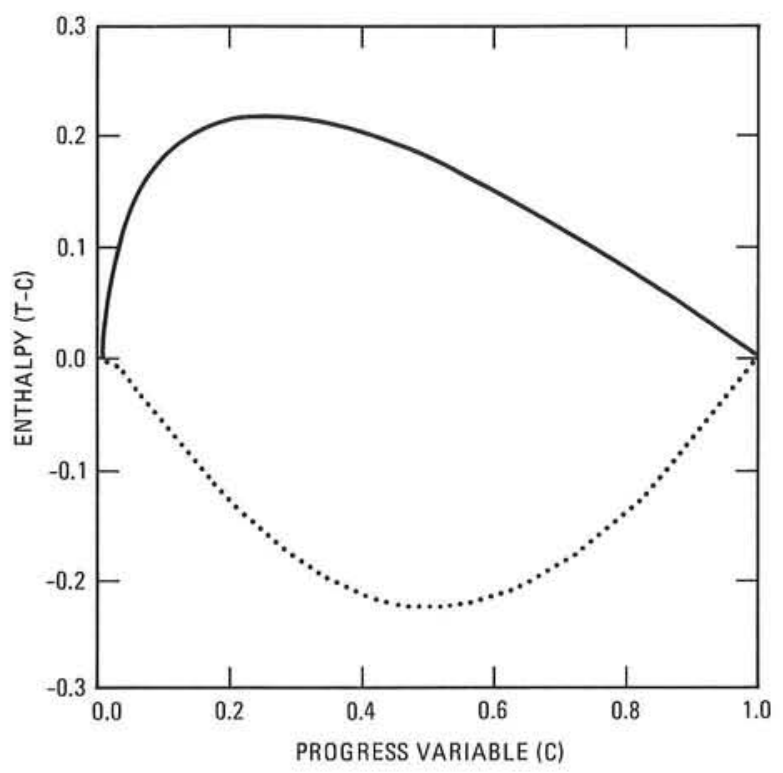

Figure 1. Calculated enthalpy of a plane laminar flame as a function of the progress variable for Lewis number of 2 (solid line) and 1/2 (dashed line).

Predictions from stability theory for the constant-density approximation give two different behaviors for non-unity Lewis number flames.

In the case of Lewis Number less than one, diffusion of the reactant from the preheat zone into the thin reaction zone is larger than heat conduction out of the reaction zone into the preheat zone. In those parts of a perturbed flame front which are convex towards the unburnt mixture, both scalar gradients will increase in the preheat zone, but the relative effect is larger for the transport of the reactant. Therefore, the enthalpy in the reaction zone will increase in this part of the flame, leading to an increase of the local flame velocity. Since the opposite is true for concave parts of the flame front, any initial perturbation of the front is increased, and this thermo-diffusive instability mechanism is believed to provide an explanation of the observed cellular structure of flames. We do find a cellular pattern in our results.

In the case of Lewis Number larger than one, heat conduction out of the reaction zone into the preheat zone is larger than diffusion of the reactant into the reaction zone. From similar arguments, as above, it follows that the flame velocity in a concave part of the flame front will increase, while it will decrease in a convex part, with respect to the unburnt mixture. Therefore, initial perturbations of a plane flame front will be reduced, leading to a stable configuration. However, at large Lewis numbers, the enhanced heat conduction from the reaction zone builds up the enthalpy in the preheat zone. The flame will eventually propagate into this higher enthalpy region and speed up. This leads to an oscillatory or pulsating instability mechanism, and we do observe this effect in our simulations.

To display the behavior of our results, contour lines of the progress variable and the excess enthalpy for one particular realization in each of the Lewis number cases are shown in Figures 2 and 3. The excess enthalpy $h^{*}$ is defined as the local enthalpy minus the enthalpy of the steady planar flame at the same progress variable value $h^{*}=h-h_{\text {planar }}$.

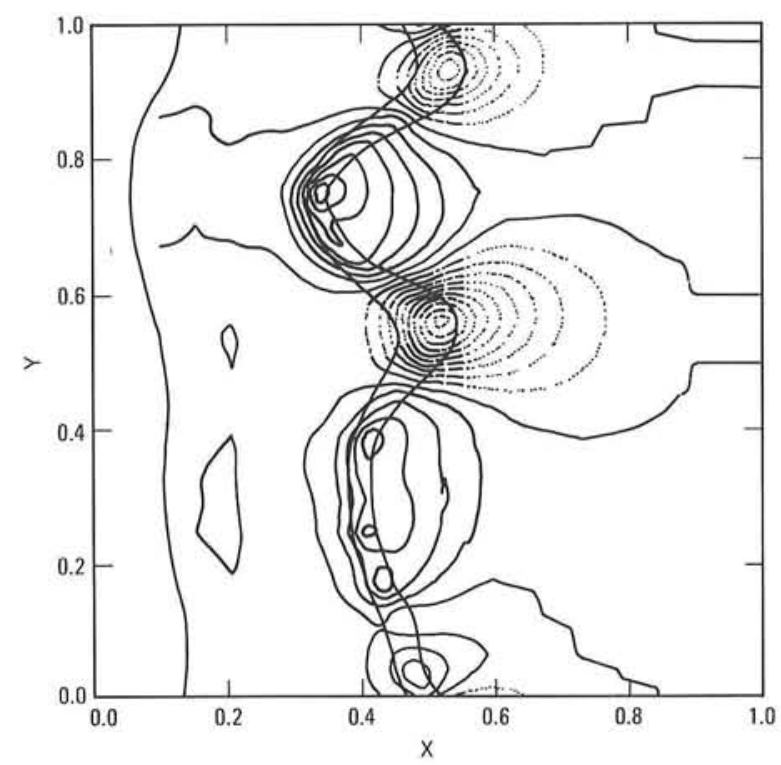

Figure 2. Contours of the progress variable, $c$ $=0.50$ and 0.99 , and contour lines of the excess enthalpy $h^{*}$ for one realization of a Lewis number of $1 / 2$ flame; positive $h^{*}$ values (including the zero value) are solid lines and negative values are dotted lines. The spacing between contour lines is $\mathbf{0 . 0 1 5}$ and the minimum value in the middle of the picture is less than $\mathbf{- 0 . 1 4}$. 
In the case of $L e$ of $1 / 2$, cellular instability is demonstrated. Figure 2 shows contours of the excess enthalpy together with the progress variable contours. Local maxima of the excess enthalpy are found in the leading parts of the flame front, while two well pronounced minima appear in the downstream troughs of the cellular pattern. This clearly demonstrates the cellular instability that has been discussed above.

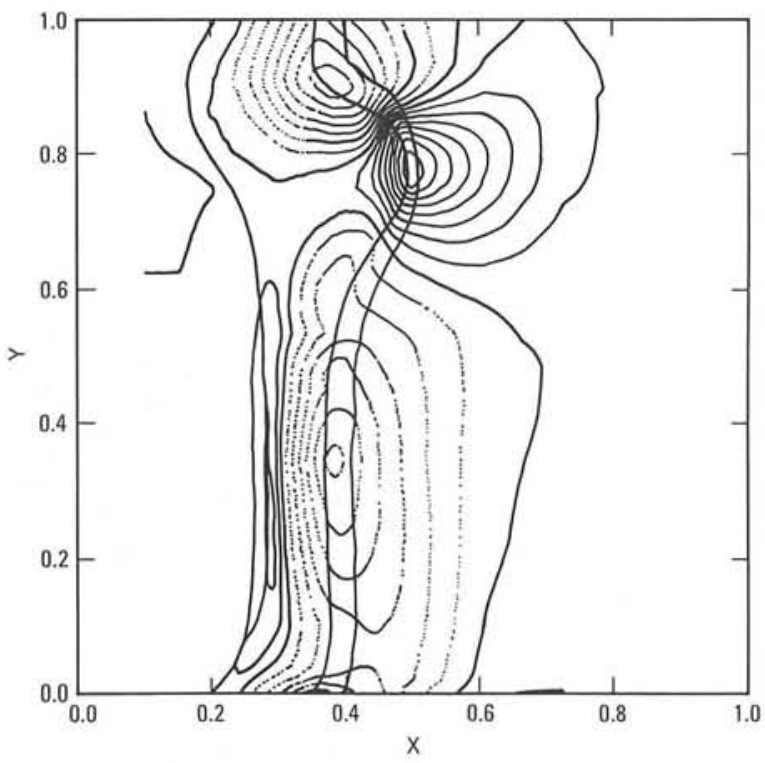

Figure 3. Lewis number of 2 flame: progress contours, $c=0.50$, and 0.99 , and contour lines of the excess enthalpy $h^{*}$; positive values (including the zero value) of $h^{*}$ are solid lines and negative values are dotted lines. The spacing between contour lines is $\mathbf{0 . 0 1 5}$.

With a $L e$ of 2, stabilization of flow field disturbances is noted. In comparison, the flames with Lewis number greater than unity are much smoother. In the upper part of Figure 3, the flame front has been pushed downstream by a large local velocity, leading to a negative curvature of the front. Due to the thermo-diffusive effect, a local maximum of the excess enthalpy builds up in the reaction zone in this part of the front. The excess enthalpy contour plots for later times show that this "hot spot" advances very rapidly with respect to the remaining part of the front. Notice also that ahead of the lower part of the flame front, where the reaction zone has negative excess enthalpy (dotted contour lines), there exists a region of positive excess enthalpy. This illustrates the effect of enthalpy deposition in the preheat zone due to differential diffusion.

To obtain a statistical description of enthalpy, we have sampled our data in terms of an excess enthalpy, $h^{*}=h-h_{\text {planar }}$, both enthalpies being at the same progress variable value, and find the resulting probability distribution functions to be independent of the value of the progress variable when $c \geq 0.8$. From this distribution, we can define a reaction-rate ratio; that is, we can compare the turbulent flame reaction rate with that of a plane flame. These ratios indicate a suppression of reaction rate for Lewis number greater than unity, and enhanced reaction for Lewis number less than unity. The values are 0.81 and 1.11 , respectively.

\section{Reference}

1. K. N. C. Bray, M. Champion, N. Dave, and P. A. Libby, "On the Thermochemistry of Premixed Turbulent Combustion in Variable Enthalpy Systems," Comb. Sci. Tech. 46, 31 (1986).

\section{Numerical Study of Vortex Reconnection ${ }^{\dagger}$}

\section{W. T. Ashurst, D. I. Meiron *}

There is currently a great deal of interest in the dynamics of vortex tubes. One wellknown method of approach is the representation of a tube as a space curve. The Biot-Savart law is then used to generate the velocity field that is used to convect the tube. In order to apply this method, assumptions are made about the core structure of the tube. Usually, it is assumed that the core remains circular and that there is no axial flow inside the core,${ }^{1}$ although more

† Sandia Report SAND86-8880 (1986); also, to appear in Phys. Rev. Lett. (1987).

* California Institute of Technology at Pasadena. 
elaborate models have been considered. It has been shown that the core will be approximately circular provided that the tubes are separated by distances that are large compared to the tube diameter.

When these approximations are violated, experiments have shown that the core dynamics become significant. There have been several experiments reported in which two vortex rings traveling in the same direction become close enough to each other that in the region of closest approach there is a reconnection of the vortex tubes and the two rings become one contorted ring. ${ }^{2-4}$ Further interaction may occur that fissions the ring into two, each made up of one-half of the original rings. This reconnection process cannot be captured by vortex methods that use continuous filaments for a description of inviscid flow, since without viscosity the vortex tube retains its identity for all time. In order for reconnection to occur, dissipative effects must be considered.

Using a Biot-Savart model of vortex filaments to provide initial conditions, a finite difference scheme for the incompressible NavierStokes equation is used in the region of closest approach of two vortex rings. In the NavierStokes solution, we see that the low pressure which develops between the interacting vorticity regions causes the distortion of the initially circular vortex cross section and forces the rearrangement of vorticity on a convective time scale which is much faster than that estimated from viscous transport.

Figure 1 shows the calculated evolution of two vortex rings which are traveling in the same direction when the response is restricted to circular vorticity cross-sections.

In the region of closest approach between the two rings, the axial stretching reduces the vortex cross-section, and hence increases the local-induced velocity by a factor of ten in the last view shown. We have taken the Lagrangian inviscid results at time-step 150 (first view in Figure 1) as the initial conditions for our NavierStokes simulation. A unit cube, with $32^{3}$ node points, is centered about the closest approach region and is used to describe the pressure field and an associated staggered grid for velocities. The vortex filament tangent vectors and incremental arclengths were used to generate the vector potential at appropriate locations so that the grid velocities are divergence free. From two time levels of the velocity field, using a backward Euler time derivative, we obtain the pressure gradients from the finite-difference momentum equations. Thus, the velocities and pressures generated by the Biot-Savart law are used as initial conditions for the Navier-Stokes solution.

We integrate the Navier-Stokes equation with a time step of $2 \times 10^{-4}$ and with a Reynolds number $(\Gamma / \nu)$ of $10^{3}$, where $\Gamma$ is the ring circulation and $\nu$ is the kinematic viscosity. We exploit the fact that the outer ring region does not appear to move during the evolution shown in Figure 1, and keep the cube boundary conditions of velocity and normal pressure gradient constant. We find that the reconnection process is completed within a small enough time interval so that these surface constraints for this volume size are not a problem.

We exhibit the reconnection process given by the Navier-Stokes solution by showing the calculated grid vorticity in Figure 2.

The ring shown in Figure 1 appears on the right side of Figure 2. The time interval of the NavierStokes calculation corresponds to the interval between steps 150 and 250 in the Biot-Savart calculation. Instead of continual core collapse shown in Figure 1, we obtain a topological change in the vorticity field with apparently little variation of core size along the newly connected filaments.

We also performed simulations at Reynolds numbers of $10,100,1,000$ and 5,000. The latter two do not appear to differ in contour plots of the solution, indicating that we may have reached the inherent numerical diffusion level in this scheme. With a Reynolds number of 100 , the reconnection still occurs, although the vorticity contours indicate more diffusion than in the higher Reynolds number case. The simulation at a Reynolds number of 10 is completely 

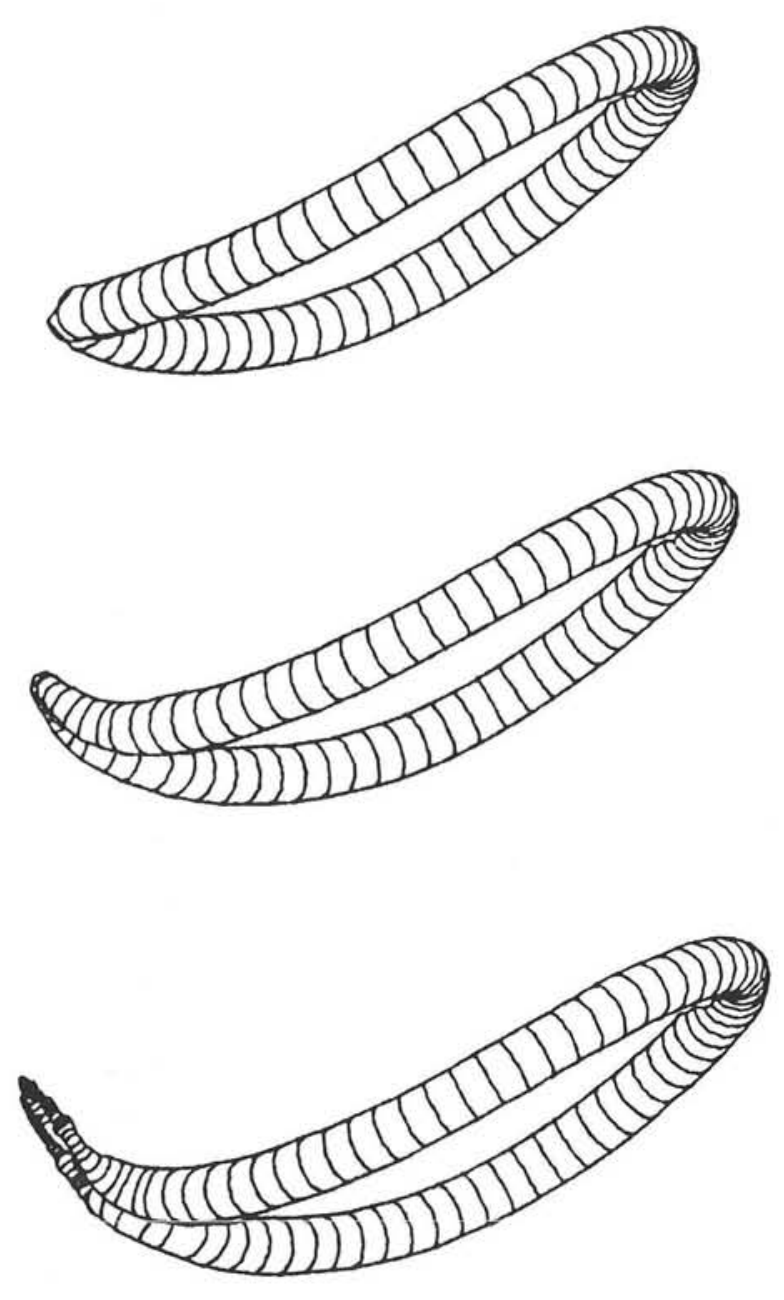

Figure 1. Lagrangian calculation using vortex tubes with round cross section to simulate interacting vortex rings. Perspective view of one vortex ring during its interaction with a mirror image ring (not shown) on the left. The observer is above the path of the approaching rings. The initial ring radius is unity, and the ring separation in the top view is 0.14. Time increases from top to bottom; time steps 150,200 , and 300 are shown; the time values are $0.1875,0.2091$, and 0.2134 . The arcs drawn on the tube are located at the filament node points and reveal the insertion of new nodes with axial stretching.

different; vorticity diffusion and cancellation is so large that vorticity disappears before it can be rotated to form reconnected tubes. Thus, we estimate that when the viscous time scale is a hundred or more times longer than the convective time scale then tube reconnection occurs.
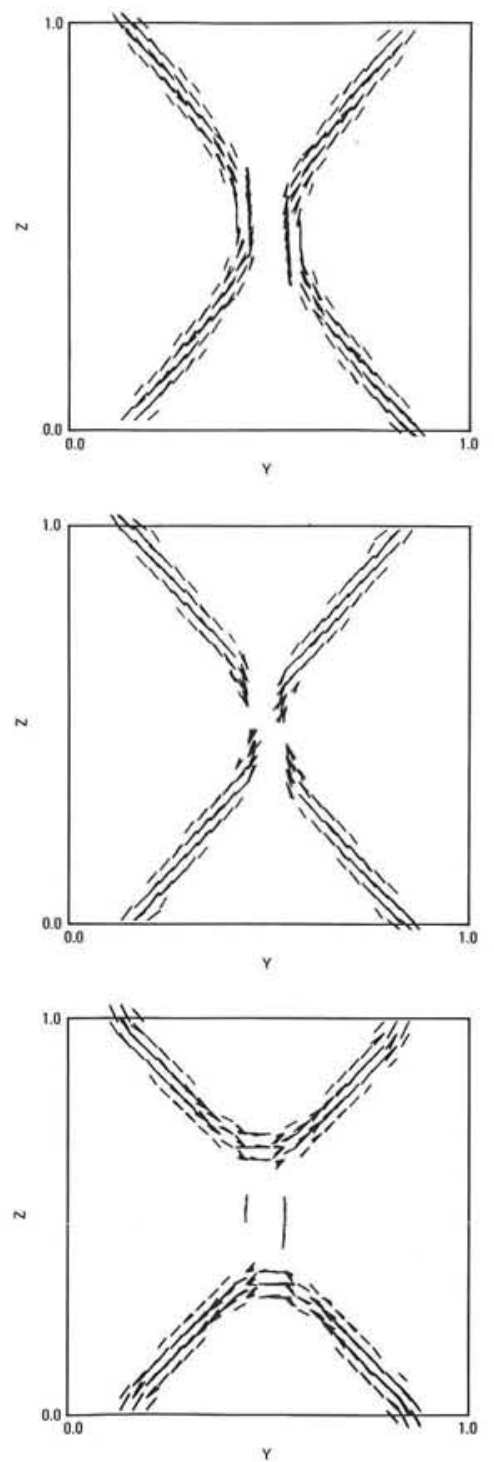

Figure 2. Navier-Stokes calculation of the reconnection of interacting vortex tubes in the region of closest approach. The view is along the $\mathrm{x}$ axis. Grid locations which have vorticity magnitude greater than 0.6 of the maximum vorticity are shown by drawing a scaled vorticity vector (maximum length is $1 / 16$ ). The times shown, from top to bottom, are $0.004,0.012$, and 0.024 from the start of the Navier-Stokes calculation with a constant time-step of $2 \times 10^{-4}$.

We infer a flow pattern by which the yortex tubes reconnect by examining the $z$ vorticity in the $x-y$ symmetry plane in the simulation for Reynolds number of 1,000 . Near the $z-y$ symmetry plane there is a line of node cells in which $d V / d y$ has a very large negative value, and both $d U / d x$ and $d W / d z$ are positive 
and comparable in value. Thus, the $z$ component of vorticity is reduced by a squeezing effect, which rotates the vorticity into another direction. This squeezing motion is caused by static pressure differences that occur when two vorticity regions of opposite sign approach each other, since they will induce a local maximum velocity between themselves, and hence a low pressure. The convective motion which results from this low pressure should scale by vorticity area $\left(\sigma^{2}\right)$ and the amount of circulation contained within that area and not by a viscous time scale. So the time scale is $\sigma^{2} / \Gamma$ rather than $\sigma^{2} / \nu$, and in our choice of parameters these values are $10^{-3}$ and 1.

Siggia and Pumir ${ }^{5}$ have shown that if one assumes that the cores remain circular when two vortex tubes approach one another, then the growth of the core due to viscosity cannot be maintained against stretching along the filament axis. Thus, the core area shrinks to zero in a finite time, resulting in a point singularity in the flow. It is, of course, impossible to make conclusive statements about singular behavior from numerical simulation of the Navier-Stokes equations, but it appears that the pressure gradients and concomitant velocities arising from the close approach of two tubes of oppositely signed vorticity are sufficiently high to disrupt the tube cores and allow rearrangement of the vorticity on a convective time scale.

\section{References}

1. D. W. Moore and P. G. Saffman, "The Motion of a Vortex Filament with Axial Flow," Philos. Trans. R. Soc. London Ser. A 272, 403 (1972).

2. P. Schatzle and D. Coles, Unpublished photographs and private communication.

3. T. Fohl and J. S. Turner, "Colliding Vortex Rings," Phys. Fluids 18, 433 (1975).

4. Y. Oshima and S. Osaka, "Interaction of Two Vortex Rings Along Parallel Axes in Air," Jour. Phys. Soc. of Japan 42, 708 (1977).

5. E. D. Siggia and A. Pumir, "Incipient Singularities in the Navier-Stokes Equations," Phys. Rev. Lett. 55, 1749 (1985).

\section{Simulation of "Tulip" Flame Formation in a Closed Vessel}

\author{
P. K. Barr
}

The formation of a "tulip" or cusped shaped flame during combustion in a closed duct has been simulated using a numerical model based on hydrodynamics. ${ }^{1}$ This flame phenomena, which has been observed experimentally for many decades, occurs during unsteady premixed laminar flame propagation in a constant volume duct. It has been observed under a variety of conditions, including different duct dimensions, ignition configurations, and fuel types. ${ }^{2}$ Since the numerical model is based on hydrodynamics, it does not include the effects of vorticity or pressure waves on the flame propagation. A comparison of the numerical predictions with laser Doppler anemometry measurements taken by Derek Dunn-Rankin, in collaboration with Robert Sawyer (University of California at Berkeley), shows close agreement, indicating that the hydrodynamics of the combustiongenerated flow is alone sufficient to cause a tulip flame.

We model the flame as a two-dimensional discontinuity separating the burned and unburned gases, which have different but spatially uniform densities. This assumption limits the calculations to low Mach number flows. In the computational model, a fixed numerical mesh records the location of the burned and unburned gas, and the flame interface is reconstructed from this information using a hybrid numerical scheme. ${ }^{3}$ This scheme is based on a combination of the Simple Line Interface Calculation method (SLIC) $)^{4,5}$ for advancement of the flame position due to either advection or self-advancement, and an algorithm from the Volume of Fluid method $(\mathrm{VOF})^{6}$ for determining the local flame orientation.

The simulation of the combustion process begins by propagating the flame normal to itself at a constant laminar burning velocity. Since combustion takes place in a closed volume, the chemical heat release causes a pressure rise. The 
pressure, which is assumed to be spatially uniform throughout the combustion chamber, rises linearly with the total amount of gas burned. The local expansion of the freshly burned gas and the global compression of the rest of the gas are treated as a set of volumetric sources and sinks that are located on the uniform computational grid. The solution to the resulting Poisson equation produces the velocity potential. This velocity field carries the flame in the advective motion step, and the process is repeated.

We restrict the computation to irrotational flow to show that vorticity is not required to generate a "tulip" flame. Since the flow field is defined by a velocity potential, no vorticity is included in the computations. The slip wall boundary condition insures that vorticity is not introduced at the walls, and the vorticity production at the flame front due to the density gradient crossed with the pressure gradient (the term $\nabla \rho \times \nabla P$ in the vorticity equation) is neglected.

The formation of a "tulip" flame with this two-dimensional planar numerical model is demonstrated in Figure 1, which presents a series of computer-generated movie frames separated by equal time intervals. The combustible mixture has the energy release and laminar flame speed of a stoichiometric methane/air mixture. The initial flame "kernel," shown in the top left frame, is prescribed since we do not include an ignition model. The succeeding frames of Figure 1, which include both the flame shape and velocity vectors at selected locations, show that the flame grows as an ellipse until it passes the center, at which time the flame reaches the side walls and the "tulip" appears. The "tulip" shape remains until the flame reaches the opposite closed end. The flame shape remains symmetric since there is no mechanism to create an asymmetry, although the solution is computed over the entire domain.

In the numerical results presented in Figure 1, as well as in experimental results, the "tulip" formation coincides with the flame arrival at the vessel side walls. By this time, the velocity at leading edge of the flame has begun to decrease as the flame approaches the closed end of the chamber, causing it to flatten.
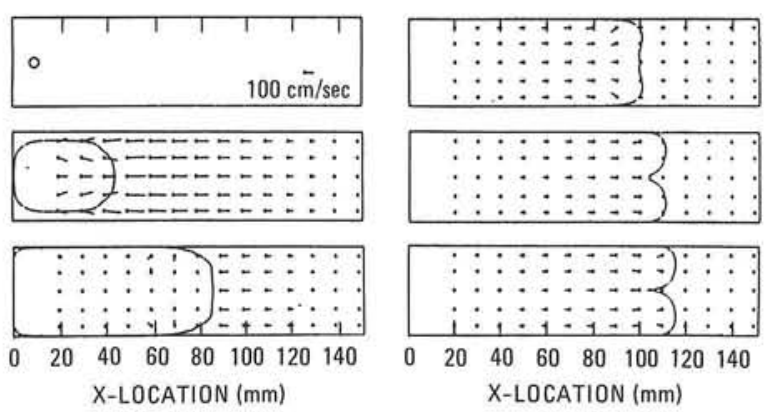

Figure 1. Numerical simulation of premixed flame propagation in a closed planar duct after line ignition. Time interval between frames is constant. Grid size is $40 \times 160$. Initial flame shape is shown in top left frame. Dots indicate the origin of the velocity vectors at selected locations.

When the flame reaches the walls a slight radial gradient in the axial velocity at the flame is produced due to the confinement of the chamber, as demonstrated in Figure 2. The radial gradient in the axial velocity results because the unburned gas, which is compressed by the expanding burned gas, is confined by the vessel sidewalls. The slightly faster flow near the walls pushes the flame ahead, creating a perturbation on the convex flame front. The continued growth of the "tulip" cusp is a result of the hydrodynamic instability of premixed flame fronts.

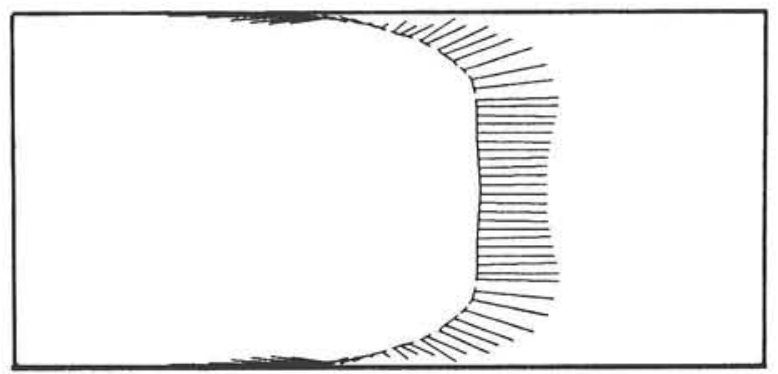

Figure 2. Velocity vectors along flame front before formation of a double-lobed "tulip." Vectors originate at the midpoint of the flame in each cell. Bulges near the wall initiate the "tulip" behavior. 


\section{References}

1. D. Dunn-Rankin, P. K. Barr, and R. F. Sawyer, "Simulation of "Tulip" Flame Formation in a Closed Vessel," Twenty-first Symposium (International) on Combustion (The Combustion Institute, 1986), in press.

2. D. Dunn-Rankin, The Interaction Between a Flame and Its Self-Generated Flow, $\mathrm{PhD}$ Thesis, University of California at Berkeley, 1985; also available as Lawrence Berkeley Laboratory Report $L B L-19582,(1985)$.

3. P. K. Barr and W. T. Ashurst, "An Interface Scheme for Turbulent Flame Propagation," Sandia Report SAND82-8773 (1984).

4. W. F. Noh and P. Woodward, "SLIC (Simple Line Interface Calculation)," Lecture Notes in Physics, Vol. 59 A. I. van de Vooren and P. J. Zandbergen, Eds. (Springer-Verlag, New York, NY, 1976), p. 330.

5. A. J. Chorin, "Flame Advection and Propagation Algorithms," J. Comp. Phys. 35, 1 (1980).

6. C. W. Hirt and B. D. Nichols, "Volume of Fluid (VOF) Method for the Dynamics of Free Boundaries," J. Comp. Phys. 39, 201 (1981).

\section{A Detailed Study of Burning Fuel Droplets ${ }^{\dagger}$}

\section{H. A. Dwyer, ${ }^{*}$ B. R. Sanders}

This article presents an investigation of the combustion behavior of individual fuel droplets in an air flowfield. This problem has been studied for many years, but modeling success has been limited because of the complexity of the many chemical and physical processes. The authors have previously studied the droplet vaporization problem without chemical reactions, ${ }^{1-5}$

$\dagger$ Twenty-first Symposium (International) on Combustion (The Combustion Institute, 1986), in press.

* University of California at Davis. and this work presents the first attempt to include global chemistry. The burning of individual droplets involves complex fluid mechanics, heat transfer, and chemical reactions, and the detailed behavior of these processes cannot be easily studied by analytical or experimental means. Therefore, there is a basic need to develop comprehensive numerical models that can aid the understanding of these basic processes through systematic studies isolating important individual parameters that often cannot be studied experimentally for small droplets. The method employed to study droplet combustion in this work is the detailed numerical solution of the equations of mass, momentum, energy, and species transport.

Droplet combustion computations have been performed over a relatively wide range of Reynolds numbers and chemical kinetic parameters. From these investigations, a detailed understanding has been gained of the flame structure and its interaction with the flowfield and surface mass transfer processes. The results of these computations show clearly that many of the assumptions made in the past, such as a thin flame surrounding the droplet, are not well founded, and that more accurate physical descriptions of individual droplet processes are needed to improve simple (practical) engineering models of droplet combustion. The present study shows that "numerical experiments" can describe with fine detail the complex problem of fuel droplet burning.

The fuel properties and physical parameters utilized in this study are those appropriate to n-decane. The solutions presented are restricted to spherical droplets near their boiling point without internal circulation. These simplifications allow for a more focused study of the influence of chemical kinetics. With the droplet near its boiling point, the surface mass transfer calculation is simplified considerably, and it is uniquely determined by the heat transfer. The boundary condition for the fuel species equation becomes a zero-gradient condition with the mass flux determined by the heat transfer. The steady-state solutions presented are consistent with experimental droplet studies utilizing 
a porous sphere to simulate a vaporizing droplet at constant Reynolds number and without internal circulation.

The equations of motion solved in this study are the following:

$$
\begin{array}{ccc} 
& \frac{\partial \rho}{\partial t}+\frac{\partial}{\partial x_{i}}\left(\rho v_{i}\right)=0 & \text { (Continuity) } \\
\frac{\partial}{\partial t}\left(\rho v_{i}\right)+ & \frac{\partial}{\partial x_{j}}\left(\rho v_{i} v_{j}\right) \\
= & -\frac{\partial P}{\partial x_{i}}+\frac{\partial}{\partial x_{j}}\left[\mu\left(\frac{\partial v_{i}}{\partial x_{j}}+\frac{\partial v_{j}}{\partial x_{i}}\right)\right] \\
- & \frac{2}{3} \frac{\partial}{\partial x_{i}}\left(\mu \frac{\partial v_{j}}{\partial x_{j}}\right) \\
\frac{\partial}{\partial t}\left(\rho C_{v} T\right) & +\frac{\partial}{\partial x_{j}}\left(\rho C_{p} v_{j} T\right) \\
= & \frac{\partial}{\partial x_{j}}\left(k \frac{\partial T}{\partial x_{j}}\right) \\
+ & \sum_{n=1}^{N} \Delta h_{n} \dot{w}_{n}^{\prime \prime \prime} \\
\frac{\partial}{\partial t}\left(\rho Y_{k}\right)+ & \frac{\partial}{\partial x_{j}}\left(\rho v_{j} Y_{k}\right) \\
= & \frac{\partial}{\partial x_{j}}\left(D_{k} \frac{\partial Y_{k}}{\partial x_{j}}\right) \\
+ & \Sigma_{n=1}^{N} C_{k n} \dot{w}_{n}^{\prime \prime \prime} \\
P=\rho R\left(Y_{k}\right) T & \text { (Energy) }
\end{array}
$$

The following notation has been employed: $\rho$-density, $v_{i}$-velocity components, $P$-pressure, $T$-temperature, $\mu$-viscosity, $k$-thermal conductivity, $D_{k}$-binary diffusion coefficient, $C_{v}$ and $C_{p}$-specific heats, $\Delta h_{n}$-heat of reaction, $\dot{w}_{n}^{\prime \prime \prime}-$ consumption rate of species $n, Y_{k}$-mass fraction, $C_{k n}$-stoichiometric coefficient, $t$-time, and $x_{i}-$ Cartesian coordinates.

The above equations were integrated using the rate expression for decane chemistry as reported by Westbrook and Dryer, ${ }^{6}$ where

$$
\dot{r}_{f}=-3.810^{11}\left[C_{10} H_{22}\right]^{1 / 4}\left[0_{2}\right]^{3 / 2} e^{(-30,000 / R T)}
$$

Figure 1 presents the isotherm pattern around a burning $\mathrm{n}$-decane droplet at a Reynolds number of ten. The maximum temperature zone does not fully enclose the droplet, although some heat release is occurring on the upstream side $(x / r<0)$. This is an intermediate case between envelope flame conditions, where a flame fully surrounds the droplet, and a wakestabilized flame for which little chemical energy is released on the upstream side of the droplet.

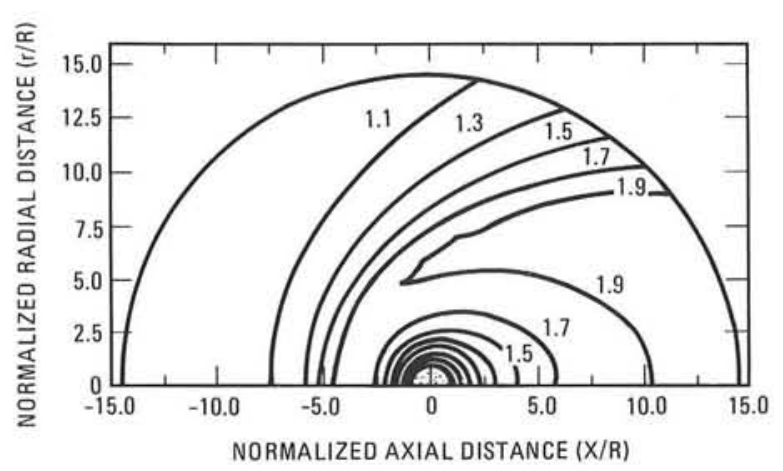

Figure 1. Isotherm pattern for burning droplet at $\mathrm{Re}=2$. Isotherms are values of $\mathrm{T} / \mathrm{T}_{\infty}$.

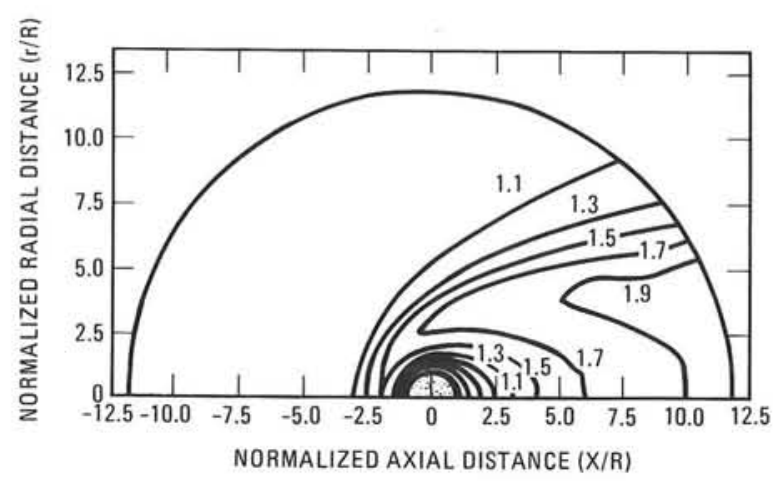

Figure 2. Isotherm pattern for burning droplet at $R e=10$. Isotherms are values of $T / T_{\infty}$.

The isotherm pattern for a Reynolds number of two is shown in Figure 2, and it can be seen that the reaction zone is spread over a region that is approximately 1000 times the volume of the droplet. For almost all pressures of practical interest, it would be difficult to achieve steady state before the droplet would have lost a significant portion of its mass. These results are for steady-state conditions, but the differences in flowfields and flame structure over the range of parameters studied suggests that quasi-steady droplet models may be in serious error. This follows from the fact that, at high initial Reynolds numbers, the velocity (hence Reynolds number) 
decays quickly, and the flame shape will transition from that of a reaction zone in the wake to a full or partial envelope flame. At small Reynolds numbers, the flames will be very diffuse relative to the diameter, and large amounts of fuel may accumulate between the surface and reaction zone.

\section{References}

1. H. A. Dwyer, R. J. Kee, P. K. Barr, and B. R. Sanders, "Transient Droplet Heating at High Peclet Number," J. Fluids Engineering 105, 83 (1983).

2. H. A. Dwyer and B. R. Sanders, "Detailed Computation of Unsteady Droplet Dynamics," Twentieth Symposium (International) on Combustion (The Combustion Institute, 1984), p. 1743.

3. H. A. Dwyer and B. R. Sanders, "Droplet Dynamics and Vaporization With Pressure as a Parameter," ASME 84-WA/HT-20 (1984).

4. G. Patnaik, W. A. Sirignano, H. A. Dwyer, and B. R. Sanders, "A Numerical Technique for the Solution of a Vaporizating Fuel Droplet," Proc. 10th International Colloquium on the Dynamics of Explosions and Reactive Systems (1985).

5. H. A. Dwyer, B. R. Sanders, and F. Raiszadek, "Ignition and Flame Propagation Studies with Adaptive Numerical Grids," Comb. Flame 52, 11 (1983).

6. C. K. Westbrook and F. L. Dryer, "Simplified Reaction mechanisms for the Oxidation of Hydrocarbon Fuels in Flames," Comb. Sci. Tech. 27, 31 (1981).

7. M. Renksizbulut and M. C. Yuen, "Numerical Study of Droplet Evaporation in a HighTemperature Stream," J. of Heat Transfer 105, 389 (1983).

\section{Interaction of Pulsating and Spinning Waves in Nonadiabatic Flame Propagation}

\author{
M. R. Booty,* S. B. Margolis, B. J. Matkowsky *
}

Nonsteady propagation of reaction fronts in premixed combustion is known to occur in both gaseous and condensed systems. Such modes of propagation often arise as bifurcations from a steady mode of burning as one or more parameters pass through critical values. ${ }^{1-2}$ In the case of premixed gaseous combustion, which is the subject of the present work, ${ }^{3}$ there are two types of instabilities, in the context of a weak thermal expansion theory, which can modify an otherwise steady, planar mode of propagation. They arise from the competing effects of thermal and mass diffusion, and occur for sufficiently low or sufficiently high values of the Lewis number, which is defined as the ratio of thermal to mass diffusivity. The first of these is a cellular instability, which occurs as a steady bifurcation for Lewis numbers less than a critical value which is less than unity. The other, with which we will be concerned here, is a pulsating instability that occurs as a Hopf bifurcation for Lewis numbers greater than another critical value which is greater than unity. ${ }^{4}$

Previous bifurcation analyses have treated the adiabatic problem. That is, the effects of heat losses, which occur through conduction and radiation, were neglected. However, the experimental studies which have corroborated nonsteady flame propagation in gaseous mixtures have been performed on nonadiabatic systems. For example, the spinning propagation of acetylene flames in long cylindrical channels ${ }^{5}$ was apparently accompanied by radiative losses due to sooting. Thus, in the present work ${ }^{3}$ we study the bifurcation of various spinning and pulsating waves for nonadiabatic flame propagation in cylindrical channels. Our analysis predicts cascades of bifurcations which exhibit a variety of quasi-periodic flame behavior.

\footnotetext{
* Both with Northwestern University, Evanston, IL.
} 
We consider the problem of premixed flame propagation through a long cylindrical channel with volumetric heat losses. In the limit of large activation energy, the reaction zone becomes a thin flame sheet whose location in nondimensional cylindrical $\left(r, \psi, x_{3}\right)$ coordinates is given by $x_{3}=\Phi(r, \psi, t)$, where $\Phi$ is to be determined. Then, introducing the moving coordinate, $z=x_{3}-\Phi(r, \psi, t)$, the model for $\Phi$, a zeroth order temperature variable $\Theta$, and a first-order enthalpy perturbation $S$ is given by

$$
\begin{aligned}
\frac{\partial \Theta}{\partial t}- & \frac{\partial \Phi}{\partial t} \frac{\partial \Theta}{\partial z}=\nabla^{2} \Theta \\
+ & {\left[1+\left(\frac{\partial \Phi}{\partial r}\right)^{2}+\frac{1}{r^{2}}\left(\frac{\partial \Phi}{\partial \psi}\right)^{2}\right]^{1 / 2} e^{(S / 2)} \delta(z) } \\
& \frac{\partial S}{\partial t}-\frac{\partial \Phi}{\partial t} \frac{\partial S}{\partial z}=\nabla^{2} S+\beta \nabla^{2} \Theta-h \Theta
\end{aligned}
$$

subject to

$$
\begin{gathered}
\Theta=S=0 \text { at } z=-\infty, \\
\Theta=1 \text { for } z>0,
\end{gathered}
$$

(where $z^{-\varepsilon} S \rightarrow 0$ as $z \rightarrow+\infty$ for some fixed $\varepsilon>0)$, and

$$
\frac{\partial \Theta}{\partial r}-\frac{\partial \Phi}{\partial r} \frac{\partial \Theta}{\partial z}=\frac{\partial S}{\partial r}-\frac{\partial \Phi}{\partial r} \frac{\partial S}{\partial z}=0 \text { at } r=R .
$$

Here, $R$ denotes the channel radius, $\beta$ is a measure of the deviation in the Lewis number from unity, $h$ is the volumetric heat loss coefficient, $\delta(z)$ is the Dirac delta function, and $\nabla^{2}$ is the Laplacian in the moving coordinate system.

A one-dimensional, time-independent solution of Equations (1)-(5), which we refer to as the basic solution, is $\Phi^{0}(t)=-a t$ where the steady planar flame speed $a$ is related to the heat loss parameter $h$ by $h=-a^{2} \ln a$. We observe that the curve $a(h)$ has the appearance of a backwards "C." Consequently, there exists a critical value $h_{c}=1 /(2 e)$ above which no steady, planar solution exists and which is sometimes interpreted as an extinction limit. For $0<h<1 /(2 e)$, there exist two solution branches. However, as determined below, the lower branch is always unstable.
We now consider the linear stability of the basic solution. We introduce perturbation quantities and find that there exist solutions to the linear stability problem of the form

$$
\phi=\Phi-\Phi^{0}(t) \sim J_{n}\left(k_{n, m} r\right) e^{i(\omega t \pm n \psi)}
$$

where the discrete radial wavenumber $k_{n, m}$ is the $m$ th root of $J_{n}^{\prime}(k R)=0$, where $n$ is the angular wavenumber and $J_{n}$ is the Bessel function of the first kind of order $n$. Thus, the values of the admissible wavenumbers $k_{n, m}$ depend on the radius $R$ of the channel according to $k_{n, m}=\hat{c}_{n, m} / R$, where $J_{n}^{\prime}\left(\hat{c}_{n, m}\right)=0$. The neutral stability boundaries, on which $\Re(i \omega)=0$, are shown in Figure 1.

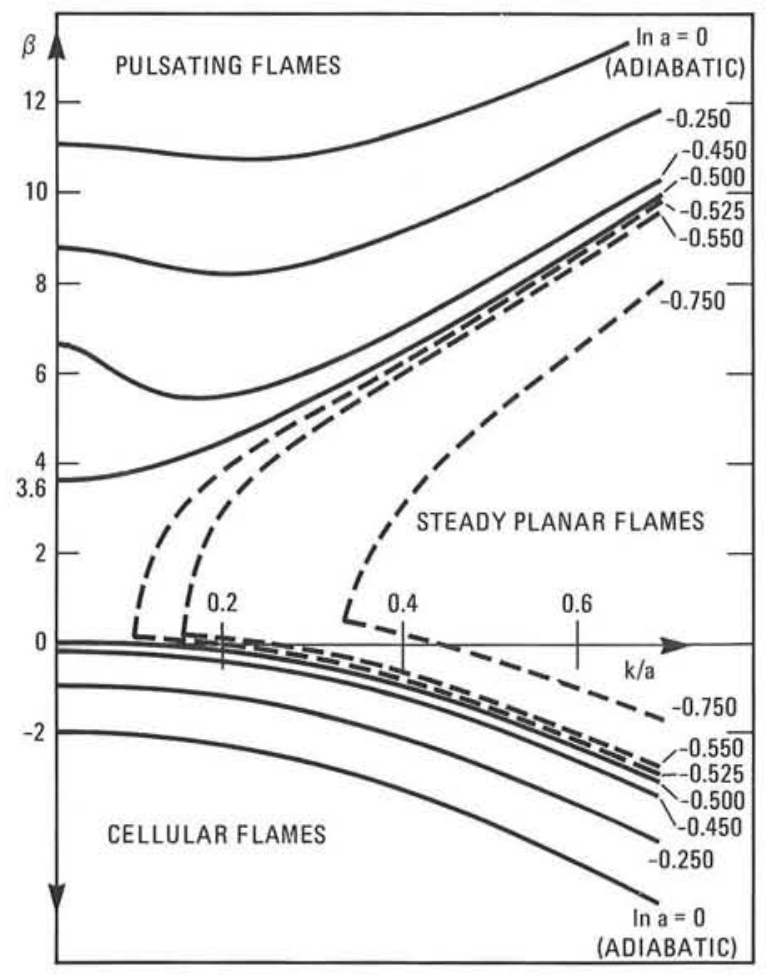

Figure 1. The family of neutral stability boundaries for various $a$.

The two types of stability boundaries correspond to the cellular $[\operatorname{Im}(i \omega)=0]$ and pulsating $[\operatorname{Im}(i \omega) \neq 0]$ instabilities discussed above. We observe that for $\ln a<-1 / 2$, corresponding to the lower branch of the basic solution, the 
pulsating and cellular boundaries intersect, and thus this branch of the basic solution is unstable to disturbances with sufficiently small wavenumbers. In particular, it is always unstable to planar pulsating disturbances since $k_{0,1}=0$. We observe that the cellular stability boundary becomes more accessible for larger values of the heat loss parameter (i.e., smaller values of $a$ ), as shown in Figure 1. The pulsating stability boundary has a local minimum which, for the adiabatic case, occurs at $k=k_{c}=1 / \sqrt{24}$, $\beta=\beta_{c}=32 / 3$. As the heat loss parameter increases from zero (i.e., as $a$ decreases from unity), both $k_{c}$ and $\beta_{c}$ decrease. In the limit that $\ln a \rightarrow-1 / 2, k_{c} \rightarrow 0$. Thus the effects of heat loss are such as to make the pulsating stability boundary more accessible.

In what follows, we consider Lewis numbers greater than unity (i.e., $\beta>0$ ) and focus on the pulsating neutral stability boundary. We note that since only discrete wavenumbers $k_{n, m}=\hat{c}_{n, m} / R$ are admissible, the basic solution does not necessarily lose stability at $\beta_{c}$. Instead, bifurcation occurs in general at a larger value $\beta_{0}$ which coincides with the smallest eigenvalue of the linear stability problem. In general, $\beta_{0}$ is a simple eigenvalue corresponding to a single wavenumber $k_{n, m}$. However, the values of the admissible wavenumbers depend on the radius $R$, and consequently, due to the minimum in the neutral stability boundary, there exist, for a given value of $h$, special values of $R$ such that $\beta_{0}$ is a double eigenvalue corresponding to two adjacent wavenumbers $k_{n_{1}, m_{1}}$ and $k_{n_{2}, m_{2}}$ with corresponding frequencies $\omega_{1}$ and $\omega_{2}$. As $R$ is perturbed away from such a special value $R_{0}$, the double eigenvalue "splits" into two neighboring simple eigenvalues, giving rise to a more complex bifurcation diagram in the neighborhood of $\beta_{0}$.

We employ a perturbation analysis in the neighborhood of a double eigenvalue by introducing the scaled bifurcation parameter $\sigma$ and the splitting parameter $\mu$ according to

$$
\beta=\beta_{0}\left(1+\sigma \epsilon^{2}\right), \quad R=R_{0}\left(1+\mu \epsilon^{2}\right) .
$$

Without loss of generality, we may set $\mu^{2}=1$, which defines the value of the small expansion parameter $\epsilon$ as $\epsilon=\left|R / R_{0}-1\right|^{1 / 2}$. We now seek solutions for the perturbation quantities in the form of power series expansions in $\epsilon$. The result of this analysis ${ }^{3}$ is that the solution for the reaction front $\Phi(r, \psi, t)$ has the long time behavior

$$
\begin{aligned}
& \Phi(r, \psi, t) \sim \\
& \left\{-1-\epsilon^{2} b_{1}\left[\left(R_{1,1}\right)^{2}+\left(R_{1,2}\right)^{2}\right]-\epsilon^{2} b_{2}\left[\left(R_{2,1}\right)^{2}+\left(R_{2,2}\right)^{2}\right]\right\} t \\
& -\epsilon a_{0}^{-1}\left[R_{1,1} e^{i\left(\omega_{1}+\epsilon^{2} \varphi_{1,1}+n_{1} \psi\right) t}+R_{1,2} e^{i\left(\omega_{1}+\epsilon^{2} \varphi_{1,2}-n_{1} \psi\right) t}\right] \\
& \quad \times J_{n_{1}}\left(k_{n_{1}, m_{1}} r\right)+\text { c.c. } \\
& -\epsilon a_{0}^{-1}\left[R_{2,1} e^{i\left(\omega_{2}+\epsilon^{2} \varphi_{2,1}+n_{2} \psi\right) t}+R_{2,2} e^{i\left(\omega_{2}+\epsilon^{2} \varphi_{2,2}-n_{2} \psi\right) t}\right] \\
& \quad \times J_{n_{2}}\left(k_{n_{2}, m_{2}} r\right)+\text { c.c. } .
\end{aligned}
$$

Equations for the real amplitudes $R_{i, j}$ and phases $\varphi_{i, j}$, which are complicated functions of the bifurcation and splitting parameters, are obtained from solvability conditions at $\mathrm{O}\left(\epsilon^{3}\right)$. These conditions determine the number and stability of the bifurcation branches in the neighborhood of the double eigenvalue $\beta_{0}$. In addition to the basic solution $R_{1,1}=R_{1,2}=$ $R_{2,1}=R_{2,2}=0$, there exist up to 15 nontrivial time-independent solutions for the real amplitudes $R_{i, j}$. Solutions Ia,b,c,d have one nonzero amplitude, solutions IIa,b,c,d,e,f have two nonzero amplitudes, solutions IIIa,b,c,d have three nonzero amplitudes, and solution IV has all four amplitudes nonzero. We remark that if the appropriate angular wavenumber is nonzero, the type I solutions represent timeperiodic spinning flames, whereas solutions IIa $\left(R_{1,1}=R_{1,2} \neq 0\right)$ and IIb $\left(R_{1,1}=R_{1,2} \neq 0\right)$ represent time-periodic standing (pulsating) modes. If the corresponding angular wavenumber is zero, these solutions represent radial (nonspinning) pulsating flames. The remaining solutions represent various quasi-periodic modes of propagation. In Figure 2, we show a typical bifurcation diagram as a function of $\sigma$ for a particular value of $a$ (or equivalently, $h$ ), and the mode numbers $n_{1}, m_{1}, n_{2}, m_{2}$. 


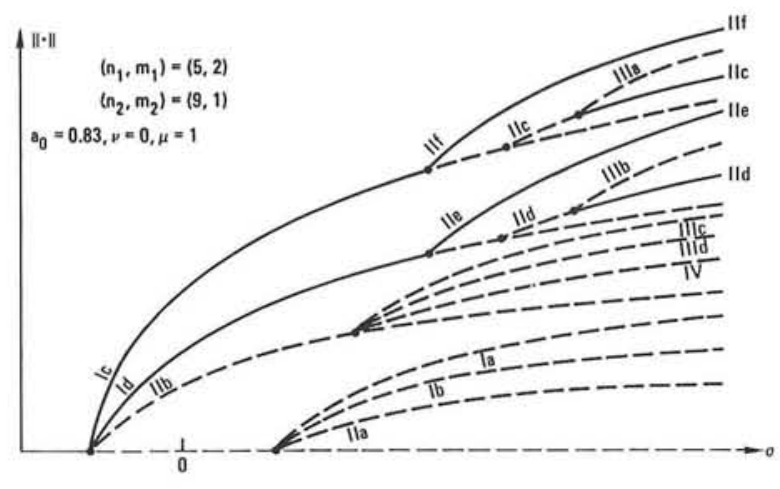

Figure 2. Bifurcation diagram near a double eigenvalue for a given pair of mode interactions and a given value of the parameter $a$. Solid (dashed) curves denote stable (unstable) solutions. The labels refer to the various equilibrium solutions of the amplitude equations.

We remark that the solutions discussed above are steady solutions of the time-dependent amplitude equations. In principle, these amplitude equations admit time-dependent solutions as well. For example, one can derive conditions on the coefficients for Hopf bifurcation from one of the secondary or tertiary branches to occur. Solutions emanating from these new bifurcation points would represent additional timedependent modes of flame propagation. Such a situation arises, for example, in the problem of condensed phase combustion, ${ }^{6}$ and an analysis of these nonsteady solutions of the amplitude equations will be reported in a forthcoming publication.

Finally, we note that the time-averaged propagation velocity is readily obtained from Equation (8) as

$$
\begin{gathered}
\lim _{T \rightarrow \infty} \frac{1}{T} \int_{0}^{T} \frac{\partial \Phi}{\partial t} d t=-1-\epsilon^{2} b_{1}\left[\left(R_{1,1}\right)^{2}+\left(R_{1,2}\right)^{2}\right] \\
-\epsilon^{2} b_{2}\left[\left(R_{2,1}\right)^{2}+\left(R_{2,2}\right)^{2}\right]+o\left(\epsilon^{2}\right) .
\end{gathered}
$$

Thus, whether the nonsteady, nonplanar modes propagate on the average slower or faster than the steadily propagating planar solution depends on the coefficients $b_{j}$ and on the amplitudes $R_{i, j}$, all of which are functions of the mode numbers $n_{j}$ and $m_{j}$ and the heat loss parameter $h$. Nonsteady combustion tends to decrease the propagation speed; however, the wrinkling of the reaction front can overcome the slowing effects of nonsteady combustion if the spatial wavenumber is sufficiently large. ${ }^{2}$ In the regimes where the primary branches are supercritical, the propagation speed is usually less $\left(b_{1}<0, b_{2}<0\right)$ than that of the basic solution.

\section{References}

1. B. Matkowsky and D. Olagunju, "Spinning Waves in Gaseous Combustion," SIAM J. Appl. Math. 42, 1138 (1982).

2. S. Margolis and B. Matkowsky, "Flame Propagation in Channels: Secondary Bifurcation to Quasi-periodic Pulsations," SIAM J. Appl. Math. 45, 93 (1985).

3. M. Booty, S. Margolis, and B. Matkowsky, "Interaction of Pulsating and Spinning Waves in Nonadiabatic Flame Propagation," to appear in SIAM J. Appl. Math. (1987).

4. S. Margolis and B. Matkowsky, "Nonlinear Stability and Bifurcation in the Transition From Laminar to Turbulent Flame Propagation," Comb. Sci. Tech. 34, 45 (1983).

5. I. Gololobov, E. Granovskii, and Yu. Gostintsev, "Two Combustion Modes at the Limit of Luminous Flame Propagation," Combust. Explos. Shock Waves 17, 22 (1981).

6. M. Booty, S. Margolis, and B. Matkowsky, "Interaction of Pulsating and Spinning Waves in Condensed Phase Combustion," SIAM J. Appl. Math. 46, 801 (1986). 


\section{On Nonadiabatic Condensed Phase Combustion}

\author{
H. G. Kaper, ${ }^{*}$ G. K. Leaf, ${ }^{*}$ S. B. Margolis, B. J. \\ Matkowsky**
}

As with most practical realizations of premixed combustion problems, the propagation of reaction fronts in condensed phase combustion, often referred to as "self-propagating hightemperature synthesis" due to its application in the synthesis of metal alloys, is generally accompanied by conductive and radiative heat losses. These losses result in measured values of the peak combustion temperature which are as much as several hundred degrees below the corresponding adiabatic value. A common way to account for these heat losses phenomenologically is to allow for a volumetric heat loss in the energy conservation equation. Here, we extend previous models of condensed phase combustion $^{1-2}$ to allow for volumetric heat losses. Aside from providing more realistic formulas for the burning velocity, we find that the burning rate for steady, planar combustion is a multivalued function of the heat loss parameter. We thus identify the critical value of the heat loss parameter at which the burning velocity has a vertical tangent as an extinction limit beyond which a steady, planar, self-propagating reaction cannot sustain itself. We also find that the effects of heat losses on steady, planar burning are destabilizing. In particular, a linear stability analysis shows that the neutral stability threshold, beyond which steady planar burning is unstable to pulsating disturbances, becomes more accessible as the heat loss parameter is increased.

In the present work, we consider both steady and nonsteady burning of the condensed powdered mixture of metal reactants. In previous work on the adiabatic problem, ${ }^{1,2}$ two different expressions for the macroscopic reaction rate were considered. In the first case (homogeneous combustion), the metal particles were assumed

* Both with Argonne National Laboratory, Argonne, IL.

** Northwestern University, Evanston, IL. to be sufficiently small so that the chemical reaction was kinetically-limited. In the second model (heterogeneous combustion), the reaction was assumed to be diffusion-limited by the formation of the product alloy which inhibits microscopic diffusion of the metal particles across contact surfaces. Both models incorporate melting phenomena, which aside from making the models more realistic, have important consequences when instability and bifurcation phenomena are considered..$^{3,4}$ Here, we find that melting has an equally significant effect on the nonadiabatic problem, parameterizing the extinction limit for steady planar burning, as well as the neutral stability boundary.

For large activation energy, a nondimensional model for the temperature $\Theta$ is given in a coordinate system attached to the moving reaction front as

$$
\begin{gathered}
\frac{\partial \Theta}{\partial t}-\frac{\partial \Phi}{\partial t} \frac{\partial \Theta}{\partial z}=\nabla^{2} \Theta-\frac{h}{\Delta} \Theta-\frac{\partial \Phi}{\partial t} \delta(z) \\
\frac{\partial \Phi}{\partial t}=-\left[1+\left(\frac{\partial \Phi}{\partial x_{1}}\right)^{2}+\left(\frac{\partial \Phi}{\partial x_{2}}\right)^{2}\right]^{1 / 2} \\
\times\left[\frac{\exp \left\{\Delta\left[\Theta\left(x_{1}, x_{2}, z=0, t\right)-1\right]\right\}-M}{1-M}\right]^{1 / 2} \\
\Theta=0 \text { at } z= \pm \infty, \quad \Theta \text { continuous at } z=0,
\end{gathered}
$$

where $x_{3}=\Phi\left(x_{1}, x_{2}, t\right)$ describes the moving front as a function of the transverse coordinates and time, and $z=x_{3}-\Phi\left(x_{1}, x_{2}, t\right)$ is the coordinate normal to the front. Here, $\delta(z)$ is the Dirac delta-function, and $\nabla^{2}$ is the Laplacian in the moving coordinate system. The parameter $\Delta$ is proportional to the activation energy of the reaction, $h$ is the volumetric heat loss parameter, and the melting parameter $M, 0 \leq M<1$, is related to the melting temperature $\Theta_{m}<1$ of the melting reactant. In the case of heterogeneous combustion, for example, $M=e^{\Delta\left(\Theta_{m}-1\right)}$.

The basic solution corresponding to a planar combustion wave propagating uniformly in the $-x_{3}$ direction is readily obtained from Equations (1)-(3) as

$$
\Theta^{0}= \begin{cases}C \exp \left[\left(\frac{1}{2} \aleph+\frac{1}{2} \sqrt{\aleph^{2}+\frac{4}{\Delta} h}\right) z\right], & z<0 \\ C \exp \left[\left(\frac{1}{2} \aleph-\frac{1}{2} \sqrt{\aleph^{2}+\frac{4}{\Delta} h}\right) z\right], & z>0,\end{cases}
$$




$$
\begin{gathered}
-\frac{\partial \Phi^{0}}{\partial t}=\aleph=\left\{\frac{\exp [\Delta(C-1)]-M}{1-M}\right\}^{1 / 2} \\
C \sqrt{\aleph^{2}+\frac{4}{\Delta} h}=\aleph,
\end{gathered}
$$

where the last relation is determined from the jump in $d \Theta^{0} / d z$ at $z=0$ implied by the deltafunction term in Equation (1). An implicit relation for the burning rate $\aleph$ (the dimensional burning rate normalized by its adiabatic value) as a function of the parameters $\mathrm{h}, \Delta$ and $M$ is obtained from Equations (5)-(6) as

$$
h=-\frac{\aleph^{2}(2+\ln \vartheta / \Delta)}{4(1+\ln \vartheta / \Delta)^{2}} \ln \vartheta, \quad \vartheta \equiv M+(1-M) \aleph^{2} .
$$

Writing $\aleph$ as an expansion $\aleph \sim a_{0}+a_{1} / \Delta+\cdots$, the first approximation $a_{0}$ for the burning rate is obtained from Equation (7) as

$$
h=-\frac{1}{2} a_{0}^{2} \ln \left[a_{0}^{2}(1-M)+M\right]
$$

In Figure 1, we plot $a_{0}$ as a function of the heat loss parameter $h$ for various values of $M$. We observe that $a_{0}(h ; M)$ is multi-valued and that no solution exists for $h>h_{c}(M)$. We thus interpret $h_{c}$ as an extinction limit beyond which steady, planar burning cannot occur, although other types of nonsteady and/or nonplanar solutions may exist beyond this limit. The function $h_{c}(M)$ is a monotonically decreasing function of $M$, with $h_{c}(0)=(2 e)^{-1}$ and $h_{c} \rightarrow 0$ as $M \rightarrow 1$. We also note that the value of $a_{0}$ at the extinction limit increases monotonically from $e^{-1 / 2}$ for $M=0$ to $2^{-1 / 2}$ in the limit $M \rightarrow 1$. The lower solution branch in Figure 1 is unstable, whereas the upper branch, which contains the adiabatic solution $a_{0}(h=0 ; M)=1$, may be stable or unstable, depending on the values of the parameters in the problem, as discussed below. In fact, our previous work on the adiabatic problem ${ }^{3,4}$ shows that this solution loses stability in favor of a pulsating or spinning mode of burning for sufficiently large values of the modified activation energy parameter $\Delta$, a phenomenon for which extensive experimental evidence exists. ${ }^{5,7}$ Finally, we remark that the nondimensional peak combustion temperature $C=\Theta(0)$ is given by $\Theta(0) \sim 1-\left(2 h / a_{0}^{2}\right) / \Delta+\cdots$, which is unity in the absence of heat losses $(h=0)$. Thus, the minimum combustion temperature occurs at the extinction limit $h_{c}(M)$, and consequently, the peak combustion temperature at extinction is a monotonically increasing function of $M$. As $M$ approaches unity, extinction occurs at nearly adiabatic conditions.

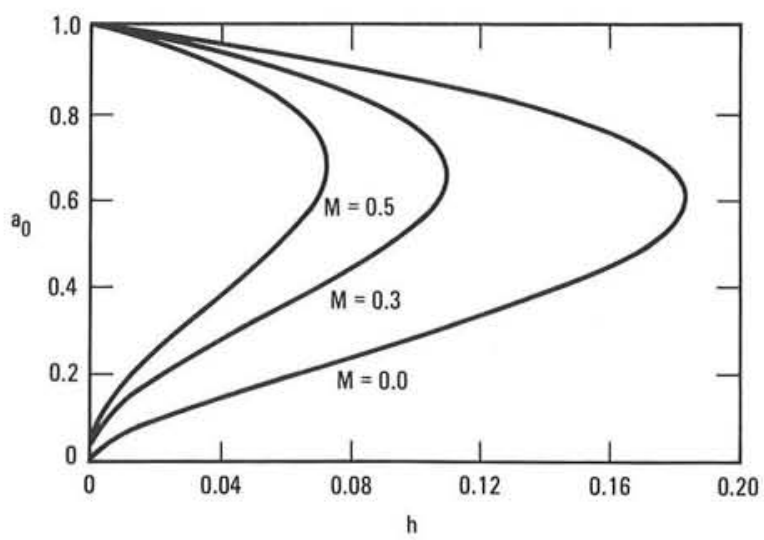

Figure 1. Leading coefficient $a_{0}$ in the burning velocity expansion plotted as a function of the heat loss parameter $h$ for various values of the melting parameter $M$.

The linear stability of steady planar combustion may be determined in a standard fashion by linearizing the nonlinear problem (1)(3) about the basic solution (4)-(7) and seeking harmonic solutions of the form

$$
\begin{gathered}
\qquad \equiv \Phi-\Phi^{0}(t)=\exp \left(i \omega t+i k_{1} x_{1}+i k_{2} x_{2}\right), \\
\theta \equiv \Theta-\Theta^{0}-\phi d \Theta^{0} / d z=f_{ \pm}(z) \exp \left(i \omega t+i k_{1} x_{1}+i k_{2} x_{2}\right) \\
\text { for } z_{<}^{>0} .
\end{gathered}
$$

The dispersion relation we obtain is given by

$$
\begin{aligned}
4(i \omega)^{3} & +\left(W^{2}+8 E-B^{2}+4 k^{2}\right)(i \omega)^{2} \\
& +2 E\left(2 E+W^{2}-W B+4 k^{2}\right)(i \omega)+4 E^{2} k^{2}=0,
\end{aligned}
$$

where we have introduced the definitions

$$
\begin{gathered}
W=\sqrt{\aleph^{2}+\frac{4}{\Delta} h}, \quad B=\frac{\Delta}{2(1-M) \aleph}\left[M+(1-M) \aleph^{2}\right], \\
E=\frac{1}{2} C \aleph B, \quad k^{2}=k_{1}^{2}+k_{2}^{2} .
\end{gathered}
$$


We anticipate that the basic solution corresponding to the lower branch in Figure 1 is always unstable [i.e., $\operatorname{Re}(i \omega)>0$ ], and we thus confine our attention to the basic solution corresponding to the upper branch in that figure. To determine the neutral stability boundary for solutions lying on this branch, we set $\operatorname{Re}(i \omega)=0$ and equate the real and imaginary parts of Equation (10) separately to zero. The result is

$$
\begin{aligned}
-\left(W^{2}+4 k^{2}\right)^{2} & +\left(W B+B^{2}-8 E\right)\left(W^{2}+4 k^{2}\right) \\
& -2 E W^{2}+(2 E-W B)\left(B^{2}-8 E\right)=0 \\
\omega^{2} & =\frac{1}{2} E\left(2 E-W B+W^{2}+4 k^{2}\right),
\end{aligned}
$$

which is readily seen to collapse to that obtained in the adiabatic limit $h=0, \aleph=C=$ $1 .^{1,2}$ We note that since $\omega \neq 0$, the basic solution loses stability to pulsating disturbances.

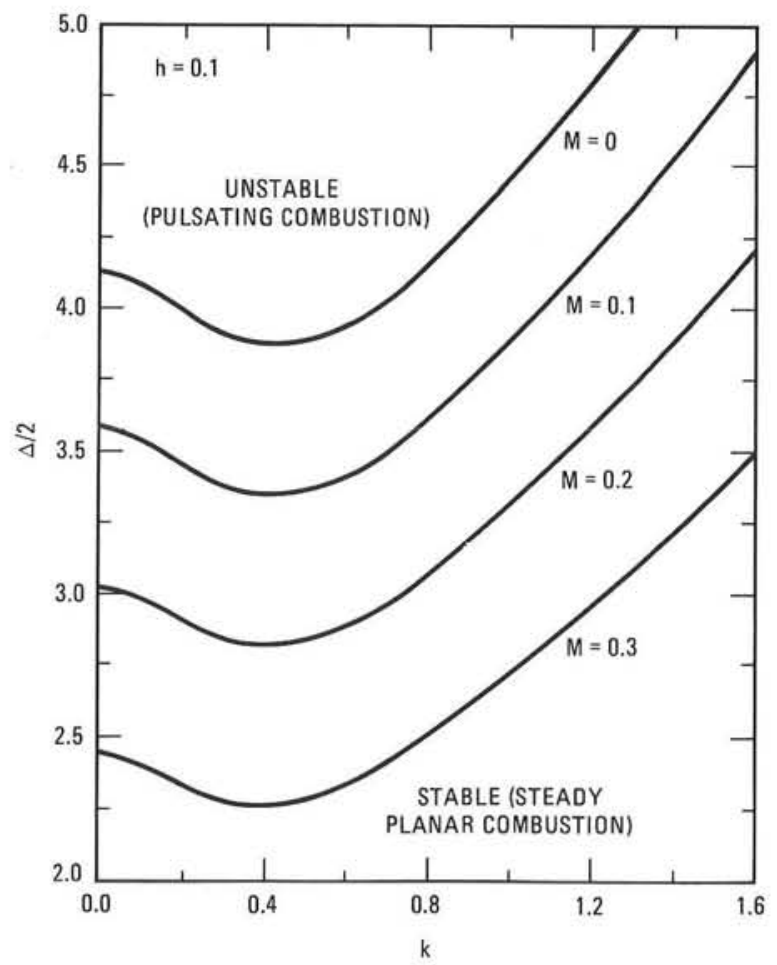

Figure 2. Neutral stability boundaries in the $(\Delta, k)$ plane for $\mathrm{h}=0.1$ and various values of the melting parameter $M$.
The neutral stability boundary, when plotted in the $(\Delta, k)$ plane, is parameterized by the melting parameter $M$ and the heat loss parameter $h$. Figures 2 and 3 show families of such curves for fixed values of $h$ and $M$, respectively. In particular, the effect of melting is shown to be destabilizing, as demonstrated in Figure 2, for arbitrary values of $h<h_{c}$.

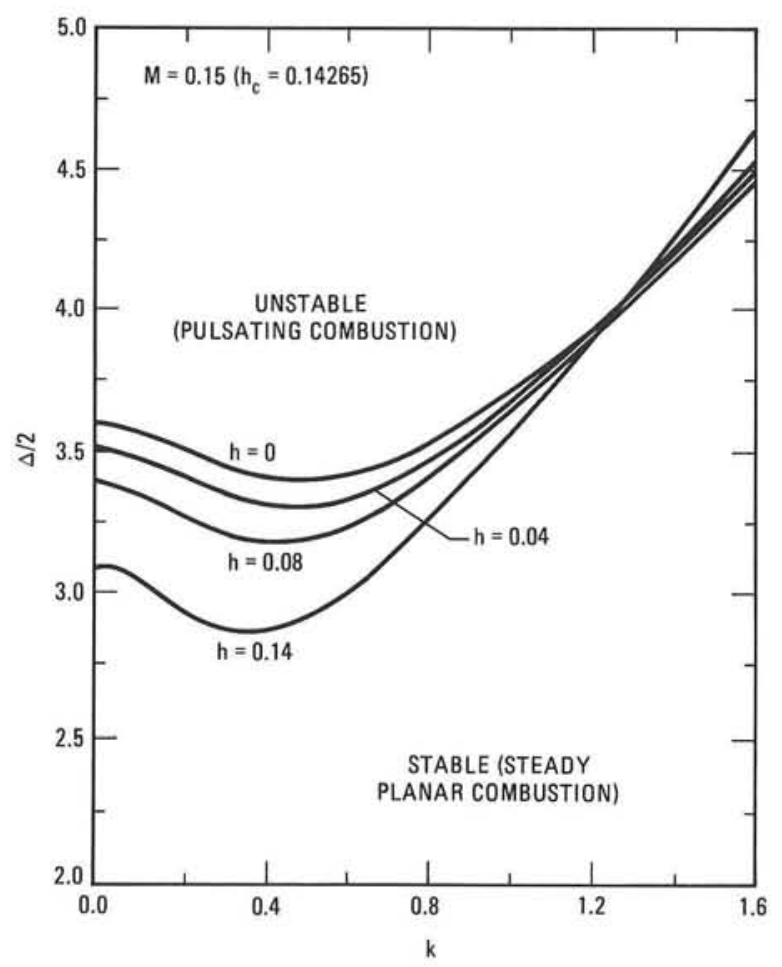

Figure 3. Neutral stability boundaries in the $(\Delta, k)$ plane for $\mathbf{M}=\mathbf{0 . 1 5}$ and various values of the heat loss parameter $h$.

Figure 3 shows that the effect of heat loss produces a similar result, at least if sufficiently small wavenumbers are admissible. However, if small nonzero wavenumber disturbances are inadmissible due to finite transverse dimensions of the sample, then heat losses may be stabilizing for sufficiently small values of $M$. We observe that the critical wavenumber at which instability first occurs decreases with increasing $h$. 


\section{References}

1. S. B. Margolis, "An Asymptotic Theory of Condensed Two-phase Flame Propagation," SIAM J. Appl. Math. 43, 351 (1983).

2. S. B. Margolis, "An Asymptotic Theory of Heterogeneous Condensed Combustion," Comb. Sci. Tech. 43, 197 (1985).

3. S. B. Margolis, H. G. Kaper, G. K. Leaf, and B. J. Matkowsky, "Bifurcation of Pulsating and Spinning Reaction Fronts in Condensed Two-phase Combustion," Comb. Sci. Tech. 43, 127 (1984).

4 M. R. Booty, S. B. Margolis, and B. J. Matkowsky, "Interaction of Pulsating and Spinning Waves in Condensed Phase Combustion," SIAM J. Appl. Math. 46, 801 (1986).

5. A. G. Merzhanov, A. K. Filonenko, and I. P. Borovinskaya, "New Phenomena in Combustion of Condensed Systems," Dokl. Phys. Chem. 208, 122 (1973).

6. A. V. Dvoryankin, A. G. Strunina, and A. G. Merzhanov, "Trends in the Spin Combustion of Thermites," Combust. Explos. Shock Waves 18, 134 (1982).

7. A. G. Strunina, A. V. Dvoryankin, and A. G. Merzhanov, "Unstable Regimes of Thermite System Combustion," Combust. Explos. Shock Waves 19, 158 (1983).

\section{Modeling of Large-Scale Flame Acceleration Experiments ${ }^{\dagger}$}

\author{
K. D. Marx
}

A new computational model for large-scale flames is developed and applied to the simulation of flame acceleration experiments. The primary objective is to circumvent the necessity for resolving turbulent flame fronts on the relatively coarse computational grids which are necessary in engineering calculations. The essence of the model is to artificially thicken the flame by increasing the appropriate diffusivities and decreasing the combustion rate, but to do this in such a way that the burn velocity and flame thickness varies with pressure, temperature, and turbulence intensity according to prespecified phenomenological characteristics. The model is particularly aimed at implementation in computer codes which simulate compressible flows. In this work, an appropriate modification of the Conchas-Spray code ${ }^{1}$ is used. It is applied to the two-dimensional simulation of hydrogenair flame acceleration experiments in which the flame speeds and gas flow velocities approach the speed of sound in the gas. It is shown that many of the features of the flame trajectories and pressure histories in the experiments are simulated quite well by the model.

The simulation of combustion processes in large experiments presents extreme demands on the capabilities of even the largest and fastest computers. In the problems considered here, it is necessary to give up the idea of resolving the flame thickness on the computational grid. We must artificially thicken the flame so that the flame thickness is equal to a few grid spacings.

Such flame thickening has been done previously by a method known as the $\beta$-transformation $^{2}$ that involves changing the length scales in the problem by artificially increasing the thermal diffusivity and diffusion coefficient and

$\dagger$ NRC NUREG/CR-4102 (1986); also, to appear in SAND Report (1987). 
decreasing the combustion rate. We have retained this essential idea. However, the original $\beta$-transformation did not work well with mixing-limited combustion models such as the Magnussen-Hjertager ${ }^{3}$ model. Hence, it was decided to effect the flame thickening in a different way. It is desirable that the burn velocity vary realistically with flow parameters such as turbulence intensity, pressure, and temperature. Our model represents an attempt to adhere to properties of turbulent flames given, for example, in Reference 4.

The approach used in this work is to endow the gas with minimum values of turbulent kinetic energy $k$ and turbulent length scale $L$. These minimum values, denoted $k_{m}$ and $L_{m}$, are used for the computation of thermal conductivity and diffusivity only. In that way, there will be minimum values of burn velocity and flame thickness.

The end result is a turbulent burn velocity $S_{T}$ which approximately satisfies

$$
S_{T}=\left(A S_{L}+B \sqrt{k}\right) p^{m} T_{u}^{n}
$$

where $S_{L}$ is laminar flame speed, $p$ is pressure, $T_{u}$ is unburned gas temperature, and $A$ and $B$ are constants chosen on the basis of experimental data.

The specific configurations to which this work has been applied are experiments performed in the FLAME facility ${ }^{5}$ at Sandia National Laboratories in Albuquerque, New Mexico. This facility consists of a reinforced concrete channel $2.44 \mathrm{~m}$ high by $1.83 \mathrm{~m}$ wide by $30.5 \mathrm{~m}$ long ( $8 \mathrm{ft}$ by $6 \mathrm{ft}$ by $100 \mathrm{ft}$ ). A comparison of the results of one calculation with the corresponding experimental data taken for a mixture of $14.5 \%$ hydrogen in air is given in Figure 1.

Considering the size of the experiment and the complexity of the computational problem, the agreement is quite good. It will be noted that there are some significant discrepancies in the flame trajectory. To some degree, these must be viewed in light of the fact that the flame velocity is extremely sensitive to the burn velocity. (Because of the geometrical factors and the feedback loop involving gas flow velocities, turbulence intensities, and combustion rates, the dependence is nonlinear.)

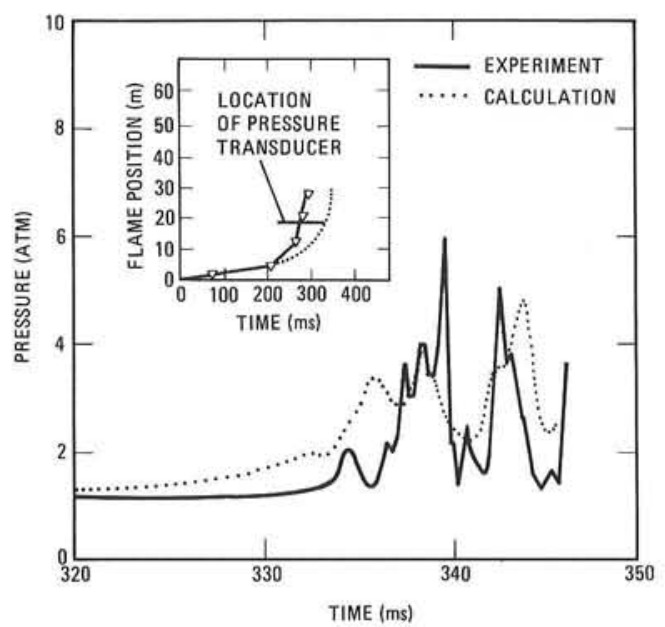

Figure 1. Comparison of computed and experimental flame trajectories (inset) and pressure histories. (The pressure shown here is that appearing at the side wall of the channel at the axial location shown in the inset). The experimental pressure signal has been shifted in time to exhibit the agreement in structure.

At a time of about $300 \mathrm{~ms}$ into the calculation, the computed flame trajectory begins to exhibit rapid acceleration. Eventually, it essentially parallels that of the experiment, while the flame traverses through axial positions from about $17 \mathrm{~m}$ through $23 \mathrm{~m}$. In this region, the computed pressures agree reasonably well with those measured experimentally. The reason for this is that the structure of the pressure history is characterized by the timing of the burnout of the gas in the successive chambers. The fact that the flame velocities approximately agree means that the chambers are burning out at about the correct rate. It should be noted that the flame velocity of about $500 \mathrm{~ms}$ is roughly the sound velocity in the burned gas. Since the gas flow velocities through the constrictions are of the same order of magnitude as the flame velocity, this is expected as a manifestation of choked flow.

Figure 2 shows the pressure in a series of three-dimensional plots over 7 of the chambers 
at 5 different times. Because of the aforementioned choking, the flame is actually not accelerating rapidly at this time. However, the pressures are still rising rapidly. It is important to note the mechanism leading to this pressure rise. As the pressure wave propagates down the channel, it hits the obstacles. Reflection of these shocks off the front face of the obstacles results in a large increase in pressure and some shock heating. This increases the burn rate, which feeds back into an increase in the overall pressure level of the wave.

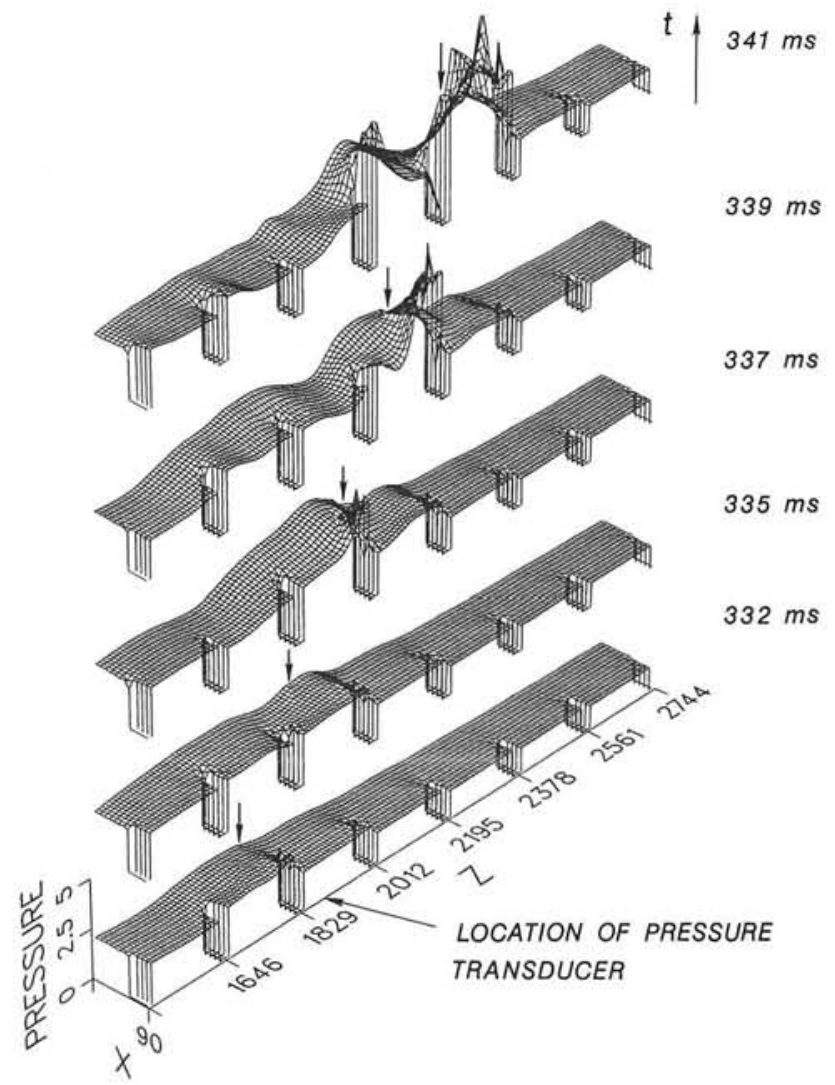

Figure 2. Three-dimensional plots of the pressure from the 8 th through the 15 th obstacle along the FLAME Facility channel. Coordinates are indicated in centimeters. The configuration has a symmetry plane at $x=0$. The short vertical arrows indicate the positions of the flame on the channel axis at the time corresponding to each plot (given on the right of the figures).

At $341 \mathrm{~ms}$, the computation exhibits a deflagration to detonation transition (DDT). Note that a sharp pressure ridge has formed all the way across the channel in the uppermost plot in
Figure 2; i.e., the pressure spike is not restricted to the region just in front of an obstacle.

Detonation was not observed experimentally at the concentration of $14.5 \% \mathrm{H}_{2}$ used in this calculation. However, it was observed at $15 \% \mathrm{H}_{2}$. The fact is that we do not claim to be able to simulate the detailed physical processes which occur in DDT. But it is reasonable to assume that the computed trends in pressure, temperature, and turbulence levels are indicative of real processes, and that what is being calculated is the evolution of a system that is progressing toward a tendency to detonate.

\section{References}

1. L. D. Cloutman, J. K. Dukowicz, J. D. Ramshaw, and A. A. Amsden, "ConchasSpray: A Computer Code for Reactive Flows with Fuel Spray," Los Alamos National Laboratory Report LA-9294-MS (1982).

2. T. D. Butler and P. J. O'Rourke, "A Numerical Method for Two Dimensional Unsteady Reacting Flows," Sixteenth Symposium (International) on Combustion (The Combustion Institute, 1977), p. 1503.

3. B. Magnussen and B. Hjertager, "On Mathematical Modeling of Turbulent Combustion with Special Emphasis on Soot Formation and Combustion," Sixteenth Symposium (International) on Combustion (The Combustion Institute, 1977), p. 719.

4. K. C. N. Bray, in Turbulent Reacting Flows, P. A. Libby and F. A. Williams, eds., (Springer-Verlag, New York, NY, 1980), p. 115.

5. M. P. Sherman, S. R. Tieszen, and W. B. Benedick, Twenty-first Symposium (International) on Combustion (The Combustion Institute, 1986), in press. 


\section{Ignition Modeling}

\section{A. E. Lutz, R. J. Kee, H. A. Dwyer, ${ }^{*}$}

A computer program is being developed to model ignition of premixed flames in closed chambers. The objectives are to investigate the effect of the nature of the source on the ignition, to quantify minimum ignition energies, and to examine the importance of pressure disturbance effects to the ignition process. In this article, we briefly describe the model and solution method, and present a simulation of the ignition of an ozone flame in a cylindrical chamber.

A significant physical aspect of premixed ignition in closed vessels is the presence of fluid motion on the acoustic timescale. Pressure waves can be generated either by the combustion front or the ignition source. Source-generated pressure waves were observed by Raffel et $a l,{ }^{1}$ in experiments where ozone flames were ignited by a laser. In anticipation of modeling efforts, the experiments were carried out in a cylindrical chamber with the laser providing a line source along the centerline, as sketched in Figure 1.

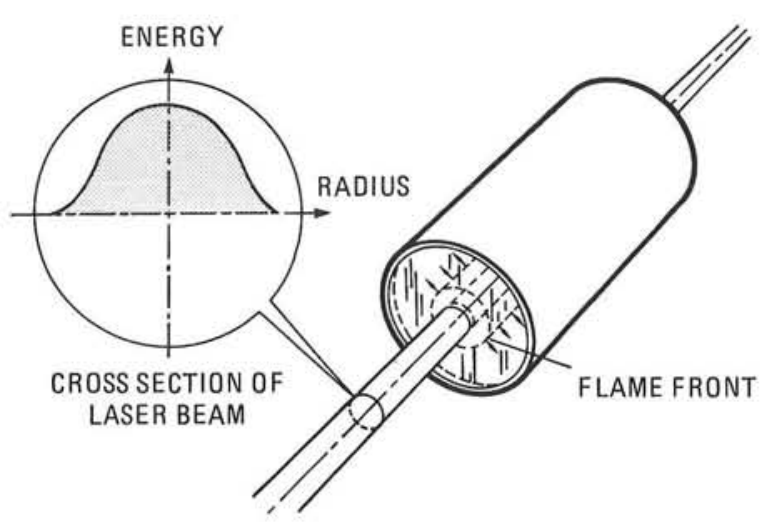

Figure 1. Schematic of model problem: laser beam passes through centerline of cylindrical chamber and a symmetric flame propagates in the radial direction.

The resulting cylindrical flame can be simulated with a one-dimensional numerical model. Oscillations in the traces of $\mathrm{O}_{3}$ concentration measured via ultraviolet absorption were caused by reflected pressure waves. The amplitude of

\footnotetext{
* University of California at Davis.
}

the oscillations was proportional to the strength of the laser. The investigators presented a onedimensional model that solves conservation equations for mass, energy, and species in a Lagrangian coordinate system, and represents the laser as a source of thermal energy. The momentum equation is replaced by the assumption that the pressure is spatially uniform within the chamber.

Even though this assumption contradicts the experimental observation, the predicted minimum ignition energies agree qualitatively with the experimental values.

The present computational model includes the effects of pressure waves that develop during the rapid energy deposition and reflect within the chamber. A more complete statement of the model problem and initial development of the model appears in Lutz et al. ${ }^{2}$

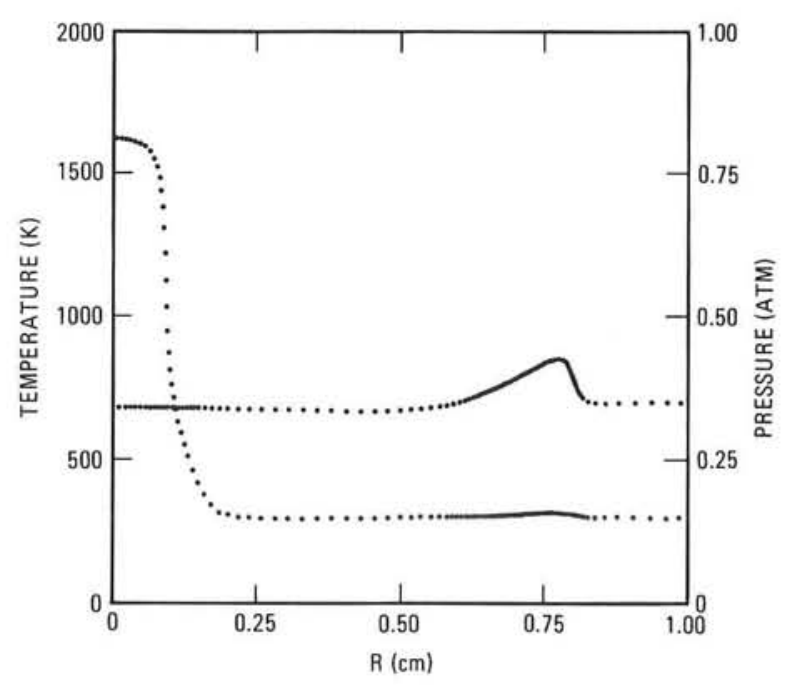

Figure 2. Temperature and pressure profiles at time equal $20 \mu \mathrm{sec}$ for a $23 \% \mathrm{O}_{3}$ in $\mathrm{O}_{2}$ mixture initially at $300 \mathrm{~K}$ and 0.35 atm. The thermal source deposited $0.17 \mathrm{~J} / \mathrm{cm}^{3}$ over a radius of 0.1 $\mathrm{cm}$ and a time duration of $1.0 \mu \mathrm{sec}$.

The model problem consists of a closed cylindrical chamber filled with a homogeneous mixture of fuel and oxidizer that is initially at rest. The ignition source acts along the centerline, such that the fluid motion and energy transfer are cylindrically symmetric. The source is a purely thermal source that has a Gaussian profile in space and is applied for a fixed period 
of time. One-dimensional conservation equations for mass, momentum, energy, and individual species, are solved along with the equation of state of an ideal gas mixture. The chemical kinetics model consists of a set of elementary reactions that proceed according to the law of mass action.

In order to perform computations on a spatial domain that is large enough to represent experiments, adaptive meshing techniques are necessary to reduce the computational expense while retaining resolution of the physical structures. The current solution method uses an adaptive meshing strategy pioneered by Winkler ${ }^{3}$ wherein an equation for the physical location of the gridpoints is coupled to transformed versions of the conservation equations and solved together via an implicit time integration technique. The mesh adjusts dynamically to resolve both the flame front and shock waves that exist in the solution.

A sample computation that closely resembles the ozone experiments of Raffel et $a l^{1}$ is presented as an example of the current capability of the model. The initial conditions consist of a mixture of $23 \% \mathrm{O}_{3}$ in $\mathrm{O}_{2}$ at $300 \mathrm{~K}$ and $0.35 \mathrm{~atm}$. The laser source is represented by a thermal source that deposits $0.17 \mathrm{~J} / \mathrm{cm}^{3}$ over a radius of $0.1 \mathrm{~cm}$ and a time duration of $1.0 \mu \mathrm{sec}$. The solution at a time of $20 \mu \mathrm{sec}$ is shown in Figure 2. The points give the discrete solution at individual meshpoints and the performance of the adaptive mesh is evident. The solution contains the flame kernel at the centerline $(r=0$ in the figure), as well as the pressure wave that was generated by the rapid energy deposition. The effect of the reflected pressure waves is seen in Figure 3, which shows the temperature at the centerline as a function of time. The temperature in the kernel rises to approximately 1500 $\mathrm{K}$ during the time duration of the source. The temperature is perturbed by the pressure waves that are repeatedly reflected at the wall and focused at the centerline. Figure 4 charts the flame propagation for the time period of Figure 3. The flame position oscillates due to the fluid motion in the chamber.

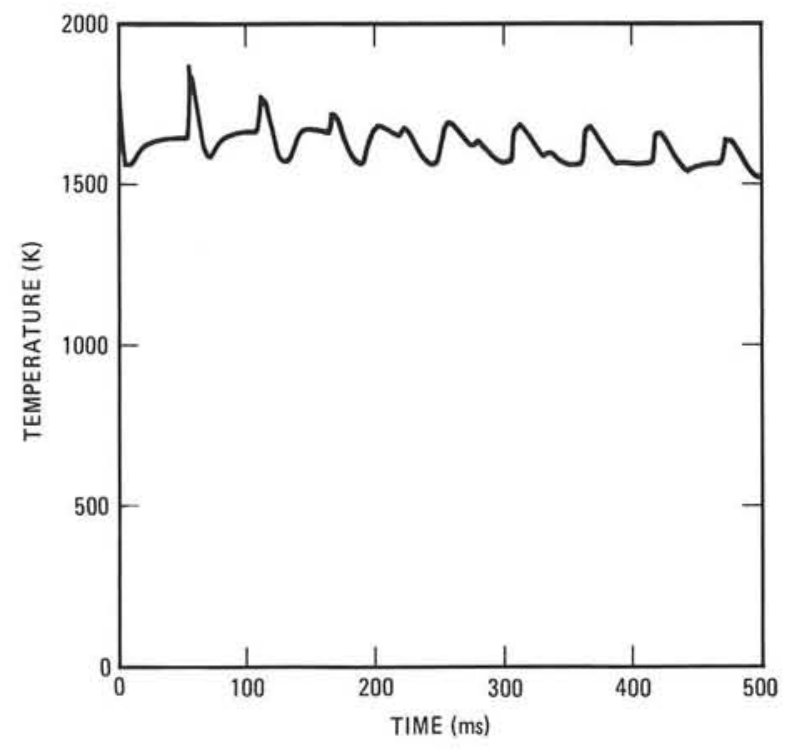

Figure 3. Temperature at the centerline of the cylindrical chamber as a function of time. The temperature in the kernel rises to approximately $1500 \mathrm{~K}$ during the time duration of the source. The temperature is perturbed by the pressure waves that are repeatedly reflected at the wall and focused at the centerline.

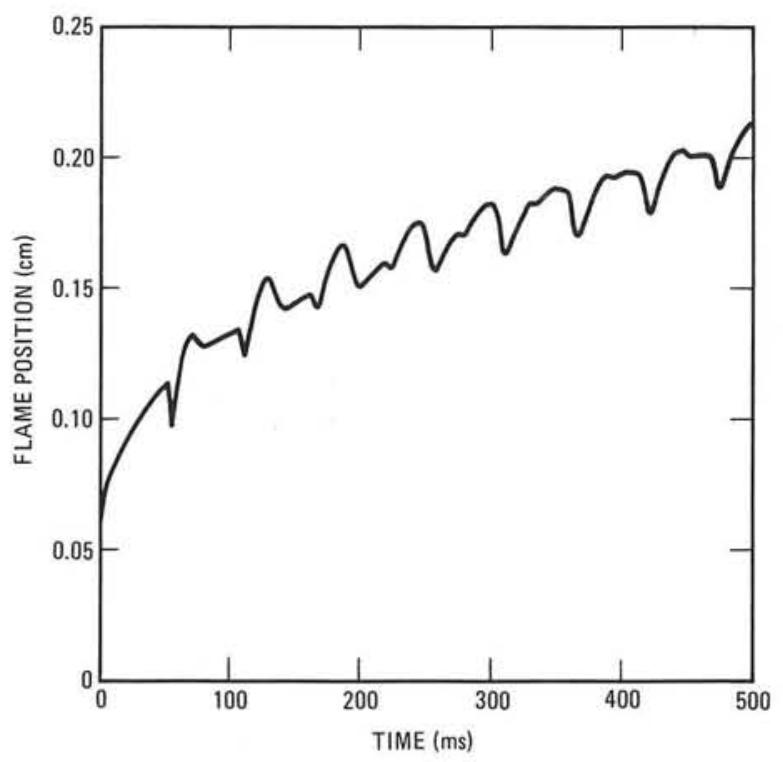

Figure 4. Flame position (as defined by point where temperature is $1000 \mathrm{~K}$ ) versus time for the period of Figure 3 . Oscillation is due to fluid motion induced by reflected pressure waves. 


\section{References}

1. B. Raffel, J. Warnatz, H. Wolff, J. Wolfrum, and R.J. Kee, "Thermal Ignition and Minimum Ignition Energy in Oxygen-Ozone Mixtures," Dynamics of Reactive Systems, Part II: Modeling and Heterogeneous Combustion (AIAA, New York, NY, 1985), p. 335.

2. A. E. Lutz, R. J. Kee, and H. A. Dwyer, "Ignition Modeling with Grid Adaptation," Dynamics of Reactive Systems, Part I: Flames and Configurations (AIAA, New York, NY, 1985), p. 79 .

3. K. A. Winkler, M. L. Norman, and M. J. Newman, "Adaptive Mesh Techniques for Fronts in Star Formation," Max-Planck-Institut fur Physik und Astrophysik, Munchen, MPA 94, (1983).

\section{New Computer Packages for Expanded Chemical Kinetics Modeling Capabilities}

\author{
R. J. Kee, J. F. Grcar, M. E. Coltrin," J. A. Miller, \\ M. D. Smooke, ${ }^{* *}$ G. Dixon-Lewis, ${ }^{* * *} J$. Warnatz, ${ }^{* * * *}$ \\ P. Glarborg, ${ }^{* * * * *}$
}

Three new Fortran computer code packages have been developed and added to our library of capabilities for modeling chemical kinetics in flowing systems. All the codes are fully integrated within the CHEMKIN modeling framework. The first is a general-purpose package to handle multicomponent transport properties. ${ }^{1}$ This package allows computation of mixture properties by a full multicomponent formulation; it supersedes our former package that incorporated only a mixture-averaged formulation. The second package is used to compute the structure

\footnotetext{
- Sandia National Laboratories Albuquerque.

* Yale University, New Haven, CT.

*.* University of Leeds, England.

*** Universität Heidelberg, West Germany.

..... Technical University of Denmark.
}

of laminar premixed flames, ${ }^{2}$ and the third computes the chemical kinetic behavior of perfectlystirred reactors. ${ }^{3}$ Each of the packages is documented by a user's manual.

Characterizing the molecular transport of species, momentum, and energy in a multicomponent gaseous mixture requires the evaluation of diffusion coefficients, viscosities, thermal conductivities, and thermal diffusion coefficients. Although evaluating pure species properties follows standard kinetic theory expressions, one can choose from a range of possibilities for evaluating mixture properties. Moreover, computing the mixture properties can be expensive, and, depending on the use of the results, it is often advantageous to make simplifying assumptions to reduce the computational cost.

For most applications, gas-mixture properties can be determined from pure species properties via certain approximate mixture averaging rules. Recently, however, we have encountered applications in which the approximate-averaging rules are not adequate. As a result, we have undertaken a software project to provide full multicomponent transport properties. ${ }^{1}$ The new package provides both the mixture-averaged forms as well as the multicomponent formulations. The multicomponent methods are based on the work of Dixon-Lewis.

The multicomponent formulation has several important advantages over the relatively simpler mixture formulas. The first advantage is accuracy. The mixture formulas are only correct asymptotically in some special cases, such as in a binary mixture, or in diffusion of trace amounts of species into a nearly pure species, or systems in which all species except one move with nearly the same diffusion velocity. A second deficiency of the mixture formulas is that overall mass conservation is not necessarily preserved when solving the species-continuity equations. To compensate for this shortcoming, one has to apply some ad hoc correction procedure. The multicomponent formulation guarantees mass conservation without any correction factors, which is a clear advantage. The only real deficiency of the multicomponent formulation is 
its computational expense. Evaluating the ordinary multicomponent diffusion coefficients involves inverting a $K \times K$ matrix, and evaluating the thermal conductivity and thermal diffusion coefficients requires solving a $3 K \times 3 K$ system of algebraic equations, where $K$ is the number of species.

The structure of the present multicomponent transport package is analogous to that of our previous transport package. That is, polynomial fits are first computed for the temperature-dependent parts of the kinetic theory expressions for pure species viscosities and binary diffusion coefficients. The coefficients from the fit are passed to a library of subroutines that can be used to return either mixture-averaged properties or multicomponent properties for an arbitrary gas mixture. This fitting procedure is used so that expensive operations, such as evaluation of collision integrals, need be done only once and not every time a property is needed.

Burner-stabilized laminar premixed flames are very often used to study chemical kinetics in a combustion environment. Such flames are effectively one dimensional and can be made very steady, thus facilitating detailed experimental measurements of temperature and species profiles. Also, laminar flame speed is often used to characterize the combustion of various fuel/oxidizer combinations. Therefore, the ability to model chemical kinetics and transport processes in these flames is critical to interpreting flame experiments and to understanding the combustion process itself.

The earliest efforts at solving premixed flame problems with realistic chemical kinetics made use of shooting techniques. However, as the kinetics becomes more complex, the shooting methods are not usable. Spalding introduced the use of implicit finite difference methods, obtaining the solution of the steady-state problem as the asymptotic limit of a transient problem. Using implicit methods relieved the stiffness and instability problems that caused the shooting methods to fail. Most subsequent treatments of the problem have utilized some variation of Spalding's approach. Both Wilde, and Kendall and Kelly introduced the idea of solving the steady-state problem directly by finite-difference boundary-value-problem techniques. Our approach ${ }^{2}$ draws on both of these ideas, using a combination of time-dependent and steady-state methods. Furthermore, we have introduced the idea of coarse-to-fine grid refinement as a means to enhance the convergence properties of the steady-state approach and as a means to provide optimal mesh placement.

This model is capable of predicting temperature and species profiles in two laminar premixed flame configurations. The first, and the one most often used for analyzing species profiles in flame experiments, is the burnerstabilized flame with a known mass flow rate. We consider two cases of the burner-stabilized flame-one where the temperature profile is known, and one in which the temperature profile is determined by the energy conservation equation. We have most often used the program when the temperatures were obtained from experiment. In this case, only the species transport equations are solved. In many flames, there can be significant heat losses to the external environment, which are of unknown or questionable origin and thus are troublesome to model. But, since the chemistry depends strongly on temperature, it is essential to know the temperatures accurately to draw conclusions about the chemical kinetics behavior. We have discovered that if a temperature profile can be measured accurately, then it is often better to use this measurement than the temperature profile obtained by solving an energy conservation equation. However, for cases where the heat losses are known to be negligible, the program can solve a burner-stabilized flame problem in which the temperatures are determined from the energy conservation equation. Comparing the two types of burner-stabilized models may provide some indication of the heat losses.

The second flame configuration that we consider is the freely-propagating adiabatic flame. In this case, there are no heat losses (by definition) and thus the temperatures should be computed from the energy equation. Flame speed depends, in part, on the transport of heat, and 
predicting the temperature distribution is an integral part of the flame-speed calculation.

Continuously-stirred-flow tank reactors have been used for many years to study the chemistry of various batch chemical processes, including combustion. Historically, the data have been analyzed in terms of overall chemical reaction rates. More recently, however, investigators have realized that well-stirred reactor data could be interpreted in terms of elementary chemical-rate processes. Since well-stirred reactors are relatively inexpensive to build and operate, and since the computational modeling is also relatively inexpensive, there is considerable value in using these reactors to aid understanding of such important problems as combustion pollutant formation. Because of the relatively small computational demands of the mathematical models, investigators with only moderate computational resources can easily consider relatively large chemical reaction mechanisms.

The stirred reactor consists of a small thermally-insulated chamber that has inlet and outlet ducts. A steady flow of fuel and oxidizer are introduced in such a way that high-intensity turbulent mixing causes the contents of the reactor to be nearly spatially uniform. This means that the rate of conversion from reactants to products is controlled by chemical reaction rates and not by mixing processes. Thus, a well-stirred reactor can be modeled as a micromixed perfectly stirred reactor (PSR), where the mixing rates are assumed to be infinitely fast.

In addition to fast mixing, the modeling of well-stirred reactors requires certain assumptions. First of all, the walls must be assumed to be noncatalytic. Furthermore, the flow through the reactor has to be characterized by a nominal residence time. This residence time has to be deduced from the flow rate and the reactor volume.

Unlike modeling flow-reactor or shock-tube experiments, which requires solving systems of time-dependent initial value problems, modeling well-stirred reactors requires solving systems of nonlinear algebraic equations. The numerical method adopted here ${ }^{3}$ is the same one that we developed and used recently for the solution of premixed flame problems. It utilizes a hybrid Newton/time-integration procedure. Both Newton iteration and time integration have been used previously to solve well-stirred reactor problems. The advantage of the present approach is that it combines the strengths of both methods. Consequently, the algorithm described is both robust and efficient. Furthermore, methods for rate-of-production analysis and sensitivity analysis greatly facilitate analysis of the computed results.

\section{References}

1. R. J. Kee, G. Dixon-Lewis, J. Warnatz, M. E. Coltrin, J. A. Miller, "A Fortran Computer Package for the Evaluation of Gas-Phase, Multicomponent Transport Properties," Sandia National Laboratories report, in preparation.

2. R. J. Kee, J. F. Grcar, M. D. Smooke, and J. A. Miller, "A Fortran Program for Modeling Steady Laminar One-dimensional Premixed Flames," Sandia Report SAND85-8240 (1985).

3. P. Glarborg, R. J. Kee, and J. A. Miller, "Kinetic Modeling and Sensitivity Analysis on Nitrogen Oxide Formation in Well-Stirred Reactors," Comb. Flame 65, 177 (1986). 

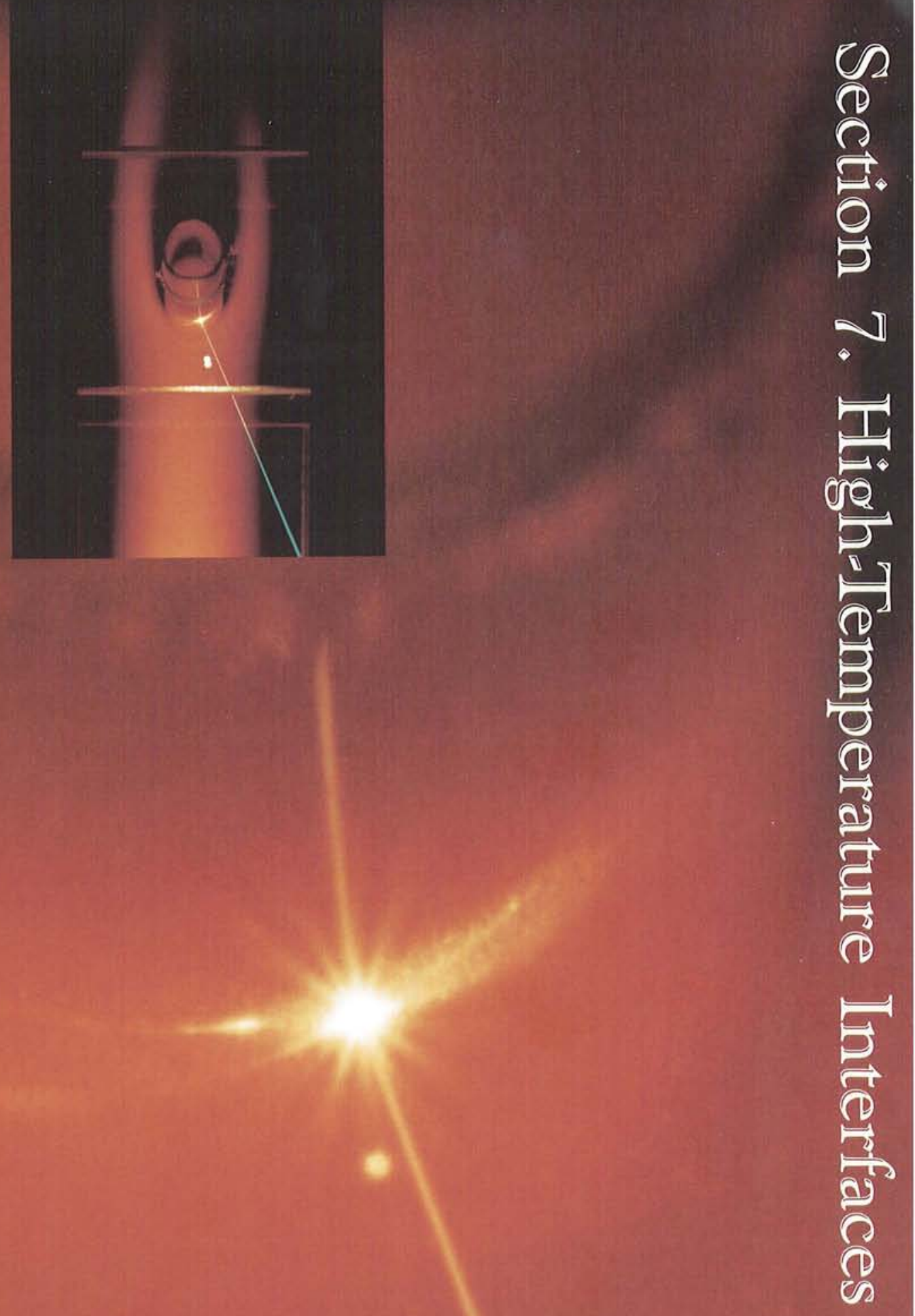
The inset photograph on the preceding page shows a deposit being grown on a platinum-rhodium substrate immersed in the exhaust-gas flow of a sodium sulfate-seeded, methane/air flame. The enlarged photograph provides an expanded view of the gaseous boundary layer surrounding the substrate and the blue argon ion laser beam used for Raman-scattering measurements. This work provides key information for understanding and controlling the corrosive deposits that result from the combustion of impure fossil fuels. The present work (see related articles, pages 7-1 and 7-4) has used in situ Raman scattering to identify deposit compositions and optical interferometry to measure film thicknesses. 


\section{Section 7 \\ High-Temperature Interfaces}

Research in high-temperature interfaces at Sandia National Laboratories is sponsored primarily by the Materials Sciences Division of the Department of Energy's Office of Basic Energy Sciences. One phase of this program involves the study of materials immersed in a combustion environment. Interdisciplinary studies involving the chemistry, physics, and fluid mechanics of such systems are performed using advanced diagnostics (emphasizing laser-based nonperturbing probes) and modern computational techniques. This effort focuses on the interface between the material surface and hostile gas flows, and includes research in the formation of thin films, adsorption and segregation studies, the deposition of flame species onto immersed material surfaces, concomitant gas/surface reactions as well as deposit/substrate corrosive attack, and the development of advanced diagnostic methods for application to these and other multiphase studies.

Throughout the studies of combustion materials, advanced and novel diagnostics are used. Laserbased methods are applied in a variety of ways, with an emphasis on Raman techniques and nonlinear optical methods that now include surface second harmonic generation (SHG), capable of probing submonolayer surface properties in situ, and CARS studies of particle-laden material processing flames. In addition, an array of conventional surface and gas-phase probes are employed to fully characterize the systems investigated.

\section{Techniques for Measuring Film Thicknesses: Interference Effects in Raman Scattering from Thin, Growing Deposits}

\section{K. F. McCarty, R. J. Anderson}

Raman spectroscopy has proven itself to be a valuable tool for the analysis of thin deposits, particularly in its ability to determine the composition of deposits and interfaces. It would be desirable to use Raman spectroscopy as a technique to measure the thickness of deposits and coatings, with the potential application being simultaneous thickness and composition measurements. In practice, however, the details of the irradiation of the film by the laser beam and the
Raman scattering process itself must be carefully considered when attempting quantitative thin film Raman measurements.

Here, the use of Raman scattering as a technique for measuring the thickness of transparent deposits has been investigated. The thicknesses of sodium sulfate $\left(\mathrm{Na}_{2} \mathrm{SO}_{4}\right)$ deposits being grown on platinum $/ 10 \%$ rhodium substrates were determined directly by in situ optical interferometric measurements performed simultaneously with in situ Raman scattering measurements. Oscillations in the intensity of the Raman scattering signal as a function of deposit thickness have been experimentally observed for deposits up to 0.8 microns thick. The Raman signal oscillations are analyzed using a model involving the superposition of the multiple reflections of the incident laser beam and the separate superposition of the multiple reflections of the scattered radiation. Conditions under which the 
oscillations can be minimized, leading to easily usable Raman signal/deposit thickness relationships, are briefly discussed.

Deposits of sodium sulfate were grown from the vapor phase on $1.27-\mathrm{cm}$ diameter cylindrical substrates clad with a platinum $/ 10 \%$ rhodium alloy. The substrates were immersed in the exhaust gases of a $\mathrm{Na}_{2} \mathrm{SO}_{4}$-seeded flame. Details of the experimental procedure are given elsewhere. ${ }^{1}$ A 488-nm, circularly-polarized argonion laser beam, operated at $400 \mathrm{~mW}$ at the sample, was used for the Raman scattering measurements, and its reflected beam was used for the interferometric thickness measurements. The laser beam was incident at about $4^{\circ}$ to the surface normal; thus, the angle of incidence was approximated as being $0^{\circ}$.

The substrates were maintained at $550^{\circ} \mathrm{C}$ during the deposit-growth process, which occurred at a rate of approximately $0.025 \mathrm{mi}-$ crons/min. The areas under the $985 \mathrm{~cm}^{-1}$ (totally symmetric vibration) sulfate Raman bands were determined by digital integration. The Raman scattered light, collected with f/2.2 efficiency at an angle of $68^{\circ}$ from the surface normal, was passed through a dichroic sheet polarizer to select light polarized either perpendicular (s-polarized) or parallel (p-polarized) to the Raman scattering plane. A polarization scrambler, placed in front of the entrance slits of the spectrometer, was used in a manner to maximize the efficiency of the gratings. The use of the scrambler for the p-polarized collection and not for the s-polarized collection increased the throughput of the s-polarized radiation by about $27 \%$ relative to the p-polarized radiation.

In the classical picture of Raman scattering, the incident laser beam establishes an electric field which induces electric dipoles in the scattering medium. The scattered radiation is produced by the induced dipoles. For scattering from thin, transparent films on top of highly reflective metallic substrates, the incident electric field arises from the coherent superposition of the multiple reflections of the incident beam. Similarly, the scattered radiation arises from the coherent superposition of the multiplyreflected radiation originating from a point in the deposit, illustrated schematically in Figure 1. The scattering contribution from a point in the film, which includes the variation of the electric field with thickness, is integrated over the film thickness to give a mathematical model of the Raman scattering.

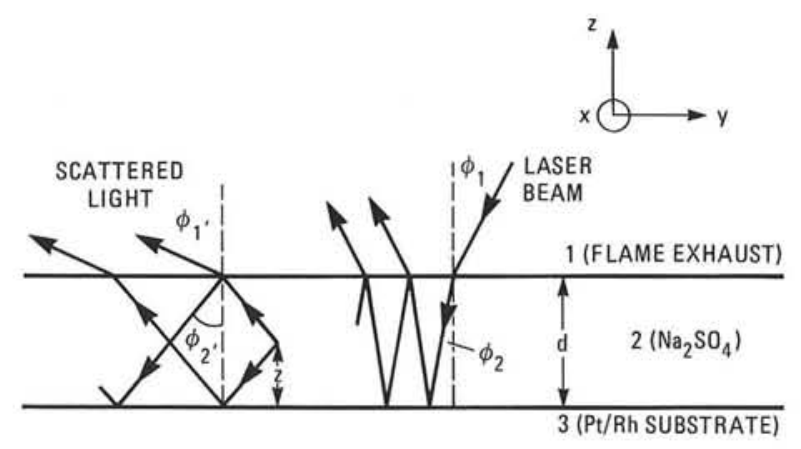

Figure 1. Multiple-beam interference effects in the irradiation of and the Raman scattering from $\mathrm{Na}_{2} \mathrm{SO}_{4}$ deposits.

The relationship between the experimentally determined s-polarized Raman signal (triangles) and the deposit thickness is illustrated in Figure 2. While generally increasing with increasing deposit thickness, the Raman scattering signal from the deposit oscillates as a function of deposit thickness. Minima occur at approximately $0.20,0.45$, and 0.62 microns.

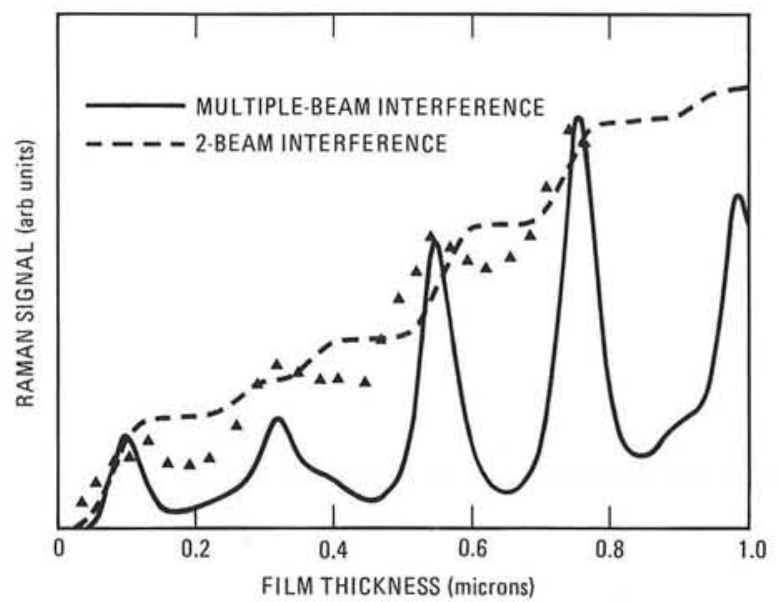

Figure 2. Raman scattering signal/deposit-thickness relationship for s-polarized collection of $\mathrm{Ra}$ man scattering: experimental data (triangles), multiple-beam interference model (solid line), and 2-beam interference model (dashed line). 
Also illustrated in Figure 2 (solid curve) is the Raman signal calculated by assuming the interference of the multiple reflections from the interfaces. The predicted relationship has been scaled to the experimental data by equating the results at 0.76 microns. The qualitative agreement between the results is good; the calculated curve predicts very well the thicknesses at which maxima and minima are observed in the Raman signal. However, the experimental data fails to exhibit the extreme degree of intensity oscillation that is present in the results of the model.

This failure is readily explainable. The model assumes the existence of perfect (in smoothness and sharpness) interfaces and relies on the complete (infinite) superposition of the reflections of the irradiating and scattered radiation. That these assumptions are not completely adhered to is apparent from the nature of the reflected laser beam intensity monitored during the growth of the deposits. Under the conditions of the model, the reflected intensity should be a periodically oscillating, undamped function. However, what was experimentally observed was a rather heavily damped, oscillating function. ${ }^{1}$ The most likely breakdown in assumptions is the condition of the interfaces with roughness occurring, particularly at the growing solid deposit/gas interface. In addition, the finite curvature of the interfaces and the solid angle of collection of the scattered radiation have not been accounted for.

The effect of a loss of reflectivity (through roughness or corrosion) was investigated for the limiting case of 2-beam interference, for which the electric field in the deposit is established by the superposition (interference) of the incident laser beam and a single reflection from the substrate. Similarly, the Raman scattered signal arises from the superposition (interference) of the directly scattered light and a single reflection from the substrate. The 2-beam result (Figure 2, dotted curve) has also been scaled to the experimental data by equating the results at 0.76 microns. This model predicts no oscillations in the Raman signal as a function of deposit thickness. Plateaus occur in the regions corresponding to the extreme minima of the multiple-beam prediction. Clearly, the conditions of the experiment lie between the 2-beam and the (infinite) multiple-beam results. Enough reflections of sufficient intensity occur to produce intensity oscillations in the Raman signal. However, the finite number and/or diminished intensity of the reflections limit the extremity of the oscillations.

Figure 3 illustrates the results of an independent experiment in which only the p-polarized Raman scattered radiation was collected. The experimental Raman scattering signals have been multiplied by a factor of 1.27 in order to compensate for differences in detection efficiencies between the s- and p-polarized light. Figures 2 and 3 are plotted on the same absolute scale, and the same scale factors applied to the multiple-beam and 2-beam calculations of Figure 2 have been applied to the corresponding calculations of Figure 3. In contrast to the experimental s-polarized collection results, the experimental p-polarized collection Raman signal exhibits no oscillations as a function of deposit thickness and is considerably reduced in absolute intensity. While the multiple-beam prediction contains oscillations in intensity, the "contrast" of the oscillations has been extremely reduced compared to the corresponding s-polarized

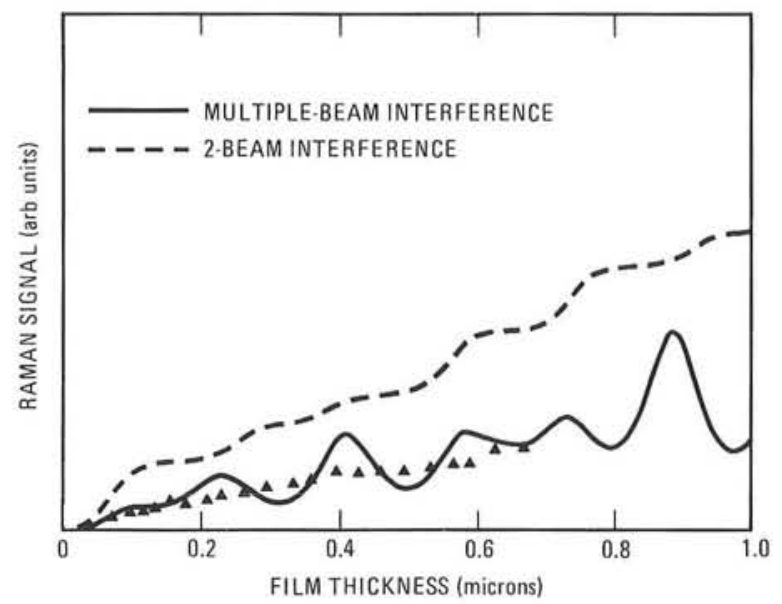

Figure 3. Raman scattering signal/deposit-thickness relationship for p-polarized collection of $\mathrm{Ra}$ man scattering: experimental data (triangles), multiple-beam interference model (solid line), and 2-beam interference model (dashed line). 
collection calculation. Given the imperfect characteristics of the experimental deposits, these small oscillations are not observed experimentally.

The large difference between the results of the s-polarized and p-polarized collections arises mainly from the greater reflectivity of the s-polarized Raman scattered radiation from the interfaces for the collection angle used $\left(68^{\circ}\right)$. The high s-polarized reflectivity results in strong interference effects in the Raman scattered radiation and this interference strongly dominates the Raman signal/deposit thickness relationship. This leads to large oscillations in the intensity relationship and a strong enhancement of the Raman signal at the intensity maxima.

When attempting to utilize Raman scattering as a deposit thickness measurement technique, it is evident that the characteristics of the deposit and the interfaces, and the details of the irradiation and the scattering processes must be carefully considered. Under perfect conditions, the deposit thickness can be a multivalued function of the Raman signal (Figure 2, solid curve). However, for less than perfect conditions (most notably, for imperfect interfaces), the severity of the oscillations is reduced (Figure 2 , experimental data). In addition, even for perfect conditions, the oscillations can be greatly minimized through the careful selection of the irradiating and scattering geometries and of the collection polarization (Figure 3, solid curve). In this manner, a nearly linear relationship between the Raman scattering signal and the deposit thickness can be obtained and utilized.

\section{References}

1. K. F. McCarty and R. J. Anderson, "Realtime Measurements of Deposit Formation From Sodium Sulfate-Seeded Flames," submitted to Comb. Sci. Tech. (1986).

\section{Deposit-Forming Reactions of Pyrite and Sodium in Seeded Flames: an In Situ Raman Study}

\section{J. C. Hamilton, K. F. McCarty}

In many operating combustion systems, elements such as sodium, sulfur, and vanadium are introduced in small quantities as fuel impurities. In coal-burning systems, solid and liquid deposits associated with these impurities frequently form on surfaces exposed to hot combustion products. These deposits cause rapid degradation of metal and ceramic parts and, additionally, reduce heat transfer, thereby lowering operating efficiencies. Pyrite $\left(\mathrm{FeS}_{2}\right)$, a common coal impurity, plays an important role in deposit formation and resulting corrosion. The experiments reported here have been conducted using light scattering to monitor in-flame reactions of pyrite with combustion products and sodium.

Deposits were formed on cylindrical Pt/ $10 \% \mathrm{Rh}$ substrates immersed in the exhaust gases of a seeded, flat-flame burner. Raman scattering was excited using the 510.6-nm line of a copper vapor laser providing short ( $<50 \mathrm{nsec}$ ) light pulses at a $5-\mathrm{kHz}$ repetition rate. Scattered light was analyzed using a triple spectrograph and a gated intensified diode array detector. Gated detection was essential to reduce detection of blackbody radiation from the incandescent particles to negligible levels. Samples of the deposits were also characterized after exposure by $\mathrm{x}$-ray diffraction analysis.

A $25 \mathrm{~cm}^{2}$ square area in the center of the burner was seeded by injecting pyrite particles or a 1:5-by-weight mixture of pyrite and sodium formate. Particles resulting from the combustion of the seed materials were confined to an area about $0.5 \mathrm{~cm}$ in diameter around the streamline of the injection point, whereas vapor-phase combustion products spread to an area about 2.5 $\mathrm{cm}$ in diameter. The equivalence ratio $(\phi)$ of the flame, which is the ratio of the stoichiometric $\mathrm{O}_{2}$ flow to the actual $\mathrm{O}_{2}$ flow, was varied between 0.98 and 1.18 . 
Figure 1 shows Raman spectra obtained from deposits during their formation from pyriteseeded flames on inert substrates held at $550^{\circ} \mathrm{C}$. In an oxygen-rich flame $(\phi=0.98)$, the deposit's Raman spectrum exhibits peaks at 273, 390, 473 , and $591 \mathrm{~cm}^{-1}$. Comparison with reference spectra allows identification of this scattering species as $\mathrm{Fe}_{2} \mathrm{O}_{3}$. Under these flame conditions, incandescent particles could be seen following the streamlines of the flame. Apparently, pyrite particles oxidized in the flame, forming $\mathrm{Fe}_{2} \mathrm{O}_{3}$ particles which deposited on the substrate.

As shown in Figure 1, the deposits formed under reducing flame conditions $(\phi=1.05$ and $\phi=1.18$ ) showed no detectable Raman spectra, and thus could not be identified by this technique.

Because hot corrosion frequently occurs in combustion systems burning fuels containing sodium impurities, investigations were undertaken coseeding pyrite and sodium formate, a convenient sodium source, into the flame. For this combination of seeding species, deposits can contain $\mathrm{Na}, \mathrm{Fe}, \mathrm{S}$, or $\mathrm{O}$, and the complexity of the deposits is greatly increased.

When sodium formate and pyrite were both added to the flame, a more complex spatial distribution of products occurred than in the flame seeded only with pyrite. Solid particles were confined to a central region of the flame, whereas vapor species had a wider spatial extent in the flame. This resulted in deposits with two distinct regions having different colors, morphologies, and chemical compositions.

Figure 2 shows Raman spectra obtained from various positions on the substrate illustrating the inhomogeneity of the deposits produced from these coseeded flames. (These deposits were formed using a low seeding level relative to those shown in Figure 3.) All the codeposition experiments reported here used oxygen-rich flame conditions, $\phi=0.98$. Near the outer edge of the deposit, the major deposit species found was a sulfate compound, apparently formed by reactions among pyrite, sodium, and oxygen. In an intermediate position, both

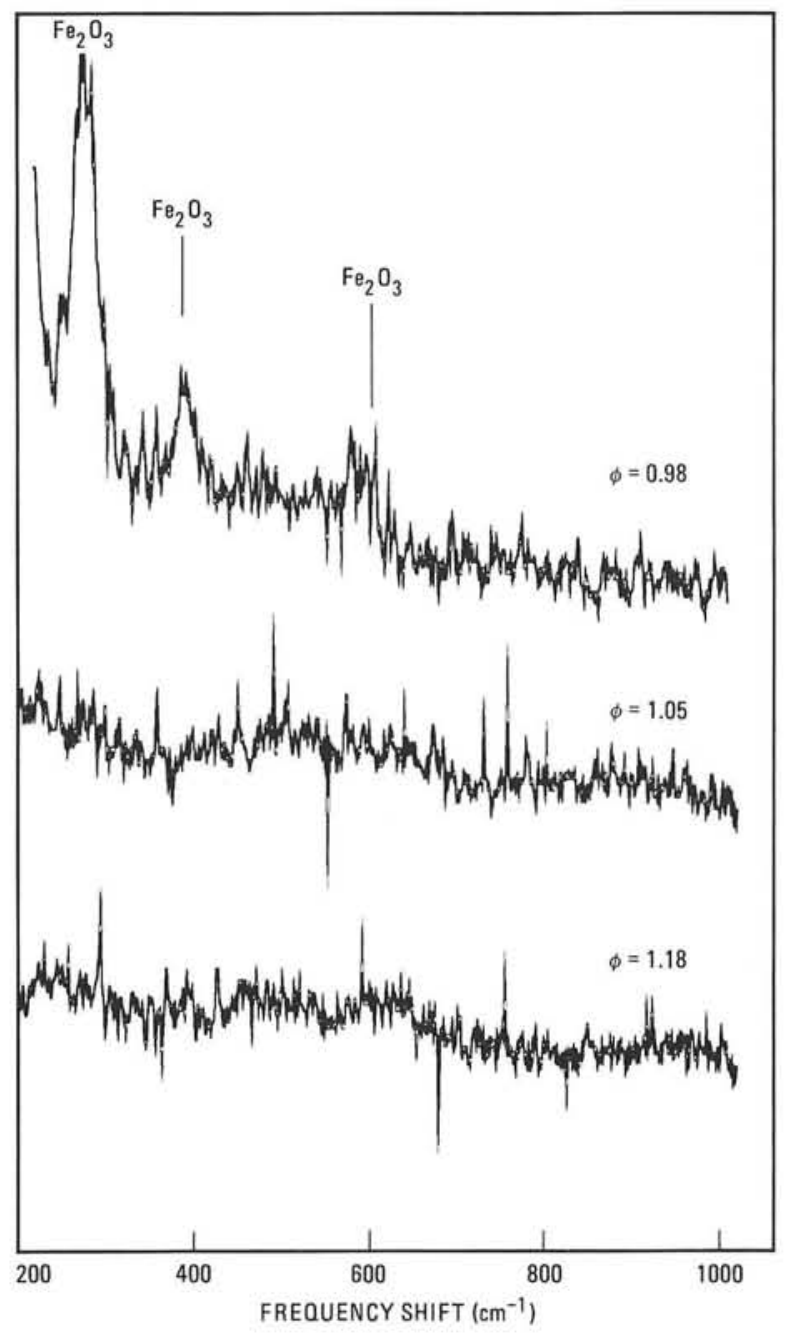

Figure 1. In situ Raman spectra collected from deposits during formation in pyrite-seeded flames with various flame stoichiometries. Deposits were formed on inert $\mathrm{Pt} / 10 \% \mathrm{Rh}$ substrate held at $550^{\circ} \mathrm{C}$ in flame.

$\mathrm{Fe}_{2} \mathrm{O}_{3}$ and sulfates were observed. In the central portion of the deposit, only $\mathrm{Fe}_{2} \mathrm{O}_{3}$ was seen. Thermodynamic calculations indicate the presence, at flame temperatures, of gas-phase deposit precursors that contain sodium and sulfur. These precursors react to form sulfates at lower temperatures. ${ }^{1}$ Expansion and diffusion apparently spread these gas-phase precursors in the flame and exhaust. Thus, the sulfate-containing deposit formed over a wider area than the $\mathrm{Fe}_{2} \mathrm{O}_{3}$ 


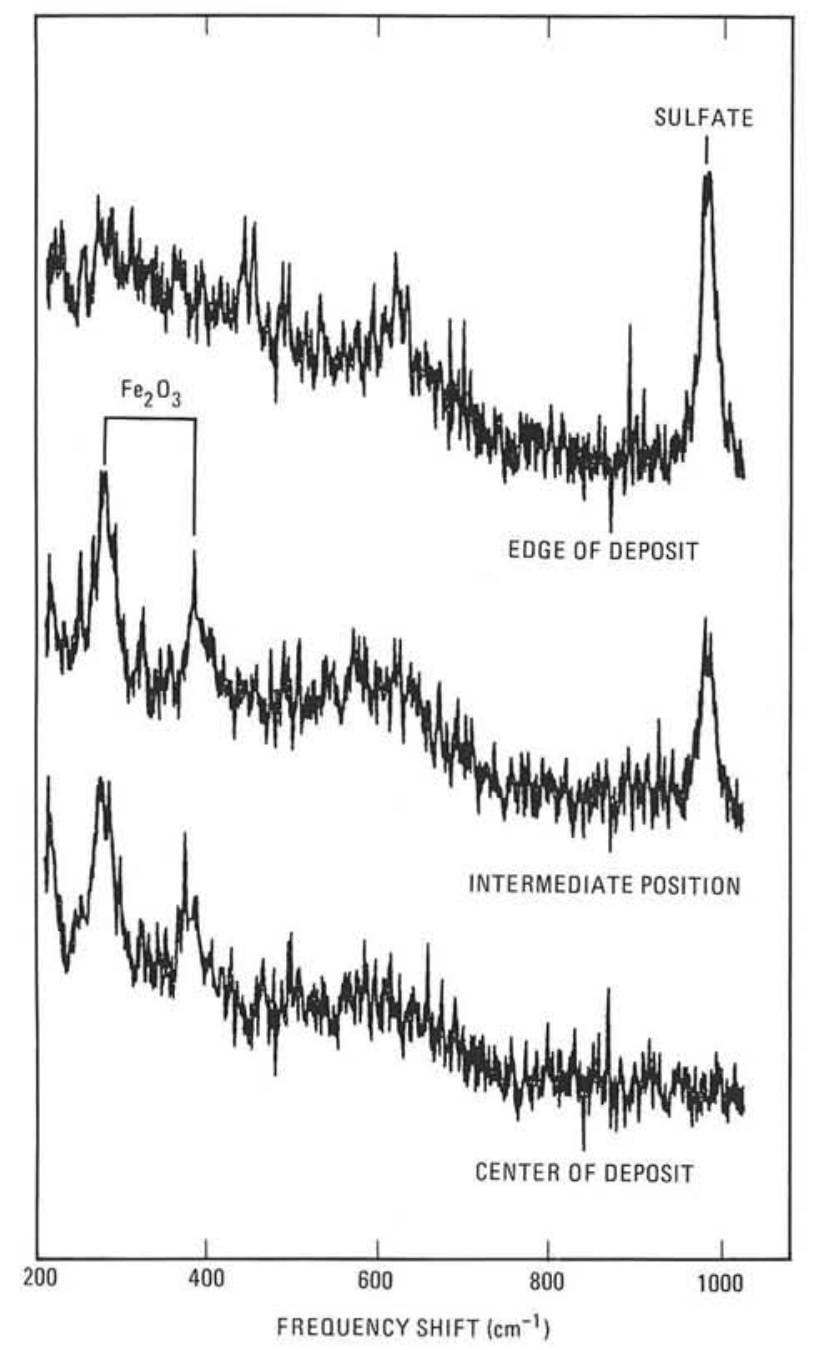

Figure 2. In situ Raman spectra collected from deposit during formation in a flame seeded with 1:5 ratio by weight of pyrite and sodium formate. Spectra are shown for three different regions of the deposit. Deposits were formed on inert $\mathrm{Pt} / 10 \% \mathrm{Rh}$ coated substrate held at $550^{\circ} \mathrm{C}$ in flame. Seeding level was lower than in Figure 3 .

(formed from particles that followed the streamlines).

When the seeding level of pyrite and sodium formate was increased, the deposit composition and morphology changed markedly. Figure 3 shows the Raman spectra obtained from the edge and from the center of the deposit formed under high seeding levels. The deposit formed at the edge shows major peaks at 444,619 , and $988 \mathrm{~cm}^{-1}$, indicating that sulfate species are

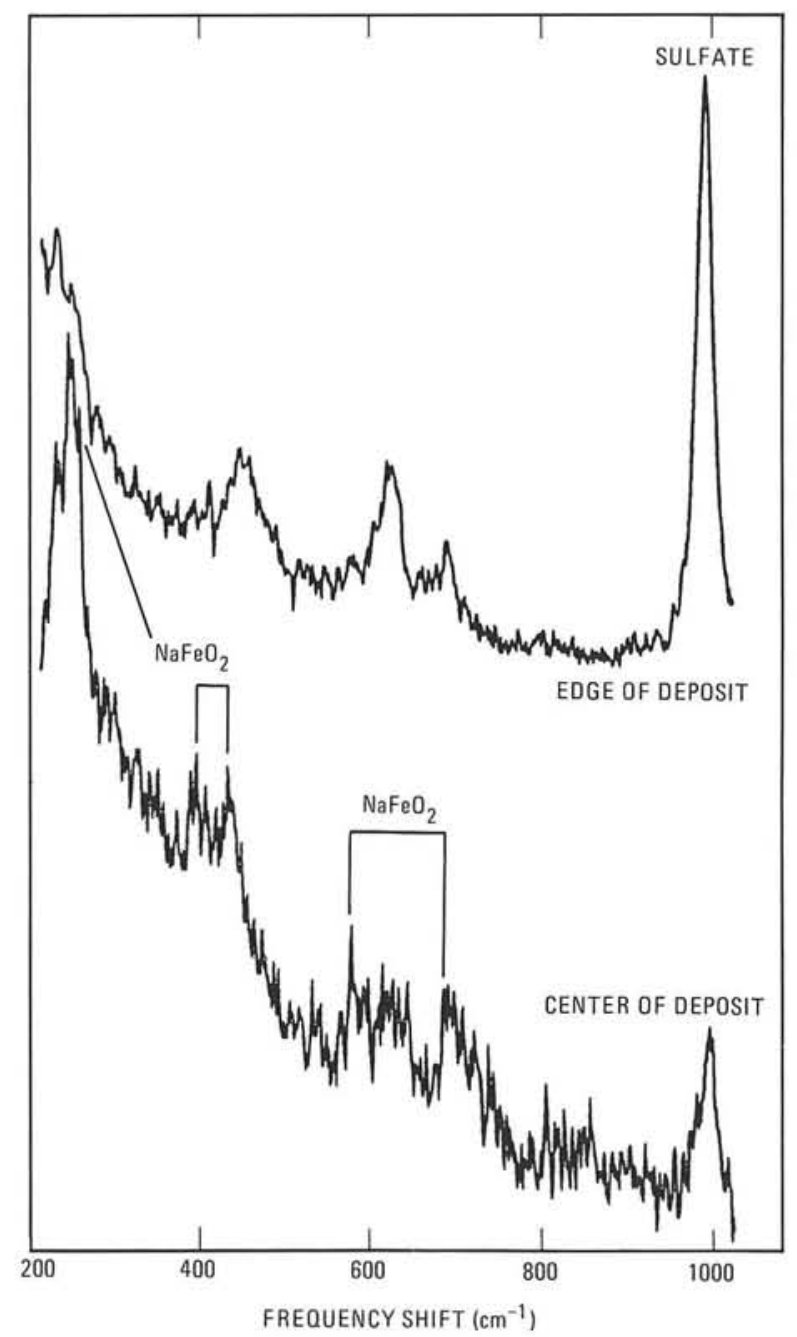

Figure 3. In situ Raman spectra collected from deposit during formation in a flame seeded with 1:5 ratio by weight of pyrite and sodium formate. Spectra are shown for two different regions of the deposit. Deposits were formed on inert $\mathrm{Pt} / 10 \% \mathrm{Rh}$ coated substrate held at $550^{\circ} \mathrm{C}$ in flame. Seeding level was greater than in Figure 2.

formed in or from the vapor phase at this seeding level. The center of the deposit shows a very different composition from that formed with a low seeding level, however. Peaks are seen at $241,393,428,574,619,688$, and $991 \mathrm{~cm}^{-1}$, indicating the presence of sodium ferrate and sulfate compounds in this deposit. 
Our observations demonstrate that chemical reactions occurring in the flame can produce compounds such as $\mathrm{Fe}_{2} \mathrm{O}_{3}, \mathrm{NaFeO}_{2}$, and mixed sulfates. The composition of the deposits is strongly influenced by the stoichiometry of the flame and the level of seeding into the flame. These compounds can combine with other substances on reactive metals to produce low-melting point, corrosive deposits. Our observations also demonstrate the utility of Raman scattering for the in situ study of surface chemical reactions between pyrite and gaseous species containing oxygen or sulfur. These reactions play an important role in coal-burning power plants and typify more complex reactions which must be understood to control long term deposition and corrosion phenomena.

\section{Reference}

1. K. L. Luthra and H. S. Spacil, "Impurity Deposits in Gas Turbines from Fuels containing sodium and Vanadium," J. Elec. Soc. 129, 649 (1982).

\section{Nonlinear Optical Surface Probe of Adsorption and High-Temperature Segregation}

\section{R. J. Anderson, J. C. Hamilton, G. W. Foltz}

The behavior of materials in combustion systems has considerable technological significance. Structural members weakened by corrosion fail, deposit-laden surfaces inhibit heat flow, and poisoned catalysts lose their chemical activity. In each case, the change in the material is initiated at the surface, and, even if thicker layers form eventually, the driving reaction proceeds at the substrate-deposit interface or at the gas-deposit interface. To probe processes at interfaces - at the monolayer level - we must have diagnostic tools that are sensitive to the electronic and vibrational structures of a material surface. A promising laser-based, nonintrusive technique for such analysis is surface nonlinear optical spectroscopy. ${ }^{1}$

Nonlinear spectroscopies are those in which frequency components of the electromagnetic field combine coherently to modify each other, mediated by the properties of the material in question. New wavelengths at combination frequencies may appear, or the intensity of one incident component may be parametrically modified by the presence of another. The lowest order process is that in which the spectral elements couple in pairs to form second harmonics and sum/difference frequencies. In the dipole approximation, this occurs only in noncentrosymmetric media, since the coupling material property must lack inversion symmetry, At the interface between two centrosymmetric materials, however, the inversion symmetry common to both is broken, and here the lowest order coupling, forbidden in the bulk, endures. Thus at sufficient intensities, the light transmitted and reflected by the interface will contain small components at sums and differences of the incident frequencies.

We have utilized the simplest manifestation of this phenomenon, surface second harmonic generation, to probe the structure of material surfaces. The sample essentially acts as a nonlinear mirror. A single, pulsed laser beam incident on an interface generates an oscillating dipole at the surface, at twice the frequency of the incident light. Light emitted by this dipole is a coherent beam that propagates along with the reflected fundamental, and can be filtered and detected. The intensity of the second harmonic is a function of the electronic structure of the reflecting interface.

Primary surface reactions in combustion systems include adsorption and high temperature segregation. Molecular adsorption precedes the formation of thicker layers, and is the first step in surface catalyzed processes. The segregation of trace contaminants (those present at a parts-per-million level in the bulk) to the surface can poison catalysts, or may weaken the 
bond to a protective scale that is eventually exfoliated. We have studied the effect of hydrogen and oxygen adsorption, and high temperature surface segregation, on second harmonic generation (SHG) from the (110) face of a Fe-18Cr$3 \mathrm{Mo}$ single crystal. Measurements were made in situ and in real time.

The 6-mm-diameter sample was polished and mounted in an ultrahigh vacuum chamber (base pressure $2 \times 10^{-10}$ Torr). The temperature of the sample manipulator was controlled by liquid nitrogen cooling combined with resistive heating. The surface temperature was monitored with a chromel-alumel thermocouple spotwelded to the crystal face. Argon-ion bombardment was used to clean the surface, and surface atomic composition was determined using Auger spectroscopy. LEED was used to confirm surface ordering, and gas exposures were regulated using high resolution leak valves. For each run, the surface was sputtered to bulk composition and annealed to form an ordered crystal face. The frequency-doubled output of a Quanta-Ray DCR II, Q-switched Nd:YAG laser pumped a dye laser that used LDS698 dye and lased at 700 $\mathrm{nm}$. A reference beam at the second harmonic wavelength was generated in a suitably oriented thin quartz crystal using a portion of the main beam. The collimated p-polarized probe beam ( $2 \mathrm{~mJ}$ or less per 5 -ns pulse, roughly $4-\mathrm{mm}$ dia.) was incident on the alloy crystal face at about 60 degrees angle of incidence. The reference beam and the light reflected from the sample were passed through copper-sulfate-solution filters to remove the fundamental, and the second harmonic $(350 \mathrm{~nm})$ component of each was detected. Sample signal, reference, sample temperature, and chamber pressure were collected and stored on a DEC PDP $11 / 23$ computer on a shot-by-shot basis.

Hydrogen and oxygen are known to adsorb dissociatively to the clean surface of iron, the major component of the alloy in question. That is, the diatomic molecules split at the surface to form adsorbed atoms. Figures 1 and 2 show the change in surface SHG during exposure of the sample at $50^{\circ} \mathrm{C}$ to $8 \times 10^{-9}$ Torr oxygen and $4 \mathrm{x}$ $10^{-8}$ Torr hydrogen, respectively.

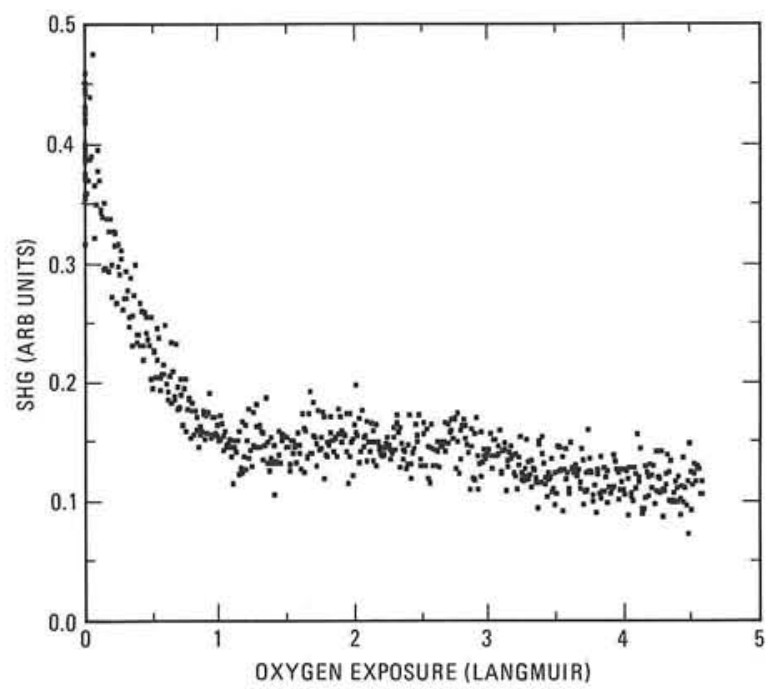

Figure 1. Intensity of the second harmonic of $700 \mathrm{~nm}$ generated at $\mathrm{Fe}-18 \mathrm{Cr}-3 \mathrm{Mo}(110)$ surface during oxygen adsorption at $50^{\circ} \mathrm{C}$.

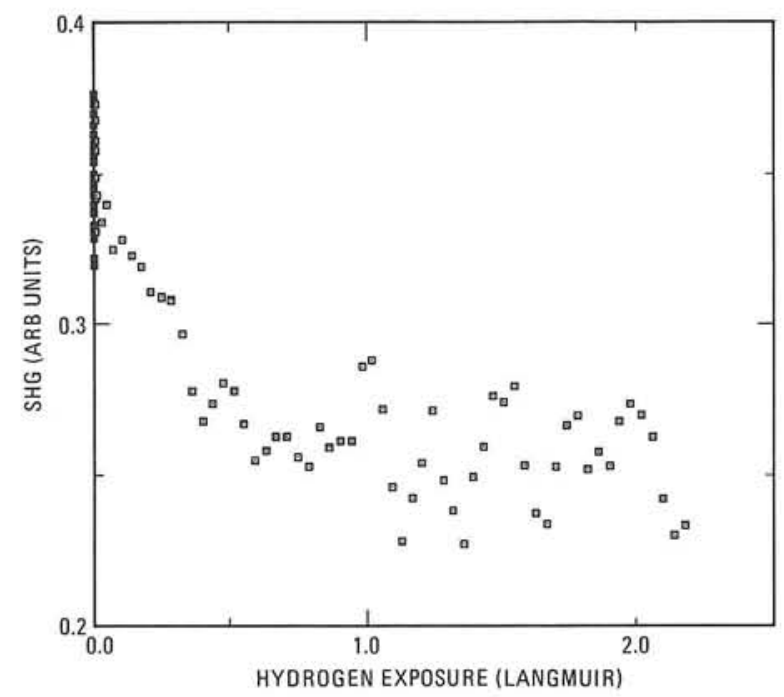

Figure 2. Intensity of the second harmonic of $700 \mathrm{~nm}$ generated at $\mathrm{Fe}-18 \mathrm{Cr}-3 \mathrm{Mo}(110)$ surface during hydrogen adsorption at $50^{\circ} \mathrm{C}$.

Oxygen adsorption reduces the signal to about one-third of its original value by one langmuir exposure, with a smaller change noticeable in the 2 to 4 langmuir range. LEED analysis of this adsorbed layer shows that it is not an ordered array at any level of exposure, in contrast to adsorption onto a clean iron crystal. The alloy is a random distribution of minor constituents (chromium and molybdenum) in the crystal lattice, which leads to a disordered, statistical array of many different sites on the crystal face. We believe that this is the reason 
for the lack of long-range order in the adsorbed layer. Nevertheless, the SHG signal shows that there is a significant change in that portion of the electronic structure of the surface probed by the process of doubling $700-\mathrm{nm}$ light. A smaller fractional change arises from the adsorption of hydrogen, but the signal loss shows the same pattern as a function of exposure. Hydrogen adsorption appears to induce a smaller change in the electronic structure measured by SHG at $700 \mathrm{~nm}$, compared to oxygen adsorption.

Figure 3 shows the change in SHG from an originally clean metal surface during heating from 50 to $950^{\circ} \mathrm{C}$ at a rate of 7.5 per minute. Also shown are the changes in surface atomic fractions of chromium and nitrogen measured during an identical run using Auger electronic spectroscopy.

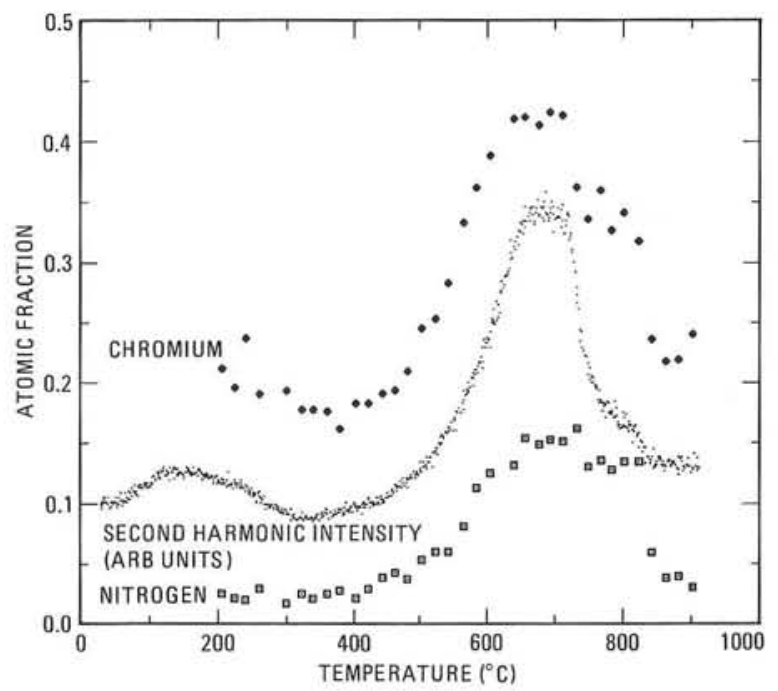

Figure 3. Intensity of the second harmonic of $700 \mathrm{~nm}$ generated at $\mathrm{Fe}-18 \mathrm{Cr}-3 \mathrm{Mo}(110)$ surface during heating in vacuum from 50 to $950^{\circ} \mathrm{C}$ at $7.5^{\circ}$ per minute. Atomic fractions of chromium and nitrogen on the surface, measured using Auger spectroscopy during an identical heating sequence, are also indicated.

While chromium is a bulk constituent of the alloy, nitrogen is a trace contaminant present in very small quantity in the bulk. As the temperature reaches $500^{\circ} \mathrm{C}$, chromium and nitrogen begin to cosegregate to the surface, increasing to maximum coverage at 650 to $700^{\circ} \mathrm{C}$, then diminishing to their bulk composition values above $850^{\circ} \mathrm{C}$. Accompanying the cosegregation is a dramatic (nearly four-fold) increase in the SHG signal. The doubling of $700-\mathrm{nm}$ light is much more efficient at the $\mathrm{Cr} / \mathrm{N}$ enriched surface, perhaps because of a change to an electronic structure that has surface interband transition energies more nearly resonant with the fundamental frequency or the second harmonic.

The observations described here demonstrate the utility of second harmonic generation for surface studies. They suggest, as well, its strong potential for bridging the gap between ultrahigh vacuum and atmospheric conditions in studies of monolayer phenomena related to questions of fundamental importance to the behavior of surfaces in systems of technological significance.

\section{Reference}

1. Y. R. Shen, The Principles of Nonlinear Optics (Wiley, New York, NY, 1984), Ch. 25.

\section{Nitrogen CARS Studies of a Flame Containing Silica Particles}

\author{
M. D. Allendorf, R. P. Lucht, E. Potkay *
}

Combustion techniques have been used to produce a variety of refractory materials. One process of industrial significance is the pyrolysis of an organosilicon compound or silicon halide in a flame to form silica $\left(\mathrm{SiO}_{2}\right)$, which is then used in the production of optical fiber for the communications industry. For example, in Vapor Phase Axial deposition (VAD), an inert gas carries silicon tetrachloride vapor to a hydrogenoxygen flame. The high temperatures produced by the flame cause hydrolysis and/or oxidationreduction to occur at a high rate, resulting in the nucleation of fine silica particles (diameter

- AT\&T Technologies, Engineering Research Center, Princeton, NJ. 
$\leq 100 \mathrm{~nm}$ ) close to the burner face. These particles continue to grow via condensation, coalescence, and agglomeration mechanisms and are deposited on a rotating boule. Additional processing is required to manufacture optical fiber. A major advantage of this process is that impurities (such as water) are almost completely excluded; this is critical to the production of low light-loss fiber.

Very few quantitative studies have been performed to characterize silica-producing flames. ${ }^{1}$ Current manufacturing processes require accurate modeling of particle nucleation and growth rates in order to improve their efficiency. One technique for measuring these rates is Diffusion Broadening Spectroscopy (DBS), which yields the distribution of particle sizes and their number density in the flame. DBS has already been shown capable of determining these parameters for silica-producing flames, but requires knowledge of the flame temperature. ${ }^{2}$ We have recently evaluated the potential of Coherent Anti-Stokes Raman Spectroscopy (CARS) as a method for obtaining a flame temperature map to be used in conjunction with DBS measurements.

The CARS pump beams were produced by the $532-\mathrm{nm}$ output of a frequency-doubled Nd:YAG laser in our Sirius laser facility; a broadband dye laser pumped by the same Nd:YAG laser produced the Stokes beam. Three-dimensional phase-matching was used. Quartz focusing lenses on either side of the burner had focal lengths of $212 \mathrm{~mm}$, producing an effective CARS sampling length of $\sim 2.5 \mathrm{~mm}$ (determined by measuring the CARS signal of a glass coverslip as it was passed through the focusing region.) Data were analyzed using the Sandia CARS code. $^{3}$

A cross-section of the burner is shown in Figure 1. It was constructed of concentric quartz tubes, with different gases flowing through each tube (Figure 1). The outer diameter of the burner was $2.3 \mathrm{~cm}$. When used to deposit silica, argon is used as a carrier gas in rings 1,2 , and 4 to prevent clogging. For these experiments, nitrogen was substituted for argon in order to make the CARS measurements. Mass flow rates were controlled by Tylan flow controllers; typical values were: $\mathrm{SiCl}_{4} / \mathrm{N}_{2}, 0.2 \mathrm{l} / \mathrm{min}$. (1.9 g $\mathrm{SiCl}_{4} / \mathrm{min}$.); $\mathrm{H}_{2}, 3.0 \mathrm{l} / \mathrm{min}$.; $\mathrm{O}_{2}, 3.5 \mathrm{l} / \mathrm{min}$.; $\mathrm{N}_{2}$, $0.5 \mathrm{l} / \mathrm{min}$. (ring 2) and $3.0 \mathrm{l} / \mathrm{min}$. (ring 4). At these flow rates, approximately $0.7 \mathrm{~g} / \mathrm{min}$ of silica were deposited in the flame. Two shrouds were employed to minimize flame disturbances: a quartz cylinder with an outer diameter of 5.0 $\mathrm{cm}$ and a larger plexiglas cylinder (outer diameter $15 \mathrm{~cm}$ ). Slots were cut in each to allow passage of the laser beams. Due to the very low velocity of these flames $(\sim 2.0 \mathrm{~m} / \mathrm{sec}$, determined by LDV), these were not sufficient to completely exclude disturbances from room air currents. An additional quartz cylinder was added (inner diameter $\sim 2.3 \mathrm{~cm}$ ) which protruded $\sim 1 \mathrm{~cm}$ above the top of the burner. Thus, measurements immediately above the top of the burner were precluded.

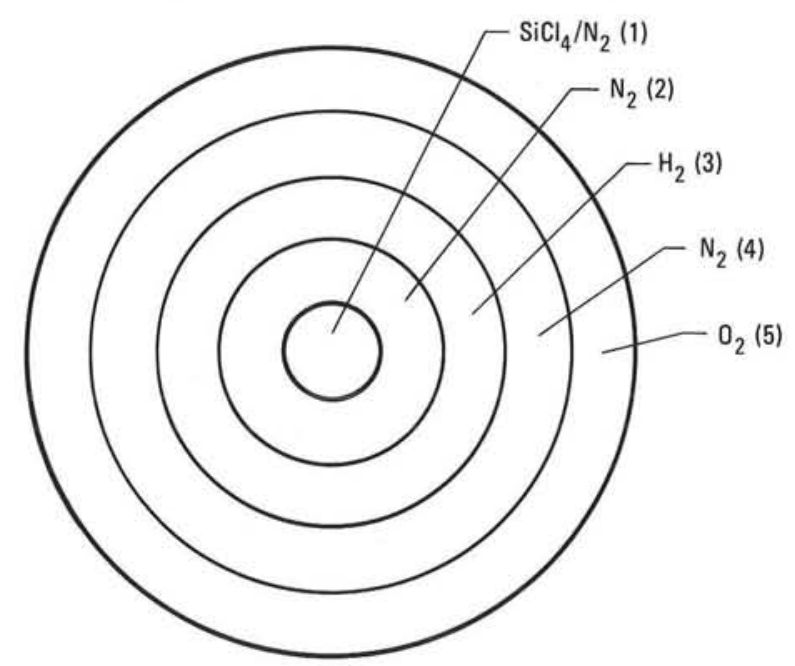

Figure 1. Cross-section of the quartz Vaporphase Axial Deposition burner used in the CARS studies. The numbers in parentheses label the rings, flow rates for which are given in the text.

Previous studies of burners of this type have shown that addition of $\mathrm{SiCl}_{4}$ to produce silica "soot" markedly changes the properties of the flame. Since we intended to use CARS 
temperature data to evaluate particle size and number densities from DBS experiments, it was crucial that measurements of the temperature be made in the presence of silica particles. Calculations based on soot sampling experiments showed that the density of these particles can be as high as $10^{13} / \mathrm{cm}^{3}$. Figure 2 shows a nitrogen CARS spectrum measured at the center of the flame, $10 \mathrm{~mm}$ above the top of the burner, in the presence of silica particles.

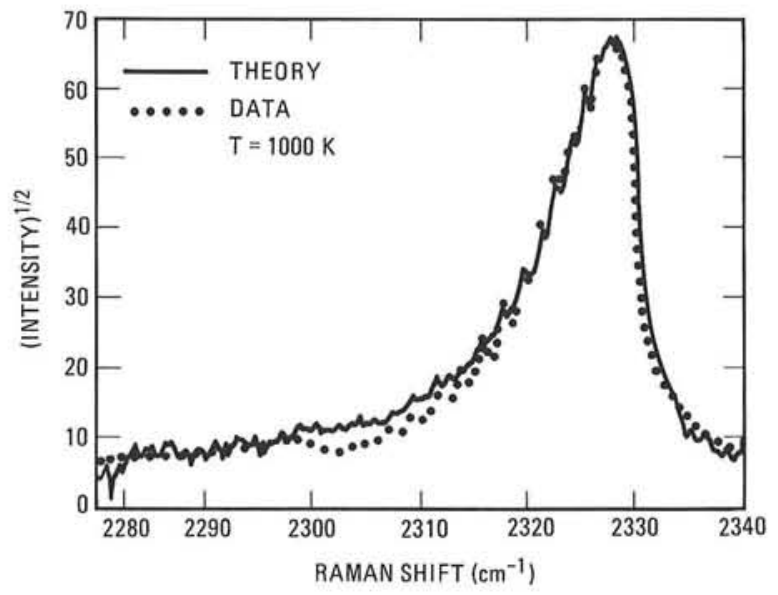

Figure 2. Nitrogen CARS spectrum in a silicasooting flame. The dashed line shows data obtained with the CARS beams focused $10 \mathrm{~mm}$ above the burner surface. The solid line represents a fit of theory to the data, from which the temperature was determined to be $1000 \mathrm{~K}$.

The introduction of silica into the flame caused immediate dielectric breakdown, and a large increase in the amount of scattered light when the pump beams were at maximum pulse energy. However, since nitrogen is plentiful at this location, CARS data could be obtained if the intensity of the pump beams was reduced so that no breakdown occurred $(5-10 \mathrm{~mJ} /$ pulse depending on the amount of $\mathrm{SiCl}_{4}$ added to the flame.) A least-squares fit of theory to the data using the Sandia CARS code indicates that the temperature at this point is approximately 1000 $\mathrm{K}$ (Figure 2, dashed line.)

At points higher in the flame, however, considerable difficulty was encountered due to lower nitrogen concentrations and much higher particle number densities. Figure 3 shows CARS spectra obtained $20 \mathrm{~mm}$ above the top of the burner, with and without $\mathrm{SiCl}_{4}$ added to produce silica.
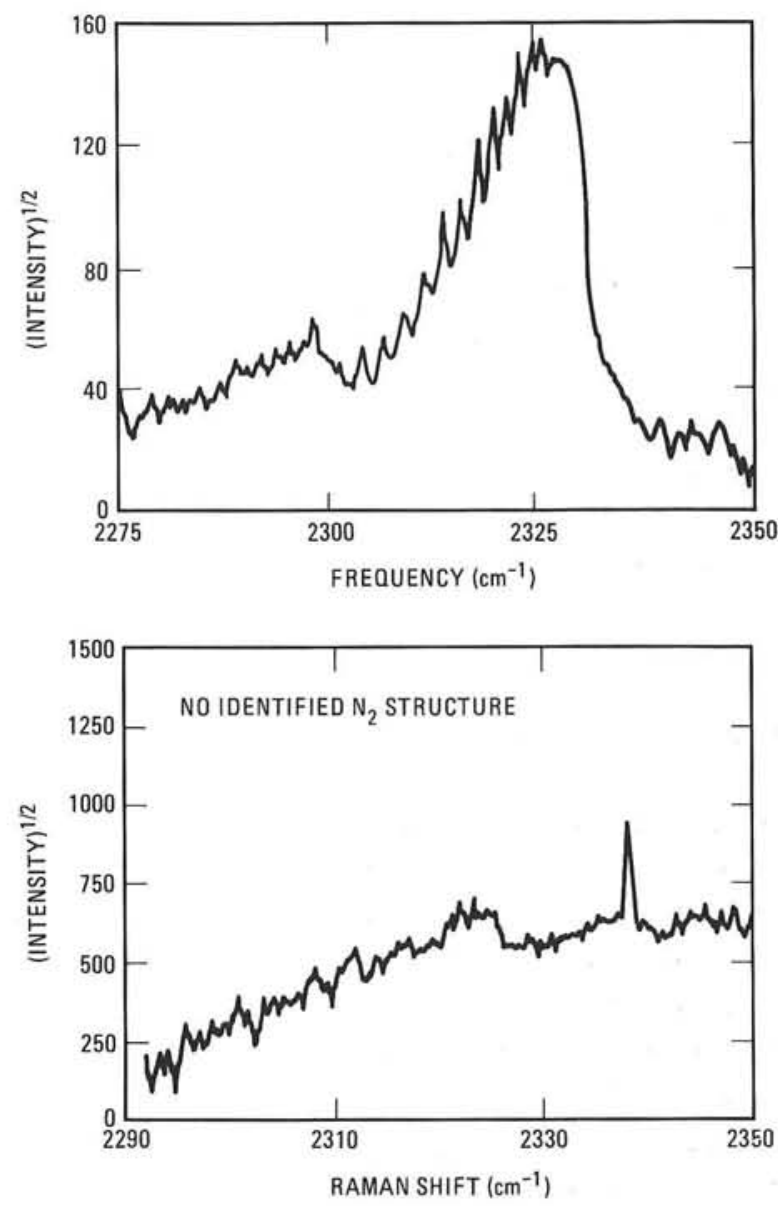

Figure 3. CARS spectra obtained with the CARS beams focused $20 \mathrm{~mm}$ above the burner surface. Top: No silica soot present in the flame. Bottom: $1.9 \mathrm{~g} / \mathrm{min} \mathrm{SiCl}_{4}$ flowing through Ring 1 producing silica soot. Pulse energies were reduced in the lower spectrum to avoid dielectric breakdown. The low concentration of nitrogen at this location produced a signal too small to be observed at these low laser pulse energies.

Although a CARS signal could be observed in the absence of silica (Figure 3, top), the addition of substantial numbers of silica particles to the flame almost completely removed any trace of that signal. This was due to two factors: scattering of the CARS signal and the pump beams by the particles and, more seriously, dielectric breakdown. Unlike carbon soot, silica particles do not absorb visible light. When high intensity CARS beams are focused into a flame containing carbon soot, soot particles are effectively vaporized so that dielectric breakdown does not occur with high frequency. In the presence of silica, however, dielectric breakdown occurred with nearly every laser shot when the 
CARS pump beams were at maximum pulse energy ( $\sim 20 \mathrm{~mJ} /$ pulse). The resulting burst of scattered light completely masks the CARS signal. The situation could not be improved by gating our optical multichannel analyzer, since the scattered light signal is coincident in time with the CARS signal. The spectrum shown at the bottom of Figure 3 was obtained with reduced pump beam intensities to prevent breakdown. Unfortunately, insufficient nitrogen exists in the flame at this point so that no CARS signal could be observed. The slope in the baseline is due to the nonresonant CARS signal with the shape of the dye laser profile.

We conclude from these studies that nitrogen is not a good candidate for CARS measurements in some parts of these flames, since its sometimes low concentration requires pulse energies in excess of the breakdown energy. Oxygen is also present in these flames and may be more abundant in parts of the flame where the concentration of nitrogen is low. It may thus be necessary to measure both oxygen and nitrogen CARS spectra to obtain a complete temperature map. High resolution oxygen CARS spectra must first be measured, however, in order to obtain oxygen line widths at temperatures comparable to those in the flame. We also intend to incorporate a double spectrometer into our CARS experiment in order to further reduce the amount of scattered light produced by the particles in the flame.

\section{References}

1. G. D. Ulrich and N. S. Subramanian, "Particle Growth in Flames III. Coalescence as a Ratecontrolling Process," Comb. Sci. Tech. 17, 119 (1977).

2. W. L. Flower, "Light Scattering Measurements of Soot Particles in Flames," SPIE 644, 28 (1986).

3. R. L. Farrow, R. P. Lucht, G. L. Clark, and R. E. Palmer, "Species Concentration Measurements Using CARS with Nonresonant Susceptibility Normalization," App. Opt. 24, 2241 (1985). 


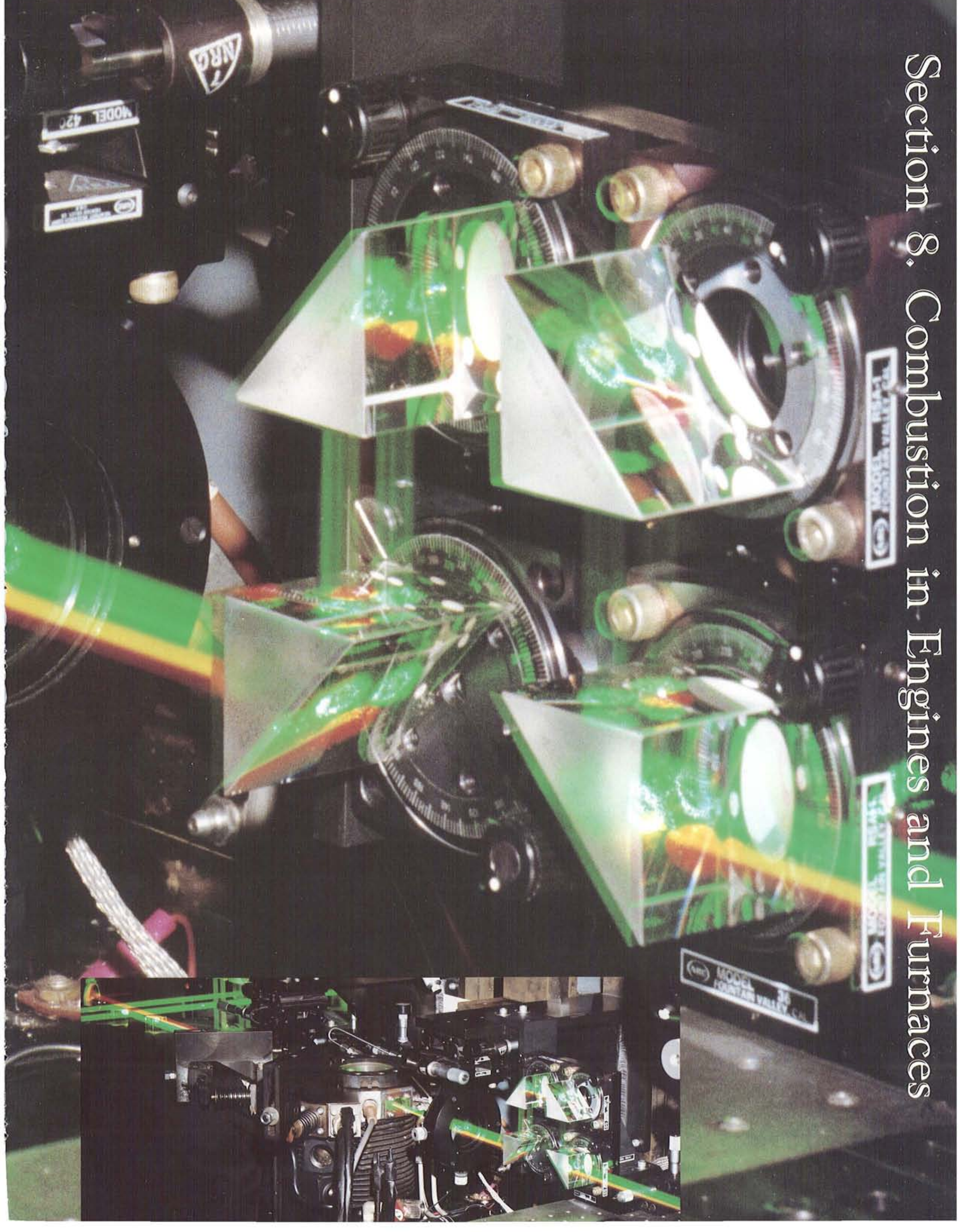


The enlarged photograph on the preceding page shows a unique lensprism optical apparatus that allows the multiple coherent anti-Stokes Raman spectroscopy (CARS) laser beams to be traversed within an internal combustion engine. As the inset photo shows, two such prism systems are used, one on each side of the engine. Vertical traversing is accomplished by separately moving the prism on each side closest to the engine. In that way, we are able to keep the lasers, spectrometers, and other optical components fixed in space and precisely aligned. With this optical arrangement, we have been able to obtain the first in-cylinder measurements of thermal boundary layer development using CARS (see related article, page 8-4). 


\section{Section 8 \\ Combustion in Engines and Furnaces}

Under the sponsorship of DOE's Energy Conversion and Utilization Technologies (ECUT) Program, Sandia National Laboratories is pursuing a broad program of research on the combustion processes in internal combustion engines. The program focuses on the application of advanced laser diagnostics to provide insight into critical problems impeding the development of improved engine concepts. The problem areas under investigation include (a) the interaction between precombustion fluid mechanics and combustion, (b) end-gas autoignition leading to engine knock, and (c) the autoignition and combustion properties of alternative fuel sprays for diesel applications. The diagnostics work involves the development of nonperturbing, laser-based methods that provide time- and space-resolved measurements inside a combustion engine.

A relatively new activity that focuses broadly on continuous combustion systems for furnace and boiler applications has begun to produce interesting new results. The program focuses on the application of advanced laser diagnostics to provide insight into critical problems impeding the development of improved furnace concepts. The initial emphasis of this program is to perform an experimental and theoretical investigation of the pulse combustion process. The goal of this experimental and theoretical program is to develop a fundamental understanding of the physics governing a gas-fired pulse combustor. The experimental work involves the use of nonperturbing laser diagnostics to provide time- and space-resolved measurements of the fluid dynamic and chemical processes controlling the combustion process. The experimental work is closely tied to the development of a one-dimensional nonlinear numerical model.

Several cooperative research groups have been established with industry partners to ensure that this research is relevant to the practical needs of industry and that the resulting technology is transferred quickly to the production sector.

\section{Direct Measurement of the Turbulent Burning Velocity in a Homogeneous- Charge Engine ${ }^{\dagger}$}

\author{
P. O. Witze, J. M. C. Mendes-Lopes*
}

A direct measurement of the turbulent burning velocity was made during premixed combustion in a spark ignition engine. The unburned gas velocity $U_{g}$ was measured using a

† SAE Paper No. 861531 (1986).

- Instituto Superior Técnico, Lisbon, Portugal. high data rate laser Doppler velocimeter system that resolved the gas motion on an individual cycle basis. The ensemble-averaged flame speed $U_{f}$ was determined from ionization probe measurements of the time of flame arrival at discrete positions along the path of flame propagation. The difference between the ensemble-averaged, cycle-resolved unburned gas velocity and the ensemble-averaged flame speed gives a direct measurement of the turbulent burning velocity $S_{T}$, since

$$
S_{T}=U_{f}-U_{g}
$$

Three different procedures for determining the turbulence intensity were compared: 
1) direct ensemble average, which ignores the existence of all forms of cyclic variations; 2) phase-shifted ensemble average, which corrects for cyclic variations in combustion-generated velocities, but does not recognize cyclic variations in the precombustion fluid motion; and, 3) trend-removing cycle-resolved analysis that utilized a third-order polynomial fit to attempt to remove all forms of cyclic variations. Each of these procedures gives progressively smaller values for the turbulence intensity, since each progressively removes a larger portion of the velocity fluctuations from the calculation of the standard deviation.

The measurements were made in the Sandia research engine, operated at $300 \mathrm{rev} / \mathrm{min}$ on a stoichiometric mixture of gaseous propane and air. To assure a pure mixture undiluted by residual combustion products, the engine was skip-fired with ignition occurring every fifth engine cycle. The spark plug was located in the side wall of the combustion chamber at the midplane of the clearance height, directly opposite a small window used to collect the scattered LDV signal. The single-component side-scatter LDV system used dual Bragg cells to discriminate flow reversals.

Shown in Figure 1 are the results for the phase-shifted computations of mean velocity and turbulence intensity, where the crank angle for each cycle was shifted slightly to give flame arrival at precisely 359.9 crank-angle degrees, which was the position of average flame arrival.

The last mean velocity data point shown in the figure is the true ensemble-averaged value of the unburned gas velocity $\bar{U}_{g}$. This measurement is not biased by cyclic variations in the combustion rate, although it is biased on the low side by tilt of the flame surface relative to the LDV probe volume.

The turbulence intensity measurements displayed in Figure 1 show an approximately constant value with the exception of the last crankangle degree nearest the flame surface. While the sudden increase shown here could be due to flame-generated turbulence before the flame, it

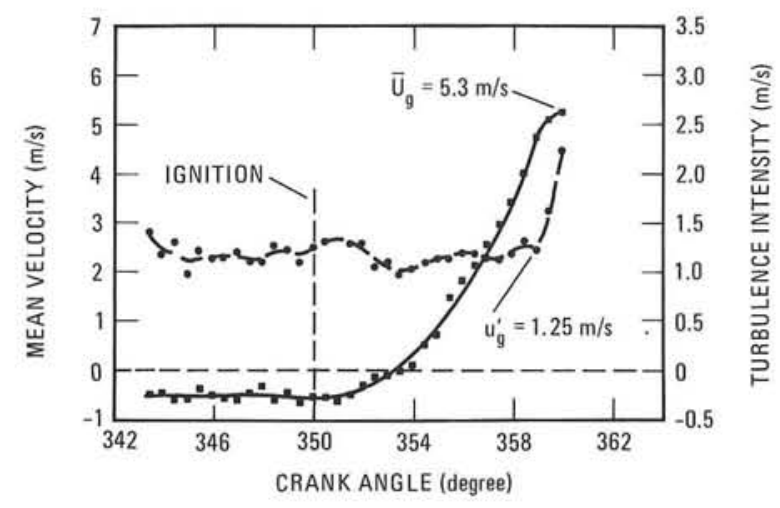

Figure 1. Phase-shifted, ensemble-averaged results for the mean velocity and turbulence intensity.

is more likely that the increase in turbulence intensity shown represents the internal structure of the flame. We have, therefore, chosen to use the turbulence intensity just prior to this sudden increase, where $u_{P}^{\prime}=1.25 \mathrm{~m} / \mathrm{s}$, to be the value for $u^{\prime}$ that we use for correlation with the turbulent burning velocity. It should be noted that this is the same value as measured at the time of ignition, which suggests that measurements of the ensemble-averaged turbulence intensity in a motored engine are a reasonable approximation to phase-shifted data from a combusting engine.

The results for the ionization probe measurements are given in Figure 2 and are compared with the results of a thermodynamic analysis performed on the pressure measurements.

At each radial location of the probe, the ensemble of flame-arrival measurements was determined for forty engine cycles. Because the model calculates the radius of an equivalent sphere separating the burned and unburned volumes, it predicts a smaller radius than indicated by the ionization probe, due to the finite thickness of the turbulent flame. The instantaneous flame speed was computed from a spline fit to the flame position data.

Figure 3 compares the turbulence intensity and burning velocity data obtained from 


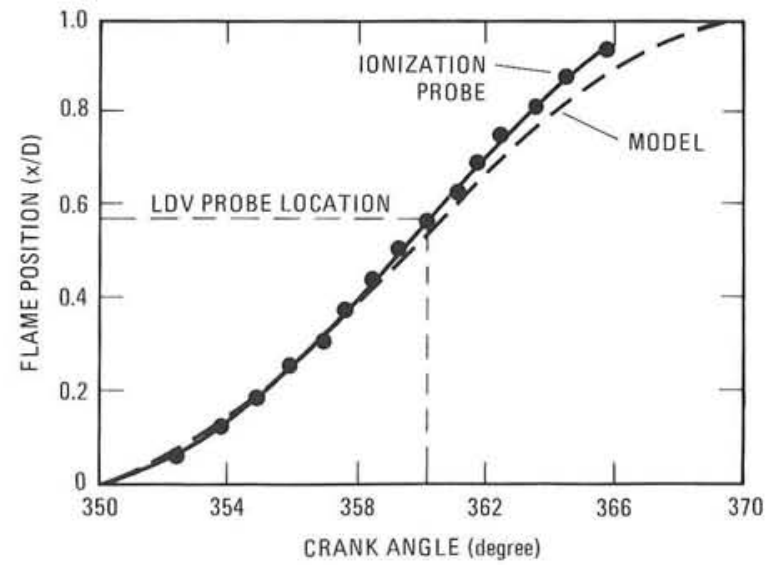

Figure 2. Comparison of ensemble-averaged ionization probe measurements and model calculations of flame position.

this study with a number of commonly used empirical models that were recently reviewed by Abraham et al. ${ }^{1}$

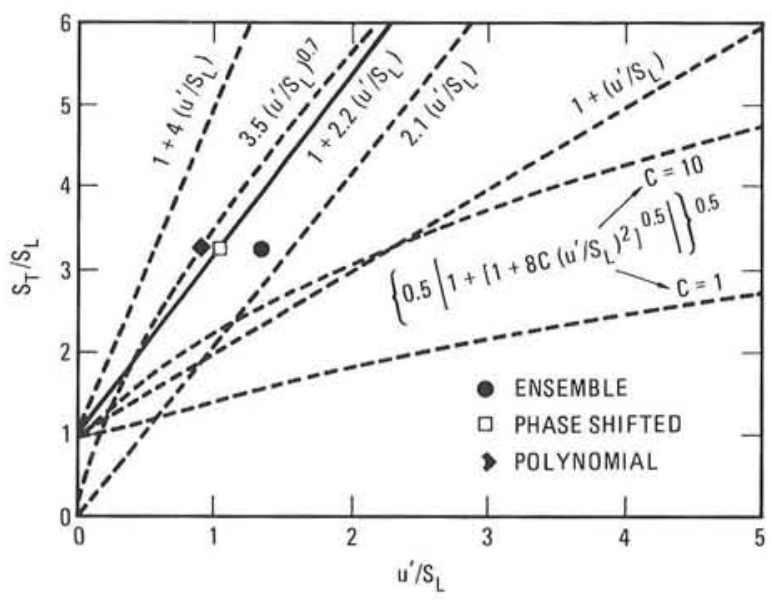

Figure 3. Comparison of turbulence-intensity measurements obtained by different analysis procedures with some commonly used models. The solid curve indicates the model that was previously developed for this particular engine.

The very wide dispersion of these theories is evidence for the need of better experimental data, since the theories were all derived from correlations with experimental measurements. The different procedures used in the present study to calculate the preflame turbulence intensity produced results differing by fifty percent. Taken by itself, this is significant, but when compared to the degree of scatter that exists among the turbulent flame speed theories, this uncertainty in the turbulence is not the major contributor to our lack of understanding.

The good agreement shown between the phase-shifted turbulence intensity and the expression

$$
S_{T} / S_{L}=1+2.2\left(u^{\prime} / S_{L}\right)
$$

might be expected, since this model was empirically determined for this particular engine using similar data processing procedures. ${ }^{2}$ At the same time, however, it is encouraging that the direct measurement of the turbulent burning velocity agrees so well.

\section{References}

1. J. Abraham, F. A. Williams, and F. V. Bracco, "A Discussion of Turbulent Flame Structure in Premixed Charges," SAE Paper No. 850345 (1985).

2. P. O. Witze, J. K. Martin, and C. Borgnakke, "Measurements and Predictions of the Precombustion Fluid Motion and Combustion Rates in a Spark Ignition Engine," Trans. SAE 92, 786, (1983). 


\section{CARS Measurements of Temperature Profiles Near a Wall in an Internal Combustion Engine ${ }^{\dagger}$}

\author{
R. P. Lucht, M. A. Maris *
}

Broadband, coherent anti-Stokes Raman scattering (CARS) measurements of burned gas temperatures near a wall in the Sandia engine have been performed. ${ }^{1} \mathrm{~A}$ lens-prism arrangement was used to traverse the probe volume perpendicular to the cylinder head wall while maintaining the alignment of the CARS optical system.

Historically, the state of gases near walls in internal combustion chambers has been of great interest to engine researchers. The temperature profile of gases near walls is important, first of all, because of heat transfer considerations. Accurate prediction of heat transfer using multidimensional modeling would be an important advance in the design of efficient engine chambers. Secondly, the possible influence of the thermal boundary layer on pollutant emission from engines has long been recognized. For many years, for instance, it was believed that the primary source of unburned hydrocarbon emissions from engines was the so-called "quench layer," a region of cool gas near engine walls. Although it is now believed that the main sources of unburned hydrocarbon emissions from engines are engine crevices, the temperature profile near the crevices will certainly affect the level of emissions. Furthermore, the thermal boundary layer is a calculated quantity in most engine heat release calculations. ${ }^{2}$ The shape and thickness of the boundary layer have a strong influence on the predicted difference between bulk (all gases in the chamber) and core (gases outside the boundary layer) temperatures in the engine.

Despite the great interest in the thermal boundary layer in engines, there have been, to

† Submitted to SAE Trans. (1986).

* Computer Genetics Corporation, Wakefield, MA. our knowledge, no previous quantitative measurements of the temperature profile near a wall in an operating internal combustion engine. We performed our temperature measurements near the cylinder head wall. The measurements were performed in the burned gases at 2, 9, and 14 crank angle degrees $(0.6,2.7$, and $4.2 \mathrm{msec})$ after peak pressure (376 CAD). The estimated spatial resolution of the measurements in the direction normal to the wall was $25-50 \mu \mathrm{m}$, and CARS spectra were obtained $25 \mu \mathrm{m}$ or less from the wall. The measurements clearly show the development of the thermal boundary layer structure during the piston expansion stroke.

The cylinder head and the apparatus for traversing the CARS probe volume normal to the surface are shown in Figure 1.

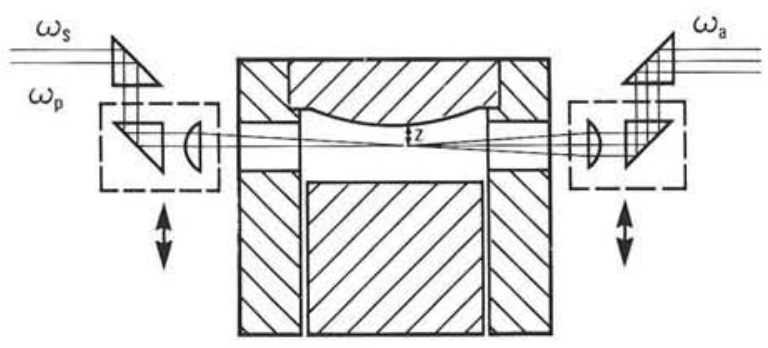

Figure 1. Convex cylinder head, and lens-prism apparatus for moving the CARS probe volume normal to the cylinder head surface.

The cylinder head was a stainless steel disk with a convex lower surface which projected into the clearance volume. The center of the disk was $7 \mathrm{~mm}$ lower than the edge of the disk, and the radius of curvature of the convex surface was $69.8 \mathrm{~mm}$.

By moving the lens and the prism closest to the lens on each side of the chamber the same distance vertically, the CARS probe volume could be moved vertically without misaligning the optical system. Dial indicators with a precision of approximately $10 \mu \mathrm{m}$ were used to measure the positions of the prisms and lenses. The position of the CARS probe volume with respect to the cylinder head surface was determined by translating the $532 \mathrm{~nm}$ beams (with the laser in the long pulse mode) until they just 
started to be blocked by the surface, as indicated by scattered light off the surface. The beams were then moved further until they were completely blocked. The distance between these two positions was approximately $50 \mu \mathrm{m}$, which represents an upper limit for the spatial resolution. The spatial resolution is probably closer to $25 \mu \mathrm{m}$, because the CARS signal depends on the square of the pump beam intensity. The absolute positions for the start of beam blockage and complete blockage were reproducible to approximately $10 \mu \mathrm{m}$ from day-to-day. The position of the CARS probe volume was referenced to the midway point.

Figure 2 shows a CARS spectrum and the theoretical fit obtained in the burned gases at $385 \mathrm{CAD}, 25 \mu \mathrm{m}$ from the wall. The spectrum was averaged for thirty laser shots. The fit to the spectrum is quite good, despite the presence of a significant amount of scattered light. At this position, both the pump and Stokes beams are partially obscured by the cylinder head wall. A vertical offset was used as one of the fitting parameters in the CARS code to account for the scattered light. Scattered light was negligible for spectra acquired further from the wall.

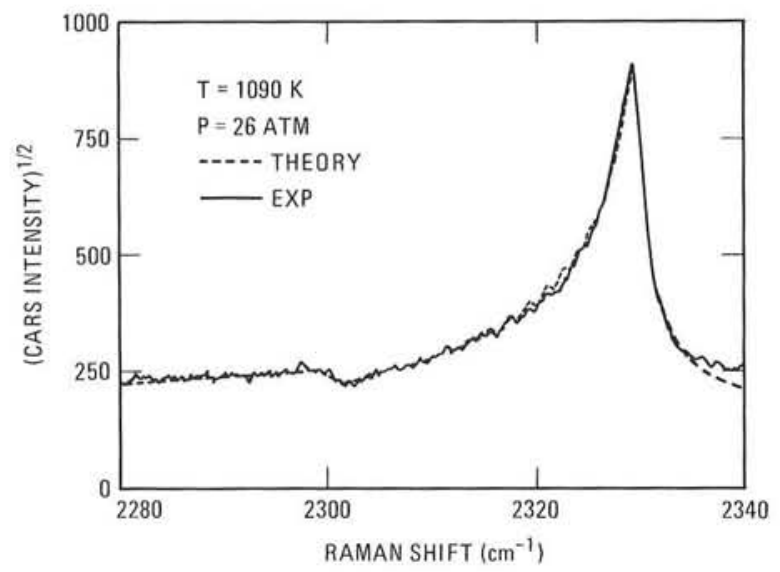

Figure 2. CARS spectrum and theoretical fit at $378 \mathrm{CAD}, 1.93 \mathrm{~mm}$ from the wall. The spectrum is the average of approximately 30 laser shots. The temperature determined from the fit is $\mathbf{2 6 6 0}$ K.

The measured temperature profile near the wall at 385 CAD is shown in Figure 3.

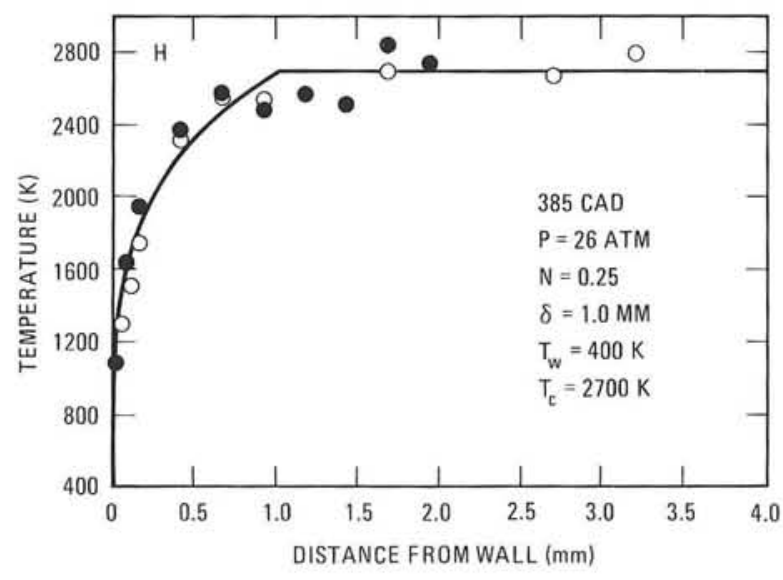

Figure 3. CARS measurements and power law calculations for the temperature profile near the cylinder head wall at $385 \mathrm{CAD}$. The dashed line is the temperature profile calculated according to equation (1) for $\delta=1.0 \mathrm{~mm}$ and $\mathrm{n}=0.25$. The solid line is the convolution of the power law temperature profile with the spatial response of the CARS probe volume, modeled as a Gaussian with a FWHM of $25 \mu \mathrm{m}$. Open and closed circles represent data acquired on different days.

The CARS data were compared with profiles calculated from power law expressions for the boundary layer temperature profile. The dashed line in each figure is the temperature profile $T(z)$ calculated from the expression

$$
T(z)=T_{w}+\left(T_{c}-T_{w}\right)(z / \delta)^{n} \quad \text { for } z \leq \delta
$$

and

$$
T(z)=T_{c} \quad \text { for } z \geq \delta
$$

where $T_{w}$ is the wall temperature, estimated to be $400 \mathrm{~K}, T_{c}$ is the freestream core temperature, $\delta$ is the boundary layer thickness, and $z$ is the distance from the wall.

The solid line in the figure is the convolution of the computed power law temperature profile (dashed line) with a function describing the spatial response of the CARS probe volume. The two profiles were virtually identical except within $0.050 \mathrm{~mm}$ of the surface. The dashed line is visible only very near the surface. Thus, it can be concluded that the spatial resolution of the measurements is sufficient to accurately measure the temperature profile at distances farther than $50 \mu \mathrm{m}$ from the wall.

The measured temperatures and computed power law profiles (convolved) are shown for all three cases in Figure 4. 


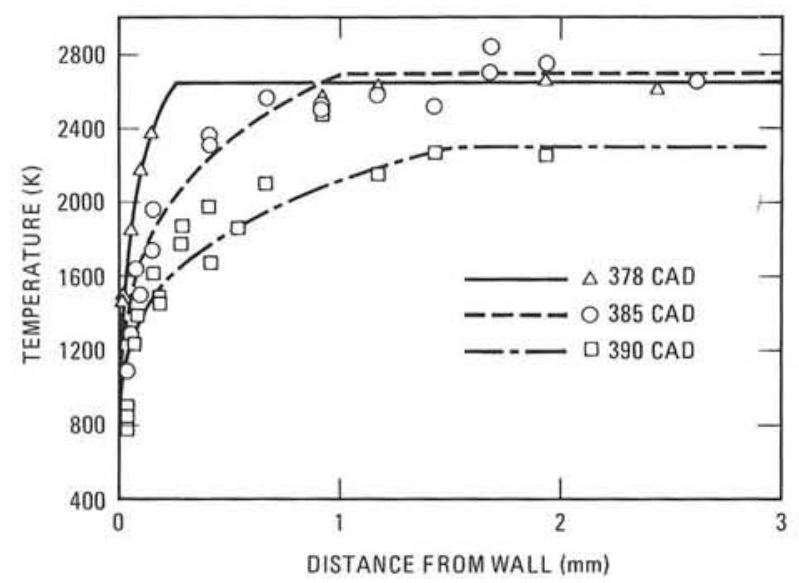

Figure 4. CARS measurements and power law calculations of the temperature profile at 378 , 385 , and $390 \mathrm{CAD}$. The solid lines are the convolved power law expressions.

The boundary layer thickness increases as the expansion stroke proceeds. The measured boundary layer thicknesses at 378,385 , and $390 \mathrm{CAD}$ were $0.25,1.0$, and $1.5 \mathrm{~mm}$, respectively.

In conclusion, we have demonstrated the capability to accurately measure burned gas temperature profiles near the cylinder head wall in an internal combustion engine. Initial measurements were performed at three different crank angles during the expansion stroke. The estimated spatial resolution of the measurements was better than $50 \mu \mathrm{m}$ normal to the cylinder head wall, and spectra were obtained 25 $\mu \mathrm{m}$ or less from the surface. Thickening of the boundary layer during the expansion stroke was clearly evident.

\section{References}

1. R. P. Lucht and M. A. Maris, "CARS Measurements of Temperature Profiles Near a Wall in an Internal Combustion Engine," submitted to SAE Trans. (1986).

2. C. R. Ferguson, R. M. Green, and R. P. Lucht, "Unburned Gas Temperatures in an Internal Combustion Engine: II. Heat Release Computations," Sandia Report SAND86-8605; also, submitted to Comb. Sci. Tech. (1986).

\section{CARS Measurements of Unburned Gas Temperatures in an Internal Combustion Engine}

\author{
R. P. Lucht, R. M. Green, R. E. Palmer, C. R. \\ Ferguson, ${ }^{*}$ R. E. Teets ${ }^{* *}$
}

We have performed broadband, coherent anti-Stokes Raman scattering (CARS) temperature measurements in the unburned gas region of an internal combustion engine operated under both knocking and non-knocking conditions. ${ }^{1}$ The motivation for performing the measurements arose from the need to understand the chemical kinetic processes which lead to autoignition of the unburned gases. Rapid heat release resulting from autoignition is responsible for engine knock, which currently limits the compression ratio and thermal efficiency of production automobile engines. In the last few years, great progress has been made in developing fundamental chemical kinetic models of the autoignition of hydrocarbon fuels, and the temperature history of the unburned gas is a critical input to such chemical kinetic models.

The Sandia optical engine was fueled with a stoichiometric n-butane/air mixture. Four spark plugs, spaced at 90 degree intervals around the chamber, ignited the mixture, confining the unburned gases to the center of the chamber. The engine was operated under both knocking and non-knocking conditions. The intake manifold was heated to $433 \mathrm{~K}$ and pressurized to 1.55 atm for knocking operation. Operating conditions for the non-knocking case were identical except that the intake manifold is pressurized to only $1.27 \mathrm{~atm}$. The engine was skip-fired, with one fired cycle followed by two non-fired cycles. Optical access was provided by two diametrically opposed fused silica windows.

The pump beams for the CARS measurements were provided by the $532-\mathrm{nm}$ second harmonic of a Nd:YAG laser. Part of the 532-nm

\footnotetext{
- Purdue University, West Lafayette, IN.

** General Motors Research Laboratories, Warren, MI.
} 
laser radiation was also used to pump a broadband dye laser centered at $607 \mathrm{~nm}$. A threedimensional phase-matching scheme was used to combine the beams at the CARS probe volume, and the estimated longitudinal spatial resolution was about $2 \mathrm{~mm}$. The CARS signal was analyzed using a $0.85-\mathrm{m}$ double spectrometer and an intensified diode array detector. The spectral resolution was approximately $0.7 \mathrm{~cm}^{-1}$.

Figure 1 shows an experimental CARS spectrum obtained in the unburned gas just prior to autoignition at a measured pressure of $21 \mathrm{~atm}$. The spectrum shown is the average of thirty laser shots. A calculated CARS spectrum, fit to the experimental spectrum, is also shown. Agreement between theory and experiment, in terms of the shape and rotational structure of the fundamental band, and the relative intensity of the hot band, is excellent.

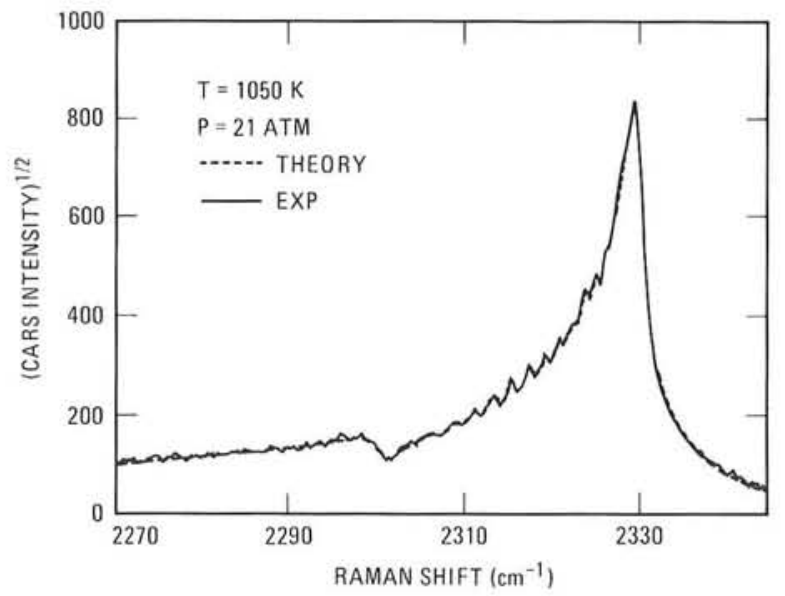

Figure 1. Experimental nitrogen CARS spectrum and the theoretical fit to the spectrum. The spectrum was obtained at 370 crank angle degrees (CAD) in the knocking engine just prior to autoignition.

To attain high accuracy in the high pressure engine environment, the nitrogen CARS spectrum must be well modeled. The accuracy of the fitted temperature is critically dependent upon knowledge of processes, such as collisional narrowing, and parameters such as nitrogen linewidths, which affect the shape of the fundamental band. We used the methodology of
Koszykowski et $a l^{2}$ to calculate collisional narrowing effects. In addition, the nonresonant susceptibility of n-butane was recently measured, ${ }^{3}$ and consequently the nonresonant susceptibility of the unburned gases as a whole is known very accurately. We also found that careful consideration of the spectral response of the intensified diode array detector was necessary for accurate determination of temperatures. Random errors in determination of the average temperature arise from dye laser spectral noise and long-term drift, from cycle-to-cycle and turbulent fluctuations in unburned gas temperatures, and from shot noise on the detector. The estimated total error for the measurements, corresponding to the standard deviation for a single averaged spectrum, is approximately $5-7 \%$.

The results of the CARS measurements of temperature in the unburned gas region of the engine operated under knocking and nonknocking conditions are shown in Figures 2 and 3 , respectively. The predictions of the heat release model ${ }^{4}$ for the Sandia engine are also shown.

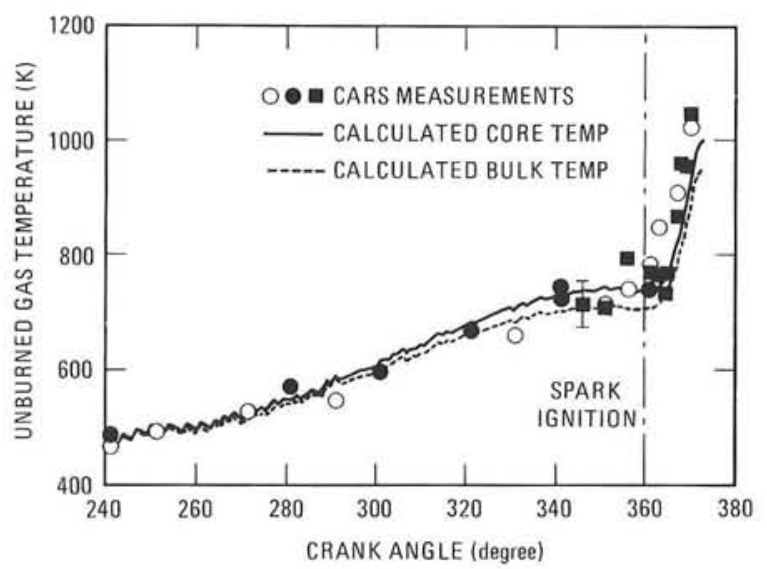

Figure 2. Comparison of CARS temperature measurements and the predictions of the heatrelease model in the knocking engine. The different symbols on the CARS measurements represent data acquired on different days. In the heatrelease model, the core gas temperature is the average temperature of the unburned gas in the engine outside the boundary layer, and the bulk gas temperature is the average temperature of the unburned gas including the boundary layer. 


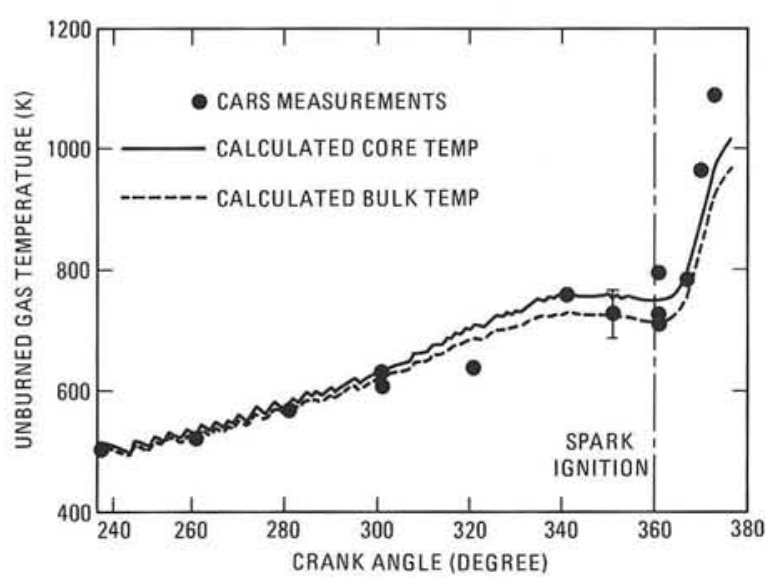

Figure 3. Comparison of CARS temperature measurements and the predictions of the heatrelease model in the non-knocking engine.

In Figures 2 and 3, the bulk gas temperature is the average temperature of the unburned gas in the cylinder, and the core gas temperature is the average temperature of the unburned gases exclusive of the boundary layer at the walls of the cylinder. Before TDC, the agreement between the CARS measurements and the core gas temperature predicted by the heatrelease model is excellent. It must be emphasized here that the CARS temperature measurements and the heat-release predictions were not normalized with respect to each other, and that the good agreement is not the result of varying undetermined parameters in the heat-release model.

After TDC, the CARS temperature is systematically higher than the calculated core gas temperature. It can be seen that the CARS temperature is about $30 \mathrm{~K}$ higher than the core gas temperature at TDC, and about $100 \mathrm{~K}$ higher at $370 \mathrm{CAD}$, just before autoignition. The measured CARS temperatures could be higher than the temperatures predicted by the heat- release model due to exothermic reactions in the unburned gas. The n-butane/air mixture is assumed to be non-reacting in the heat release model, although some reactions must be occurring because the mixture autoignites. A further assumption in the heat release model is that the core gas is isothermal. If gradients do exist in the core gas, the temperature would be highest at the center of the chamber, where the CARS probe volume is located, and the CARS temperature would be higher than the predicted core temperature.

\section{References}

1. R. P. Lucht, R. M. Green, R. E. Palmer, R. E. Teets, and C. R. Ferguson, "Unburned Gas Temperature in an Internal Combustion Engine: I. CARS Temperature Measurements," Sandia Report SAND86-8749; also, submitted to Comb. Sci. Tech. (1986).

2. M. L. Koszykowski, R. L. Farrow, and R. E. Palmer, "Calculation of Collisionally Narrowed Coherent Anti-Stokes Raman Spectroscopy Spectra," Opt. Lett. 10, 478 (1985).

3. R. L. Farrow, R. P. Lucht, and L. A. Rahn, "Measurements of the Nonresonant Susceptibilities of Gases Using Coherent Anti-Stokes Raman Spectroscopy," submitted to JOSA B (1986).

4. C. R. Ferguson, R. M. Green, and R. P. Lucht, "Unburned Gas Temperatures in an Internal Combustion Engine: II. Heat Release Computations," Sandia Report SAND86-8605; also, submitted to Comb. Sci. Tech. (1986).

\section{Autoignition of Methanol and Ethanol Sprays Under Diesel Engine Conditions}

\section{L. Siebers, C. F. Edwards}

Methanol and ethanol are being considered as alternative fuels for diesel engines. Many studies have shown that these fuels are suitable replacements for gasoline in homogeneous-charge engines. However, elimination of the gasoline fraction of the distillates currently used is insufficient to achieve energy independence; those distillate fractions now used for diesel, gas turbine, utility and other applications must also be supplanted. Among these applications, the diesel engine is perhaps the most challenging, 
but also one with a significant potential benefit. The challenge stems from the poor ignition quality exhibited by alcohol fuels in diesel engines. As a result, successful adaption of the diesel engine to alcohol fuels will require accurate knowledge of alcohol fuel autoignition and combustion characteristics over a broad range of conditions. The benefit from the use of alcohol fuels would be a significant reduction in particulate emission from diesel engines.

The ignition characteristics of methanol and ethanol were measured under simulated diesel engine conditions in a constant-volume combustion vessel. ${ }^{1}$ Neat methanol and ethanol and methanol and ethanol with $10 \%$ water by volume were examined. The ignition characteristics of isooctane and normal hexadecane (cetane) were also measured, providing reference data for low and high quality diesel fuels, respectively.

The diesel simulation consisted of two steps. First, pressures and temperatures that occur at the time of fuel injection in a diesel engine were generated in a constant-volume combustion vessel by burning a premixed charge of lean $\mathrm{C}_{2} \mathrm{H}_{4} / \mathrm{H}_{2} / \mathrm{O}_{2} / \mathrm{N}_{2}$ in the vessel. The equivalence ratio of the premixed charge was chosen so that the combustion products contained $21 \%$ oxygen. Second, a liquid fuel was injected into the premixed combustion products at a preselected pressure and temperature condition. Autoignition and combustion of the fuel spray ensued as it would in a diesel engine.

The pressure and temperature conditions at injection were chosen to lie on four isentropes in the pressure-temperature domain. The four isentropes represent compression from bottomdead-center (BDC) conditions of one atmosphere and $325 \mathrm{~K}, 375 \mathrm{~K}, 450 \mathrm{~K}$, and $550 \mathrm{~K}$, respectively, to compression ratios as high as 23:1.

Figure 1 shows the ignition delay data for methanol. The data are labeled by the BDC temperature of the isentrope the data lie on. Ignition delay is defined as the time to recover the pressure loss from fuel evaporation. The ethanol data, not shown here, is very nearly identical to the methanol data shown in Figure $1 .^{1}$

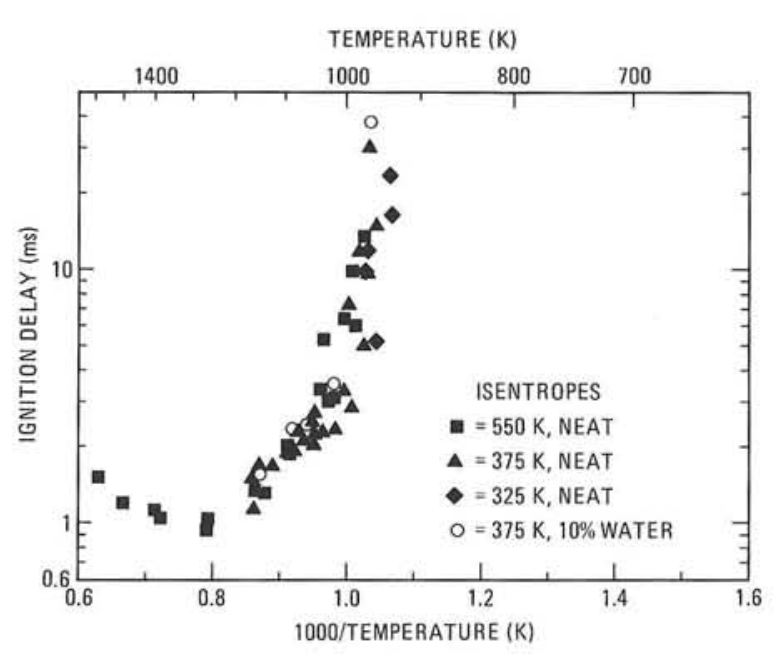

Figure 1. Methanol ignition delay as a function of temperature.

Several important features of the methanol and ethanol ignition delay data can be noted. First, the ignition delays of these alcohol fuels are independent of pressure, since the data on each isentrope for a given temperature (i.e., different pressures for a given temperature) are the same. Second, the ignition delays of both fuels are unaffected by 10 percent water. Third, the minimum ignition temperatures for methanol and ethanol are approximately $950 \mathrm{~K}$. Fourth, large random variations occur in the ignition delay for temperatures less than $1100 \mathrm{~K}$. These variations are a feature of low ignition quality fuel (noted earlier during the cetane rating of such fuels). Finally, at approximately $1300 \mathrm{~K}$ the ignition delay reaches a minimum of $1.0 \mathrm{~ms}$ and increases with further increases in temperature. It was also noted that the ignition delays appear insensitive to the type of injector used and that the trends in ignition delay, based on luminosity, are similar to the trends in ignition delay, based on pressure (see Figure 1). ${ }^{1}$

The ignition delay of cetane is shown in Figure 2 for comparison. The ignition delay decreases with increasing temperature and pressure for temperatures higher than approximately $1050 \mathrm{~K}$. At $1050 \mathrm{~K}$, the ignition delay data on all but the $550 \mathrm{~K}$ isentrope reaches a minimum of $1.2 \mathrm{~ms}$. The $550 \mathrm{~K}$ isentrope data reaches a minimum at a higher temperature. Above a 
temperature of $1200 \mathrm{~K}$, the ignition delay is independent of pressure as was the isooctane ignition delay at high temperatures.

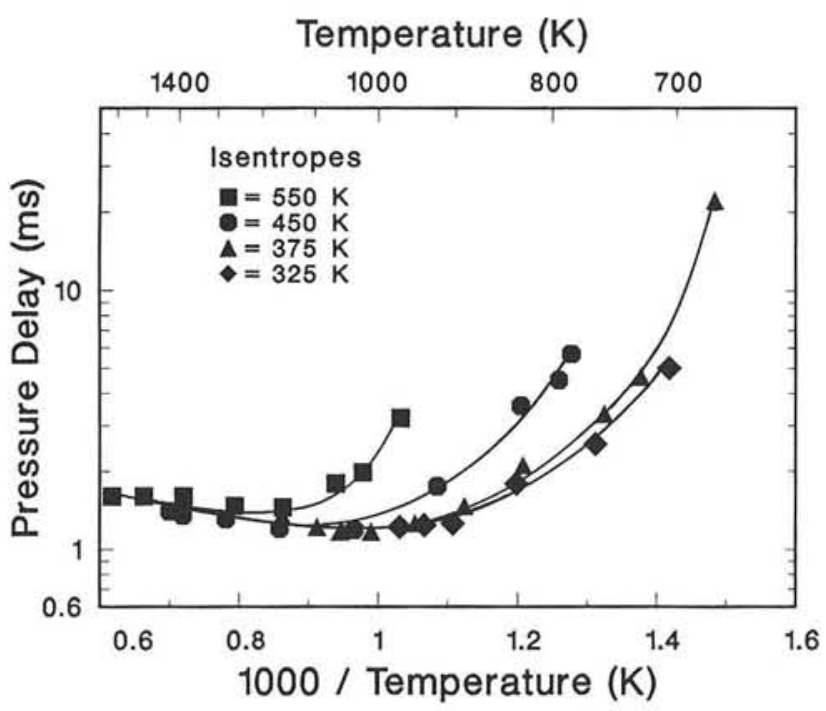

Figure 2. Cetane ignition delay as a function of temperature.

An important feature of the data is that the cetane and the alcohol ignition delays become nearly equal and independent of temperature for temperatures above approximately $1100 \mathrm{~K}$, in spite of the large differences in cetane number. This is also true for isooctane. The limit in the ignition delay is believed to be governed by physical processes associated with injection, atomization, evaporation, and/or spray mixing; the kinetic portion of the ignition delay being short in comparison. If such limits in ignition delay are reached in engines running at high temperatures, the chemistry of the fuels will not be a significant factor in the autoignition process, except during cold start. This insensitivity of the ignition delay to a wide range of fuel types indicates that a diesel engine designed to run on alcohol fuels might be very fuel tolerant from an ignition-quality point of view.

The region of the pressure-temperature domain in which the ignition delay characteristics of methanol and ethanol were examined is shown in Figure 3, along with the important features of the methanol and ethanol data.

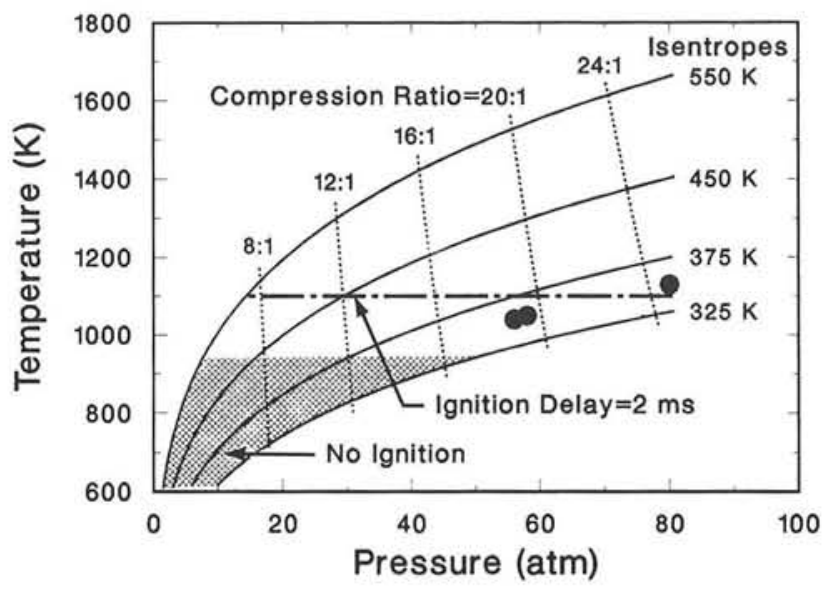

Figure 3. Temperature and pressure conditions covered for methanol and ethanol. The crosshatched region is the region of no ignition. The broken line represents the conditions required for a two millisecond ignition delay. The data points show the locations of TDC conditions for four diesel engines run on methanol or ethanol.

The conditions for which methanol and ethanol could not be ignited are cross-hatched. This region covers all pressures for temperature less than approximately $950 \mathrm{~K}$. The region above the broken line in Figure 3 defines the conditions necessary to have an ignition delay of less than two milliseconds, a typical ignition delay in an engine (12 CAD at $1000 \mathrm{RPM})$. The two millisecond line corresponds to a horizontal line at a temperature of $1100 \mathrm{~K}$. To achieve this temperature at TDC in a naturally aspirated diesel engine with a BDC temperature of $350 \mathrm{~K}$ would require a compression ratio in excess of 23:1, assuming isentropic compression.

Also shown in Figure 3 is the approximate location of the TDC conditions, assuming isentropic compression, for four diesel engines run on methanol or ethanol (the solid circles). ${ }^{1}$ Three of the diesel engines are located below a temperature of $1100 \mathrm{~K}$, in the region where ignition delay is long and has a very strong temperáture dependence (two are located at the same point, $56 \mathrm{~atm}$ and $1040 \mathrm{~K}$ ). Their locations in this region explain why ignition assists were required under all operating conditions for these three engines. Only the engine design of Toepel et al, ${ }^{2}$ represented by the solid circle above 
a temperature of $1100 \mathrm{~K}$, was able to run (under certain load conditions) without an ignition assist. Toepel et al ${ }^{2}$ reported that ignition assistance was only needed under light load conditions, where the engine ran rough, and under cold start conditions. The cold- start characteristics of the alcohols are very poor because they are much more sensitive to temperature than other fuels for temperatures less than $1100 \mathrm{~K}$.

The results suggest that to use alcohol fuels in a spray-combustion mode in diesel engines without ignition assists, ignition improvers, etc., a chamber temperature above $1100 \mathrm{~K}$ will be required at injection. Providing this temperature level is not a simple task. To cold start an engine at room temperature $(295 \mathrm{~K})$ and achieve this temperature would require compression ratios in excess of 23:1. It is, therefore, not likely that an alcohol diesel engine would be built without cold-start ignition assists (i.e., glow plugs, spark plugs, hot surfaces, etc.). However, operation on alcohol fuels at all load conditions once an engine is warmed up is conceivable. Such an engine would require compression ratios in the range of 19:1 to 21:1, depending on the amount of intake air preheating through exhaust gas recirculation, exhaust gas residual, waste heat recovery, or some other means. These conditions were very nearly achieved by Toepel $e t$ $a l^{2}$ Their modification of a conventional engine design required ignition assistance by glow plugs only during cold-start and light load operation. If intake air heating is used, the intake air pressure will also have to be boosted to maintain engine power output. Boosting the intake air pressure alone will have little effect on the ignition characteristics of the alcohol fuels, because of their pressure independence. Achieving temperatures above $1100 \mathrm{~K}$ will also result in acceptable rates-of-pressure-rise for alcohol fuels in the premixed burn stage of combustion. Finally, since the ignition delays of the wide range of ignition-quality fuels tested here become very comparable above $1100 \mathrm{~K}$, a diesel engine designed to run on alcohol fuels will potentially be more fuel tolerant than current engines and run on a wider variety of fuels.

\section{References}

1. D. L. Siebers and C. F. Edwards, "Autoignition of Methanol and Ethanol Sprays Under Diesel Engine Conditions," presented at the SAE International Congress and Exposition (1987), Paper No. 870588.

2. R. R. Toepel, J. E. Bennethum, and R. E. Heruth, "Development of Detroit Diesel Allison 6V-92TA Methanol Fueled Coach Engine," SAE Transactions 92 (4), 959 (1983).

\section{Particle Seeding for Mie Scattering Measurements in Combusting Flows ${ }^{\dagger}$}

\author{
P. O. Witze, T. A. Baritaud *
}

The application of laser Doppler velocimetry (LDV) to combustion studies in reciprocating engines requires the introduction of seed particles that can survive temperatures as high as $2800 \mathrm{~K}$, and yet must be both non-abrasive to the cylinder wall and non-adhering to window surfaces. Earlier studies showed that neither $\mathrm{TiO}_{2}$ nor $\mathrm{Al}_{2} \mathrm{O}_{3}$ were satisfactory, even though these materials are commonly used in combustion experiments. When used in an engine, $\mathrm{Al}_{2} \mathrm{O}_{3}$ acts as a polishing compound, with the result that the cylinder wall becomes too smooth to retain lubricants. In contrast, while $\mathrm{TiO}_{2}$ is not as abrasive, its scattering efficiency deteriorates severely at the high temperatures encountered in engines, such that it was not possible to make velocity measurements in the burned gas region of the flow.

Although $\mathrm{TiO}_{2}$ has been used successfully in some LDV studies with combustion, problems have often been reported. In contrast, $\mathrm{Al}_{2} \mathrm{O}_{3}$ is commonly used without mention of any notable difficulties. Several comparative studies have

$\dagger$ Third International Symposium of Applications of Laser Anemometry to Fluid Mechanics (1986).

- Institut Français du Pétrole, Rueil-Malmaison, France. 
been made of the Mie scattering performance of these two materials. Moss ${ }^{1}$ and Shepherd and Moss $^{2}$ investigated gas density in turbulent premixed flames by measuring the scattered light intensity from a probe volume large enough to contain many particles. They found that the scattered light intensity from $\mathrm{TiO}_{2}$ particles in the burned gas regions was three-times less than could be explained by volume expansion of the gas; no measurable loss was observed for $\mathrm{Al}_{2} \mathrm{O}_{3}$. Kennedy ${ }^{3}$ reported a similar experiment in a laminar premixed hydrogen flame, except that the scattered light was obtained from single particles. He confirmed the previous observations, except that he also noted an equivalence ratio dependency with the $\mathrm{TiO}_{2}$; for a very lean mixture there was no loss in scattered intensity, suggesting a temperature dependence of the scattering efficiency.

In the present study, simultaneous measurements of LDV data rate and scattered light intensity were made using an argon-ion laser. The LDV measurements were made using the $514.5 \mathrm{~nm}$ line, and the scattered light intensity measurements were made using the $488 \mathrm{~nm}$ line.

Table I summarizes the properties of the nine materials considered as seeding candidates. The nominal particle diameters shown are the values provided by the suppliers. The vaporization and sublimation temperatures given are for ambient conditions; these values significantly increase at the elevated pressures found in an engine. With the exception of $\mathrm{NaCl}$, each of the materials listed has been successfully used for LDV measurements with combustion. $\mathrm{NaCl}$ was included in the matrix because it is soluble in water, and thus served as a control particle that could be continuously varied in size. The procedure used to introduce solid particles into the flow was to atomize a water dispersion of the seed material. A constant volume ratio of 10 parts of powder to 400 parts of water was used for all seed materials.

The inclusion of $\mathrm{ZrF}_{4}$ in the test matrix requires further explanation. We inadvertently discovered this material while searching for a tracer particle that would survive compression heating in an engine, and yet disappear rapidly behind the flame front. The $873 \mathrm{~K}$ sublimation temperature of $\mathrm{ZrF}_{4}$ seemed ideal; however, preliminary tests in a steady flame, and later studies in an engine, showed that it survived combustion with no measurable loss in scattering efficiency. Thermogravimetric analysis revealed that weight loss begins by $373 \mathrm{~K}$, and ends by $823 \mathrm{~K}$. We now believe that the $\mathrm{ZrF}_{4}$ undergoes a low temperature reaction, producing $\mathrm{ZrO}_{2}$ and hydrofluoric acid. While the latter product is obviously undesirable, the particles that are formed have yielded excellent LDV data rates during combustion.

Table I

Matrix of Seed Materials Included in the Study

\begin{tabular}{|c|c|c|c|c|}
\hline Material & $\begin{array}{l}\text { Size } \\
(\mu \mathrm{m})\end{array}$ & $\begin{array}{c}\text { Melt } \\
\text { Temp. }\end{array}$ & $\begin{array}{c}\text { Boil } \\
\text { Temp. }\end{array}$ & $\begin{array}{l}\text { Mohs } \\
\text { Hard }\end{array}$ \\
\hline $\begin{array}{l}\text { Aluminum } \\
\text { oxide } \\
\mathrm{Al}_{2} \mathrm{O}_{3}\end{array}$ & $\begin{array}{c}0.05 \\
0.5\end{array}$ & $2300 \mathrm{~K}$ & $3250 \mathrm{~K}$ & 9 \\
\hline $\begin{array}{l}\text { Boron } \\
\text { nitride } \\
\text { BN } \\
\end{array}$ & 0.5 & - & $\begin{array}{c}\text { Subl. } \\
3300 \mathrm{~K}\end{array}$ & 2.0 \\
\hline $\begin{array}{l}\text { Magnesium } \\
\text { oxide } \\
\mathrm{MgO}\end{array}$ & $<1.0$ & $3125 \mathrm{~K}$ & $3900 \mathrm{~K}$ & 6 \\
\hline $\begin{array}{l}\text { Silicon } \\
\text { carbide } \\
\text { SiC } \\
\end{array}$ & 1.5 & - & $\begin{array}{c}\text { Subl. } \\
3000 \mathrm{~K}\end{array}$ & 9.2 \\
\hline $\begin{array}{l}\text { Sodium } \\
\text { chloride } \\
\mathrm{NaCl}\end{array}$ & - & $1074 \mathrm{~K}$ & $1686 \mathrm{~K}$ & 2 \\
\hline $\begin{array}{l}\text { Talc } \\
\begin{array}{c}3 \mathrm{MgO}_{4} \cdot \mathrm{SiO}_{2} \\
\quad \cdot \mathrm{H}_{2} \mathrm{O}\end{array} \\
\end{array}$ & $?$ & $?$ & $?$ & 1 \\
\hline $\begin{array}{l}\text { Titanium } \\
\text { dioxide } \\
\mathrm{TiO}_{2} \\
\end{array}$ & 0.22 & $2100 \mathrm{~K}$ & $\begin{array}{c}2800- \\
3300 \mathrm{~K}\end{array}$ & $\begin{array}{l}5.5- \\
6.0\end{array}$ \\
\hline $\begin{array}{l}\text { Zirconium } \\
\text { fluoride } \\
\mathrm{ZrF}_{4} \\
\end{array}$ & $<0.4$ & - & $\begin{array}{l}\text { Subl. } \\
873 \mathrm{~K}\end{array}$ & $?$ \\
\hline $\begin{array}{l}\text { Zirconium } \\
\text { oxide } \\
\mathrm{ZrO}_{2}\end{array}$ & 1.5 & $3000 \mathrm{~K}$ & $5300 \mathrm{~K}$ & 6.5 \\
\hline
\end{tabular}

Because the combustion chamber of a reciprocating engine is a difficult environment for conducting research, initial characterization of the LDV seed particles was performed in a 
steady air jet. Figure 1 summarizes the LDV data rates measured in the jet core for each of the materials listed in Table I.

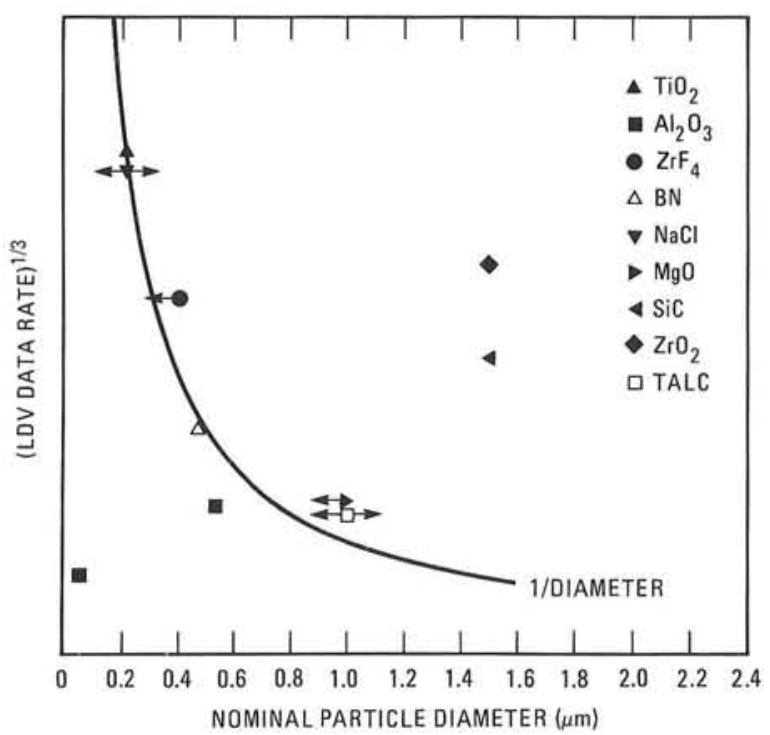

Figure 1. LDV data rates for various seed particles in a steady jet. The arrows indicate uncertainties in the specified particle size.

With the exception of the smallest ( 0.05 $\mu \mathrm{m})$ and the largest $(1.5 \mu \mathrm{m})$ particles studied, it is seen that the cube root of the data rate, or particle flux, decreases inversely with the particle diameter. This is the expected behavior if it is assumed that the LDV data rate depends on just the particle number density, and is independent of scattering efficiency.

The low data rate obtained using the 0.05 $\mu \mathrm{m} \mathrm{Al}_{2} \mathrm{O}_{3}$ is unexpected, since it would seem that a dispersion of particles this small should behave like the solution of $\mathrm{NaCl}$. Similarly, the high data rates obtained using $1.5 \mu \mathrm{m} \mathrm{SiC}$ and $\mathrm{ZrO}_{2}$ particles are also surprising. Compared to the $0.22 \mu \mathrm{m} \mathrm{TiO}_{2}$, there should be approximately 300 times fewer particles of this larger size, such that the probability of an LDV event should be proportionally less. Additional tests have led us to conclude that the $\mathrm{Al}_{2} \mathrm{O}_{3}$ agglomerates into particles on the order of $1 \mu \mathrm{m}$, and that the $\mathrm{SiC}$ and $\mathrm{ZrO}_{2}$ powders contain a large number of very small particles in the $0.3 \mu \mathrm{m}$ range, in addition to the larger specified nominal sizes.
Results for the light-scattering efficiency of these materials during combustion are summarized in Figure 2. The measurements are compared to the normalized theoretical particle number density, which was calculated from the known piston motion. In general, prior to ignition and after the completion of combustion, the measured scattered-light intensity and theoretical particle-number density should coincide if the scattering cross section and total number of particles is not affected by combustion. During combustion, the situation is somewhat more complicated because of the large density gradient across the flame surface. Ideally, before the flame, the scattered-light intensity should be high because of the high particle number density, while behind the flame, there should be a corresponding reduction in light scattered from the burned gas region.

Referring to Figure 2, the excellent performance of $\mathrm{ZrF}_{4}$ is clearly evident. The $\mathrm{ZrO}_{2}$ also performed well, although there was some loss in signal level from the postflame region. The $\mathrm{Al}_{2} \mathrm{O}_{3}$ and $\mathrm{BN}$ results also suggest some loss in scattering efficiency behind the flame, although these results are less reliable because the scattered light levels were very low. Finally, the results for $\mathrm{TiO}_{2}$ and $\mathrm{NaCl}$ definitively show that these materials cannot be used for postflame measurements. The result for lean $\mathrm{TiO}_{2}$ is consistent with Kennedy's ${ }^{3}$ result, in that the scattering efficiency improves in a cooler flame. Although the precise reason is not known, it is evident that the light scattering efficiency of $\mathrm{TiO}_{2}$ particles is severely reduced at high temperatures, and it appears that the critical temperature is the melting point. 


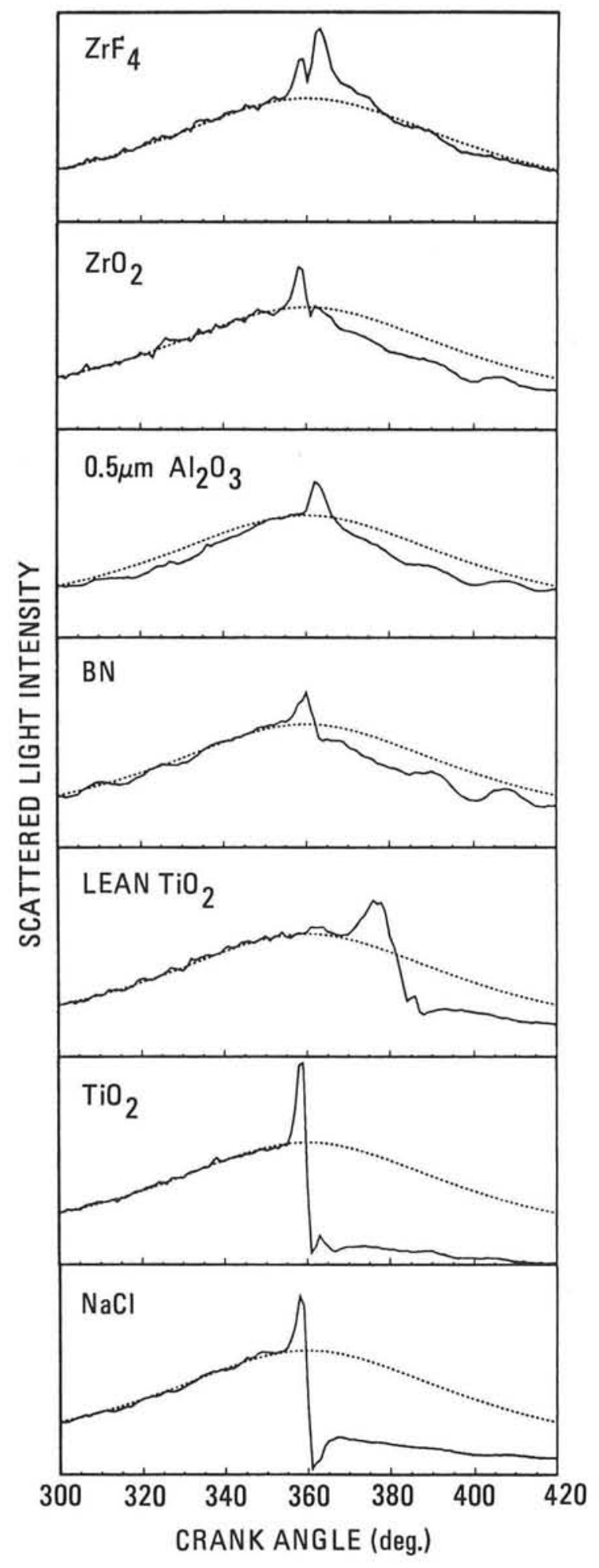

Figure 2. Scattered light intensity during premixed combustion in a spark-ignited engine. The propane-air mixture was stoichiometric except for the one lean case indicated. The dotted line represents the theoretical particle density determined from the piston motion.

\section{References}

1. J. B. Moss, "Simultaneous Measurements of Concentration and Velocity in an Open Premixed Turbulent Flame," Comb. Sci. Tech. 22, 119 (1980).

2. I. G. Shepherd and J. B. Moss, "Measurements of Conditioned Velocities in a Turbulent Premixed Flame," AIAA J. 20, 566 (1982).

3. I. M. Kennedy, "Some Aspects of Seeding Flames with Refractory Oxide Particles," Comb. Sci. Tech. 27, 247 (1982).

\section{Response of a Pulse Combustor to Changes in Fuel Composition}

\author{
J. O. Keller, C. K. Westbrook*
}

The response of a valved pulse combustor to changes in fuel chemistry is reported. Guided by a detailed chemical kinetics model, experiments were performed to determine the response of this pulse combustor to changes in fuel composition. The objective of this study was to use physically-similar fuels whose compositions would lead to large differences in their homogeneous ignition delay times, thereby isolating the effects of different chemical-ignition delay times on the behavior of the combustor.

The two fuels chosen for this study were pure methane and methane mixed with $15 \%$ ethane. These fuels exhibit significantly different homogeneous chemical-ignition delay times - the chemical-ignition delay time for pure methane being substantially longer than for methane mixed with small quantities of ethane. ${ }^{1}$ Their physical properties, however, are essentially the same. Hence, the careful selection of these fuels ensured that only the chemical-ignition delay times were significantly affected. Acceleration of methane ignition by addition of small

\footnotetext{
* Lawrence Livermore National Laboratory,
} Livermore, CA. 
amounts of ethane or other species has been studied extensively, ${ }^{1-4}$ and is of considerable practical importance, since natural gas consists primarily of methane with 5-15\% ethane.

The lean-stable operating limit of this combustor was determined. Measurements in the combustor were also made of spatially integrated, ensemble-averaged chemiluminescence and ensemble-averaged pressures. The energy release rate was determined from the measurements of $\mathrm{OH}^{*}$ chemiluminescence. These measurements, combined with the modeling of the chemical-ignition delay time, enabled the determination of the effects of the chemical-ignition delay on the coupled energy release and fluid dynamic processes that govern this pulse combustor's operation.

The detailed chemical kinetic model was used to calculate the chemical induction times for two fuels which differed only slightly in their composition. The model also helped validate the use of measured $\mathrm{OH}^{*}$ chemiluminescence to represent the energy release. This development allowed the measurement of Rayleigh's efficiency, which provides a measurement of the relativephase relationship between the resonant pressure wave and the energy release. The response of this pulse combustor to changes in chemical induction times was explained by a change in Rayleigh's efficiency between the two fuels examined. The methane mixed with ethane fuel had a lower Rayleigh's efficiency for all of the cases studied than did the slower pure methane fuel (see Table I). Hence, for the pulse combustor under current study, the slow fuel reinforces the resonant pressure waves more than does the faster fuel.
Table I

The Fraction $(\eta)$ of the Available Energy Which Goes Into the Pulsations

(Rayleigh's Efficiency) and the RMS Value of Pressure for Various Equivalence Ratios and Fuel Types

\begin{tabular}{|c|c|c|c|c|}
\hline \multicolumn{3}{|c|}{$\begin{array}{l}\text { Fuel Type } \\
\text { Pure } \mathrm{CH}_{4}\end{array}$} & \multicolumn{2}{|c|}{$\begin{array}{c}\text { Fuel Type } \\
\mathrm{CH}_{4} \text { mixed with } \\
15 \% \mathrm{C}_{2} \mathrm{H}_{6}\end{array}$} \\
\hline $\begin{array}{l}\text { Equiv. } \\
\text { Ratio }\end{array}$ & $\begin{array}{l}\eta \\
\%\end{array}$ & $\begin{array}{c}\text { (P'rms) } \\
\mathrm{kPa}\end{array}$ & $\begin{array}{l}\eta \\
\%\end{array}$ & $\begin{array}{c}\text { (P'rms) } \\
\mathrm{kPa}\end{array}$ \\
\hline 0.8 & 0.80 & 7.76 & 0.54 & 7.35 \\
\hline 0.6 & 0.88 & 7.48 & 0.53 & 6.80 \\
\hline 0.522 & 0.88 & 7.12 & 0.49 & 6.16 \\
\hline 0.473 & 0.82 & 6.53 & NA & NA \\
\hline
\end{tabular}

\section{References}

1. C. K. Westbrook, "An Analytical Study of the Shock Tube Ignition of Mixtures of Methane and Ethane," Comb. Sci. Tech. 20, 5 (1979).

2. C. K. Westbrook and W. J. Pitz, "Effects of Propane on Ignition of Methane-Ethane-Air Mixtures," Comb. Sci. Tech. 33, 315 (1983).

3. R. W. Crossley, E. A. Dorko, A. Burcat, and G. B. Skinner, "The Effect of Higher Alkanes on the Ignition of Methane-Oxygen-Argon Mixtures in Shock Waves," Comb. Flame 19, 373 (1972).

4. C. S. Eubank, M. J. Rabinowitz, W. C. Gardiner, Jr., and R. E. Zellner, "Shock-Initiated Ignition of Natural Gas-Air Mixtures," Eighteenth Symposium (International) on Combustion (The Combustion Institute, 1981), p. 1767. 


\section{Systematic Study of the Oscillating Flow in the Tail Pipe of a Pulse Combustor}

\author{
J. E. Dec, ${ }^{*}$ P. K. Barr, J. O. Keller
}

The enhancement of heat transfer caused by the oscillating flow in a pulse combustor is well known; however, the causes of this increased heat transfer are not understood. A comprehensive study to understand the oscillating heat transfer in the pulse combustor's tail pipe has begun. An initial study of tail pipe velocities has been conducted. Time-resolved velocity profiles showed that the velocity is quite uniform across the tail pipe at all times through the combustor cycle (see Figure 1). The effects of varying the mass flux and equivalence ratio on the magnitude of the tail pipe velocity oscillations were also studied. These velocity oscillations were compared with the combustion chamber pressure oscillations, and the two methods of measuring pulsation magnitude were found to correlate qualitatively. In order to eliminate unknowns caused by the stratification of the fuelair charge, this study was conducted for both conventional (i.e., separate fuel and air) and premixed injection. It was found that increases in the energy release, created either by increasing the mass flux or the equivalence ratio, corresponded to decreases in pulsation magnitude for both injection systems. Another effect of the increased energy release was to increase the resonant frequency through the higher gas temperatures in the combustor. It is believed that this higher frequency causes the energy release to occur out-of-phase with the pressure wave, resulting in the lower pulsation magnitudes.

This decrease in pulsations with increasing energy release was also studied with a nonlinear computer model described below. Using a normalized energy-release rate obtained from chemiluminescence measurements of the combustor operating in a non-premixed mode and an adiabatic flame temperature corresponding to the equivalence ratio of the completely

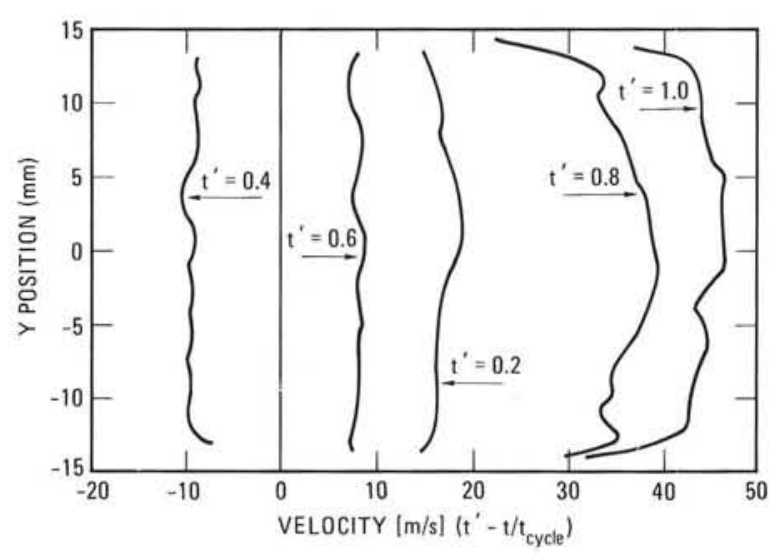

Figure 1. Tail pipe velocity profiles at various normalized times during the combustor cycle.

mixed fuel and air, and using quasi-steady estimates for heat transfer and friction coefficients, we found that the pulsations, though periodic, are moderate in amplitude when the system is pulsed at the experimentally-observed operating frequency. In rerunning the simulation, using a lower value for the adiabatic flame temperature (since it is observed that the combustor is actually burning at a lower equivalence ratio), as well as using better estimates for the heat transfer coefficients (changed to increase the energy loss), we found that the pulsations are much stronger than in the case with more energy release and lower energy losses. A comparison of a computer-generated velocity profile taken at a location in the tail pipe compares well with the experimentally-measured profile, as can be found in Figure 2.

Using the integral of the product between the spatially integrated energy release and the pressure, which is a measure of pulsation stability, we can numerically determine the operating frequency of the system. We do this by forcing the system at different frequencies, and locating the frequency corresponding to a maximum in the phase-relation integral. At this frequency, the pulsations are observed to be both the strongest, and the most in-phase with the energy release. We found that for the set of parameters used in the first simulation (higher energy release and lower energy losses) the combustor performance is a maximum at $62 \mathrm{~Hz}$, whereas for the case with lower energy release

- University of Michigan at Ann Arbor 


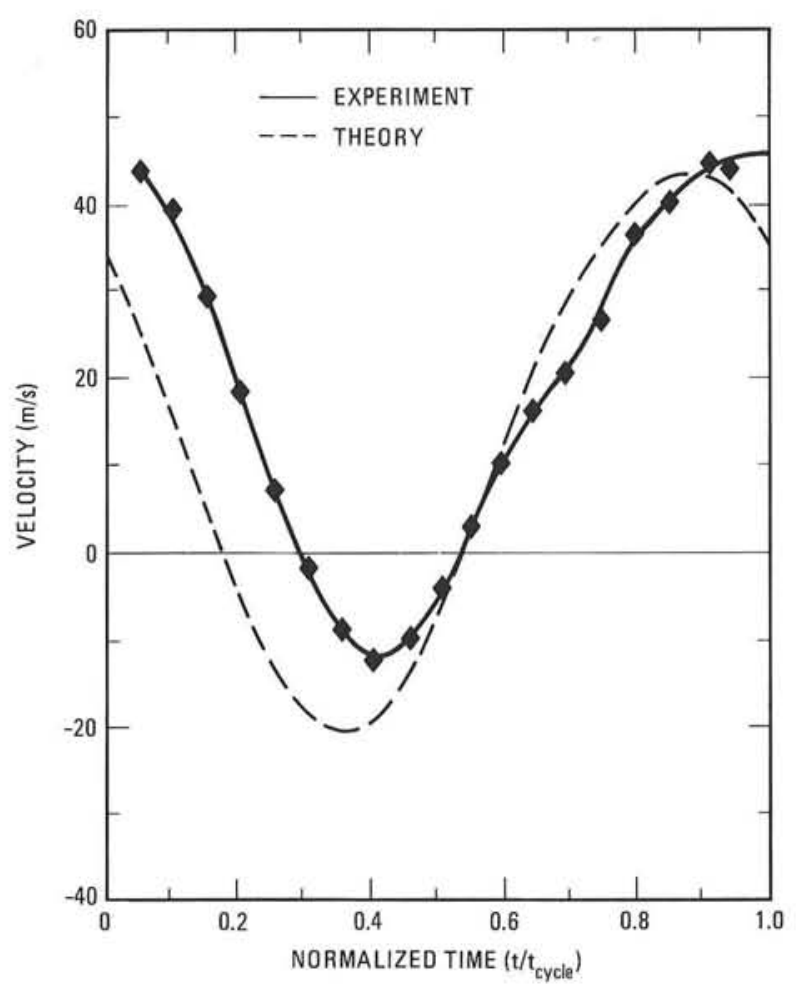

Figure 2. Experimental and calculated velocity profiles within the tail pipe $400 \mathrm{~mm}$ downstream of the combustor.

and higher energy losses the system peaks at $50 \mathrm{~Hz}$. When the temperature in the tail pipe is higher, the sound speed increases; and in order to keep the pressure waves in phase with the combustion process, the operating frequency increases.

\section{Mixing of Hot Products and Cold Reactants in a Pulse Combustor}

\author{
T. T. Bramlette
}

It has long been known that fluid dynamic mixing is important to the operation of pulse combustors, largely as a consequence of testing a variety of configurations that had different "mixing" characteristics. However, the precise role of mixing has never been quantified either experimentally or theoretically.

We have recently applied the mixing model of Rife and Heywood ${ }^{1,2}$ to pulse combustors to study the mixing of a premixed jet of fuel and air that is injected into hot combustion products. In the present work, this model is extended in two ways. First, it has been demonstrated that the model works well for gaseous jets of modest density ratios (the ratio of the density of the jet to the density of the medium into which it is injected) if one assumes that the rate of penetration is proportional to the rate one would obtain with a quasi-steady approximation. A single proportionality-constant of order unity works well for a variety of jets with different density ratios, injection velocities, and nozzle configurations. Typical results are shown in Figure 1 where the theoretical penetration rate is compared with the experimental data of Witze. $^{3}$

Second, the original model was for the case of constant injection velocity for a specified period of time. It is postulated that if the injection velocities vary with time (as is the case with pulse combustors), the process may be approximated as the injection of a constant velocity jet, where the injection velocity and injection duration are chosen to match the total mass and momentum of the temporally-varying jet. The temporally-varying jet velocity is thus modeled in an integral sense.

We are interested in the rate at which the jet mixes to a specified temperature, in this case $1100 \mathrm{~K}$, a temperature at which the premixed 


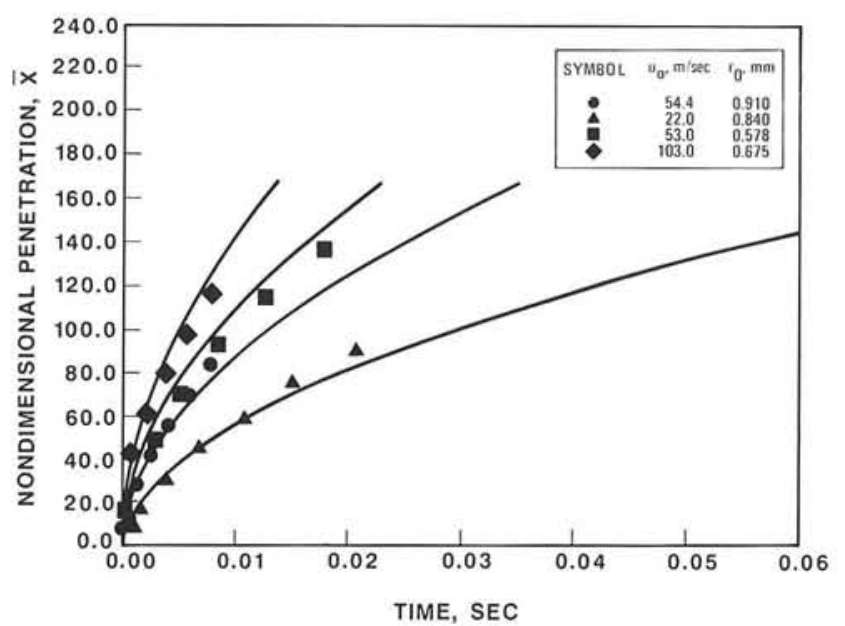

Figure 1. Comparison of the theoretical penetration rate of an impulsively-started gaseous jet with the experimental data of Witze. ${ }^{3}$ These data are for the injection of air into air; comparisons have also been made with the unpublished data of Green, ${ }^{4}$ which are for the injection of freon into air. In all cases, very good agreement is obtained.

reactants would have a chemical ignition delay of approximately $1 \mathrm{~ms}$. This mixing rate is presented as a function of time in Figure 2. Several points are of interest. For these conditions, the time scale for mixing is on the order of 10 ms. If one assumes instantaneous mixing, the energy release occurs approximately $1 \mathrm{~ms}$ after the mixing takes place, and the reaction is complete within approximately $1 \mathrm{~ms} .{ }^{5}$ However, it is known from $\left\langle\mathrm{OH}^{*}\right\rangle$ chemiluminescence measurements ${ }^{5}$ that the energy release in the Sandia pulse combustor occurs throughout the pulse combustor cycle, with the most intense combustion occurring in the last $10 \mathrm{~ms}$ of the $20 \mathrm{~ms}$ cycle. The similarity of the profiles for mixing rate and energy release is encouraging, and suggests that fluid dynamic mixing plays a significant role in the determination of the timing and rate of energy release. We plan to conduct parametric variations with this model and to verify the results by comparison with experimental data obtained with the Sandia pulse combustor.

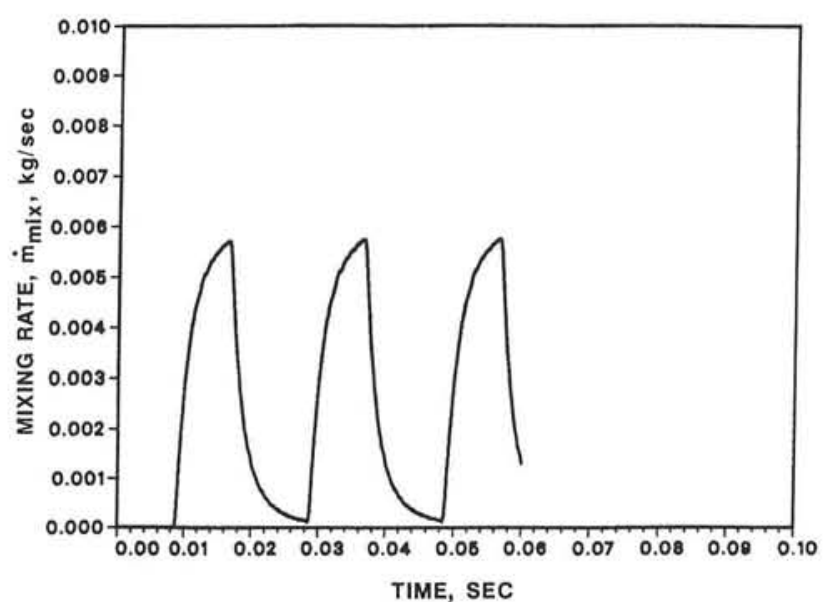

Figure 2. Theoretical prediction of the mixing rate of a premixed transient jet into hot combustion products. The similarity of this profile to the $\left\langle\mathrm{OH}^{*}\right\rangle$ chemiluminescence measurements of Keller and Westbrook ${ }^{5}$ suggests that fluid dynamic mixing plays a significant role in the determination of the timing and rate of energy release.

\section{References}

1. J. M. Rife, "Photographic and Analytical Study of Diesel Combustion with a Rapid Compression Machine," Ph.D. Thesis, (Massachusetts Institute of Technology, Boston, MA, 1974).

2. J. M. Rife and J. B. Heywood, "Photographic and Analytical Study of Diesel Combustion with a Rapid Compression Machine," $S A E$ Transactions 83, 2942 (1974).

3. P. O. Witze, "The Impulsively Started Incompressible Turbulent Jet," Sandia Report SAND80-8617 (1980).

4. H. Green, Private Communication, Imperial College, London, England.

5. J. O. Keller and C. K. Westbrook, "Response of a Pulse Combustor to Changes in Fuel Composition," Twenty-First Symposium (International) on Combustion (The Combustion Institute, 1986), in press. 


\section{Development of a}

\section{Nonlinear Model of Pulse} Combustion Processes ${ }^{\dagger}$

\author{
P. K. Barr
}

A one-dimensional numerical model of a pulse combustor has been developed to simulate the nonlinear wave motion resulting from the periodic combustion process. The model predicts the influences of the system geometry and operating parameters on the resulting wave structure within the combustor. Although many of the processes that take place in the pulse combustor are three-dimensional and are dominated by complex turbulent transport phenomena, the large length-to-diameter ratio of these combustors allows the wave dynamics to be accurately described by a one-dimensional model. The model is based on the unsteady equations of continuity, momentum, and energy, and is solved with variable-area geometry, along with the perfect gas equation of state. Losses due to friction and heat transfer are also included. The numerical method used to solve the equations, MacCormack's explicit predictor-corrector algorithm, assures the propagation of nonlinear pressure waves at the correct amplitude and speed.

The model was designed to simulate the experimental pulse combustor being studied at Sandia National Laboratories. The simplified combustor consists of a combustion chamber and tail pipe joined by a short transition section. The combustion process is simulated by introducing heat uniformly in the combustion chamber at a rate obtained from chemiluminescence measurements taken in the Sandia pulse combustor. The injection of fuel and air is also prespecified by experimentally measured rates.

Although the frequency at which we repeatedly impose both the heat release and reactant injection is specified a priori, the theoretical operating frequency of the pulse combustor is found by identifying the frequency that produces the strongest, most stable pulsations. The pulsation strength and stability

\footnotetext{
† To be presented at the Second ASME/JSME Thermal En-
} gineering Joint Conference, March 1987. are quantified from the integrated phase relation between the heat release and the combustion chamber pressure,${ }^{1}$ which is referred to as the Rayleigh efficiency. To determine the operating frequency of the pulse combustor, the simulation is repeated at different frequencies and the value of the Rayleigh efficiency is computed after the pulsations have become repeatable. Large positive values of the Rayleigh efficiency occur when the peaks in the pressure coincide with the peaks in the heat release. The forcing frequency that produces the maximum value of the Rayleigh efficiency identifies the operating frequency.

This procedure has been confirmed by simulations of the Sandia experimental pulse combustor with this one-dimensional model. Using an energy release corresponding to the combustible mixture used in the experiment, and selecting reasonable values of heat transfer and friction, the numerical prediction of the Rayleigh efficiency peaks at a frequency corresponding to the experimentally observed operating frequency of the pulse combustor, as shown in Figure 1. The cyclic velocity profile at this frequency shows good agreement with the experimentally measured profile, as shown in Figure 2 of the article in this Annual Report by Dec, Barr, and Keller (p. 8-16).

The Rayleigh efficiency can be thought of as a multidimensional surface that quantifies the pulse combustor performance for a range of operating parameters. One parameter to which the combustor is highly sensitive is the gas temperature. By changing the equivalence ratio of the mixture, we can investigate the variation of operating frequency with energy content of the fuel. We do this by using the same forcing functions (the same time-resolved normalized heat release and mass injection profiles) and change the amount of energy released during combustion to account for the differences in the adiabatic flame temperatures. We find that the operating frequency increases when we increase the equivalence ratio (and thus the energy release) up to the stoichiometric condition while maintaining a constant mass flux, as shown in Figure 


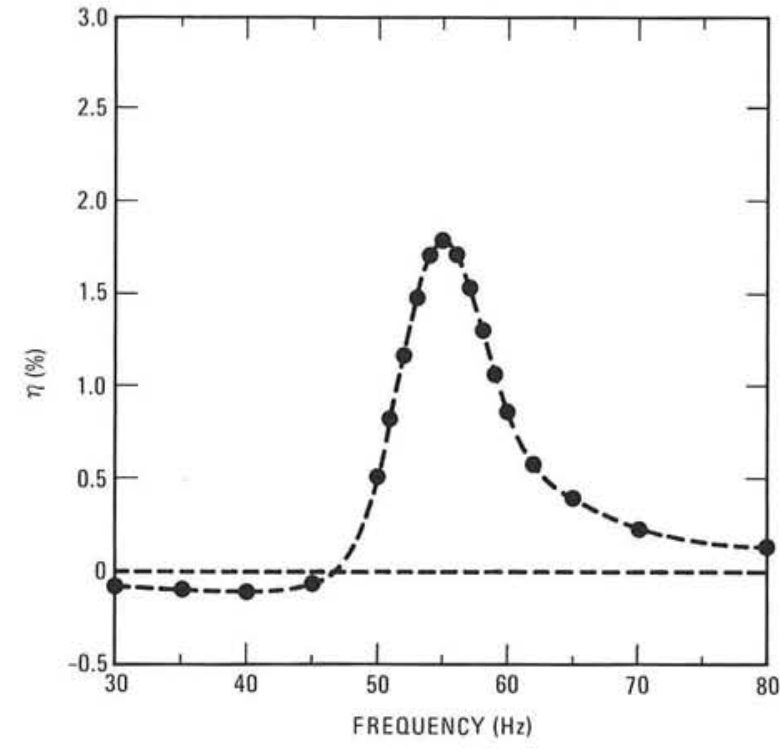

Figure 1. The Rayleigh efficiency $\eta$ as a function of frequency. The operating frequency is identified from the maximum of $\eta$.

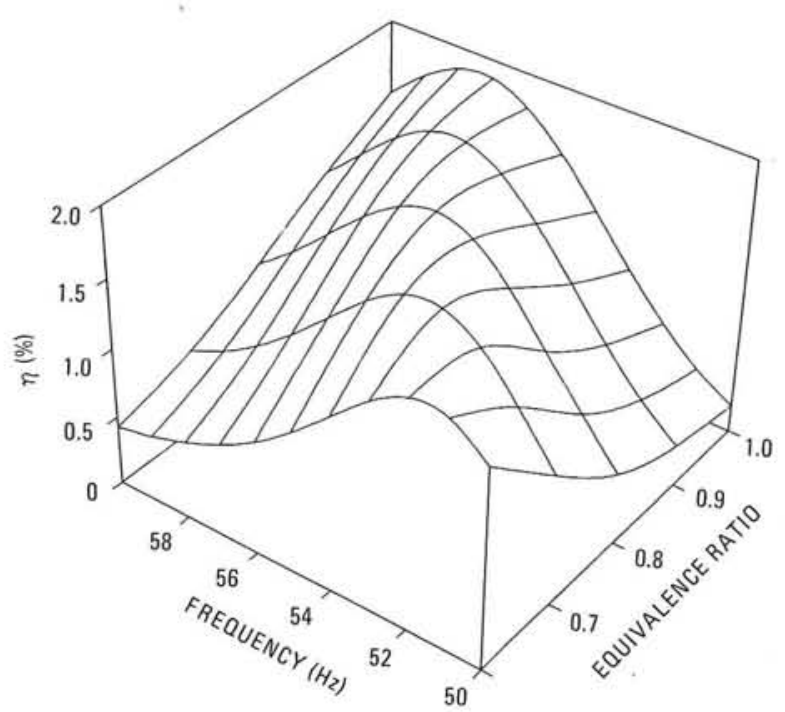

Figure 2. Sensitivity of the Rayleigh efficiency $\eta$ to equivalence ratio and pulsation frequency at a constant mean mass injection rate. The operating frequency increases with increasing equivalence ratio, as identified by the shift in the $\eta$ peaks for each fuel mixture.

2. This finding is supported by the experimental results of Dec and Keller. ${ }^{2}$ However, in these numerical results the maximum Rayleigh efficiency also increases with increasing equivalence ratio, counter to the experimental results. This difference may be caused by the changes in the time-resolved heat release and mass injection profiles observed in the experimental combustor over this range of conditions.

We have also used the Rayleigh efficiency to quantify the sensitivity of pulse combustor performance to changes in other system parameters, such as friction and heat loss. In these numerical simulations, we have independently varied parameters that are not easily controlled in operating pulse combustors. We can thus obtain useful design information by systematically mapping this surface and using the insight gained to affect more rational design changes.

\section{References}

1. J. O. Keller and C. K. Westbrook, "Response of a Pulse Combustor to Changes in Fuel Composition," Sandia Report SAND86-863 (1986); also, Twenty-first Symposium (International) on Combustion (The Combustion Institute, 1986), in press.

2. J. E. Dec and J. O. Keller, "The Effect of Fuel Burn Rate on Pulse Combustor Tail Pipe Velocities," Sandia Report SAND86-8757 (1986).; also, Proceedings of the 1986 International Gas Research Conference (1986), in press. 


\section{Two-Dimensional Flame Visualization in an Internal Combustion Engine}

\section{R. M. Green, T. A. Baritaud*}

Over the past several decades, automotive engineers have significantly improved their understanding of the physical phenomena occurring in the combustion chamber of a spark ignition engine. Laser- based diagnostic techniques have provided a unique new insight into the fluid mechanic, thermodynamic, and chemical processes. Along with this progress in experimental techniques, computer simulation has evolved towards sophisticated two- and threedimensional models. Unfortunately, due to a poor understanding of the structure of the flame zone, current combustion models cannot accurately predict the combustion process.

Measurements ${ }^{1,2}$ of flame structure in an Internal Combustion (I. C.) engine have revealed a wrinkled flame having structures varying from a few millimeters down to a tenth of a millimeter, depending on the engine speed. The existence of pockets of unburned mixture was observed in the burned gas region with a flame interface between fresh and burned gas having a width of only a few tenths of a millimeter. In this study, we have obtained two-dimensional Mie scattering images from a thin, planar sheet of laser light. Because of the density difference across the flame front, the scattered light is more intense in the cold unburned mixture than in the hot burned gases. This effect allows the flame front to be precisely located. Our results were obtained for a freely propagating flame in premixed combustion for low-to-moderate engine speed.

The experiments were conducted in the Sandia optical research engine. A fused silica window in the side wall of the combustion chamber allows the passage of a 9-mm-wide sheet of light through the combustion chamber normal to the mean propagation direction of the flame. The disk-shaped combustion chamber

\footnotetext{
* Institut Français du Pétrole, Rueil-Malmaison, France.
}

permits full optical access through a sapphire window. The circular beam of a frequency doubled Nd:YAG laser is transformed to a $9-\mathrm{mm}$ planar sheet of light by two cylindrical lenses, while a third lens, having a long focal length, focuses the laser sheet at the middle of the chamber. The region of the laser sheet forming the scattering image is located in the center of the combustion chamber as shown in Figure 1.

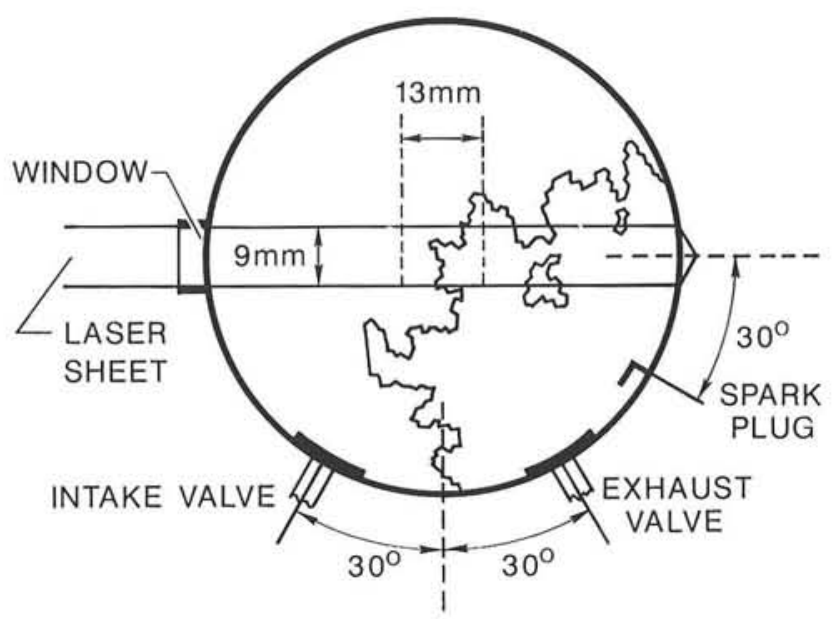

Figure 1. A plan view of the engine combustion chamber illustrating the laser sheet entering through a window in the sidewall of the chamber. The $9 \times 13 \mathrm{~mm}$ section of the laser sheet in the center of the chamber was imaged on the vidicon camera.

The image is obtained using an intensified vidicon camera positioned above the combustion chamber. The image intensifier is time-gated with a $1 \mathrm{~ms}$ gating window, to reduce the flame emission background and improve the signal-tonoise.

The particle seeding of the flowfield is accomplished by the production of $\mathrm{TiO}_{2}$ from the reaction of $\mathrm{TiCl}_{4}$ and water. A simple device to implement this technique was installed in our intake system and provided a sufficiently dense seeding from the small amount of moisture in the intake air.

In order to define the flame contour from the Mie-scattering intensity data, it is necessary to optimize the quality of the raw image by rejecting bad data from isolated pixels and 
subtracting the background intensity. Following this, the location of the flame front can be identified and the flame contour defined. The low intensity region of the image corresponds to the burned gas, while the high intensity region represents the initial fuel-air mixture. For the present data, the light intensity change across the flame zone was about a factor of three. This is the result of both the density decrease and a change in scattering properties of the titanium dioxide across the flame front.

In Figure 2, we present five typical images representing engine speeds ranging from 300 to $1500 \mathrm{rpm}$. These images are shown in an orientation similar to that of Figure 1; that is, the flame is propagating from the spark plug located below the lower right corner. At 300 rpm, the flame front shape appears generally continuous and displays a number of smooth wrinkles. At an engine speed of $600 \mathrm{rpm}$, the flame front structure is more convoluted, and even somewhat disrupted. A further increase of the engine speed to $900 \mathrm{rpm}$ results in large scale structures dominating the shape of the flame, and leading to peninsulas or islands of unburned mixture in the burned gas region. In addition, the small scale wrinkles in the flame shape seem to be enhanced. Furthermore, since there is burned gas located at the top of the image while fresh gas is located in the lower part, the combustion zone must occupy the full field of view of the camera.

At $1200 \mathrm{rpm}$, small scale corrugations in the flame front are more numerous, and regions of flame front curvature of only several pixels (equivalent to a few tenths of a millimeter) are obvious. However, we were not able to determine the extent of the large scales of the flame structure, since the reaction region is larger than the field of view. Very little data were obtained at $1500 \mathrm{rpm}$ because of the rapid fouling of the windows. Here, pockets of both burned gas and fresh mixture are observed, with sizes ranging from a few tenths of a millimeter to a few millimeters.

The images of the combustion zone obtained in this study have revealed that even at $300 \mathrm{rpm}$, pockets or islands can be observed. In addition, these features are sometimes observed quite far from the main flame front, indicating a very disrupted structure. At low rpm, the pockets are nearly always fresh mixture within the burned gas, suggesting that they are created by wrinkles having sides that are merging as a result of combustion progress. When the engine speed is higher, pockets of burned gas inside of fresh mixture gas are observed more frequently.
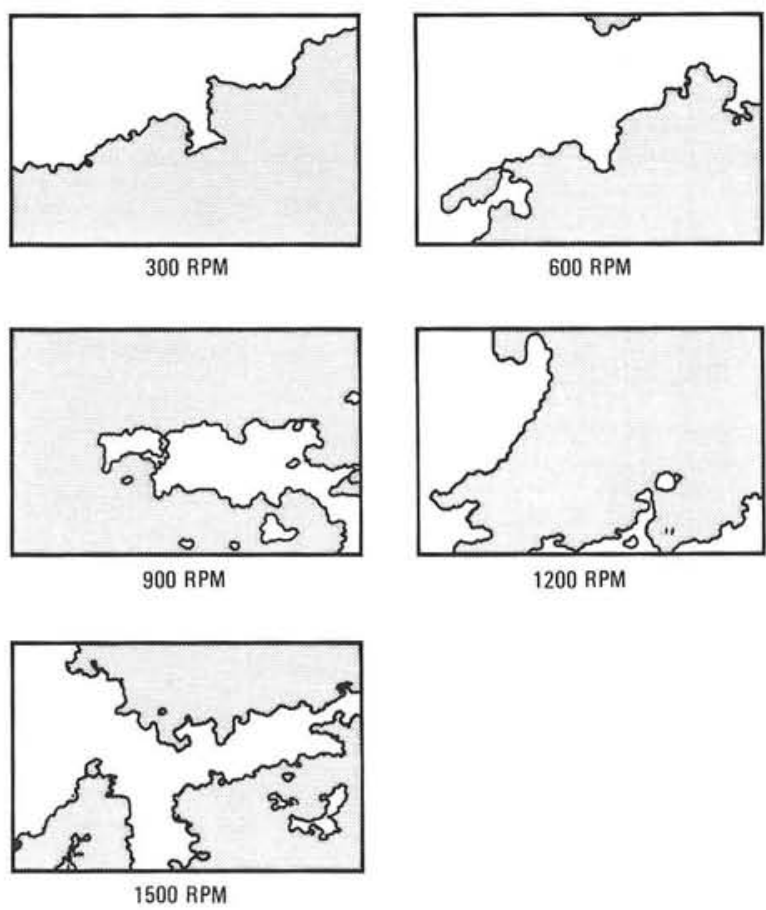

Figure 2. Five Mie-scattering images illustrating the nature of the turbulent flame structure as a function of engine speed (or turbulence intensity). The shaded region is hot combustion products and the light region is fresh unburned mixture.

The images obtained from Mie scattering within a thin sheet of laser light have provided a unique new two-dimensional insight into the nature of the turbulent flame structure in a spark ignition engine. The most obvious feature of the flame structure images is the correlation of flame front corrugations with engine speed. These corrugations produce an increase in flame surface area, and thus a faster global combustion rate. In order to correlate the current flame structure data with the characteristics of the precombustion flowfield, it will be necessary to 
define quantitative measures of the flame wrinkles, corrugations and area properties observed in the present experiments.

\section{References}

1. P. O. Witze and F. R. Vilchis, "Stroboscopic Laser Shadowgraph of the Effect of Swirl on Homogeneous Combustion in a Spark-Ignited Engine," SAE Trans. 90, 979 (1981).

2. J. R. Smith, "Turbulent Flame Structure in a Homogeneous Charge Engine," SAE Trans. 91, 150 (1982). 


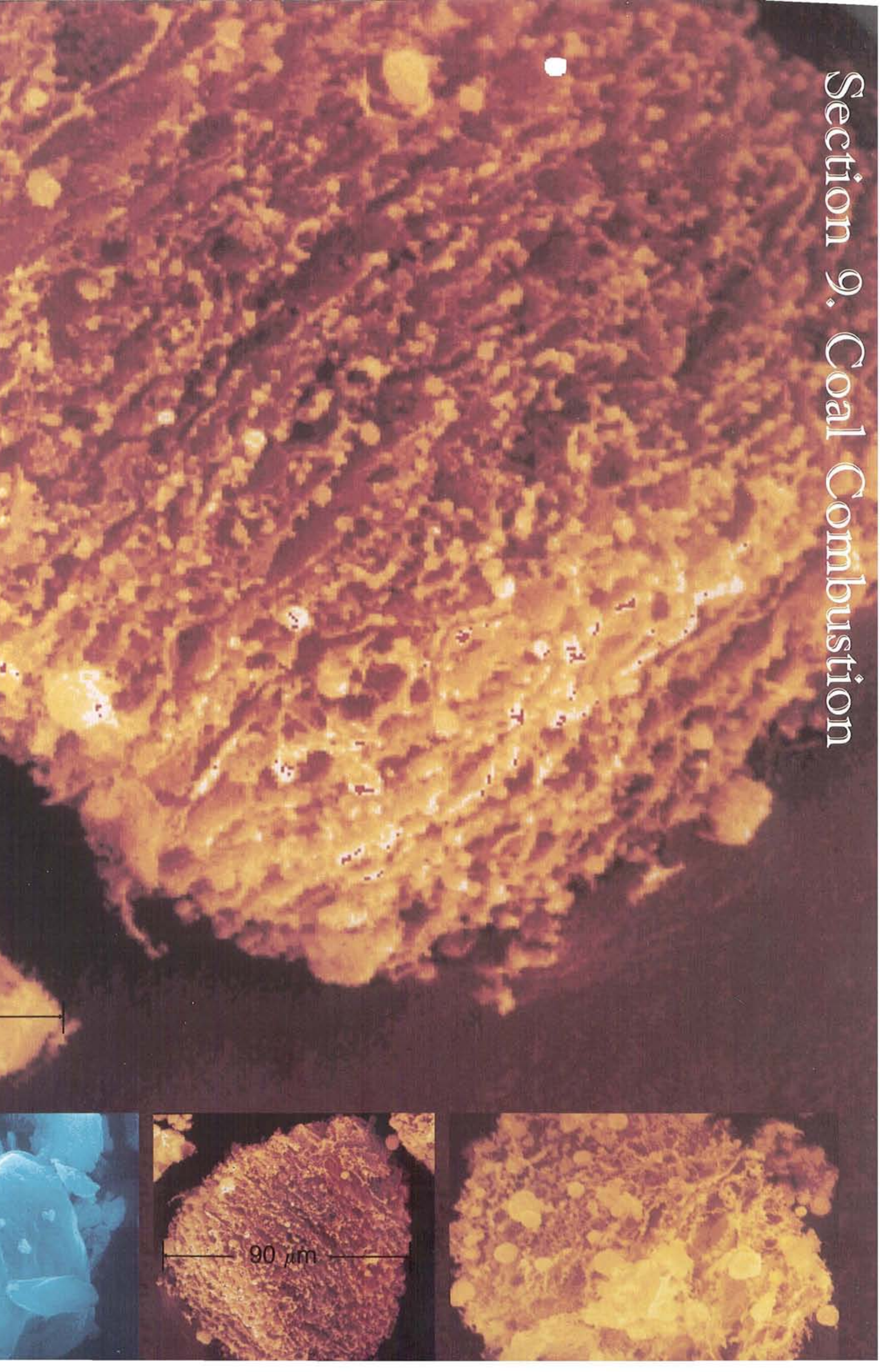


In our fundamental studies of the combustion of pulverized coals, electron optical methods are applied to obtain detailed physical and chemical information. The figure shows a sequence of scanning electron microscope (SEM) images of bituminous coal particles at various stages of their combustion lifetimes. At the left is an unreacted particle, while the other two images (the one in the middle is enlarged at the top) are typical of the residual $100 \mu \mathrm{m}$ char particles removed from our bench scale reactors after 50 to $100 \mathrm{msec}$ of oxidation in $6 \% \mathrm{O}_{2}$ at $1500 \mathrm{~K}$. The information gleaned from our SEM studies includes the evolution of char morphology and the fate of mineral species (which appear as bright, nearly spherical dots in the SEM images) as a function of coal chemistry, combustion environment, and residence time (see related article, page 9-19). 


\section{Section 9 \\ Coal Combustion}

Under the sponsorship of the Department of Energy's Office of Fossil Energy, a broad program of fundamental research on the combustion of pulverized coals has been developed at Sandia National Laboratories. These studies are aimed at predicting and measuring the rates and mechanisms of the devolatization of coals and coal/liquid mixtures and of the subsequent oxidation of the residual chars. Special emphasis is given to determining the high temperature transformation and evolution of mineral species imbedded in the original coal. Experimental studies use well-controlled laboratory flow reactors to obtain spatially and temporally resolved information during the combustion of size-classified samples of well-characterized coal. The reactors are instrumented with advanced optical detection systems, including a Sandia-patented, particle-sizing pyrometer, for in situ observation of particle size, temperature, and velocity, and with laser spectroscopic systems for detection of gas phase species and temperatures. Theoretical work is underway to describe the experimentally observed details of single-particle combustion and to assist in interpreting the observed transformations of mineral species. Extensive data of this type are critical to the understanding and evolution of properly engineered techniques for increased coal use.

Diagnostic research involves the development of laser-based optical and physical sampling techniques to monitor local detail of particle-laden flames in coal- and synfuel-fired combustors and hightemperature process flow streams. This work includes research on novel optical particle sizing and counting methods, on coherent anti-Stokes Raman scattering for measuring gas phase temperature flows, and on laser spark spectroscopy for determining the elemental composition of droplets and particulates entrained in high-temperature media. This work is conducted cooperatively with universities and industry.

\section{Experimentally Determined Overall Burning Rates of Coal Chars ${ }^{\dagger}$}

\section{R. E. Mitchell}

Optically obtained measurements of the size, temperature, and velocity of coal char particles burning in controlled environments are used to determine overall particle burning rates per unit external surface area. From the overall particle burning rates, chemical reaction rate

\footnotetext{
$\dagger$ To appear in Combustion Science and
} Technology (1987). coefficients which include the combined effects of pore diffusion and the intrinsic chemical reactivity of the coal chars are evaluated. In this paper, the parameters which describe the chemical reaction rate coefficients for the chars of three coals are presented. The effect of the value assumed for the apparent particle density, and the effect of the value employed for the apparent reaction order with respect to the oxygen partial pressure are considered. Constant-density and constant-diameter modes of burning are employed as limiting burning scenarios.

Pulverized coal particles in the size range 50 to $200 \mu \mathrm{m}$ are injected into a laminar flow reactor at a loading rate low enough to assure 
that the gas conditions in the reactor are not appreciably altered due to consumption of the particles. Prior to particle injection, the oxygen partial pressure and gas temperature and velocity profiles along the reactor centerline are determined. The transparent walls of the flow reactor allow luminous emission from a burning particle to be monitored by a particle-sizing pyrometry system. ${ }^{1}$ From the luminous emission, the size, temperature and velocity of the emitting body are determined. At selected heights in the reactor, 200 to 400 single particles are monitored. The data are grouped into roughly tenmicron-size ranges and the average temperature and velocity in each size range is determined.

The mass, momentum, and energy conservation equations are used to describe the char particle as it burns in the laminar flow reactor. It is assumed that the particles are in the Stokes flow regime. In the energy equation, allowance is made for the thermal inertia of the particle, the heat generated at the particle surface due to reaction, and the conductive and radiative heat losses.

In order to account for the boundary layers along the reactor walls, an empirical relation is used to describe the gas velocity in the central core of the reactor. The size and velocity measurements obtained with spherical, non-friable graphite particles are used to determine the two constants which appear in the relation. ${ }^{2}$ With the graphite particles, any errors in the measurements associated with non-sphericity effects are negligible, and any heat effects due to devolatilization and combustion of the volatiles are avoided.

The equations are applicable only after coal devolatilization because the mass and heat transfer effects associated with volatiles release and combustion are omitted in the analysis. Since only very dilute particle streams are considered, conditions in the free-stream are specified independently, as a function of the axial position in the reactor.

For given parameters that describe the overall particle-burning rate as a function of particle size and temperature, the governing set of differential equations are numerically integrated, simultaneously, from a starting height to a selected height, to yield the particle mass, temperature, and velocity at the height selected. At the starting height, the particle apparentdensity, diameter, temperature, and velocity are specified. The measured gas temperature is imposed during the integration.

The overall particle-burning rate is expressed in terms of the particle size and temperature by assuming that the single film model $^{3}$ of a burning carbon sphere, with carbon monoxide as the sole heterogeneous reaction product, applies. For such a model, the overall particleburning rate per unit external surface area, q, can be expressed in terms of a diffusional and a chemical reaction rate coefficient, $\mathrm{k}_{\mathrm{d}}$ and $\mathrm{k}_{\mathrm{c}}$, respectively, as follows:

$$
\mathrm{q}=\mathrm{k}_{\mathrm{d}}\left(\mathrm{P}_{\mathrm{g}}-\mathrm{P}_{\mathrm{s}}\right)=\mathrm{k}_{\mathrm{c}} \mathrm{P}_{\mathrm{s}}^{\mathrm{n}}
$$

The oxygen partial pressure in the free-stream and at the particle surface are denoted by $\mathrm{P}_{\mathrm{g}}$ and $\mathrm{P}_{\mathrm{s}}$, respectively, and $\mathrm{n}$ is the apparent reaction order with respect to the oxygen partial pressure. The diffusional reaction rate coefficient is expressed as:

$$
\mathrm{k}_{\mathrm{d}}=48 \mathrm{D}_{\mathrm{ox}} / \mathrm{R}^{\prime} \mathrm{T}_{\mathrm{m}} \mathrm{d}
$$

where $\mathrm{D}_{\mathrm{ox}}$ is the effective diffusion coefficient of oxygen in the boundary layer surrounding the particle of diameter $\mathrm{d}, \mathrm{R}^{\prime}$ is the gas constant, and $T_{m}$ is the mean temperature between the gas and particle.

Our objective is to express the chemical reaction rate coefficient in Arrhenius form:

$$
\mathrm{k}_{\mathrm{c}}=\mathrm{A}_{\mathrm{a}} \exp \left(-\mathrm{E}_{\mathrm{a}} / \mathrm{RT}_{\mathrm{p}}\right)
$$

Values of $\mathrm{A}_{\mathrm{a}}$ and $\mathrm{E}_{\mathrm{a}}$ which yield calculated temperatures that agree with the measured temperatures at the various measurement heights are determined. The procedure involves using the measured particle size, temperature and velocity interrelations in the energy equation to calculate the overall particle-burning rate for each measured particle size. Estimates of the mass and the temperature gradient of each particle size at each measurement height are calculated using the governing conservation equations. A reaction order is specified and the calculated burning rates and measured particle sizes and temperatures are used in equations (1) and (2) 
to determine values for $\mathrm{k}_{\mathrm{c}}$ corresponding to each of the size-temperature pairs. A least-squares procedure is then employed to determine the parameters which correlate the $\mathrm{k}_{\mathrm{c}}$ values and the particle temperatures. An iterative procedure is required, for the values calculated for the particle mass and its temperature gradient depend upon the values used for $A_{a}$ and $E_{a}$.

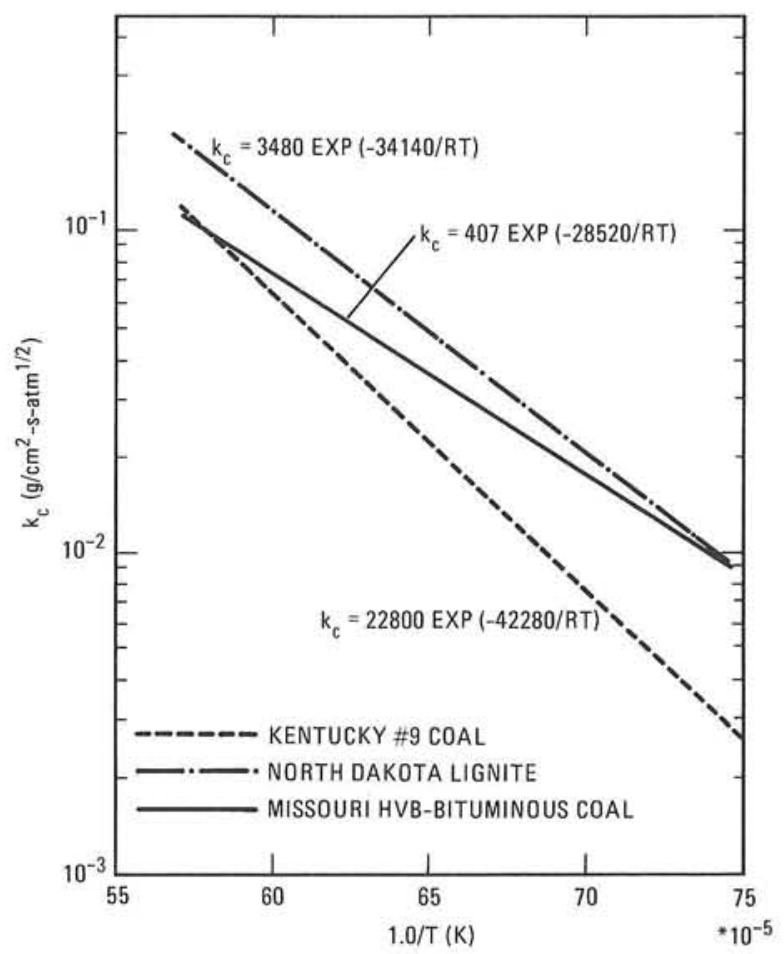

Figure 1. Arrhenius fits to the chemical reaction rate coefficients for the three coals investigated.

Figure 1 shows the Arrhenius fits to the chemical reaction rate coefficients for the chars of three coals. For each of the coals employed, the value determined for the activation energy is in the range expected for burning limited by the combined effects of pore diffusion and the intrinsic chemical reactivity of the particle material.

The parameters shown in the figure were determined by employing an oxygen reaction order of one-half and by assuming that the particles burn at constant density $\left(\rho=0.5 \mathrm{~g} / \mathrm{cm}^{3}\right)$. Table I shows the values obtained for the Arrhenius parameters when the initial apparent density of the char particle is varied. Values are shown for both constant density and constant diameter modes of burning. Also shown are statistical parameters which indicate the "goodness" of the least-squares fits. The column headed $\mathrm{e}\left(\mathrm{k}_{\mathrm{c}}\right)$ is the root mean-square difference between values of $\mathrm{k}_{\mathrm{c}}$ determined from the measurements and values calculated using the Arrhenius parameters from the least-squares fit. The column headed $\mathrm{e}(\mathrm{T})$ is the root meansquare difference between measured and calculated temperatures for a given particle size. In general, the smaller these statistical parameters, the better the fit to the data.

Note that regardless of the mode of burning hypothesized, the fit to the chemical reactionrate coefficients improves as the value assumed for the initial char density is increased to 0.7 $\mathrm{g} / \mathrm{cm}^{3}$. This is an indication of the importance of including the thermal inertia term in the energy equation. For constant density burning, the differences between measured and calculated temperatures increase as the value assumed for the char density exceeds $0.5 \mathrm{~g} / \mathrm{cm}^{3}$. For constant diameter burning, when the initial char density is specified as $0.3 \mathrm{~g} / \mathrm{cm}^{3}$ or less, the calculations result in the burnout of particles whose initial diameter is less than $100 \mu \mathrm{m}$ before a height of $25.4 \mathrm{~cm}$ in the reactor. This was not observed experimentally and is the cause of the large values for $\mathrm{e}(\mathrm{T})$ when the density is specified to be less than $0.3 \mathrm{~g} / \mathrm{cm}^{3}$. These observations support an initial char density in the range 0.3 to $0.5 \mathrm{~g} / \mathrm{cm}^{3}$, and burning at constant density is favored over burning at constant diameter.

Table II shows the values obtained for the Arrhenius parameters that describe the chemical reaction-rate coefficient when the apparent reaction order with respect to the oxygen partial pressure is varied. The values calculated for 
Table I

Arrhenius Parameters for the Chemical Reaction Rate Coefficient:* Variations in the Initial Apparent Density of the Char

\begin{tabular}{|c|c|c|c|c|}
\hline \multicolumn{5}{|c|}{ Constant Density Burning } \\
\hline $\begin{array}{c}\rho \\
\left(\mathrm{g} / \mathrm{cm}^{3}\right)\end{array}$ & $\begin{array}{c}\mathrm{A}_{\mathrm{a}} \\
\left(\mathrm{g} / \mathrm{cm}^{2}-\mathrm{s}-\mathrm{atm}^{\frac{1}{2}}\right)\end{array}$ & $\begin{array}{c}\mathrm{E}_{\mathrm{a}} \\
\text { (cal/mole) }\end{array}$ & $\begin{array}{c}\mathrm{e}\left(\mathrm{k}_{\mathrm{c}}\right) \\
\left(\mathrm{g} / \mathrm{cm}^{2}-\mathrm{s}-\mathrm{atm}^{\frac{1}{2}}\right)\end{array}$ & $\begin{array}{l}\mathrm{e}(\mathrm{T}) \\
(\mathrm{K})\end{array}$ \\
\hline "eq"** & $3.96 \times 10^{1}$ & 21020 & 0.058 & 26 \\
\hline 0.1 & $5.53 \times 10^{2}$ & 22120 & 0.056 & 25 \\
\hline 0.3 & $3.56 \times 10^{2}$ & 27950 & 0.051 & 20 \\
\hline 0.5 & $4.07 \times 10^{2}$ & 28520 & 0.050 & 22 \\
\hline 0.7 & $9.78 \times 10^{2}$ & 31350 & 0.048 & 29 \\
\hline 0.9 & $2.07 \times 10^{3}$ & 33850 & 0.051 & 32 \\
\hline \multicolumn{5}{|c|}{ Constant Diameter Burning } \\
\hline $\begin{array}{c}\rho \\
\left(\mathrm{g} / \mathrm{cm}^{3}\right)\end{array}$ & $\begin{array}{c}\mathrm{A}_{\mathrm{a}} \\
\left(\mathrm{g} / \mathrm{cm}^{2}-\mathrm{s}-\mathrm{atm}^{\frac{1}{2}}\right)\end{array}$ & $\begin{array}{c}\mathrm{E}_{\mathrm{a}} \\
\text { (cal/mole) }\end{array}$ & $\begin{array}{c}\mathrm{e}\left(\mathrm{k}_{\mathrm{c}}\right) \\
\left(\mathrm{g} / \mathrm{cm}^{2}-\mathrm{s}-\mathrm{atm}^{\frac{1}{2}}\right)\end{array}$ & $\begin{array}{l}\mathrm{e}(\mathrm{T}) \\
(\mathrm{K})\end{array}$ \\
\hline "eq"** & $3.96 \times 10^{1}$ & 21020 & 0.058 & 26 \\
\hline 0.1 & $4.09 \times 10^{1}$ & 21130 & 0.058 & 149 \\
\hline 0.3 & $8.34 \times 10^{1}$ & 23440 & 0.051 & 86 \\
\hline 0.5 & $2.60 \times 10^{2}$ & 27020 & 0.050 & 30 \\
\hline 0.7 & $7.31 \times 10^{2}$ & 30360 & 0.047 & 23 \\
\hline 0.9 & $1.85 \times 10^{3}$ & 33420 & 0.046 & 29 \\
\hline
\end{tabular}

Table II

Arrhenius Parameters for the Chemical Reaction Rate Coefficient:* Variations in Oxygen Reaction Order

\begin{tabular}{ccccc}
\hline \multicolumn{5}{c}{ Constant Density Burning } \\
\hline $\mathrm{n}$ & $\begin{array}{c}\mathrm{A}_{\mathrm{a}} \\
\left(\mathrm{g} / \mathrm{cm}^{2}-\mathrm{s}-\mathrm{atm}^{n}\right)\end{array}$ & $\begin{array}{c}\mathrm{E}_{\mathrm{a}} \\
(\mathrm{cal} / \mathrm{mole})\end{array}$ & $\begin{array}{c}\mathrm{e}\left(\mathrm{k}_{\mathrm{c}}\right) \\
\left(\mathrm{g} / \mathrm{cm}^{2}-\mathrm{s}_{-} \mathrm{atm}^{n}\right)\end{array}$ & $\begin{array}{c}\mathrm{e}(\mathrm{T}) \\
(\mathrm{K})\end{array}$ \\
\hline 0.3 & $2.61 \times 10^{2}$ & 30000 & 0.024 & 23 \\
0.4 & $3.38 \times 10^{2}$ & 29360 & 0.035 & 22 \\
0.5 & $4.07 \times 10^{2}$ & 28520 & 0.050 & 22 \\
0.6 & $5.50 \times 10^{2}$ & 28000 & 0.071 & 21 \\
0.7 & $7.64 \times 10^{2}$ & 27540 & 0.099 & 21 \\
0.8 & $1.11 \times 10^{3}$ & 27180 & 0.142 & 21 \\
0.9 & $1.78 \times 10^{3}$ & 27080 & 0.213 & 21 \\
1.0 & $5.16 \times 10^{3}$ & 28690 & 0.378 & \\
\hline
\end{tabular}


$\mathrm{e}\left(\mathrm{k}_{\mathrm{c}}\right)$ increase as the value specified for the reaction order is increased. The data clearly support an apparent reaction order less than unity. Considering both $\mathrm{e}\left(\mathrm{k}_{\mathrm{c}}\right)$ and $\mathrm{e}(\mathrm{T})$, an apparent order of 0.5 or less appears to correlate the data best. Consistent with previous studies, ${ }^{4,5}$ an apparent order of 0.5 is suggested.

\section{References}

1. D. A. Tichenor, R. E. Mitchell, K. R. Hencken, and S. Niksa, "Simultaneous in situ Measurement of the Size, Temperature and Velocity of Particles in a Combustion Environment," Twentieth Symposium (International) on Combustion (The Combustion Institute, 1984), p. 1213.

2. S. Niksa, R. E. Mitchell, K. R. Hencken, and D. A. Tichenor, "Optically Determined Temperatures, Sizes and Velocities of Individual Carbon Particles Under Typical Combustion Conditions," Comb. Flame 60, 183 (1984).

3. M. A. Field, D. W. Gill, B. B. Morgan, and P. G. W. Hawksley, Combustion of Pulverized Coal (BCURA, Leatherhead, England, 1967), p. 186.

4. B. C. Young and I. W. Smith, "The Kinetics of Combustion of Petroleum Coke Particles at 1000 to $1800 \mathrm{~K}$ : The Reaction Order," Eighteenth Symposium (International) on Combustion (The Combustion Institute, 1981), p. 1249.

5. R. E. Mitchell and W. J. McLean, "On the Temperature and Reaction Rate of Burning Pulverized Fuels," Nineteenth Symposium (International) on Combustion (The Combustion Institute, 1982), p. 1113.

\section{Experimentally Determined Overall Burning Rates of Pulverized-Coal Chars in Specified $\mathrm{O}_{2}$ and $\mathrm{CO}_{2}$ Environments ${ }^{\dagger}$}

\author{
R. E. Mitchell, O. H. Madsen *
}

The burning of coal chars under conditions characteristic of pulverized-coal combustion is a complex phenomenon involving the effects of heterogeneous and homogeneous reactions. In developing models of the burning phenomenon, critical issues concern the primary reaction product formed at the particle surface and the extent to which any carbon monoxide formed is oxidized in the boundary layer surrounding the particle. Several studies support the formation of carbon monoxide at the surfaces of particles having diameters in the pulverized-coal-size range. The important heterogeneous reactions are characterized by

$$
\begin{aligned}
& \mathrm{C}+\frac{1}{2} \mathrm{O}_{2} \longrightarrow \mathrm{CO} \\
& \mathrm{C}+\mathrm{CO}_{2} \longrightarrow 2 \mathrm{CO}
\end{aligned}
$$

Carbon monoxide conversion in the boundary layer is characterized by

$$
2 \mathrm{CO}+\mathrm{O}_{2} \longrightarrow 2 \mathrm{CO}_{2}
$$

Although the rate of the $\mathrm{C}-\mathrm{O}_{2}$ reaction is several orders of magnitude faster than the rate of the $\mathrm{C}-\mathrm{CO}_{2}$ reaction, if the oxygen concentration near the particle surface is substantially reduced due to $\mathrm{CO}$ oxidation in the boundary layer, then reaction (r2) can compete favorably with reaction ( $\mathrm{r} 1$ ) in governing the overall heterogeneous oxidation rate.

The single-film ${ }^{1}$ and double-film ${ }^{2}$ models for a burning carbon particle represent limiting cases for the effects of the homogeneous reaction. In the single-film model, the particle is consumed via reactions with oxygen and no reaction occurs in the boundary layer. Either CO

$\dagger$ Twenty-first Symposium (International) on Combustion (The Combustion Institute, 1986), in press.

* Technical University of Denmark, Lyngby, Denmark. 
or $\mathrm{CO}_{2}$ can be the product formed at the particle surface, but any $\mathrm{CO}$ formed is oxidized only after it has been transported to the free-stream. In the double-film model, the conversion of $\mathrm{CO}$ to $\mathrm{CO}_{2}$ (via reaction ( $\mathrm{r} 3$ )) at a flame front in the boundary layer prevents any oxygen from reaching the particle surface, thus allowing reaction (r2) to govern the particle consumption rate.

The continuous-film models of Caram and Amundson $^{3}$ and Sundaresan and Amundson ${ }^{4}$ indicate that for particles having diameters less than $50 \mu \mathrm{m}$ (and perhaps as large as $100 \mu \mathrm{m}$ ) burning in environments free of $\mathrm{CO}_{2}$, the singlefilm model with $\mathrm{CO}$ formation at the particle surface is an acceptable model. Here, experimental evidence which supports this theoretical conclusion is presented. The overall burning rates of coal chars, determined from a heat balance analysis employing measured values for particle size, temperature, and velocity, are found to be essentially unaffected by the carbon dioxide content of the ambient gas. The results support the single-film model with $\mathrm{CO}$ as the sole heterogeneous-reaction product for coal char particles as large as $130 \mu \mathrm{m}$ burning under typical pulverized-coal combustion conditions.

The combustion of single coal particles is observed in a vertical, entrained flow reactor which is rectangular in cross-section. The reactor is fed by an array of diffusion flamelets supported by $\mathrm{H}_{2} / \mathrm{CH}_{4} / \mathrm{O}_{2} / \mathrm{N}_{2} / \mathrm{CO}_{2}$ mixtures. Gas temperatures along the reactor centerline are determined using a platinum-vs-platinum $/ 13 \%$ rhodium thermocouple prior to injection of the particles. Oxygen levels in the reactor are calculated, assuming that the burned gases are in chemical equilibrium. In selected environments, the calculated oxygen levels are confirmed by measurement using a gas-sampling probe and a solid-state oxygen analyzer. Parameters which describe the gas velocity profile in the potential core of the reactor are determined from measurements of particle size and velocity employing spherical, nonfriable graphite particles. ${ }^{5}$

At the base of the reactor, coal particles are injected along the centerline at the feedgas temperature and velocity, and at a loading low enough to assure that the gas conditions in the reactor are not appreciably altered due to consumption of the particles. The transparent walls of the flow reactor allow luminous emission from the burning particles to be monitored by a particle-sizing pyrometry system. ${ }^{6}$ Analysis of the transmitted light intensity from a single particle traversing the image of a coded-aperture focused onto the reactor centerline yields the particle size and its temperature and velocity. Measurements are obtained at heights above the reactor inlet of $12.7,19.1$, and $25.4 \mathrm{~cm}$ which correspond to residence times of approximately 80,100 , and $125 \mathrm{~ms}$, respectively.

The $\mathrm{O}_{2} / \mathrm{CO}_{2}$ mole-percent ratio in the four gaseous environments employed in the experiments reported are 3.0/2.1, 6.0/2.1, 3.0/8.0, and $6.0 / 8.0$. In addition to the oxygen and carbon dioxide levels, each environment consisted of 16 mole-percent water with the balance being nitrogen.

The hvb-bituminous coal employed in this study is from Missouri (approximately 38\% fixed carbon, $8 \%$ moisture, $33 \%$ volatiles, and $21 \%$ ash), and was obtained from the Penn State Coal Data Base (PSOC-287). The "as received" coal was ground and screened using a Ro-Tap testing sieve shaker. Particles passing through the 115-mesh screening surface and retained on the 150-mesh surface were aerodynamically classified using a Vortec classifier. This yielded particles not only of similar size (mean diameter of $115 \mu \mathrm{m}$ ), but similar in density as well.

The Arrhenius parameters that describe the chemical reaction rate coefficient $\left(\mathrm{k}_{c}\right)$ are determined from the particle size, temperature, and velocity measurements. ${ }^{7}$ For each measured particle size, a value for the overall burning rate is calculated by using the size, temperature, and velocity measurements and estimates of the mass and the temperature gradient in the particle energy-balance equation. These calculated particle-burning rates and the measured particle sizes and temperatures are used in the equations that relate the flux of oxygen across the boundary layer surrounding the particle to the consumption rate of oxygen at the surface 
due to reaction, in order to determine values for $\mathrm{k}_{c}$ corresponding to each of the size-temperature pairs. A reaction order of one-half is specified. A least-squares procedure is then employed to determine the Arrhenius parameters, $\mathrm{A}_{a}$ and $\mathrm{E}_{a}$, which correlate the $\mathrm{k}_{c}$ values and the particle temperatures.

Estimates of the mass and the temperature gradient of each particle size are obtained by integrating numerically, the governing mass, energy, and momentum conservation equations. An iterative procedure is required because the values calculated for the mass and the temperature gradient depend upon the values selected for the rate parameters. For the first iteration, the particles are assumed to be in thermal equilibrium with the surrounding gas. Accordingly, the overall particle-burning rate is calculated from the energy balance equation with the thermal inertia contribution set to zero.

Figure 1 shows values determined for the chemical reaction-rate coefficients for the bituminous coal char particles burning in the gaseous environments employed. Calculated overall particle-burning rates are within 50 to $85 \%$ of the diffusion-limited rates. The line in the figure represents a least-squares fit to the calculated rate coefficients. The value determined for the activation energy (20000 cal/mole) is in the range expected for burning, limited by the combined effects of pore diffusion and the intrinsic chemical reactivity of the particle material. Within the error incurred in the measurements, the data support the conclusion that the different $\mathrm{CO}_{2}$-level environments exhibit the same temperature dependence for the chemicalreaction rate coefficients. The apparent activation energy falls in the range 17400 to 28500 $\mathrm{cal} / \mathrm{mole}$, with $20000 \mathrm{cal} / \mathrm{mole}$ being the likely value.

In order to assess the assumption that particles burn at constant density, the Thiele modulus is estimated from the overall particleburning rate. The extent to which diffusion effects within the particle are significant is determined by the value of the Thiele modulus, $\phi$. For a given particle size, a large value of $\phi$ indicates that the depth of penetration of oxygen is low and, therefore, only a small fraction of the total surface area available for reaction is utilized, meaning that the effectiveness factor $\eta$, is small. Reaction is primarily on or near the particle external surface. As the Thiele modulus decreases, the oxygen penetration depth increases and the effectiveness factor asymptotically approaches unity. Reaction occurs within the particle causing a decrease in the particle density.

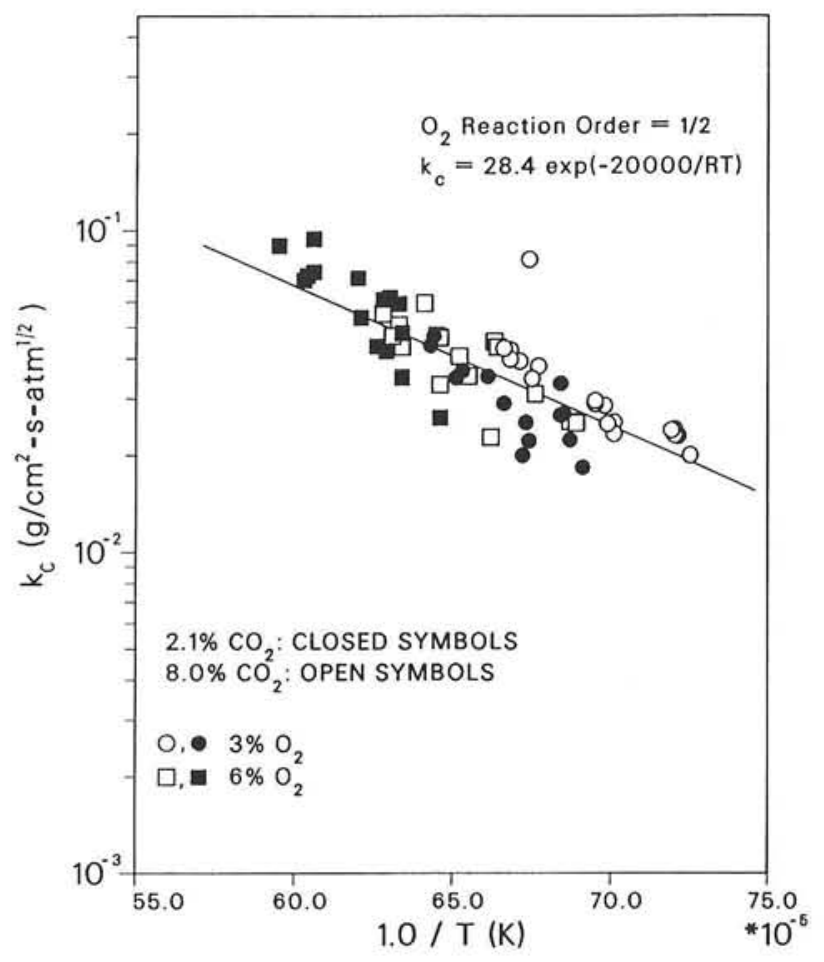

Figure 1. Chemical reaction-rate coefficients as a function of particle temperature

The relation between $\phi$ and $\eta$ depends upon the order of the reaction. Irrespective of the order, for $\phi^{2} \eta \geq 5$, pore diffusion effects limit the particle burning rate, meaning that the Thiele modulus is large. As this product is increased, the reaction rate increases and the oxygen penetration depth (and thus the effectiveness factor) decreases. Within the range of uncertainty in the values employed in determining the effective pore-diffusion coefficients and the overall particle-burning rates, the values calculated for $\phi^{2} \eta$ for the various particle sizes 
exceed 100. The Thiele modulus for each particle size is therefore in a range which indicates significant pore-diffusion effects with little penetration of oxygen into the particle pores. This supports the constant density burning assumption employed in the analysis.

\section{References}

1. M. A. Field, D. W. Gill, B. B. Morgan, and P. G. W. Hawksley, Combustion of Pulverized Coal (BCURA, Leatherhead, England, 1967), p. 186.

2. S. P. Burke and T. E. W. Schumann, "The Mechanism of Combustion of Solid Fuel," Proceedings of the International Conference on Bituminous Coal, 485 (1932).

3. H. S. Caram and N. R. Amundson, "Diffusion and Reaction in a Stagnant Boundary Layer About a Carbon Particle," Industrial and Engineering Chemical Fundamentals 16, 171 (1977).

4. S. Sundaresan and N. R. Amundson, "Diffusion and Reaction in a Stagnant Boundary Layer about a Carbon Particle. 6. Effect of Water Vapor on the Pseudo-Steady-State Structure," Industrial and Engineering Chemical Fundamentals 19, 351 (1980).

5. S. Niksa, R. E. Mitchell, K. R. Hencken, and D. A. Tichenor, "Optically Determined Temperatures, Sizes, and Velocities of Individual Carbon Particles Under Typical Combustion Conditions," Comb. Flame 60, 183 (1984).

6. D. A. Tichenor, R. E. Mitchell, K. R. Hencken, and S. Niksa, "Simultaneous in situ Measurement of the Size, Temperature, and Velocity of Particles in a Combustion Environment," Twentieth Symposium (International) on Combustion (The Combustion Institute, 1984), p. 1213.

7. R. E. Mitchell, "Experimentally Determined Overall Burning Rates of Coal Chars," to appear in Comb. Sci. Tech. (1987).

\section{Agglomeration During Combustion of Coal/Water Slurries as a Function of Coal Rank ${ }^{\dagger}$}

\author{
D. Dunn-Rankin, J. Hoornstra, ${ }^{*}$ T. H. Fletcher, \\ D. J. Holve
}

Coal/water slurry (CWS) fuel utilization is confined presently to long-residence-time combustor applications, such as utility boilers. There is continuing interest, however, in extending CWS application to shorter residencetime combustion systems, such as medium-speed diesels and turbines. One problem associated with using coal slurries in these traditionally liquid-fuel-fired, short-residence-time combustors is the formation of coal agglomerates during atomization of the CWS. These agglomerates are relatively large and take a long time to burn completely, thereby increasing residence time requirements for efficient combustion. The atomization of CWS generates agglomerates because many coal particles are contained in a single CWS droplet, and, as the water in the drop evaporates, capillary forces bring these particles together to form an agglomerate. It is not clear, however, what happens when the capillary liquid that initially draws the agglomerate together also evaporates. There is photographic evidence that, for a bituminous caking coal, the agglomerates melt and fuse into a single entity, ${ }^{1}$ suggesting that the agglomerate- binding mechanism ultimately relates to the inherent caking properties of the coal constituent of the slurry. In the present work, we examine this hypothesis by comparing the combustion behavior of caking bituminous CWS agglomerates to the combustion behavior of agglomerates formed of non-caking, lower rank, sub-bituminous and lignite CWS. These lower rank coals display no caking tendency under standard test conditions prescribed by the American Society for Testing and Materials (ASTM).

\footnotetext{
$\dagger$ Accepted by Fuel (1986).

* Netherlands Energy Research Foundation-ECN-Petten,
} The Netherlands. 
This experimental investigation has two parts: first, we compare the particle size distribution (PSD) of the atomized coal/water slurry to the PSD of the dry parent-coal constituent of the slurry. This comparison locates a specific feature of the PSD that is attributable to agglomeration. Second, we observe the evolution of this characteristic feature, during the combustion of CWS with different rank, to determine the influence of coal rank on the behavior of the CWS agglomerates.

The experimental apparatus consists of a laboratory-scale, laminar-flow reactor, a slurryatomization system, and an in situ optical particle-counter-sizer velocimeter (PCSV). Descriptions of all of these components and their operation have appeared previously. ${ }^{2,3}$

The flow reactor contains two flat flame burners $(20 \mathrm{~mm} \times 40 \mathrm{~mm}$ ) bridged by a slot flame $(2 \mathrm{~mm} \times 40 \mathrm{~mm})$. The reactor is fired with pre-mixed gas (methane and air) that produces a uniform temperature (approximately $1700 \mathrm{~K})$ and oxygen concentration $(3-5 \%)$ in the exhaust plume of the burner combination. The concentration and temperature environment is similar to that found in industrial furnaces. The CWS is introduced into the slot flame by a round pattern air-atomized nozzle assembly.

The PCSV uses two laser beams to measure two particle-size ranges $(0.3-4 \mu \mathrm{m}$ and $4-120 \mu \mathrm{m})$ at relatively high-number density $\left(10^{6} / \mathrm{cc}\right)$. The instrument produces a particlesize distribution by measuring the Mie-scattered light from individual particles. The instrument also measures particle velocity by timing the particle passage across the beam. The instrument accuracy is approximately $10 \%$ in both size and number density.

We use three CWS of different rank in the study (Table I): a lignite CWS, a physically beneficiated sub-bituminous CWS, and a bituminous CWS provided by different manufacturers (lignite - North Dakota Energy Research Center; sub-bituminous-AMAX Corporation; bituminous-United Coal Company). For comparison, AMAX Corporation also provided the parent pulverized coal of the sub-bituminous CWS.

\section{Table I}

Rank and Proximate Analyses of CWS on a Dry Weight Basis

\begin{tabular}{lccc}
\hline \multicolumn{1}{c}{ Rank } & Ash(\%) & $\begin{array}{c}\text { Fixed } \\
\text { Carbon(\%) }\end{array}$ & $\begin{array}{c}\text { Volatile } \\
\text { Matter(\%) }\end{array}$ \\
\hline Bituminous & 1.1 & 63.8 & 35.1 \\
Sub-bituminous & 5.6 & 49.7 & 44.7 \\
Lignite & 15.1 & 46.8 & 38.1 \\
\hline
\end{tabular}

Figure 1 compares the PSD of atomized sub-bituminous coal/water slurry to the PSD of the dry parent pulverized coal (PC). The measurements were made under ambient conditions (300 K). The CWS PSD shows a nearly uniform distribution in the large particles with a small local peak around 15 microns, while the dry coal has a decreasing number density with increasing particle size. Furthermore, there are no particles larger than $25 \mu \mathrm{m}$ measured in the PC PSD. The large-particle region of the CWS PSD represents agglomerates, and is typical for CWS particle-size distributions. The decreasing distribution with increasing particle size is typical for dry coal PSD's. The formation of the largeparticle characteristic region of the CWS PSD derives from the atomization process, ${ }^{4}$ and we use the behavior of this large-particle region to distinguish the behavior of the coal/water slurry agglomerates during further combustion.

Figure 2 compares the particle-size distribution evolution during combustion for two slurries of different rank coal. Three stages of the slurry size distribution evolution are shown in the figure: (1) the initial size distribution (10 mm COLD), (2) the PSD after evaporation and devolatilization (10 mm HOT), and (3) the PSD during char oxidation (70 $\mathrm{mm}$ HOT). The break between the large and small particle ranges (Figure 2) is an artifact of the PCSV size ranges selected. The break is bridged by a dotted line to indicate a continuous distribution.

There are few differences between the initial distributions of the slurries examined. The 

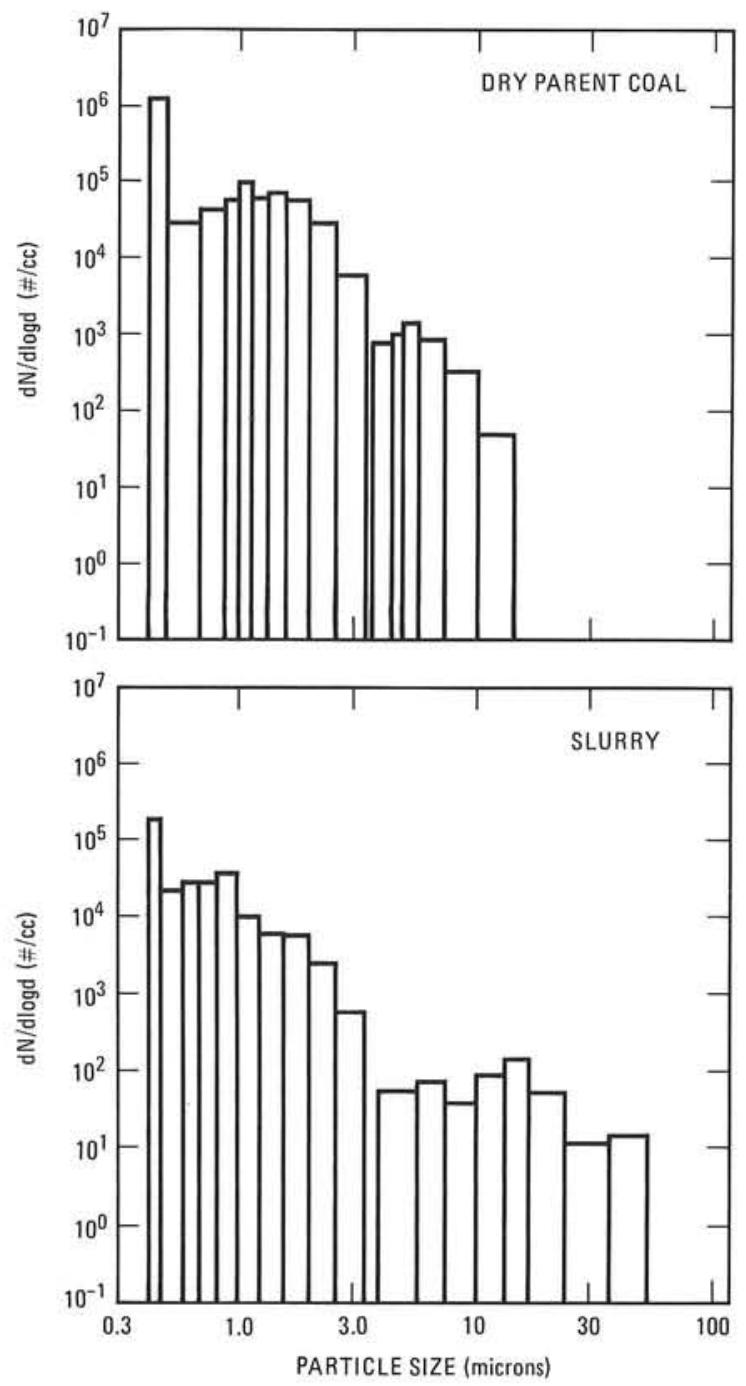

Figure 1. Comparison of particle-size distribution measurements under ambient conditions (300 K) of: a) dry parent pulverized coal and b) atomized coal/water slurry, showing the characteristic region of the particle-size distribution that indicates CWS agglomeration.

bituminous CWS has fewer of the smallest particles and slightly larger particles than the lower rank CWS, but the shape of the distributions is similar. Both of the initial PSD's show the characteristic local peak around 15 microns, described above as characteristic of the atomization process.
In a well-mixed coal/water slurry, the size distribution within an agglomerate will reflect the size distribution of the parent dry coal. Consequently, if the agglomerates break up after the slurry water evaporates, the slurry particlesize distribution should collapse to the PSD of the parent dry coal. However, our results show that the PSD's of the slurries are similar even after the water has evaporated. The characteristic agglomerate region is still evident for both coal slurries shown, indicating that the agglomerates have not broken up to form the parent coal distribution. The persistence of agglomerates is expected for bituminous coal because it is classified as a caking or agglomerating coal, but the agglomerates of the non-caking lignite also appear to remain intact after the water evaporates. Evidently, the mechanism binding the agglomerates together does not depend entirely on the inherent caking properties of the coal.

Differences between the evolution of the agglomerates for the different CWS become more apparent at the $70 \mathrm{~mm}$ measurement position. For these large particles, the bituminous CWS shows little change from the $10-\mathrm{mm}$ residence height due to limited burnout and swelling. However, the lower rank coal/water slurry exhibits a marked decrease in large particles. The decrease indicates a greater extent of burnout or disaggregation among the low-rank agglomerates than occurs for the bituminous agglomerates. This result suggests that the lowrank coal agglomerates, while definitely formed during atomization, are more tenuously bound than the well-fused agglomerates typical of bituminous coals. This tenuous binding may allow the low-rank agglomerates to flake and burn in a more rapid fragmentary mode. 

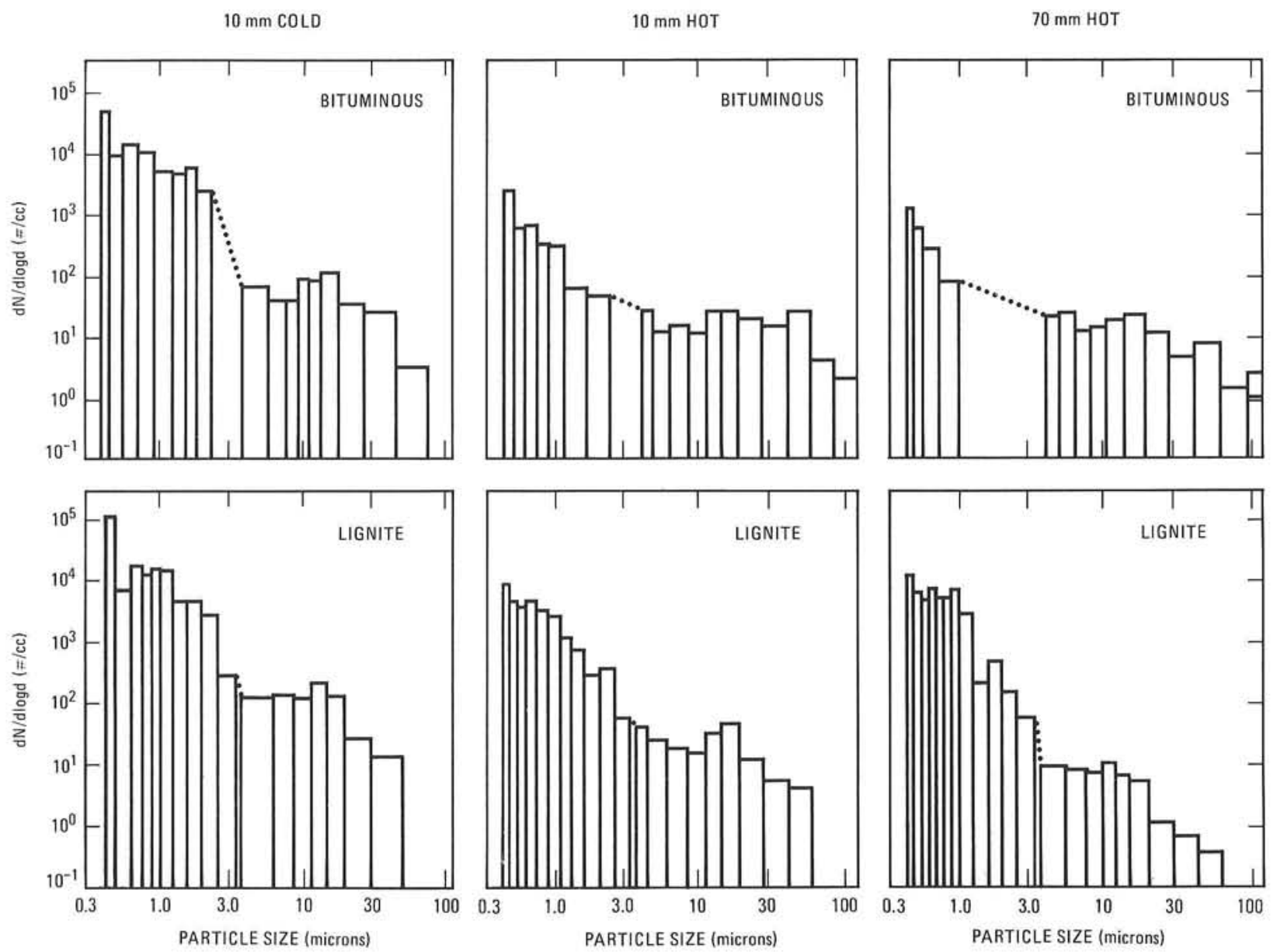

Figure 2. Particle size distribution of two coal/water slurries at three stages of evolution during combustion: 1) initial condition (10 mm COLD); 2) after devolatilization and evaporation (10 $\mathrm{mm} \mathrm{HOT}$ ); and 3) during char oxidation (70 mm HOT): a) Caking bituminous coal/water slurry, b) Non-caking lignite/water slurry.

\section{References}

1. K. J. Matthews and P. J. Street, "Combustion Histories of Various Coal-Water Fuels," Proceedings of the Sixth International Symposium on Coal Slurry Combustion and Technology (1984), p. 109.

2. D. J. Holve and P. L. Meyer, "Coal/Water Slurries: Fuel Preparation Effects on Atomization and Combustion," Sandia Report SAND848886; also, to appear in Comb. Sci. Tech. (1987).
3. D. J. Holve, "A Single Particle Counting Diagnostic System for Measuring Fine Particulates at High Number Densities in Research and Industrial Applications," Sandia Report SAND83-8246.

4. D. Dunn-Rankin, J. Hoornstra, and D. J. Holve, "Coal Rank Influences on Particle Size and Particle Volume Distributions During Coal/Water Slurry Combustion," Sandia Report SAND86-8769; also, Proceedings of the Eighth International Symposium on Coal Slurry Fuels Preparation and Utilization, (1986), in press. 


\section{Numerical Simulation of Particle-Size Distribution Evolution During Pulverized Char Combustion ${ }^{\dagger}$}

\section{Dunn-Rankin, A. R. Kerstein}

Particle size plays a major role in pulverized coal and coal/water slurry combustion. However, because typical pulverized-coal fuel is extremely polydisperse, it is better to relate combustion behavior to changes in the entire particle size distribution (PSD) than to changes in the mean particle size. Ignition, stability, combustion efficiency, and pollutant formation are all strongly influenced by the fuel particlesize distribution.

A complex interaction of competing physical processes determines the evolution of the particle-size distribution during combustion. Swelling, oxidation, and fragmentation contribute to the PSD evolution, and, consequently, it is difficult to extract the relative importance of the competing routes from experimental determinations of the particle-size distribution alone.

In this work, we explore supplementary information that can be obtained from numerical simulations of particle-size distribution evolution during combustion. Specifically, the numerical simulations help determine the role of fragmentation in PSD evolution. We use two models to study fragmentation during char combustion. The first is a percolation model that superposes the simulated combustion of individual char particles to build up the behavior of a particlesize distribution. The percolation model is mechanistic in nature, and we use this model to examine the more fundamental aspects of the fragmentation influence. The second model directly simulates the effects of combustion and fragmentation on the entire size distribution. This kinetic simulation (or population balance) has the nature of a transfer function that allows

\footnotetext{
$\dagger$ Submitted to Combustion and Flame (1986).
}

prediction of the influence of different fragmentation scenarios on PSD evolution. ${ }^{1}$

The percolation model consists of the simulated burning of individual particles. The timehistories of these single-particle simulations are then superposed to predict PSD evolution during combustion.

The percolation model represents a particle of the initial feedstock or char as a cluster of connected line segments (called "bonds" in the percolation literature) that have been randomly placed on a cubic lattice. The random process governing the construction of the oxidation of the particle consists of removing line segments (i.e., particle mass) from the perimeter of the cluster at a specified rate. Fragmentation occurs upon removal of a bond that links two sub-clusters together. This fragment pair further oxidizes and fragments until an entire distribution of cluster sizes is produced from a single parent cluster or particle. We determine the evolution of the entire size distribution by superposing, at a fixed time, the fragment populations resulting from the percolative oxidation of different initial clusters.

Results of the percolation simulation for two different initial particle-size distributions are shown in Figure 1. The PSD evolution is shown in terms of total mass fraction burned, which is a fundamental quantity of the simulation that provides a reasonable comparative base for different initial conditions.

Figures 1a-e shows the simulation of PSD evolution of an initial monodispersion during percolative oxidation. Kerstein and Edwards ${ }^{2}$ noted that an initial distribution of this shape eventually relaxes to a self-preserving $1 / d^{3}$ power-law form, up to a cut-off size. This relaxation of a monodispersion to a $1 / d^{3}$ distribution from Kerstein and Edwards appears in Figures 1a-e, with coarser size bins for convenience of comparison between cases.

Interestingly, the $1 / d^{3}$ initial distribution of Figure 1f is not self-preserving, though it eventually relaxes to the $1 / d^{3}$ form at higher 

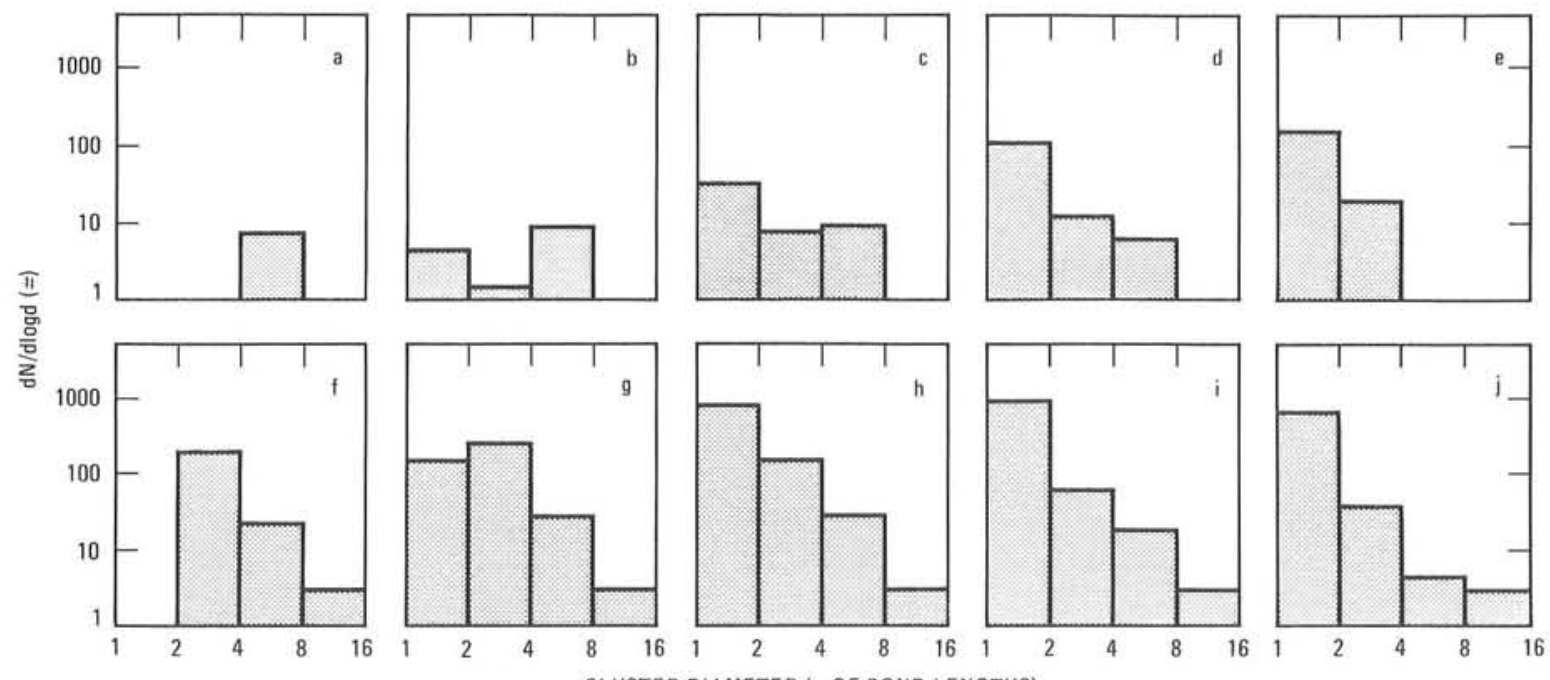

Figure 1. Percolation simulations of particle-size distribution evolution during char oxidation using three initial distributions: a monodispersion (a-e), and a power-law distribution ( $\mathrm{f}-\mathrm{j})$. The distributions are reported at different stages of particle burnout.

burnout (Figure 1j). The deviation from the $1 / d^{3}$ shape results because each monodispersion making up the initial distribution relaxes to the self-preserving distribution shape at a different rate. This result suggests that the selfpreserving power-law distribution cannot occur until all of the clusters have undergone a morphological pre-treatment from oxidation during the transient period of PSD evolution. The time required for this morphological pre-treatment depends on the particle size, and consequently, burnout of the largest particles controls the relaxation of the entire distribution to the $1 / d^{3}$ shape.

Kinetic modeling or the population balance method has been applied to many size degradation processes. In particular, simulations of grinding processes have made extensive use of kinetic modeling. ${ }^{3}$ For this work, we adopt the nomenclature outlined for simulating grinding processes by Austin et al,,$^{3}$ except that we include the influence of combustion. The model solves the following system of differential equations:

$$
\frac{d N_{i}}{d t}=-C_{i} N_{i}+C_{i-1} N_{i-1}-S_{i} N_{i}+\sum_{\substack{j=1 \\ i>1}}^{i-1} b_{i j} S_{j} N_{j}
$$

where $N_{i}$ is the number of particles in the $i$ th discrete-size class. In the above formulation, increasing size index corresponds to decreasing particle size, with $N_{1}$ representing the number density of the largest particles. The $n$th bin is the lower size cutoff of the simulation, and this bin is a particle sink that accumulates any entering particles.

The first two terms on the right hand side of the rate equation (1) represent the sinks and sources caused by particle burning. $C_{i}$ is the rate at which particles leave size bin $i$ due to combustion, and $C_{i-1} N_{i-1}$ is the rate at which particles in the next larger size class burn to particles of size $i$. Similarly, the second pair of terms on the right hand side of the rate equation represent the influence of fragmentation. $S_{i}$ is the rate of fragmentation of size $i$ particles, and $b_{i j} S_{j} N_{j}$ is the rate at which particles enter size $i$ from the fragmentation of $j$-sized 
particles. The $b_{i j}$ are the elements of the fragmentation progeny matrix. This matrix describes the number of particles that enter size class $i$ from the fragmentation of a single particle in size class $j$. The number of degrees of freedom in the fragmentation-progeny distribution is very large, and, consequently, we restrict our study to two limiting cases of fragmentation behavior, and we acquire a power-law-based progeny matrix from the percolation model of char oxidation described above. The two limiting forms of fragmentation that we investigate are termed attritive fragmentation and breakage. Attritive fragmentation refers to the loss of very fine particles from the surface of a large particle without noticeable size reduction of the parent particle. Contrary to attritive fragmentation, breakage implies that a particle splits into only a few relatively large fragments.

For the initial distribution of the kinetic simulations, we use the simple power-law (i.e., $d N / d \log d=$ constant $\left./ d^{3}\right)$ that has been measured $^{4}$ and predicted as the distribution resulting from percolative fragmentation (Figure 1).

Figure 2 shows the PSD evolution of the initial power-law distribution with both fragmentation and combustion. Attritive fragmentation begins to influence the distribution shape when a sufficient number of fine particles have burned. The evolution of the PSD during breakage and burning is very similar to the evolution of the PSD with burning alone, except that the large particle concentration decreases more rapidly with breakage fragmentation included. Power-law fragmentation similarly facilitates large particle oxidation, but also affects the small particle concentration. The power-law progeny matrix generates a larger number of fragments than either the attritive or breakage progeny matrices, and, after an initial transient, the contribution to each size class from powerlaw fragmentation approximately balances the loss in each size class due to burning. This produces a self-preserving power-law distribution reminiscent of the progeny matrix power-law form. However, the power law of the predicted distribution is closer to $1 / d^{2}$ than it is to the progeny matrix relationship of $1 / d^{3}$.
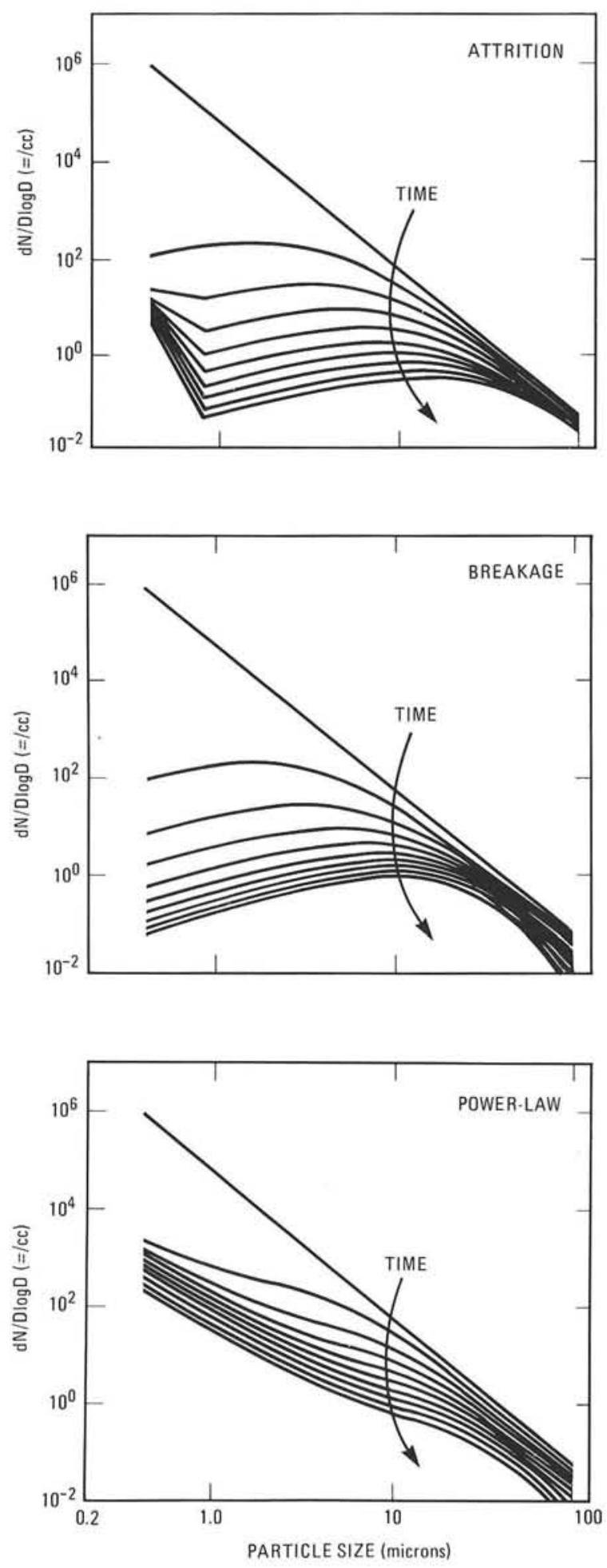

Figure 2. Kinetic simulation of initial power-law distribution evolution with three fragmentation modes: attritive fragmentation, breakage fragmentation, and power-law fragmentation. The evolution with fragmentation and burning are shown. Curves are shown at $10 \mathrm{~ms}$ intervals. 


\section{References}

1. D. Dunn-Rankin and A. R. Kerstein, "Numerical Simulation of Particle Size Distribution Evolution During Pulverized Coal Combustion," Sandia Report SAND86-8944 (1986); also, presented at the Western States Section (The Combustion Institute, 1986).

2. A. R. Kerstein and B. F. Edwards, "Percolation Model for Simulation of Char Oxidation and Fragmentation Time-Histories," accepted by Chemical Engineering Science (1986).

3. L. G. Austin, R. R. Klimpel, and P. T. Luckie, Process Engineering of Size Reduction: Ball Milling, (Society of Mining Engineers, New York, NY, 1984).

4. D. J. Holve, "In situ Measurements of Flyash Formation from Pulverized Coal." Comb. Sci. Tech. 44, 269 (1986).

\section{Laser Spark Spectroscopy For In Situ Analysis of Particles in Combustion Flows}

\section{K. Ottesen}

A challenging area for coal combustion diagnostics is that of determining the composition of entrained coal, char, and mineral matter particles in the combustion zone. We are continuing the development of optical techniques for the simultaneous measurement of the size, velocity, and elemental composition of particles in combustion flows. We use laser spark spectroscopy ${ }^{1}$ to determine the elemental composition of the particles. (Measurements on uniform size water droplets containing sodium at known concentrations have been conducted previously, ${ }^{2}$ and sizing measurements, using a two-color light scattering technique, are described elsewhere. ${ }^{3}$ )
The two laser beams, which are used to make sizing measurements, are also used to trigger a high power Nd:YAG laser when a particle is centered in the confocal volume of the three beams. The laser energy deposited in the particle rapidly heats the material forming a plasma. The hot elemental species contained in this "spark" produce a series of intense emission lines that are detected using a grating spectrometer with an optical multichannel detector array.

Optimum results are obtained by gating the detector array 2 microseconds after the initiation of the spark. This allows the very hot species in the plasma to decay into the neutral or singly-ionized state with a considerable simplification in the number of emission lines and their interpretation. Our work has used a detection window of 2 microseconds.

Experiments have been conducted on raw coal particles entrained in a room temperature nitrogen flow. This material is a high volatile bituminous Kentucky No. 11 coal, and we have used the classified size fraction in the $+30 /-50$ $\mu \mathrm{m}$ size range. An independent semi-quantitative spectrochemical analysis of a bulk sample indicated the following composition ranges: greater than $1000 \mathrm{ppm}$ of $\mathrm{Al}, \mathrm{Fe}, \mathrm{Ca}$, and $\mathrm{Si} ; 100-1000$ ppm of $\mathrm{K}, \mathrm{Mg}, \mathrm{Na}$, and $\mathrm{Ti}$; and less than 100 ppm of $\mathrm{Mn}$ and $\mathrm{Cr}$.

All of these elements except $\mathrm{Mn}$ and $\mathrm{Cr}$ were observed in the single particle laser spark experiments. In addition to the species mentioned above, we also identified $\mathrm{Li}, \mathrm{Ba}$, and $\mathrm{H}$. Representative spectra for four single particles are shown in Figures 1a-d. CN resulting from the recombination of $\mathrm{C}$ and $\mathrm{N}$ atoms was also observed, as were $\mathrm{N}$ and $\mathrm{O}$ emission lines.

The shot-to-shot reproducibility of relative line intensities was a concern. We have investigated the correlation of intensities for two Fe lines (2601 $\AA$ and $2634 \AA$ ). The results for 80 successive particles are shown in Figure 1a. To a first approximation, this plot should yield a straight line with a slope proportional to the ratio of the line strengths for these two transitions. This was clearly the case (Figure 2a). 

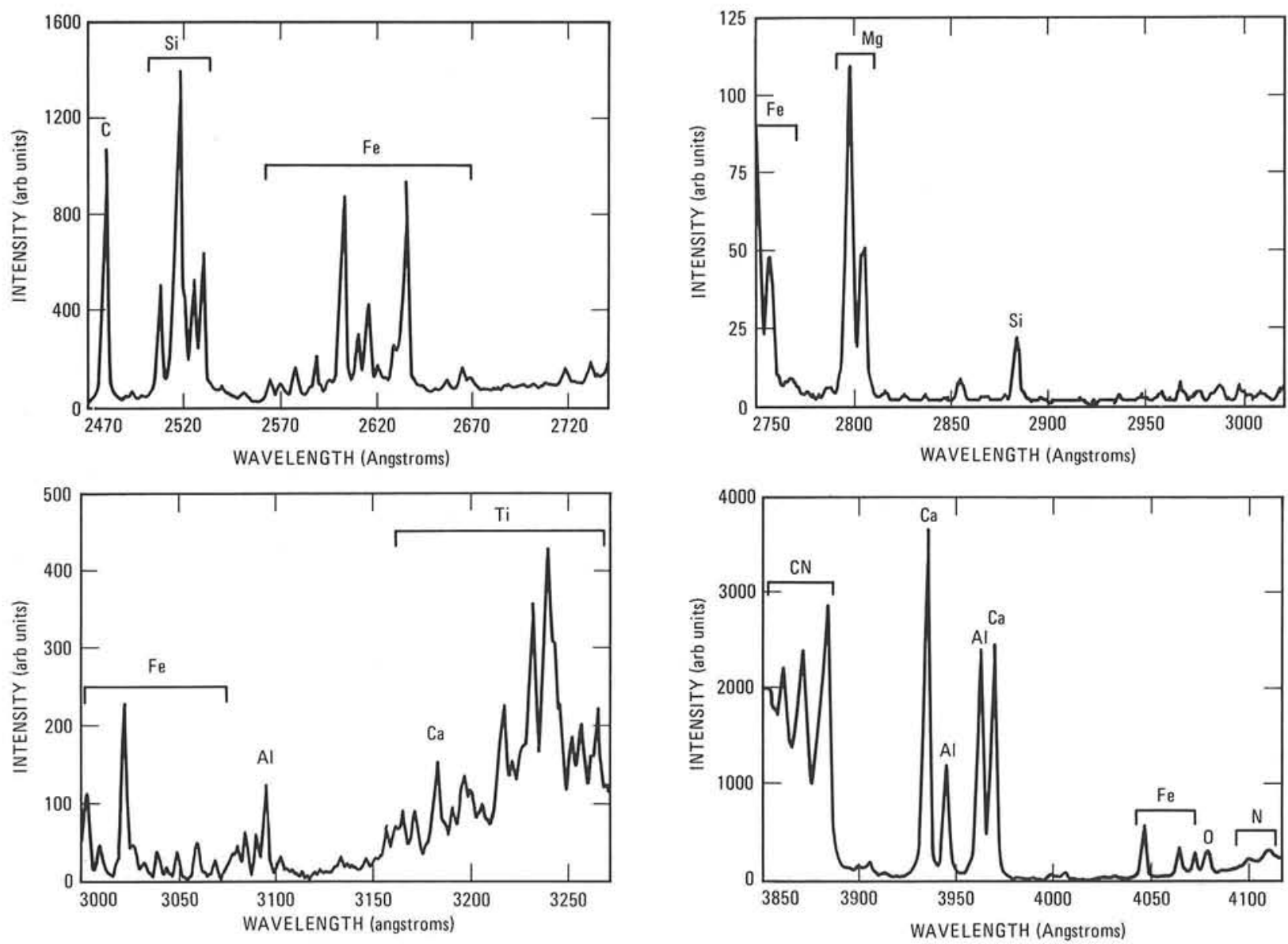

Figure 1. Representative emission spectra following laser breakdown of four single coal particles in different wavelength regions.
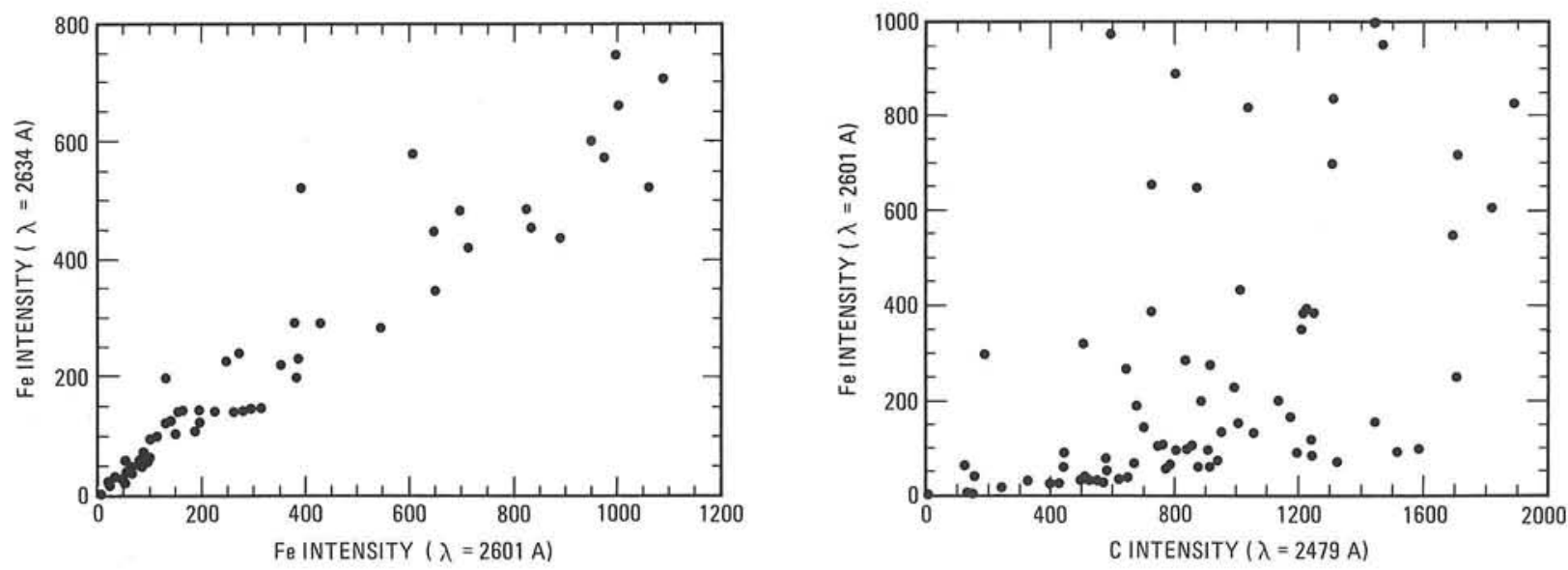

Figure 2. Correlation of spark emission intensities on a particle-by-particle basis in a series of 80 single particles: a) for two Fe-emission lines; b) for a single $\mathrm{Fe}-$ and a single C-emission line. 
The small scatter indicates a small variation in the plasma temperature from shot to shot.

In a similar study, the intensity of the Fe transition at $2601 \AA$ was correlated with the intensity of a C transition at $2475 \AA$ for the same set of 80 particles (Figure 2b). Preliminary examination of the data indicates that there may be two distinct populations in this correlation: a clustering of points along the $\mathrm{X}$-axis with low $\mathrm{Fe}$ intensities which may be related to iron bound in the organic matrix and distributed fairly homogeneously; and scattered points with high Fe intensities which may correspond to discrete inclusions of iron-bearing minerals in individual coal particles.

A primary research goal in the present study is the simultaneous measurement of size and composition of individual coal particles. Recent work has demonstrated this capability, and preliminary data are being acquired to determine size effects on the observed emission spectra of raw coal particles at room temperature.

Considerable additional work remains to be done on the question of calibration. We are in the process of developing a code to predict the relative intensities of analytical lines as a function of particle size, relative elemental concentrations, and plasma temperature. This code will be validated against a group of well characterized coals and ashes. Matrix effects will be considered, and an internal standard method for the experimental spectra will be developed.

The laser spark technique appears to be a promising method for on-line elemental analysis of particles in combustion flows. When these results are combined with the simultaneous particle size and velocity measurements, the resulting comprehensive statistical picture should be of particular importance in understanding the evolution and eventual fate of inorganic species as ash and pollutants in pulverized coal combustion.

\section{References}

1. R. W. Schmieder, "Techniques and Applications of Laser Spark Spectroscopy," Sandia Report SAND83-8618 (1983).

2. D. K. Ottesen and J. C. F. Wang, "NonIntrusive Optical Techniques for the Study of Pulverized Fuels," ACS Div. of Fuel Chem. Preprints 31, 136, (1986).

3. J. C. F. Wang and K. R. Hencken, "In Situ Particle Size Measurements Using a Two-Color Laser Scattering Technique," Sandia Report SAND85-8869 (1985).

\section{Determination of Coal Devolatilization Rates at High Temperatures and Heating Rates}

\section{T. H. Fletcher}

Current models of pulverized coal devolatilization utilize global rates that span seven orders of magnitude. Much of the controversy in these rates arises from the fact that particle temperatures have not been measured during devolatilization. A flameless, inert gas flow reactor has been developed to provide maximum optical access for measurement of particle temperatures during devolatilization.

As shown in Figure 1, electric heaters are used to control the gas temperature in the flow reactor. The maximum gas temperature currently attainable is $1300 \mathrm{~K}$, due to significant radiant heat loss from the final heater through the transparent flow reactor walls.

The major optical diagnostic in this experiment is an improved version of the particlesizing pyrometer ${ }^{1}$ currently being used in our char combustion studies. Reflecting optics are used in the microscope, allowing detection of 


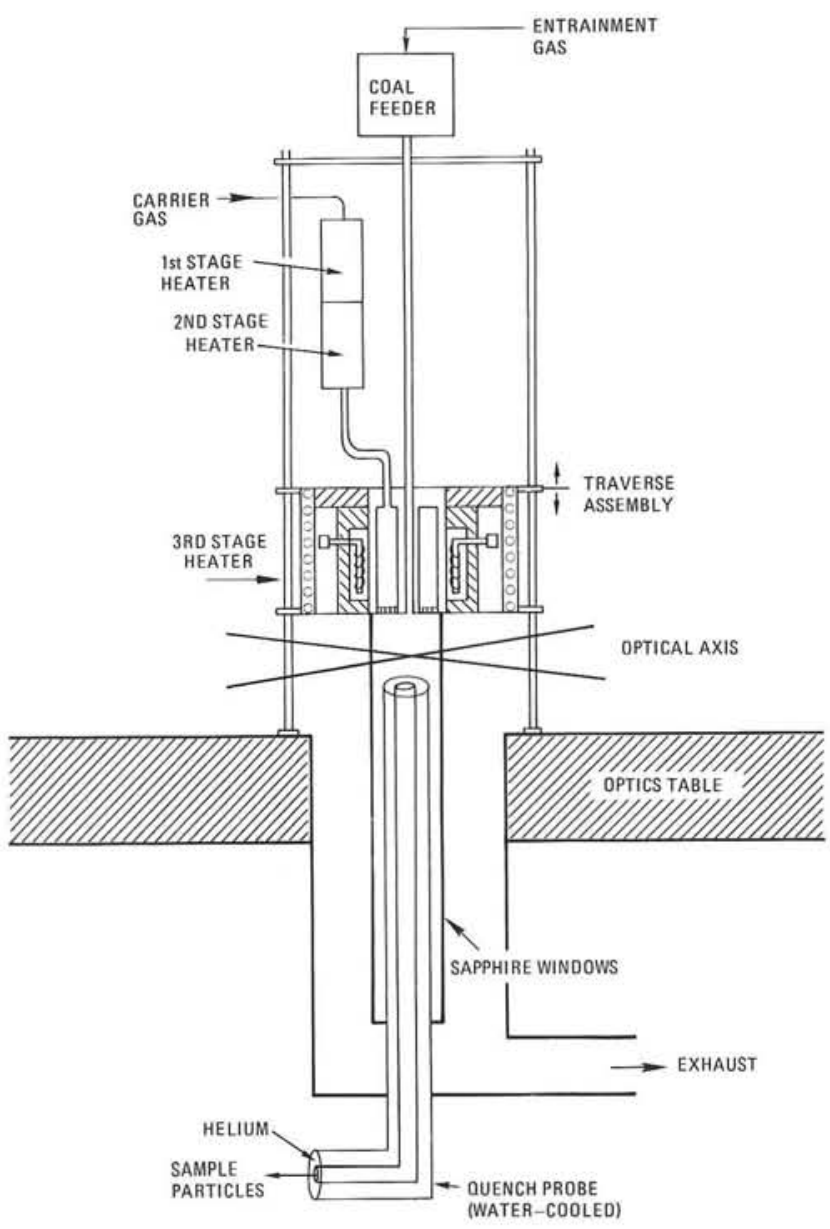

Figure 1. Schematic of flameless flow reactor for studying coal devolatilization rates in inert environments. both visible and infrared radiation from the particles without chromatic aberrations. A schematic of the electro-optical layout is shown in Figure 2. Radiant emission from particles in the flow reactor is focused on a particle-sizing mask. The coded light signal is then measured at two different wavelengths (1.6 and $2.2 \mu \mathrm{m})$ using indium arsenide detectors. This diagnostic allows simultaneous measurement of particle size, temperature, and velocity during devolatilization.

Solid samples are collected using a heliumquench probe and analyzed for mineral content. Titanium content will be used as a tracer to compute weight loss. Coal particles are sieved and then aerodynamically classified prior to use in order to achieve a narrow size distribution. Tars and aerosols generated during devolatilization are aerodynamically separated from the partially-reacted coal particles in order to obtain a more accurate determination of weight loss.

High-speed cinematography is used to observe the soot cloud surrounding the particle during devolatilization. The size of the soot cloud will be observed at various oxygen concentrations in the flow reactor. The soot cloud size is a measure of the size of the flame produced by the combustion of volatiles produced by the coal. Experimental observations will be compared with calculations of volatile combustion. ${ }^{2}$

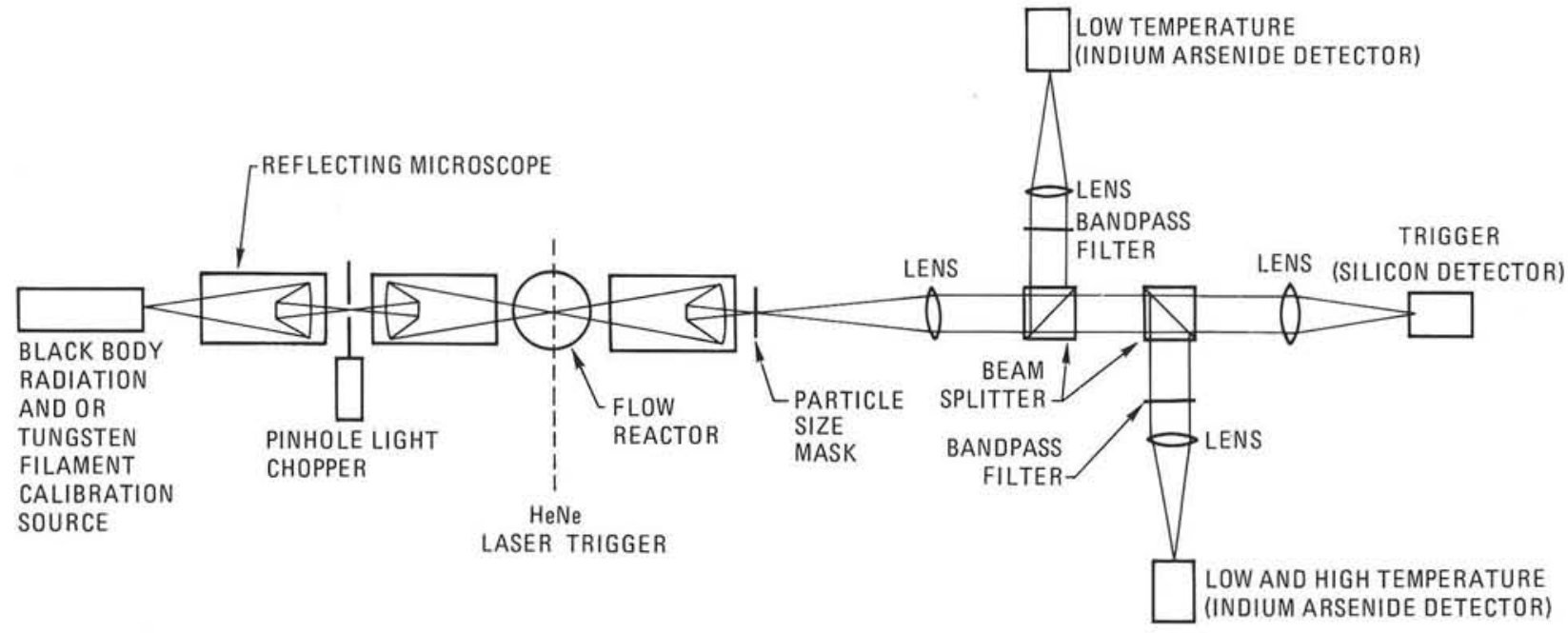

Figure 2. Optical system for in situ measurement of particle size, temperature, and velocity. Reflecting optics are used to eliminate chromatic aberrations. 


\section{References}

1. D. A. Tichenor, R. E. Mitchell, K. R. Hencken, and S. Niksa, "Simultaneous In Situ Measurement of the Size, Temperature and Velocity of Particles in a Combustion Environment," Twentieth Symposium (International) on Combustion (The Combustion Institute, 1984), p. 1213.

2. S. P. Musarra, T. H. Fletcher, S. Niksa, and H. A. Dwyer, "Heat and Mass Transfer in the Vicinity of a Devolatilizing Coal Particle," Comb. Sci. Tech. 45, 289 (1986).

\section{Evolution of Char Morphology and Mineral Matter Transformations During the Controlled Combustion of a Bituminous Coal}

\section{A. Nissen, F. A. Greulich}

One of the major problems resulting from the combustion of coal is the formation of mineral deposits on boiler walls and heat transfer surfaces. These deposits are caused by inorganic material that enters the furnace in association with the coal, either as inherent or as extraneous mineral matter. Accumulation of these deposits on heat-transfer surfaces leads to reduced output and alters the flow patterns of combustion gases. Coupled with this are the problems of disposing of enormous quantities of ash residue as well as atmospheric pollution caused by the unretained ash. In this context, it is obvious that mineral matter is undesirable and detrimental to the efficient and effective use of coal. On the other hand, it is quite possible that various mineral species in the coal matrix catalyze many of the organic transformations that take place during combustion. ${ }^{1}$ Consequently, coal beneficiation may have unknown and perhaps deleterious effects on the combustion process. The high temperature chemistry of extraneous, uncombined mineral matter is reasonably well understood. However, the need for a detailed understanding of the nature and distribution of the mineral matter initially present in coal, its subsequent transformation(s), and the distribution and form of the transformed inorganic material is an important and unresolved issue.

Certainly one of the most powerful tools that has been developed to provide detailed compositional information is the automated scanning electron microscope (SEM). The ability of this instrument to provide microanalytical and structural information on the composition and size distribution of mineral matter in coal has been amply demonstrated. ${ }^{2-4}$ In the present study, the scanning electron microscope was used to evaluate the evolution of char particle morphology as well as to determine the distribution, composition, and structure of mineral matter during the combustion process.

In order to begin to gain an understanding of the types of mineral transformations and the mechanisms of ash formation in coal, we have undertaken a systematic study of coal chars that have been produced under well-defined conditions. Because they were readily available, we have chosen to examine the chars produced during the controlled combustion of PSOC-287 in a laminar flow reactor. ${ }^{5}$ PSOC-287 is a hvbbituminous coal from Missouri having $20 \mathrm{wt} \%$ ash and a total sulfur content of $5.4 \mathrm{wt} \%$. The coal was burned in an atmosphere containing $\approx 6$ mole $\%$ oxygen at a peak temperature of $1550 \mathrm{~K}$. Samples were withdrawn with the isokinetic probe at 77,102 , and 124 msec.

The char particles exhibit the rounded, pock-marked appearance seen in bituminous coal (Figure 1). The rounded edges of the char particles in Figure 1b indicate that, during this stage in the combustion, the coal has softened and become very plastic. The large voids on the surface of individual particles represent places where, because of the plastic nature of the matrix, volatiles formed in the interior of the particle could escape. The external appearance of the char particles is reflected in their internal 

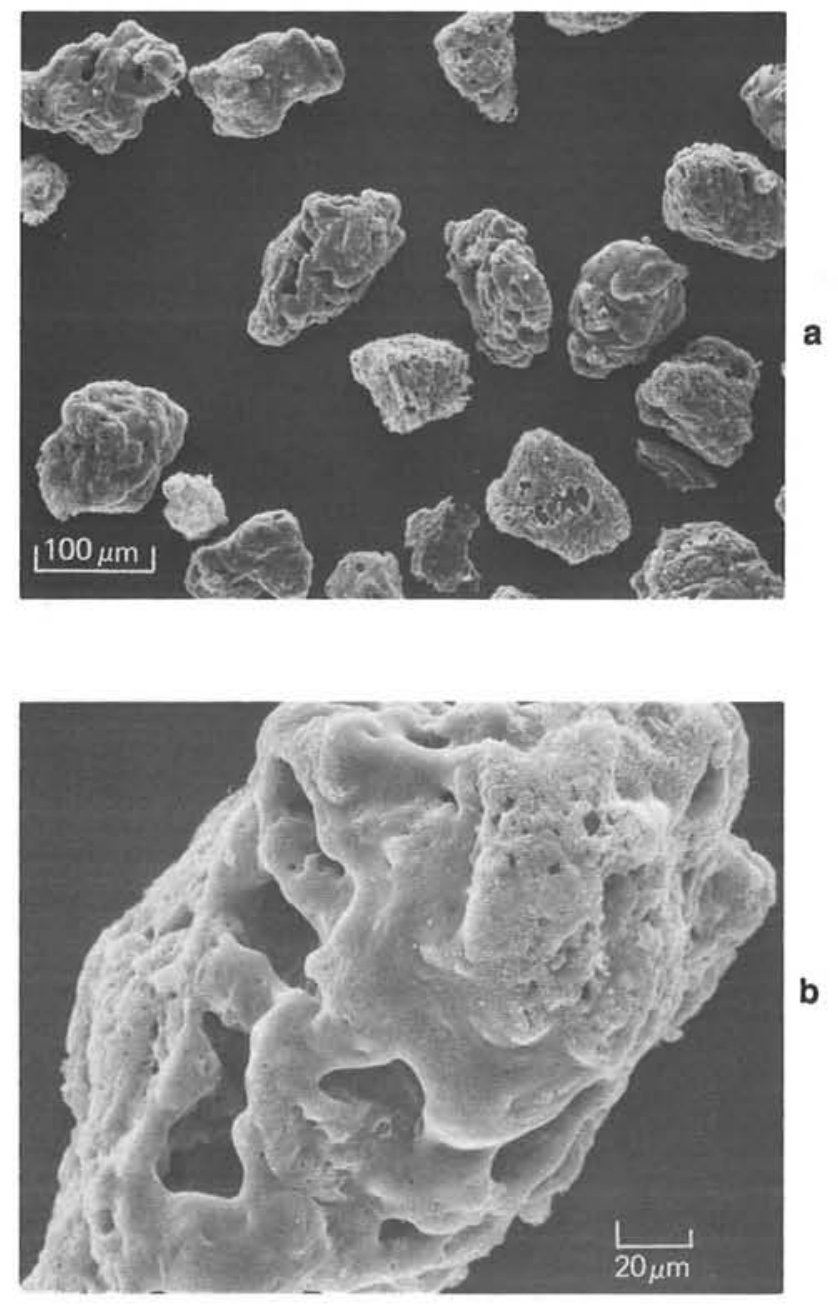

Figure 1. SEM image of char particle: a) 77msec sample; b) higher magnification. Deformation and rounding of the char particle shows its plastic nature.

structure. Figure 2 is a backscattered electron image of a cross-sectioned char particle.

Large internal voids are seen in those areas where volatile combustion products were produced. Their size may reflect a combination of deformation of the plastic matrix by internal pressure generated by volatile combustion products and oxidation by the combustion atmosphere. In the backscatter mode, discrete mineral particles are seen as bright spots against the background organic material. At this magnification, there is very little evidence of mineral matter at the surface of the char particle. Discrete mineral particles are, however, visible in the interior. Energy Dispersive X-ray analysis

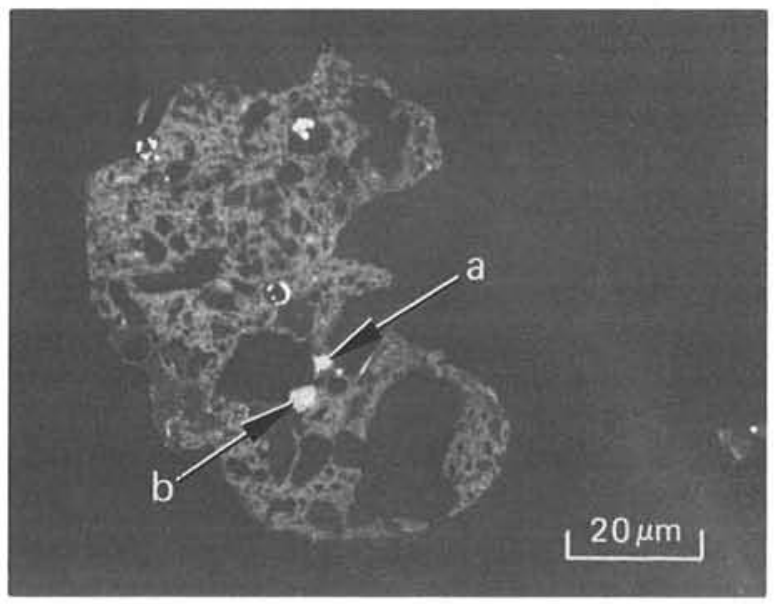

Figure 2. Backscatter electron image of a crosssectioned char particle showing the presence of (a) $\mathrm{SiO}_{2}$ and (b) iron-substituted illite in the interior.

of two of them (arrows in Figure 2) shows that these are (a) $\mathrm{SiO}_{2}$ and (b) what appears to be a partially iron-substituted illite.

As Allen has shown, ${ }^{5}$ the devolatilization process in bituminous coal is characterized by the formation of low density organic filaments. Close examination of the filaments show that they are covered with a fine mineral matter fuzz whose principal inorganic constituents are $\mathrm{Fe}$ and $\mathrm{S}$ with minor amounts of $\mathrm{A} 1$ and Si. As the volatile organic matter is ejected, minerals on the surface of the char particle adhere to it and are carried along. This process is shown in more detail in Figure 3.

While the mineral species associated with the surface of the char retain some sulfur, those that have been ejected have completely lost sulfur. Analysis of the mineral matter on the surface of such particles show only iron, from which we infer that the $\mathrm{Fe} / \mathrm{S}$ species on the surface have been oxidized to iron oxide with the concurrent release of sulfur oxides.

These data show that for the Missouri bituminous coal, mineral matter, as well as part of the organic matrix, is ejected from the burning char particle as volatile organic compounds are expelled. The filaments are composed of a porous organic matrix upon which is deposited small amounts of mineral matter, principally $\mathrm{Fe}$ and $\mathrm{S}$ compounds. While the mineral matter 

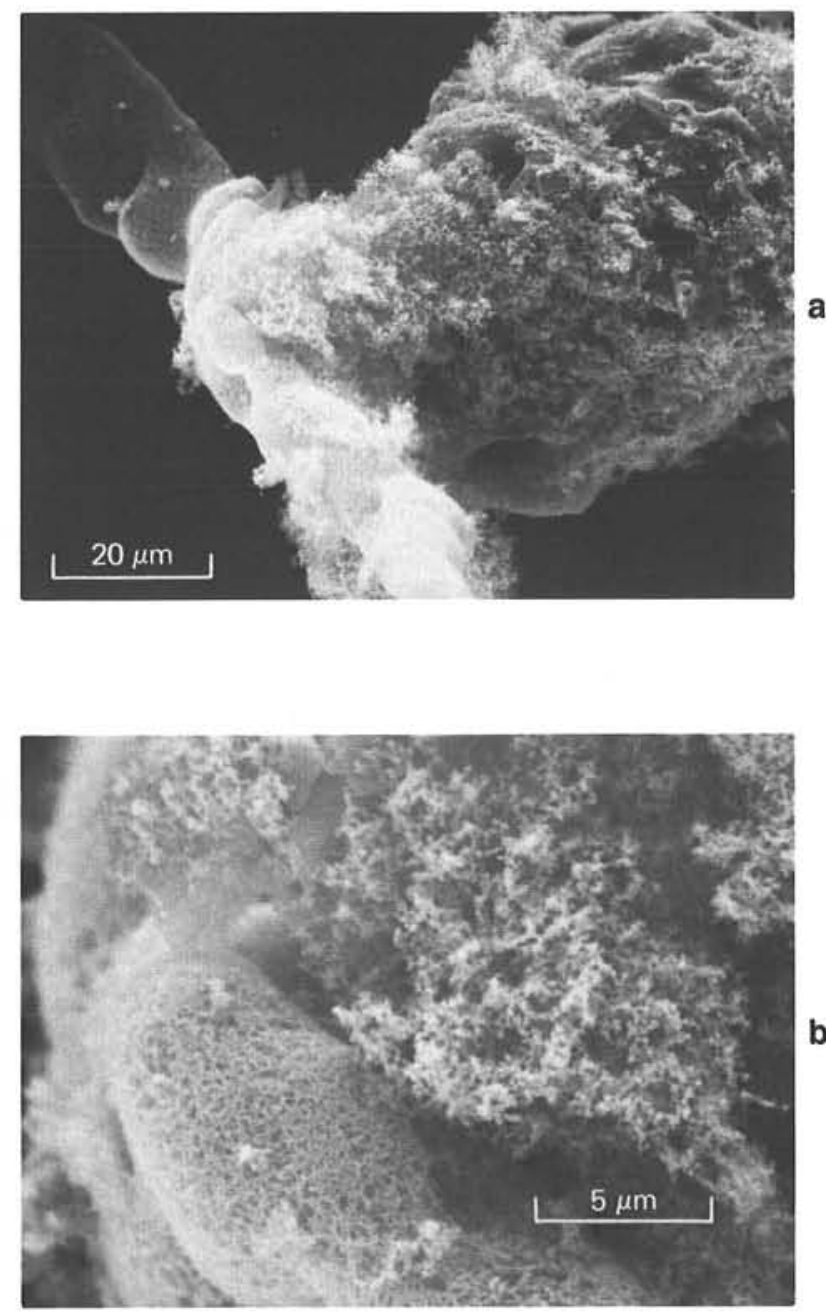

Figure 3. SEM image of a char particle: a) the emergence of a porous filament and the accompanying fine-textured mineral coating: b) higher magnification showing the point of emergence of the filament from the body of the char particle and the porous nature of the filament.

associated with char particles retains some of its sulfur, mineral particles that have become separated (for example, those on the surface of the ejected organic filaments), appear to have been oxidized to iron oxide. This probably reflects differences in the composition of the atmosphere close to, and at some distance from, the burning particles. The interior of the char particles is very porous, which may be a consequence of the generation and loss of volatile organic compounds coupled with internal oxidation. In order to determine whether these observations are peculiar to Missouri bituminous coal or are generic to bituminous coals themselves, we will extend these studies to a set of Alberta bituminous coal chars that were produced under controlled and well characterized conditions.

\section{References}

1. R. G. Herman, G. W. Simmons, D. A. Cole, V. Kuzmicz, and K. Klier, "Catalytic Action of Minerals in the Low Temperature Oxidation of Coal," Fuel 63, 673 (1984).

2. L. A. Harris, D. N. Braski, and C. S. Yust, Microstructural Science, Vol. 5,(Elsevier, New York, NY, 1976), p. 351.

3. R. M. Allen and J. B. Vander Sande, "Analysis of Sub-Micron Mineral Matter in Coal via Scanning Transmission Electron Microscopy," Fuel 63, 24 (1984).

4. R. J. Quann and A. F. Sarofim, "A Scanning Electron Microscopy Study of the Transformations of Organically Bound Metals During Lignite Combustion," Fuel 65, 40 (1986).

5. R. M. Allen and R. E. Mitchell, Proceedings of the International Conference on Coal Science (International Energy Agency, 1985), p. 401.

\section{Percolation Model for Simulation of Char Oxidation and Fragmentation Time-Histories ${ }^{\dagger}$}

\section{A. R. Kerstein, B. F. Edwards*}

A computational model has been developed to represent the evolution of char morphology during oxidation. The model is designed primarily to identify the effect of fragmentation on particle burning-rate time-histories. The

$†$ To appear in Chemical Engineering Science (1987).

- Presently affiliated with Department of Physics, West Virginia University at Morgantown. 
effort was motivated by recently reported ${ }^{1}$ measurements of single-particle burning-rate timehistories. Measured time-histories exhibited features which were interpreted as evidence of particle fragmentation.

The computations involve the simulated burning of char particles, based on a model of particle macrostructure consisting of randomly selected links of a cubic lattice. The twodimensional analog of this model is illustrated in Figure 1a.

\section{a}

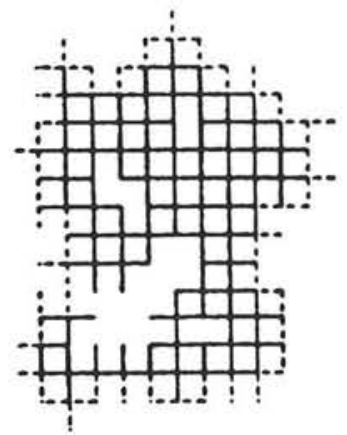

b

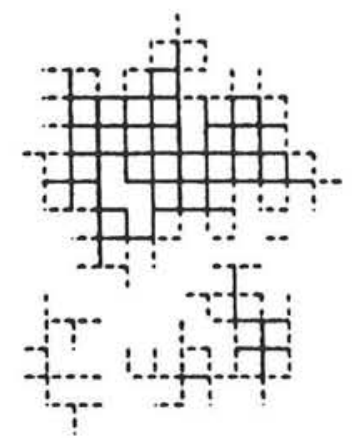

Figure 1. Two-dimensional example of particle macrostructure. Perimeter links (dashed line segments) are removed at a rate dependent on particle size, representing surface burnout. Link removal may convert interior links (solid line segments) into perimeter links. (a) Initial configuration. (b) Configuration after partial burnout, exhibiting four fragments.

Unbroken line segments represent solid material in the particle interior. Broken line segments represent solid material on the particle perimeter which is accessible to oxygen diffusing in from the ambiance and is therefore ignited. Upon ignition, a link is randomly assigned a burning time, determining the epoch at which the link is consumed, i.e., removed from the network. Link removal may result in interior links becoming part of the perimeter, as illustrated by comparison of the initial configuration, Figure 1a, and the configuration after partial burnout, Figure 1b.

Figure 1 also illustrates the fragmentation process, namely the separation of the initial configuration of interconnected links into several (in this case, four) distinct fragments.

Within this modeling framework, there are several mechanisms which influence the overall burning rate. Link removal tends to reduce the particle perimeter, hence reducing the number of burning links. However, occasionally the opening of an interior void due to link removal may cause a sudden increase in the number of burning links, hence increasing the burning rate. The burning rate of a particle is the product of the number of burning links times the burning rate per link. The latter quantity has an assumed power-law dependence on the total number of links in the particle. This represents the power-law mass dependence of the burning rate per unit surface, a characteristic feature of hightemperature (film-diffusion-limited) char oxidation. In the high-temperature oxidation regime, the burning rate is a negative power of mass, so fragmentation results in total burning (summed over fragments) at a higher rate than for the unfragmented particle.

For randomly generated initial configurations on a cubic lattice, simulated burning-rate time-histories were computed and the results were compared to experimental burning-rate time-histories measured by Sundback et al. ${ }^{1}$ Features of the measured histories, in particular, sharp increases in the burning rate (interpreted as fragmentation events), were reproduced quantitatively based on adjustment of two parameters in the model. These parameters are the number of links present initially, which was set to approximately 400 , and the exponent $\mathrm{b}$ in the $\mathrm{n}^{-b}$ dependence of the burning rate per link on the number $n$ of links in the particle. (b 
was set to 0.3.) A simulated burning-rate timehistory corresponding to these parameter values is shown in Figure 2.

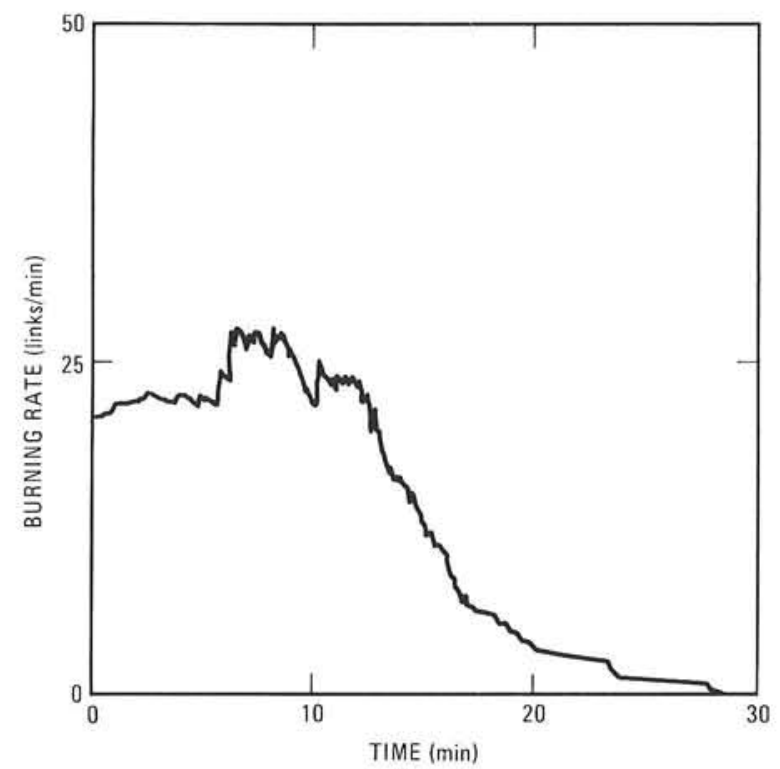

Figure 2. Simulated time-history of overall burning rate for a three-dimensional realization with 400 links initially.

For simulations at these parameter values, fragment number distributions were also computed at various conversion levels. At high conversion (73 percent), the number of distributions were found to exhibit $\mathrm{n}^{-1}$ functional dependence, consistent with the functional dependence of fragment number distributions for partially reacted char measured by Holve et al..$^{2,3}$ In the latter experiments, the $\mathrm{n}^{-1}$ functional dependence was exhibited by the feedstock as well as the partially reacted char. The invariance of the functional dependence during burnout was interpreted as indicative of the absence of fragmentation. However, the computed results indicate that the measured distributions are not inconsistent with particle-size evolution dominated by the fragmentation process. Additional measurements are therefore needed in order to unambiguously quantify the role of fragmentation in the generation of fine char particles, and ultimately, fine fly ash.

\section{References}

1. C. A. Sundback, J. M. Beer, and A. F. Sarofim, "Fragmentation Behavior of Single Coal Particles in a Fluidized Bed," Twentieth Symposium (International) on Combustion (The Combustion Institute, 1985), p. 1495.

2. D. J. Holve, "In situ Measurements of Flyash Formation from Pulverized Coal," Comb. Sci. Tech. 44, 269 (1985).

3. D. J. Holve, K. Gomi, and T. H. Fletcher, "Comparative Combustion Studies of Ultrafine Coal/Water Slurries," to appear in Comb. Sci. Tech. (1987). 
The enlarged photograph on the preceding page shows the combustion chamber of our gas-fired pulse combustor (see inset). This pulse combustor is a "Helmholtz" type, operating with fuel-air mixture and an external flapper valve. Pulse combustion represents an area of strong technology transfer at the Combustion Research Facility. The pulse combustion laboratory hosts several visitors from this country and abroad. The pulse combustion laboratory also interacts with other national laboratories, universities, and industry. This past year, for instance, John Dec, a $\mathrm{PhD}$ candidate from the University of Michigan, performed dissertation work in resonant heat transfer on this pulse combustor (see related article, page 8-16). 


\section{Section 10 \\ Technology Transfer}

Transferring technology to universities, industry, and other government laboratories is an important part of activities at the Combustion Research Facility. The majority of this transfer occurs through publications. However, the Facility also participates in other transfer activities such as an extensive visiting scientist program, which this fiscal year included more than fifty long-term visitors. This program encourages visiting scientists and engineers to take advantage of our unique resources, become familiar with our technology, and bring new information back to their home institutions.

The Facility also periodically offers short courses and workshops (no formal courses, however, were conducted this past year). These courses typically last two to three days and are intended to familiarize industry, government, and university attendees with the advanced technology developed here. Laboratory sessions use the Facility's experimental and computational facilities to offer a unique hands-on learning experience.

The technology developed at the Facility is also shared with industry, universities, and other government laboratories through several cooperative working groups. In these groups, progress in fundamental combustion science is exchanged among the various representatives. The fundamental technology can then be adapted by industry to applied research leading to improved combustion processes.

\section{Cooperative Working Groups}

\section{G. B. Drummond}

Technology transfer is effected not only through the visiting scientist program and short courses, but by participating in cooperative working groups with U.S. industry, universities, and other national laboratories. Five joint working groups are now active as shown in Table I.

On a global scale, the Combustion Research Facility is active in two programs of the International Energy Agency. In the internal combustion engine and furnace program, the Facility participates with representatives from Canada, Italy, Japan, Norway, Sweden, the United Kingdom, and West Germany in an exchange of research progress in the areas of advanced piston engine technology, in furnace and combustor technology, and in fundamental combustion studies. Some forty-nine projects are under way in this program. The second, a program in coal combustion sciences, involves the Facility and other U.S. researchers with representatives of Australia, Canada, and the Netherlands. Both of the programs are conducted at some of the most outstanding research institutions of the world. 
Table I

Active Working Groups Providing Technology Transfer

Topic:

Participants:

Direct-Injection Stratified-Charge

Combustion

Dilute Homogeneous-Charge

Combustion

Diesel Combustion

Engine Knock

Aerothermochemistry of Turbulent Combustion
General Motors, Princeton University, Lawrence Livermore National Laboratory, Los Alamos National Laboratory, Sandia National Laboratories

Ford, Science Applications, Inc., S-Cubed, Imperial College, Massachusetts Institute of Technology, University of California, Berkeley, University of Wisconsin, Integral Technologies, Inc., Pennsylvania State University, Sandia National Laboratories

Cummins, Exxon, Navistar, S-Cubed, TRW, Purdue University, Los Alamos National Laboratory, Sandia National Laboratories

General Motors, Drexel University, Princeton University, UNOCAL, Lawrence Livermore National Laboratory

Sandia National Laboratories

General Electric Company, California Inst. of Technology, Cornell University, University of California, Davis, University of California, San Diego, University of Sydney (Australia), Sandia National Laboratories

\section{Visiting Scientist Program}

\section{G. B. Drummond}

The Combustion Research Facility is a national facility for studying fundamental problems in energy-related combustion. An important element of the Facility charter provides for promoting the development and application of new combustion research tools (laser diagnostics, computational tools, etc.) with the active involvement of the combustion research community at large. The Visiting Scientist Program enables scientists from universities, industry, and other national laboratories to join in research at the Combustion Research Facility and thus take advantage of the unique equipment and resources here. Research support provided to visiting scientists includes technicians and 
engineering staff dedicated to the project, computer time, shop work, and other purchases necessary to support the project. The visitor generally provides his or her own salary, travel, and living expenses. So long as results of a visitor's research are available in the open literature, there is no cost imposed for use of the Facility. Support for proprietary research is available, but only on a full cost-recovery basis.

Visitors usually elect to work primarily on collaborative projects or to use some preexisting experimental setup to do their own research. However, they are free to execute their own research projects.

Since the Facility opened early in 1981 we have had almost 4300 technical visits (see Figure 1). Over 4100 of these visits were brief, typically for one or two days. These short-term visitors were here either to learn something about the technical scope of the CRF program, or to discuss specific combustion research problems of their own with the Facility staff. There were about 150 non-programmatic visitors this year, including school classes, a high school honors chemistry class, and the Department of Comerce's 1986 Commerce and Science Technology Fellows.

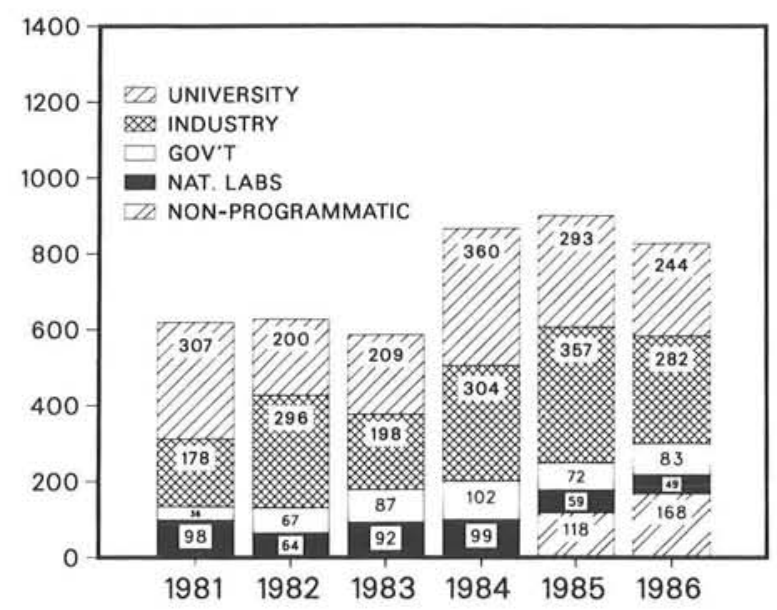

Figure 1. Number of visitors to Facility since 1981.
The list of visiting scientists who have spent two weeks or more at the Facility now exceeds 240, as shown in Figure 2. Those visiting in FY86 (listed in Table I) have engaged in a wide variety of research projects, and have included representatives from universities, industry, other national laboratories, and governmental facilities. About $22 \%$ of those listed are from foreign institutions. To encourage graduate students with an interest in combustion science, we have since 1982 hosted a limited number of graduate students here doing thesis work under the guidance of a faculty advisor and a mentor assigned from the Sandia staff.

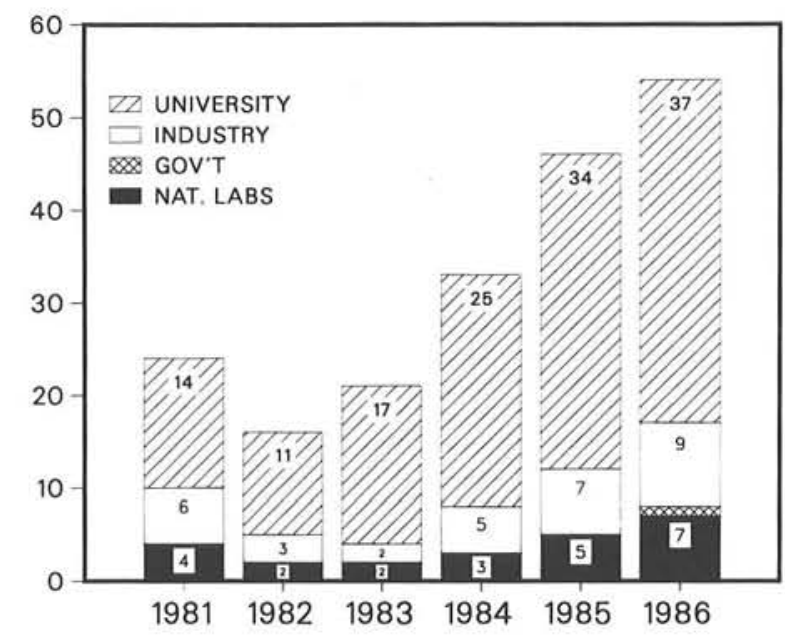

Figure 2. Number of long term visiting scientists.

Another kind of long-term visitor comes to the Facility through the Sandia Research Associate (post-doctoral) Program. Sandia Laboratories accepts a small number of postdoctoral candidates in its research programs each year for a one-year appointment (renewable to two years). The Combustion Research Facility draws physicists, chemists, mathematicians, and engineers who wish to work in the field of combustion research. Candidates are expected to move to industry and universities when their term of appointment ends. In this way, the Program provides us another means of technology transfer outside the Facility. 


\section{Table I}

Combustion Research Facility Visiting Scientists

October 1, 1985-September 30, 1986

\begin{tabular}{|c|c|c|c|}
\hline Visitor & Affiliation & Term of Stay & Collaborative Project \\
\hline R. Axelbaum* & UC Davis & Oct 1-Sept 30 & Thesis support \\
\hline $\begin{array}{l}\text { L. Baxter* } \\
\text { T. Bowman }\end{array}$ & $\begin{array}{l}\text { Brigham Young Univ. } \\
\text { Stanford }\end{array}$ & $\begin{array}{l}\text { Sept 1-Sept } 30 \\
15 \% \text { of year }\end{array}$ & $\begin{array}{l}\text { Coal devolitization studies } \\
\text { Soot formation }\end{array}$ \\
\hline M. Branch & Univ. of Colorado & June 10 -June 21 & Combustion chemistry \\
\hline N. Brown & UC Berkeley & $10 \%$ of year & Theoretical chemistry \\
\hline J. Buck* & UC Santa Cruz & Oct $1-$ Dec 30 & Molecular beam experiments \\
\hline N. Cernasky & Drexel Univ. & Mar 10-Mar 21 & Engine knock studies \\
\hline A. Chambers & $\begin{array}{l}\text { Alberta Research } \\
\quad \text { Council }\end{array}$ & June 16-Aug 15 & Char burning rate studies \\
\hline T. Cool & Cornell Univ. & Mar 31-Apr 30 & REMPI ionization detection \\
\hline J. Dec* & $\begin{array}{l}\text { Univ. of Michigan } \\
\text { (GRI Fellow) }\end{array}$ & Oct 1 -Sept 30 & Pulse combustion studies \\
\hline A Droege** & Univ. of Oregon & Oct 1 -Sept 30 & Combustion chemistry \\
\hline D. Dudek*. & MIT & June 15-Aug 19 & Single particle levitation \\
\hline D. Dunn-Rankin & UC Berkeley & Nov 1-Sept 30 & CARS coal combustion \\
\hline H. Dwyer & UC Davis & $20 \%$ of year & Turbulence modeling \\
\hline B. Edwards** & Stanford & Oct 1 -Aug 8 & Percolation analysis \\
\hline C. Ferguson & Purdue University & Oct 1-May 23 & Diesel spray studies \\
\hline D. Foster & Univ. of Wisconsin & Sept 2-Sept 30 & LDV engine diagnostics \\
\hline P. Glarborg & Tech. Inst. Denmark & Jan 20-Feb 10 & Combustion chemistry \\
\hline V.-P. Heiskanen & $\begin{array}{l}\text { Tech Research Cen- } \\
\text { ter Finland }\end{array}$ & Feb 1-May 30 & Coal combustion study \\
\hline J. Hoornstra & Netherlands Energy & Oct 1-June 6 & CARS coal combustion \\
\hline M. Jarzembski* & $\begin{array}{l}\text { New Mexico State } \\
\text { University }\end{array}$ & June $3-$ July 2 & Spark spectroscopy \\
\hline N. Lilliheie & Tech. Inst. Norway & Sept 1-Sept 30 & Combustion chemistry \\
\hline B. Magnussen & Tech. Inst. Norway & Oct 1-Dec 17 & Turbulent flames \\
\hline P. Magre & ONERA & Oct 1-Aug 15 & Turbulent combustion studies \\
\hline M. Maris & $\begin{array}{l}\text { Computer Genetics } \\
\text { (DOE Ind. Fellow) }\end{array}$ & Sept 8-Sept 30 & CARS code \\
\hline D. Meiron & Cal Tech & $\begin{array}{l}\text { June 9-July } 30 \\
\text { Sept } 1 \text {-Sept } 30\end{array}$ & Turbulence modeling \\
\hline M. Namazian & Altex & Oct 1 -Sept 30 & $\begin{array}{l}\text { Two-dimensional shear } \\
\text { layer studies }\end{array}$ \\
\hline R. Pandey & Jackson State & May 30-Aug 9 & Percolation analysis \\
\hline C. Parker & Jackson State & $\begin{array}{l}\text { Feb 1-Aug } 15 \\
\text { (cont.) }\end{array}$ & Auto ignition chemistry \\
\hline
\end{tabular}


Table I (cont.)

Combustion Research Facility Visiting Scientists

October 1, 1985-September 30, 1986

\begin{tabular}{|c|c|c|c|}
\hline Visitor & Affiliation & Term of Stay & Collaborative Project \\
\hline D. Parker & UC Santa Cruz & Oct 1 -Jan 15 & Molecular beam experiments \\
\hline N. Peters & Univ. of Aachen & Mar 11-Mar 28 & Turbulence modeling \\
\hline P. Petropoulos & $\begin{array}{l}\text { UC Berkeley } \\
\text { (Altex) }\end{array}$ & July 1 -Sept 30 & $\begin{array}{l}\text { Two-dimensional shear } \\
\text { layer modeling }\end{array}$ \\
\hline L. Radziemski & $\begin{array}{l}\text { New Mexico State } \\
\text { University }\end{array}$ & June 22-July 30 & Spark spectroscopy \\
\hline G. Rosasco & $\begin{array}{l}\text { National Bureau } \\
\text { of Standards }\end{array}$ & Mar 1-Mar 30 & IRS of combustion \\
\hline R. Sawyer & UC Berkeley & May 8-July 31 & Turbulent combustion imaging \\
\hline O. Smith & UCLA & July 1-Aug 30 & Combustion chemistry \\
\hline T. Spliganin** & Wesleyan Univ. & Oct 1 -Sept 30 & Molecular dynamics studies \\
\hline R. Strickland & Univ. of Arizona & July 1-July 30 & Hybrid image processing \\
\hline L. Talbott* & UC Berkeley & July 1-July 30 & $\begin{array}{c}\text { Two-dimensional imaging } \\
\text { of } \mathrm{CH} / \mathrm{OH} \text { radicals }\end{array}$ \\
\hline J. Wallace & Univ. of Toronto & July 1-Sept 30 & Diesel alternative fuels study \\
\hline B. Waters* & Purdue Univ. & July 1 -Sept 26 & Char reactivity measurements \\
\hline F. Weinberg & Imperial College & Apr 7-Apr 18 & Plasma-torch ignition studies \\
\hline \multicolumn{4}{|c|}{$\begin{array}{l}\text { * Graduate Student } \\
\text { ** Sandia Post-doctoral } \\
\text { *** Undergraduate Student }\end{array}$} \\
\hline
\end{tabular}


Appendix 


\section{Publications 1986}

\section{Refereed Journal Articles}

G. S. Agarwal and R. L. Farrow, "Theoretical Modeling of Two-Color Coherent Anti-Stokes Raman Spectroscopy Spectra Measured with a Frequency-Doubled, Multimode Pump Laser," J. Opt. Soc. Am. B 3, 1596 (1986).

R. M. Allen, R. W. Carling, and J. B. VanderSande, "Microstructural Changes in Coal During Low Temperature Ashing," Fuel 65, 321 (1986).

R. C. Armstrong and S. B. Margolis, "Nonhomogeneous Propellants as Random Media," Comb. Sci. Tech. 52, 59 (1986).

M. R. Booty, S. B. Margolis, and B. J. Matkowsky, "Interaction of Pulsating and Spinning Waves in Condensed Phase Combustion," SIAM J. Appl. Math. 46, 801 (1986).

R. J. Cattolica and S. R. Vosen, "Two-Dimensional Fluorescence Imaging of a Flame-Vortex Interaction," Comb. Sci. Tech. 48, 77 (1986).

N. P. Cernansky, R. M. Green, W. J. Pitz, and C. K. Westbrook, "Chemistry of Fuel Oxidation Preceding End-Gas Autoignition," Comb. Sci. Tech. 50, 3 (1986).

D. W. Chandler and R. L. Farrow, "Measurements of Rotational Energy Transfer Rates for HD (v=1) in Collisions with Thermal HD," J. Chem. Phys. 85, 810 (1986).

D. W. Chandler and L. R. Thorne, "Measured Radiative Lifetimes for $\mathrm{H}_{2}$ and HD in the E,F ${ }^{1} \Sigma_{g}^{+}$Electronic State," J. Chem. Phys. 85, 1733 (1986).

M. E. Coltrin, R. J. Kee, and J. A. Miller, "A Mathematical Model of Silicon Chemical Vapor Deposition: Further Refinements and the Effects of Thermal Diffusion," J. Electrochem. Soc. 133, 1206 (1986).

D. J. DeFrees, J. S. Binkley, M. J. Frisch, and A. D. McLean, "In N-Protonated Hydrogen Isocyanide, $\mathrm{H}_{2} \mathrm{NC}^{+}$, an Observable Interstellar Species?" J. Chem. Phys. 85, 5194 (1986).

A. T. Droege and F. P. Tully, "Hydrogen-Atom Abstraction from Alkanes by OH. III. Propane," J. Phys. Chem. 90, 1949 (1986).

A. T. Droege and F. P. Tully, "Hydrogen-Atom Abstraction from Alkanes by OH. V. n-Butane," J. Phys. Chem. 90, 5937 (1986).

H. A. Dwyer, A. Lutz, and R. J. Kee, "Numerical Calculation of the Interaction of Pressure Waves and Flames," Reacting Flows, G. S. S. Ludford, ed., Lecture Notes in Applied Mathematics, AMS Vol. 24 (1986).

R. L. Farrow and R. P. Lucht, "High-Resolution Measurements of Saturated Coherent AntiStokes Raman Spectroscopy Lineshapes," Opt. Lett. 11, 374 (1986).

W. L. Flower, "The Effect of Elevated Pressure on the Rate of Soot Production in Laminar Diffusion Flames," Comb. Sci. Tech. 48, 31 (1986). 
W. L. Flower, "Light-Scattering Measurements of Soot Particles in Flames," SPIE 644, 28 (1986).

P. Glarborg, J. A. Miller, and R. J. Kee, "Kinetic Modeling and Sensitivity Analysis of Nitrogen Oxide Formation in Well-Stirred Reactors," Comb. Flame 65, 177 (1986).

J. E. M. Goldsmith, "Photochemical Effects in 205-nm, Two-Photon-Excited Fluorescence Detection of Atomic Hydrogen in Flames," Opt. Lett. 11, 416 (1986).

J. E. M. Goldsmith and R. J. M. Anderson, "Laser-Induced Fluorescence Spectroscopy and Imaging of Molecular Oxygen in Flames," Opt. Lett. 11, 67 (1986).

J. E. M. Goldsmith and N. M. Laurendeau, "Two-Photon-Excited Fluorescence Measurements of $\mathrm{OH}$ Concentration in a Hydrogen-Oxygen Flame," Appl. Opt. 25, 276 (1986).

J. C. Hamilton, J. C. Farmer, and R. J. Anderson, "In Situ Raman Spectroscopy of Anodic Films Formed on Copper and Silver in Sodium Hydroxide Solution," J. Electrochem. Soc. 133, 739 (1986).

P. Ho, M. E. Coltrin, J. S. Binkley, and C. F. Melius, "A Theoretical Study of the Heats of Formation of $\mathrm{Si}_{2} \mathrm{H}_{\mathrm{n}}(\mathrm{n}=0-6)$ Compounds and Trisilane," J. Phys. Chem. 90, 3399 (1986).

D. J. Holve, "In Situ Measurements of Fly Ash Formation from Pulverized Coal," Comb. Sci. Tech. 44, 269 (1986).

S. C. Johnston, R. W. Dibble, R. W. Schefer, W. T. Ashurst, and W. Kollmann, "Laser Measurements and Stochastic Simulations of Turbulent Reacting Flows," AIAA J. 24, 918 (1986).

A. R. Kerstein, "Computational Study of Propagating Fronts in a Lattice-Gas Model," J. Stat. Phys. 45, 921 (1986).

A. R. Kerstein and A. L. R. Bug, "Scaling Theory of Pore Growth in a Reactive Solid," Phys. Rev. B 34, 1754 (1986).

A. R. Kerstein and B. F. Edwards, "Crossover from Contact Propagation to Chemical Propagation in First-Passage Percolation," Phys. Rev. B 33, 3353 (1986).

A. M. F. Lau, "AC-Stark Splitting in the Multiphoton Excitation of Atomic Hydrogen in Flames: Abnormal Peak Asymmetry due to Pressure Broadening," Phys. Rev. A 33, 3602 (1986).

R. P. Lucht, R. L. Farrow, and R. E. Palmer, "Acetylene Measurements in Flames by Coherent Anti-Raman Scattering," Comb. Sci. Tech. 45, 261 (1986).

R. P. Lucht, D. W. Sweeney, and N. M. Laurendeau, "Time-Resolved Fluorescence Investigation of Rotational Transfer in $\mathrm{A}^{2} \Sigma^{+}(\mathrm{v}=0) \mathrm{OH}, "$ Appl. Opt. 25, 4086 (1986).

S. B. Margolis and R. C. Armstrong, "Two Asymptotic Models for Solid Propellant Combustion," Comb. Sci. Tech. 47, 1 (1986).

J. A. Miller, "Non-Statistical Effects and Detailed Balance in Quasi-Classical Trajectory Calculations of the Thermal Rate Coefficient for $\mathrm{O}+\mathrm{OH} \rightarrow \mathrm{O}_{2}+\mathrm{H}$," J. Chem. Phys. 84, 6170 (1986). 
J. A. Miller and D. W. Chandler, "A Theoretical Analysis of the Overtone-Induced Isomerization of Methyl Isocyanide," J. Chem. Phys. 85, 4502 (1986).

J. A. Miller, C. Parrish, and N. J. Brown, "A Statistical-Theoretical Investigation of the Thermal Rate Coefficient and Branching Ratio for the Reaction $\mathrm{O}+\mathrm{HCN} \rightarrow$ Products," J. Phys. Chem. 90, 3339 (1986).

S. P. Musarra, T. H. Fletcher, S. Niksa, and H. A. Dwyer, "Heat and Mass Transfer in the Vicinity of a Devolatizing Coal Particle," Comb. Sci. Tech. 45, 289 (1986).

S. J. Niksa and A. R. Kerstein, "The Distributed-Energy Chain Model for Rapid Coal Devolatilization Kinetics: Part I: Formulation," Comb. Flame 66, 95 (1986).

D. W. Noid, S. K. Knudson, M. L. Koszykowski, and R. J. Renka, "Semiclassical Calculation of Molecular Vibrations from ab initio Potentials Using Splines: Ozone," J. Phys. Chem. 90, 6135 (1986).

D. K. Ottesen and J. C. F. Wang, "Non-intrusive Optical Techniques for the Study of Pulverized Coal Particles During Devolatilization and Combustion," ACS Div. of Fuel Chem. Preprints 31, 136 (1986).

R. A. Perry and D. L. Siebers, "Rapid Reduction of Nitrogen Oxides in Exhaust Gas Streams," Nature 324, 657 (1986).

L. A. Rahn and D. A. Greenhalgh, "High-Resolution Inverse Raman Spectroscopy of the $\nu_{1}$ Band of Water Vapor," J. Mol. Spectrosc. 119, 11 (1986).

L. A. Rahn and R. E. Palmer, "Studies of Nitrogen Self-Broadening at High Temperature with Inverse Raman Spectroscopy," J. Opt. Soc. Am. B 3, 1164 (1986).

C. M. Rohlfing and R. L. Martin, "A Theoretical Study of the Isovalent Diatomics $\mathrm{C}_{2}, \mathrm{Si}$, and SiC," J. Phys. Chem. 90, 2043 (1986).

G. F. Schils and D. W. Sweeney, "Iterative Technique for the Synthesis of Optical-Correlation Filters," J. Opt. Soc. Am. A 3, 1433 (1986).

R. L. Schmitt and L. A. Rahn, "Diode-Laser-Pumped Nd:YAG Laser Injection Seeding System," Appl. Opt. 25, 629 (1986).

J. A. Sell and R. J. Cattolica, "Linear Imaging of Gas Velocity Using the Photothermal Deflection Effect," Appl. Opt. 25, 1420 (1986).

D. L. Siebers and T. M. Dyer, "The Autoignition and Combustion of Coal-Water Slurry Under Simulated Diesel Engine Conditions," ASME J. of Engineering for Gas Turbines and Power 108, 654 (1986).

O. I. Smith and D. W. Chandler, "An Experimental Study of Probe Distortions to the Structure of One-Dimensional Flames," Comb. Flame 63, 19 (1986).

D. L. Snavely, R. N. Zare, J. A. Miller, and D. W. Chandler, "Methyl Isocyanide Isomerization Kinetics: Determination of Collisional Deactivation Parameters Following C-H Overtone Excitation," J. Phys. Chem. 90, 3544 (1986). 
T. A. Spiglanin, R. A. Perry, and D. W. Chandler, "Photodissociation of Studies of HNCO: Heat of Formation and Product Branching Ratios," J. Phys. Chem. 90, 6184 (1986).

T. Suzuki, L. D. Smoot, T. H. Fletcher, and P. J. Smith, "Prediction of High-Intensity Pulverized Coal Combustion," Comb. Sci. Tech. 45, 167 (1986).

R. (F. P.) Trebino, C. E. Barker, and A. E. Siegman, "Tunable-Laser-Induced Gratings for the Measurement of Ultrafast Phenomena," IEEE J. Quantum Electron. QE-22, 1413 (1986).

R. (F. P.) Trebino, E. K. Gustafson, and A. E. Siegman, "Fourth-Order Partial-Coherence Effects in the Formation of Integrated-Intensity Gratings with Pulsed Light Sources," J. Opt. Soc. Am. B S, 1295 (1986).

F. P. Tully, A. T. Droege, M. L. Koszykowski, and C. F. Melius, "Hydrogen-Atom Abstraction from Alkanes by OH. II. Ethane," J. Phys. Chem. 90, 691 (1986).

F. P. Tully, J. E. M. Goldsmith, and A. T. Droege, "Hydrogen-Atom Abstraction from Alkanes by OH. IV. Isobutane," J. Phys. Chem. 90, 5932 (1986).

J. V. Volponi, W. J. McLean, R. M. Fristrom, Z. A. Munir, "Determination of H, OH, and O Species Concentrations by Deuterium Scavenging," Comb. Flame 65, 243 (1986).

J. C. F. Wang and K. R. Hencken, "In Situ Particle Size Measurements Using a Two-Color Laser Scattering Technique," Appl. Opt. 25, 653 (1986).

P. O. Witze and T. M. Dyer, "Laser Measurement Techniques Applied to Turbulent Combustion in Piston Engines," Experiments in Fluids, Vol. 4, 81 (1986).

\section{Other Publications}

R. M. Allen and R. E. Mitchell, Proceedings of the International Conference on Coal Science (International Energy Agency, 1985), p. 401.

R. J. Anderson, J. C. Hamilton, and G. W. Foltz, "Optical Second Harmonic Generation Studies of Surface Adsorption and Segregation on Fe-18, Cr-3, Mo(110)," to appear in Opt. Soc. Amer. (1987).

R. C. Armstrong, "Theory for Complex Diffusion Limited Reactions," submitted to J. Chem. Phys. (1986).

R. C. Armstrong and S. B. Margolis, "Modification of Solid Propellant Deflagration Rates due to Random Nonhomogeneities," Twenty-first Symposium (International) on Combustion (The Combustion Institute, 1986), in press.

W. T. Ashurst, "Vortex Simulation of Unsteady Wrinkled Laminar Flames," to appear in Comb. Sci. Tech. (1987).

W. T. Ashurst, A. R. Kerstein, R. M. Kerr, and C. H. Gibson, "Alignment of Vorticity and Scalar Gradient with Strain Rate in Simulated Navier-Stokes Turbulence," accepted by Phys. Fluids (1986). 
W. T. Ashurst and D. I. Meiron, "Numerical Study of Vortex Reconnection," Sandia Report SAND86-8880 (1986); also, to appear in Phys. Rev. Lett. (1987).

W. T. Ashurst, N. Peters, and M. D. Smooke, "Numerical Simulation of Turbulent Flame Structure With Non-Unity Lewis Number," Sandia Report SAND86-8838 (1986); also, to appear in Comb. Sci. Tech. (1987).

R. L. Axelbaum, C. K. Law, and W. L. Flower, "Effects of Diffusional Stratification and Oxygen Addition on Soot Formation in a Counterflow Diffusion Flame," submitted to Comb. Flame (1986).

R. Behrens, Jr., "New Simultaneous Thermogravimetry and Modulated Molecular Beam Mass Spectrometry Apparatus for Quantitative Thermal Decomposition Studies," to appear in Rev. Sci. Inst. (1987).

M. R. Booty, S. B. Margolis, and B. J. Matkowsky, "Interaction of Pulsating and Spinning Waves in Nonadiabatic Flame Propagation," to appear in SIAM J. Appl. Math. (1987).

R. J. Cattolica, "Visualization of Flame Propagation by Laser-Fluorescence Imaging of Nitrogen Dioxide," to appear in Comb. Sci. Tech. (1987).

R. J. Cattolica, "Combustion-Torch Ignition: Fluorescence Imaging of $\mathrm{NO}_{2}$," Twenty-first Symposium (International) on Combustion (The Combustion Institute, 1986), in press.

R. J. Cattolica and N. Klein, "Plasma-Torch Ignition of Liquid Propellant," Sandia Report SAND868647 (1986); also, submitted to Comb. Sci. Tech. (1986).

R. J. Cattolica and S. R. Vosen, "Combustion-Torch Ignition: Fluorescence Imaging of OH Concentration," to appear in Comb. Flame (1987).

N. P. Cernansky, R. M. Green, W. J. Pitz, and C. K. Westbrook, "Chemistry of Fuel Oxidation Preceding End-Gas Autoignition," accepted by Comb. Sci. Tech. (1986).

D. W. Chandler, D. H. Parker, and J. O. Buck, "Double-Resonance Laser-Ionization Spectroscopy of Molecular Hydrogen in the Region of the Second Dissociation Limit," submitted to J. Phys. Chem. (1986).

J.-Y. Chen, "Second-Order Conditional Modeling of Turbulent Nonpremixed Flames with a Composite PDF," Spring Meeting of Western States Section (The Combustion Institute, 1986); also, submitted to Comb. Flame (1986).

J.-Y. Chen, F. C. Gouldin, and J. L. Lumley, "Second-Order Modeling of a Turbulent Nonpremixed $\mathrm{H}_{2}$-Air Jet Flame with Intermittency and Conditional Averaging," accepted by Comb. Sci. Tech. (1986).

J.-Y. Chen, J. L. Lumley, and F. C. Gouldin, "Modeling of Wrinkled Laminar Flames with Intermittency and Conditional Statistics," Twenty-first Symposium (International) on Combustion (The Combustion Institute, 1986), in press.

J.-Y. Chen, T.-S. Shih, and J. L. Lumley, "Second Order Modeling of Passive Scalar in a Turbulent Shear Flow," submitted to J. Fluid Mech. (1986). 
J. E. Dec and J. O. Keller, "High Speed Thermometry Using Two-Line Atomic Fluorescence," Sandia Report SAND86-8622 (1986); also, Twenty-first Symposium (International) on Combustion (The Combustion Institute, 1986), in press.

J. E. Dec and J. O. Keller, "The Effect of Fuel Burn Rate on Pulse Combustor Tail Pipe Velocities," Sandia Report SAND86-8757 (1986); also, Proceedings of the 1986 International Gas Research Conference (1986), in press.

R. W. Dibble, M. B. Long, and A. R. Masri, "Two-Dimensional Imaging of $\mathrm{C}_{2}$ in Turbulent Nonpremixed Jet Flames," Progress in Astronautics and Aeronautics, Vol. 105, edited by J. R. Bowen et al (AIAA, New York, NY, 1986), p. 99.

R. W. Dibble, V. Hartmann, R. W. Schefer, and W. Kollman, "Conditional Sampling of Velocity and Scalars in Turbulent Flames Using Simultaneous LDV-Raman Scattering," accepted by J. of Experiments in Fluids (1986).

R. W. Dibble, W. Kollmann, M. Farschi, and R. W. Schefer, "Second-Order Closure of Turbulent Nonpremixed Flames: Scalar Dissipation and Heat Release Effects," Twenty-first Symposium (International) on Combustion (The Combustion Institute, 1986), in press.

R. W. Dibble, P. Magre, R. W. Schefer, J.-Y. Chen, V. Hartmann, and W. Kollman, "Simultaneous Mixture Fraction and Velocity Measurements in a Turbulent Nonpremixed Flame," submitted to Comb. Sci. Tech. (1986).

R. W. Dibble, R. W. Schefer, J.-Y. Chen, V. Hartmann, and W. Kollman, "Velocity and Density Measurements in a Turbulent Nonpremixed Flame with Comparison to a Numerical Model," submitted to Comb. Flame (1986).

A. T. Droege and F. P. Tully, "Hydrogen-Atom Abstraction from Alkanes by OH. VI. Cyclopentane and Cyclohexane," submitted to J. Phys. Chem. (1986).

D. R. Dudek, T. H. Fletcher, J. P. Longwell, and A. F. Sarofim, "Natural Convection-Induced Drag Forces on Spheres at Low Grashof Numbers: Comparison of Theory with Experiment," submitted to Internat. J. Heat and Mass Transfer (1986).

D. Dunn-Rankin, P. K. Barr, and R. F. Sawyer, "Simulation of 'Tulip' Flame Formation in a Closed Vessel," Twenty-first Symposium (International) on Combustion (The Combustion Institute, 1986), in press.

D. Dunn-Rankin, J. Hoornstra, T. H. Fletcher, and D. J. Holve, "Agglomeration During Combustion of Coal/Water Slurries as a Function of Coal Rank," accepted by Fuel (1986).

D. Dunn-Rankin, J. Hoornstra, and D. J. Holve, "Coal Rank Influences on Particle-Size and Particle-Volume Distributions During Coal/Water Slurry Combustion," Sandia Report SAND868769 (1986); also, Proceedings of the Eighth International Symposium on Coal Slurry Fuels Preparation and Utilization (1986), in press.

D. Dunn-Rankin and A. R. Kerstein, "Numerical Simulation of Particle-Size Distribution Evolution During Pulverized Char Combustion," Sandia Report SAND86-8944 (1986); also, accepted by Comb. Flame (1986); also, presented at the Western States Section (The Combustion Institute, 1986). 
H. A. Dwyer, T. T. Bramlette, J. O. Keller, and B. R. Sanders, "A Numerical Model of a Pulse Combustor," Progress in Astronautics and Aeronautics, Vol. 105, edited by J. R. Bowen et al (AIAA, New York, NY, 1986).

H. A. Dwyer and B. R. Sanders, "A Detailed Study of Burning Fuel Droplets," Twenty-first Symposium (International) on Combustion (The Combustion Institute, 1986), in press.

R. L. Farrow, "High-Resolution CARS Measurements in a Flame," Twenty-first Symposium (International) on Combustion (The Combustion Institute, 1986), in press.

R. L. Farrow, "Accurate Measurements of Combustion Species Concentrations Using CARS," Advanced Instrumentation for Aero Engine Components, AGARD Conference Preprint No. 399 (AGARD, Neuilly sur Seine, France, 1986), p. 3-1.

R. L. Farrow and R. P. Lucht, "High-Resolution CARS for Combustion Diagnostics," Proceedings of the Tenth International Conference on Raman Spectroscopy, edited by W. L. Peticolas and B. Hudson (University of Oregon Press, Eugene, OR, 1986), p. 15-27.

R. L. Farrow, R. P. Lucht, and L. A. Rahn, "Measurements of the Nonresonant Susceptibilities of Gases Using Coherent Anti-Stokes Raman Spectroscopy," submitted to JOSA B (1986).

M. Farschi, W. Kollmann, R. W. Dibble, and R. W. Schefer, "Second-Order Closure for Turbulent Nonpremixed Flames," Turbulent Shear Flows V, edited by J. Whitelaw, B. Launder, F. Durst, and F. W. Schmidt (Springer-Verlag, New York, NY, 1986).

P. M. Fauchet, W. L. Nighan, Jr., and R. (F. P.) Trebino, "Characterization of Ultrashort Laser Pulses by the Method of Self-Diffraction," Advances in Laser Science-I, edited by W. C. Stwalley and M. Lapp, (AIP, New York, NY, 1986), p. 588.

C. R. Ferguson, R. M. Green, and R. P. Lucht, "Unburned Gas Temperatures in an Internal Combustion Engine: II. Heat Release Computations," Sandia Report SAND86-8605 (1986); also, submitted to Comb. Sci. Tech. (1986).

W. L. Flower and C. T. Bowman, "Soot Production in Axisymmetric Laminar Diffusion Flames at Pressures From One to Ten Atmospheres," Twenty-first Symposium (International) on Combustion (The Combustion Institute, 1986), in press.

W. F. Flower and C. T. Bowman, "Observations on the Soot Formation Mechanism in Laminar Ethylene-Air Diffusion Flames at One and Two Atmospheres," to appear in Comb. Sci. Tech. (1987).

W. L. Flower and A. J. Hurd, "In Situ Measurement of Flame-Formed Silica Particles Using Dynamic Light Scattering," submitted to App. Opt. (1986).

G. W. Foltz and C. F. Melius, "Studies of Isotopic Exchange Between Gaseous Hydrogen and Palladium Hydride Powder," submitted to J. Catal. (1986).

C. H. Gibson, W. T. Ashurst, and A. R. Kerstein, "Mixing of Strongly Diffusive Passive Scalars Like Temperature by Turbulence," submitted to J. Fluid Mech. (1986). 
J. E. M. Goldsmith, "Multiphoton Excitation Techniques for Combustion Diagnostics," Advances in Laser Science-I, edited by W. C. Stwalley and M. Lapp, (AIP, New York, NY, 1986), p. 279.

F. C. Gouldin, R. W. Schefer, S. C. Johnston, and W. Kollmann, "Nonreacting Turbulent Mixing Flows," submitted to Prog. Energy Comb. Sci. (1986).

F. J. Grcar, R. J. Kee, M. D. Smooke, and J. A. Miller, "A Hybrid Newton/Time Integration Procedure for the Solution of Steady, Laminar, One-Dimensional, Premixed Flames," Twentyfirst Symposium (International) on Combustion (The Combustion Institute, 1986), in press.

J. C. Hamilton, "In Situ Spectroscopy of Pyrite Oxidation and Resulfidation," Electrochemical Society, Vol. 86-1 (Extended Abstracts of the Spring Meeting, 1986).

J. C. Hamilton, R. J. Anderson, K. F. McCarty, and A. S. Nagelberg, "Reaction Monitoring on Metal and Ceramic Surfaces Using Laser-Light Scattering," SPIE Proceedings 644 Remote Sensing, 48 (1986).

J. C. Hamilton, K. F. McCarty, A. S. Nagelberg, "In Situ Raman Spectroscopy of High-Temperature Pyrite Reactions Related to Deposit Formation from Coal," Proceedings of the Symposium of Fundamental Aspects of High Temperature Corrosion II (Electrochemical Society, 1986), in press.

S. J. Harris, A. M. Weiner, R. J. Blint, and J. E. M. Goldsmith, "Concentration Profiles in Rich and Sooting Ethylene Flames," Twenty-first Symposium (International) on Combustion (The Combustion Institute, 1986), in press.

D. J. Holve, K. Gomi, and T. H. Fletcher, "Comparative Combustion Studies of Ultrafine Coal/Water Slurries," to appear in Comb. Sci. Tech. (1987).

D. J. Holve and P. L. Meyer, "Coal/Water Slurries: Fuel Preparation Effects on Atomization and Combustion," accepted by Comb. Sci. Tech. (1986).

J. Hruby, R. R. Steeper, G. Evans, and C. Crow, "An Experimental and Numerical Study of Flow and Convective Heat Transfer in a Freely Falling Curtain of Particles," accepted by J. Fluids Mech. (1986).

A. J. Hurd and W. L. Flower, "In Situ Study of Fractal Silica Aggregates in a Flame," submitted to J. Colloid and Interface Sci. (1986).

J. O. Keller and C. K. Westbrook, "Response of a Pulse Combustor to Changes in Fuel Composition," Sandia Report SAND86-863 (1986); also, Twenty-first Symposium (International) on Combustion (The Combustion Institute, 1986), in press.

A. R. Kerstein, "Percolation Model of Polydisperse Composite Solid Propellant Combustion," submitted to Comb. Flame (1986).

A. R. Kerstein and B. F. Edwards, "Percolation Model for Simulation of Char Oxidation and Fragmentation Time-Histories," accepted by Chem. Engineering Sci. (1986).

M. L. Koszykowski, L. A. Rahn, and R. E. Palmer, "Studies of High-Resolution Inverse Raman Spectroscopy Lineshapes for $\mathrm{N}_{2}$," Proceedings of the Eighth International Conference on Lineshapes, (Walter de Gruyter, Berlin, 1986). 
N. M. Laurendeau and J. E. M. Goldsmith, "Comparison of Laser-Induced Fluorescence Methods for Measurement of Hydroxyl Concentration in Flames," submitted to Appl. Opt. (1986).

M. B. Long, R. L. Schmitt, and R. W. Dibble, "Two-Dimensional Imaging of Fluorescence from CH Radical in Turbulent Nonpremixed Flames," submitted to Opt. Lett. (1986).

R. P. Lucht, "Three-Laser Coherent Anti-Stokes Raman Scattering Measurements of Two Species," accepted by Opt. Lett. (1986).

R. P. Lucht, R. M. Green, R. E. Palmer, R. E. Teets, and C. R. Ferguson, "Unburned Gas Temperature in an Internal Combustion Engine: I. CARS Temperature Measurements," Sandia Report SAND86-8749; also, submitted to Comb. Sci. Tech. (1986).

R. P. Lucht and M. A. Maris, "CARS Measurements of Temperature Profiles Near a Wall in an Internal Combustion Engine," submitted to SAE Trans. (1986).

R. P. Lucht, R. E. Palmer, and M. A. Maris, "Simultaneous Acquisition of Pure Rotational and Vibrational Nitrogen Spectra Using Three-Laser CARS," to appear in Opt. Lett. (1987).

T. S. Lundgren and W. T. Ashurst, "Area Varying Waves on Curved Vortex Tubes," submitted to J. Fluid Mech. (1986).

A. E. Lutz, R. J. Kee, and H. A. Dwyer, "Ignition Modeling with Grid Adaption," Dynamics of Reactive Systems Part I: Flames and Configurations, (AIAA, New York, NY, 1986), p. 78.

S. B. Margolis, F. A. Williams, and R. C. Armstrong, "Influences of Two-Phase Flow in the Deflagration of Homogeneous Solids," to appear in Comb. Flame (1987).

P. Marshall, A. Fontijn, and C. F. Melius, "High-Temperature Photochemistry and BAC-MP4 Studies of the Reaction Between Ground-State H Atoms and $\mathrm{N}_{2} \mathrm{O}$," submitted to J. Chem. Phys. (1986).

K. D. Marx, "Air Currents Driven by Water Sprays in Reactor Containment Buildings," Sandia Laboratories NRC Report NUREG/CR-4102 (1986).

A. R. Masri, R. W. Bilger, and R. W. Dibble, "Fluorescence in Laminar and Turbulent Nonpremixed Flames of Methane," submitted to Comb. Flame (1986).

A. R. Masri, R. W. Bilger, and R. W. Dibble, "Turbulent Nonpremixed Flames of Methane Near Extinction: Probability Density Functions," submitted to Comb. Flame (1986).

A. R. Masri, R. W. Dibble, and R. W. Bilger, "Turbulent Nonpremixed Flames of Methane Near Extinction: Mean Structure from Raman Measurements," submitted to Comb. Flame (1986).

K. F. McCarty and R. J. Anderson, "Real-Time Measurements of Deposit Formation From Sodium Sulfate-Seeded Flames," submitted to Comb. Sci. Tech. (1986).

C. F. Melius and J. S. Binkley, "Thermochemistry of the Decomposition of Nitramines in the Gas Phase," Twenty-first Symposium (International) on Combustion (The Combustion Institute, 1986), in press. 
J. A. Miller and C. F. Melius, "A Theoretical Analysis of the Reaction Between Hydroxyl and Hydrogen Cyanide High Temperature," Twenty-first Symposium (International) on Combustion (The Combustion Institute, 1986), in press.

R. E. Mitchell, "Experimentally Determined Overall Burning Rates of Coal Chars," to appear in Comb. Sci. Tech. (1987).

R. E. Mitchell and O. H. Madsen, "Experimentally Determined Overall Burning Rates of Pulverized-Coal Chars in Specified $\mathrm{O}_{2}$ and $\mathrm{CO}_{2}$ Environments," Twenty-first Symposium (International) on Combustion (The Combustion Institute, 1986), in press.

M. Namazian, J. Kelly, R. W. Schefer, S. C. Johnston, and M. B. Long, "Flow and Mixing Structures in Bluff-Body Stabilized Gas Flames," to appear in Proceedings of the International Gas Research Conference (1987).

M. Namazian, R. L. Schmitt, and M. B. Long, "Two-Wavelength, Single Laser CH and $\mathrm{CH}_{4}$ Imaging in a Turbulent Diffusion Flame," submitted to Opt. Lett. (1986).

S. Niksa and A. R. Kerstein, "On the Role of Macromolecular Configuration in Rapid Coal Devolatilization," accepted by Fuel (1986).

S. Niksa, A. R. Kerstein, and T. H. Fletcher, "Predicting Devolatilization at Typical Coal Combustion Conditions with the Distributed-Energy Chain model," accepted by Comb. Flame (1986).

D. H. Parker, J. O. Buck, and D. W. Chandler, "Double-Resonance Laser-Ionization Spectroscopy of Molecular Hydrogen in the Region of the Second Dissociation Limit," submitted to J. Phys.

Chem. (1986).

R. A. Perry, "The Kinetics of the Reaction of the NCO Radical with Ethene and Oxygen Over the Temperature Range 295-662 K," Twenty-first Symposium (International) on Combustion (The Combustion Institute, 1986), in press.

N. Peters and R. J. Kee, "The Computation of Stretched Laminar Methane-Air Diffusion Flames Using a Reduced Four-Step Mechanism," accepted by Comb. and Flame (1986).

L. A. Rahn and R. E. Palmer, "Application of High-Resolution Coherent Raman Spectroscopy to Combustion Measurement," Proceedings of the Tenth International Conference on Raman Spectroscopy, edited by W. L. Peticolas and B. Hudson (University of Oregon Press, Eugene, OR, 1986), p. 15-26.

E. A. Rohlfing and D. W. Chandler, "Laser Spectroscopy of Jet-Cooled Chlorinated Aromatic Hydrocarbons," Advances in Laser Science-II, edited by M. Lapp, W. C. Stwalley, and G. A. Kenney-Wallace, (AIP, New York, NY, 1986), in press.

C. M. Rohlfing and P. J. Hay, "The Structure and Bonding of $\mathrm{F}_{2} \mathrm{O}_{2}$," submitted to J. Chem. Phys. (1986).

J. T. Salmon, R. P. Lucht, D. W. Sweeney, and N. M. Laurendeau, "Laser-Saturated Fluorescence Measurements of $\mathrm{NH}$ in a Premixed Subatmospheric $\mathrm{CH}_{4} / \mathrm{N}_{2} \mathrm{O} /$ Ar Flame," Twenty-first Symposium (International) on Combustion (The Combustion Institute, 1986), in press. 
R. W. Schefer, V. Hartmann, and R. W. Dibble, "Conditional Sampling of Velocity in a Turbulent Nonpremixed Propane Jet," submitted to AIAA J. (1986).

P. J. Smith and T. H. Fletcher, "A Study of Two Chemical Reaction Models in Turbulent Coal Combustion," submitted to Comb. Sci. Tech. (1986).

T. A. Spiglanin and D. W. Chandler, "Rotational Excitation of $\mathrm{NH}\left(\mathrm{a}^{1} \Delta\right)$ From Photodissociation of HNCO," submitted to J. Chem. Phys. (1986).

T. A. Spiglanin, D. W. Chandler, and D. H. Parker, "Mass-Resolved Laser Ionization Spectroscopy of HCl," submitted to Chem. Phys. Lett. (1986).

T. A. Spiglanin, R. A. Perry, and D. W. Chandler, "Internal State Distribution of CO from HNCO Photolysis," submitted to J. Chem. Phys. (1986).

R. R. Steeper, P. A. Jensen, and D. Dunn-Rankin, "Using Mie-Scattering for Measuring Size Changes of Individual Combusting Particles," submitted to J. Physics E: Sci. Instru. (1986); also, presented at Spring Meeting, Western States Section, (The Combustion Institute, 1986).

L. R. Thorne, M. C. Branch, D. W. Chandler, R. J. Kee, and J. A. Miller, "Nitric Oxide-Hydrocarbon Interactions in Low-Pressure Flames," Twenty-first Symposium (International) on Combustion (The Combustion Institute, 1986), in press.

R. (F. P.) Trebino and R. L. Farrow, "CARS Measurements of Temperature Profiles and Pressure in a Tungsten Lamp," Proceedings of the Tenth International Conference on Raman Spectroscopy, edited by W. L. Peticolas and B. Hudson (University of Oregon Press, Eugene, OR, 1986), p. 15-12.

R. (F. P.) Trebino, "Collision-Induced Resonances in Four-Wave-Mixing and Induced-Grating Processes," Advances in Laser Science-II, edited by M. Lapp, W. C. Stwalley, and G. A. KenneyWallace, (AIP, New York, NY, 1986), in press.

P. O. Witze, and T. A. Baritaud, "Particle Seeding for Mie-Scattering Measurements in Combusting Flows," to appear in Laser Anemometry in Fluid Mechanics-III (Ladoan, Lisbon, Portugal, 1987), in press.

P. O. Witze and J. K. Martin, "Cyclic-Variation Bias in Spark-Ignition Engine Turbulence Measurements," Laser Anemometry in Fluid Mechanics-II, edited by R. J. Adrian et al (Ladoan, Lisbon, Portugal, 1986), p. 419.

\section{Selected Presentations}

G. S. Agarwal and R. L. Farrow, "Theoretical Modeling of Two-Color CARS Spectra Measured with a Frequency-Doubled, Multimode Pump Laser," 1986 International Laser Science Conference, Seattle, WA, October 20-24, 1986.

R. J. Anderson, J. C. Farmer, and G. W. Foltz, "Detection of Monolayer Adsorbates Using Surface Second Harmonic Generation." 169th Meeting of the Electrochemical Society, Boston, MA, May 4-9, 1986. 
R. J. Anderson, J. C. Farmer, G. W. Foltz, and J. C. Hamilton, "Surface Second Harmonic Studies of Monolayer Adsorbates at Metal Surfaces," Invited paper FB4, Joint Meeting of the Division of Atomic, Molecular, and Optical Physics and the Division of Chemical Physics, Eugene, OR, June 18-20, 1986.

R. J. Anderson and J. C. Hamilton, "Optical Studies of High Temperature Surface Reactions in Ultrahigh Vacuum," Invited Paper, International Laser Science Conference, Seattle, WA, October 20-24, 1986.

R. L. Axelbaum, C. K. Law, and W. L. Flower, "Effects of Diffusional Stratification and Oxygen Addition on Soot Formation in a Counterflow Diffusion Flame," Spring Technical Meeting of the Western States and Canadian Sections, The Combustion Institute (1986), Paper No. 86-67.

T. A. Baritaud and R. M. Green, "A 2-D Flame Visualization Technique Applied to the I.C. Engine," SAE International Congress and Exposition, Paper No. 860025, Detroit, MI, February 1986.

J. E. Dec, "The Effect of Fuel Burn Rate on Pulse Combustor Tail Pipe Velocities," International Gas Research Conference, Toronto, Canada, September 1986.

R. L. Farrow, "Species Concentration Measurements with Coherent Anti-Stokes Raman Spectroscopy," Invited Paper, 37th Pittsburgh Conference and Exposition on Analytical Chemistry and Applied Spectroscopy, Atlantic City, NJ, March 10-14,1986.

R. L. Farrow, "Accurate Measurements of Combustion Species Concentrations Using CARS," AGARD (NATO) PEP 67th Symposium on Advanced Instrumentation for Aero Engine Components, Philadelphia, PA, May 19-23, 1986.

R. L. Farrow and R. P. Lucht, "High-Resolution Coherent Anti-Stokes Spectroscopy Measurements for Combustion Diagnostic Development," CLEO/IQEC '86, San Francisco, CA, June 913, 1986.

R. L. Farrow, "High-Resolution Coherent Anti-Stokes Raman Spectroscopy Measurements of Carbon Monoxide Linewidths in a Flame," Twenty-first Symposium (International) on Combustion, Munich, West Germany, August 3-8, 1986.

R. L. Farrow and R. P. Lucht, "High-Resolution CARS for Combustion Diagnostics," Tenth International Conference on Raman Spectroscopy, Eugene, OR, August 31 - September 5, 1986.

R. L. Farrow, "Effects of Fluctuating Laser Fields and Saturation on CARS Spectra," 1986 International Laser Science Conference, Seattle, WA, October 20-24, 1986.

C. R. Ferguson, R. M. Green, and R. P. Lucht, "Unburned Gas Temperatures in an Internal Combustion Engine: II. Heat Release Computations," Spring Meeting of the Western States Section, The Combustion Institute, Banff, Alberta, Canada, April 27-30, 1986.

C. R. Ferguson and R. P. Lucht, "Unburned Gas Temperatures in an Internal Combustion Engine: Thermodynamic Modeling and CARS Measurements," Fourteenth DOE/Diesel Cooperative Program Meeting, Livermore, CA, May 5, 1986. 
J. E. M. Goldsmith, "Multiphoton Excitation Techniques for Combustion Diagnostics," Invited Paper, Gordon Research Conference on Multiphoton Processes, New London, NH, June 9-13, 1986.

J. E. M. Goldsmith, "Photochemical Effects in Laser-Based Optical Detection of Radicals in Flames," Invited talk, National Bureau of Standards, Washington, DC, October 9, 1986.

J. E. M. Goldsmith and L. A. Rahn, "Doppler-Free Two-Photon-Excited Fluorescence Spectroscopy of OH in Flames," 1986 International Laser Science Conference, Seattle, WA, October 20-24, 1986.

J. F. Grcar, R. J. Kee, M. D. Smooke, and J. A. Miller, "A Hybrid Newton/Time-Integration Procedure for the Solution of Steady, Laminar, One-Dimensional, Premixed Flames," Twentyfirst Symposium (International) on Combustion, Munich, West Germany, August 3-8, 1986.

J. C. Hamilton and R. J. Anderson, "Reaction Monitoring on Metal and Ceramic Surfaces Using Laser-Light Scattering," Invited Paper, SPIE 1986 Technical Symposium, Southeast, on Optics and Optoelectronics, Orlando, FL, March 31-April 4, 1986.

J. C. Hamilton, "In Situ Spectroscopy of Pyrite Oxidation and Resulfidation," Electrochemical Society Spring Meeting, Boston, MA, May 4-9, 1986.

J. C. Hamilton, R. J. Anderson, and G. W. Foltz, "Optical Second-Harmonic Generation from Surface Segregants and Adsorbed Oxygen on an Fe-18Cr-3Mo (100) Surface," Invited talk, Material Research Society Meeting, Boston, MA, December 1-6, 1986.

S. J. Harris, A. M. Weiner, R. J. Blint, and J. E. M. Goldsmith, "Concentration Profiles in Rich and Sooting Ethylene Flames," Spring Meeting of the Western States Section, The Combustion Institute, Banff, Alberta, Canada, April 27-30, 1986.

S. J. Harris, A. M. Weiner, R. J. Blint, and J. E. M. Goldsmith, "Concentration Profiles in Rich and Sooting Ethylene Flames," Twenty-First Symposium (International) on Combustion, Munich, West Germany, August 3-8, 1986.

A. M. F. Lau, "Abnormal Peak Asymmetry in AC-Stark Doublets due to Pressure Broadening," CLEO/IQEC '86, San Francisco, CA, June 9-13, 1986.

N. M. Laurendeau and J. E. M. Goldsmith, "Comparison of Laser-Induced Fluorescence Methods for Measurement of Hydroxyl Concentration in Flames," Spring Meeting of the Western States Section, The Combustion Institute, Banff, Alberta, Canada, April 27-30, 1986.

R. P. Lucht, R. M. Green, R. E. Palmer, and C. R. Ferguson, "CARS Temperature Measurements in the Unburned Gas Region of an Internal Combustion Engine," CLEO/IQEC '86, San Francisco, CA, June 9-13, 1986.

R. P. Lucht, R. M. Green, R. E. Palmer, R. E. Teets, and C. R. Ferguson, "Unburned Gas Temperatures in an Internal Combustion Engine: I. CARS Temperature Measurements," Spring Meeting of the Western States Section, The Combustion Institute, Banff, Alberta, Canada, April 27$30,1986$. 
R. P. Lucht and R. L. Farrow, "Saturation of CARS Resonances Using Multi-Mode Lasers: Calculation of Saturation Lineshapes and Intensities," 1986 International Laser Science Conference, Seattle, WA, October 20-24, 1986.

R. P. Lucht, "Three-Laser CARS Measurements of Two Species," 1986 International Laser Science Conference, Seattle, WA, October 20-24, 1986.

W. J. McLean, T. T. Bramlette, T. M. Dyer, and J. O. Keller, "New Diagnostic Techniques for In Situ measurements in Furnaces," Aluminum Association Workshop, Washington, DC, April 1986.

J. A. Miller, "Modeling Nitric Oxide Formation in Combustion," Ninth International Symposium on Gas Kinetics, Bordeaux, France, July 20-25, 1986.

J. A. Miller, C. F. Melius, and N. J. Brown, "Theoretical Prediction of Rate Coefficients for Reactions Involving Intermediate Complex Formation," Ninth International Symposium on Gas Kinetics, Bordeaux, France, July 20-25, 1986.

J. A. Miller and C. F. Melius, "A Theoretical Analysis of the Reaction Between Hydroxyl and Hydrogen Cyanide at High Temperature," Twenty-First Symposium (International) on Combustion, Munich, West Germany, August 3-8, 1986.

R. A. Perry, "Kinetic Measurements of the NCO Radical Reaction with Ethene and Oxygen over an Extended Temperature Range," Ninth International Symposium on Gas Kinetics, Bordeaux, France, July 20-25, 1986.

R. A. Perry, "Kinetics of the Reaction of NCO with Ethene and Oxygen," 191st National Meeting of the American Chemical Society, New York, NY, April 1986.

R. A. Perry, "Kinetics of the Reaction of NCO with Ethene and Oxygen over the Temperature Range 295-662 K," Twenty-First Symposium (International) on Combustion, Munich, West Germany, August 3-8, 1986.

L. A. Rahn, " $\mathrm{N}_{2}$ Raman Linewidths in an $\mathrm{H}_{2}-\mathrm{O}_{2}-\mathrm{N}_{2}$ Flame by Inverse Raman Spectroscopy," CLEO/IQEC '86, San Francisco, CA, June 9-13, 1986.

L. A. Rahn and R. E. Palmer, "Application of High-Resolution Coherent Raman Spectroscopy to Combustion Measurements," Invited Paper, Tenth International Conference on Raman Spectroscopy, Eugene, OR, August 31-September 5, 1986.

E. A. Rohlfing and D. W. Chandler, "Laser Spectroscopy of Jet-Cooled Chlorinated Aromatic Hydrocarbons," Second International Laser Science Conference, Seattle, WA, October 20-24, 1986.

T. A. Spiglanin, R. A. Perry, and D. W. Chandler, "Photodissociation Dynamics of HNCO," Seventeenth Conference on Photochemistry, Boulder, CO, June 22-26, 1986.

T. A. Spiglanin, R. A. Perry, and D. W. Chandler, "Photodissociation Dynamics of HNCO," Ninth International Symposium on Gas Kinetics, Bordeaux, France, July 20-25, 1986. 
T. A. Spiglanin and D. W. Chandler, "Rotational Energy Distributions of CO Produced by Photofragmentation of HNCO," Dynamics of Molecular Photofragmentation (Faraday Discussion No. 82), Bristol, Great Britain, September 15-17, 1986.

T. A. Spiglanin, R. A. Perry, and D. W. Chandler, "Dynamics and Energetics of HNCO Photodissociation," 192nd National Meeting of the American Chemical Society, Anaheim, CA, September 1986 .

L. R. Thorne, M. C. Branch, D. W. Chandler, R. J. Kee, and J. A. Miller, "Hydrocarbon/Nitric Oxide Interactions in Low-Pressure Flames," Twenty-first Symposium (International) on Combustion, Munich, West Germany, August 3-8, 1986.

L. R. Thorne and O. I. Smith, "The Structure of Cyanogen-Nitrogen Dioxide Premixed Flames," Fall Meeting of the Western States Section, The Combustion Institute, 1986.

D. A. Tichenor, K. R. Hencken, and R. W. Bickes, "Sapphire Fiber Optic Probe," Advisory Group for Aerospace Research and Development Conference, May 1986.

R. (F. P.) Trebino and R. L. Farrow, "CARS Measurements of Temperature Profiles and Pressure in a Tungsten Lamp," Tenth International Conference on Raman Spectroscopy, Eugene, OR, August 31-September 5, 1986.

R. (F. P.) Trebino and L. A. Rahn, "Observation of Long-Lived Collision-Induced Coherences and Ground-State-Spin Gratings in a Flame," 1986 International Laser Science Conference, Seattle, WA, October 20-24, 1986.

F. P. Tully and A. T. Droege, "Kinetic and Mechanistic Studies of Hydrocarbon Oxidation," Seventeenth Informal Conference on Photochemistry, Boulder, CO, June 1986.

F. P. Tully, A. T. Droege, and J. E. M. Goldsmith, "Hydroxyl-Radical Reactions with Unsaturated Hydrocarbons: Effects of Deuterium Substitution," Ninth International Symposium on Gas Kinetics, Bordeaux, France, July 1986.

C. K. Westbrook and J. O. Keller, "Chemical Kinetic Analysis of Pulse Combustors," International Gas Research Conference, Toronto, Canada, September 1986.

P. O. Witze and J. M. C. Mendes-Lopes, "Direct Measurement of the Turbulent Burning Velocity in a Homogeneous-Charge Engine," SAE Fuels and Lubricants Meeting, Paper No. 861531, Philadelphia, PA, October 1986.

P. O. Witze and T. A. Baritaud, "Particle Seeding for Mie-Scattering Measurements in Combusting Flows," Third International Symposium on Applications of Laser Anemometry to Fluid Mechanics, Lisbon, Portugal, July 1986. 
Issued by Sandia National Laboratories, operated for the United States Department of Energy by Sandia

\section{Corporation.}

NOTICE: This report was prepared as an account of work sponsored by an agency of the United States Government. Neither the United States Government nor any agency thereof, nor any of their employees, nor any of the contractors, subcontractors, or their employees, makes any warranty, express or implied, or assumes any legal liability or responsibility for the accuracy, completeness, or usefulness of any information, apparatus, product, or process disclosed, or represents that its use would not infringe privately owned rights. Reference herein to any specific commercial product, process, or service by trade name, trademark, manufacturer, or otherwise, does not necessarily constitute or imply its endorsement, recommendation, or favoring by the United States Government, any agency thereof or any of their contractors or subcontractors. The views and opinions expressed herein do not necessarily state or reflect those of the United States Government, any agency thereof or any of their contractors or subcontractors. 